\title{
Cau'r bwlch rhwng polisi ac arfer mewn perthynas â Chymraeg Ail laith: Astudiaeth ar sut orau y gellir cefnogi cyflwyno'r Gymraeg fel ail iaith yn Ilwyddiannus yng nghyd-destun ysgolion uwchradd cyfrwng Saesneg
}

\author{
Lovell, Alexander E.
}

How to cite:

Lovell, Alexander E. (2018) Cau'r bwlch rhwng polisi ac arfer mewn perthynas â Chymraeg Ail laith: Astudiaeth ar sut orau y gellir cefnogi cyflwyno'r Gymraeg fel ail iaith yn Ilwyddiannus yng nghyd-destun ysgolion uwchradd cyfrwng Saesneg. Doctoral thesis, Swansea University.

http://cronfa.swan.ac.uk/Record/cronfa50742

Use policy:

This item is brought to you by Swansea University. Any person downloading material is agreeing to abide by the terms of the repository licence: copies of full text items may be used or reproduced in any format or medium, without prior permission for personal research or study, educational or non-commercial purposes only. The copyright for any work remains with the original author unless otherwise specified. The full-text must not be sold in any format or medium without the formal permission of the copyright holder. Permission for multiple reproductions should be obtained from the original author.

Authors are personally responsible for adhering to copyright and publisher restrictions when uploading content to the repository.

Please link to the metadata record in the Swansea University repository, Cronfa (link given in the citation reference above.) 


\section{Cau'r bwlch rhwng polisi ac arfer mewn perthynas â Chymraeg Ail Iaith:}

Astudiaeth ar sut orau y gellir cefnogi cyflwyno'r Gymraeg fel ail iaith yn llwyddiannus yng nghyd-destun ysgolion uwchradd cyfrwng Saesneg.

Alexander E. Lovell

Cyflwynwyd i Brifysgol Abertawe er mwyn diwallu gofynion y Radd Doethur mewn Athroniaeth

Prifysgol Abertawe

2018 


\section{Crynodeb}

Ymddengys yn y byd addysg yng Nghymru fod consensws nad yw addysgu na dysgu Cymraeg fel ail iaith yn effeithiol yn gyffredinol (W. G. Lewis, 2010b, para. 1). Mae hyn wedi cael ei gefnogi mewn adroddiadau blynyddol diweddar gan Estyn a ganfu ostyngiad graddol yn safonau Cymraeg Ail Iaith. Mae adolygiad Davies (2013, t. 1) o Gymraeg Ail Iaith yng Nghyfnodau Allweddol 3 a 4, Un Iaith $i$ Bawb, wedi atgyfnerthu'r consensws hwn, gan nodi mai Cymraeg Ail Iaith yw'r pwnc lle ceir y lefelau cyrhaeddiad isaf. Pwysleisiodd Davies (2013, t. 1) fod angen newid sylfaenol, a hynny ar frys, am ei bod yn "unfed awr ar ddeg ar Gymraeg ail iaith." Er bod nifer o bolisiau iaith ac addysg wedi' $u$ gweithredu gan Lywodraeth Cymru o fewn y pedwar degawd diwethaf, ac er bod achosion unigol o ddysgu Cymraeg yn rhagorol mewn ysgolion uwchradd Saesneg (Estyn, 2014b, t. 44), ymddengys nad yw darpariaeth y gyfundrefn bresennol ar gyfer addysgu Cymraeg fel ail iaith yn ddigonol o hyd i gynhyrchu siaradwyr sydd yn medru defnyddio'r Gymraeg y tu hwnt i'r dosbarth dysgu.

O gofio nod uchelgeisiol Llywodraeth Cymru (2017b) i gyrraedd miliwn o siaradwyr erbyn 2050, mae'n amlwg bellach nad yw darpariaeth bresennol Cymraeg Ail Iaith yn ddigonol i gynyddu nifer y bobl sy'n defnyddio'r Gymraeg. Ymddengys felly fod angen ystyried ffyrdd newydd o gefnogi cyflwyno'r Gymraeg fel ail iaith yn llwyddiannus mewn ysgolion cyfrwng Saesneg, os ydym i wireddu gweledigaeth uchelgeisiol Llywodraeth Cymru. Wrth ystyried y gagendor rhwng polisi ac arfer, cyflwynir yr achos dros newid pellgyrhaeddol yng nghyfundrefn Cymraeg Ail Iaith, er mwyn cefnogi'r brif ddadl fod angen canolbwyntio o'r newydd ar ddysgu cynnwys ac iaith mewn ffordd integredig maes o law mewn ysgolion cyfrwng Saesneg yng Nghymru. 


\section{Datganiadau}

\section{CYHOEDDIAD}

Nid yw'r gwaith hwn wedi'i dderbyn o'r blaen yn gyffredinol ar gyfer unrhyw radd ac nid yw'n cael ei gyflwyno ar yr un pryd mewn ymgeisyddiaeth ar gyfer unrhyw radd.

Llofnod (ymgeisydd)

Dyddiad

\section{DATGANIAD 1}

Canlyniad fy ymchwiliadau fy hun yw'r traethawd ymchwil hwn, ac eithrio lle nodir yn wahanol. Cydnabyddir ffynonellau eraill drwy droednodiadau sy'n rhoi cyfeiriadau amlwg. Atodir llyfryddiaeth.

Llofnod (ymgeisydd)

Dyddiad

\section{DATGANIAD 2}

Trwy hyn, rhoddaf ganiatâd i'm traethawd ymchwil, os caiff ei dderbyn, fod ar gael i’w lungopïo ac i'w fenthyca rhwng llyfrgelloedd, ac i'r teitl a'r crynodeb fod ar gael i sefydliadau allanol.

Llofnod (ymgeisydd)

Dyddiad 


\section{Cynnwys}

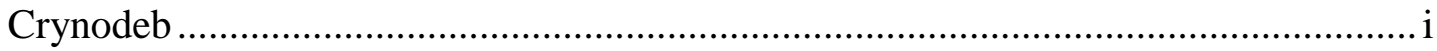

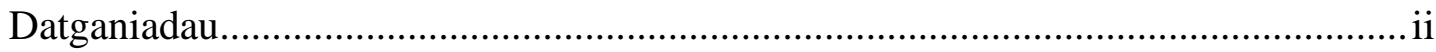

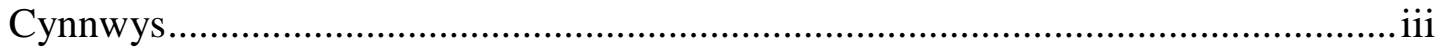

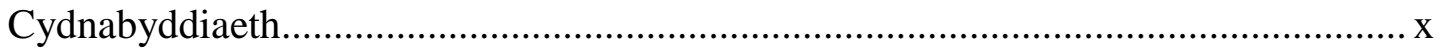

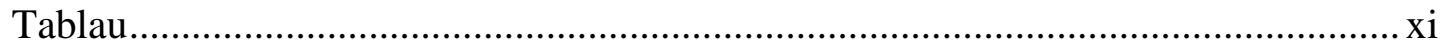

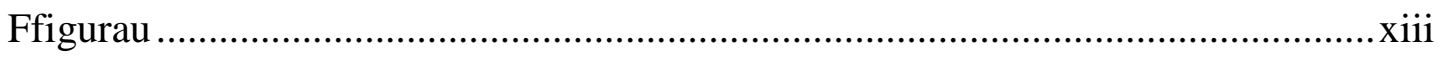

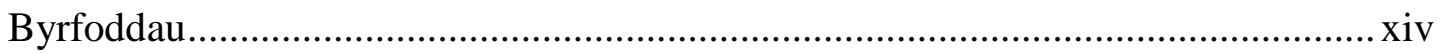

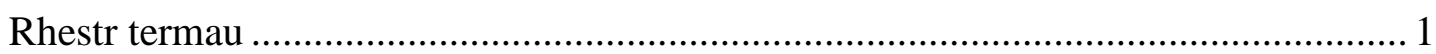

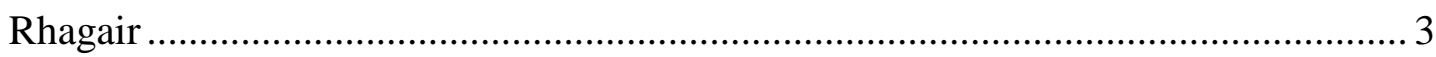

Pennod 1: Polisi ac arfer o ran addysgu'r Gymraeg mewn addysg cyfrwng Saesneg. 5

1.1. Olrhain hanes y deddfau iaith arwyddocaol yng Nghymru ........................... 5

1.1.1. Deddf yr Iaith Gymraeg 1967 ..................................................................... 5

1.1.2. Deddf yr Iaith Gymraeg 1993 .............................................................. 7

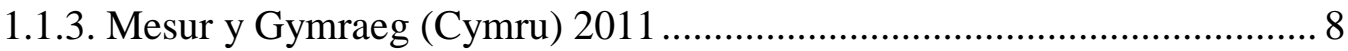

1.2. Y datblygiadau diweddar ym mholisi addysg Cymru .................................. 8

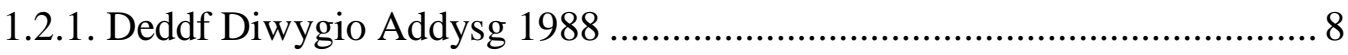

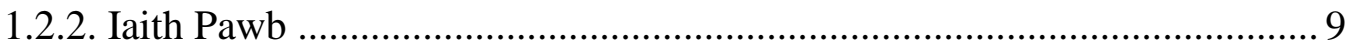

1.2.3. Dysgu ac addysgu Cymraeg fel ail iaith (2010) .................................... 12

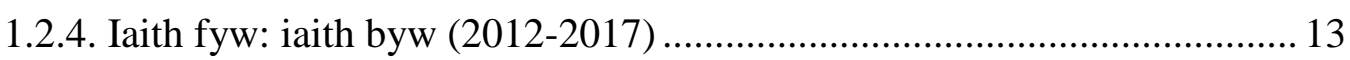

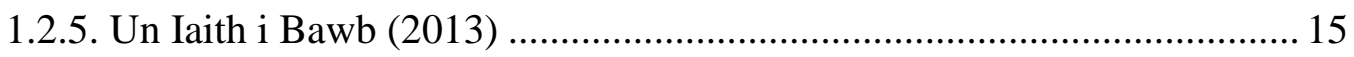

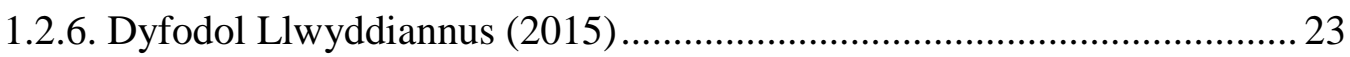

1.2.7. Cymraeg 2050: Miliwn o siaradwyr (2017) ............................................ 25

1.3. Y canfyddiadau diweddar parthed arfer ym maes Cymraeg Ail Iaith ............26

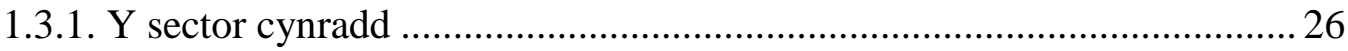

1.3.2. Y cyfnod pontio: Dilyniant rhwng y sectorau cynradd ac uwchradd....... 31

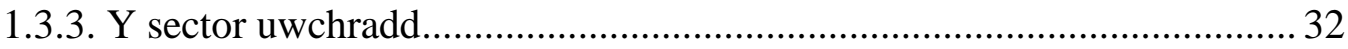


1.4. Casgliadau ar bolisi ac arfer o ran addysgu'r Gymraeg fel ail iaith .35

Pennod 2: Diffinio'r termau priodol ac esbonio eu harwyddocâd ...............................36

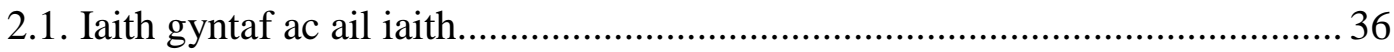

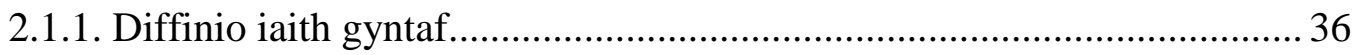

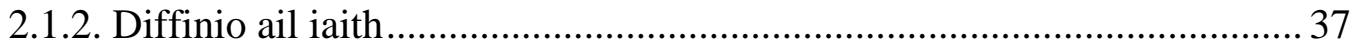

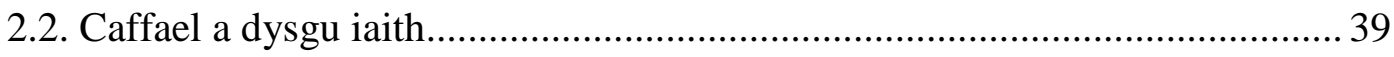

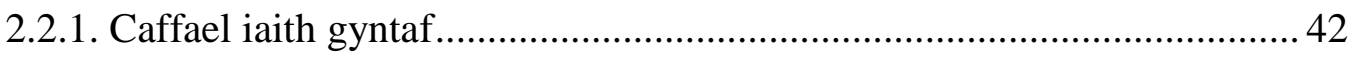

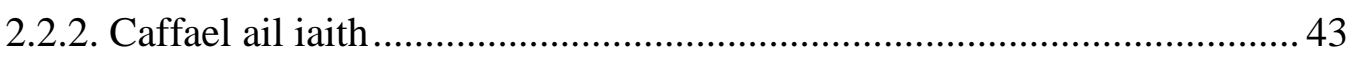

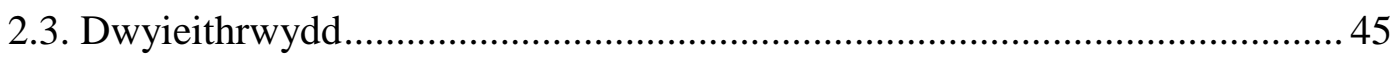

2.3.1. Disgrifio dwyieithrwydd yn hytrach na'i ddiffinio ................................. 46

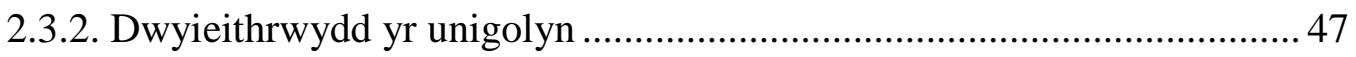

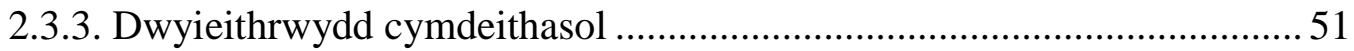

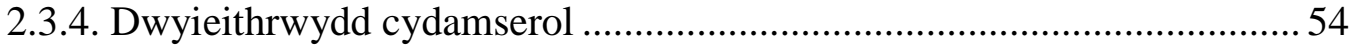

2.3.5. Dwyieithrwydd cynyddol a dwyieithrwydd gostyngol ............................55

2.4. Dwyieithrwydd yng nghyd-destun yr ymchwil ...........................................56

2.5. Addysg ddwyieithog yng nghyd-destun yr ymchwil .................................. 56

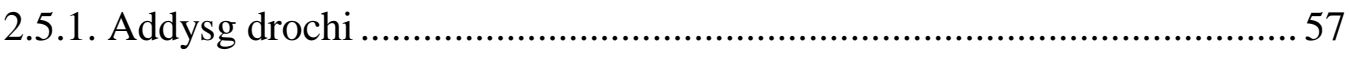

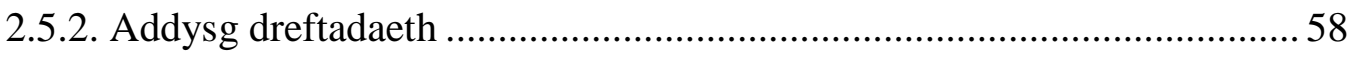

2.5.3. Addysg iaith dramor fodern ac addysg ail iaith ................................... 59

2.5.4. Content and Language Integrated Learning ......................................... 60

2.6. Addysg cyfrwng Cymraeg, addysg ddwyieithog a Chymraeg Ail Iaith ......... 61

2.6.1. Diffinio ysgolion yn ôl eu darpariaeth cyfrwng Cymraeg ........................ 61

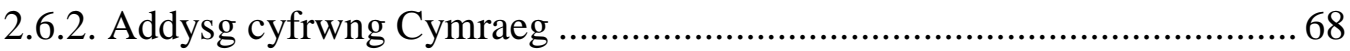

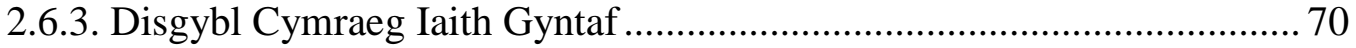

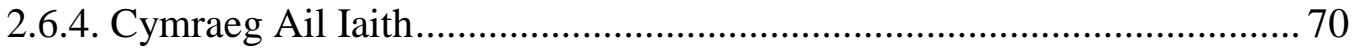

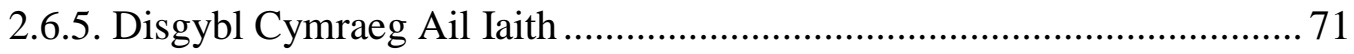

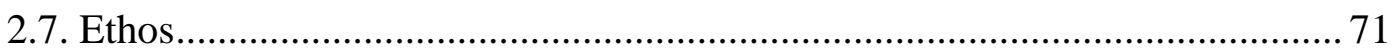




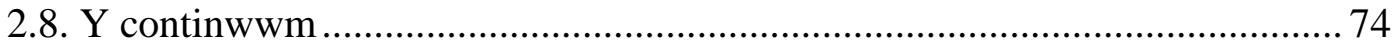

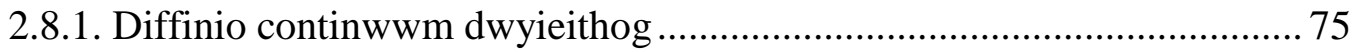

2.8.2. Diffinio'r continwwm Cymraeg a'r continwwm ieithyddol ................... 78

Pennod 3: Manteision posibl dwyieithrwydd.................................................... 81

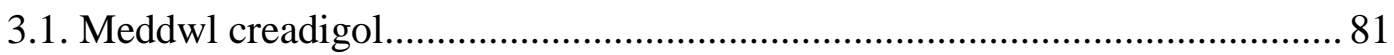

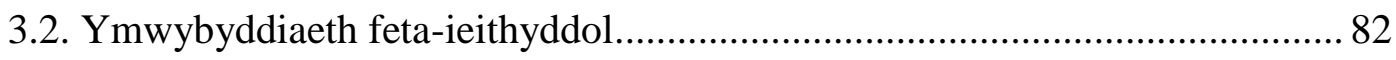

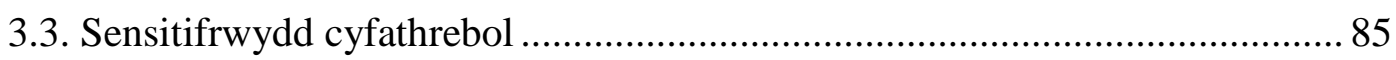

3.4. Dwyieithrwydd megis sylfaen i ddysgu ieithoedd eraill .............................. 86

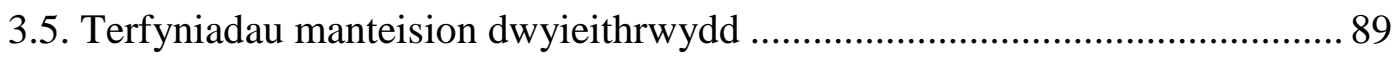

Pennod 4: Y gymhariaeth ryngwladol: Tystiolaeth o Ganada, Gwlad y Basg a'r

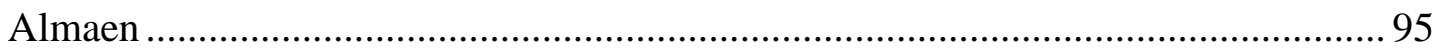

4.1. Trosolwg o addysg drochi a CLIL ar lwyfan rhyngwladol.......................... 95

4.2. Sefyllfa'r Ffrangeg yn y gyfundrefn addysg yng Nghanada.........................96

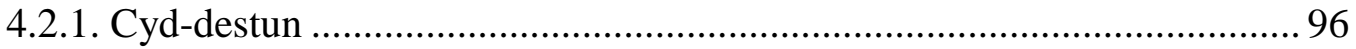

4.2.2. Canfyddiadau addysg drochi yng Nghanada......................................... 99

4.3. Sefyllfa'r Fasgeg yng Ngwlad y Basg ..................................................... 103

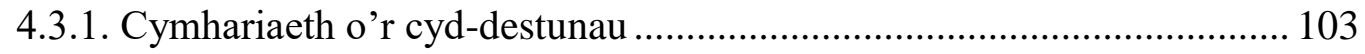

4.3.2. Sefyllfa'r Fasgeg yn y gyfundrefn addysg yng Ngwlad y Basg ............ 105

4.3.3. Sefyllfa addysg ddwyieithog Gwlad y Basg ....................................... 105

4.3.4. Canfyddiadau ar addysg drochi yng Ngwlad y Basg .......................... 107

4.3.5. Casgliadau ar addysg drochi yng Nghanada a Gwlad y Basg................ 111

4.4. Sefyllfa'r Saesneg mewn addysg ddwyieithog yn yr Almaen ...................... 113

4.4.1. Rhesymeg o ran canolbwyntio ar CLIL yn yr Almaen ......................... 115

4.4.2. Diffinio addysg drochi a CLIL yn yr Almaen..................................... 115

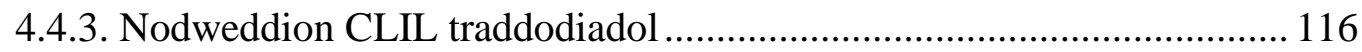

4.4.4. Canfyddiadau parthed CLIL traddodiadol .......................................... 118 
4.4.5. Casgliadau ar CLIL yn yr Almaen a'r tu hwnt

Pennod 5: Y gymhariaeth genedlaethol: Cynlluniau trochi a dysgu dwys yng Nghymru 125

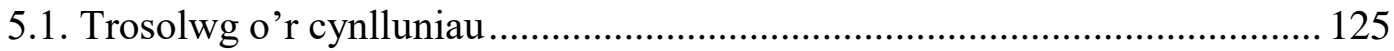

5.2. Cynllun Trochi Hwyr Ysgol Maes Garmon (1994-1999) ............................. 127

5.2.1. Cefndir y Cynllun Trochi Hwyr ....................................................... 127

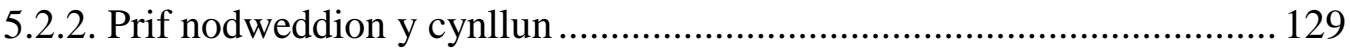

5.2.3. Gwerthusiad o'r cynllun trochi hwyr ................................................. 131

5.3. Cynllun Peilot Trochi a Dysgu Dwys Bwrdd yr Iaith Gymraeg (2003 -2008)

5.3.1. Cyd-destun y Cynllun Trochi Canolig

5.3.2. Gwerthusiad cyntaf Cynllun Peilot Bwrdd yr Iaith Gymraeg (2003 2006)

5.3.3. Ail werthusiad Cynllun Peilot Bwrdd yr Iaith Gymraeg (2007 - 2008) 136

5.4. Cynllun Ysgol X: Y Cwrs Carlam ............................................................ 138

5.4.1. Cyd-destun y Cwrs Carlam ................................................................ 138

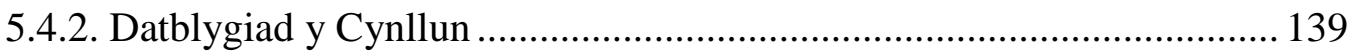

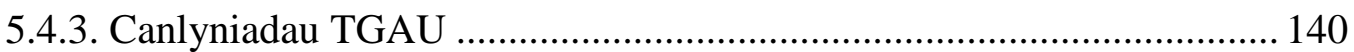

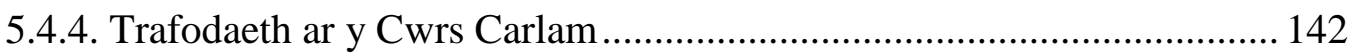

5.5. Casgliadau ar y cynlluniau trochi a dysgu dwys ....................................... 145

Pennod 6: Cynllun methodolegol yr ymchwil ....................................................... 146

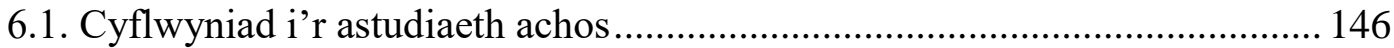

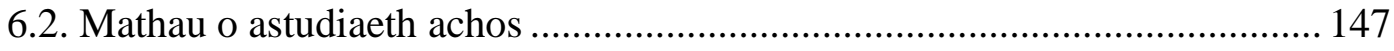

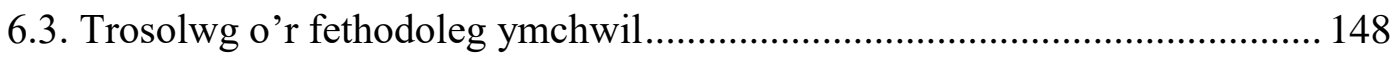

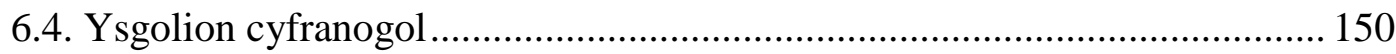

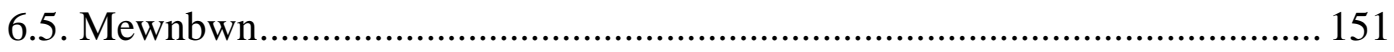

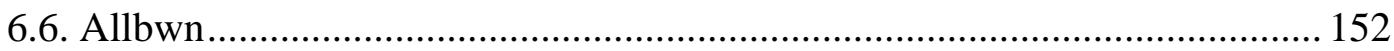

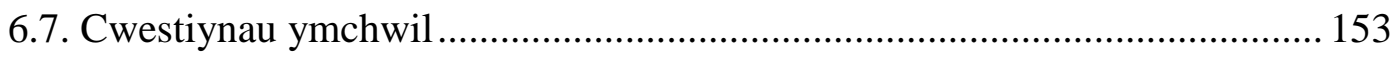


6.8. Dulliau dadansoddi. 153

6.9. Dulliau ymchwil 154

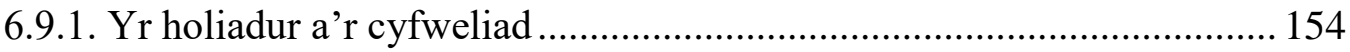

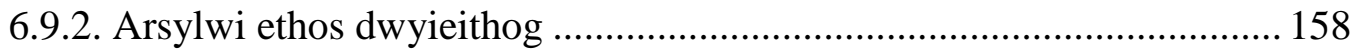

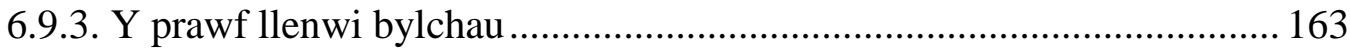

6.9.4. Sgoriau darllen Saesneg ................................................................... 174

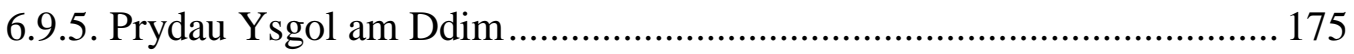

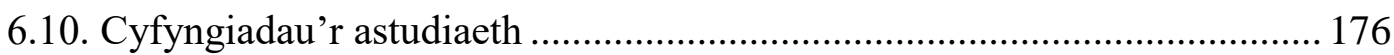

Pennod 7: Canlyniadau i: Yr holiadur, y cyfweliadau a'r arsylwadau ar ethos ....... 178

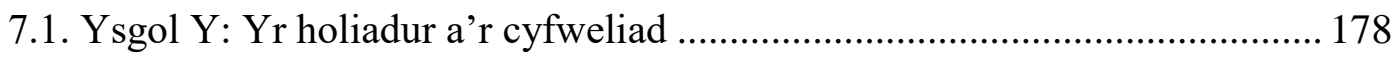

7.1.1. Gwybodaeth sosio-economaidd a sosio-ieithyddol.............................. 178

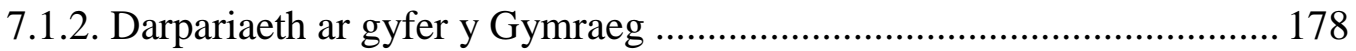

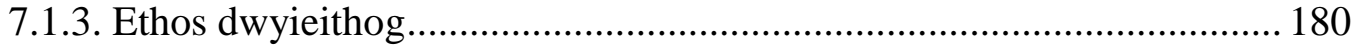

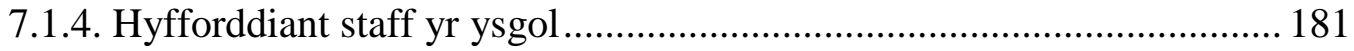

7.1.5. Pontio rhwng y sector cynradd a'r sector uwchradd ............................ 183

7.2. Ysgol X: Yr holiadur a'r cyfweliad .......................................................... 184

7.2.1. Gwybodaeth sosio-economaidd a sosio-ieithyddol............................. 184

7.2.2. Darpariaeth ar gyfer y Gymraeg ..................................................... 184

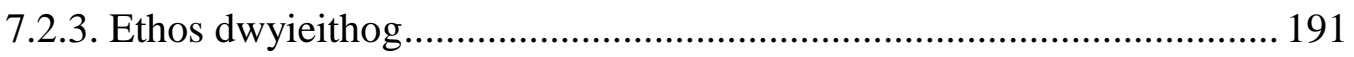

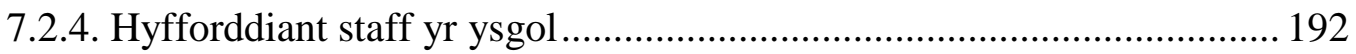

7.2.5. Pontio rhwng y sector cynradd a'r sector uwchradd ............................ 193

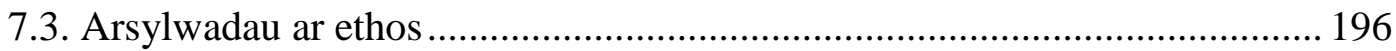

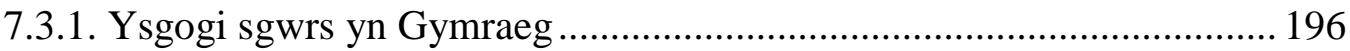

7.3.2. Casglu dogfennaeth a theithio o amgylch yr ysgolion .......................... 196

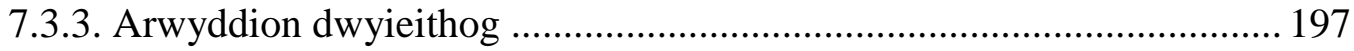

7.3.4. Galwadau ffôn i'r ysgolion .................................................................. 199

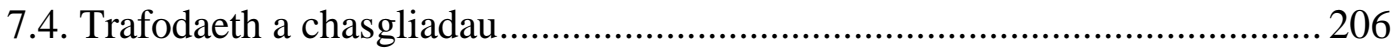


7.4.2. Ethos dwyieithog

7.4.3. Hyfforddiant staff yr ysgolion.

7.4.4. Pontio rhwng y sector cynradd a'r sector uwchradd

Pennod 8: Canlyniadau ii: Y prawf llenwi bylchau, y prawf darllen Saesneg a'r data

Prydau Ysgol am Ddim

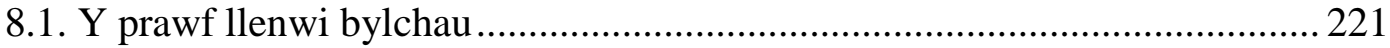

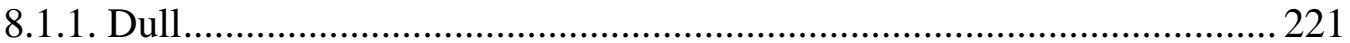

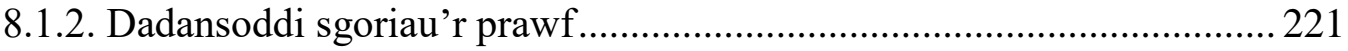

8.1.3. Dadansoddi dibynadwyedd a dilysrwydd y prawf ................................ 241

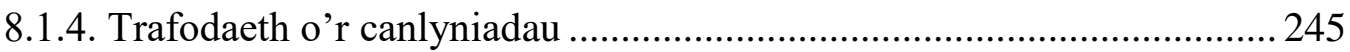

8.1.5. Casgliadau ar y prawf llenwi bylchau fel mesurydd hyfedredd ieithyddol cyffredinol yn y Gymraeg ......................................................................... 246

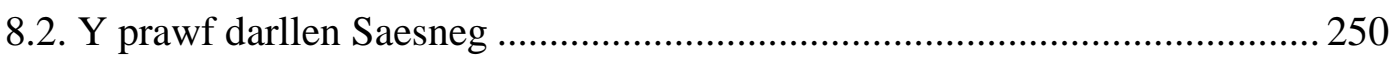

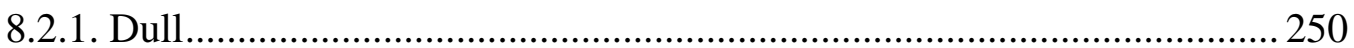

8.2.2. Dadansoddi sgoriau darllen Saesneg.................................................... 251

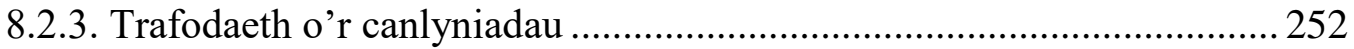

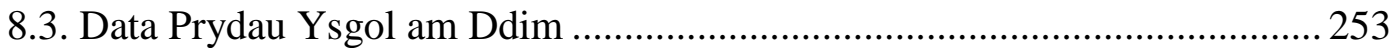

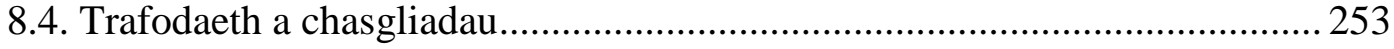

8.4.1. Proffiliau ieithyddol y disgyblion cyfranogol ...................................... 254

8.4.2. Proffiliau sosio-economaidd y disgyblion cyfranogol .......................... 255

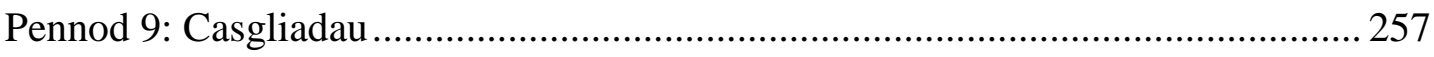

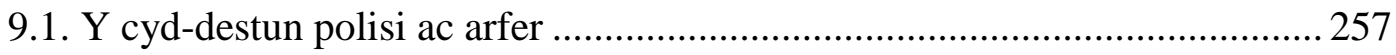

9.2. Yr ymchwil ryngwladol: Addysg drochi a CLIL ........................................ 257

9.3. Yr ymchwil genedlaethol: Cynllun Ysgol X............................................. 259

9.4. Prif ganfyddiadau'r ymchwil empirig a'u cyfraniad at y llenyddiaeth ......... 259

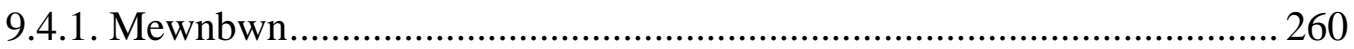

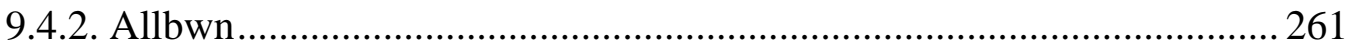




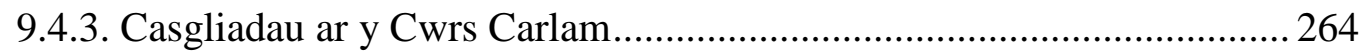

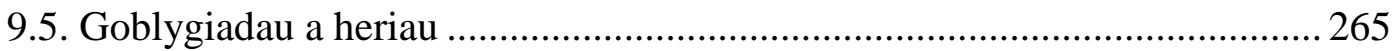

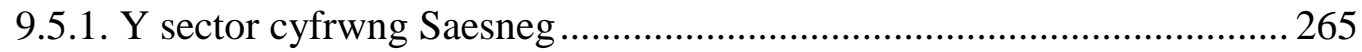

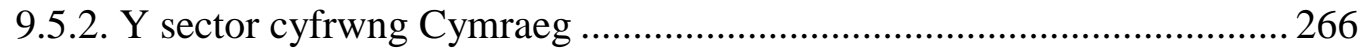

9.5.3. Cwricwlwm i Gymru a'r continwwn Cymraeg..................................... 268

Pennod 10: Argymhellion ........................................................................... 270

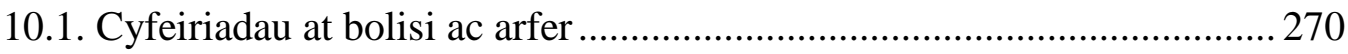

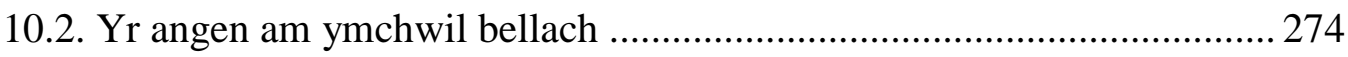

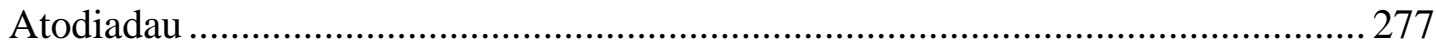




\section{Cydnabyddiaeth}

Yn gyntaf, hoffwn ddiolch yn ddiffuant i Ymddiriedolaeth James Pantyfedwen a'r Ganolfan Hyfforddiant Doethurol ar gyfer Ieithoedd Celtaidd (a ariennir gan yr AHRC) am noddi'r gwaith ymchwil hwn. Heb gymorth ariannol yr ysgoloriaethau hyn, ni fyddai wedi bod yn bosibl gwireddu'r ddoethuriaeth hon.

Heb os, ni allwn fod wedi cynnal y gwaith ymchwil heb gyfraniad yr ysgolion. Hoffwn ddiolch i'r holl ddisgyblion, penaethiaid adran ac ymarferwyr eraill a gymerodd ran yn ymchwil. Diolch hefyd i'r ysgolion am y croeso cynnes a'r gefnogaeth yn ystod y gwaith maes.

I'm goruchwylwyr, Mr. Steve Morris, Yr Athro Wilson Macleod a'r Athro Tudur Hallam, rwyf yn ddiolchgar iawn am eich diddordeb, eich arbenigedd a'ch cefnogaeth barhaus dros y pedair blynedd diwethaf. Diolch yn benodol i Mr. Steve Morris a'm hysbrydoli yn ystod y radd is-raddedig i barhau â'm hastudiaethau ar lefel ôl-raddedig.

Yn olaf, hoffwn ddiolch i'm teulu. Roedd eu cefnogaeth yn ystod y pedair blynedd diwethaf yn ganolog i'r gwaith hwn. Diolch yn benodol i Jade am ei chariad a'i hamynedd, yn enwedig dros yr wyth mlynedd diwethaf. 


\section{Tablau}

Tabl 1. Datblygiad y Cwrs Carlam rhwng 2001 - 2006.................................. 139

Tabl 2. Canlyniadau Cymraeg Ail Iaith Ysgol X 2013-2015........................... 142

Tabl 3. $\quad$ Ysgogi sgwrs yn Gymraeg yn Ysgol Y ac Ysgol X.................................. 196

Tabl 4. Nifer yr arwyddion uniaith Saesneg, dwyieithog ac uniaith Gymraeg a

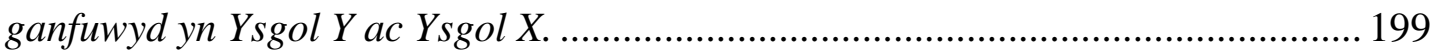

Tabl 5. Galwadau ffôn i Ysgol Y ac Ysgol X pan oeddent ar agor. .................... 200

Tabl 6. Galwadau ffôn i Ysgol Y ac Ysgol X pan oeddent ar gau....................... 200

Tabl 7. $\quad$ Nifer y cyfranogwyr fesul dosbarth ..................................................... 221

Tabl 8. Amrediad a gwasgariad sgoriau cyfanswm y tri dosbarth .................... 223

Tabl 9. $\quad$ Sgoriau cymedrig Rhan A, Rhan B a chyfanswm fesul dosbarth........... 224

Tabl 10. Sgoriau cymedrig y tri dosbarth ar gyfer Geiriau Cynnwys a Geiriau Swyddogaethol.

Tabl 11. Sgoriau cymedrig geiriau cynnwys y tri dosbarth fesul mathau o air... 226

Tabl 12. Sgoriau cymedrig geiriau swyddogaethol y tri dosbarth fesul mathau o air.

Tabl 13. Nifer a chanran y cyfranogwyr a atebodd yr eitemau sy'n cymell enw yn gywir fesul dosbarth

Tabl 14. Nifer a chanran y cyfranogwyr a atebodd yr eitemau sy'n cymell ansoddair yn gywir fesul dosbarth

Tabl 15. Nifer a chanran y cyfranogwyr a atebodd yr eitemau sy'n cymell berf yn gywir fesul dosbarth

Tabl 16. Nifer a chanran y cyfranogwyr a atebodd yr eitemau sy'n cymell berfenw yn gywir fesul dosbarth.....

Tabl 17. Nifer a chanran y cyfranogwyr a atebodd yr eitemau sy'n cymell adferf yn gywir fesul dosbarth.

Tabl 18. Nifer a chanran y cyfranogwyr a atebodd yr eitemau sy'n cymell rhagenw yn gywir fesul dosbarth

Tabl 19. Nifer a chanran y cyfranogwyr a atebodd yr eitemau sy'n cymell arddodiad yn gywir fesul dosbarth 238

Tabl 20. Nifer a chanran y cyfranogwyr a atebodd yr eitemau sy'n cymell cysylltair yn gywir fesul dosbarth 
Tabl 21. Nifer a chanran y cyfranogwyr a atebodd yr eitemau sy'n cymell y fannod yn gywir fesul dosbarth.

Tabl 22. Cydberthyniad Spearman ar gyfer Sgôr Rhan A, Sgôr Rhan B a Sgôr Gyfanswm.

Tabl 23. Dadansoddi cysondeb mewnol yr eitemau a gallu'r eitemau $i$ wahaniaethu

Tabl 24. Nifer a chanran y disgyblion cyfranogol. 251

Tabl 25. Sgoriau darllen Saesneg fesul dosbarth. 252

Tabl 26. Hawl i Brydau Ysgol am Ddim fesul dosbarth. 253

Tabl 27. Trosolwg o broffiliau ieithyddol a sosio-economaidd y disgyblion cyfranogol fesul dosbarth 


\section{Ffigurau}

Ffigur 1. Continwwm dwyieithog Valdés (2003) ............................................... 76

Ffigur 2. Continwwm dwyieithog Grosjean $(2010$, t. 40) ..................................... 78

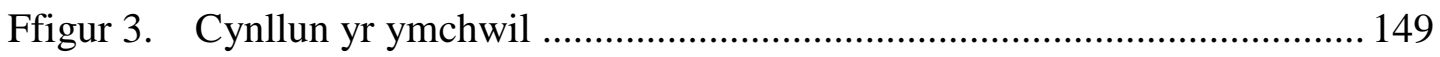

Ffigur 4. Strwythur yr astudiaeth ............................................................. 150 


\section{Byrfoddau}

\begin{tabular}{|c|c|}
\hline AAA & Anghenion Addysgol Arbennig \\
\hline $\mathrm{ABaCH}$ & Addysg Bersonol a Chymdeithasol \\
\hline $\mathrm{BICS}$ & Basic Interpersonol Communication Skills \\
\hline CAI & Cymraeg Ail Iaith \\
\hline $\mathrm{CA}$ & Cyfnod Allweddol \\
\hline CALP & Cognitive Academic Language Proficiency \\
\hline CBI & Content Based Instruction \\
\hline CIG & Cymraeg Iaith Gyntaf \\
\hline CLIL & Content and Language Integrated Learning \\
\hline CS & Cyfnod Sylfaenol \\
\hline CYGB & Cymuned Ymreolaethus Gwlad y Basg \\
\hline ITM & Ieithoedd Tramor Modern \\
\hline MAT & Mwy Abl a Thalentog \\
\hline $\mathrm{LlC}$ & Llywodraeth Cymru \\
\hline $\mathrm{LICC}$ & Llywodraeth Cynulliad Cenedlaethol \\
\hline PYDd & Prydau Ysgol am Ddim \\
\hline TAG & Tystysgrif Addysg Gyffredinol \\
\hline TGAU & Tystysgrif Gyffredinol Addysg Uwchradd \\
\hline
\end{tabular}




\section{Rhestr termau}

Addysg Dreftadaeth

Addysg Drochi

Addysg Foddi

Addysg Iaith Dramor Fodern

Education

Damcaniaeth Caffael-Dysgu

Damcaniaeth Arolygu

Damcaniaeth y Trothwy Ieithyddol

Dwyieithrwydd Ar Wahân

Dwyieithrwydd Cydamserol

Dwyieithrwydd Cyfartal

Dwyieithrwydd Cyflawn

Dwyieithrwydd Cytbwys

Dwyieithrwydd Cynyddol

Dwyieithrwydd Gostyngol

Dwyieithrwydd Hyblyg
Heritage Education

Immersion Education

Submersion Education

Modern Foreign Language

Acquisition-learning Distinction (Krashen, 1982)

Monitor Theory

(Krashen, 1982)

Threshold Theory

(Cummins, 1976)

Separate Bilingualism

(Blackledge \& Creese, 2010)

Simultaneous Bilingualism

(Swain, 1972)

Equalingualism

(Baetens-Beardsmore, 1986)

Full Bilingualism

(Romaine, 1995)

Balanced Bilingualism

(Romaine, 1995)

Additive Bilingualism (Lambert, 1974)

Subtractive Bilingualism (Lambert, 1974)

Flexible Bilingualism

(Blackledge \& Creese, 2010) 
Dyfais Caffael Iaith

(LAD)

Dysgu Cynnwys ac Iaith Integredig

Gramadeg Cyffredinol

Hyfedredd Iaith Gwybyddol

Academaidd

Hyfedredd Ieithyddol Cyffredinol

Prawf Llenwi Bylchau

Pwnc Craidd

Pwnc Sylfaenol

Rhyngiaith

Sgiliau Cyfathrebol Rhyngbersonol Sylfaenol

Y Camsyniad Cymharu

Y Safbwynt Rhyngweithiol / Gwybyddol
Language-Acquisition Device (Chomsky, 1965)

Content and Language

Integrated Learning

Universal Grammar

(Chomsky, 1965)

Cognitive Academic Language Proficiency (CALP)

(Cummins, 1979a)

General Language Proficiency

Cloze Test

Core Subject

Foundation Subject

Interlanguage

(Selinker, 1972)

Basic Interpersonol

Communication Skills (BICS)

(Cummins, 1979a)

The Comparative Fallacy

(Bley-Vroman, 1983)

The Interactionalist / Cognitive Perspective

(Ellis, 2015; Lightbown \&

Spada, 2015) 


\section{Rhagair}

Ceir i'r traethawd doethurol hwn ddwy brif ran, sef yr ymchwil ragarweiniol (Penodau 1-5) a'r ymchwil empirig (Penodau 6-8), gyda chryn bwyslais ar yr ymchwil ragarweiniol. Diben rhan gyntaf y traethawd yw gosod y gwaith ymchwil hwn yn ei gyd-destun ehangach gan archwilio'r gagendor sy'n bodoli rhwng polisi ac arfer ym maes Cymraeg Ail Iaith (Pennod 1) a chyflwyno'r prif dermau a ddefnyddir yn y traethawd, yn ogystal â'u harwyddocâd yng nghyd-destun yr ymchwil hon (Pennod 2). Ceir trafodaeth ar fanteision (ac anfanteision) posibl dwyieithrwydd i atgyfnerthu'r ddadl dros ddatblygu sgiliau dwyieithog dysgwyr y sector cyfrwng Saesneg ymhellach (Pennod 3). I gloi rhan gyntaf y traethawd, trafodir ymchwil rhyngwladol (Pennod 4) a chenedlaethol (Pennod 5) ar ddysgu dwys, addysg drochi a CLIL (Content and Language Integrated Learning) er mwyn gosod sail a chyddestun i'r ymchwil empirig a gyflwynir yn ail ran y traethawd. Canolbwyntir yn benodol ar gynllun unigryw a gynigir mewn ysgol uwchradd cyfrwng Saesneg yn $\mathrm{Ne}$ Cymru, a adwaenir yn yr ymchwil hon fel Ysgol X. Mae'r cynllun hwn, a adwaenir fel y Cwrs Carlam, yn cyflwyno pum pwnc cwricwlaidd yn ddwyieithog i garfan o ddisgyblion Mwy Abl a Thalentog yng Nghyfnod Allweddol 3 (Blynyddoedd 7, 8 a 9), gyda'r nod o gyfoethogi'r dysgu. Yn ogystal â'i darpariaeth ychwanegol i'r Gymraeg, mae gan Ysgol X hanes o ran datblygu ethos dwyieithog cryf a threfniadau pontio effeithiol, a threfnu hyfforddiant iaith Gymraeg i'w staff.

Yn ail ran y traethawd, cyflwynir astudiaeth achos gyfunol sy'n amlygu gwahaniaethau rhwng Ysgol X ac un ysgol uwchradd cyfrwng Saesneg gymharus arall (a adwaenir fel Ysgol Y yn yr ymchwil hon) a thair carfan o ddysgwyr ar draws y ddwy ysgol gyfranogol hyn. Yn Pennod 7, cyflwynir dadansoddiad o'r gwahanol fewnbwn a geir yn y naill ysgol a'r llall, gan gymharu'r canfyddiadau hyn â chanfyddiadau'r ymchwil ragarweiniol. Yn Pennod 8, cyflwynir dadansoddiad o'r gwahanol allbwn a geir ar draws y ddwy ysgol gyfranogol, gan ganolbwyntio'n benodol ar amlygu proffiliau ieithyddol a sosio-economaidd (i) disgyblion y Cwrs Carlam yn Ysgol X; (ii) disgyblion Cymraeg Ail Iaith Set 1 Ysgol X; a (iii) disgyblion Cymraeg Ail Iaith Set 1 Ysgol Y. Yn ogystal ag archwilio mewnbwn ac allbwn y ddwy ysgol, nodir mai diben yr astudiaeth hon yw canfod tystiolaeth i gefnogi'r drafodaeth yn rhan gyntaf y traethawd, sef sut orau y gellir cefnogi 
cyflwyno'r Gymraeg fel ail iaith yn llwyddiannus mewn ysgolion uwchradd cyfrwng Saesneg.

I gloi'r traethawd, crynhoir prif ganfyddiadau'r ymchwil a'r goblygiadau a'r heriau ar gyfer y sector cyfrwng Saesneg a Chymraeg (Pennod 9), cyn cyflwyno argymhellion yr ymchwilydd o ran cyfeiriadau at bolisi ac arfer yn y dyfodol a'r angen am ymchwil bellach (Pennod 10). 


\section{Pennod 1: Polisi ac arfer o ran addysgu'r Gymraeg mewn addysg cyfrwng}

\section{Saesneg}

Meysydd cyfredol sy'n datblygu trwy'r amser yw'r rhai dan sylw yn y traethawd hwn, ac felly y mae llwyth offynonellau ac adnoddau ar gael i dynnu arnynt wrth ymchwilio. Wrth ystyried hynny, penderfynwyd mai addas fyddai trafod y llenyddiaeth fwyaf cyfoes yn y maes, yn enwedig ers pasio Deddf Diwygio Addysg yn 1988 gan fod hyn yn ddeddf addysg arwyddocaol iawn, deddf "y gwelir ei heffeithiau mewn ysgolion hyd heddiw" (Pritchard \& Butt, 2009, t. 1). Serch hynny, allweddol yw nodi yma na ddigwyddodd y datblygiadau deddf iaith ac addysg diweddar hyn mewn gwacter. Digwyddodd y datblygiadau hyn o fewn cyd-destun ehangach a chyfnod arbennig o newid i'r Gymraeg yn ystod yr ugeinfed ganrif a'r unfed ganrif ar hugain. Ers diwedd yr ugeinfed ganrif, gwelwyd nifer o ddatblygiadau yn hanes deddfwriaethol Cymru a fyddai'n cael dylanwad ar addysgu'r Gymraeg mewn addysg, yn ogystal â chodi statws y Gymraeg yn fwy cyffredinol.

Penderfynwyd rhannu'r bennod hon felly yn dair is-adran. Yn gyntaf, olrheinir y deddfau iaith arwyddocaol ers Deddf yr Iaith Gymraeg 1967 hyd at Mesur y Gymraeg (Cymru) 2011, er mwyn rhoi cyd-destun digon cadarn i'r ymchwiliad hwn. Yn ail, archwilir y datblygiadau polisi addysg diweddar hyn a thrafodir eu pwysigrwydd yng nghyd-destun addysgu'r Gymraeg fel ail iaith, gan ganolbwyntio ar bolisïau a deddfwriaeth addysg ers pasio Deddf Diwygio Addysg 1988 yn benodol. Yn drydydd, trafodir canfyddiadau diweddar Estyn, ynghyd ag unrhyw randdeiliaid allweddol eraill, ar arfer a safonau ym maes Cymraeg Ail Iaith (CAI o hyn ymlaen). Yn ogystal â thrafod y cyhoeddiadau a'r deddfau arwyddocaol, cyfeirir at ddeunyddiau beirniadol eraill er mwyn dangos ymwybyddiaeth o'r hyn sydd wedi'i ysgrifennu yn y maes eisoes er mwyn strwythuro dadl ddilys yn y gwaith.

\subsection{Olrhain hanes y deddfau iaith arwyddocaol yng Nghymru}

\subsubsection{Deddf yr Iaith Gymraeg 1967}

Catalydd i'r newidiadau cyfoes mewn deddfwriaeth a pholisi iaith oedd pasio Deddf yr Iaith Gymraeg 1967. Mae G. Lewis (2008, t. 85) wedi nodi mai dyma oedd "y datblygiad gwirioneddol arwyddocaol cyntaf" yn neddfwriaeth y Gymraeg, am i'r ddeddf nodi'r canlynol: 
(1) In any legal proceeding in Wales or Monmouthshire the Welsh language may be spoken by any party, witness or other person who desires to use it... (Deddf yr Iaith Gymraeg 1967)

Er gwaethaf arwyddocâd y cymal arwyddocaol a'i ddylanwad ar gyflwyno rhai hawliau i'r siaradwr Cymraeg yn y llys, ni wnaeth y ddeddf hon eto gydnabod y ddwy iaith yn gyfartal â'i gilydd, gan nodi'r canlynol:

... in case of any discrepancy between an English and a Welsh text the English text shall prevail. (Deddf yr Iaith Gymraeg 1967)

Ar ben hyn, nodir nad oes sôn yn Deddf yr Iaith Gymraeg 1967 am hawliau ieithyddol yr unigolyn, dilysrwydd y Gymraeg mewn gweinyddiaeth gyhoeddus (er bod y ddwy iaith "yr un mor ddilys â'i gilydd” mewn achosion cyfreithiol (G. Lewis, 2008, t. 85)), na'r un cyfeiriad chwaith at statws swyddogol y Gymraeg yng Nghymru.

Er gwaethaf ymdrech y Swyddfa Gymreig i dawelu'r ymgyrch dros ddeddfwriaeth newydd drwy sefydlu Cyngor yr Iaith Gymraeg yn 1977, ni fyddai sefydlu'r Cyngor yn ymateb digonol gan y Llywodraeth. Daeth y Cyngor i ben ymhen misoedd oherwydd diffyg swyddogaeth bendant (G. Lewis, 2008, t. 85). Yng ngoleuni datblygiadau eraill yn ystod yr wythdegau, megis sefydlu'r orsaf deledu Gymraeg gyntaf yn 1982 a phasio Deddf Diwygio Addysg 1988, ailddechreuodd Cymdeithas yr Iaith ymgyrchu dros ddeddf iaith newydd, un a fyddai'n trin y Gymraeg ar sail ei bod yn gyfartal â'r Saesneg. Ymatebodd Llywodraeth San Steffan i'r ymdrechion hyn drwy sefydlu Bwrdd Iaith cyntaf yn 1988, a'r prif nod oedd adrodd ar yr angen am ddeddf newydd ar gyfer y Gymraeg.

Er bod nifer o ddiwygiadau yn ystod yr wythdegau o ran addysg, y gyfraith, gweinyddiaeth gyhoeddus a llywodraeth leol, nid oedd y datblygiadau hyn yn ddigonol yn ôl Colin H. Williams (2014, t. 250), a ddisgrifiodd y datblygiadau hyn fel rhai "piecemeal, insufficient, and rudimentary." Er gwaetha'r datblygiadau a welwyd erbyn yr wythdegau, ni chafodd dau o'r prif alwadau, sef datganoli gwleidyddol a sicrhau hawliau pellach i ddefnyddio'r Gymraeg ym meysydd cyhoeddus Cymru, eu hateb, a byddai'n rhaid aros tan y nawdegau cyn i'r galwadau hyn gael eu bodloni (C. H. Williams, 2014, t. 251). Ni fyddai newid pellgyrhaeddol yn neddfwriaeth Cymru yn digwydd felly am ryw chwe mlynedd ar hugain, nes i 
ddeddf iaith newydd gael ei phasio yn 1993 a nes i Gynulliad Cenedlaethol Cymru gael ei ffurfio, yn unol â Deddf Llywodraeth Cymru ym 1998. Cyn ffarwelio â'r ganrif a'r mileniwm, byddai gan Gynulliad Cenedlaethol Cymru y cyfrifoldeb am lunio polisi addysg yng Nghymru.

\subsubsection{Deddf yr Iaith Gymraeg 1993}

Mae'n debyg mai pasio Deddf yr Iaith Gymraeg 1993 oedd y datblygiad mwyaf arwyddocaol o ran deddfu'r iaith Gymraeg yn ystod y pedwar degawd diwethaf, gan y cyflwynwyd y cysyniad "y dylid trin y Gymraeg a'r Saesneg ar y sail eu bod yn gyfartal” (Deddf yr Iaith Gymraeg 1993). Mae Colin. H. Williams (2014, t. 245) wedi cydnabod arwyddocâd y ddeddf hon wrth gasglu: "[t]he passage of the Welsh Language Act of 1993 provided a statutory framework for the treatment of English and Welsh on the basis of equality and was a turning point in the history of Welsh language promotion."

Fel y noda Williams uchod, dyma'r tro cyntaf i'r Gymraeg gael ei thrin ar y sail ei bod yn gyfartal â'r Saesneg. Yn ogystal â symboleiddio newid o ran statws cyfartal rhwng y Gymraeg a'r Saesneg, sicrhawyd hefyd hawl llwyr yr unigolyn i ddefnyddio'r Gymraeg yn y llys. Amlinellwyd yn Adran 22 Deddf yr Iaith Gymraeg 1993 "mewn unrhyw achosion cyfreithiol yng Nghymru gellir siarad Cymraeg gan unrhyw barti, tyst neu berson arall sy'n dymuno'i defnyddio... a gwneir unrhyw ddarpariaeth angenrheidiol ar gyfer cyfieithu yn unol â hyn." Prif ddatblygiad arall a ddeilliodd o'r ddeddfwriaeth hon oedd sefydlu Bwrdd yr Iaith Gymraeg fel corff statudol. Cafodd y Bwrdd ei sefydlu yn Adran 1 y ddeddf a byddai ganddo'r cyfrifoldeb am hyrwyddo a hybu'r Gymraeg yng Nghymru. Ymhlith ei swyddogaethau, roedd gan y Bwrdd y pwer i orfodi cyrff cyhoeddus i gyflwyno cynllun iaith i'w weithredu er mwyn dangos eu hymrwymiad i ddarparu gwasanaethau cyhoeddus yn Gymraeg. Gwnaeth y Bwrdd hyn am dros ugain mlynedd hyd at 2012, pan gafodd y Bwrdd ei ddiddymu a'i ddisodli gan swyddfa newydd Comisiynydd y Gymraeg, yn unol â Mesur y Gymraeg (Cymru) 2011. Er pwysiced Deddf yr Iaith Gymraeg 1993 yn nhermau cyflwyno statws cyfartal rhwng y Gymraeg a'r Saesneg a sefydlu Bwrdd yr Iaith Gymraeg i hyrwyddo a hybu'r Gymraeg, gellir beirniadu'r ddeddf hon am sawl gwendid, gan gynnwys diffyg hawliau i'r unigolyn, rhwystr Bwrdd yr Iaith Gymraeg rhag gorfodi cyrff y Goron, 
diffyg statws swyddogol i'r Gymraeg yng Nghymru, ynghyd ag amwysedd rhai o'i chymalau.

\subsubsection{Mesur y Gymraeg (Cymru) 2011}

Ar ôl ffurfio Cymru'n Un yn 2007, sef glymblaid rhwng Llafur a Phlaid Cymru yng Nghynulliad Cenedlaethol Cymru, addawyd y byddai'r glymblaid yn gweithio tuag at gyflwyno "Mesur Cynulliad newydd i gadarnhau statws swyddogol ar gyfer y Gymraeg a'r Saesneg, hawliau ieithyddol o safbwynt darparu gwasanaethau a sefydlu swydd Comisiynydd Iaith." (Llywodraeth Cynulliad Cymru, 2007a, tt. 3536). Ar 9 Chwefror 2011, cafodd y Mesur gydsyniad brenhinol, sef Mesur y Gymraeg (Cymru) 2011. Yn sgil pasio'r Mesur, daeth y Gymraeg yn iaith swyddogol yng Nghymru am y tro cyntaf, cafodd y Bwrdd ei ddiddymu yn lle Comisiynydd Iaith newydd, ynghyd â chynlluniau iaith a fyddai'n cael eu disodli gan safonau iaith newydd, sefydlwyd Tribiwnlys y Gymraeg, yn ogystal â nifer o newidiadau eraill. Daeth statws y Gymraeg yn neddfwriaeth Cymru yn gryfach unwaith eto. Er na roes hawliau ieithyddol i'r unigolyn, roedd yn datblygiad hollbwysig o ran sicrhau statws swyddogol i'r Gymraeg a'r egwyddor "na ddylid trin y Gymraeg yn llai ffafriol na'r Saesneg” (Mesur y Gymraeg (Cymru) 2011). Wedi sicrhau amodau ffafriol i'r iaith Gymraeg a'i statws megis iaith swyddogol Cymru, mae Mesur y Gymraeg yn gosod sylfaen gadarn ar gyfer datblygiadau pellach yn y dyfodol, nid yn unig o ran deddfu iaith, ond hefyd o ran deddfu addysg yng Nghymru.

\subsection{Y datblygiadau diweddar ym mholisi addysg Cymru}

\subsubsection{Deddf Diwygio Addysg 1988}

Gellid dadlau mai Deddf Diwygio Addysg 1988 oedd y diwygiad mwyaf dylanwadol ar addysg Cymru ar ôl i'r ddeddf addysg gael ei phasio yn 1944¹․ O dan Adran 1 Deddf Diwygio Addysg 1988, sefydlwyd y Cwricwlwm Cenedlaethol ('y cwricwlwm' o hyn ymlaen) a fyddai'n creu cwricwlwm safonol yn ysgolion Cymru a chyflwyno gofyniadau statudol am y tro cyntaf ar addysg i ddysgwyr o dan 14 oed

\footnotetext{
${ }^{1}$ Roedd Deddf Addysg 1944, a adwaenir hefyd fel 'Deddf Addysg Butler' ar ôl y Gweinidog Addysg R.A. Butler a arweiniodd y ddeddfwriaeth, yn ddeddf ddylanwadol a gyflwynodd addysg uwchradd yn rhad ac am ddim ar gyfer pob plentyn ym Mhrydain, yn ogystal â chodi oedran gadael yr ysgol i 15 oed a galluogi Awdurdodau Addysg Lleol (AALl) sefydlu tri math newydd o ysgol uwchradd sef Ysgol Ramadeg, Ysgol Uwchradd Fodern ac Ysgol Dechnegol. (Parliment UK., d.d.). Yn benodol i addysgu'r Gymraeg yng Nghymru, bu pasio'r ddeddf yn bwysig i alluogi AALl i ddarparu ysgolion cyfrwng Cymraeg am y tro cyntaf (Jones, 2001, t. 9).
} 
yng Nghymru. Cyn hynny, addysg grefyddol ac addysg gorfforol oedd yr unig bynciau gorfodol, o ganlyniad i basio Deddf Addysg 1944. Câi'r Gymraeg, fodd bynnag, ei chyflwyno a'i dysgu mewn ychydig o ysgolion ar draws Cymru yn unig, heb unrhyw ofyniadau statudol ar ysgolion i addysgu Cymraeg (L1C, 2013b).

Ar ben creu cwricwlwm cenedlaethol safonol i'w weithredu, lluniwyd y Cyfnodau Allweddol, o dan Adran 3 Deddf Diwygio Addysg 1988. Byddai'n strwythuro'r dysgu a chyflwyno targedau cyrhaeddiad penodol i'w cyrraedd erbyn diwedd pob un Cyfnod Allweddol (CA o hyn ymlaen). Fel un o bynciau sylfaenol CA1, 2 a 3 y cwricwlwm, daeth Cymraeg yn bwnc gorfodol i'w addysgu mewn ysgolion cyfrwng Saesneg, ac yn bwnc craidd mewn ysgolion cyfrwng Cymraeg o 1990 ymlaen yng Nghymru. Yng ngoleuni datblygiadau dylanwadol ar y pryd, megis pasio deddf iaith newydd yn 1993 (gweler 1.1.2. Deddf yr Iaith Gymraeg 1993) a datblygu Cwricwlwm Cymreig ar wahân i'r un yn Lloegr, byddai'r Gymraeg yn dod yn bwnc sylfaenol yn CA4 yn 1999. Ac o ganlyniad i ddatganoli yng Nghymru yn 1999, wedi i Deddf Llywodraeth Cymru 1998 gael ei phasio, byddai'r gyfundrefn addysg Gymreig bellach yn un annibynnol (LIC, 2013b). Am y tro cyntaf erioed, byddai gan bob disgybl oed ysgol yng Nghymru yr hawl i ddysgu'r Gymraeg naill ai mewn perthynas ag addysg cyfrwng Cymraeg, trwy ei hastudio fel pwnc craidd, sef Cymraeg Iaith Gyntaf (CIG o hyn ymlaen), gan astudio pynciau eraill trwy gyfrwng y Gymraeg, neu mewn perthynas ag addysg cyfrwng Saesneg, gan astudio'r iaith fel pwnc sylfaenol, sef CAI, yn unig.

\subsubsection{Iaith Pawb}

Ar ôl i Gynulliad Cenedlaethol Cymru gael ei sefydlu yn 1999, cododd disgwyliadau am i'r Llywodraeth gymryd cyfrifoldeb dros ddatblygu dwyieithrwydd Cymru a hyrwyddo'r Gymraeg a'i ffyniant (Y Pwyllgor Diwylliant a'r Pwyllgor Addysg a Dysgu Gydol Oes, 2002, t. 3). Yn wir, o dan Adran 32(c) Deddf Llywodraeth Cymru (1998) rhoes Adran 32(c) Deddf Llywodraeth Cymru (1998) y grym i'r Llywodraeth “do anything it considers appropriate to support the Welsh language." Yn 2002, cyhoeddodd y Pwyllgor Diwylliant a'r Pwyllgor Addysg a Dysgu Gydol Oes adroddiad, sef Ein Hiaith: Ei Dyfodol, er mwyn adolygu polisi'r iaith Gymraeg. Yn ogystal â nodi ystod fawr o argymhellion o ran sut y gellir cyflawni'r heriau sy'n wynebu Cymraeg mewn polisi ac addysg, nododd yr adroddiad mai Llywodraeth Cynulliad Cymru (LiCC o hyn ymlaen), ynghyd â'r cyrff a'r asiantaethau priodol, a 
oedd yn gyfrifol am gyflawni'r heriau hyn er mwyn sicrhau ffyniant yr iaith Gymraeg a hybu dwyieithrwydd Cymru (Y Pwyllgor Diwylliant a'r Pwyllgor Addysg a Dysgu Gydol Oes, 2002, t. 3). Cyflwynir gweledigaeth y Llywodraeth yn glir, sef gweld Cymru'n datblygu'n wlad ddwyieithog:

Mewn Cymru wirioneddol ddwyieithog fe fydd y Gymraeg a'r Saesneg yn ffynnu ac yn cael eu trin yn gyfartal. Ystyr Cymru ddwyieithog yw gwlad lle mae pobl yn gallu dewis byw eu bywydau trwy gyfrwng un neu'r ddwy iaith; gwlad lle mae presenoldeb dwy iaith genedlaethol, ac ieithoedd a diwylliannau amrywiol eraill yn ffynhonnell balchder a nerth (Y Pwyllgor Diwylliant a'r Pwyllgor Addysg a Dysgu Gydol Oes, 2002, t. 5).

Wrth gyhoeddi ei datganiad polisi, Dyfodol dwyieithog: A bilingual future yn 2002, cyflwynodd LlCC (2003, t. 1) ei chynllun gweithredu cenedlaethol cyntaf ar gyfer datblygu "Cymru gwbl ddwyieithog", sef Iaith Pawb. Roedd y Llywodraeth bellach am roi'r cyfle i bawb yng Nghymru ddefnyddio'r naill iaith a'r llall yn eu bywydau bob dydd (LlCC, 2003, t. 1). Yn y cynllun, nododd LlCC (2003, t. 11) ei bod am weld y "ganran o bobl Cymru sy'n gallu siarad Cymraeg” gynyddu "5 pwynt canran o'r ffigwr a ddaw i'r amlwg o gyfrifiad 2001" erbyn 2011. Dyma drobwynt yn agwedd a pholisi Llywodraeth Cymru (LlC o hyn ymlaen), gan mai hon oedd strategaeth gyntaf y Llywodraeth ynghylch datblygu twf siaradwyr dwyieithog yn y Gymraeg ac yn y Saesneg. O hyn ymlaen, byddai ffyniant y Gymraeg a dwyieithrwydd yn ganolbwynt i bolisi iaith y wlad, ac yn ei thro, byddai rôl addysg, yn benodol addysg cyfrwng Cymraeg, yn cael ei gwerthfawrogi yn y broses o wireddu'r weledigaeth uchelgeisiol hon:

Mae addysg trwy gyfrwng y Gymraeg wedi ehangu'n gyson a dymuna Llywodraeth y Cynulliad annog y duedd hon. Cydnabyddir rôl hollbwysig addysg trwy gyfrwng y Gymraeg a dulliau trochi ieithyddol yn y broses o ddatblygu sgiliau dwyieithog cadarn ymhlith disgyblion. (L1CC, 2003, t. 40).

Pwysig yma yw nodi mor uchelgeisiol oedd ymrwymiad y Llywodraeth i ddatblygu Cymru gwbl ddwyieithog. Pwysleisiodd y Prif Weinidog ar y pryd, Rhodri Morgan, a'r Gweinidog dros Ddiwylliant, Chwaraeon a'r Gymraeg ar y pryd, Jenny Randerson, natur yr her o'u blaen, ynghyd â'u hymrwymiad, yng nghyflwyniad Iaith Pawb: 
Efallai na fydd yn waith hawdd, ac efallai na fyddwn yn cyflawni ein targedau dros nos, ond byddwn yn gwneud y cyfan sydd o fewn ein gallu i greu'r amodau cywir lle y gall yr iaith Gymraeg dyfu a blodeuo ym mhob agwedd o fywyd Cymru. Cam pwysig yn y broses honno yw'r cynllun gweithredu hwn. (LlCC, 2003, t.2).

Wedi lansio'r cynllun cenedlaethol yn 2003, cyhoeddwyd adroddiad yn flynyddol gan y Llywodraeth am y saith mlynedd nesaf er mwyn ystyried ei pherfformiad o ran gweithredu gweledigaeth Iaith Pawb. Er pwysiced y cynllun o ran dangos ymrwymiad LICC i'w gweledigaeth i greu gwlad wirioneddol ddwyieithog, prin oedd y manylion sy'n ystyried sut y byddai LICC yn mynd ati i wella CAI a datblygu ei rôl ar gyfer creu Cymru ddwyieithog. Mae Colin H. Williams (2005) wedi beirniadu'r cynllun hwn, gan ei ddisgrifio megis cynllun sydd ond yn llawn rhethreg ac sydd heb drafod sut y mae'r Llywodraeth yn bwriadu monitro effeithiau polisïau. Mae ef yn mynd yn ei flaen i nodi'r canlynol:

There is almost no detail in Iaith Pawb on developing Welsh within the statutory sector, whether in terms of the provision of additional bilingual schools, in the boosting of the Welsh L2 sector, or in using Welsh as the medium for the teaching of a wide range of subjects.

(C. H. Williams, 2005, t. 16).

Gyda golwg ar CAI yn benodol, mae Williams hefyd wedi amlygu'r diffyg trafodaeth ar addysgu'r Gymraeg i ddysgwyr ail iaith fel un o'r prif wendidau. Dywedodd Williams (Awst 2013, t. 21) fod "[t]he discussion on education in particular is a major weakness... There is also far too little on L2 learners..." Gellir nodi'r diffygion hyn wrth ddarllen y bedwaredd bennod yn Iaith Pawb. Mewn byr eiriau, cydnabu'r ddogfen "rôl hollbwysig addysg trwy gyfrwng y Gymraeg a dulliau trochi ieithyddol yn y broses o ddatblygu sgiliau dwyieithog cadarn ymhlith disgyblion." (LlCC, 2003, t. 40). Eir ymlaen i nodi bod angen "edrych yn fanylach ar rai materion" ynglŷn ag addysgu CAI, ond ni wnaethpwyd hynny ar y pryd (LlCC, 2003, t. 40).

Mae'r diffyg sôn am CAI hefyd yn amlwg wrth astudio adroddiad blynyddol olaf Iaith Pawb (LlCC, 2010a). Nid aeth yr adroddiad hwn ati i fanylu ar rôl addysg CAI yn y broses o wireddu gweledigaeth Iaith Pawb ychwaith. Yn hytrach, cafwyd trafodaeth ar addysg cyfrwng Cymraeg a phwysigrwydd strategaeth newydd, sef Strategaeth Addysg Cyfrwng Cymraeg (2010). Roedd y canolbwynt hwn ar lunio 
strategaeth ar gyfer datblygu addysg cyfrwng Cymraeg hefyd i'w weld pan ffurfiwyd clymblaid gan Blaid Llafur a Phlaid Cymru yn 2007 a chyhoeddwyd ganddynt ar y cyd eu hawydd cyffredin ar gyfer llywodraethu Cymru, yn Cymru'n Un (2007a). Er i'r glymblaid ymrwymo'n glir i greu strategaeth ar gyfer cynllunio addysg cyfrwng Cymraeg, ni chafwyd yn y ddogfen hon drafodaeth benodol ar sut y byddai'r llywodraeth newydd yn ymrwymo i wella safonau CAI mewn ysgolion cyfrwng Saesneg.

Er pwysiced y dogfennau Cymru'n Un ac Iaith Pawb o ran mynegi awydd y Llywodraeth am bolisi iaith agored, cynhwysol, a chanolbwyntio ar ddwyieithrwydd megis eiddo'r gymdeithas, ymddengys nad oedd y naill na'r llall yn cynnig strategaeth ddigon manwl ar gyfer cynllunio'n rhagweithiol er mwyn datblygu Cymru ddwyieithog. Yn fwy penodol, nid ystyriwyd pa rôl a fyddai gan CAI i’w chwarae yn y strategaeth honno. Yn y bôn, dyma bolisi a oedd yn mynegi dyhead a delfryd (C. H. Williams, 2005). O ganlyniad, gellid dadlau y byddai'n well trin Iaith Pawb megis fframwaith bras ac arwydd o fwriad da gan LlCC ar y pryd (C.H. Williams, Awst 2013, t. 22).

\subsubsection{Dysgu ac addysgu Cymraeg fel ail iaith (2010)}

Yn yr un flwyddyn â lansiad Strategaeth Addysg Cyfrwng Cymraeg, cyhoeddwyd adroddiad allweddol ar CAI gan Y Pwyllgor Menter a Dysgu, sef Dysgu ac addysgu Cymraeg fel ail iaith (2010). Yng ngoleuni canfyddiadau cyfres o adroddiadau ar y pryd gan Estyn, a nododd wendidau difrifol yn y ffordd yr addysgir y Gymraeg fel ail iaith (Cynulliad Cenedlaethol Cymru, 30 Medi 2010), cynhaliwyd cyfarfod gan Y Pwyllgor Menter a Dysgu ar 15 Ionawr 2009 a ffurfiwyd Grŵp Rapporteur er mwyn ymchwilio i ddwyieithrwydd yng Nghymru. Un o'r tri maes strategol y byddai'r Grŵp yn canolbwyntio arno fyddai dysgu ac addysgu'r Gymraeg fel ail iaith mewn ysgolion cyfrwng Saesneg (Y Pwyllgor Menter a Dysgu, 2010, t. 11). Yn adroddiad y Pwyllgor, ceir ymdrech i ddiffinio dwyieithrwydd a thrafod ei arwyddocâd i Gymru, ynghyd ag amlygu'r heriau a wyneba'r Llywodraeth o ran addysgu CAI a chynnig argymhellion i'r Llywodraeth am sut y dylai fynd ati i unioni'r problemau hynny. Mae'n amlwg yn yr adroddiad fod y Pwyllgor yn rhannu'r un weledigaeth â'r Llywodraeth am ddatblygu dyfodol dwyieithog ar gyfer Cymru, ac mae'n cydnabod bod angen gwella'r ffordd y cyflwynir y Gymraeg yn y sector cyfrwng Saesneg er mwyn gwireddu uchelgais Iaith Pawb. Er enghraifft, cyfeirir yn yr adroddiad at 
Strategaeth Addysg Cyfrwng Cymraeg (2010) a'r rôl sydd gan CAI i'w chwarae wrth wireddu nodau LICC yn y strategaeth honno:

Mae'r strategaeth hefyd yn ceisio codi safonau addysgu Cymraeg fel ail iaith er mwyn i'r 'rhai nad ydynt yn dewis addysg cyfrwng Cymraeg hefyd gael cyfleoedd gwell i ddatblygu sgiliau iaith Gymraeg sy'n cyfoethogi eu profiad o fyw mewn gwlad ddwyieithog'. (Y Pwyllgor Menter a Dysgu, 2010, t. 13)

\subsubsection{Iaith fyw: iaith byw (2012-2017)}

Er mwyn adeiladu ar waith Iaith Pawb, lansiodd Leighton Andrews, Gweinidog Addysg a Sgiliau ar y pryd, strategaeth newydd yn 2012. Cafodd y strategaeth hon, sef Iaith fyw: Iaith byw (2012), ei gweithredu ym mis Mawrth 2012 ar ôl i’r Llywodraeth drafod a llunio strategaeth ddrafft ar y Gymraeg rhwng 13 Rhagfyr 2010 a 4 Chwefror 2011 (LlC, 2011). Yn ôl y strategaeth hon, byddai LlC (2012b, t. 14) yn canolbwyntio ar ddwy elfen graidd, sef caffaeliad a defnydd o'r Gymraeg, er mwyn gwireddu ei nod hirdymor o "weld y Gymraeg yn ffynnu yng Nghymru."

Yn wahanol i'r cynllun blaenorol, Iaith Pawb, ceir yn Iaith fyw: Iaith byw fwy o fanylder wrth i'r strategaeth ganolbwyntio ar chwe maes strategol, sef y teulu, plant a phobl ifanc, y gymuned, y gweithle, gwasanaethau Cymraeg a'r seilwaith. Yr hyn a geir yn y strategaeth hon felly yw gwaith gweithredol parhaol sydd yn canolbwyntio'n fwy ar weld yr iaith a'i defnydd yn ffynnu yn hytrach na chanolbwyntio ar adeiladu cymdeithas ddwyieithog, fel y mae Iaith Pawb yn ei wneud (C. H. Williams, Awst 2013, t. 19). Fodd bynnag, mae Iaith fyw: Iaith byw wedi'i feirniadu gan Selleck (2013). Nododd fod natur gynhwysol ac agored Iaith $P a w b$ ar goll yn y strategaeth hon:

From the outset, the document seems to be more exclusionary at the level of policy than Iaith Pawb. Despite the criticisms that can be, and have been levelled at Iaith Pawb... there remains a sense of openness and tolerance in that text that is noticeably absent from the newer consultation document. (Selleck, 2013, t. 38)

Â Selleck yn ei blaen i dybio a fyddai polisi o 'ddwyieithrwydd hyblyg' (flexible bilingualism $)^{2}$, sy'n canolbwyntio ar ddefnyddio a datblygu'r ddwy iaith ar y cyd yn

\footnotetext{
${ }^{2}$ Dyma'r term a ddefnyddir gan Blackledge a Creese (2010) er mwyn disgrifio'r defnydd a datblygiad
} o ddwy iaith ar y cyd mewn addysg. 
hytrach na pholisi o 'ddwyieithrwydd ar wahân' (separate bilingualism) ${ }^{3}$, yn fwy addas ar gyfer y sefyllfa addysg ddwyieithog sy’n bodoli yng Nghymru. Yn ôl Selleck, rhaid ystyried a fyddai polisi iaith mwy hyblyg o'r fath yn fwy addas yng nghyd-destun addysgu disgyblion ail iaith sydd heb fawr o gyswllt â'r Gymraeg: Ys gofynnodd hi: "[w]ould, for example, flexible bilingualism provide the appropriate environment for pupils with limited exposure to Welsh in a curricular context to develop a full range of skills in Welsh?” (Selleck, 2013, t. 38).

Er bod Iaith fyw: Iaith byw yn cydnabod rhan addysg yn y broses o ddatblygu defnydd o'r Gymraeg, yn enwedig addysg cyfrwng Cymraeg (L1C, 2012b, t. 15), cafodd y cyfle i fanylu ar rôl ysgolion cyfrwng Saesneg yn y broses honno ei golli yn y strategaeth hon. Roedd hefyd ddiffyg manylder o ran sut y byddai'r Llywodraeth yn unioni diffygion darpariaeth Gymraeg yn y gyfundrefn addysg cyfrwng Saesneg. Mae hyn yn amlwg wrth ddarllen y datganiad polisi newydd Iaith byw: iaith fyw Bwrw mlaen (2014), sydd yn adeiladu ar strategaeth Iaith fyw: Iaith byw. Ni chaed manylder ar rôl CAI na sut y byddai LlC yn sicrhau bod newid yn y system gyfredol. Aeth y datganiad cyn belled â dweud y canlynol yn unig:

Mae'n rhaid i'r system gyfredol o addysgu a dysgu Cymraeg mewn ysgolion cyfrwng Saesneg newid... Fodd bynnag, gan fod yr Athro Graham Donaldson wrthi'n cynnal adolygiad ehangach o'r cwricwlwm a threfniadau asesu yng Nghymru, bydd unrhyw newidiadau o ran y cwricwlwm yn cael eu hystyried fel rhan o'r adolygiad ehangach hwn. (LlC, 2014, t. 20).

Yn ei ymateb i Iaith fyw: Iaith byw - Bwrw mlaen, cydnabu Dyfodol i'r Iaith (2014) nad yw'r gyfundrefn bresennol o addysgu'r Gymraeg fel ail iaith yn llwyddo o hyd, ac awgrymir bod angen strategaethau pellach er mwyn ystyried:

... diwygio dysgu'r Gymraeg yn yr ysgolion cyfrwng Saesneg presennol i gynnwys dysgu trwy gyfrwng y Gymraeg mewn rhai pynciau a defnyddio'r Gymraeg y tu allan i'r dosbarth... I gryfhau'r ddarpariaeth, mae angen symud oddi wrth y syniad o ysgolion Saesneg, a gweld llwybr o gontinwwm ieithyddol sy'n symud tuag at ehangu darpariaeth ysgolion dwyieithog a Chymraeg. (Dyfodol i'r Iaith, 2014, t. 6).

Yng ngoleuni adroddiadau blynyddol diweddar gan Estyn (gweler, er enghraifft, Estyn, 2007, 2008, 2009, 2010, 2011, 2012b, 2013, 2014, 2015), sydd wedi amlygu

\footnotetext{
${ }^{3}$ Dyma'r term a ddefnyddir gan Blackledge a Creese (2010) er mwyn disgrifio'r defnydd a datblygiad o ddwy iaith ar y cyd mewn addysg.
} 
diffygion parhaus o ran safon gyffredinol CAI, cyhoeddodd Leighton Andrews ym mis Mai 2012 gynllun gwerth $£ 400,000$ dros bedair blynedd ar gyfer gwella safonau a chyrhaeddiad CAI trwy weithgareddau tymor byr a thymor hir. Mae gan y cynllun, sef Cynllun Gweithredu Cymraeg Ail Iaith, ddau brif nod, sef "codi safonau addysgu a chyrhaeddiad dysgwyr yng Nghyfnod Allweddol 2 gan adeiladu ar waith y Cyfnod Sylfaen" a "sicrhau bod gan ysgolion uwchradd yr adnoddau a'r capasiti ynghyd â chymwysterau addas i’w cynnig er mwyn adeiladu ar bolisi Cymraeg ail iaith ysgolion cynradd.” (LlC, 2012a, tt. 1-2). Yn ogystal â chyhoeddi'r cynllun gweithredu hwn, cyhoeddodd Andrews ym mis Gorffennaf 2012 ei fod yn sefydlu grŵp annibynnol i adolygu CAI yn CA3 a 4. Gorchwyl y Grŵp oedd trafod y pwyntiau canlynol:

- Sut i ddarparu profiad dysgu positif i ddysgwyr Cymraeg ail iaith mewn ysgolion cyfrwng Saesneg;

- Sut i godi statws Cymraeg ail iaith fel pwnc a chael gwared ar rwystrau ar lefel leol, ranbarthol a chenedlaethol;

- A yw cymwysterau (cymwysterau sydd eisoes ar gael a/neu ffurfiau eraill posibl o achredu) yn annog neu'n rhwystro datblygiad sgiliau iaith Gymraeg trosglwyddadwy;

- Y ffordd orau o ddatblygu sgiliau iaith Gymraeg dysgwyr fel eu bod nhw'n gallu trosglwyddo eu sgiliau a defnyddio'r Gymraeg yn y gweithle, y gymuned a'r teulu; a

- Sut i fynd ati i gynllunio'r gweithlu er mwyn sicrhau bod gan bob ysgol uwchradd yr adnoddau a'r capasiti i gynnig darpariaeth Cymraeg ail iaith o safon. (L. Andrews, 2012, 17 Gorffennaf, t. 2)

\subsubsection{Un Iaith i Bawb (2013)}

Yn yr adroddiad Un Iaith i Bawb, a gyhoeddwyd ym mis Medi 2013, agorir y rhagair gan yr Athro Sioned Davies, sef cadeirydd y grŵp adolygu, wrth iddi bwysleisio difrifoldeb sefyllfa CAI:

Os ydym o ddifrif ynglŷn â datblygu siaradwyr Cymraeg a gweld yr iaith yn ffynnu, rhaid newid cyfeiriad, a hynny fel mater o frys cyn ei bod yn rhy hwyr. (Davies, 2013, t. 1)

Pwysleisia Davies (2013) o'r cychwyn cyntaf nad yw cyrhaeddiad presennol CAI yn CA3 a 4 yn ddigon cadarnhaol, a bod diffyg mwynhad yn y pwnc ymhlith disgyblion. Yn ôl Davies, rhaid newid cyfeiriad i sicrhau y bydd disgyblion y sector 
cyfrwng Saesneg yn derbyn addysg effeithiol a dymunol yn y Gymraeg, os yw'r iaith i ffynnu. Mae'r adroddiad yn cyflwyno rhyw bedwar ar hugain o argymhellion i LlC ynghylch sut mae gwella CAI yn CA3 a 4, ond ymhlith yr argymhellion hyn, pwysleisir mai'r prif ffactor yw sicrhau bod statws a phwysigrwydd i CAI megis pwnc craidd yn y cwricwlwm er mwyn creu sylfaen i sicrhau gwelliannau pellach (Davies, 2013, t. 43).

O blith yr argymhellion sy'n cael eu cynnig yn adolygiad Davies, mae sawl argymhelliad nodedig, sef argymhellion 6, 15, 14, 10 a 24. Un o'r argymhellion pennaf a gyflwynir yn yr adroddiad yw Argymhelliad 6 sydd yn galw ar y Llywodraeth i ystyried adolygu'r rhaglen astudio bresennol a chynnwys CAI yn y Fframwaith Llythrennedd Cenedlaethol. Wrth gyflwyno un rhaglen ddysgu, yr hyn a gyfeirir ato fel 'continwwm Cymraeg', byddai holl ddisgyblion Cymru yn cael eu hasesu yn erbyn un fframwaith er mwyn hwyluso'r dilyniant ieithyddol rhwng y Cyfnodau Allweddol, hwyluso mesur galluoedd disgyblion yn y Gymraeg a disodli'r term ‘Cymraeg Ail Iaith' (Davies, 2013, tt. 26-27). Nid yw'r argymhelliad hwn yn un newydd. Yn ei adolygiad polisi o'r Gymraeg, Ein Hiaith, Ei Dyfodol (2002), roedd y Pwyllgorau Diwylliant, ac Addysg a Dysgu Gydol Oes o'r farn:

... [y d]ylai ysgolion cynradd ac uwchradd cyfrwng Saesneg ddatblygu polisïau ar gyfer gwneud mwy o ddefnydd o'r Gymraeg fel cyfrwng dysgu mewn pynciau priodol, gan ystyried cyfarwyddyd priodol, dymuniadau rhieni, a'r staff gyda hyfforddiant addas sydd ar gael (Y Pwyllgor Diwylliant a'r Pwyllgor Addysg a Dysgu Gydol Oes, 2002, t. 13).

Eir ymlaen i nodi bod angen "ymchwil drylwyr i ymarferoldeb mesur hyfedredd disgyblion mewn Cymraeg ar hyd continwwm ieithyddol" (Y Pwyllgor Diwylliant a'r Pwyllgor Addysg a Dysgu Gydol Oes, 2002, t. 13). Cafodd y cysyniad o gyflwyno un continwwm Cymraeg ei argymell hefyd gan ysgolheigion megis Baker a Jones:

New strategies are needed, for example establishing a continuum from early language learning to full fluency, and to move from the current separation of Welsh first- and second-language lessons and Welsh-medium content teaching to establish a concurrent use of both languages in teaching and learning contexts; evolving a bilingual approach in classrooms rather than language separation. (Baker \& Jones, 2000, t. 135). 
Mae gweithredu un continwwm dysgu yn argymhelliad sydd wedi'i gefnogi gan Gymdeithas yr Iaith Gymraeg (2015, 25 Chwefror). Mynegodd y Gymdeithas fod angen sefydlu un continwwm Cymraeg er mwyn disodli'r drefn bresennol o ddysgu Cymraeg fel ail iaith a chyflwyno cyfrannau o addysg drwy gyfrwng y Gymraeg ymhob ysgol yng Nghymru. I'r un perwyl, cydnabu Dyfodol i'r Iaith (2014) fod angen cyflwyno cyfran o addysg drwy gyfrwng y Gymraeg mewn ysgolion cyfrwng Saesneg yn ei ymateb i Iaith fyw: iaith byw - Bwrw mlaen. Yn ogystal â mwy o ddefnydd o'r Gymraeg megis iaith cyfrwng mewn ysgolion cyfrwng Saesneg a gweithredu un continwwm dysgu, awgrymodd adroddiad Un Iaith $i$ bawb hefyd y dylid ystyried darparu cyfnodau dwys o ddysgu'r Gymraeg mewn ysgolion cynradd ac uwchradd cyfrwng Saesneg, yn Argymhelliad 15 yr adroddiad (Davies, 2013, t. $35)$.

Un o flaenoriaethau eraill adroddiad Davies yw'r anghysondeb yn nifer yr oriau a neilltuir ar gyfer addysgu Cymraeg fel ail iaith. Yn Argymhelliad 14, mae'r Grŵp yn galw ar y Llywodraeth i baratoi canllawiau i ysgolion er mwyn sicrhau bod digon o oriau dysgu ac ymarfer yn cael eu gweithredu ar gyfer disgyblion, gydag Awdurdodau Lleol yn adrodd yn rheolaidd ar yr amser y mae ysgolion yn ei glustnodi i addysgu'r Gymraeg trwy eu Cynlluniau Strategol ar gyfer y Gymraeg mewn Addysg. Mae'r Grŵp hefyd yn awgrymu disodli Cwrs Byr CAI, gan ddatblygu cymwysterau newydd sydd yn gyfwerth â TGAU mewn rhaglen astudio ddiwygiedig, yn Argymhelliad 10. Yn yr adroddiad cylch gwaith gan Estyn (2007), sef Arfarniad o'r cwrs byr Cymraeg ail iaith, nodwyd pryderon ynglŷn â'r nifer o oriau a dreulir yn addysgu'r Gymraeg, yn enwedig yn y Cwrs Byr lle y nodwyd bod cyn lleied ag awr o addysg yn y Gymraeg bob pythefnos mewn rhai o'r ysgolion a adolygwyd. Nid yw sefyllfa'r Cwrs Llawn yn gadarnhaol ychwaith, gan y gellir ond disgwyl derbyn rhwng awr i dair awr a hanner o addysg yn y Gymraeg bob wythnos (W. G. Lewis, 2010a, para. 4.5). Rhaid nodi hefyd fod sefyllfa'r Cyfnodau Allweddol cynt yn debyg iawn, lle ceir amrywiaeth yn nifer yr oriau addysgu a neilltuir i'r Gymraeg, rhwng hanner awr i ddwy awr a hanner yr wythnos yn y Cyfnod Sylfaen (CS o hyn ymlaen), deugain munud i ddwy awr a hanner yn CA2, ac un i ddwy awr yn CA3 (W. G. Lewis, 2010a, para. 4.2).

Yn Adroddiad Blynyddol 2012-2013 Estyn (2014b, t. 44), codwyd unwaith eto'r pryderon hyn o ran nifer yr oriau cyswllt yn achos CAI TGAU. Mae'r cyflwyniad i'r 
canfyddiadau ar CAI yn cynnig llun digalon, tebyg i'r un a geir yn adroddiad Davies, wrth i Estyn (2014b, t. 44) fynegi'n ddiamau "nad yw cyrsiau TGAU mewn Cymraeg ail iaith yn cynhyrchu disgyblion dwyieithog na phobl ifanc sy'n ddigon hyderus i ddefnyddio'r iaith yn eu bywydau bob dydd." Mae'r diffyg amser yn y dosbarth i ddysgu'r Gymraeg yn llwyddiannus wedi bod yn un o bryderon cyson Estyn ers rhai blynyddoedd, fel y nodir yn Adroddiad Blynyddol 2007-2008:

Nid yw llawer o ddisgyblion yn gwneud gystal ag y gallen nhw mewn Cymraeg fel ail iaith yng nghyfnod allweddol 4 am nad ydyn nhw'n cael digon o amser addysgu, ac mae ansawdd yr addysgu'n wael yn gyffredinol. (Estyn, 2009, t. 11).

Rhannwyd y pryderon hynny o ran yr nifer gyfyngedig o oriau a neilltuir i'r Gymraeg megis pwnc â Jones, Lewis, George, Thomas a Laugharne (2009). Ar adeg lle'r oedd cwrs newydd ar gyfer CAI ar fin cael ei lansio, credai Jones ac eraill (2009, t. 112) na fyddai nifer yr oriau dysgu yn y cwrs newydd hwn yn ddigonol o hyd. Mae Cen Williams (2002) hefyd wedi beirniadu'r nifer gyfyngedig o oriau dysgu a neilltuir i addysgu'r Gymraeg fel ail iaith. Wrth gloi ei adroddiad Ennill Iaith, pwysleisia Williams (2002, t. 53) bwysigrwydd ystyrlondeb o ran dysgu iaith yn effeithiol, gan nodi bod “profiadau'r gorffennol wedi dangos mai wrth ddefnyddio'r iaith yn gyfrwng y daeth y llwyddiant mwyaf dwys a pharhaol." Mae'r model cyfredol ar gyfer cyflwyno'r Gymraeg a'r Ieithoedd Tramor Modern (ITM o hyn ymlaen) yn y rhan fwyaf o ysgolion cyfrwng Saesneg yng Nghymru, a adwaenir fel 'y dull bwydo' (the drip-feed method) gan Baker (2011), wedi'i feirniadu gan sawl ysgolhaig. Cydnebydd Lightbown a Spada (2006), er enghraifft, fod angen digonedd o oriau cyswllt er mwyn dysgu iaith yn effeithlon. Wrth drafod yr oedran delfrydol a nifer yr oriau y dylid eu neilltuo ar gyfer addysgu'r ail iaith mewn addysg ddwyieithog, noda Lightbown a Spada (2006, t. 74): “[o]ne or two hours a week will not produce advanced second language speakers, no matter how young they were when they began." Mae Archibald ac eraill (2006, t. 3) hefyd yn nodi bod angen nifer sylweddol o oriau cyswllt ag ail iaith er mwyn cyflawni dwyieithrwydd ffwythiannol a rhuglder mewn ail iaith, gan gasglu: "[1]earning a second language for 95 hours per year for six years will not lead to functional bilingualism and fluency in the second language. Expectations must be realistic." 
Mae'r union nifer o oriau cyswllt sydd eu hangen i ddod yn rhugl yn yr ail iaith yn dir dadleuol. Un targed rhesymegol yw'r hyn a gynnig Gruffudd a Morris (2012) yng nghyd-destun canolfannau Cymraeg i Oedolion. Wrth dynnu ar y ffigwr a nodir gan Brifysgol Caergrawnt ar gyfer dysgu Saesneg drwy ESOL, hynny yw, oddeutu 1,000 - 1,200, i gyrraedd lefel uchaf Fframwaith Cyfeirio Cyffredin Ewrop, sef lefel C2, a'r nifer o oriau dysgu a gynigir mewn canolfannau iaith yng Ngwlad y Basg, sef rhwng 1,500 - 1,800, mae Gruffudd a Morris (2012) yn argymell mai oddeutu 1,500 awr yw'r nifer o oriau cyswllt sydd ei hangen er mwyn i siaradwyr ail iaith ddod yn rhugl yn y Gymraeg trwy gyrsiau Cymraeg i Oedolion. Nodir hefyd fod Estyn (2007) wedi cyferbynnu'r nifer o oriau cyswllt â'r Gymraeg yn y cyrsiau dwys ar gyfer oedolion â'r nifer o oriau cyswllt a geir wrth ddilyn y cwrs byr:

...mae'r dyraniad amser a argymhellir o ddwy awr a hanner bob pythefnos ar gyfer y cwrs byr yn parhau i gymharu'n anffafriol â'r oriau cyswllt wythnosol mewn cyrsiau Cymraeg dwys ar gyfer oedolion. Mae angen tua phedair awr yr wythnos o amser cyswllt ar ddysgwyr (gan gynnwys cyswllt ffurfiol ac anffurfiol) i ddod yn rhugl mewn iaith. (Estyn, 2007, t. 14)

Mae cymhariaeth ddiddorol arall, rhwng dysgu Cymraeg fel ail iaith a dysgu ITM, o ran nifer yr oriau cyswllt a geir yn ffurfiol ac yn anffurfiol, wedi'i chodi gan Jones ac eraill (2009). Yn achos CAI ac ITM, caiff yr iaith ei chyflwyno fel pwnc cwricwlaidd trwy gyfrwng y Saesneg, a hynny fel arfer trwy'r dull bwydo. Mae Jones ac eraill (2009, t. 114) o'r farn fod angen ymchwil bellach er mwyn archwilio pedagogeg CLIL (Content and Language Integrated Learning, CLIL o hyn ymlaen) ${ }^{4}$ a chanfod a oes potensial ar gyfer dysgu'r Gymraeg fel ail iaith, ac ITM, drwy ddefnyddio'r iaith darged ei hun ar gyfer dysgu cynnwys y cwricwlwm, yn hytrach na dysgu'r ail iaith fel pwnc mewn gwersi penodedig yn unig, lle nad oes digonedd o gyfleoedd i ddysgu'r iaith mewn modd pwrpasol. Trwy fabwysiadu pedagogeg CLIL felly, y gred yw y bydd yr amser cyswllt â'r ail iaith yn cynyddu wrth i'r ail iaith gael ei defnyddio mewn ffordd ystyrlon.

\footnotetext{
${ }^{4}$ Dyma derm a fathwyd ar ôl i aelodau'r rhywdwaith EuroCLIC gynnal fforymau agored yn y Forum for Mainstream Bilingual Education yn 1996 (Marsh, 2002, t. 63). Mae CLIL yn "generic umbrella term which would encompass any activity in which a foreign language is used as a tool in the learning of a non-language subject in which both language and the subject have a joint curricular role" (Marsh, 2002, t. 58). Mae Jones ac eraill (2009) yma'n estyn ystyr CLIL i gwmpasu dysgu cynnwys mewn addysg cyfrwng Saesneg trwy gyfrwng y Gymraeg. (Gweler 2.5.4. Content and Language Integrated Learning.)
} 
Mewn arolwg cenedlaethol cynhwysfawr gan Board a Tinsley (2015), amlygwyd diffygion o ran yr amser a'r cyfleoedd a neilltuir i addysgu ITM mewn ysgolion uwchradd yng Nghymru. Daeth Board a Tinsley (2015, t. 57) i'r casgliad fod yr amser a neilltuir i addysgu ITM yn CA3, ynghyd â'r diffyg cyfleoedd ar gyfer disgyblion sy'n dymuno astudio ieithoedd yn CA4 i wneud hynny, yn cyfrannu at y sefyllfa bresennol. Daeth Board a Tinsley (2015, t. 57) hefyd i'r casgliad fod y sefyllfa sy'n wynebu ITM mewn ysgolion uwchradd yng Nghymru yr un mor ddifrifol â'r hyn sy'n wynebu CAI, gan nodi: “[o]s yw’r Athro Davies wedi nodi ei bod hi'n 'unfed awr ar ddeg' ar Gymraeg ail iaith, yna mae hi hefyd yn mynd yn hwyr iawn ar ieithoedd tramor modern."

Gan mai pwnc sylfaenol yw CAI, yn hytrach na phwnc craidd fel CIG, a chan y caiff yr amser a neilltuir i astudio'r Gymraeg mewn ysgolion cyfrwng Saesneg ei benderfynu gan yr ysgolion eu hunan yn lleol, fel pob pwnc arall yn y cwricwlwm (Y Pwyllgor Menter a Dysgu, 2010, t. 17), nid syndod yw canfod bod nifer yr oriau cyswllt â'r Gymraeg mewn llawer o achosion yn annigonol. Wrth gymharu canfyddiadau Lewis (2010a) â'r nifer o oriau dysgu a argymhellir uchod gan Gruffudd a Morris (2012) er enghraifft, mae'n amlwg nad yw'r amser a dreulir yn addysgu CAI yn ddigonol i ddatblygu sgiliau dwyieithog disgyblion erbyn diwedd eu haddysg orfodol.

Thema arall a ddaw i'r amlwg wrth ddarllen adroddiad Davies (2013) yw'r cysyniad o gysylltu dysgwyr ail iaith â'r gymuned Gymraeg y tu allan i'r dosbarth ffurfiol. Yn Argymhelliad 24 yr adroddiad, gelwir ar LlC i sicrhau cyfleoedd anffurfiol i ddisgyblion ymarfer eu Cymraeg a chlywed yr iaith yn cael ei siarad, y tu mewn yn ogystal â'r tu allan i'r ysgol. Gan na ellir dibynnu ar addysg ar ei phen ei hun wrth adfer a gwarchod iaith, rhaid hefyd ystyried ei defnyddio a sut mae ei throsglwyddo i'r cartref a'r gymuned er mwyn integreiddio siaradwyr newydd i'r gymuned Gymraeg. Gan werthfawrogi pwysigrwydd y gymuned a'r cartref ar lwyddiant adfer iaith, dywed Jones (1997):

Nid yw twf ysgolion Cymraeg na chynnydd yn y sector ail iaith o angenrheidrwydd yn gwarantu y bydd y Gymraeg yn cael ei throsglwyddo drwy'r cartref i blant y disgyblion presennol. Dyna'r broses naturiol o drosglwyddo iaith a'r ffordd fwyaf dibynadwy o fesur llwyddiant polisïau adfer iaith o fewn y gyfundrefn addysg. (Jones, 1997, t. 379) 
Mae eraill (Gruffudd \& Morris, 2012; Jones, 2011) hefyd wedi nodi pwysigrwydd trosglwyddo'r iaith o'r dosbarth i'r gymuned. Awgryma Gruffudd a Morris (2012) mai trwy ddarparu canolfannau Cymraeg, a fyddai'n creu llwyfan i ddysgwyr ail iaith bontio rhwng yr ysgol uwchradd a'r coleg neu'r brifysgol, yn ogystal â bod yn hwb lleol i'r Gymraeg, y gellir cysylltu dysgwyr a siaradwyr rhugl â'i gilydd yn y gymuned. Yn ystod Eisteddfod 2014, cyhoeddodd y Prif Weinidog Carwyn Jones y byddai L1C yn buddsoddi $£ 1.25$ miliwn mewn colegau, prifysgolion ac Awdurdodau Lleol er mwyn iddynt ddatblygu canolfannau Cymraeg o'r fath (BBC Cymru Fyw, 2014, 6 Awst). At hynny, cyhoeddwyd dri mis yn ddiweddarach y byddai $£ 1$ miliwn pellach yn cael ei ychwanegu er mwyn bodloni'r galw am gymorth ariannol i ddatblygu rhagor o ganolfannau iaith (LlC, 2014b). Trwy fanteisio ar ganolfannau o'r fath mewn cymunedau cymharol ddi-Gymraeg felly, gellir dadlau fod cyfle yma i ddisgyblion mewn ysgolion cynradd ac uwchradd cyfrwng Saesneg gysylltu fwyfwy â'r gymuned leol â siaradwyr rhugl yn y Gymraeg. Yng nghyd-destun y traethawd hwn, ymddengys yn glir fod angen rhoi ystyriaeth i'r defnydd allgyrsiol, cymunedol o'r Gymraeg o amgylch yr ysgol a'r tu hwnt, yn ogystal â'i defnydd fel iaith addysgol yn y dosbarth, wrth ystyried sut orau y gellir cefnogi cyflwyno'r Gymraeg fel ail iaith.

Thema arall i'w nodi yn adroddiad Un iaith i bawb yw'r pryder nad yw'r Gymraeg yn cael ei haddysgu mewn ffordd sydd yn ei gwneud yn berthnasol, diddorol nac yn fuddiol i ddysgwyr y tu hwnt i'r dosbarth. Mae ysgogi disgyblion yn y dosbarth yn holl bwysig yn y broses o addysgu'r ail iaith yn llwyddiannus, a daw hyn trwy sicrhau bod cynnwys y dysgu'n briodol, diddorol ac yn heriol i'r disgyblion, a bod amgylchedd dysgu cadarnhaol yn ei le (Lightbown \& Spada, 2006, t. 64). Caiff y pryder hwn ei godi gan Davies (2013) yn rhagair yr adolygiad, wrth iddi dynnu ar dystiolaeth a dderbyniwyd gan y Grŵp Adolygu:

Profiad diflas dros ben ydyw i lawer iawn ohonynt yn ôl y dystiolaeth - nid ydynt yn gweld y pwnc yn berthnasol nac o unrhyw fudd iddynt. Nid ydynt yn ddigon hyderus i ddefnyddio'r Gymraeg y tu allan i'r dosbarth - yn wir, prin iawn yw'r cyfleoedd i wneud hynny - ac nid oes unrhyw gymhelliad felly i ddysgu'r iaith. (Davies, 2013, t. 1)

Nid ffaith newydd yw hon, wrth gwrs. Mae'r pryder hwn wedi cael ei godi gan eraill megis Lewis (2010b, para. 12), a gyfeiriodd at ddiffyg ymgysylltu â'r Gymraeg 
ymhlith disgyblion CA4 yn benodol. I'r un perwyl, nodwyd mai "anfodlonrwydd ymhlith disgyblion CA4 yw un o'r meysydd sy'n cynnig yr her fwyaf' yn Ein Hiaith, Ei Dyfodol (Y Pwyllgorau Diwylliant, ac Addysg a Dysgu Gydol Oes, 2002, t. 60).

Fodd bynnag, yr hyn a gesglir yn bennaf o'r adroddiad hwn yw'r angen am newid sylfaenol ar raddfa uchelgeisiol o ran sut y cyflwynir y Gymraeg mewn addysg cyfrwng Saesneg. Fel y noda Davies (2013) yng nghasgliad ei hadroddiad:

Rhaid i'r newidiadau sydd eu hangen i hwyluso'r gwelliant hwn gael eu cefnogi drwy system addysg gyfan Cymru; mae llwyddiant yn dibynnu ar athrawon unigol yn cael cefnogaeth gan eu hadrannau, eu hysgolion a'u hawdurdodau lleol; ac mae gan Lywodraeth Cymru, ar lefel genedlaethol, ran allweddol o ran newid y cwricwlwm a'r trefniadau asesu ac o ran darparu hyfforddiant i hwyluso'r newidiadau. (Davies, 2013, t. 43).

Mae'r awydd am newid uchelgeisiol wedi'i rannu gan nifer o ysgolheigion sydd wedi cytuno nad yw ymdrechion diweddaraf y Llywodraeth wedi bod yn ddigon uchelgeisiol yn eu hanfod hyd yma er mwyn gwireddu ei gweledigaeth am weld y Gymraeg yn ffynnu. Yn ei drafodaeth, A fydd y Cymry Cymraeg mewn pryd?, mae Jones (2011) wedi nodi diffyg uchelgais o ran cynllunio ar gyfer y Gymraeg megis ffactor i'w hystyried yn ddwys. Mae Jones (2011) yn mynd yn ei flaen i feirniadu cynnydd y Gymraeg mewn polisi ac addysg wrth ddisgrifio datblygiadau'r ganrif ddiwethaf fel a ganlyn:

Sefydlwyd yn y ganrif ddiwethaf ryw fath o uniongrededd protestiol. Y diwylliant protest. Nid protest, ond y ddibyniaeth ar arfer protest. Rhoddwyd gormod o bwyslais ar statws, ar iaith swyddogol, ac ar rym deddf. Heblaw hynny, tybiwyd y byddai'r cynnydd enbyd o araf mewn addysg yn ddigonol. (Jones, 2011, t. 16)

Yn ôl Jones (2011), mae'n rhaid i'r Cymry Cymraeg sylweddoli'r darlun mwy. Ni ellir gwneud cynnydd sylweddol yn y broses o adfer yr iaith os nad ydynt yn newid strategaeth, gan nad “yw'r cynlluniau gwleidyddol, cyfreithiol, cymdeithasol, nac addysgol presennol yn ddigon uchelgeisiol ar gyfer 'adfer' yr iaith Gymraeg, er pwysiced ydynt o 'hawliau'.” (Jones, 2011, t. 3). Er mwyn ennill tir yn y meysydd gwleidyddol, cyfreithiol, cymdeithasol ac addysgol felly, dadleua Jones fod angen i'r ymdrechion hyn fod yn fwy uchelgeisiol yn eu hanfod. 


\subsubsection{Dyfodol Llwyddiannus (2015)}

Yn ogystal â'i bwysigrwydd megis adolygiad annibynnol cyfoes o CAI yn CA3 a 4, mae adroddiad Davies a'u canfyddiadau at ei gilydd wedi ffurfio casgliad o dystiolaeth sylweddol ac argymhellion cynhwysfawr a gafodd eu hystyried gan yr adolygiad diweddar o'r cwricwlwm, sef Dyfodol llwyddiannus (2015). Yn 2014, cyhoeddwyd gan y Gweinidog Addysg a Sgiliau ar y pryd Huw Lewis (2014, 12 Mawrth) y byddai adolygiad cynhwysfawr o'r cwricwlwm yn cael ei gynnal er mwyn adeiladu ar ymgynghoriad diweddar y Llywodraeth ar wella safonau llythrennedd a rhifedd yn y cwricwlwm. Mae'r adroddiad, a gafodd ei gynnal dan arweiniad yr Athro Grahame Donaldson, yn tynnu ar sawl adroddiad annibynnol unigol, gan gynnwys Un Iaith i Bawb, wrth gyflwyno argymhellion ar gyfer diwygio'r cwricwlwm.

Mewn ymateb i'r pedwar ar hugain o argymhellion a gyflwynwyd yn yr adolygiad, cafodd deg argymhelliad mewn perthynas â'r Gymraeg yn y cwricwlwm eu cyflwyno yn adolygiad Donaldson (2015, t. 115) o'r cwricwlwm, Dyfodol Llwyddiannus. Datganodd Huw Lewis (2015, 15 Hydref) y byddai'n derbyn pob un o'r argymhellion mewn perthynas â'r Gymraeg. O blith yr argymhellion hyn fodd bynnag, nodir mai ond tri argymhelliad sy'n nodedig:

22. Dylai ysgolion ganolbwyntio o'r newydd ar ddysgu Cymraeg yn bennaf fel ffordd o gyfathrebu, yn enwedig cyfathrebu a deall yr iaith lafar.

26. Dylai ysgolion cyfrwng Cymraeg weithio fel canolbwynt ar gyfer y Gymraeg, er mwyn helpu athrawon ac ymarferwyr mewn ysgolion cyfrwng Saesneg.

28. Dylai Llywodraeth Cymru alinio cymwysterau iaith Gymraeg yn 16 oed â'r pwyslais arfaethedig ar siarad a gwrando a defnyddio'r iaith yn y gweithle. (Donaldson, 2015, t. 60)

Er bod Argymhelliad 22 yn galw ar ysgolion i ganolbwyntio ar gyflwyno'r Gymraeg fel ffordd o gyfathrebu gan ganolbwyntio'n fwy ar y sgiliau cyfathrebol, nodir bod hyn eisoes wedi bod yn digwydd mewn nifer o gyrsiau dysgu Cymraeg y tu hwnt i addysg. Mae cyrsiau ar-lein megis Say Something in Welsh, llyfrau hunan-ddysgu megis Live Welsh gan Gruffudd (2012), meddalwedd dysgu iaith megis Rosetta Stone, ynghyd â dosbarthiadau iaith megis Cariad@iaith a Chymraeg i Oedolion yn enghreifftiau o gyrsiau Cymraeg lle canolbwyntir ar ddeall a defnyddio'r iaith lafar. 
Mae'r argymhelliad hwn felly yn dangos symudiad ym maes addysg cyfrwng Saesneg tuag at ganolbwyntio o'r newydd ar ddatblygu sgiliau llafar. Fodd bynnag, er pwysiced datblygu sgiliau cyfathrebol, rhaid hefyd sicrhau nad yw'r pwyslais hwn ar lafaredd yn y cwricwlwm newydd yn dod ar draul datblygu'r sgiliau ysgrifennu, gan fod y sgiliau ysgrifennu yn hollbwysig wrth greu gweithlu dwyieithog yn y dyfodol.

Er bod Dyfodol Llwyddiannus yn cynnig cyfle arbennig i ddiwygio'r gyfundrefn addysg gyfan yng Nghymru, mae rhai carfannau pwyso wedi beirniadu'r adolygu gan awgrymu nad yw ei argymhellion yn cyflwyno newidiadau gwirioneddol bellgyrhaeddol ar gyfer addysgu'r Gymraeg yn y sector cyfrwng Saesneg. Er bod Dyfodol i'r Iaith (2015, para. 2) yn croesawu'r pwyslais cynyddol ar sgiliau cyfathrebu, mae'n feirniadol o adroddiad Donaldson am nad yw'n cydnabod "rôl addysg Gymraeg wrth lunio gwlad ddwyieithog" na'r angen am "ehangu addysg Gymraeg ar raddfa fawr.” Ceir ymateb tebyg i hyn gan Rieni dros addysg Gymraeg (RhAG) sydd hefyd yn beirniadu'r diffyg cyfeiriad tuag at addysg Gymraeg. Dywed Cyfarwyddwr Datblygu RhAG, Ceri Owen (2015b):

Mae'n dda bod yr adroddiad am weld y Gymraeg yn cael ei datblygu'n iaith drafod mewn ysgolion Saesneg. Y ffordd orau o wneud hyn yw defnyddio'r iaith fel cyfrwng, a dyw'r adroddiad ddim yn cynnig ffordd ymlaen yn hyn o beth. (RhAG, 2015b, para. 3).

Mae Cymdeithas yr Iaith Gymraeg (2015, 8 Mai) hefyd wedi cynnig ymateb i adroddiad Donaldson. Er ei bod yn cefnogi'r angen am i ysgolion cyfrwng Cymraeg gydweithio gydag ysgolion cyfrwng Saesneg ac am roi pwyslais cynyddol ar y sgiliau siarad a gwrando yn Gymraeg, nododd y Gymdeithas nad yw'r adroddiad yn manylu ar sut y gellir rhoi argymhellion adroddiad Davies ar waith y tu mewn i fframwaith y cwricwlwm newydd. Dadleuwyd hefyd fod angen integreiddio'r Gymraeg ar draws y cwricwlwm cyfan ar yr un raddfa ag y mae'r adroddiad yn ei wneud â'r elfen ddigidol. Ys nododd Cymdeithas yr Iaith Gymraeg (2015, 25 Chwefror, para. olaf): “[t]ra bod yr adroddiad yn rhoi cryn bwyslais ar integreiddio'r elfen ddigidol ar draws y cwricwlwm, nid yw'n trin y Gymraeg yn yr un modd." 


\subsubsection{Cymraeg 2050: Miliwn o siaradwyr (2017)}

Efallai mai un o'r datblygiadiau diweddar mwyaf arwyddocaol ym mholisi iaith ac addysg yng Nghymru oedd lansiad y strategaeth newydd ar gyfer y Gymraeg yn 2017. Ar ôl cynnal ymgynghoriad ar ei strategaeth ddrafft rhwng 1 Awst a 31 Hydref 2016 (2017c), lansiodd LIC (2017b) ei strategaeth newydd ar gyfer y Gymraeg, sef Cymraeg 2050: Miliwn o siaradwyr, ym mis Gorffennaf 2017. Yn ogystal â chanolbwyntio ar gaffaeliad a defnydd o'r Gymraeg, fel y nodwyd yn y strategaeth flaenorol Iaith fyw: Iaith byw, mae Cymraeg 2050 hefyd yn canolbwyntio ar sut mae creu amodau ffafriol i'r iaith.

Sut bynnag, yn wahanol i'r strategaeth flaenorol, ceir yn y strategaeth gyfredol ymrwymiad cryfach i ddatblygu'r Gymraeg, wrth i LlC (2017b) osod ei gweledigaeth uchelgeisiol o gyrraedd miliwn o siaradwyr Cymraeg erbyn 2050. O ran addysgu'r Gymraeg, ceir ffocws newydd ar CAI, wrth i LlC (2017b, t. 38) nodi bod "cyfraniad pwysig i'w wneud gan y sector cyfrwng Saesneg" at wireddu'r weledigaeth uchelgeisiol hon. Cydnabyddir bellach fod angen "[g]weddnewid sut rydym yn addysgu Cymraeg i ddysgwyr yr ysgolion hynny”, a hynny drwy ddatblygu "un continwwm ar gyfer addysgu'r Gymraeg, gan bwysleisio dysgu Cymraeg yn bennaf fel modd o gyfathrebu, yn enwedig cyfathrebu ar lafar" (LlC, 2017b, t. 38).

Er bod y strategaeth newydd wedi dangos ymrwymiad cryfach ar ran y Llywodraeth i ddatblygu'r Gymraeg mewn addysg cyfrwng Saesneg, mae'r Pwyllgor Diwylliant, y Gymraeg a Chyfathrebu (2017) wedi mynegi ei bryder ynglŷn â dehongliad LlC o’r cysyniad o'r 'continwwm ieithyddol'. Yn ôl y Pwyllgor (2017, t. 5), "gwella rhuglder disgyblion ym mhob ysgol" yw'r flaenoriaeth wrth ddatblygu'r continwwm ieithyddol, nid "canolbwyntio gormod ar newid categori iaith yr ysgol." Ymddengys felly fod angen i LlC roi ystyriaeth lawnach i'r cysyniad o gyflwyno 'un continwwm Cymraeg' cyn ei gyflwyno ym mhob ysgol yng Nghymru. (Am drafodaeth lawnach, gweler 2.8. Y continwwm.)

Wrth i LlC ddatblygu ei strategaeth newydd ar gyfer yr iaith, gosododd hefyd ei blaenoriaethau ar gyfer y strategaeth newydd ar gyfer y Gymraeg mewn addysg yn Strategaeth Addysg Cyfrwng Cymraeg: y camau nesaf (2016c). Gan ddiwygio'r strategaeth flaenorol Strategaeth Addysg cyfrwng Cymraeg (2010), a amlinellwyd yn 
Gwerthusiad o'r Strategaeth Addysg Cyfrwng (2016b), cyhoeddodd LlC ei strategaeth newydd ar gyfer addysg cyfrwng Cymraeg a Chymraeg dros y pedair blynedd nesaf, sef Y Gymraeg mewn Addysg: Cynllun Gweithredu 2017-2021, ym mis Rhagfyr 2017. Unwaith eto, ceir yn y ddogfen hon ffocws pellach ar rôl ysgolion cyfrwng Saesneg yn y broses o wireddu'r uchelgais a osodwyd yn Cymraeg 2050. Wrth drafod argymhellion adroddiad Donaldson (2015) ar gyfer rôl y Gymraeg yn y cwricwlwm newydd, noda LlC y canlynol:

Derbyniom argymhellion yr adolygiad a chytunom y byddai continwwm dysgu Cymraeg yn cael ei ddatblygu fel rhan o'r cwricwlwm a'r trefniadau asesu newydd i Gymru. Bydd y continwwm hwn yn sail ar gyfer addysgu a dysgu Cymraeg ac ieithoedd eraill yn y dyfodol. (LlC, 2017ch, t. 10)

Er na cheir manylder o ran sut y bydd LlC yn cyflwyno'r continwwm Cymraeg arfaethedig, ynghyd â'i disgwyliadau ar gyfer y sector cyfrwng Saesneg ynglŷn â chyrhaeddiad mewn Cymraeg, ymddengys fod y ddwy strategaeth yn gosod sylfaen cadarn ar gyfer gwella rôl y Gymraeg mewn addysg cyfrwng Saesneg, yn enwedig yng ngoleuni'r diwygiad presennol o'r Cwricwlwm Cenedlaethol.

\subsection{Y canfyddiadau diweddar parthed arfer ym maes Cymraeg Ail Iaith}

Er i adroddiad Sioned Davies (2013) ganolbwyntio'n benodol ar CA3 a 4, nodir nad yw heriau'r Cyfnodau Allweddol hyn yn digwydd mewn gwagle. Rhaid sicrhau bod y CS a CA2 hefyd yn ddigon cryf (Davies, 2013, t. 2). Ystyrir yma'r Gymraeg a'i dilyniant o'r sector cynradd i'r sector uwchradd, gan roi ystyriaeth i'r canfyddiadau ar arfer yn y ddau sector. Er bod nifer o heriau gwahanol yn cael eu hwynebu gan y ddau sector, nodir eu bod hwythau yn rhannu rhai o'r un heriau â'i gilydd yn ystod y Cyfnod Pontio. ${ }^{5}$ Oherwydd hynny, rhennir y bennod hon yn dair er mwyn trafod heriau'r sector cynradd, y Cyfnod Pontio, a'r sector uwchradd, yn eu tro.

\subsubsection{Y sector cynradd}

Yn yr adroddiad blynyddol diweddaraf gan Estyn (2018, t. 66), nodwyd nad yw safonau CAI mewn ysgolion cynradd wedi gwella ryw lawer ers y saith mlynedd ddiwethaf, a hynny mae'n debyg, oherwydd nifer o wendidau. Er bod athrawon yn aml yn cynllunio'n briodol ar gyfer datblygu'r Gymraeg o fewn gwersi Cymraeg

\footnotetext{
${ }^{5}$ Hynny yw'r cyfnod rhwng gadael yr ysgol gynradd (diwedd CA2) ac ymuno âr ysgol uwchradd (dechrau CA3).
} 
ffurfiol, ymddengys o ganfyddiadau adroddiadau blynyddol diweddar Estyn (2018, 2017, 2016b) nad yw medrau disgyblion y sector cynradd yn cael eu hestyn y tu allan i'r gwersi hyn. Nododd Estyn (2016b) fod hyn yn effeithio ar allu disgyblion ar lafar, ynghyd â'u hyder cyffredinol wrth siarad Cymraeg:

...mae llai o sylw'n cael ei roi i atgyfnerthu medrau llafar disgyblion a hyrwyddo'r defnydd o'r Gymraeg y tu allan i wersi. O ganlyniad, nid yw llawer o ddisgyblion yn defnyddio'r Gymraeg yn hyderus, ac mae'n parhau'n faes i'w wella mewn llawer o ysgolion. (Estyn, 2016b, t. 42)

Nodwyd hyn hefyd yn yr adroddiad blynyddol blaenorol (Estyn, 2015), wrth ychwanegu bod diffyg cyfleoedd i ddisgyblion ddatblygu eu medrau ysgrifennu y tu allan i'r gwersi Cymraeg. Yn ogystal, adroddodd Estyn (2015) fod diffyg hyder ymhlith disgyblion o ran defnyddio'r Gymraeg wrth drafod pynciau bob dydd. Nododd Estyn (2015, t. 63): “[y]n y rhan fwyaf o ysgolion, nid yw disgyblion yn defnyddio'u medrau llefaredd ac ysgrifennu Cymraeg ymhellach na gwersi Cymraeg. Nid oes hyder ganddynt ac maent yn amharod i gyfranogi mewn sgyrsiau byr ynglŷn â phynciau bob dydd." Cafodd y diffygion o ran datblygu llefaredd Cymraeg disgyblion eu bwysleisio unwaith eto yn yr adroddiad blynyddol diwethaf: "Mewn rhyw chwech o bob deg ysgol cyfrwng Saesneg, nid oes digon o ffocws ar ddatblygu medrau llefaredd Cymraeg disgyblion mewn gwersi Cymraeg, mewn meysydd eraill o'r cwricwlwm ac yn ystod gweithgareddau anffurfiol." (Estyn, 2018, t. 69).

Yn ogystal â diffyg hyder o ran siarad ac ysgrifennu'r Gymraeg y tu hwnt i'r gwersi ffurfiol, ymddengys fod angen i ysgolion cynradd ganolbwyntio'n arbennig ar sgiliau darllen disgyblion, er mwyn i ddisgyblion ddatblygu'r hyn a drafodir ar lafar ac yn ysgrifenedig yn well:

Mae gan y rhan fwyaf o ddisgyblion fedrau darllen nad ydynt wedi'u datblygu gystal â'u medrau siarad ac ysgrifennu, ac mae llawer yn ei chael hi'n anodd trafod yr hyn maent wedi'i ddarllen. Ychydig iawn o ddisgyblion sydd â llyfr darllen Cymraeg. (Estyn, 2015, t. 63)

Mae'r canfyddiadau hyn yn datgelu bod safonau cyflawniad presennol disgyblion cynradd mewn CAI yn gyffredinol yn is na'r disgwyl. Nid maes trafod newydd yw safonau isel CAI yn y sector cynradd, fodd bynnag. Yn ei arolwg o'r Gymraeg fel ail iaith yn y sector cynradd, sef Arolwg o'r Gymraeg fel ail iaith yng Nghyfnodau Allweddol 2 a 3 a'r Cyfnod Pontio, adroddodd Estyn (2004, t. 3) na "fu cynnydd 
sylweddol mewn safonau da" yn y sector cynradd "er bod y gwaith anfoddhaol wedi gostwng." Ymddengys mai un o'r rhesymau am safonau isel yn y sector cynradd yw diffyg hyfforddiant $\mathrm{i}$ athrawon. Ar y cyfan, nid oes gan athrawon cynradd ddigon o hyder ynglŷn â datblygu defnyddio'r Gymraeg o fewn gwersi Cymraeg na'r tu hwnt i'r gwersi ffurfiol hyn: “[m]ae'r rhan fwyaf o ysgolion yn ymateb i anghenion hyfforddi staff yn briodol. Fodd bynnag, mae diffyg hyder ymhlith staff o ran addysgu Cymraeg fel ail iaith ac mae cyfleoedd i wella'r medrau hyn yn gyfyngedig yn aml." (Estyn, 2015, t. 69).

Mae astudiaeth gan W. Gwyn Lewis (2010a) yn atgyfnerthu'r canfyddiad hwn. Yn ei astudiaeth ar ysgolion cynradd ac uwchradd, canfu fod rhyw $40 \%$ o athrawon cynradd o'r farn nad yw'r cyfleoedd i ddatblygu'n broffesiynol, sef mynychu gweithgareddau Hyfforddiant Mewn Swydd (HMS) er mwyn datblygu eu medrau yn y Gymraeg a'u methodoleg addysgu Cymraeg fel ail iaith, yn ddigonol. Cafodd y pryder hwn ynglŷn â darparu hyfforddiant i athrawon mewn ysgolion cyfrwng Saesneg ei nodi eisoes gan Cennard Davies mewn adroddiad i'r Pwyllgor Menter a Dysgu (2010). Roedd Davies o'r farn fod angen darparu hyfforddiant er mwyn i'r athrawon hyn yn y sector cyfrwng Saesneg ddatblygu eu Cymraeg, yn arbennig ar adeg pan fo angen cynnydd yn y nifer o athrawon sy'n medru'r Gymraeg. Ys dywedodd Davies (d.d., para. 4): "[i]n the sixties, there were intensive courses available to introduce the Welsh language to non-Welsh speaking teachers. Unfortunately, those courses have more or less disappeared, at a time when the demand for Welsh teachers is growing."

Adroddodd Estyn (2004) nad yw gwaith disgyblion ar ddiwedd CA2 yn ddigon heriol er mwyn iddynt adeiladu ar y cynnydd a wneid yn y cyfnod blaenorol. Ar ben hyn, nodwyd bod diffyg arbenigedd gan athrawon o ran addysgu Cymraeg fel ail iaith, yn enwedig mewn ysgolion cynradd yn yr ardaloedd di-Gymraeg, a hynny oherwydd bod "gan gyfran sylweddol o athrawon sy'n addysgu yng nghyfnodau allweddol 1 a 2 wybodaeth gyfyngedig o'r Gymraeg" eu hunain (Estyn, 2004, t. 24). Oherwydd hyn, cred Estyn fod gan athrawon ddisgwyliadau isel iawn, yn arbennig yn ystod Blynyddoedd 5 a 6 . Ymddengys felly nad yw disgyblion CA2 yn cael eu herio'n ddigonol: “[m]ae diffyg arbenigedd athrawon yn fwyaf amlwg ar ddiwedd cyfnod allweddol 2. Yn aml mae gan ysgolion ddiffyg athrawon Cymraeg arbenigol 
ym Mlynyddoedd 5 a 6 i alluogi disgyblion i gyflawni eu llawn botensial yn y pwnc.” (Estyn, 2004, t. 24).

Cadarnhawyd y pryder hwn o ran diffyg hyfforddiant i athrawon cynradd yn adroddiad y Pwyllgor Menter a Dysgu (2010), a gasglodd dystiolaeth oddi wrth Gareth Wyn Roberts a'r Gwasanaeth Addysg a Gwella Ysgolion (ESIS). Yn hytrach na chondemnio 'disgwyliadau isel' athrawon fodd bynnag, mae Roberts yn canmol athrawon cynradd gan eu bod yn cyflwyno'r Gymraeg yn frwdfrydig hyd eu gallu. Er hynny, cyfeddyf nad oes gan y rhan fwyaf o'r athrawon hyn yr arbenigedd i addysgu ail iaith yn effeithiol:

Problem fawr yw bod athrawon ysgol cynradd [sic] sy'n addysgu amrediad o bynciau, gydag eithriadau prin, heb y sgiliau arbenigol i addysgu ail iaith, pa un ai ydynt yn siarad Cymraeg ai peidio eu hunain.

(Roberts, 2010, 25 Mawrth, "Gwella safonau yn y Gymraeg (ail iaith) ar y lefel gynradd", para. 4)

Er yr awgryma'r dystiolaeth fod y rhan fwyaf o ddisgyblion yn y CS yn mwynhau dysgu'r Gymraeg ac yn datblygu agweddau cadarnhaol ati (Estyn, 2013, 2014, 2015, 2017), a bod gan lawer y gallu i ofyn ac ateb cwestiynau syml gyda hyder (Estyn, 2015), ymddengys na chaiff disgyblion eu herio'n ddigonol wrth iddynt gyrraedd CA2, a hynny oherwydd diffyg hyder ymhlith athrawon i addysgu'r Gymraeg i ddisgyblion hŷn (Estyn, 2013a, "Rhan 3: Adroddiad sector”, t. 3)

Cadarnhaodd arolygiadau Estyn (2003) ac OldBell 3 a Dateb (2011) ar waith Athrawon Bro fod achosion, yn enwedig yn yr ardaloedd cymharol ddi-Gymraeg yng Nghymru, lle mae athrawon cynradd yn dibynnu'n helaeth ar arbenigedd gwasanaeth Athrawon Bro i gyflwyno'r Gymraeg. Yn adroddiad Estyn (2003, t. 23) ar wasanaeth Athrawon Bro, galwyd am i Athrawon Bro "gynghori athrawon dosbarth", yn hytrach nag "addysgu dosbarthiadau yn uniongyrchol", gan y byddai athrawon cynradd yn parhau i orddibynnu ar y gwasanaeth yn hytrach na datblygu eu medrau Cymraeg eu hunain. Er i adroddiad OldBell3 a Dateb (2011) nodi symudiad o ran ffocws gwaith yr Athrawon Bro ers adroddiad Estyn (2003), mae'n ymddangos fod ysgolion cynradd yn dal i ddibynnu'n helaeth ar y cymorth hwn. Mae canfyddiad Lewis (2010a, para. 9.1.) fod 96\% o'r prifathrawon ysgolion cynradd a holwyd o'r farn na fyddai'n bosibl cyflwyno CAI heb gymorth yr Athrawon Bro hefyd yn cadarnhau'r tueddiad hwn. Casglodd adroddiad OldBell3 a Dateb $(2011$, t. 86) fod 
“angen i Awdurdodau Lleol symud disgwyliadau ysgolion, fel eu bod yn cymryd cyfrifoldeb dros eu capasiti iaith eu hunain, yn hytrach na dibynnu ar Athrawon Bro i gau bylchau yn sgiliau ieithyddol eu staff."

Maes trafod pwysig arall yw'r diffyg oriau addysgu a neilltuir yn y sector cynradd cyfrwng Saesneg i gyflwyno'r Gymraeg. Fel y cyfeiriwyd ati eisoes (gweler 1.2.5. Un Iaith i Bawb) mae Lewis (2010a, "Primary sector", para. 4.2) wedi nodi yn ei bapur i'r Pwyllgor Menter a Dysgu (2010) fod yr amser a neilltuir i addysgu'r Gymraeg bob wythnos yn y CS a CA2 yn amrywio'n fawr, rhwng hanner awr i ddwy a hanner a rhwng pedwar deg munud i ddwy awr a hanner, yn ôl eu trefn. Dangosodd astudiaeth Lewis y credai rhyw 75\% o'r prifathrawon a holwyd nad yw'r nifer o oriau a neilltuir i Gymraeg fel ail iaith yn yr ysgol yn ddigonol i gyflawni gofynion y Rhaglenni Astudio, yn arbennig o ran darllen ac ysgrifennu yn CA2. Er i Estyn (2015) sylwi ar gynnydd mewn ychydig o ysgolion cynradd o ran defnyddio'r Gymraeg yn ddyddiol neu mewn sesiynau byrion, ymddengys nad yw hyn yn digwydd yn y rhan fwyaf o achosion. Fel y dangosodd Lewis (2010a, "Primary sector", para. 4.2), mae rhyw 90\% o'r ysgolion cynradd yn ei astudiaeth yn cyflwyno'r Gymraeg mewn slot amser dynodedig yn unig, fel petai'n wers CAI. Nodir bod 52\% o'r rheini a holwyd yn yr astudiaeth hon o blaid cyflwyno rhannau'r cwricwlwm yn ddwyieithog, ar yr amod na fyddai hyn ar draul datblygiad y Saesneg. Ymddengys o'r canfyddiadau fod y cyswllt â'r Gymraeg a'i defnydd ffurfiol ac anffurfiol yn y sector cynradd yn gyfyngedig iawn, a hynny, debyg iawn, oherwydd nad yw cyfran sylweddol o'r athrawon yn y sector hwn yn ddigon cymwys i gyflwyno'r Gymraeg fel ail iaith yn effeithiol (Roberts, 2010, 25 Mawrth), nac yn ddigon hyderus i ddefnyddio'r iaith y tu hwnt i'r gwersi Cymraeg (Estyn, 2015, 2016b). Mae'n amlwg fod y diffyg cymhwyster a hyder wrth gyflwyno Cymraeg fel ail iaith fel pwnc, yn arbennig yn CA2, yn deillio'n bennaf o ddiffyg hyfforddiant o ran methodoleg addysgu Cymraeg fel ail iaith (Y Pwyllgor Menter a Dysgu, 2010; C. Davies, d.d.; Estyn, 2004, 2015; Lewis, 2010a; Roberts, 2010, 25 Mawrth). Mae'r llenyddiaeth hefyd yn datgelu bod diffyg sgiliau yn y Gymraeg yn gyffredinol ymhlith athrawon cynradd, yn arbennig yn yr ardaloedd cymharol ddi-Gymraeg (Estyn, 2004), a chaiff hyn yn ei dro effaith ar hyder athrawon cynradd wrth gyflwyno'r Gymraeg fel iaith achlysurol. Fel yr awgrymodd arolygiadau Estyn (2003) ac OldBell3 a Dateb (2011), ni ddylai athrawon ddibynnu ar gymorth 
gwasanaeth Athrawon Bro er mwyn cyflwyno'r Gymraeg yn effeithiol. Yn hytrach, mae angen sicrhau'r hyfforddiant priodol i athrawon, yn ogystal â mynediad ato, er mwyn ymateb i'r galw am athrawon sy'n medru addysgu'r Gymraeg yn effeithiol (C. Davies, d.d.). Mae'n hollbwysig hefyd y caiff yr athrawon hyn eu hyfforddi hyd at safon lle maent yn gallu cynllunio'r Gymraeg yn effeithiol nid yn unig rhwng CA 1 a 2, ond hefyd rhwng CA 2 a 3, er mwyn sicrhau dilyniant ieithyddol disgyblion o'r sector cynradd i'r sector uwchradd.

\subsubsection{Y cyfnod pontio: Dilyniant rhwng y sectorau cynradd ac uwchradd}

Mae arwyddocâd sicrhau dilyniant rhwng y sector cynradd a'r sector uwchradd wedi'i amlygu orau mewn arolygiadau gan Estyn (2004) a'r Pwyllgor Menter a Dysgu (2010). Yn ei adroddiad thematig, Arolwg o'r Gymraeg fel ail iaith yng Nghyfnod Allweddol 2 a 3 a'r cyfnod pontio, nododd Estyn (2004) y dylai ysgolion cynradd ac uwchradd fel ei gilydd gynllunio'n effeithiol ar y cyd er mwyn i ddisgyblion adeiladu ar yr hyn a ddysgwyd yn y cynradd ac osgoi ailadrodd gwaith. Wrth wneud hyn, nododd Estyn (2004, t. 7) y gall y cynlluniau pontio gorau, fel y rhai a arsylwodd yn ei arolwg, sicrhau dilyniant gwell ac mae hyn yn "ennyn diddordeb disgyblion fel eu bod yn edrych ymlaen at barhau â'r gwaith yn eu hysgol uwchradd." Yn achos yr ysgolion a arsylwyd gan Estyn, lle mae cynllunio effeithiol ar y cyd ar waith, gellir nodi o'r llenyddiaeth fod pum prif agwedd i'w hystyried er mwyn hwyluso dilyniant rhwng y ddau sector, sef cynllunio gwaith ar y cyd, arsylwi ar ddosbarthiadau eraill, rhannu arbenigedd rhwng y ddau sector, trosglwyddo a defnyddio data rhwng sectorau a chynnal gweithgareddau allgyrsiol i fabwysiadu cysylltiadau rhwng y sectorau (Estyn, 2004).

Er bod Estyn (2004) yn adrodd mai nifer fechan o ysgolion sydd yn ymgorffori'r agweddau uchod ar gynllunio'n effeithiol ar y cyd, mae'n amlwg nad yw cynllunio ar y cyd ymhob clwstwr o ysgolion yr un mor effeithiol. Nododd Roberts (2010, 25 Mawrth), yn ei adroddiad i'r Pwyllgor Menter a Dysgu (2010), ei bryder ef o ran disgyblion yn colli cymhelliant wrth iddynt drosglwyddo o'r cynradd i'r uwchradd. Yn aml, mae'r ysgol uwchradd yn “[a]iladrodd yn hytrach nag atgyfnerthu'r sgiliau iaith a'r wybodaeth o'r sector gynradd" ac mae "hyn yn golygu bod llawer o ddysgwyr yn teimlo eu bod yn camu'n ol [sic] yn hytrach na symud ymlaen wrth ddysgu'r Gymraeg” (Roberts, 2010, 25 Mawrth, t. 3). Mae hyn wedi'i gadarnhau hefyd mewn papur arall i'r Cynulliad gan Uned Datblygu'r Gymraeg L1CC (2010, 27 
Mai). Adroddodd yr Uned fod y trefniadau cyfredol ar gyfer pontio'r ddau sector yn annigonol a bod hyn wedi arwain at sefyllfa lle mae disgyblion CA3 yn aml yn ailadrodd gwaith blaenorol, yn hytrach nac adeiladu ar yr hyn a ddysgwyd yn CA2. Mae ailadrodd gwaith blaenorol yn "dadrithio disgyblion” (Y Pwyllgor Menter a Dysgu, 2010, t. 19) ac yn sgil hynny, mae disgyblion yn colli cymhelliant. Mae Gwasanaeth Addysg a Gwella Ysgolion (ESIS) hefyd wedi mynegi pryderon ynglŷn â'r trefniadau pontio, wrth ychwanegu y dylai ysgolion uwchradd, yn ogystal ag ysgolion cynradd, "ysgwyddo rhywfaint o gyfrifoldeb am faterion sy'n ymwneud â dwyieithrwydd yn yr ysgolion cynradd sy'n eu bwydo" (fel y dyfynnwyd yn Y Pwyllgor Menter a Dysgu, 2010, t. 19). I'r un perwyl, mae adroddiad Davies (2013, t. 27) yn casglu bod "angen mwy o waith eto i rannu arfer da ac i sicrhau bod gan bob ysgol drefniadau pontio effeithiol ar gyfer Cymraeg ail iaith."

Mae'r llenyddiaeth ddiweddar felly yn awgrymu nad yw cyfran sylweddol o ysgolion yn cynllunio'n ddigonol er mwyn sicrhau dilyniant ieithyddol parhaol i'r Gymraeg fel ail iaith. Mewn nifer o achosion, mae'n ymddangos y caiff y cyfle hwn i sicrhau dilyniant ieithyddol ei golli, a hynny oherwydd diffyg cydweithrediad effeithiol rhwng y ddau sector. Mae'r llenyddiaeth hefyd yn dangos mor bwysig yw ystyried CAI yn ei gyfanrwydd, o'r cynradd i'r uwchradd, gan y caiff gwendidau'r naill Gyfnod Allweddol ddylanwad ar y llall. Wrth gadw hyn mewn cof felly, rhaid ystyried sut y gall model newydd posibl sicrhau dilyniant i'r Gymraeg, o'r cynradd i'r uwchradd. Rhaid ystyried sut y gall y model hwn ymgorffori'r agweddau allweddol ar gynllunio'n effeithiol ar y cyd rhwng ysgolion cynradd ac uwchradd, fel y'u gosodwyd gan Estyn (2004), er mwyn gwella a chysoni dilyniant CAI.

\subsubsection{Y sector uwchradd}

Daw darlun llawnach o sefyllfa CAI i'r amlwg wrth archwilio canfyddiadau diweddaraf Estyn ar y sector uwchradd. Er bod Estyn (2015, 2016b, 2017, 2018) wedi nodi gwelliant o ran safonau dysgu yn CA 3 a 4 mewn ysgolion yng Nghymru yn ddiweddar, ymddengys fod y gwelliant hwn, yn enwedig yn CA3, wedi dechrau “o sylfaen isel.” (Estyn, 2015, t. 51). Nododd Estyn (2014b, t. 43) mewn yn o’i adroddiadau blynyddol mai ond 27\% o ddisgyblion a safodd Gwrs Llawn CAI yn 16 oed rhwng 2012 a 2013, gyda 35\% yn sefyll y Cwrs Byr. O ystyried bod 15\% o ddisgyblion yn 2013 wedi sefyll arholiadau mewn CIG, nodir na fu bron i chwarter o ddisgyblion sefyll arholiad mewn Cymraeg yn 2013 (Estyn, 2014b, t. 43). Nid oedd y 
sefyllfa yn gadarnhaol y flwyddyn flaenorol ychwaith, gan y gellir nodi na safwyd arholiad mewn Cymraeg gan 21.9\% o'r holl ddisgyblion yng Nghymru yn y flwyddyn honno (Davies, 2013, t. 1). Er y bu ychydig o gynnydd o ran nifer y disgyblion sydd wedi cofrestru am y Cwrs Llawn yn ddiweddar (Estyn, 2018, t. 81), nododd Estyn (2018, t. 38) yn ei adroddiad blynyddol diwethaf fod y rhan fwyaf o ddisgyblion mewn ysgolion cyfrwng Saesneg rhwng 2010 a 2016 wedi ennill cymhwyster Cwrs Byr TGAU mewn Cymraeg.

Mae ffigyrau Estyn yn awgrymu bod tuedd i ddisgyblion mewn ysgolion cyfrwng Saesneg ddilyn y Cwrs Byr, lle yn ôl Lewis (2010a) ceir rhwng hanner awr yr wythnos hyd at awr a hanner o gyswllt â'r Gymraeg ar ei fwyaf. Yn wir, o'r rhai sydd yn dilyn y Cwrs Byr, ond nifer fach ohonynt sydd yn gwneud cynnydd digonol yn y Gymraeg (Estyn, 2007, t. 3). Ar ôl gostyngiad sylweddol rhwng 2004-2006 o ran y nifer o ddisgyblion a oedd yn dilyn y Cwrs Llawn, canfuwyd bod nifer y disgyblion a oedd yn dilyn y Cwrs Byr yn uwch na'r rhai a oedd yn dilyn y Cwrs Llawn yn y rhan fwyaf o ysgolion am y tro cyntaf yn 2006 (Estyn, 2007, t. 3). Mae Estyn (2007) wedi priodoli'r gostyngiad sylweddol yn nifer y disgyblion sy'n dilyn y Cwrs Llawn i'r ffactorau canlynol:

- Ni chaiff disgyblion eu cynghori i ddilyn y cwrs (byr neu lawn) a fydd yn eu helpu i gyflawni eu potensial. Yn benodol, nid oes digon o gymorth ar gyfer disgyblion i ddewis y cwrs llawn;

- Er bod gan y rhan fwyaf o'r disgyblion a gafodd eu cyfweld agweddau cadarnhaol tuag at ddysgu'r Gymraeg a'u bod yn cydnabod gwerth parhau i wneud hynny yng nghyfnod allweddol 4, mae'r dewisiadau pwnc yng nghyfnod allweddol wedi'u trefnu mewn ffordd sy'n golygu bod y cwrs TGAU llawn Cymraeg ail iaith yn cystadlu â dewisiadau pwnc poblogaidd eraill; ac

- Mewn rhai o'r ysgolion, nid yw'r cwrs llawn ar gael i ddisgyblion ei astudio, yn aml oherwydd nad oes gan yr ysgol ddigon o athrawon Cymraeg ail iaith arbenigol. (Estyn, 2007, tt. 3-4).

Nid yw'n syndod felly fod Un iaith $i$ bawb wedi argymell dileu'r Cwrs Byr yn llwyr er mwyn annog ysgolion i neilltuo mwy o oriau i addysgu'r Gymraeg (Davies, 2013, t. 31).

Yn ogystal â'r diffygion o ran darpariaeth, rhaid hefyd dynnu sylw at y diffygion o ran hyfforddi athrawon y sector uwchradd. Mae'r angen am fuddsoddiad sylweddol 
mewn hyfforddiant i athrawon eisoes wedi'i fynegi, er enghraifft, gan C. Williams (2008) a C. Davies (2010). Fel y maent yn ei gydnabod, rhaid sicrhau bod digon o athrawon cymwys yn eu lle os yw ysgolion uwchradd cyfrwng Saesneg yn mynd i allu cynnig elfennau cwricwlaidd trwy gyfrwng y Gymraeg. Fel y nododd Estyn (2018, t. 40) yn ei adroddiad blynyddol diwethaf: “[n]id yw bron i chwarter o athrawon sy'n cael eu cyflogi i addysgu Cymraeg wedi'u hyfforddi i addysgu'r pwnc." Mae cyflwyno staff cymwys i addysgu'r Gymraeg yn her fawr yn y sector cyfrwng Saesneg, ac un o'r prif rhwystrau, ymddengys, yw'r amrywiaeth rhwng y tair canolfan addysg athrawon o ran ansawdd yr hyfforddiant ar gyfer addysgu'r Gymraeg mewn lleoliadau cyfrwng Saesneg (Estyn, 2018, t. 40).

Yn ogystal â'r angen i gyflogi rhagor o athrawon Cymraeg Ail Iaith arbenigol yn y sector uwchradd, nodir hefyd fod angen cryfhau'r ddarpariaeth hyfforddi Cymraeg dwys ar gyfer athrawon di-Gymraeg (C. Davies, 2010, Y Pwyllgor Menter a Dysgu, 2010 , t. 27) er mwyn i'r athrawon hyn allu defnyddio'r Gymraeg yn achlysurol yn y dosbarth ac ar draws yr ysgol. Er bod y Cynllun Sabothol, er enghraifft, yn cynnig cyrsiau hyfforddiant iaith Gymraeg a methodoleg addysgu Cymraeg fel ail iaith i athrawon a chynorthwywyr dosbarth yn y sector cynradd ac uwchradd, nid yw'r Cynllun yn cynnig cwrs ar fethodoleg addysgu dwyieithog i athrawon pynciol mewn ysgolion cyfrwng Saesneg yn benodol (Cynrychiolydd ar ran y Cynllun Sabothol, cyfathrebiad personol, Mawrth 22, 2017). Ar ben hyn, nodir nad oes cwrs ar gael sy'n targedu staff cynorthwyol a'u sgiliau Cymraeg yn y gweithle yn benodol (Cynrychiolydd ar ran y Cynllun Sabothol, cyfathrebiad personol, Mawrth 22, 2017). Mae'r dystiolaeth at ei gilydd yn awgrymu'n gryf nad yw disgyblion ar draws y sector cyfrwng Saesneg yn cael yr un cyfleoedd i ennill cymhwyster TGAU mewn Cymraeg trwy astudio'r Cwrs Llawn, a hynny, ymddengys, oherwydd nad yw nifer o ysgolion cyfrwng Saesneg yn hyrwyddo statws y pwnc gan annog disgyblion i ddewis y Cwrs Llawn. At hyn, mae Davies (2013, t. 1) wedi nodi nad yw CAI yn bwnc deniadol i lawer o ddisgyblion, gan ddweud mai "profiad diflas dros ben ydyw i lawer iawn ohonynt yn ôl y dystiolaeth - nid ydynt yn gweld y pwnc yn berthnasol nac o unrhyw fudd iddynt”. Pwysleisia'r Pwyllgor Menter a Dysgu (2010, t. 31) hefyd “... n[a]d yw'n ddigon addysgu Cymraeg fel ail iaith yn unig, ond y dylid ei defnyddio mewn agweddau eraill ar fywyd yr ysgol, ac y dylai'r Gymraeg ac ymwybyddiaeth o Gymru dreiddio drwy holl ethos yr ysgol." Mae'r diffygion hyn i 
gyd yn cynnig nifer o heriau i'r sector uwchradd, nid yn unig o ran safonau addysgu Cymraeg fel ail iaith, ond hefyd o ran mwynhad yn y pwnc a pherthnasrwydd yr iaith i fywydau disgyblion CAI. Er mwyn sicrhau bod disgyblion CAI yn parhau i fwynhau astudio'r Gymraeg yn yr ysgol uwchradd, rhaid ystyried felly sut y gall model newydd posibl wneud yn sicr bod pwrpas ac ystyrlonrwydd i ddysgu'r Gymraeg fel ail iaith.

\subsection{Casgliadau ar bolisi ac arfer o ran addysgu'r Gymraeg fel ail iaith}

Er y bu nifer fawr o ddatblygiadau arwyddocaol ynglŷn â pholisi a deddfwriaeth, yn enwedig yn ystod y degawd diwethaf, mae'n amlwg o'r testunau uchod fod y ddarpariaeth gyfredol ar gyfer addysgu'r Gymraeg mewn perthynas ag addysg cyfrwng Saesneg yn ddiffygiol ac nad yw'n llwyddo i ddatblygu sgiliau dwyieithog yn ddigonol erbyn i ddisgyblion orffen addysg orfodol. Os yw LlC am gyflawni ei nod uchelgeisiol o gyrraedd miliwn o siaradwyr Cymraeg, bydd yn rhaid iddi barhau i ystyried y rôl y mae addysg cyfrwng Saesneg yn benodol yn ei chwarae o ran creu siaradwyr newydd a sut y gall diwygio'r gyfundrefn gyfrannu at yr uchelgais. Er nad yw addysg ynddi ei hun yn ateb cyflawn i ddwyieithrwydd yng Nghymru, mae'n amlwg fod gan addysg rôl ganolog yn y broses o greu siaradwyr newydd yn y Gymraeg. Ymddengys felly mai drwy newid sylfaenol yn y gyfundrefn addysg, o ran addysgu'r Gymraeg yng nghyswllt addysg cyfrwng Saesneg yn benodol, y gellir sicrhau sylfaen gadarn wrth ddatblygu Cymru wirioneddol ddwyieithog. Ar y sylfaen hon wedyn, rhaid sicrhau defnyddio'r ddwy iaith y tu hwnt i'r ysgol, yn y gymuned a'r cartref, a hefyd sicrhau cyfleoedd i ddefnyddio'r ddwy iaith ar gyfer gweithlu dyfodol Cymru. 


\section{Pennod 2: Diffinio'r termau priodol ac esbonio eu harwyddocâd}

Mae'r meysydd dan sylw yn y traethawd hwn yn feysydd cyfoes sydd yn datblygu'n barhaus. Defnyddir yn y meysydd hyn dermau a all gael eu dehongli'n wahanol o'r naill ysgolhaig i'r llall. O ganlyniad i natur gyfnewidiol ymchwil meysydd caffael iaith a dwyieithrwydd, a natur agored dehongli'r termau, ni cheir yn y traethawd hwn ymdrech i ddiffinio'n bendant y cysyniadau a thermau priodol. Yn hytrach, cyflwynir y gwahanol ddiffiniadau a dehongliadau a ddefnyddir gan ysgolheigion o'r termau priodol, cyn esbonio ystyr y termau hyn, ynghyd â'u harwyddocâd, yng nghyddestun yr ymchwil hon.

\subsection{Iaith gyntaf ac ail iaith}

\subsubsection{Diffinio iaith gyntaf}

Cyn mynd ati i egluro'r gwahaniaethau rhwng y cysyniadau o ddysgu a chaffael ail iaith, rhaid yn gyntaf ddiffinio'r hyn a olygir gan y termau 'iaith gyntaf' ac 'ail iaith' (yn nhermau dysgu iaith). Mae Crystal (1997a, t. 427) yn diffinio iaith gyntaf megis "[t]he language first acquired as a child (mother tongue, native language), or preferred in a multilingual situation." Yn syml iawn, dyma'r iaith sy'n cael ei chaffael a dyma'r iaith a siaredir yn gyntaf yn blentyn ifanc. Yn aml, ceir cyfystyron yn lle'r ymadrodd 'iaith gyntaf' megis 'mamiaith' ac 'iaith frodorol' ac yn aml y mae cysyniad y term 'iaith gyntaf' yn gysylltiedig â'r iaith a siaredir yn y cartref, sef 'iaith y cartref' ac 'iaith y gymuned' (Thompson, 2004). Mae Thompson (2004, t. 418) yn mireinio'r diffiniad cyffredinol o ‘famiaith' ymhellach wrth gynnig “...the term mother tongue is the first language that the child learns, and inherent in this description is the assumption that the learning takes place in a naturalistic way, i.e. not through formal teaching."

Fodd bynnag, dylid bod yn ofalus wrth ddefnyddio'r term 'mamiaith' a'r term 'iaith gyntaf' o safbwynt gallu a defnydd y siaradwr o'r iaith. Gall defnyddio'r termau hyn gyfeirio at iaith gryfaf y siaradwr neu'r iaith sy'n well ganddo ac felly mae'n bosibl nad yr iaith a ddysgwyd yn gyntaf, sef y famiaith, yw iaith gryfaf y siaradwr neu'r iaith sy'n well ganddo bob tro (Romaine, 1995; Thompson, 2004). Mae'n bosibl y bydd yr ail iaith yn datblygu'n iaith gryfaf i'r siaradwr neu'n iaith sy'n well ganddo dros amser, ac felly'r ail iaith wedyn fydd iaith gyntaf y siaradwr (Saville-Troike, 2006). 
At ddiben yr ymchwil hon, defnyddir y term 'iaith gyntaf' yng nghyd-destun y gyfundrefn addysg yng Nghymru wrth gyfeirio at y rhai sydd yn dysgu'r Gymraeg drwy gyfrwng y Gymraeg, mewn ysgolion cyfrwng Cymraeg, hynny yw 'disgyblion Cymraeg Iaith Gyntaf (CIG)', yn ogystal â'r cyd-destun caffael iaith, wrth gyfeirio at yr iaith gyntaf a ddysgir, sef 'disgyblion Cymraeg iaith gyntaf'. (Am drafodaeth lawnach, gweler 2.6.3. Disgybl Cymraeg Iaith Gyntaf.) Ni chaiff y term 'mamiaith' ei ddefnyddio gan ei bod yn bosibl i siaradwyr etifeddu dwy 'famiaith,' a gallai dryswch godi wrth ddefnyddio'r term hwn. Yn dilyn arfer W. Gwyn Lewis (2008), defnyddir 'addysg dreftadaeth' wrth gyfeirio at raglenni addysg cyfrwng Cymraeg yng Nghymru sy'n cael eu hanelu'n bennaf at "gynnal, cadarnhau ac ymestyn" Cymraeg siaradwyr o gartrefi Cymraeg eu hiaith. (Am drafodaeth lawnach ar addysg dreftadaeth, gweler 2.5.2. Addysg dreftadaeth.)

\subsubsection{Diffinio ail iaith}

Ar sail diffiniad 'iaith gyntaf' felly, a ddylid diffinio 'ail iaith' yn nhermau iaith sydd wedi'i dysgu ar ôl yr iaith gyntaf? Mae Lightbown a Spada (2006, t. 30) yn cefnogi'r dehongliad hwn: "By definition, all second language learners, regardless of age, have already acquired at least one language.” Ar sail y dehongliad hwn, gellir esbonio ail iaith megis unrhyw iaith sydd wedi'i dysgu ar ôl yr iaith gyntaf a gafodd ei chaffael. Ceir diffiniad tebyg o ail iaith gan Crystal (1997a, t. 436), sy'n ychwanegu bod gan yr ail iaith, fel arfer, rôl swyddogol mewn cymuned: "A non-native language, esp. one that has an official role in a country."

O'r ddau ddiffiniad uchod felly, ymddengys fod gwahaniaeth sylfaenol rhwng y siaradwr ail iaith a'r siaradwr iaith gyntaf, a'r ffordd y maent yn dysgu'r iaith darged. Mae gennym yma'r cysyniad bod gan y siaradwr ail iaith ddealltwriaeth o un iaith o leiaf yn barod cyn iddo ddysgu'r ail iaith, lle nad yw'r siaradwr iaith gyntaf yn gyfarwydd ag unrhyw iaith cyn dysgu ei iaith gyntaf. Ymhelaetha Lightbown a Spada (2006, tt. 30-31) ar hyn wrth iddynt nodi bod y pum nodwedd ganlynol yn wahanol ym mhrofiad y siaradwr ail iaith a'r siaradwr iaith gyntaf: i) dealltwriaeth flaenorol o un iaith o leiaf eisoes, ii) aeddfedrwydd gwybyddol ${ }^{6}$, iii) ymwybyddiaeth

\footnotetext{
${ }^{6}$ Dyma fesur o allu'r siaradwr i ymgymryd â thasgau'n ymwneud â chofio, casglu gwybodaeth o rywbeth a datrys problemau (Lightbown \& Spada, 2006, t. 29).
} 
feta-ieithyddol ${ }^{7}$, iv) adnabyddiaeth o'r byd, a v) phryder o ran gwneud camgymeriadau ieithyddol. Oherwydd bod y siaradwr ail iaith ddealltwriaeth sylfaenol gadarn o un iaith o leiaf a ddefnyddir yn y gymdeithas, yn ogystal ag adnabyddiaeth o'r byd, ymddengys ei fod yn adeiladu ar ddealltwriaeth sylfaenol yr iaith gyntaf wrth ddysgu'r iaith darged ${ }^{8}$.

Fodd bynnag, nid yw'r diffiniadau uchod yn ddigon manwl nac yn ddigon eglur er mwyn esbonio sefyllfaoedd cymhleth dysgu'n ddwyieithog a gall cwestiynau fel a ganlyn godi: Beth am blant a gaiff eu magu gan siaradwyr dwy iaith wahanol? Ai caffael y ddwy iaith ar yr un pryd y mae'r plentyn yn ei wneud, neu ddysgu un ar ôl y llall? Wrth ystyried enghreifftiau o blant sydd yn caffael mwy nag un iaith ar yr un pryd, mae'n amlwg nad yw'r diffiniadau uchod yn ddigonol wrth gategoreiddio'r iaith gyntaf a'r ail iaith. Er enghraifft, pe bai gan blentyn un rhiant sy'n siarad Cymraeg ag ef, a chanddo un rhiant sy'n siarad Saesneg ag ef, ai Cymraeg fyddai iaith gyntaf y plentyn neu Saesneg? Pe bai plentyn arall yn mynychu meithrinfa Gymraeg ac wedyn ysgol gynradd Gymraeg, er mai Saesneg yw iaith y cartref, ai siaradwr 'Cymraeg iaith gyntaf' fyddai'r plentyn ynteu siaradwr ail iaith? Wrth fwrw sylw ar Diffinio ysgolion yn ôl y ddarpariaeth cyfrwng Cymraeg (L1CC, 2007b, t. 8), mae'n amlwg o safbwynt addysgol mai siaradwr iaith gyntaf fyddai'r plentyn gan fod disgwyl i ruglder disgyblion ysgolion cynradd Cymraeg sy'n dod o gartrefi diGymraeg fod yn ddigonol er mwyn gallu trosglwyddo i ysgolion uwchradd cyfrwng Cymraeg: "Fel rheol, disgwylir y bydd disgyblion, ni waeth beth yw iaith y cartref, yn gallu trosglwyddo'n hawdd i ddarpariaeth uwchradd cyfrwng Cymraeg..." Mae gennym sefyllfa gymhleth iawn lle mae dysgu dwy iaith (neu ragor) yn gallu digwydd ar yr un pryd (gweler 2.3.4. Dwyieithrwydd cydamserol) ac mewn achosion o'r fath ni fyddai'r diffiniadau uchod yn bodloni'r enghreifftiau hyn. Cydnebydd Thompson (2004, tt. 418-419) gymhlethdod y sefyllfa hon ac mae'n priodoli

\footnotetext{
${ }^{7}$ Dyma ymwybyddiaeth y siaradwr o'r iaith ei hun a'i chydrannau ieithyddol. Os yw'r siaradwr wedi datblygu lefel uchel o ymwybyddiaeth feta-ieithyddol mewn iaith, disgwylir gweld gallu i ddiffinio geiriau, esbonio rheolau gramadegol, yn ogystal â deall cysylltiadau rhwng y naill iaith a'r llall (Johnstone, 2004, t. 191; Lightbown \& Spada, 2006, t. 29). Diffinia Baker (1996, t. 122) hyn fel "the ability to think about and reflect upon the nature and functions of language."

${ }^{8}$ Yr hyn a gyfeirir ato yma'n fras yw 'ymyrraeth'. Digwydd ymyrraeth yn ystod y broses o gaffael ail iaith wrth i'r dysgwr ail iaith dynnu ar nodweddion yr iaith gyntaf er mwyn ceisio dyfalu natur yr ail iaith a gall hyn gael effaith gadarnhaol neu negyddol ar y dysgu (Cherrington, 2004, t. 633-635).
} 
cymhlethdod diffinio'r term i ffactorau megis symudedd cynyddol yr unigolyn, arferion cymdeithasol newidiol a pholisïau cynllunio ieithyddol cenedlaethol. Yng nghyd-destun yr ymchwil hon, defnyddir y term 'ail iaith' wrth gyfeirio'n benodol at y rhai sydd yn siarad Saesneg iaith gyntaf ac sydd yn dysgu'r Gymraeg megis pwnc addysgol mewn addysg cyfrwng Saesneg, sef 'disgyblion ail iaith', neu ‘ddisgyblion Cymraeg Ail Iaith’ (gweler 2.6.5. Disgybl Cymraeg Ail Iaith), a hefyd wrth gyfeirio at y model o addysgu'r Gymraeg yn bwnc ail iaith mewn ysgolion cyfrwng Saesneg ei hun, sef ‘Cymraeg Ail Iaith' (gweler 2.6.4. Cymraeg Ail Iaith). Defnyddir hefyd y term 'siaradwyr newydd' yn gyfystyr er mwyn cyfeirio at unigolion sydd yn dysgu Cymraeg neu ieithoedd eraill fel ail iaith.

\subsection{Caffael a dysgu iaith}

Y mae nifer fawr o ddamcaniaethau sydd wedi cael eu cynnig gan ysgolheigion ynghylch sut mae caffael iaith yn digwydd. Yn wir, mae'n faes rhyngddisgyblaethol iawn sydd yn cyffwrdd ag agweddau ar ieithyddiaeth, seicoleg, ieithyddiaeth gymdeithasegol, seicoieithyddiaeth, niwroleg, ynghyd ag eraill. O ran esbonio'r broses caffael iaith, y mae tair prif ddamcaniaeth, sef yr ymagwedd ymddygiadol (behaviourist approach), y gallu cynhenid (innatism approach) a'r ymagwedd rhyngweithiol-datblygiadol (interactional / developmental approach). Nid ymdrech i drafod y damcaniaethau gwahanol hyn yn fanwl mo'r adran hon. Yn hytrach, anelir at gyflwyno egwyddorion sylfaenol caffael iaith a dysgu iaith sydd wedi'u cynnig er mwyn egluro ystyr y ddau derm hyn ac esbonio'n well y gwahaniaeth rhwng y dysgwr iaith gyntaf a'r dysgwr ail iaith a sut y maent yn dysgu neu'n caffael iaith. Felly, beth a olygir gan y termau 'caffael iaith' a 'dysgu iaith'? A oes gwahaniaeth? Mae Crystal (1997a, t. 430) yn diffinio 'caffael iaith' fel " $[\mathrm{t}]$ he process of learning a first language in children" neu' $r$ "analogous process of gaining a foreign or second language." Yn ei hanfod, gellir diffinio ‘caffael iaith' yn gyffredinol megis y broses o godi iaith, naill ai'n iaith gyntaf, ynteu'n ail iaith ar ôl caffael yr iaith gyntaf. Mae ysgolheigion megis Lightbown a Spada (2006) a Saville-Troike (2006) yn defnyddio'r term 'caffael iaith' yn yr ystyr hon. Yn ogystal â chyfeirio'n gyffredinol at y broses o godi iaith fodd bynnag, mae'r term caffael iaith wedi cael ei ddehongli megis proses gynhenid, naturiol o ddysgu iaith yn yr isymwybod, sydd yn digwydd ar wahân i ddysgu ffurfiol. Fel y mae Krashen (1982, t. 10) yn ei esbonio yn ei 


\section{Ddamcaniaeth Caffael-Dysgu (Acquisition-learning distinction): "Language}

acquisition is a subconscious process; language acquirers are not usually aware of the fact that they are acquiring language, but are only aware of the fact that they are using the language for communication."

Proses naturiol yw caffael iaith, lle mae dysgu iaith yn digwydd yn yr isymwybod wrth ddefnyddio'r iaith darged ar gyfer cyfathrebu. Mae hyn yn bosibl oherwydd bod gan y dysgwr allu cynhenid yn yr ymennydd i ddysgu iaith o'i enedigaeth ${ }^{9}$. Nid yw nodweddion ieithyddol (megis geirfa a rheolau gramadeg) yn ganolbwynt wrth gaffael iaith, yn hytrach, canolbwyntir ar gyfleu ystyr trwy gyfathrebu. Mae caffael iaith felly yn arfer digwydd mewn cyd-destunau anffurfiol. Fel yr esbonia Baker (2001, t. 113), "[a]cquisition in informal contexts may be a more subconscious process where communication is a means and not a focus nor an end in itself."

Mae 'dysgu iaith' felly, yn ôl y fath feini prawf, yn broses wahanol ymwybodol o ddysgu iaith a'i nodweddion ieithyddol. Yn rhesymegol, mae hyn yn arfer digwydd mewn cyd-destun ffurfiol, addysgol, megis dosbarth iaith. Esbonia Krashen (1982, t. 10) ‘ddysgu iaith’ yn ei Ddamcaniaeth Caffael-Dysgu megis “conscious knowledge of a second language, knowing the rules, being aware of them, and being able to talk about them. In non-technical terms, learning is "knowing about" a language, known to most people as "grammar", or "rules"." Yn ogystal â datgan bod caffael iaith a dysgu iaith yn ddwy broses sy'n bodoli ar wahân i'w gilydd, mae Krashen (1982) yn gwahaniaethu ymhellach rhwng swyddogaethau'r ddwy broses yn ei Ddamcaniaeth Arolygu (Monitor Theory). Mae Krashen yn damcaniaethu mai ‘caffael iaith’ yw beth sydd wrth wraidd dealltwriaeth a rhuglder ail iaith gan mai dyma'r broses sy'n galluogi'r siaradwr i gynhyrchu ymadroddion naturiol ar lafar. 'Dysgu iaith' yw'r broses sy'n arolygu'r iaith y mae'r siaradwr yn ei chynhyrchu. Mewn geiriau eraill, 'dysgu' yw'r broses sy'n cywiro ac adolygu'r hyn y mae'r siaradwr wedi'i 'chaffael' yn ei ail iaith.

Er gwaetha'r dehongliadau o 'gaffael iaith' a 'dysgu iaith' a gynnig Krashen, mae nifer o ysgolheigion erbyn hyn yn defnyddio'r ddau derm yn gyfystyr (er enghraifft,

\footnotetext{
${ }^{9}$ Yn ôl Damcaniaeth y Gallu Cynhenid (Innatist Theory), mae gan blant o'u genedigaeth wybodaeth gynhenid o'r hyn sydd yn gyffredin ymhob iaith o'u enidigaeth, a elwir yn 'Gramadeg Cyffredinol' (Universal Grammar). Mae'r wybodaeth gynhenid hon yn digwydd yn rhan o'r ymennydd a elwir yn Ddyfais Caffael Iaith, neu 'LAD' (Language-Acquisition Device).
} 
Lightbown \& Spada 2006; Macaro 2010; Saville-Troike 2006). Gan wrthod damcaniaeth Krashen, mae Macaro (2010) yn dadlau nad oes angen gwahaniaethu rhwng caffael iaith a dysgu iaith gan fod meddwl y dysgwr yn ymwybodol wrth i'r ddwy broses ddigwydd. Dadleua felly na ellir yn wir ddysgu iaith yn yr isymwybod:

Nor is a distinction nowadays being made (as Stephen Krashen once attempted to make) between acquisition, which was supposedly subconscious, and learning which was supposedly conscious. It is now generally accepted that totally subconscious acquisition/learning could really only take place when you are asleep, in a coma or through some kind of subliminal device such as is sometimes (illegally) attempted in advertising. (Macaro, 2010, t. 5)

Ni waeth sut y diffinnir 'caffael' a 'dysgu' iaith fodd bynnag, mae'n amlwg o'r diffiniadau uchod fod cyd-destun y dysgu'n bwysig wrth gaffael neu ddysgu iaith. Gall dysgu iaith ddigwydd mewn ffordd anffurfiol, er enghraifft, wrth wrando ar siaradwyr rhugl ac wrth siarad â nhw mewn sefyllfa gymdeithasol y tu allan i'r dosbarth. Dyma'r lle y byddai ‘caffael iaith' yn digwydd yn ôl Damcaniaeth y Gallu Cynhenid. Mewn sefyllfa ffurfiol, addysgol, lle mae'n amlwg y canolbwyntir ar ddysgu'r iaith ei hun yn fwriadol a bod y dysgwr yn dysgu'r cynnwys mewn ffordd ffurfiol, ymwybodol ac uwch ieithyddol, dyma'r lle mae 'dysgu iaith' fel arfer yn digwydd yn ôl Damcaniaeth y Gallu Cynhenid. Pwysig nodi yma na ellir cyfyngu'r broses o ddysgu anffurfiol, isymwybodol, neu 'gaffael iaith', a'r broses o 'ddysgu iaith' mewn modd ffurfiol, bwriadol i sefyllfaoedd ffurfiol ac anffurfiol gan y gall 'dysgu iaith anffurfiol' ddigwydd mewn sefyllfa ffurfiol a gall 'dysgu iaith ffurfiol' ddigwydd mewn sefyllfa anffurfiol (Baker, 2001). Er enghraifft, gall 'dysgu iaith anffurfiol' ddigwydd mewn dosbarth iaith wrth siarad yn naturiol a heb ganolbwyntio ar nodweddion ieithyddol megis gramadeg a rheolau iaith, ac yr un modd gall 'dysgu iaith ffurfiol' ddigwydd mewn sefyllfa anffurfiol, naturiol, wrth ofyn i rywun am reolau gramadeg, er enghraifft, mewn sgwrs naturiol, anffurfiol (Baker, 2001, t. 114).

At ddiben y traethawd hwn, bwriedir defnyddio'r ddau derm 'caffael iaith' a 'dysgu iaith' yn gyfystyr, fel y mae eraill yn ei wneud (gweler Lightbown \& Spada 2006; Macaro 2010; Saville-Troike 2006) gan nad diben yr ymchwil hon yw trafod sut mae caffael iaith a dysgu iaith yn digwydd yn yr ymennydd. 


\subsubsection{Caffael iaith gyntaf}

Wrth fwrw sylw eto ar ddiffiniadau Crystal (1997b, t. 430) uchod, gwelir ei fod yn cyfeirio at y ddau fath o gaffael iaith. Wrth gymryd ei ddiffiniad cyntaf, gellir diffinio caffael iaith megis y broses o ddysgu'r iaith gyntaf yn blentyn ifanc iawn, ac mae'n debyg fod caffael iaith gyntaf bron bob amser yn dechrau wrth i'r plentyn gael ei fagu trwy gyfrwng iaith y cartref.

\subsubsection{Gallu Cynhenid}

Mae Chomsky (1993) yn priodoli gallu'r plentyn i gaffael ei iaith gyntaf i'r hyn a eilw yn 'Gramadeg Cyffredinol'10 (Universal Grammar). Diffinnir Gramadeg Cyffredinol yn nhermau "a characterization of the child's pre-linguistic initial state" a gellir ei weld yn “cognitive organ” sy'n nodweddiadol ym modau dynol (Chomsky, 2007, t. 1). Mae O'Grady, Dobrovolsky a Katamba (1996, t. 734) wedi diffinio Gramadeg Cyffredinol megis "system of categories, mechanisms and constraints shared by all human languages and considered to be innate". Yn y system neu 'organ' hwn felly, ymddengys fod 'formal universals', sef egwyddorion a pharamedrau, ynghyd â 'substantive universals', sef categorïau a nodweddion geirfaol. Fodd bynnag, nid oes cytundeb rhwng ysgolheigion ar beth yn union y mae'r 'formal universals' a'r 'substantive universals' yn ei gynnwys (Dąbrowska, 2015). Mae Koster (2013, t. 17) wedi symleiddio diffinio Gramadeg Cyffredinol a’i swyddogaeth wrth ei gymharu â 'chist offer' sy'n llawn o'r nodweddion ieithyddol a geir ym mhob iaith. Mae'r iaith gyntaf wedyn yn tynnu ar y nodweddion hyn er mwyn iddi gael ei chaffael (Koster, 2013, t. 17). Mae'r wybodaeth gynhenid hon yn cael ei lleoli mewn rhan o'r ymennydd a adwaenir fel y Ddyfais Caffael Iaith neu 'LAD' (Language-Acquisition Device).

Yn ôl y ddamcaniaeth hon felly, caiff plentyn ei eni gyda gwybodaeth gynhenid o'r hyn sy'n gyffredinol ymhob iaith, ac oherwydd y wybodaeth gynhenid hon, y gred ymhlith damcaniaethwyr megis Chomsky yw bod gan y plentyn y gallu i adeiladu ei iaith gyntaf o gwmpas y wybodaeth gynhenid sydd yn yr ymennydd. Oherwydd y system gynhenid felly, cred Chomsky (1975, t. 4) hyn nad yw mewnbwn yr un mor bwysig a bod modd i blentyn gaffael iaith "on relatively slight exposure and without specific training."

\footnotetext{
${ }^{10}$ Defnyddir yma'r term Cymraeg a fathwyd gan Thomas a Webb-Davies (2017).
} 


\subsubsection{Y safbwynt rhyngweithiol / gwybyddol}

Yn fwy diweddar, mae damcaniaeth Gramadeg Cyffredinol wedi cael ei herio gan ddamcaniaethwyr a ddaw at y maes o safbwynt arall, sef y safbwynt rhyngweithioldatblygiadol (interactivist-developmental perspective) (Lightbown \& Spada, 2013) neu'r safbwynt gwybyddol-rhyngweithiol (interactivist-cognitive perspective) (Ellis, 2015). Mae'r safbwynt hwn, sydd wedi ei seilio i raddau helaeth ar waith y seicolegydd Vygotsky ar ddatblygiad gwybyddol, ${ }^{11}$ yn honni bod damcaniaethwyr y gallu cynhenid wedi rhoi gormod o bwyslais ar gymhwysedd terfynol yr unigolyn, yn hytrach na chanolbwyntio ar sut y mae caffaeliad iaith yr unigolyn yn datblygu dros amser (Lightbown \& Spada, 2013, t. 24). Yn groes i ddamcaniaethwyr y gallu cynhenid, cred damcaniaethwyr y safbwynt rhyngweithiol fod yr hyn sydd ei angen ar yr unigolyn er mwyn dysgu iaith yn cael ei gynnwys yn yr iaith ei hun, a bod y mewnbwn a geir wrth ddod mewn cyswllt â'r iaith honno, hynny yw, trwy ryngweithio, yn sicrhau y gall yr unigolyn gaffael yr iaith. Yn ôl y ddamcaniaeth hon felly, mae gan fewnbwn rôl hollbwysig ym mhroses caffael iaith ac mae'n honni bod angen mewnbwn er mwyn i'r unigolyn gaffael ei iaith gyntaf.

\subsubsection{Caffael ail iaith}

Er y gall damcaniaethau uchod egluro, i ryw raddau, y cyflwr cyn-ieithyddol sy'n galluogi caffael iaith gyntaf, mae'r ddadl yn parhau ymhlith ysgolheigion o ran beth yw'r sail ieithyddol ar gyfer caffael ail iaith, hynny yw, ai'r iaith gyntaf yw'r sail i gaffael yr ail iaith, neu Ramadeg Cyffredinol? (Haznedar \& Gavruseva, 2013; Saville-Troike, 2006). Mae Saville-Troike (2006, “L1 versus L2 learning”) er enghraifft, yn cynnig tri chyflwr yn natblygiad yr iaith gyntaf a hefyd yr ail iaith, sef 'y cyflwr cychwynnol' (initial state), 'y rhyng-gyflyrau' (intermediate states), a'r 'cyflwr terfynol' (final state). Ar gyfer y 'cyflwr cychwynnol', mae Saville-Troike (2006, t. 17) yn rhestru'r canlynol megis y ffactorau posibl sy'n creu sail i gaffael ail iaith, sef gallu cynhenid, dealltwriaeth o'r iaith gyntaf, adnabyddiaeth o'r byd, a sgiliau rhyngweithio. Mae Saville-Troike yma'n ychwanegu dau ffactor arall sy'n hwyluso caffael ail iaith, un ym maes datblygiad gwybyddol, sef dealltwriaeth o'r iaith gyntaf a sgiliau rhyngweithio, ac un ym maes datblygiad meta-ieithyddol, sef

\footnotetext{
${ }^{11}$ Gwnaeth Vygotsky ddamcaniaethu fod datblygiad gwybyddol (ac ieithyddol) yn digwydd pan fo'r plentyn yn rhyngweithio'n gymdeithasol. Erbyn hyn, mae syniadau Vygotsky ar ddatblygiad gwybyddol plant wedi dylanwadu'n fawr ar ymchwil ym maes caffael ail iaith a'r honiad mai proses yw caffael iaith, yn hytrach na'r cyflwr terfynol (Ellis, 2015; Lightbown \& Spada, 2013).
} 
adnabyddiaeth o'r byd. Ymddengys felly fod y cyflwr cyn-ieithyddol caffael ail iaith yn wahanol i gyflwr cyn-ieithyddol caffael iaith gyntaf. Wrth gaffael ail iaith mae'r siaradwr yn dibynnu ar ei ddealltwriaeth o'i iaith gyntaf, ei ymwybyddiaeth fetaieithyddol, ei sgiliau rhyngweithio ac, o bosibl, ei allu cynhenid i ddysgu iaith. O ran caffael iaith gyntaf, fodd bynnag, ymddengys fod y siaradwr yn dibynnu o'r cychwyn cyntaf ar fewnbwn ieithyddol ei amgylchfyd, gan nad ydyw wedi datblygu ymwybyddiaeth feta-ieithyddol, sgiliau rhyngweithio na dealltwriaeth o unrhyw iaith arall.

Er nad yw'n glir beth yn union yw cyflwr ieithyddol yr ymennydd wrth gaffael iaith gyntaf a chaffael ail iaith, mae'n amlwg fod y ddwy broses yn wahanol i'w gilydd ac nad yw'r ddwy broses yn cyd-fynd (Saville-Troike, 2006), er bod nodweddion tebyg rhyngddynt. Mae gwahaniaethau o ran cyd-destun y dysgu, hynny yw, rhwng nodweddion y dysgwr iaith gyntaf a'r dysgwr ail iaith, a hefyd rhwng yr amgylchfyd y mae'r dysgwr yn caffael yr iaith ynddo (Lightbown \& Spada, 2006). Nodir hefyd fod gan y siaradwr uniaith a'r siaradwr dwyieithog gymwyseddau a sgiliau gwahanol (Saville-Troike, 2006) ac felly, nid yw'n bosibl cymharu caffael ail iaith â chaffael iaith gyntaf. Mae Cook (2010) yn esbonio'r gwahaniaeth rhwng caffael iaith gyntaf a chaffael ail iaith fel a ganlyn:

L2 learners already have at least one other language in their minds; the initial language state of their minds is in principle different from the L1 child because of the first language they already know, however variously this may be interpreted. (Cook, 2010, t. 147)

Ymddengys felly fod caffael ail iaith yn digwydd wedi i'r siaradwr gaffael o leiaf un iaith arall eisoes. A dyfynnu Saville-Troike (2006, t. 193) ymhellach, mae'r term 'ail iaith' yn cyfeirio at "both to the study of individuals and groups who are learning a language subsequent to learning their first one as young children, and to the process of learning that language."

Cyn cloi'r is-bennod hon, mae'n bwysig pwysleisio eto rôl mewnbwn yn y broses o gaffael ail iaith. Fel y trafodwyd yn Pennod 1, mae sicrhau bod digonedd o oriau cyswllt â'r ail iaith yn hollbwysig os yw'r dysgwr i gaffael yr iaith yn effeithiol. Nid yw cyflwyno'r ail iaith am ychydig o oriau, yn arbennig mewn cyd-destunau addysgol lle ceir defnydd o'r dull bwydo (Baker, 2011) i gyflwyno'r iaith fel pwnc yn unig, yn ddigonol er mwyn sicrhau dwyieithrwydd ffwythiannol (Archibald ac 
eraill, 2006; Lightbown \& Spada, 2006). Er bod nifer o ffactorau eraill sydd yn effeithio ar y broses o gaffael ail iaith, mae'r dystiolaeth yn dangos bod cydberthynas rhwng amlder a natur y mewnbwn y mae'r plentyn yn ei dderbyn yn yr iaith darged a pha mor gyflym y mae'n caffael yr iaith (Gathercole \& Hoff, 2007, t. 120). Mae astudiaethau yng Nghymru, er enghraifft, wedi dangos cydberthynas rhwng dangosiad i'r Gymraeg yn y cartref ac agweddau ar gaffael yr iaith, megis strwythurau gramadegol a geirfa (Gathercole \& Thomas, 2005) a morffoleg enwau lluosog (Thomas ac eraill, 2013). Ac yn fwy perthnasol i'r ymchwil hon, ymddengys fod cydberthynas rhwng dangosiad i'r Gymraeg yn yr ysgol a chaffael yr iaith (Gathercole \& Thomas, 2005). Mewn astudiaeth o blant mewn ysgolion cyfrwng Cymraeg a dwyieithog o gefndiroedd ieithyddol gwahanol (cartrefi Cymraeg eu hiaith; cartrefi cymysg a chartrefi Saesneg eu hiaith), canfu Gathercole a Thomas (2005) fod iaith yr ysgol yn ffactor allweddol wrth gefnogi caffaeliad plant o'r Gymraeg, yn arbennig pan fo'r plant yn dod o gefndiroedd di-Gymraeg, lle na cheir mewnbwn Cymraeg naturiol yn y cartref.

Er nad oes cytundeb ymhlith ysgolheigion o ran sut yn union y mae plant yn caffael ail iaith yn effeithiol, mae ymchwil diweddar yn tueddu i gefnogi damcaniaethau'r safbwynt rhyngweithiol-datblygiadol, sy'n honni bod mewnbwn yn chwarae rôl bwysig yn y broses hon. Ys dywed Pike ac Young-Scholten (2009, t. 1) mewn byr eiriau: "as long [as] there is input, acquisition will occur." Gwaith athrawon, mae'n amlwg, yw sicrhau bod y mewnbwn hwn yn ystyrlon, o ansawdd dda, ac yn digwydd cyn amled â phosibl.

\subsection{Dwyieithrwydd}

Mae'r astudiaeth o ddwyieithrwydd yn faes mawr ac iddo sawl agwedd wahanol. Yn wir, mae'r maes wedi datblygu'n un rhyngddisgyblaethol (Hamers \& Blanc, 2000, xi), ac fel y meysydd caffael iaith a chaffael ail iaith, mae dwyieithrwydd yn cyffwrdd ag agweddau seicoleg, ieithyddiaeth gymdeithasegol, niwrowyddoniaeth, cymdeithaseg, addysgeg ymhlith eraill. Mae ysgolheigion wedi trafod dwyieithrwydd o safbwyntiau'r disgyblaethau hyn, wrth gynnig nifer o ddamcaniaethau a diffiniadau. Er gwaethaf terfynau eang astudiaethau dwyieithrwydd, noda Delyth Jones (1997, tt. 11-12) fod tuedd ymhlith ysgolheigion i rannu'r maes yn ddwy, megis 'dwyieithrwydd yr unigolyn' a 'dwyieithrwydd cymdeithasol'. Dyma'r hyn y bwriedir ei wneud wrth gyflwyno disgrifiadau 
dwyieithrwydd yn y traethawd hwn, cyn trafod pwysigrwydd dwyieithrwydd yng nghyd-destun y gwaith dan sylw.

\subsubsection{Disgrifio dwyieithrwydd yn hytrach na'i ddiffinio}

Mae diffinio'r enw 'dwyieithrwydd' a'r ansoddair 'dwyieithog' yn bendant yn her fawr i'r ysgolhaig a'r siaradwr unigol. Yn wir, mae dadl barhaus sydd yn dal i fodoli ymhlith yr unigolion a honna eu bod yn ddwyieithog, amlieithog neu'n unieithog, yn ogystal â'r arbenigwyr yn y maes sydd wedi cynnig gwahanol ddiffiniadau ar sail gwahanol feini prawf. Yr unig beth y gellir ei ddweud yn bendant am ddwyieithrwydd yw bod "[b]ilingualism as a concept has open-ended semantics" (Baetens-Beardsmore, 1986, t. 1). Mae Hoffmann (1991, t. 14), er enghraifft, wedi atgyfnerthu'r gred hon gan ddweud: "Whether one is considering it at a societal or an individual level, one has to accept that there can be no clear cut-off points. As bilingualism defies delimitation, it is open to a variety of descriptions, interpretations and definitions."

Oherwydd bod nifer fawr o ddiffiniadau y gellir eu cynnig, ymddengys nad oes un diffiniad safonol o'r term. Nid ymdrech i ailddiffinio'r term nac i drafod y gwahanol ddamcaniaethau a methedolegau posibl mo hon chwaith (gweler, er enghraifft, Thomas \& Mennen, 2014), ond yn hytrach, ystyrir y gwahanol ddiffiniadau a gynigir gan yr ysgolheigion er mwyn disgrifio dwyieithrwydd ac egluro ei arwyddocâd yng nghyd-destun y gwaith ymchwil hwn.

Fel y nodwyd eisoes, mae'n bosibl rhannu maes dwyieithrwydd yn syml yn ddwy ran, megis 'dwyieithrwydd yr unigolyn' a 'dwyieithrwydd cymdeithasol' (gweler, er enghraifft, Baker, 2011; Hamers \& Blanc, 2000; Hoffmann, 1991; Jones, 1997; Romaine, 1995). Mae'r naill faes astudiaeth yn ystyried agweddau seicoieithyddol a niwroieithyddol megis seicoleg dwyieithrwydd ${ }^{12}$, caffael ail iaith, effeithiau dwyieithrwydd (ieithyddol, gwybyddol, addysgol a niwrolegol, er enghraifft) a datblygiad y siaradwr dwyieithog o ran ei ddeallusrwydd a phersonoliaeth (D. Jones, 1997, t. 12), ac y mae'r llall yn ystyried agweddau ieithyddol-gymdeithasol wrth

\footnotetext{
${ }^{12}$ Mae 'seicoleg dwyieithrwydd' yn astudio sut y mae'r ddwy iaith (neu fwy) yn digwydd yn yr ymenydd. Noda McDonough (2004, tt. 494-495) fod seicoleg dwyieithrwydd yn gofyn cwestiynau megis: Sut y mae'r ieithoedd yn rhyngweithio â'i gilydd, sut y mae'r ieithoedd yn rhyngweithio â gwybyddiaeth a meddwl, a sut a pham mae'r siaradwyr eisiau defnyddio'r ieithoedd. Mae lleoliad yr ieithoedd yn yr ymennydd yn ystyriaeth seicolegol arall ym maes dwyieithrwydd (Jones, 1997).
} 
ymdrin â chynnal iaith, dewis iaith, cyfnewid cod, iaith a hunaniaeth, cynllunio ieithyddol ac addysg ddwyieithog (D. Jones, 1997, t. 12). Mae'r traethawd hwn yn cyffwrdd ag agweddau ar y ddau faes astudiaeth hyn, yn benodol caffael ail iaith, effeithiau dwyieithrwydd, cynllunio ieithyddol ac addysg ddwyieithog.

\subsubsection{Dwyieithrwydd yr unigolyn}

\subsubsection{Gallu}

Mae'n amlwg fod tuedd ymhlith arbenigwyr cynnar ym maes dwyieithrwydd i geisio diffinio a mesur dwyieithrwydd yr unigolyn ar sail hyfedredd y siaradwr yn ei ail iaith o'i gymharu â'r iaith gyntaf, neu ddefnydd y siaradwr o'r ddwy iaith. O ganlyniad i hyn, y mae sawl enghraifft annigonol a gynigwyd gan ysgolheigion nad ydynt yn bodloni pob agwedd ar ddwyieithrwydd. Yn unol â thraddodiad yr ysgolheigion cyfoes sy'n trafod maes dwyieithrwydd yn ei ystyr ehangach (Baker, 2001; Hamers \& Blanc, 2000; Hoffmann, 1991; D. Jones, 1997; Romaine, 1995), cyfeirir yma eto at rhai enghreifftiau defnyddiol a gynnig Delyth Jones (1997) er mwyn amlygu'r anhawster sy'n codi wrth ddiffinio'n union ddwyieithrwydd.

Yn gyntaf, ystyrir Bloomfield (1933), ysgolhaig Americanaidd cynnar ym maes ieithyddiaeth, megis man cychwyn wrth ddiffinio dwyieithrwydd yr unigolyn. Yn ôl Bloomfield (1933, t. 56), mae angen "native-like control of two or more languages" er mwyn i siaradwr fod yn ddwyieithog. Hefyd gan rannu safbwynt tebyg o ran gallu'r siaradwr dwyieithog, mae Oestreicher $(1974$, t. 9) wedi diffinio dwyieithrwydd megis "complete mastery of two different languages without interference." Wrth edrych ar y safbwynt eithafol arall, cynigiodd MacNamara (1969, t. 82) fod siaradwr dwyieithog yn "person who, for example, is an educated native speaker of English and who can also read a little French.” Cymerodd Haugen (1953) yntau safbwynt tebyg iawn i MacNamara (1969). Yn ôl Haugen (1953, t. 7), pan all siaradwr un iaith greu ymadroddion ystyrlon mewn iaith arall, yna gellir ei ystyried yn siaradwr dwyieithog.

Yr hyn a geir o'r ddau ddiffiniad uchod yw dau safbwynt gwahanol iawn yn eu hanfod. Ar y naill law, gellir diffinio person dwyieithog megis siaradwr sydd â sgiliau cyflawn mewn dwy iaith. Ar y llall law, gellir diffinio person dwyieithog megis siaradwr sydd ag unrhyw lefel o allu mewn mwy nag un iaith. Pe byddid yn derbyn diffiniad MacNamara, byddai siaradwr Saesneg sydd â'r gallu i gynhyrchu 
cyfarchion syml yn unig yn y Gymraeg, megis 'shwmae', 'bore da' a 'sut wyt ti', yn ddwyieithog. Pe byddid yn derbyn diffiniad Oestreicher ar y llaw arall, tasg heriol iawn fyddai dod o hyd i siaradwyr sydd â hyfedredd uchel a chytbwys yn y ddwy iaith, a thasg anos fyth fyddai mesur eu hunion alluoedd yn y ddwy iaith er mwyn profi eu bod yn gallu defnyddio'r ddwy iaith yn gyfartal.

Yr hyn y cyfeirir ato yma yw'r cysyniadau o 'ddwyieithrwydd cychwynnol' (incipient bilingualism), hynny yw, dwyieithrwydd yn ei gamau cychwynnol wrth i'r siaradwr ddod i gysylltiad â mwy nag un iaith, a 'dwyieithrwydd cytbwys' (balanced bilingualism), ‘dwyieithrwydd cyflawn' (full bilingualism) (Romaine, 1995, t. 6), neu yng ngeiriau Baetens-Beardsmore (1986, t. 9) 'dwyieithrwydd cyfartal' (equalingualism), hynny yw, hyfedredd uchel a chytbwys mewn dwy iaith. Mae disgrifio siaradwr dwyieithog, fel y gwna MacNamara (1969) uchod, yn golygu y gall siaradwr sydd newydd ddechrau dysgu ail iaith gael ei ystyried yn 'ddwyieithog'. Mae disgrifio siaradwr dwyieithog, fel y gwna Oestriecher uchod, fodd bynnag, yn golygu bod yn rhaid i'r siaradwr feistroli'r ddwy iaith yn gyfartal cyn iddo gael ei ystyried yn 'ddwyieithog'. Mae Hamers a Blanc (2000) yn trin dwyieithrwydd cytbwys megis lefel gyraeddadwy o ddwyieithrwydd, er nad yw'r cydbwysedd hwn yn cael ei ddosbarthu'n gyfartal ar draws pob pau neu swyddogaeth yn y ddwy iaith. Mae Thomas a Mennen (2014) fodd bynnag yn defnyddio'r term ar gyfer disgrifio siaradwyr sydd â hyfedredd tebyg yn y ddwy iaith. Gwahaniaethodd Thaler a Sunstein (2008) rhwng siaradwyr dwyieithog cytbwys a siaradwyr dwyieithog cychwynnol wrth gyfeirio at ddwy system o feddwl y mae seicolegwyr a niwrowyddonwyr yn eu galw'n 'System Awtomatig',13 (Automatic System) a 'System Adlewyrchol'14 (Reflective System). Mae siaradwyr dwyieithog cytbwys, yn debyg i siaradwyr uniaith, yn cynhyrchu iaith mewn ffordd naturiol heb feddwl yn fwriadol am yr hyn a ddywedir wrth ddefnyddio'r 'System Awtomatig'. Mae siaradwyr dwyieithog cychwynnol, fodd bynnag, yn dibynnu ar y 'System Adlewyrchol', hynny yw, cynhyrchu iaith mewn ffordd ymwybodol, fwriadol, wedi meddwl yn ofalus am yr hyn a ddywedir.

\footnotetext{
${ }^{13}$ Defnyddir yma'r term Cymraeg a fathwyd gan Elias (2014, Rhagfyr).

${ }^{14}$ Defnyddir yma'r term Cymraeg a fathwyd gan Elias (2014, Rhagfyr).
} 
Mae sawl ysgolhaig (Baker 2011; Hoffman 1991; Romaine 1995) wedi beirniadu'r cysyniad o ddwyieithrwydd cytbwys, gan nodi mai cysyniad delfrydol yn unig ydyw oherwydd bod siaradwyr dwyieithog fel arfer yn defnyddio eu dwy iaith ar gyfer swyddogaethau gwahanol ac mewn peuoedd gwahanol, a bod gan siaradwyr dwyieithog lefelau amrywiol o ruglder yn eu dwy iaith, fel arfer. Prin iawn felly yw dod ar draws siaradwyr sy'n gwbl gytbwys yn eu dwy iaith. O'r diffiniadau uchod, ymddengys felly nad yw'n ddigonol disgrifio dwyieithrwydd yn nhermau pendant gan fod diffiniadau o'r fath yn gorsymleiddio gallu'r person dwyieithog i siarad yr ail iaith ac nid ydynt yn cymryd i ystyriaeth y safon o hyfedredd y dylid ei chyrraedd er mwyn siarad iaith yn ystyrlon ac yn bwrpasol. O ganlyniad, mae tueddiad ymhlith ysgolheigion erbyn hyn i ystyried dwyieithrwydd yr unigolyn ar lefelau amrywiol ar hyd graddfa continwwm, gyda dysgwyr newydd ar yr naill ben o'r continwwm hwnnw, a siaradwyr dwyieithog 'cytbwys' yn eu dwy iaith ar y llall (D. Crystal, 2010a, t. 374). (Am drafodaeth lawnach, gweler 2.8. Y continwwm.)

Y cwestiwn mawr sy'n codi o'r ddau safbwynt uchod felly yw pa mor 'fedrus' neu 'rugl' y dylai'r siaradwr fod yn yr ail iaith er mwyn bod yn ddwyieithog? Dyma wendid yn strategaeth gyfredol LlC ar gyfer y Gymraeg. Ni cheir yn strategaeth Cymraeg 2050 ddiffiniad clir o'r hyn a olygir gan y term 'siaradwyr Cymraeg'; ai siaradwyr rhugl yn y Gymraeg a olygir, neu ddefnyddwyr rheolaidd o'r iaith? Oherwydd yr anhawster sy'n codi wrth geisio diffinio hyfedredd siaradwr yn ei ail iaith, cred Baker $(2011$, t. 7) fod dosbarthu siaradwyr megis siaradwyr uniaith neu siaradwyr dwyieithog yn rhy syml. Rhaid ystyried hyfedredd y siaradwr o ran ei sgiliau darllen, ysgrifennu, gwrando a siarad, ynghyd â'r sgiliau sy'n deillio o'r sgiliau hyn megis ynganiad, geirfa, arddull a chywirdeb gramadegol. Nid yw bod yn 'fedrus' neu'n 'rhugl' mewn mwy nag un iaith yn feini prawf digonol wrth ddiffinio dwyieithrwydd unigol, gan y gall 'rhuglder' a 'hyfedredd' mewn iaith amrywio o berson i berson yn dibynnu ar y lefelau gwahanol a gyrhaeddir yn y pedair sgil.

\subsubsection{Defnydd}

Fel y nodwyd eisoes, mae arbenigwyr y maes hefyd wedi cyfeirio at y defnydd o'r ddwy iaith a wneir wrth ddiffinio dwyieithrwydd yr unigolyn. Cyfeirir yma eto at enghraifft a gynnig Delyth Jones (1997), sef diffiniad Weinreich (1968). Yn ôl Weinreich $(1968$, t. 1): “The practice of alternately using two languages will be called bilingualism and the person involved, bilingual." Fodd bynnag, y broblem a 
wynebir wrth dderbyn y diffiniad hwn yw nad yw'n ystyried hyfedredd y siaradwr dwyieithog yn y ddwy iaith, ac nid yw'n manylu ar y swyddogaethau na'r peuoedd y defnyddir y ddwy iaith ar eu cyfer ychwaith.

Ar sail amrywiaeth y diffiniadau a gynigiwyd felly, ymddengys nad yw diffinio dwyieithrwydd unigol yn hawdd ac mae nifer helaeth o gwestiynau a phroblemau'n codi wrth geisio labelu siaradwyr yn uniaith neu'n ddwyieithog. Crynhoa Baker (2001, t. 5) gymhlethdod diffinio dwyieithrwydd unigol yn addas iawn wrth ddefnyddio cyfatebiaethau i olwynion beic ac ysbienddrych: “...the two wheels of bilingualism exist in different sizes and styles. The two lenses of bilingualism will vary in strength and size."

\subsubsection{Ffactorau eraill i'w hystyried}

Gan nad yw'n bosibl diffinio siaradwr dwyieithog ar sail gallu a defnydd yn y ddwy iaith yn unig, rhaid hefyd gymryd i ystyriaeth ffactorau posibl eraill sy'n effeithio ar ei allu a'i ddefnydd o'r ddwy iaith. O blith y ffactorau posibl y gellir eu hystyried, dyma'r ffactorau sydd fwyaf dylanwadol:

1. Datblygiad yr iaith gyntaf a'r ail iaith

2. Trefn a chanlyniad dysgu'r ieithoedd

3. Defnydd a swyddogaeth yr ieithoedd

4. Nodweddion ieithyddol megis cyfnewid cod a benthyg geiriau

5. Hyfedredd yn yr ieithoedd

6. Agweddau tuag at yr ieithoedd gan y siaradwr

7. Pwysau mewnol (e.e. seicolegol) ac allanol (e.e. cymdeithasol) ar y siaradwr

8. Amgylchedd y person dwyieithog

9. Cydnabyddiaeth y siaradwr o ddiwylliannau'r ddwy iaith. (Cyfieithiad o Hoffmann, 1991, t. 31)

Wrth ystyried mor gymhleth yw diffinio'n bendant y termau 'dwyieithrwydd' a 'dwyieithog', mae Hoffmann (1991) yn awgrymu mai llunio proffil ar sail y ffactorau hyn yw'r peth mwyaf buddiol. Mae Baker (2011) hefyd yn cytuno y byddai coethi'r gwahaniaethau a'r nodweddion a geir wrth drafod dwyieithrwydd yn fuddiol, yn hytrach na diffinio'n bendant y termau dwyieithrwydd a dwyieithog. Fel 
y noda Baker (2011, t. 15), mae diffinio'n bendant pwy sy'n ddwyieithog yn "essentially elusive and ultimately impossible."

\subsubsection{Dwyieithrwydd cymdeithasol}

Wedi trafod dwyieithrwydd ar lefel unigol, ymarferol, neu'r lefel feicro (Hamers \& Blanc, 2000, t. 273) ystyrir nesaf ddwyieithrwydd ar lefel gymdeithasol, neu'r lefel facro (Hamers \& Blanc, 2000, t. 273). Wrth ystyried dwyieithrwydd ar y lefel gymdeithasol, cymerir yma safbwynt yr ieithydd cymdeithasegol sy'n gofyn cwestiynau megis: Sut y mae'r ieithoedd yn rhyngweithio â'i gilydd mewn cymdeithas? Ar gyfer pa swyddogaethau y defnyddir ieithoedd mewn cymdeithas? Pa iaith yw'r un fwyafrifol a ph'un yw'r iaith leiafrifol? Ni cheir yma ond nifer fach o'r cwestiynau ieithyddol-gymdeithasegol posibl. Y prif bynciau o ddiddordeb i'r ieithydd cymdeithasegol felly, fel y nodwyd eisoes gan Jones (1997, t. 12), yw cynnal iaith, dewis iaith, cyfnewid iaith, iaith a hunaniaeth, cynllunio ieithyddol ac addysg ddwyieithog. Unwaith eto, ni fwriedir trafod pob agwedd ar ddwyieithrwydd cymdeithasol na'r pynciau sydd o ddiddordeb i'r ieithydd cymdeithasegol yma, ond yn hytrach, bwriedir egluro ystyr ac arwyddocâd y term 'dwyieithrwydd cymdeithasol' yng nghyd-destun y darn hwn o ymchwil.

\subsubsection{Diffinio 'iaith fwyafrifol' ac 'iaith leiafrifol'}

Cyn gwahaniaethu rhwng dwyieithrwydd yr unigolyn a dwyieithrwydd megis ffenomen gymdeithasol, rhaid egluro yn y traethawd hwn beth a olygir gan y termau 'iaith fwyafrifol' ac 'iaith leiafrifol'. Ceir nifer o rwystrau wrth geisio diffinio 'iaith fwyafrifol' ac 'iaith leiafrifol' oherwydd gall yr ansoddeiriau 'mwyafrifol' a 'lleiafrifol' gael eu dehongli mewn dwy ffordd wahanol. Ar y naill law, gall 'mwyafrifol' a 'lleiafrifol' awgrymu rhywbeth am y nifer sy'n siarad dwy iaith wahanol mewn cymuned. Yn yr ystyr hon, iaith sy'n cael ei siarad gan fwyafrif o'r boblogaeth mewn cymuned fyddai 'iaith fwyafrifol' ac iaith sy'n cael ei siarad gan leiafrif o'r boblogaeth mewn cymuned fyddai 'iaith leiafrifol'. Diffinia Romaine (2000, t. 35) 'iaith leiafrifol' fel iaith "with a relatively small number of speakers living within the domain of a more widely spoken language, whose knowledge is usually necessary for full participation in society." Fodd bynnag, ceir trafferth wrth ddehongli'r termau yn y ffordd hon. Er enghraifft, nid yw Cymraeg yn iaith leiafrifol 
mewn rhai ardaloedd yng Nghymru, megis Gwynedd ac Ynys Môn ${ }^{15}$, oherwydd bod y mwyafrif o'r boblogaeth yn yr ardaloedd hyn yn ei siarad hi (Baker \& Jones, 1998, t. 100).

Ar y llaw arall, gall 'mwyafrifol' a 'lleiafrifol' gyfeirio at statws a bri dwy iaith wahanol mewn cymuned. Wrth ddehongli 'iaith fwyafrifol' ac 'iaith leiafrifol' yn y ffordd hon, cydnabyddir bod yr ieithoedd yn gysylltiedig â'r grwpiau sy'n eu siarad hwy yn y gymuned. Yn yr ystyr hon felly, gellir diffinio 'iaith fwyafrifol' megis iaith y grŵp mwyaf grymus mewn cymuned sydd â dylanwad llywodraethol, ac 'iaith leiafrifol' megis iaith unrhyw grŵp sydd â llai o bwer a statws na'r grŵp pennaf mewn cymuned. Pwysleisia Baker a Jones (1998, t. 703) bwysigrwydd 'statws' wrth ddiffinio iaith fwyafrifol megis "[a] high status language usually (but not always) spoken by a majority of the population of a country." Mae Skutnabb-Kangas a McCarty (2008) hefyd yn pwysleisio pwysigrwydd 'statws' yr iaith, wrth ddiffinio 'iaith leiafrifol' fel a ganlyn:

[A] [1] anguage that is not the dominant language of a territorial unit such as a state, because the speakers of the language have less power (they have been minoritised), and the language is generally spoken by a smaller number of people. Power relations - not numbers - constitute the defining characteristic of 'minority' languages... (Skutnabb-Kangas \& McCarty, 2008, t. 10)

Wrth ddefnyddio'r fath ddiffiniadau mae'n bosibl deall sut mae Cymraeg yn iaith leiafrifol, o safbwynt statws, yng nghyd-destun Cymru gyfan, er ei bod hi'n iaith fwyafrifol, o ran nifer y siaradwyr Cymraeg, mewn ychydig o ardaloedd yng Nghymru.

At ddiben y traethawd hwn, diffinnir 'iaith fwyafrifol' ac 'iaith leiafrifol' fel y gwneir gan Baker a Jones (1998) a Skutnabb-Kangas a McCarty (2008). Mae iaith fwyafrifol felly yn iaith sydd â statws uchel mewn cymuned neu wlad, ac sydd yn arfer cael ei siarad gan fwyafrif o'r boblogaeth, ond nid bob tro. Mae iaith leiafrifol yn iaith sydd â statws is o'i chymharu â statws iaith fwyafrifol y gymuned neu wlad, ac sydd yn arfer cael ei siarad gan nifer lai o'r boblogaeth, ond nid bob tro.

\footnotetext{
${ }^{15}$ Gweler (Ystadegau'r Boblogaeth a'r Cyfrifiad, 2012) am ffigyrau Cyfrifiad ar gyfer siaradwyr Cymraeg fesul Awdurdod Lleol.
} 


\subsubsection{Gwahaniaethu rhwng dwyieithrwydd yr unigolyn a dwyieithrwydd cymdeithasol}

Fel y soniwyd eisoes, mae cryn duedd ym maes dwyieithrwydd i wahaniaethu rhwng dwyieithrwydd yr unigolyn a dwyieithrwydd cymdeithasol oherwydd bod agweddau'r ddau yn perthyn i feysydd gwahanol, sef seicoieithyddiaeth ac ieithyddiaeth gymdeithasegol, yn ôl eu trefn. Mae'n bosibl defnyddio'r term a ddefnyddiwyd gan Hamers a Blanc (2000) sef 'dwyieithedd' (bilinguality) wrth gyfeirio at allu'r unigolyn i gyfathrebu drwy ddau 'god'16 neu ddwy 'iaith', ac wedyn defnyddio'r term 'dwyieithrwydd' wrth gyfeirio at bresenoldeb dwy iaith (neu ragor) mewn cymuned. Cynigiwyd termau eraill gan ysgolheigion er mwyn gwahaniaethu rhwng dwyieithrwydd unigol a dwyieithrwydd cymdeithasol. Mae Fishman (1972, 1980) yn defnyddio'r termau 'diglosia' ${ }^{17}$ a 'dwyieithrwydd'. Defnyddia Fishman y term 'diglosia' wrth gyfeirio at gasgliad o bobl sydd yn defnyddio dau 'god' neu ddwy 'iaith' ar gyfer gwahanol swyddogaethau mewn cymdeithas. Defnyddia ef y term 'dwyieithrwydd' wedyn wrth gyfeirio at ddefnydd yr unigolyn o fwy nag un 'cod' neu 'iaith'. Oherwydd gall dwyieithrwydd unigol a dwyieithrwydd cymdeithasol, neu 'diglosia', ddigwydd ar wahân i'w gilydd, mae'n bosibl categoreiddio cymdeithasau yn ôl y bedair sefyllfa sosioieithyddol ganlynol a gynnig Fishman (1980):

1. Diglosia a dwyieithrwydd unigol

2. Diglosia heb ddwyieithrwydd unigol

3. Dwyieithrwydd unigol heb ddiglosia

4. Dim dwyieithrwydd na diglosia (cyfieithiad o Fishman, 1980, t. 6)

Er mwyn i gymunedau fod yn ddwyieithog felly, rhaid bod casgliad o unigolion a all siarad mwy nag un iaith. Ac i'r gwrthwyneb, er mwyn i unigolion fod yn ddwyieithog, rhaid bod casgliad o siaradwyr eraill i gyfathrebu â hwy mewn mwy

\footnotetext{
${ }^{16}$ Mae cod yn eitem ieithyddol a all fod yn acen, arddull ieithyddol, tafodiaith neu iaith (Holmes, 1992, t. 9).

${ }^{17}$ Mae diglosia yn derm a fathwyd gan Ferguson (1959) er mwyn cyfeirio at bresenoldeb dau god, amrywiad neu dafodiaith wahanol a fodola mewn cymdeithas ar gyfer gwahanol swyddogaethau. Fel arfer, cyfeirir at amrywiad uchel ' $\mathrm{H}$ ' ac amrywiad isel 'L' (High variation; Low variation). Datblygwyd y cysyniad hwn gan Fishman $(1972,1980)$ er mwyn cwmpasu’r term 'iaith’ yn y diffiniad.
} 
nag un iaith. Yr hyn a geir yma felly yw cydbwysedd rhwng yr unigolion sy'n gorfod siarad yr ieithoedd a'r gymuned y mae'n rhaid wrthi er mwyn cynnal nifer yr unigolion dwyieithog. Heb siaradwyr dwyieithog sy'n siarad mwy nag un iaith â'i gilydd, ni cheir cymdeithas ddwyieithog. Ac i'r gwrthwyneb, heb gymuned ddwyieithog sy'n caniatáu i siaradwyr siarad mwy nag un iaith â'i gilydd, ni cheir siaradwyr dwyieithog. Gall y cydbwysedd hwn fod yn ansefydlog, yn arbennig os yw cymdeithas mewn sefyllfa o shifft ieithyddol o un iaith i'r llall. ${ }^{18}$ Trwy ymdrechion yr ieithydd cymdeithasegol i gynnal iaith a chynllunio'n ieithyddol fodd bynnag, gellir gwrthdroi shifft ieithyddol a chadw iaith leiafrifol mewn cymdeithas ddwyieithog rhag tranc iaith, ac felly, gwarchod, cynnal a chynyddu nifer yr unigolion dwyieithog mewn cymdeithas yn eu tro.

Er bod y ddau fath hyn o ddwyieithrwydd yn elfennol wahanol (Hoffmann, 1991, t. 13), mae'n bwysig cofio nad ydynt yn digwydd mewn gwagle. Er bod dwyieithrwydd unigol a dwyieithrwydd cymdeithasol yn ymwneud ag agweddau gwahanol ar ddwyieithrwydd, maent yn gysylltiedig, a gall y naill gael effaith ar y llall (Baker, 2011, tt. 2-3). Fel yr esbonia Romaine (1995, t. 23): "It is not possible to make a neat separation between bilingualism as a societal and individual phenomenon..."

\subsubsection{Dwyieithrwydd cydamserol}

Er bod y diffiniadau uchod wedi cynnig gwahaniaeth clir rhwng caffael iaith gyntaf a chaffael ail iaith, nid yw'n bosibl defnyddio'r meini prawf damcaniaethol hyn wrth ddisgrifio siaradwr sydd yn caffael dwy iaith gyntaf (neu ragor) ar yr un pryd. Enghraifft o hyn yw siaradwr dwyieithog yn y Fasgeg a'r Sbaeneg yng Ngwlad y Basg. Mae nifer o siaradwyr dwyieithog yn caffael ieithoedd ar yr un pryd o oedran ifanc, mewn cyd-destunau ffurfiol ac anffurfiol, oherwydd presenoldeb a statws y Sbaeneg a'r Fasgeg yn y gymuned hon. Mae hyn yn wir hefyd yng Nghymru, gyda siaradwyr sydd wedi caffael y Gymraeg a'r Saesneg o oedran ifanc, yn arbennig y rhai sydd wedi derbyn addysg ffurfiol mewn ysgol cyfrwng Cymraeg neu ysgol ddwyieithog, lle mae presenoldeb Cymraeg ar ei uchaf. Er gwaetha'r ffaith mai Cymraeg yw prif iaith cyfrwng, neu un o brif ieithoedd cyfrwng yr addysg yn yr ysgolion hyn, mae'n bosibl i blant gaffael y Saesneg a'r Gymraeg ar yr un pryd o

\footnotetext{
${ }^{18}$ Dyma'r broses lle mae cymuned o siaradwyr uniaith, siaradwyr yr iaith leiafrifol fel arfer, yn mabwysiadu iaith arall yn raddol dros amser (Jones, 1997, t. 124).
} 
oedran ifanc oherwydd presenoldeb a dylanwad y Saesneg y tu hwnt i'r ysgol, yng Nghymru, ac yn rhyngwladol. Yn yr achosion hyn, mae'n bosibl disgrifio'r broses lle mae caffael dwy iaith gyntaf (neu ragor) yn digwydd megis "simultaneous multilingualism" (Saville-Troike, 2006, t. 13), neu'r hyn y mae Swain (1972) yn ei ddisgrifio'n ddwyieithrwydd megis iaith gyntaf, hynny yw, 'dwyieithrwydd cydamserol'.

\subsubsection{Dwyieithrwydd cynyddol a dwyieithrwydd gostyngol}

Yng nghyd-destun y traethawd hwn, cyfeirir yn bennaf at gaffael neu ddysgu ail iaith, yn bennaf y Gymraeg, mewn ysgolion at ddiben ychwanegu at yr iaith gyntaf, sef y Saesneg yn achos disgyblion CAI. Dyma'r hyn a elwir yn 'ddwyieithrwydd cynyddol' (additive bilingualism) (Lambert, 1974; Baker, 2011; Baker \& Jones, 1998; García, 2009), neu 'ddwyieithrwydd esgynnol' (D. Jones, 1997). Bwriad y fath addysg yw sicrhau llythrennedd deuol a dwyieithrwydd yn yr iaith leiafrifol (yr ail iaith) a'r iaith fwyafrifol (yr iaith gyntaf), heb effaith negyddol ar yr iaith gyntaf, wrth fwrw pwyslais arbennig ar yr ail iaith (Baker \& Jones, 1998). (Am drafodaeth lawnach, gweler 2.5. Addysg ddwyieithog yng nghyd-destun yr ymchwil.)

Pwysig hefyd yw nodi bod enghreifftiau o gaffael / dysgu ail iaith mewn cyddestunau lle mae'r ail iaith yn cael ei chaffael ar draul yr iaith gyntaf, a dyma'r hyn a elwir yn 'ddwyieithrwydd gostyngol' (subtractive bilingualism) (Lambert, 1974; Baker, 2011; Baker \& Jones, 1998; García, 2009), neu ‘ddwyieithrwydd enciliol’ (D. Jones, 1997). Enghraifft o hyn yw disgybl sy'n siarad iaith leiafrifol megis iaith gyntaf, ond sy'n derbyn ei addysg yn gyfan gwbl drwy gyfrwng iaith fwyafrifol y gymuned. Dyma'r math o addysg a elwir yn 'addysg foddi' (submersion education). Bwriad y fath addysg yw cymathu siaradwyr iaith leiafrifol â chymuned o siaradwyr iaith fwyafrifol wrth eu haddysgu drwy gyfrwng yr iaith fwyafrifol (gweler 2.5. Addysg ddwyieithog yng nghyd-destun yr ymchwil). Mewn achosion o'r fath lle mae'r iaith fwyafrifol yn gyfrwng yr addysg ac nid yw'r disgybl yn derbyn unrhyw addysg ffurfiol trwy gyfrwng ei iaith gyntaf, yr iaith leiafrifol, mae perygl y gall rhuglder y disgybl yn ei iaith gyntaf wanhau, neu hyd yn oed gael ei golli yn gyfan gwbl, wrth i'r iaith fwyafrifol gymryd lle'r iaith leiafrifol. 


\subsection{Dwyieithrwydd yng nghyd-destun yr ymchwil}

Yn y traethawd hwn, defnyddir y term 'dwyieithrwydd unigol' neu 'ddwyieithrwydd yr unigolyn' wrth gyfeirio at ddwyieithrwydd ar lefel yr unigolyn. O ran cyfeirio at berson neu ddisgybl sydd yn adnabod mwy nag un iaith ac/neu sydd yn defnyddio mwy nag un iaith, defnyddir y termau 'siaradwr dwyieithog', 'unigolyn dwyieithog' a 'disgybl dwyieithog' yn gyfystyr. O ran cyfeirio at lefel gymdeithasol o ddwyieithrwydd, defnyddir y term 'dwyieithrwydd cymdeithasol' a defnyddio'r term 'cymdeithas ddwyieithog' wrth gyfeirio at gymuned/cymdeithas sydd â thrigolion dwyieithog. Gan nad yw'r traethawd hwn yn canolbwyntio'n benodol ar sefyllfa sosio-ieithyddol Cymru a gwledydd eraill, credir na fydd angen gwahaniaethu rhwng sefyllfaoedd lle mae diglosia yn bresennol ai peidio.

Mae'n bwysig hefyd nodi y defnyddir y termau 'dwyieithrwydd' a 'dwyieithog' yn lle'r termau 'amlieithrwydd' ac 'amlieithog', hyd yn oed wrth gyfeirio at unigolion a chymdeithasau sydd yn siarad mwy na dwy iaith. Er y gellir defnyddio'r termau hyn yn gyfystyr, credir mai synhwyrol fydd dilyn arfer ysgrifenwyr eraill yn y maes megis Baker (1996, 2011), García (2009), Romaine (1995) a Hoffmann (1991), gan mai cyd-destunau lle y ceir dwy iaith mewn addysg fydd canolbwynt y traethawd hwn.

\subsection{Addysg ddwyieithog yng nghyd-destun yr ymchwil}

Mae'r defnydd o'r label 'addysg ddwyieithog' yn asgwrn cynnen ym maes addysg gan fod modd defnyddio'r label hwn ar gyfer nifer o wahanol fodelau o addysg. Yn syml iawn, mae'n bosibl diffinio 'addysg ddwyieithog' fel y mae Andersson a Boyer (1978) a Skutnabb-Kangas a McCarty (2008) yn ei wneud, megis defnyddio dwy iaith (neu ragor) yn ieithoedd cyfrwng mewn pynciau addysgol, ar wahân i’r pynciau hynny sy'n astudio'r ieithoedd hyn eu hunain. Fodd bynnag, mae diffinio 'addysg ddwyieithog' yn y ffordd hon yn gorsymleiddio cymhlethdod addysg ddwyieithog. Fel y mae Baker (2011, t. 207) yn ei esbonio'n syml: "Bilingual education is a simplistic label for a complex phenomenon."

Er mwyn deall beth a olygir gan y term 'addysg ddwyieithog', rhaid deall cyd-destun yr addysg, ynghyd ag amcanion a chanlyniadau'r rhaglen addysg. Er bod modd rhannu addysg ddwyieithog yn dri math (Scott \& Beadle, 2014), yn ddeng math (Baker, 2011; Baker \& Jones, 1998), neu hyd yn oed yn naw deg math (Mackey, 
1970, fel y dyfynnwyd yn Baker, 2011), ni cheir yma drafodaeth ar bob un o'r gwahanol fodelau posibl o addysg ddwyieithog. Yn hytrach, canolbwyntir yma ar ddisgrifio'r rhaglenni addysg ddwyieithog sy'n berthnasol i'r ymchwil hon. Wrth gofio y gall 'addysg ddwyieithog' gyfeirio at nifer fawr o raglenni addysg, defnyddir y term hwn yn unol ag arfer García (2009), megis term ymbarél sy’n cyfeirio at unrhyw addysg lle y ceir defnydd o ddwy iaith, neu ragor, megis ieithoedd cyfrwng yn yr addysg, at ddiben mabwysiadu dwyieithrwydd cynyddol. Yn yr ystyr hon felly, nid rhaglenni dwyieithog mo 'rhaglenni addysg foddi'. Mae 'addysg foddi' yn anelu at gymathu siaradwyr iaith leiafrifol y gymuned â siaradwyr iaith fwyafrifol y gymuned wrth gyflwyno addysg trwy gyfrwng yr iaith fwyafrifol yn unig. Canlyniad y fath addysg yw unieithrwydd a sefyllfa o ddwyieithrwydd gostyngol gan nad oes ymdrech fwriadol i gynnal iaith gyntaf y disgybl (Baker, 2011, t. 209; Baker \& Jones, 1998, t. 470). Yng nghyd-destun y traethawd hwn, gall 'addysg ddwyieithog' gyfeirio at raglenni addysg lle y caiff dwy iaith, neu ragor, eu gwahanu mewn addysg, neu lle y caiff y ddwy iaith eu defnyddio ar y cyd (García, 2009). Dyma'r hyn y mae Blackledge a Creese (2010) wedi'i enwi'n 'ddwyieithrwydd ar wahân' (separate bilingualism) a 'dwyieithrwydd hyblyg' (flexible bilingualism), yn ôl eu trefn.

Er bod modd defnyddio labeli megis 'addysg deirieithog' neu 'addysg amlieithog' wrth gyfeirio at addysg lle y ceir defnydd o dair iaith neu ragor, credir y bydd yn synhwyrol defnyddio'r term 'addysg ddwyieithog' oherwydd bod y term hwn yn "more grounded in theory, research, practice, and reality than 'multilingual education'.” (García, 2009, t. 11).

\subsubsection{Addysg drochi}

Math o addysg ddwyieithog sydd â'i gwreiddiau yn y 1960au yn St. Lambert, Montreal, Canada yw addysg drochi. Er bod 'addysg drochi' yn gysylltiedig yn hanesyddol â chyflwyno iaith fwyafrifol megis cyfrwng addysg i siaradwyr iaith fwyafrifol arall, mae rhaglenni trochi wedi lledaenu i gyd-destunau eraill lle cyflwynir iaith leiafrifol neu frodorol megis iaith cyfrwng at ddiben cynnal yr iaith honno (Skutnabb-Kangas \& McCarty, 2008, t. 3). O ganlyniad, mae'n bosibl gwahaniaethu rhwng dau fath o raglen addysg drochi erbyn hyn, sef 'rhaglenni trochi i siaradwyr iaith fwyafrifol' a 'rhaglenni trochi i siaradwyr iaith dreftadaeth / leiafrifol' (Skutnabb-Kangas \& McCarty, 2008). Mae'n bosibl hefyd labelu'r rhaglen 
olaf hon yn 'addysg cynnal iaith', 'addysg iaith dreftadaeth' (Baker, 1993, 2011; Baker \& Jones, 1998) neu 'addysg iaith y gymuned' (Johnstone, 2002). Ni fydd angen gwahaniaethu'n fanwl rhwng y gwahanol fathau o addysg drochi yn y traethawd hwn, ond gwahaniaethir rhwng y ddau brif fath o 'raglen drochi' wrth ddefnyddio'r termau 'addysg drochi' ac 'addysg dreftadaeth', yn unol ag arfer Baker (2011), Baker a Jones (1993), Johnstone (2002) a Lewis (2008).

Mewn rhaglenni addysg drochi i siaradwyr iaith fwyafrifol, anelir at drochi disgyblion o gartrefi iaith fwyafrifol y gymuned mewn iaith dramor fodern neu iaith leiafrifol, trwy ddefnyddio'r ail iaith fel iaith cyfrwng yr addysg. Yn y rhaglenni hyn, rhoir pwyslais cychwynnol ar yr ail iaith (Baker, 2011, t. 210; Baker \& Jones, 1998, t. 470), gyda defnydd cynyddol o'r iaith gyntaf, yn unol â'r math o raglen. Amcanion ieithyddol y rhaglenni hyn yw sicrhau llythrennedd deuol a dwyieithrwydd yn iaith fwyafrifol y gymuned (iaith gyntaf y siaradwr) ac yn yr iaith dramor fodern / iaith leiafrifol (ail iaith y siaradwr). Er bod rhaglenni o'r fath fel arfer yn wirfoddol (Day \& Shapson, 1996, t. 1), mae enghreifftiau lle nad yw'r fath addysg yn wirfoddol. Enghraifft o hyn yw addysg drochi yn y Gymraeg yng Ngwynedd, gogledd Cymru, lle nodir bod bron pob ysgol gynradd ac uwchradd y sir, naill ai'n ysgol cyfrwng Cymraeg neu'n ysgol ddwyieithog. Mae polisi addysg Gwynedd yn blaenoriaethu'r Gymraeg megis iaith cyfrwng addysg y sir, ac mae hyn yn amlwg o'i chynllun strategol ar gyfer y Gymraeg mewn addysg sydd am "sicrhau datblygiad y Gymraeg fel pwnc a chyfrwng dysgu o oedran cyn ysgol ymlaen yn ogystal â hyrwyddo'r defnydd a wna ein plant a'n pobl ifanc o'r Gymraeg fel iaith gymdeithasol." (Cyngor Gwynedd, 2014, t. 3). Y gwir am sefyllfa addysg cyfrwng Cymraeg Gwynedd yw nad yw addysg drochi yn wirfoddol, a hynny o reidrwydd. Dyma sefyllfa gymhleth a nodir mewn nifer o sefyllfaoedd addysg yng Nghymru, lle y caiff y Gymraeg ei chynnal a'i gwarchod megis iaith dreftadaeth, sydd o dan fygythiad ieithoedd mwyafrifol megis y Saesneg, yn ogystal â'i chyflwyno megis iaith drochi i siaradwyr Cymraeg ail iaith (Lewis, 2008). Mae sefyllfa addysg ddwyieithog yng Nghymru felly'n un gymhleth iawn gan fod 'amrywiaeth caleidosgopig' yn bodoli ledled y wlad erbyn hyn (Baker, 1993; B. Jones, 2010).

\subsubsection{Addysg dreftadaeth}

Mewn rhaglenni trochi i siaradwyr iaith dreftadaeth, neu 'addysg dreftadaeth', anelir at warchod a chynnal iaith dreftadaeth neu iaith leiafrifol y gymuned, wrth ddatblygu 
hefyd iaith fwyafrifol y gymuned, trwy ddefnyddio'r iaith leiafrifol megis iaith cyfrwng wrth gyflwyno cynnwys addysgol. Yn y rhaglenni hyn, rhoir pwyslais arbennig ar ddatblygu'r iaith leiafrifol (Baker, 2011, t. 210; Baker \& Jones, 1998, t. 470). Amcanion ieithyddol y rhaglenni hyn yw sicrhau llythrennedd deuol a dwyieithrwydd cyflawn yn yr iaith dreftadaeth yn ogystal ag iaith fwyafrifol y gymuned. Mae'r rhaglenni hyn yn wirfoddol (Skutnabb-Kangas \& McCarty, 2008, t. 7), ac fel arfer mae gan rieni ddewis mewn perthynas â'r math hwn o addysg (Baker, 2011, t. 235), ond nid yw'r fath addysg ar gael ym mhob achos.

\subsubsection{Addysg iaith dramor fodern ac addysg ail iaith}

Dyma raglenni a gyflwynir ym maes addysg i ddisgyblion iaith fwyafrifol y gymuned er mwyn iddynt ddatblygu hyfedredd mewn iaith dramor fodern neu ail iaith, trwy ei hastudio megis pwnc yn unig, fel arfer. Ni cheir defnydd o'r iaith darged yn bwrpasol ac ystyrlon megis iaith cyfrwng wrth gyflwyno cynnwys mewn pynciau neu fodiwlau eraill. Cyfeiria García (2009) at y rhaglenni hyn megis 'rhaglenni addysgu iaith traddodiadol'. Gan gyfieithu Baker (1993), bathodd D. Jones (1997) y label 'prif ffrwd ynghyd â dysgu iaith dramor' ar gyfer rhaglenni o'r math. Mae'n bosibl gwahaniaethu rhwng y ddau fath hyn o raglenni traddodiadol, sef 'addysg iaith dramor fodern' ac 'addysg ail iaith' (Baker, 2011; Baker \& Jones, 1998). Lle y gellir disgrifio addysg drochi ac addysg dreftadaeth yn fathau 'cryf' o addysg ddwyieithog, gellir disgrifio addysg iaith dramor fodern ac addysg ail iaith yn fathau 'gwan' o addysg ddwyieithog oherwydd bod canlyniadau'r fath addysg yn aml yn gyfyngedig. Ys dywed Baker (2011, t. 218): "Mainstream education rarely produces functionally bilingual children. A very limited knowledge of a foreign language tends to be the typical outcome for the mass of the language majority."

Er bod Baker yn trin 'rhaglenni addysgu iaith traddodiadol' megis math o addysg ddwyieithog, ni chred García (2009) mai rhaglenni dwyieithog yw'r rhain gan nad yw'r rhaglenni hyn yn cyflwyno'r iaith darged mewn modd ystyrlon sy'n gwneud defnydd ohoni megis cyfrwng addysg. Yn ôl García (2009, t. 6), ni all 'rhaglenni addysgu iaith traddodiadol" "provide a general education, teach in two or more languages, develop multiple understandings about languages and cultures," neu "foster appreciation for human diversity." I'r un perwyl, mae Skutnabb-Kangas a McCarty (2008, t. 4) hefyd yn diffinio'r rhaglenni hyn yn "[n]on-forms of bimultilingual education." Gan na cheir defnydd o ddwy neu ragor o ieithoedd megis 
ieithoedd cyfrwng yr addysg yn y rhaglenni hyn, nid ystyrir 'addysg iaith dramor fodern' nac 'addysg ail iaith' yn rhaglenni dwyieithog yn y traethawd hwn.

\subsubsection{Content and Language Integrated Learning}

Math o addysg ddwyieithog a ddatblygodd yn ystod y 1990au yn Ewrop mewn ymateb i'r galw cynyddol am well addysg ail iaith / addysg iaith dramor fodern yw 'Content and Language Integrated Learning' (CLIL o hyn ymlaen) (Marsh, 2002). Er y caiff CLIL ei gysylltu i raddau helaeth ag addysgu Saesneg fel iaith dramor (Dalton-Puffer, Nikula, \& Smit, 2010; Whittaker \& Llinares, 2011), mae amrywiaeth fawr o raglenni CLIL yn bodoli erbyn hyn (gweler 4. Y gymhariaeth ryngwladol: Tystiolaeth o Ganada, Gwlad y Basg a'r Almaen). Gan ymdebygu i addysg drochi ac addysg dreftadaeth, mae rhaglenni CLIL yn cyflwyno'r ail iaith trwy ei hintegreiddio â chynnwys addysgol a thrwy ei defnyddio megis iaith cyfrwng yr addysg. Yn ôl Coyle (2006), Pérez-Cañado (2012) a Surmont, Struys, van den Noort a van de Craen (2016), gellir ystyried CLIL fel ymagwedd sydd â dau nod, sef dysgu cynnwys a dysgu iaith, lle nad yw'r naill nod yn bwysicach na'r llall. Yn y rhaglenni hyn, addysgir rhwng 10\% a 50\% o'r cwricwlwm trwy gyfrwng yr iaith darged (Baker, 2011, t. 245). Yn hytrach na sicrhau cymhwysedd brodorol (native-like competence) neu gymhwysedd sydd bron yn frodorol (near-native competence) yn yr ail iaith / iaith dramor, fel y mae rhaglenni addysg drochi llawn yn anelu ato, diben rhaglenni CLIL yw sicrhau cymhwysedd ffwythiannol yn yr ail iaith / iaith dramor (Muñoz, 2002; Pérez-Cañado, 2012).

Er bod rhai ysgolheigion yn dadlau mai cyrchddull sydd ar wahân i addysg drochi yw CLIL (Lasagabaster \& Sierra, 2010), mae eraill o'r farn nad yw CLIL yn unigryw yn nhermau methodoleg o'i gymharu â 'Content Based Instruction' (CBI o hyn ymlaen) (Cenoz, Genesee, \& Gorter, 2014; Coyle, Hood, \& Marsh, 2010; DaltonPuffer, 2007; Van de Craen, Ceuleers, \& Mondt, 2007), a chred rhai ysgolheigion fod y gwahaniaethau hyn yn gyd-destunol yn unig (Cenoz, 2015; Cenoz ac eraill, 2014; Paran, 2013). Ymddengys felly mai gwell yw trin CLIL megis term ymbarél sy'n cyfeirio at nifer o gyrchddulliau addysgol mewn addysg ddwyieithog lle cyflwynir ail iaith trwy ei hintegreiddio â chynnwys (Cenoz ac eraill, 2014; Mehisto, Marsh, \& Frigols, 2008). Er y cydnabyddir yn y traethawd hwn fod CLIL yn derm ymbarél, rhaid nodi y caiff y term ei ddefnyddio yn y llenyddiaeth ryngwladol wrth gyfeirio at raglenni dwyieithog penodol y gellid eu labelu'n well megis 'addysg 
drochi rannol hwyr' (gweler 4. Y gymhariaeth ryngwladol: Tystiolaeth o Ganada, Gwlad y Basg a'r Almaen). Oherwydd hynny, defnyddir yn y traethawd hwn y labeli 'addysg drochi rannol hwyr' a 'CLIL' yn gyfystyr.

\subsection{Addysg cyfrwng Cymraeg, addysg ddwyieithog a Chymraeg Ail Iaith}

Yn y sector cynradd a'r sector uwchradd, nodir bod sawl gwahaniaeth yn bodoli yn y ffordd y cyflwynir y Gymraeg yn ysgolion Cymru, yn arbennig o ran cyfrwng neu gyfryngau'r dysgu, nifer yr oriau cyswllt â'r iaith, faint o bwyslais a roddir arni megis iaith gyfathrebu feunyddiol yn yr ysgol a hefyd yr arholiadau ffurfiol a sefir. Er gwaetha'r gwahaniaethau hyn rhwng yr ysgolion, gellir nodi bod tri llwybr bras y gellir eu dilyn yng nghyfundrefn addysg Cymru, a cheir gwahanol raddau o gyswllt â'r Gymraeg ymhob un. Y tri llwybr hyn yw addysg cyfrwng Cymraeg, addysg ddwyieithog ac addysg cyfrwng Saesneg. Nid yw'r tri llwybr hyn mor sefydlog na phendant ag y byddid yn ei ddisgwyl, ac o ganlyniad, mae'n bosibl rhannu'r mathau o ysgol ymhellach, fel y gwelir yn yr is-adran isod.

\subsubsection{Diffinio ysgolion yn ôl eu darpariaeth cyfrwng Cymraeg}

Yn swyddogol, diffinnir gan Adran 105(7) o Ddeddf Addysg 2002 'ysgol Gymraeg ei hiaith' yn nhermau ysgol lle y caiff mwy na hanner y pynciau sylfaenol, gan gynnwys addysg grefyddol, ac eithrio'r Gymraeg a'r Saesneg, eu haddysgu yn llawn neu'n rhannol drwy gyfrwng yr iaith Gymraeg. Noder fodd bynnag nad yw'r diffiniad cyfreithiol uchod yn manylu ar "natur ddarpariaeth ieithyddol ar draws y cwricwlwm" ac yn wir, nid dyna oedd bwriad y diffiniad (LICC, 2007b, t. 3). Bwriad y diffiniad cyfreithiol hwn oedd gwahaniaethu rhwng yr ysgolion lle mae Cymraeg yn bwnc craidd ac yn gyfrwng dysgu â'r ysgolion lle mae Cymraeg yn bwnc sylfaenol yn unig (LlCC, 2007b). Fodd bynnag, ers diffinio ysgolion yn unol â'r diffiniad hwn, mae dryswch wedi codi ynglŷn â labelu a chymharu ysgolion yn ôl eu gwahanol raddau o ddarpariaeth ar gyfer Cymraeg, megis iaith cyfrwng dysgu ac iaith sgyrsiol bob dydd yn yr ysgol. Cafodd yr angen am set newydd o ddiffiniadau ei nodi gan Bwyllgor Addysg a Dysgu Gydol Oes y Cynulliad (2002) yn ei adolygiad polisi o'r Gymraeg, sef Ein hiaith: Ei dyfodol, ac unwaith eto gan LICC (2003), yn ei strategaeth gyntaf ar gyfer y Gymraeg, sef Iaith Pawb, fel mater o flaenoriaeth.

O dan arweiniad Bwrdd yr Iaith Gymraeg a LlCC, ac ar sail gwaith blaenorol gan grŵp o arbenigwyr o dan arweiniad y Bwrdd, cafodd sawl cyfarfod ymgynghori ei 
gynnal er mwyn ystyried diffiniadau newydd ar gyfer ysgolion yn ôl eu darpariaeth cyfrwng Cymraeg. Yn 2007, ailddiffiniwyd y gwahanol fathau o ysgolion yng Nghymru yn ôl eu darpariaeth cyfrwng Cymraeg yn yr adroddiad Diffinio ysgolion yn ôl y ddarpariaeth cyfrwng Cymraeg (L1CC, 2007b) ar sail dibenion blaenorol y Pwyllgor Addysg a Dysgu Gydol Oes, sef pennu amcanion clir ar gyfer addysgwyr a disgyblion mewn perthynas â chanlyniadau ieithyddol, rhoi gwybodaeth glir i rieni a'u plant am y dewisiadau sydd ar gael, helpu Awdurdodau Addysg Lleol i ddatblygu strategaethau ar gyfer y ddarpariaeth cyfrwng Cymraeg, a gwerthuso cryfderau a gwendidau'r modelau gwahanol o addysgu iaith o'u cymharu â'i gilydd (LlCC, 2007b, tt. 4-5).

\subsubsection{Categoriau ieithyddol ysgolion y sector cynradd}

Yn unol â Diffinio ysgolion yn ôl y ddarpariaeth cyfrwng Cymraeg (2007b), ceir pum prif gategori ar gyfer ysgolion cynradd Cymru, sef:

i. Ysgol Gynradd Cyfrwng Cymraeg

ii. Ysgol Gynradd Ddwy Ffrwd

iii. Ysgol Gynradd Drawsnewidiol: cyfrwng Cymraeg ond â defnydd sylweddol o'r Saesneg

iv. Ysgol Gynradd cyfrwng Saesneg yn bennaf ond â defnydd sylweddol o'r Gymraeg

v. Ysgol Gynradd cyfrwng Saesneg yn bennaf

Caiff y diffiniadau uchod eu seilio ar dair ystyriaeth benodol, sef:

i. y cyfrwng addysgu ym mhob cyfnod allweddol: h.y. canran y cwricwlwm (cynradd) a chanrannau'r pynciau (uwchradd) a addysgir drwy gyfrwng y Gymraeg a'r Saesneg;

ii. $\quad y r$ iaith/ieithoedd a ddefnyddir i gyfathrebu â'r disgyblion y tu allan i'r cwricwlwm, ethos yr ysgol, yr iaith a ddefnyddir yn yr ysgol o ddydd i ddydd, a'r iaith/ieithoedd a ddefnyddir i gyfathrebu â'r rhieni; ac

iii. y canlyniadau arferol a ddisgwylir ar gyfer disgyblion sy'n mynychu pob math o ddarpariaeth o ran eu datblygiad addysgol.

(LlCC, 2007b, t. 6) 
Diffinia LlCC (2007b, t. 8) ysgol gynradd cyfrwng Cymraeg megis ysgol sy’n cyflwyno cyfleoedd i ddisgyblion y CS ddysgu trwy'r Gymraeg (heb fanylu ar faint o Gymraeg), ac sy'n cyflwyno o leiaf 70\% o'r cwricwlwm trwy gyfrwng y Gymraeg yn CA2. Defnyddir y Saesneg ar gyfer cyflwyno Saesneg megis pwnc ac weithiau agweddau ar bynciau eraill. O ran iaith yr ysgol, Cymraeg yw'r iaith a ddefnyddir ar gyfer cyfathrebu â'r disgyblion trwy'r dydd ac ar gyfer gweinyddiaeth yr ysgol, ond mae Saesneg a Chymraeg yn cael eu defnyddio wrth siarad â rhieni. Mae disgwyl i ddisgyblion y categori hwn drosglwyddo i ysgol uwchradd cyfrwng Cymraeg.

Mae ysgol gynradd ddwy ffrwd, yn ôl y diffiniadau newydd (LlCC, 2007b, t. 8), yn ysgol lle mae dau fath o ddarpariaeth yn cael ei weithredu, ochr yn ochr â'i gilydd, yn yr ysgol. Mae'r naill ddarpariaeth yn dilyn strwythur Categori (i), sef cyfrwng Cymraeg, ac mae'r llall yn dilyn strwythur Categori (v), sef cyfrwng Saesneg yn bennaf. O ran iaith yr ysgol, mae'r ysgol yn defnyddio'r ddwy iaith ar gyfer cyfathrebu â disgyblion, ond nodir mai natur y ddarpariaeth y mae'r disgybl yn ei chael sy'n pennu pa iaith y mae'r staff yn defnyddio ag ef. Mae pa fath o ysgol uwchradd y mae'r disgybl yn trosglwyddo iddi yn dibynnu ar y lwybr a ddilynodd y disgybl yn y cynradd. Fel arfer, disgwylir i ddisgyblion y ffrwd Gymraeg drosglwyddo i ysgol uwchradd cyfrwng Cymraeg ac i ddisgyblion y ffrwd Saesneg drosglwyddo i ysgol uwchradd cyfrwng Saesneg.

Mae ysgol gynradd drawsnewidiol yn gategori newydd a luniwyd ar gyfer ysgolion sydd wrthi'n trosglwyddo i naill ai Categori (i) neu Gategori (ii), a dylid ei drin yn gategori "dros dro" oherwydd nad yw'n "fodel priodol er mwyn sicrhau bod disgyblion yn hyddysg yn y Gymraeg a’r Saesneg” (L1CC, 2007b, t. 6). Yn y categori hwn, mae LlCC (2007b, tt. 8-9) yn nodi bod yr ysgolion hyn yn addysgu trwy gyfrwng y Saesneg neu'r Gymraeg i wahanol raddau, ond disgwylir i'r ysgolion hyn ddarparu meysydd trwy gyfrwng y Gymraeg yn bennaf i ddisgyblion y CS, a thros 50\%, ond hyd at 70\%, o'r cwricwlwm trwy gyfrwng y Gymraeg yn CA2. Cymraeg yw'r iaith a ddefnyddir o ddydd i ddydd yn yr ysgol, ac mae pwyslais arbennig ar greu ethos Cymraeg trwy'r ysgol. Wrth gofio mai categori trawsnewidiol yw hwn, disgwylir i'r ysgolion hyn symud tuag at ddefnyddio mwy o'r Gymraeg yn y cwricwlwm ac o gwmpas yr ysgol er mwyn galluogi disgyblion i drosglwyddo i ysgol uwchradd cyfrwng Cymraeg, yn arbennig ar gyfer y rhai sy'n dod o gartrefi Cymraeg (LlCC, 2007b, t. 9). 
O’r disgyblion yng Nghymru a gâi eu haddysgu yn y sector cynradd yn ystod y flwyddyn 2016/2017 (206,764), ymddengys mai ond 10.2\% (21,069) a oedd yn rhugl ac a oedd wedi dod o gartrefi Cymraeg (Stats Cymru, 2017a). Golyga hyn fod 89.8\% $(185,695)$ o ddisgyblion cynradd Cymru, p'un ai ydynt yn gallu siarad Cymraeg yn rhugl neu beidio, yn dod o gartrefi di-Gymraeg (Stats Cymru, 2017a). Yn drawiadol iawn, bach iawn yw'r rhai sydd yn derbyn 'addysg cynnal iaith' neu 'addysg iaith dreftadaeth' ar gyfer eu Cymraeg. Heb os, disgyblion o gartrefi di-Gymraeg yw'r mwyafrif yn y tri chategori uchod, lle y ceir 'addysg drochi' yn y Gymraeg i wahanol raddau.

Wrth fwrw sylw ar ganlyniadau'r cyfrifiad ysgolion diweddaraf (L1C, 2017a), mae'n glir iawn mai derbyn addysg yn ysgolion Categorïau (iv) a (v) yw profiad y mwyafrif o ddisgyblion cynradd Cymru. Ymddengys mai ond 24.1\% $(66,612)$ o ddisgyblion sy'n mynychu ysgolion cynradd cyfrwng Cymraeg ${ }^{19}$ (LlC, 2017a, Tabl 2), gyda 75.9\% (210,328) o ddisgyblion yn mynychu ysgolion cyfrwng Saesneg (Categorïau 4 a 5), allan o'r 276,940 o ddisgyblion posibl mewn ysgolion a gynhelir (L1C, 2017a, Tabl 5). Yng Nghategori (iv), mae disgwyl i ddisgyblion y CS gael addysg yn y naill iaith neu'r llall, ond gyda phwyslais ar y Saesneg. Er bod y Gymraeg yn cael ei defnyddio megis cyfrwng dysgu, ni ddisgwylir gweld mwy na 50\% o gwricwlwm CA2 yn gyfrwng Cymraeg, a dim llai na 20\%. Er y disgwylir i'r ysgolion hyn ymrwymo i greu ethos Cymraeg cryf, gellir disgwyl i'r ddwy iaith gael eu siarad o gwmpas yr ysgol i wahanol raddau. Yn y pen draw, "cyd-destun ieithyddol yr ysgol sy'n pennu iaith neu ieithoedd yr ysgol o ddydd i ddydd" (L1CC, 2007b, t. 9).

Mae Categori (v), sef ysgol gynradd cyfrwng Saesneg yn bennaf, yn cyflwyno addysg trwy gyfrwng y Saesneg yn y CS a CA2, gyda'r Gymraeg yn cael ei chyflwyno fel ail iaith o fewn yr amser penodedig a roddir iddi yn yr amserlen. Er ei bod yn bosibl i'r Gymraeg gael ei chyflwyno megis cyfrwng dysgu ar gyfer agweddau ar bynciau, disgwylir i'r ysgolion hyn ddarparu llai nag 20\% o'r addysg trwy gyfrwng y Gymraeg. Yn achos y ddau gategori olaf, mae disgyblion yr ysgolion hyn yn arfer trosglwyddo i ysgol uwchradd cyfrwng Saesneg yn bennaf. Fodd bynnag, noda LlCC (2007b, t. 9) fod disgwyl i ddisgyblion Categori (iv) gaffael

\footnotetext{
${ }^{19}$ Yr ysgolion y mae LIC (2017a) yn cyfeirio atynt hwy wrth ddefnyddio'r ymadrodd 'ysgolion cynradd cyfrwng Cymraeg' yn Canlyniadau'r Cyfrifiad ysgolion 2017 yw ysgolion Categorïau (i), (ii) a (iii). (Gweler LlC, 2017a, Tabl 2.)
} 
"gwell sgiliau o safbwynt y Gymraeg fel ail iaith" oherwydd elfen gryfach o'r Gymraeg, a bod "rhai o'r disgyblion yn gallu dilyn nifer fechan o bynciau drwy gyfrwng y Gymraeg ar y lefel uwchradd os yw'r dewis hwnnw ar gael.”

\subsubsection{Categoriau ieithyddol ysgolion y sector uwchradd}

Yn unol â diffiniadau LICC (2007b), ceir pedwar prif gategori o ysgolion uwchradd Cymru, sef:

i. Ysgol uwchradd cyfrwng Cymraeg

ii. Ysgol uwchradd ddwyieithog

iii. Ysgol uwchradd cyfrwng Saesneg yn bennaf ond â defnydd sylweddol o'r Gymraeg

iv. Ysgol uwchradd cyfrwng Saesneg yn bennaf

Yn debyg i gategorïau'r sector cynradd, mae gan y sector uwchradd gategorïau cyfrwng Cymraeg, cyfrwng Saesneg yn bennaf ond â defnydd sylweddol o'r Gymraeg, a chyfrwng Saesneg yn bennaf. Yn lle categori dwy ffrwd, ceir categori ysgol uwchradd ddwyieithog, sydd yn cynnwys pedwar is-gategori gwahanol, Categorïau (2A), (2B), (2C) a (2Ch), er mwyn egluro beth yw'r cydbwysedd rhwng y ddarpariaeth cyfrwng Cymraeg a'r ddarpariaeth cyfrwng Saesneg yn yr ysgol, ac a yw'r ysgol yn cynnig yr un ddarpariaeth trwy gyfrwng y Saesneg (L1CC, 2007b, t. 12).

Yng Nghategori (i), sef ysgol uwchradd cyfrwng Cymraeg, disgwylir i ddisgyblion gael addysg cyfrwng Cymraeg ymhob pwnc, gan gynnwys Addysg Grefyddol ac $\mathrm{ABaCh}$, a'u hasesu ymhob pwnc trwy gyfrwng y Gymraeg, ac eithrio Saesneg. Fel rheol disgwylir i'r disgyblion hyn gael eu hasesu yn Gymraeg ac i ddewis, os yw'n briodol, addysg ôl-16 drwy gyfrwng y Gymraeg. Cymraeg yw iaith bob dydd a gweinyddiaeth yr ysgol.

Fel y soniwyd eisoes, mae gan gategori ysgol ddwyieithog bedwar is-gategori, gyda phob categori'n esbonio faint o ddarpariaeth cyfrwng Cymraeg / Saesneg a geir yn yr ysgol. Yn wahanol i ysgol cyfrwng Cymraeg, gall darpariaeth Gymraeg ysgol ddwyieithog amrywio: 
Categori 2A - Addysgir o leiaf 80\% o'r pynciau ac eithrio'r Gymraeg a'r Saesneg drwy gyfrwng y Gymraeg yn unig i bob disgybl. Mae un neu ddau o bynciau yn cael eu haddysgu i rai o'r disgyblion yn Saesneg neu yn y naill iaith a'r llall.

Categori 2B - Addysgir o leiaf 80\% o'r pynciau (ac eithrio'r Gymraeg a'r Saesneg) drwy gyfrwng y Gymraeg ond fe'u haddysgir drwy gyfrwng y Saesneg hefyd.

Categori 2C - Addysgir 50 - 79\% o bynciau (ac eithrio'r Gymraeg a Saesneg) drwy gyfrwng y Gymraeg ond fe'u haddysgir drwy gyfrwng y Saesneg hefyd.

Categori 2CH - Addysgir pob pwnc (ac eithrio'r Gymraeg a'r Saesneg) i bob disgybl gan ddefnyddio'r naill iaith a'r llall.

(LlCC, 2007b, tt. 12-13).

Oherwydd gwahaniaethau ieithyddol ysgolion dwyieithog, mae iaith neu ieithoedd cyfathrebu a gweinyddu'r ysgol yn dibynnu ar gyd-destun ieithyddol unigol yr ysgol. Noda LlCC (2007b, t. 13) ei bod yn disgwyl y byddai disgyblion y pedwar categori yn gallu "symud ymlaen yn hawdd" at astudiaethau ôl-16 yn y pynciau cyfrwng Cymraeg a ddilynwyd ganddynt yn CA3 a 4.

O'r holl ddisgyblion uwchradd yng Nghymru yn ystod y flwyddyn academaidd 2016/2017 (174,812), ymddengys mai ond $10.1 \%(17,630)$ a oedd yn rhugl ac a oedd wedi dod o gartrefi Cymraeg (Stats Cymru, 2017a). Golyga hyn fod 89.9\% $(157,182)$ o ddisgyblion cynradd Cymru, p'un ai ydynt yn gallu siarad Cymraeg yn rhugl neu beidio, yn dod o gartrefi di-Gymraeg (Stats Cymru, 2017a). Eto, ychydig iawn yw'r sawl sy'n derbyn 'addysg cynnal iaith' ar gyfer y Gymraeg.

Wrth fwrw sylw ar ganlyniadau'r cyfrifiad ysgolion diweddaraf, gellir nodi mai ond $20.0 \%(34,986)$ o ddisgyblion sy'n mynychu ysgolion uwchradd cyfrwng Cymraeg (LlC, 2017a, Tabl 2) o'i gymharu ag 80.0\% $(139,826)$ o ddisgyblion sy'n mynychu ysgolion cyfrwng Saesneg (Categorïau iii a iv), allan o'r 174,812 o ddisgyblion mewn ysgolion a gynhelir (LlC, 2017a, Tabl 5). Yng Nghategorïau (iii) a (iv), mae disgwyl i'r ddau fath hyn o ysgol gyflwyno pob pwnc trwy gyfrwng y Saesneg, gyda'r Gymraeg yn cael ei chyflwyno naill ai'n bwnc ail iaith yn unig (Categori iv) neu'n iaith cyfrwng dysgu ac asesu ar gyfer $20 \%$ hyd at $49 \%$ o'r addysg (Categori iii). Fel y gwelir yn achos ysgolion uwchradd dwyieithog, mae sefyllfa ieithyddol ysgolion Categori (iii) yn pennu pa iaith a ddefnyddir wrth gyfathrebu â disgyblion ac wrth weinyddu'r ysgol. O ran ysgolion Categori (iv) fodd bynnag, er bod modd i ddisgyblion ddilyn "un neu ddau bwnc (a allai gynnwys y Gymraeg fel ail iaith) 
drwy gyfrwng y Gymraeg neu drwy gyfrwng y naill iaith a'r llall” (L1CC, 2007b, tt. 13-14), cydnebydd LICC (2007b, t. 14) y byddai'r rhan fwyaf o ddisgyblion y categori hwn yn cael eu hasesu drwy gyfrwng y Saesneg gan ddilyn eu hastudiaethau ôl-16 hefyd yn Saesneg.

\subsubsection{Rhwystrau posibl wrth ddiffinio ysgolion yn ôl eu darpariaeth cyfrwng Cymraeg}

Nod y Pwyllgor Addysg a Dysgu Gydol Oes wrth lunio'r ddogfen wybodaeth Diffinio ysgolion yn ôl eu darpariaeth cyfrwng Cymraeg oedd gosod set newydd o ddiffiniadau a fyddai'n "hawdd eu hadnabod ar gyfer y modelau gwahanol o ddarpariaeth ieithyddol sydd ar gael yn ysgolion Cymru" (L1CC, 2007b, t. 4). Er bod y diffiniadau yn egluro'n well y ddarpariaeth Gymraeg a Saesneg a geir yn ysgolion Cymru, gellir dadlau nad ydynt yn ddigonol am nad ydynt yn disgrifio'n union gyfrwng yr addysgu yn yr ysgol ym mhrofiad y disgyblion gael yn yr ysgol (LICC, 2007b, t. 6). Er enghraifft, er y gallai ysgol yng Nghategori ysgol uwchradd cyfrwng Saesneg yn bennaf sy'n defnyddio'r Gymraeg yn sylweddol gynnig rhywfaint o’i haddysg (rhwng 20\% a 49\%) trwy gyfrwng y Gymraeg, mae disgwyl i'r ysgol hon hefyd gynnig yr un pynciau trwy gyfrwng y Saesneg (LlCC, 2007b). O ganlyniad, mae'n bosibl y gallai disgyblion ddewis addysg trwy gyfrwng y Saesneg yn unig ac felly ni fyddai'r disgyblion hyn yn derbyn unrhyw addysg trwy gyfrwng y Gymraeg, er bod yr ysgol ei hun yn gwneud defnydd sylweddol o'r Gymraeg. Mae'n bosibl hefyd ddadlau y gellid diffinio ysgol cyfrwng Saesneg yn bennaf lle ceir defnydd sylweddol o'r Gymraeg, ac sy'n darparu addysg trwy gyfrwng y Gymraeg hyd at y canran uchaf posibl yn y categori hwn (49\%), yn ysgol ddwyieithog oherwydd ei hethos dwyieithog. Enghraifft o ysgol bosibl yw Ysgol X (gweler 5.4. Cynllun Ysgol $\mathrm{X}$ : Y Cwrs Carlam). Yn swyddogol, caiff yr ysgol ei diffinio'n ysgol cyfrwng Saesneg yn bennaf lle ceir defnydd sylweddol o'r Gymraeg (L1C, 2016a) am fod yr ysgol yn cynnig peth darpariaeth ddwyieithog a chyfrwng Cymraeg yn CA3, ac am ei bod yn rhoi blaenoriaeth i greu ethos Cymraeg ac i hyrwyddo'r defnydd allgyrsiol o’r Gymraeg (Estyn, 2012c). Ar ôl arolygiad Ysgol X yn 2006, cynigodd Estyn (2006) y radd uchaf i'r ysgol ar gyfer ei dwyieithrwydd yn CA3 a 4, sef 'gradd 1'. Chwe mlynedd yn ddiweddarach, cynigodd Estyn (2012e) y radd uchaf ar gyfer darpariaeth Gymraeg Ysgol X, sef ‘ardderchog', yn ei arolygiad dilynol o'r ysgol. 
Rhwystr arall sy'n codi yw nad yw'r diffiniadau hyn yn gwahaniaethu rhwng ysgolion sy'n cynnig addysg cyfrwng Cymraeg ar gyfer cynnal iaith dreftadaeth disgyblion o gartrefi Cymraeg iaith gyntaf, sef 'ysgolion Cymraeg traddodiadol', ac ysgolion cyfrwng Cymraeg ar gyfer trochi disgyblion o gartrefi di-Gymraeg neu gartrefi ieithyddol-gymysg yn y Gymraeg, sef 'ysgolion Cymraeg trochi'. Fel y nododd Lewis (2008):

Addysg drochi yw addysg Gymraeg i $99.8 \%$ o blant cynradd yng Nghasnewydd; nid addysg drochi yw hi i bob un yng Ngwynedd / Ceredigion / Sir Gaerfyrddin, ond cyfuniad o addysg gyfoethogi ac addysg drochi. Dyma'r cyfle a'r her sy'n wynebu datblygiad y gyfundrefn heddiw. (W. G. Lewis, 2008, t. 11).

O ganlyniad, mae nifer o ysgolion ar draws Cymru, yn arbennig y rhai yn yr ardaloedd ieithyddol-gymysg, yn cynnwys cymysgedd o ddisgyblion o gefndiroedd ieithyddol gwahanol sydd ag anghenion ieithyddol gwahanol i'w bodloni, ac mae hyn yn cynnig her fawr i ysgolion cynradd cyfrwng Cymraeg ar draws Cymru, yn arbennig o ran methodoleg addysgu (W. G. Lewis, 2008).

Er mai'r ysgolion eu hunain yw'r rhai sy'n penderfynu i ba gategori y maent yn perthyn wrth iddynt lenwi'r Cyfrifiad Ysgolion bob blwyddyn (L1CC, 2007b, t. 2), rhaid cofio nad oes gan y diffiniadau hyn sail cyfreithiol. O safbwynt Deddf Addysg 2002, ceir ond dau gategori swyddogol o ysgol yng Nghymru. Hynny yw, ysgolion lle mae Cymraeg yn bwnc craidd ac mae'r Gymraeg yn gyfrwng addysgu ar gyfer dros 50\% o'r addysg, ac ysgolion lle mae Cymraeg yn bwnc sylfaenol ac mae'r Gymraeg yn gyfrwng addysgu ar gyfer llai na 50\% o'r addysg. Er bod y diffiniadau hyn yn egluro'n well y gwahanol ddarpariaethau posibl a geir yn ysgolion Cymru, defnyddir y diffiniadau hyn yn y traethawd hwn yn ofalus.

\subsubsection{Addysg cyfrwng Cymraeg}

Mae'n bosibl defnyddio label ‘addysg Gymraeg' wrth gyfeirio'n gyffredinol at unrhyw addysg sy'n cael ei chyflwyno trwy gyfrwng y Gymraeg, ac felly gall y label 'ysgol Gymraeg' gyfeirio at amryw o gategoriau ieithyddol gwahanol o ysgolion sydd yn cyflwyno elfennau o'r cwricwlwm, ar wahân i Saesneg a Chymraeg, trwy gyfrwng y Gymraeg neu drwy gyfrwng y Gymraeg a'r Saesneg (L1CC, 2007b, tt. 34). Oherwydd y dryswch posibl wrth ddefnyddio'r labeli ‘addysg Gymraeg' ac 'ysgol Gymraeg', penderfynwyd mai gwell fyddai defnyddio'r diffiniadau a restrir 
gan LlCC (2007b). Yng nghyd-destun yr ymchwil hon felly, defnyddir y label 'addysg cyfrwng Cymraeg' wrth gyfeirio at unrhyw ddarpariaeth addysg, heblaw am y pynciau Saesneg a Chymraeg, sy'n cael ei chyflwyno trwy gyfrwng y Gymraeg yn unig, a'r label 'ysgol cyfrwng Cymraeg' wrth gyfeirio at ysgolion cynradd yng Nghategoriau (i) a (ii), ac ysgolion uwchradd yng Nghategori (i). Wrth gyfeirio at ysgolion cynradd yng Nghategori (iii) ac ysgolion uwchradd yng Nghategori (ii), defnyddir y label 'ysgol ddwyieithog'. Er ei bod yn bosibl cael peth addysg cyfrwng Cymraeg neu ddwyieithog mewn ysgolion uwchradd yng Nghategori (iv), ni chyfeirir at ysgolion o'r fath fel 'ysgolion dwyieithog' gan nad yw hyn yn cyfateb i ddiffiniad LICC (2007b) o ysgol uwchradd ddwyieithog (gweler 2.6.1.2. Categorïau ieithyddol ysgolion y sector uwchradd).

Gan mai ysgolion cyfrwng Saesneg mewn ardaloedd cymharol ddi-Gymraeg yng Nghymru $^{20}$ yw canolbwynt yr ymchwil dan sylw, ni ragwelir y bydd angen gwahaniaethu'n fanwl rhwng y gwahanol fathau o ysgolion cyfrwng Cymraeg. Fodd bynnag, mae'n bosibl gwahaniaethu'n fras rhwng ddau brif fath o ysgolion cyfrwng Cymraeg, sef 'ysgolion Cymraeg traddodiadol' mewn ardaloedd Cymraeg traddodiadol, sy'n denu'r rhan fwyaf o'u disgyblion o gartrefi Cymraeg iaith gyntaf, ac 'ysgolion Cymraeg trochi' mewn ardaloedd cymharol ddi-Gymraeg, sy'n denu'r rhan fwyaf o'u disgyblion o gartrefi di-Gymraeg, neu gartrefi ieithyddol-gymysg. Diben addysg mewn 'ysgol Gymraeg draddodiadol' yw “cynnal, cadarnhau ac ymestyn” Cymraeg disgyblion o gartrefi Cymraeg (W. G. Lewis, 2008, t. 10). Dyma'r math o addysg y mae Baker (2011) a Johnstone (2002) yn ei labelu yn 'addysg cynnal iaith', 'addysg iaith dreftadaeth' neu 'addysg iaith gymuned'. I'r gwrthwyneb, diben addysg mewn 'ysgol Gymraeg drochi' yw trochi siaradwyr Saesneg eu hiaith gyntaf yn yr iaith darged (W. G. Lewis, 2008, t. 10), sef y Gymraeg, trwy ei defnyddio megis cyfrwng dysgu ac addysgu. Fodd bynnag, fel y nodwyd eisoes yn is-adran flaenorol, y mae'r sefyllfa yn gymhlethach o lawer, gan fod nifer fawr o ysgolion Cymru yn cynnwys cymysgedd o ddisgyblion o gefndiroedd ieithyddol amrywiol, ac felly nid yw'n hawdd gwahaniaethu a diffinio ysgolion cyfrwng Cymraeg ar sail cefndir ieithyddol y disgyblion sy'n mynychu'r ysgolion hyn.

\footnotetext{
${ }^{20}$ At ddiben y traethawd hwn, diffinnir 'ardaloedd cymharol ddi-Gymraeg' yn gymunedau (sef cydrannau o'r awdurdodau unedol) lle y caiff y Gymraeg ei siarad gan lai nag $20 \%$ y boblogaeth yn y cymunedau hynny (gweler Comisiynydd y Gymraeg, d.d.)
} 


\subsubsection{Disgybl Cymraeg Iaith Gyntaf}

Ar sail y diffiniadau ar gyfer ysgolion a luniwyd gan LlCC (2007b), defnyddir y label 'disgybl Cymraeg Iaith Gyntaf (CIG)' wrth gyfeirio at ddisgybl cynradd sy'n derbyn addysg yng Nghategorïau (i), (ii), a (iii). O ran y sector uwchradd, defnyddir y label 'disgybl Cymraeg Iaith Gyntaf' wrth gyfeirio at ddisgybl sy'n derbyn addysg yng Nghategorïau (i), a (ii), gan fod disgyblion y tri chategori hyn yn debygol o sefyll arholiad TGAU CIG, yn ogystal ag arholiadau trwy gyfrwng Cymraeg yn y pynciau sy'n cael eu haddysgu trwy gyfrwng yr iaith honno. Nid yw 'iaith gyntaf' yn y cyd-destun hwn yn cyfeirio at yr iaith a gafodd ei chaffael gan y disgybl yn gyntaf, nac iaith y cartref, oherwydd mae'n bosibl y daw disgyblion y categorïau hyn o gefndiroedd di-Gymraeg neu gartrefi ieithyddol-gymysg (gweler 2.6.1.3. Rhwystrau posibl wrth ddiffinio ysgolion yn ôl eu darpariaeth cyfrwng Cymraeg). Yn hytrach, mae 'iaith gyntaf' yma'n cyfeirio at yr iaith a ddefnyddir yn bennaf ar gyfer addysgu, gweinyddu a chyfathrebu. Er mwyn amrywio rhwng y ddwy ystyr, defnyddir 'disgybl Cymraeg Iaith Gyntaf' yng nghyd-destun addysg, hynny yw, wrth gyfeirio at ddisgybl sydd yn neu sydd wedi astudio'r cwrs CIG, a 'disgybl Cymraeg iaith gyntaf' yng nghyd-destun caffael iaith, hynny yw, wrth gyfeirio at ddisgybl sydd wedi caffael y Gymraeg fel iaith gyntaf.

\subsubsection{Cymraeg Ail Iaith}

Wrth gyfeirio at y gyfundrefn addysg lle y cyflwynir y Gymraeg megis pwnc ail iaith (neu mewn perthynas ag amser penodedig yn achos ysgolion cynradd) yn unig, defnyddir yr ymadrodd 'Cymraeg Ail Iaith (CAI)'. Er bod modd defnyddio'r ymadrodd 'addysg Gymraeg ail iaith' (gweler, er enghraifft, BBC Cymru Fyw, 2014, 15 Mai) credir mai gwell fydd osgoi defnyddio'r ymadrodd hwn oherwydd bod 'addysg Gymraeg' wedi'i hen ddefnyddio wrth gyfeirio at yr addysg a geir mewn ysgolion cyfrwng Cymraeg. Mae Rhieni dros addysg Gymraeg, mudiad cenedlaethol a sefydlwyd yn 1952, yn defnyddio'r ymadrodd 'addysg Gymraeg' yn y cyd-destun hwn. Ceir enghraifft o hyn yn ei adroddiad blynyddol 2013-2014: "Prif amcanion y mudiad yw cefnogi a datblygu ysgolion Cymraeg ledled Cymru trwy roi cyfle cyfartal i'r rhai sy'n dymuno rhoi addysg Gymraeg i'w plant a'u caniatáu i gaffael dwyieithrwydd cyflawn.” (RhAG, 2015a, t. 3). Oherwydd y dryswch a allai godi wrth ddefnyddio ‘addysg Gymraeg' ac ‘addysg Gymraeg ail iaith' felly, defnyddir 'Cymraeg Ail Iaith (CAI)' yn y traethawd hwn. 


\subsubsection{Disgybl Cymraeg Ail Iaith}

Ar sail diffiniadau LICC (2007b) ar gyfer ysgolion, defnyddir yr ymadrodd 'disgybl Cymraeg Ail Iaith (CAI)' wrth gyfeirio at ddisgybl sy'n derbyn addysg trwy gyfrwng y Saesneg ac sy'n astudio'r Gymraeg fel pwnc yn unig mewn ysgolion cynradd Categorïau (iv) a (v), ac ysgolion uwchradd Categoriau (iii) a (iv). Unwaith eto, nid yw'r ymadrodd 'disgybl Cymraeg Ail Iaith' yn cyfeirio at yr iaith a gafodd ei chaffael yn gyntaf gan y disgybl, gan ei bod yn bosibl, er enghraifft, i ddisgyblion o gartrefi Cymraeg eu hiaith sydd wedi cael addysg gynradd trwy gyfrwng y Gymraeg, fynychu ysgol uwchradd cyfrwng Saesneg. Er mwyn amrywio rhwng y ddwy ystyr hyn a geir yma, defnyddir yr ymadrodd 'Cymraeg Ail Iaith' wrth gyfeirio at yr ystyr addysgol, a'r ymadrodd 'Cymraeg ail iaith' wrth gyfeirio at yr iaith a gafodd ei chaffael yn ail gan yr unigolyn.

\subsection{Ethos}

\subsubsection{Diffinio ethos}

Mae dod i gasgliad pendant o ran beth a olygir gan y term 'ethos' yn drafferthus iawn, a hynny oherwydd digon niwlog ac amwys yw ei ystyr (Donnelly, 2000; Solvason, 2005). Fel y mynega Donnelly (2000, t. 134): "Ethos is a fashionable but nebulous term often employed by organisational theorists, educationists and theologists to describe the distinctive range of values and beliefs, which define the philosophy or atmosphere of an organisation." Yn aml, defnyddir termau eraill yn lle 'ethos', termau a gaiff eu defnyddio'n gydgyfnewidiol, megis "spirit” (Torrington \& Weightman, 1989, t. 17), "climate” (Everard, 1986, t. 22; Lynch, 1987, t. 59; Rutter, Maughan, Mortimore, Ouston, \& Smith, 1979, tt. 18, 153), "atmosphere" (Rutter ac eraill, 1979, t. 55), “ambience” (Lynch, 1987, t. 59) a "culture" (Everard, 1986, t. 22). Mae geiriaduron hefyd yn defnyddio rhai o'r geiriau uchod wrth ddiffinio ystyr y term. Er enghraifft, diffinia Oxford Dictionaires Online (“ethos”, d.d.) 'ethos' fel "[ $\mathrm{t}]$ he characteristic spirit of a culture, era, or community as manifested in its attitudes and aspirations." I'r un perwyl, diffinia Geiriadur Prifysgol Cymru ("ethos", 1966) 'ethos' fel “[y]sbryd (natur, naws) neu awyrgylch moesol sy'n nodweddiadol o gymdeithas neilltuol."

Ymddengys o'r gwahanol gynigion uchod felly fod y term 'ethos' yn adlewyrchu rhyw naws, awyrgylch, neu ysbryd a deimlir mewn cymuned, neu ysgol yn y cyd- 
destun hwn. Yn yr ystyr hon felly, gellir disgrifio 'ethos' megis cysyniad haniaethol, sy'n awgrymu ei fod yn anfesuradwy. Yn ogystal â hynny, awgryma'r diffiniadau uchod hefyd y caiff y teimlad hwn ei rannu gan bawb yn y gymuned, ar lefel anffurfiol, trwy weithrediadau ac ymddygiad y gymuned o ddydd i ddydd. Dyma sut mae Allder (1993) yn diffinio 'ethos'. Yn ei hastudiaeth sy'n cymharu ystyr y pedwar gair cydgyfnewidiol uchod ag ystyr ethos, daw Allder i'r casgliad mai 'ethos' yw:

... the unique, pervasive atmosphere or mood of the organisation which is brought about by activities or behaviour, primarily in the realm of social interaction and to a lesser extent in matters to do with the environment, of members of the school, and recognised initially on an experiential rather than a cognitive level. (Allder, 1993, t. 69)

Mae Solvason (2005) hefyd yn cytuno ag Allder (1993) mai awyrgylch a deimlir mewn cymuned yw ethos, ond mae hithau'n pwysleisio bod ethos yn deillio o egwyddorion a rheolau anffurfiol y gymuned sy'n cael eu hamlygu trwy ei gweithrediadau beunyddiol. Dadleua Solvason (2005, t. 86) fod rhai ysgolheigion yn aml yn camddefnyddio'r term 'ethos' wrth gyfeirio at egwyddorion a rheolau anffurfiol cymuned, lle dylid defnyddio'r term 'diwylliant' yn hytrach, yn ei barn hi. Yn syml, noda Solvason (2005, t. 87): "We recognize and comprehend the school culture, whereas we experience the ethos." Daw Solvason i'r un casgliad â Deal a Peterson (1999). 'Ethos' (neu 'ddiwylliant' yn ôl ei therminoleg hi) yw:

... [a] school's own unwritten rules and traditions, norms and expectations that seem to permeate everything; the way people act, how they dress, what they talk about or avoid talking about, whether they seek out colleagues for help or don't, and how teachers feel about their work and their students. (Deal \& Peterson, 1999, tt. 2-3, fel y dyffynnwyd yn Solvason, 2005, t. 93)

Mae Torrington a Weightman (1989) fodd bynnag yn defnyddio'r termau 'ethos' a 'diwylliant' mewn modd gwahanol. Yn groes i ddehongliad Solvason (2005), cred Torrington a Weightman (1989, t. 18) mai "characteristic spirit and belief of an organisation" yw 'diwylliant yr ysgol', a bod 'diwylliant' yn deillio o'r "norms and values that are generally held about how people should treat each other, the nature of working relationships that should be developed and attitudes to change." "Ethos yr ysgol' yw "a more self-conscious expression of specific types of objective in relation 
to behaviour and values" (Torrington \& Weightman, 1989, t. 18). Yn ôl Torrington a Weightman (1989) felly, mae'r term 'ethos' yn cyfeirio at yr hyn a fynegir yn ffurfiol, megis datganiadau ffurfiol a wneir gan brifathro'r ysgol.

Mewn adolygiad o'r amryw dermau a ddefnyddir yn llenyddiaeth y maes, mae Glover a Coleman (2005) yn ceisio darganfod p'un ai termau cydgyfnewidiol yw'r termau hyn, neu'n hytrach dermau ac iddynt ystyron gwahanol. Maent yn dod i'r casgliad canlynol:

- climate, the measurable input and outcome features of the school experience;

- ethos, the more subjective values and principles underpinning policy and practice;

- culture, the integration of environmental, organisational and experiential features of school existence to offer a context for teaching and learning, and its subsequent improvement. (Glover \& Coleman, 2005, tt. 265-266)

Mae astudiaeth Glover a Coleman felly yn rhoi hygrededd i ddehongliad Torrington a Weightman (1989) o 'ethos' megis set o egwyddorion goddrychol sydd wrth wraidd polisi ffurfiol yr ysgol. Fodd bynnag, mae diffiniad Glover a Coleman hefyd yn cydnabod bod ethos wrth wraidd arfer hefyd, ac awgryma hyn fod modd gwahaniaethu rhwng dwy lefel wahanol o ethos, sef 'ethos anffurfiol' ac 'ethos ffurfiol'. O’u cymharu, mae dehongliadau Torrington ac Weightman (1989) a Solvason (2005) yn methu â gwahaniaethu'n glir rhwng y lefel anffurfiol a'r lefel ffurfiol, neu'r "externally imposed practice and the more informal climate of internally evolved features of relationships" (Glover \& Coleman, 2005, t. 258).

Mae Donnelly (2000) yn gwahaniaethu'n gliriach fyth rhwng dwy lefel ethos. Yn ôl Donnelly, mae'n bosibl diffinio 'ethos' o ddau safbwynt damcaniaethol. O'r 'safbwynt cadarnhaol' (positivist viewpoint), gwelir 'ethos' megis mynegiad ffurfiol o'r hyn yr hoffai'r sefydliad ei gyflawni mewn polisi. O'r 'safbwynt gwrthgadarnhaol' (anti-positivist viewpoint), gwelir 'ethos' megis mynegiad anffurfiol aelodau'r sefydliad. Mae hyn yn deillio o ryngweithio rhwng aelodau'r ysgol o ddydd i ddydd. Mae Donnelly (2000) yn cydnabod nad yw ethos anffurfiol y staff yn cyd-fynd ag ethos ffurfiol yr ysgol bob tro, ac mewn achosion o'r fath nid yw'n bosibl creu ethos cyson ar gyfer yr ysgol gyfan. Mae Solvason (2005) hefyd yn cytuno bod gweithredu un 'diwylliant' (ethos) cyfan yn anodd iawn, yn arbennig 
mewn ysgolion uwchradd, lle mae rhwydweithiau cymhleth iawn yn bodoli rhwng adrannau'r ysgol. Mae'n bosibl i'r adrannau hyn, neu grwpiau eraill y tu mewn i'r ysgol, ffurfio agweddau gwahanol a allai wrthdaro ag egwyddorion swyddogol yr ysgol (Solvason, 2005, t. 89). I'r un perwyl, mae Glover a Coleman (2005) yn cydnabod nad yw ethos disgyblion yr ysgol bob tro'n cyd-fynd ag ethos ffurfiol yr ysgol. Amlygwyd mor bwysig yw sicrhau cytundeb rhwng yr ethos anffurfiol a'r ethos ffurfiol mewn astudiaeth Ph.D. gan Selleck (2012) o ysgol ddwyieithog ac ysgol cyfrwng Saesneg yng Nghymru. O’i sgyrsiau ethnograffig â disgyblion o gartrefi Saesneg eu prif iaith mewn ysgol ddwyieithog, noda Selleck $(2012,2013)$ fod enghreifftiau lle mae disgyblion yn cwestiynu pam mae'n rhaid iddynt ddefnyddio'r Gymraeg trwy'r amser er mai ysgol ddwyieithog yw hi. Er mai datblygu Saesneg a Chymraeg y disgyblion yn gyfartal yw polisi ffurfiol yr ysgol, mae'r athrawon yn blaenoriaethu defnyddio'r Gymraeg trwy'r amser ar y lefel lafar. Os yw ysgol am greu ethos cynhwysol, neu "whole-school culture" yng ngeiriau Solvason (2005, t. 89), ymddengys fod yn rhaid sicrhau bod yr ethos ffurfiol a fynegir mewn polisi ac ethos ymarferol yr ysgol yn cyd-fynd â'i gilydd. (Donnelly, 2000, t. 137; Solvason, 2005, t. 90).

Fel y gwelwyd, mae diffinio 'ethos' yn bwnc llosg ac nid oes cytundeb o ran beth yn union a olygir gan y term hwn. Fodd bynnag, mae dealltwriaeth Donnelly (2000) a Solvason (2005) o'r gwahaniaeth rhwng disgwyliadau ffurfiol mewn polisi ysgol ac arferion aelodau'r ysgol yn cynnig dehongliad cliriach o'r term ethos. Felly, yn gyson â dehongliad Donnelly (2000) o 'ethos' megis “formal and informal expressions of school members" (t. 136), defnyddir yn y traethawd hwn y term 'ethos ffurfiol' wrth gyfeirio at yr hyn a fynegir yn ffurfiol, boed ar lafar neu'n ysgrifenedig, megis mewn polisi, datganiadau'r pennaeth, dogfennaeth a deunyddiau marchnata'r ysgol er enghraifft, a'r term 'ethos anffurfiol' wrth gyfeirio at yr hyn a fynegir yn anffurfiol, boed ar lafar neu'n ysgrifenedig, megis credoau, ymddygiadau, ac arferion aelodau'r ysgol o ddydd i ddydd.

\subsection{Y continwwm}

Cyn cloi'r bennod hon, mae'n angenrheidiol rhoi sylw i un o'r cysyniadau mwyaf niwlog a ymddengys trwy gydol y traethawd hwn, sef 'continwwm'. Dyma derm sydd wedi'i ddefnyddio'n aml gan ysgolheigion a llunwyr polisi fel ei gilydd, ond 
nid yw'n eglur o hyd beth yn union a olygir gan y term, yn enwedig yng nghyddestun cyflwyno'r Gymraeg a'r ieithoedd modern yn y cwricwlwm newydd.

\subsubsection{Diffinio continwwm dwyieithog}

Mae Geiriadur Termau'r Coleg Cymraeg Cenedlaethol (d.d.) yn diffinio 'continwwm' fel: 'Llinell sy'n dechrau ar un pegwn eithafol ac sy'n cyrraedd yr eithaf arall (e.e., tristwch - hapusrwydd)." Mae'r diffiniad uchod yn ystyried 'continwwm' fel un llinell ac iddi ddau ben deubarthol. Wrth ystyried y diffiniad hwn yng nghyd-destun dysgu'r Gymraeg, mae'n bosibl disgrifio dysgwyr newydd fel rhai ar un pegwn eithaf, a siaradwyr dwyieithog sydd â sgiliau 'cytbwys' yn y ddwy iaith fel rhai ar y pegwn eithaf arall. Fodd bynnag, gallai'r dehongliad hwn awgrymu bod cyfyngiadau penodol ar y broses o ddysgu iaith, ac felly gellid dadlau bod hyn yn cefnogi'r safbwynt ffracsiynol / uniaith (fractional / monolingual view) o ddwyieithrwydd.

Fodd bynnag, mae Gweiadur (d.d.) yn diffinio ‘continwwm' fel “rhywbeth y gellir canfod undod sylfaenol iddo o fewn amrywiaeth o fân newidiadau”. Er bod amrywiaeth o fân newidiadau rhwng y ddau begwn, mae'r diffiniad yn cydnabod bod y pwyntiau hyn yn fwy cyffredin nag yn wahanol i'w gilydd. I'r un perwyl, mae Hornberger (2003) yn cydnabod bod y pwyntiau rhwng dau begwn continwwm yn gysylltiedig â’i gilydd. Yn ôl diffiniad Hornberger, gwell ystyried 'continwwm' fel llinell annherfynol, ddiddiwedd, ac iddi nifer o fân newidiadau ansefydlog:

The notion of continuum is intended to convey that, although one can identify (and name) points of the continuum, those points are not finite, static, or discrete. There are infinitely many points on the continuum; any single point is inevitably and inextricably related to all other points; and all the other points have more in common than not with each other.

(Hornberger, 2003, t. xiv).

Mae dehongliad Hornberger yn cyd-fynd â'r safbwynt holistaidd o ddwyieithrwydd unigol a gefnogir gan ysgolheigion megis Baker (2011), Hoffman (1991), Grosjean (1985, 2008) a Romaine (1995). O ddefnyddio dehongliad Hornberger (2003) o'r term 'continwwm', gellid ystyried y 'continwwm dwyieithog' ar lefel yr unigolyn, fel y broses gydol oes o ddysgu a defnyddio ieithoedd. Nid yw'r broses hon o reidrwydd yn un gynyddol bob tro. Hynny yw, mae'n bosibl i'r unigolyn symud yn ôl ac ymlaen ar hyd y continwwm, yn dibynnu ar ei hyfedredd yn yr ieithoedd. Ar 
naill ben y continwwm, ceir dysgwyr newydd i'r iaith, ac ar y llall, ceir siaradwyr dwyieithog hyfedr mewn dwy iaith (neu ragor). Fodd bynnag, fel y mae dehongliad Hornberger yn ei awgrymu, nid oes cyfyngiadau penodol ar y broses o ddysgu iaith. Mae Valdés (2003) wedi cynnig dehongliad o'r hyn y mae'n ei alw'r 'continwwm dwyieithog', gan ganolbwyntio ar hyfedredd y siaradwr mewn mwy nag un iaith. Yn ôl Valdés, ceir 'unieithrwydd' ar y naill begwn a'r llall, gyda 'dwyieithrwydd cytbwys' yng nghanol y continwwm:

A $\quad$ A $\quad$ Ab Ab Ab Ab AB BA Ba Ba Ba Ba Ba Monolingual
Bilingual
B

Monolingual

Ffigur 1. Continwwm dwyieithog Valdés (2003)

(Addaswyd o Expanding definitions of giftedness, Valdés, 2003, t. 36; Foundations of bilingual education and bilingualism, Baker, 2011, t. 8)

Wrth ddefnyddio'r continwwm hwn, gellir ystyried siaradwyr uniaith Saesneg yn naill ben y continwwm (Iaith A), a siaradwyr uniaith Gymraeg yn y llall (Iaith B). Rhwng dau ben y continwwm, gellir nodi siaradwyr Saesneg iaith gyntaf sy’n medru'r Gymraeg fel ail iaith i wahanol lefelau $\left(A_{b}-A b\right)$, siaradwyr dwyieithog 'cytbwys' Saesneg-Cymraeg (AB) a Chymraeg-Saesneg (BA), a siaradwyr Cymraeg iaith gyntaf sy'n medru'r Saesneg fel ail iaith i wahanol lefelau $\left(\mathrm{Ba}-\mathrm{B}_{\mathrm{a}}\right)$. Fodd bynnag, oherwydd presenoldeb a statws y Saesneg fel iaith fwyafrifol yng Nghymru a'r tu hwnt bellach, prin iawn yw'r sawl y gellir eu labelu'n siaradwyr uniaith Gymraeg. Nodir hefyd mai prin iawn yw'r sawl sydd â sgiliau cyflawn cytbwys yn y naill iaith a'r llall (gweler 2.3.2. Dwyieithrwydd yr unigolyn). Er bod dehongliad Valdés yn ddefnyddiol wrth ystyried sut y gall hyfedredd siaradwr dwyieithog yn y naill iaith a'r llall ddatblygu drwy gydol ei fywyd, nid yw'n ddigonol i fesur hyfedredd siaradwyr amlieithog sy'n medru tair iaith neu ragor. Nid yw'r fframwaith hon yn rhoi sylw i ddefnydd y siaradwr o'i ieithoedd ychwaith. Oherwydd y 
rhwystrau wrth ddiffinio unigolion dwyieithog yn ôl continwwm sy'n canolbwyntio ar allu yn eu hieithoedd, gellid dadlau mai gwell yw "to move away from the multicolored canvas of proficiency levels to a portrait of the everyday use of the two languages by individuals..." (Baker, 2011, t. 8).

Wrth drafod gwahanol fframweithiau damcaniaethol ar gyfer addysg ddwyieithog, er enghraifft, cyfeiria García (2009, t. 118) at y cysyniad o 'gontinwwm dwyieithog', ar lefel yr unigolyn, gan gydnabod bod gan fyfyrwyr eu continwwm dwyieithog eu hunain. Fodd bynnag, awgryma García (2009, t. 118) fod modd ymestyn y cysyniad hwn ymhellach: “...different children, families and communities find themselves at various points on a bilingual continuum depending on life circumstances, and as they interact with other speech communities.” Mae dehongliad García o'r term 'continwwm' yn awgrymu bod cymunedau dwyieithog, yn ogystal â'u trigolion dwyieithog, ar daith ieithyddol drosiadol wrth iddynt symud yn ôl ac ymlaen rhwng dau begwn y continwwm, sef unieithrwydd a dwyieithrwydd, neu amlieithrwydd. Mae'r dyfyniad uchod hefyd yn awgrymu bod defnydd yr unigolyn dwyieithog o'i ieithoedd yn y gymuned yn ystyriaeth hollbwysig.

Mae Grosjean (2010) hefyd wedi dehongli'r continwwm dwyieithog ar lefel yr unigolyn. Yn wahanol i Valdés (2003) fodd bynnag, mae Grosjean yn canolbwyntio ar ddefnydd siaradwyr dwyieithog o'u hieithoedd, yn hytrach na'u gallu yn yr ieithoedd. Yn ei ôl ef, ceir dau begwn, neu ddau fodd ieithyddol, sef y 'modd uniaith' (monolingual mode) a'r 'modd dwyieithog' (bilingual mode). Pan fo'r siaradwr dwyieithog yn defnyddio un o'i ieithoedd wrth gyfathrebu â siaradwyr uniaith yr iaith honno, er enghraifft, gellir disgrifio'r siaradwr fel petai yn y modd uniaith. Pan fo'r siaradwr dwyieithog yn cyfathrebu â siaradwyr dwyieithog drwy ddefnyddio y naill iaith a'r llall, gellir disgrifio'r siaradwr fel petai yn y modd dwyieithog. Gan ddibynnu ar y cyd-destun, y person y mae'r siaradwr dwyieithog yn siarad ag ef, a'r pwnc trafod dan sylw, gall y siaradwr dwyieithog symud yn ôl ac ymlaen ar hyd y continwwm hwn drwy'r dydd (Grosjean, 2010, t. 42). Yn y ffigur isod, gwelir sut mae Grosjean yn dychmygu'r 'continwwm dwyieithog'.

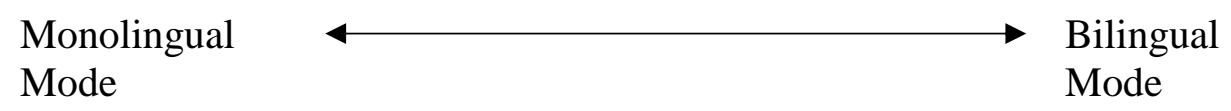


Ffigur 2. Continwwm dwyieithog Grosjean (2010)

I'r un perwyl â Grosjean (2010), mae Hornberger (2003) wedi cynnig dehongliad o'r continwwm dwyieithog ar sail defnydd siaradwyr o'u hieithoedd. Yn ei gwaith ar ddatblygu continua ar gyfer llythrennedd deuol, mae Hornberger (2003) yn nodi'r 'continwwm uniaith-dwyieithog' fel un o'r tri chontinwwm y mae hi'n eu cynnig ar gyfer diffinio cyd-destunau datblygu llythrennedd deuol. Fel y mae Hornberger yn ei gydnabod, mae'n bosibl ystyried y continwwm ar lefel facro, sef defnydd a swyddogaeth ieithoedd mewn cymuned ddwyieithog, yn ogystal ag ar lefel feicro, sef defnydd unigolion dwyieithog o'u hieithoedd. Yn ôl Horberger:

...the difference between monolingual and bilingual individuals is not so much that bilinguals possess two complete sets of functions and uses of language, one for each language. Rather, bilinguals switch languages according to specific functions and uses, whereas monolinguals switch styles in the same context. (Hornberger, 2003, tt. 13-14)

Gwelir o'r gwahanol ddehongliadau a gynigiwyd uchod nad oes un ffordd o ddiffinio'r 'continwwm dwyieithog'. Fodd bynnag, mae'n amlwg fod nifer o ysgolheigion, megis Hornberger (2003), García (2009), Grosjean (2010) a Baker (2011), am symud i ffwrdd o ganolbwyntio ar hyfedredd siaradwyr dwyieithog yn y naill iaith a'r llall, tuag at greu proffil ieithyddol o ddefnyddio'r ieithoedd. At ddiben y traethawd hwn, defnyddir yr ymadrodd 'continwwm dwyieithog' wrth gyfeirio at y broses o ddysgu ieithoedd ar lefel yr unigolyn yn benodol, hynny yw, dilyniant ieithyddol y dysgwr mewn addysg a'r tu hwnt.

\subsubsection{Diffinio'r continwwm Cymraeg a'r continwwm ieithyddol}

Mewn adroddiad diweddar gan LIC (2017ch), nodwyd y cynllun gweithredu ar gyfer y Gymraeg mewn addysg dros y pedair blynedd nesaf. Yn yr adroddiad, mae LlC (2017ch, t. 10) yn datgan ei bod wedi cytuno "y byddai continwwm dysgu Cymraeg yn cael ei ddatblygu fel rhan o'r cwricwlwm a'r trefniadau asesu newydd i Gymru" ac y byddai'r “...continwwm hwn yn sail ar gyfer addysgu a dysgu Cymraeg ac ieithoedd eraill yn y dyfodol." Mae'n mynd yn ei blaen i nodi y “[b]ydd cwricwlwm newydd a fydd yn ymgorffori fframwaith ar gyfer datblygu sgiliau ar hyd continwwm, capasiti gwell o ran athrawon, ac ymchwil i addysgeg effeithiol yn llywio addysgu a dysgu." (LlC, 2017ch, t. 14). Awgrymir gan y dyfyniadau uchod 
fod y 'continwwm Cymraeg' yn cael ei ddiffinio fel fframwaith y gellir seilio trefniadau dysgu, addysgu ac asesu ar gyfer Cymraeg arno. Mae Davies (2013) a Lewis (2010a) hefyd wedi dehongli'r continwwm Cymraeg yn y ffordd hon.

Gellir cynnig un diffiniad arall cyn cloi'r bennod hon. Mewn adroddiad diweddar gan Y Pwyllgor Diwylliant, y Gymraeg a Chyfathrebu (2017), cafodd yr ymadrodd 'continwwm ieithyddol' ei ddefnyddio yng nghyd-destun symud ysgolion o'r naill gategori iaith i'r llall. Amlygwyd pryder hefyd am y dehongliad hwn o'r 'continwwm ieithyddol' yn y strategaeth newydd ar gyfer y Gymraeg:

Mae'r strategaeth yn sôn am symud ysgolion ar hyd y continwwm ieithyddol. Yn ein barn ni, y mater allweddol yw sicrhau bod mwy o ddisgyblion yn symud ar hyd y continwwm dysgu tuag at fod yn rhugl ar draws addysg cyfrwng Cymraeg a Saesneg. Mae yna berygl ein bod yn canolbwyntio gormod ar newid categori iaith yr ysgol yn hytrach na gwella rhuglder disgyblion ym mhob ysgol. (Y Pwyllgor Diwylliant, y Gymraeg a Chyfathrebu, 2017, t. 5)

O'r dyfyniad uchod, daw i'r amlwg y gall 'continwwm ieithyddol' neu 'gontinwwm Cymraeg' gael eu dehongli yng nghyd-destun categoreiddio ieithyddol ysgolion yng Nghymru. Ar naill ben y continwwm, ceir ysgolion cyfrwng Saesneg, ac ar y llall ceir ysgolion cyfrwng Cymraeg, gyda'r gwahanol fathau o ysgolion dwyieithog rhwng y ddau begwn. Er y nodwyd uchod bod Lewis (2010a) yn cyfeirio at y cysyniad o gontinwwm fel fframwaith asesu, nodir ei fod yntau'n defnyddio'r ymadrodd 'continwwm dwyieithog' wrth gyfeirio at yr amrywiaeth eang o fodelau addysg ddwyieithog yng Nghymru: “... o'r wers 'Gymraeg ail iaith' mewn ysgol cyfrwng Saesneg yn naill begwn y continuum (pwyslais yr awdur) dwyieithog i addysg a gyflwynir bron yn gyfan gwbl trwy gyfrwng y Gymraeg (ac eithrio Saesneg fel pwnc) yn y pegwn arall.” (W.G. Lewis, 2011, t. 68). Yn ogystal â fframwaith asesu felly, gellir hefyd ddehongli'r continwwm ieithyddol fel cysyniad gwleidyddol-niwtral ar gyfer y broses o symud ysgolion cyfrwng Saesneg tuag at fod yn ysgolion dwyieithog a chyfrwng Cymraeg.

Yr hyn sydd yn amlwg o'r llenyddiaeth yw nad oes cysondeb o ran defnyddio'r term 'continwwm' a bod angen i LlC gynnig diffiniad clir o'r hyn a olygir gan y term, cyn iddi weithredu'r continwwm Cymraeg arfaethedig. Caiff hyn ei amlygu gan Gymwysterau Cymru (d.d.), sy'n nodi'r canlynol: 
Mae gennym ddiddordeb penodol yn y ffordd o ddiffinio continwwm ar gyfer caffael iaith a meithrin sgiliau iaith a wnaiff helpu i sicrhau dysgu ac addysgu effeithiol, yn ogystal ag asesiad dibynadwy, dilys ac ymarferol. Mae'r gwaith hwn ar gamau cynnar o hyd a hyd nes y caiff ei ddatblygu ymhellach, ni fydd modd nodi gofynion dylunio cymhwyster neu gymwysterau newydd i gefnogi'r camau y bwriedir eu cymryd. (Cymwysterau Cymru, d.d., t. 2)

Er y gall 'continwwm Cymraeg' a 'chontinwwm ieithyddol' gael eu defnyddio'n gyfnewidiol, at ddiben y traethawd hwn defnyddir yr ymadrodd 'continwwm Cymraeg' wrth gyfeirio at y fframwaith asesu ar gyfer yr iaith Gymraeg mewn ysgolion y mae LlC wrthi'n ei ddatblygu fel rhan o'r cwricwlwm newydd. Defnyddir yr ymadrodd 'continwwm ieithyddol' yng nghyd-destun categoreiddio ysgolion yn ôl eu darpariaeth cyfrwng Cymraeg, gydag ysgolion cyfrwng Saesneg yn bennaf ar naill ben y continwwm ieithyddol ac ysgolion cyfrwng Cymraeg ar y llall (gweler 2.6.1. Diffinio ysgolion yn ôl eu darpariaeth cyfrwng Cymraeg). 


\section{Pennod 3: Manteision posibl dwyieithrwydd}

Yn y bennod hon, rhoir ystyriaeth benodol i'r manteision posibl o fod yn ddwyieithog, y terfyniadau posibl ar y manteision hyn, yn ogystal â'r anfanteision posibl o fod yn ddwyieithog. Diben y drafodaeth hon yw cynnig tystiolaeth bellach i'r ddadl a gyflwynir yn yr ymchwil dros newid sylfaenol i'r ffordd y cyflwynir y Gymraeg mewn perthynas ag addysg cyfrwng Saesneg er mwyn cyflawni nod uchelgeisiol cyfredol LlC o gyrraedd miliwn o siaradwyr erbyn 2050.

Yn ogystal â'r manteision posibl o fod yn ddwyieithog y gellid eu hystyried yn fwy cyffredinol, megis cyfathrebu â'r teulu a'r gymuned mewn mwy nag un iaith, mabwysiadu dau ddiwylliant (neu ragor), mwynhau dwy lenyddiaeth (neu ragor), cryfhau hunaniaeth yr unigolyn, a dod yn fwy cydymdeimladol a goddefgar o ran ieithoedd a diwylliannau eraill (Baker, 2010, tt. 1-2), mae casgliad sylweddol o ymchwil sy'n awgrymu bod nifer o fanteision posibl pellach, megis manteision deallusol a gwybyddol, manteision addysgol, a hyd yn oed manteision iechyd meddwl. Er gwaethaf cryfder y dystiolaeth bresennol o blaid manteision dwyieithrwydd, rhaid hefyd bwyso a mesur terfyniadau'r manteision hyn, a'r anfanteision posibl, sydd wedi'u hymchwilio. Cyflwynir rhai o'r prif ganfyddiadau mewn perthynas â'r manteision hyn, yn ogystal â'r anfanteision posibl, er mwyn cryfhau'r ddadl o blaid datblygu sgiliau dwyieithog disgyblion o fewn addysg cyfrwng Saesneg.

\subsection{Meddwl creadigol}

Mae meddwl creadigol wedi bod yn rhan o'r drafodaeth ar ddwyieithrwydd ers chwedegau'r ganrif ddiwethaf. Oherwydd bod gan siaradwyr dwyieithog fwy nag un gair am wrthrychau a chysyniadau, credir yn sylfaenol y gall siaradwyr dwyieithog ystyried gwrthrychau a'u labelu'n fwy hyblyg, a hynny gyda dimensiynau ychwanegol, na siaradwyr uniaith. Mae Baker (2011, t. 149) yn nodi enghraifft ddiddorol iawn o sut y gall hyn ddigwydd ar gyfer siaradwyr dwyieithog yn y Gymraeg a'r Saesneg, wrth gynnig y gair 'ysgol' sydd â dwy ystyr wahanol, sef yr ysgol a ddringir a'r ysgol a fynychir fel disgybl. Mae'n bosibl y gallai siaradwyr dwyieithog ystyried 'ysgol' felly yn drosiadol megis ysgol a ddringir i lwyddo'n academaidd. Nid yw hyn yn wir o ran y gair Saesneg, sef 'school', sydd heb ystyr 
drosiadol debyg. O ganlyniad, dadleuir bod gan siaradwyr dwyieithog amrywiaeth ehangach o gysylltiadau â geiriau na siaradwyr uniaith (Baker, 2011, t. 149).

Oherwydd yr hyblygrwydd hwn o ran meddwl am gysyniadau a gwrthrychau mewn dwy iaith wahanol, mae'n bosibl felly fod dwyieithrwydd yn fantais wrth feddwl yn greadigol, ac mae nifer fawr o astudiaethau wedi atgyfnerthu'r gred hon (Kharkhurin, 2011; Ricciardelli, 1992; Simonton, 2008). Mae astudiaeth gan Kharkhurin (2011), o 90 o fyfyrwyr prifysgol dwyieithog o gefndiroedd amrywiol, gyda lefelau gwahanol o hyfedredd yn y Saesneg, yn enghraifft o ymchwil ddiweddar ar greadigrwydd y siaradwr dwyieithog. Yn ei astudiaeth, canfu Kharkhurin (2011) fod y siaradwyr dwyieithog mwyaf galluog (yn ieithyddol) yn eu dwy iaith yn well o ran eu perfformiad creadigol yn y Saesneg na'r siaradwyr dwyieithog llai galluog. Mae'r canfyddiadau hyn yn gyson â chanfyddiadau ymchwil flaenorol (Ricciardelli, 1992) a ganfu fod siaradwyr dwyieithog rhugl yn y ddwy iaith yn arddangos perfformiad creadigol gwell na siaradwyr dwyieithog llai rhugl yn eu dwy iaith, a gwell fyth na siaradwyr uniaith. Mae'r canfyddiadau hyn hefyd yn gyson â Damcaniaeth y Trothwy Ieithyddol (Threshold Theory) ${ }^{21}$ sy'n damcaniaethu mai ond siaradwyr dwyieithog 'cytbwys' a all elwa ar fanteision gwybyddol cadarnhaol.

\subsection{Ymwybyddiaeth feta-ieithyddol}

Fel y soniwyd eisoes, mae gan siaradwyr dwyieithog ddwy eirfa, ac o'r herwydd mae'n bosibl y gall siaradwyr dwyieithog weld ystyr geiriau'n gyfnewidiol mewn modd mwy hyblyg na siaradwyr uniaith. Yn y bôn, mae gwrthrychau a'u labeli yn bethau ar wahân i'w gilydd i siaradwyr dwyieithog, tra bod siaradwyr uniaith yn eu gweld yn anwahanadwy (Baker, 2011, t. 151). Mewn astudiaeth gan Ianco-Worrall (1972) a gynhaliodd ddau arbrawf ar ddwy garfan o blant, 4-6 oed a 7-9 oed, dangoswyd bod y siaradwyr dwyieithog yn yr Affricaneg a'r Saesneg rhwng 4-6 oed yn fwy tebygol o ddewis geiriau ar sail semanteg, lle mae siaradwyr uniaith yr un grŵp oedran yn fwy tebygol o ddewis geiriau ar sail ffonoleg, hyd at 7 oed pan

\footnotetext{
${ }^{21}$ Dyma ddamcaniaeth a gafodd ei chyflwyno gan Cummins (1976) a Toukomaa a Skutnabb-Kangas (1977). Prif egwyddor y ddamcaniaeth hon yw bod dau drothwy ar gyfer hyfedredd mewn iaith. Mae'r trothwy cyntaf yn sicrhau na fydd anfanteision wrth fod yn ddwyieithog ac mae'r ail drothwy yn sicrhau y bydd manteision wrth fod yn ddwyieithog. Mae angen felly gyrraedd lefel o hyfedredd mewn iaith, sef yr ail drothwy, cyn i'r siaradwr dwyieithog allu elwa ar fanteision posibl dwyieithrwydd. Fodd bynnag, nid yw'r ddau drothwy ieithyddol hyn erioed wedi'u diffinio'n benodol (Williams, d.d., t. 13), ac nid yw rhai ysgolheigion (e.e. Takakuwa, 2005) yn cefnogi'r ddamcaniaeth hon.
} 
fyddent yn dewis geiriau ar sail semanteg hefyd. Mae'r canfyddiadau hyn yn awgrymu bod siaradwyr dwyieithog ifanc yn well o ran cadw ystyr a ffurf geiriau ar wahân, lle mae siaradwyr uniaith ifanc yn fwy tebygol o gysylltu ystyr â ffurf. Ar sail y canfyddiadau hyn, casglodd Ianco-Worrall (1972, t. 1398) fod galluoedd semanteg siaradwyr dwyieithog 22 yn datblygu'n ddwy i dair blynedd yn gynharach na galluoedd semanteg siaradwyr uniaith. Fodd bynnag, mae Bialystok (2001b) wedi rhybuddio nad oes digon o dystiolaeth i gasglu bod ymwybyddiaeth feta-ieithyddol yn datblygu'n rhwyddach ac yn gyflymach mewn plant dwyieithog nag mewn plant uniaith. Serch hynny, cyfeddyf Bialystok fod plant dwyieithog yn perfformio'n well mewn tasgau meta-ieithyddol. Mae hi'n casglu mai rheolaeth siaradwyr dwyieithog dros sylwgarwch sy'n ganolog i'w gallu hwy i ddatrys problemau meta-ieithyddol:

Rather than attributing this advantage to privileged access to a domain of knowledge, however, the benefit is traced to a specific cognitive process that develops more readily in bilingual children. This process, control of attention, is central to certain metalinguistic problems, and it is in solving these problems that bilingual children excel (Bialystok, 2001b, t. 180).

Mae ymchwil gan Bialystok (1987a, 1987b, 1997, 2001a, 2001b) wedi astudio'r berthynas rhwng dwyieithrwydd ac ymwybyddiaeth feta-ieithyddol. Ymddengys o'r ymchwil ganddi fod plant dwyieithog 5 i 9 oed yn perfformio'n well o ran darganfod brawddegau yn anghywir o ran gramadeg (Bialystok, 1987a) a hefyd o ran cyfrif nifer y geiriau mewn brawddegau (Bialystok, 1987b), o'u cymharu â phlant uniaith. Mae canfyddiadau'r ddwy astudiaeth hyn yn awgrymu bod gan blant dwyieithog cytbwys allu uwch o ran dadansoddi'r hyn y maent yn ei wybod am eu hieithoedd a bod ganddynt ddealltwriaeth well o'r cysyniad o brint. Mae Bialystok (1997) wedi atgyfnerthu'r canfyddiadau hyn ymhellach yn ei hastudiaeth ar dair carfan o blant 4 a 5 oed. Astudiodd Bialystok un garfan o blant uniaith Saesneg, a dwy garfan o blant dwyieithog, Ffrangeg-Saesneg a Mandarin-Saesneg. Darganfuwyd bod y plant dwyieithog yn well o ran deall y cysyniad o brint, gan eu bod wedi deall cysyniad geiriau print heb ymyrraeth ystyr y lluniau. Mae'n bosibl felly y gall plant sydd â hyfedredd cytbwys mewn mwy nag un iaith godi sgiliau darllen yn gyflymach na siaradwyr uniaith oherwydd yr ymwybyddiaeth feta-ieithyddol uwch hon (Baker \&

\footnotetext{
${ }^{22}$ Mae 'siaradwyr dwyieithog' yma'n cyfeirio at siaradwyr sydd wedi caffael dwy iaith mewn cartrefi lle mae'r naill riant yn siarad un iaith â hwy, ac mae'r llall yn siarad iaith arall â hwy (Ianco-Worrall, 1972, t. 1391)
} 
Jones, 1998, t. 73). At hyn, mae Durgunoğlu, Nagy a Hancin-Bhatt (1993) wedi darganfod bod ymwybyddiaeth ffonolegol, gallu meta-ieithyddol arall, sydd wedi’i datblygu mewn mwy nag un iaith yn cyfrannu at gaffael sgiliau darllen. Yn eu hastudiaeth ar blant 7 oed, darganfu Durgunoğlu, Nagy a Hancin-Bhatt fod ymwybyddiaeth ffonolegol yn iaith gyntaf y plant, sef y Sbaeneg, yn cefnogi adnabod geiriau yn yr ail iaith, sef Saesneg, ac felly mae'n bosibl fod hyn yn gallu cefnogi darllen yn y Saesneg.

Nid yw'n glir eto, fodd bynnag, a yw tebygrwydd rhwng systemau sain mewn dwy iaith yn cynorthwyo datblygiad ffonolegol yn yr ail iaith. Dyma'r hyn a noda Bialystok a Herman (1999, t. 41), sy'n credu nad yw'n glir eto a fyddai hyn yn digwydd gyda dwy iaith sydd â systemau sain gwahanol fel Tsieinëeg a Sbaeneg. Cred Bialystok a Herman (1999) mai'r tebyg yw fod dwyieithrwydd yn cael effaith ar lythrennedd plant dwyieithog, ond nid yw'r cysylltiad rhyngddynt eto'n eglur. Esbonia Bialystok a Herman (1999) fod caffael llythrennedd mewn iaith yn broses sy'n ddibynnol ar brofiadau cymdeithasol, gwybyddol ac ieithyddol y plentyn. Mae datblygu llythrennedd yn digwydd mewn ffordd gymdeithasol wrth i blant wrando ar storïau ac adrodd storïau eu hunain, er enghraifft. Mae datblygu llythrennedd mewn ffordd wybyddol yn digwydd wrth ddatblygu'r cysyniad o brint, ac yn ieithyddol drwy ddatblygu ymwybyddiaeth ffonolegol. Er bod cyswllt rhwng dwyieithrwydd â'r agweddau hyn ar ddatblygu llythrennedd yn gynnar, daw Bialystok a Herman (1999, t. 41) i'r casgliad fod "[e]ach of the three areas of competence follows a complex path of growth and bears a different link to bilingualism." Darganfu Abu-Rabia a Sanitsky (2010) fodd bynnag dystiolaeth yn fwy diweddar sy'n profi y gall dysgu dwy iaith sydd ag orgraffau gwahanol hwyluso caffael ieithoedd eraill, a bod gallu ffonolegol siaradwyr teirieithog hyd yn oed yn uwch o'u cymharu â siaradwyr dwyieithog. Dônt i'r casgliad hwn: "experience with a number of orthographies enhances the phonological awareness of readers and ultimately their reading accuracy in alphabetic orthographies, regardless of orthographic depth." (Abu-Rabia a Sanitsky, 2010, t. 195).

Yn ogystal â'r posibilrwydd o gefnogi llythrennedd cynnar, mae'n bosibl hefyd y gall dwyieithrwydd gael effaith gadarnhaol ar ddatblygu rhifedd cynnar. Mewn astudiaeth gan Bialystok a Codd (1997), dangoswyd bod siaradwyr dwyieithog ifanc, 4-5 oed, yn well na'u cyfoedion uniaith o ran datrys problemau ynglŷn â phrifoledd 
lle bo angen sylw manwl. Fel y trafodir nes ymlaen yn y bennod hon (gweler 3.3. Sensitifrwydd cyfathrebol), mae sylwgarwch siaradwyr dwyieithog i fanylion yn sgìl bwysig hefyd wrth gyfathrebu, gan fod yn rhaid i siaradwyr dwyieithog fod yn ymwybodol o ofynion y gwrandäwr. Fel yr esbonia Baker (2001, t. 153), rhaid bod yn ymwybodol o'r iaith a'r sefyllfa y defnyddir iaith ynddi. Mae'n debyg felly fod y sylwgarwch gwell a ddaw wrth fod yn ddwyieithog yn fantais posibl i blant dwyieithog ifanc wrth iddynt ddysgu cysyniadau rhif a'i natur symbolaidd (Baker, 2001, t. 151).

\subsection{Sensitifrwydd cyfathrebol}

Yn ogystal â'r manteision gwybyddol posibl o fod yn ddwyieithog, mae ymchwil wedi dangos bod gan siaradwyr dwyieithog 'sensitifrwydd' neu 'ymwybyddiaeth' gyfathrebol uwch na siaradwyr uniaith. Credir bod siaradwyr dwyieithog yn fwy sensitif i gyd-destunau cyfathrebol wrth ddefnyddio iaith oherwydd bod mwy o angen iddynt fonitro'r sefyllfa gyfathrebol o'u cwmpas trwy'r amser (Yow \& Markman, 2011, t. 13). Mae sensitifrwydd cyfathrebol yn arbennig o bwysig mewn sefyllfaoedd lle mae angen tawelu meddwl rhywun, neu wneud i rywun deimlo'n gartrefol. Mae Baker a Jones (1998, t. 56) yn cynnig enghraifft dda lle mae sensitifrwydd cyfathrebol uwch yn hollbwysig, sef staff dwyieithog mewn ysbyty. Mae staff dwyieithog, sy'n dewis cyfathrebu trwy gyfrwng iaith eu cleifion, yn gallu gwneud i'w cleifion deimlo'n gartrefol oherwydd bod gan y claf ddewis o ran pa iaith yr hoffai ei defnyddio wrth gyfathrebu â'r staff. Mae ymchwil hefyd wedi dangos y gall anawsterau cyfathrebol effeithio'n negyddol ar y gofal iechyd a geir gan glaf sy'n siarad iaith leiafrifol (Irvine ac eraill, 2006; Owen \& Morris, 2012). Felly mae'n bwysig yng nghyd-destun Cymru, lle mae cynnig gwasanaethau iechyd trwy gyfrwng yr iaith leiafrifol yn her (Irvine ac eraill, 2006), y caiff siaradwyr sydd â medrau uchel yn y Gymraeg a'r Saesneg eu cyflogi yn y sectorau hynny lle mae sensitifrwydd cyfathrebol yn hollbwysig.

Genesee, Tucker a Lambert (1975) a Ben-Zeev (1977) oedd yr ysgolheigion cyntaf i ddadlau bod manteision cyfathrebol posibl wrth fod yn ddwyieithog. Yn astudiaeth Genesee ac eraill (1975) ar blant uniaith a phlant dwyieithog 5-8 oed, darganfuwyd bod plant dwyieithog yn fwy ymwybodol o anghenion cyfathrebol y gwrandäwr. Daeth hyn i'r amlwg wrth i'r siaradwyr dwyieithog geisio trosglwyddo mwy o wybodaeth i'r gwrandäwr wrth gyfathrebu na'r siaradwyr uniaith yn yr un prawf 
(Genesee ac eraill, 1975, t. 1013). Casglodd Genesee ac eraill (1975, t. 1013) fod plant a gaiff eu haddysgu drwy gyfrwng eu hail iaith, yn arbennig y rhai sy'n dilyn rhaglen addysg drochi gyflawn, yn fwy sensitif i anghenion eraill na phlant a gaiff eu haddysgu drwy gyfrwng eu hiaith gyntaf. Mewn astudiaeth debyg arall, darganfu Ben-Zeev (1977) y gallai plant dwyieithog Saesneg-Sbaeneg o ddosbarth is godi cliwiau yn yr arbrawf a chywiro eu gwallau eu hunain yn well na phlant uniaith. At ei gilydd, dangosodd y ddwy astudiaeth hyn duedd i siaradwyr dwyieithog fod yn fwy ymwybodol o'r cyd-destun cyfathrebol o'u cwmpas wrth gyfathrebu'n rhyngbersonol, a hynny er gwaethaf anfanteision eu cefndir weithiau.

Yn fwy diweddar, mewn cyfres o astudiaethau gan Yow a Markman (2011) ar blant dwyieithog ac uniaith, dangoswyd bod plant dwyieithog yn ymateb yn well i arwyddion cyfeiriadol dieiriau (non-verbal referential gestures), megis pwyntio at rywbeth neu syllu ar rywbeth. Gan fod angen i siaradwyr dwyieithog ifanc fonitro'r cyd-destun cyfathrebol o'u cwmpas trwy'r amser a chan eu bod yn ymateb i ofynion ieithyddol y cyd-destun yn well, mae'n debyg fod plant dwyieithog ifanc yn codi'r cliwiau dieiriau hyn, sy’n hwyluso cyfathrebu llwyddiannus, o’u cwmpas yn well na phlant uniaith. A dyfynnu Yow a Markman (2011):

...it remains plausible that the experience of growing up bilingual improves children's sensitivity to referential gestures. In particular, we suggest that such improvement stems from bilingual children's self-generated efforts to communicate effectively. (Yow \& Markman, 2011, t. 28)

Mae canfyddiadau ymchwil Yow a Markman (2011) felly yn cefnogi'r ddamcaniaeth flaenorol, sef bod gan siaradwyr dwyieithog sensitifrwydd cyfathrebol uwch o oedran cynnar na'u cyfoedion uniaith.

\subsection{Dwyieithrwydd megis sylfaen i ddysgu ieithoedd eraill}

Mae ymchwil ddiweddar ym maes dwyieithrwydd hefyd wedi canolbwyntio ar effaith dwyieithrwydd ar hwyluso dysgu ieithoedd ychwanegol. Mewn arolwg cenedlaethol ar addysgu ITM yn ysgolion uwchradd Cymru, amlygodd Board a Tinsley (2015) sefyllfa ieithyddol unigryw Cymru a'i photensial i fanteisio ar ddwyieithrwydd yn y Gymraeg a'r Saesneg er mwyn hwyluso dysgu ITM:

Fel cenedl ddwyieithog, mae gan Gymru fantais o ran dysgu ieithoedd eraill, gan fod ymchwil yn dangos, ar ôl dysgu un iaith ychwanegol, fod myfyrwyr yn ei chael hi'n haws caffael ieithoedd eraill. (Board \& Tinsley, 2015, t. 9). 
Mae'r hyn a noda Board a Tinsley (2015) uchod wedi'i danlinellu eisoes yn adroddiad Dyfodol Llwyddiannus, wrth i Donaldson (2015) ymrwymo i fanteisio ar hybu dysgu ITM drwy ddatblygu'r Gymraeg yn gynharach:

Ym marn yr Adolygiad, bydd y gallu i ddysgu ieithoedd tramor modern yn cael ei hybu drwy ddatblygu'r Gymraeg yn gynharach, ac mae'n argymell felly y bydd y drydedd a/neu bedwaredd iaith yn cael ei chyflwyno yn y flwyddyn gyntaf yn yr ysgol uwchradd, neu'n gynharach lle mae amser, amgylchiadau ac arbenigedd yn caniatáu. (Donaldson, 2015, t. 48).

Astudiodd Cenoz a Valencia (1994) 320 o fyfyrwyr, 48\% yn uniaith Sbaeneg a 52\% yn ddwyieithog yn y Sbaeneg a'r Fasgeg, rhwng 17-19 oed, er mwyn darganfod pa fath o effaith a gâi addysg cyfrwng Basgeg ar gaffael Saesneg fel trydedd iaith. Wedi darganfod mai deallusrwydd, oedran, cymhelliant a dangosiad i'r iaith yw'r rhagfynegyddion a oedd yn sicrhau'r cyfraniad mwyaf sylweddol at gyrhaeddiad yn y Saesneg (Cenoz \& Valencia, 1994, t. 202), mesurwyd effaith dwyieithrwydd ar wahân i'r ffactorau cyfnewidiol hyn er mwyn darganfod a ydy dwyieithrwydd ei hun yn cael effaith ar gyrhaeddiad yn y Saesneg. Fel y casglodd Cenoz a Valencia (1994):

...bilingualism has a positive mediating effect on third language learning... Furthermore, there were no interaction effects between bilingualism and the other predictors, so the effect of bilingualism was obtained regardless of the effects of the cognitive, sociostructural, social psychological, and educational variables. (Cenoz \& Valencia, 1994, t. 204)

Yn ogystal â'r rhagfynegyddion cyfnewidiol sydd yn amlwg yn gallu cael effaith ar gaffael trydedd iaith felly, mae Cenoz a Valencia wedi dangos bod dwyieithrwydd, megis ffactor ar wahân i'w hystyried, yn gallu cyfrannu'n gadarnhaol at ddysgu trydedd iaith mewn cyd-destun dwyieithrwydd ychwanegol (additive bilingualism). Mae'r dystiolaeth hefyd yn cefnogi'r ddamcaniaeth y gall dysgu trwy gyfrwng yr ail iaith, sef y Fasgeg, gael dylanwad cadarnhaol ar ddysgu trydedd iaith, sef y Saesneg, yn ogystal â'r ail iaith, ar gyfer siaradwyr Sbaeneg eu hiaith gyntaf:

The acquisition and reinforcement of heritage languages within the educational system and the use of the minority language as the language of instruction can improve linguistic proficiency, not only in a second language (Basque), but also in a third (English) (Cenoz \& Valencia, 1994, t. 206). 
Mae Cenoz a Valencia (1994, t. 205) yn priodoli manteision dwyieithrwydd megis dealltwriaeth strwythurol o ddwy iaith ac, o ganlyniad, ymwybyddiaeth fetaieithyddol sydd wedi'i datblygu'n well, ynghyd â sensitifrwydd cyfathrebol uwch, i esbonio hyfedredd ieithyddol uwch yn y drydedd iaith ymhlith siaradwyr sydd eisoes â dwy iaith. At y rhain, ychwanega Cenoz (2013, t. 76) ffactorau eraill, megis profiad wrth ddysgu ieithoedd eraill a repertoire ieithyddol ehangach o ieithoedd, at y manteision sydd gan siaradwyr dwyieithog wrth ddysgu ieithoedd ychwanegol.

Mae ymchwil Cenoz a Valencia wedi'i hatgyfnerthu mewn astudiaethau tebyg diweddarach gan eraill (Abu-Rabia \& Sanitsky, 2010; Sanz, 2000). Canolbwyntiodd Sanz (2000), er enghraifft, ar gymharu gallu myfyrwyr ysgol uwchradd dwyieithog yn y Sbaeneg a'r Gatalaneg i gaffael Saesneg megis trydedd iaith â gallu myfyrwyr uniaith Sbaeneg i gaffael Saesneg megis ail iaith. Canfu Sanz (2000) ganfyddiadau tebyg iawn i'r hyn a ganfuwyd gan Cenoz a Valencia (1994) o'r blaen, sef bod llythrennedd mewn dwy iaith yn cael effaith gadarnhaol ar gaffael trydedd iaith. Unwaith eto, dangoswyd canfyddiadau tebyg gan Abu-Rabia a Sanitsky (2010) a astudiodd 82 o fyfyrwyr, 40 o fyfyrwyr teirieithog a 42 o fyfyrwyr dwyieithog, gradd 6 o ysgolion yn Israel. Cafodd y ddwy garfan o fyfyrwyr, un garfan o fyfyrwyr Rwsieg iaith gyntaf, Hebraeg eu hail iaith, a Saesneg eu trydedd iaith, a charfan arall o fyfyrwyr Hebraeg iaith gyntaf a Saesneg eu hail iaith, eu hasesu mewn cyfres o brofion a astudiodd agweddau gwybyddol, meta-ieithyddol a gwybyddiaeth iaith, yn y tair iaith. Er gwaethaf gwahaniaethau o ran ffonoleg ac orgraff rhwng y tair iaith, canfu Abu-Rabia a Sanitsky (2010, t. 195) fod gallu ffonolegol siaradwyr teirieithog yn well na siaradwyr dwyieithog, ac felly bod "experience with a number of orthographies enhances the phonological awareness of readers and ultimately their reading accuracy in alphabetic orthographies, regardless of orthographic depth." Mae'r dystiolaeth hon felly yn cefnogi canfyddiadau ymchwil flaenorol, sef bod gan siaradwyr dwyieithog fantais ar siaradwyr uniaith o ran dysgu ieithoedd eraill, a bod siaradwyr teirieithog (neu amlieithog) yn manteisio ymhellach fyth ar siaradwyr dwyieithog o ran hynny.

Yn ei adolygiad o'r ymchwil ar effaith dwyieithrwydd ar gaffael trydedd iaith, mae Cenoz (2013, t. 77) yn nodi cymhlethdod y sefyllfa. Er y caiff dwyieithrwydd ddylanwad cadarnhaol ar ddysgu trydedd iaith mewn nifer o achosion, noda mai proses gymhleth iawn sydd yn ddibynnu ar ystod o ffactorau eraill, megis 
deallusrwydd, statws sosio-economaidd, cymhelliant a dangosiad i'r iaith, yw caffael iaith. Oherwydd hynny, mae'n bosibl y gall y ffactorau hyn fod yn bwysicach na dwyieithrwydd fel ffactor a'u bod hyd yn oed yn cuddio effaith gyflawn dwyieithrwydd ar ddysgu trydedd iaith.

\subsection{Terfyniadau manteision dwyieithrwydd}

Mae'r ymchwil gyfredol ar ddwyieithrwydd wedi awgrymu'n gyson fod nifer o fanteision posibl i fod yn ddwyieithog, gan gynnwys manteision gwybyddol, deallusol, addysgol ac iechyd meddwl, ynghyd â'r manteision a drafodwyd yn yr isadran flaenorol. Er hynny, dylid cadw mewn cof fod terfyniadau ar y canfyddiadau cadarnhaol hyn a hefyd fod nifer o astudiaethau sydd wedi mynegi anfanteision posibl o ran bod yn ddwyieithog, gan gynnwys rhai astudiaethau ar siaradwyr dwyieithog Cymraeg-Saesneg.

Mae bellach ystod fawr o dystiolaeth sy'n cadarnhau bod sgiliau llafar siaradwyr dwyieithog ar y cyfan yn wannach na sgiliau llafar siaradwyr uniaith (Bialystok ac eraill, 2012, t. 240). Yn benodol, mae nifer o astudiaethau wedi adrodd yn gyson bod siaradwyr dwyieithog ifanc yn cynhyrchu llai o eiriau yn eu dwy iaith na siaradwyr uniaith (gweler, er enghraifft, Bialystok, Luk, Peets, \& Yang, 2010; Pearson, Fernandez, \& Oller, 1993), ac o ganlyniad, nid yw geirfaoedd siaradwyr dwyieithog yn eu dwy iaith mor estynedig o'u cymharu â geirfa siaradwyr uniaith mewn un iaith. Mae'n debyg hefyd fod hyn yn wir ar gyfer pob oedran, drwy gydol oes y siaradwr dwyieithog (Bialystok, 2001a; Bialystok \& Craik, 2010). Er bod gan siaradwr dwyieithog ifanc ddwy eirfa anghyflawn, gan nad ydynt wedi meistroli'r un iaith na'r llall i'r un graddau â siaradwyr uniaith, dadleua Bialystok (2001a) fod y siaradwyr hyn wedi caffael digon o'r ddwy iaith er mwyn cyfathrebu ar lefel wynebol ${ }^{23}$ yn y naill a'r llall iaith, ac mae hyn yn agor mwy o bosibiliadau wrth gyfathrebu. Mae Bialystok, Luk, Peets a Yang (2010, t. 530) hefyd wedi disgrifio'r ffordd y mae'r siaradwr dwyieithog yn caffael dwy eirfa yn drosiadol wrth ddweud mewn byr eiriau: "[b]ilingual children are constructing the world through two telescopes, and their two vocabularies provide the lenses."

${ }^{23}$ Cyfeirir yma at yr hyn a ddiffiniwyd gan Cummins (1979a) yn BICS (Basic Interpersonal Communication Skills). Mae hyn yn wahanol i'r hyn y mae Cummins yn diffinio'n CALP (Cognitive Academic Language Proficiency), hynny yw, dysgu iaith trwy ei hastudio yn ffurfiol. 
Cyfeirir yma at yr hyn a adwaenir gan Grosjean (2008) fel y 'Complementarity

Principle'. Gan fod siaradwyr dwyieithog ifanc yn adeiladu dwy eirfa wahanol wrth ddefnyddio ei ddwy iaith, fel arfer ar gyfer peuoedd gwahanol, caiff yr eirfa gyflawn y mae'r siaradwr dwyieithog yn ei chaffael ei rhannu ar draws y ddwy iaith hynny. Gan fod caffael geirfa yn gysylltiedig â chyd-destun felly, ni fydd y siaradwr dwyieithog nodweddiadol yn gwybod pob gair yn eu dwy iaith oherwydd nad yw siaradwyr dwyieithog yn aml yn gytbwys yn eu dwy iaith. Felly, yn hytrach nac ystyried dwy eirfa'r siaradwr dwyieithog ar wahân, a'u cymharu'n anffafriol ag un eirfa'r siaradwr uniaith, dylid ymgymryd ag ymagwedd holistaidd er mwyn trin y ddwy eirfa fel rhan o eirfa gyflawn y siaradwr. Os nad yw'r siaradwr dwyieithog yn gwybod geiriau mewn un iaith, mae'n debyg ei fod yn gwybod y geiriau hyn yn y 1lall, ynghyd â'r geiriau y maent yn gwybod yn y ddwy iaith, ac felly mae'n bosibl mai siaradwyr dwyieithog sydd â'r eirfa fwyaf ar y cyfan (Bialystok ac eraill, 2010, t. $530)$.

Yn ogystal â'r diffygion o ran geirfa'r siaradwr dwyieithog, mae hefyd yn bosibl y gall anawsterau godi wrth adalw geiriau i'w cynhyrchu ar lafar. Mae ymchwil gan Gollan ac Acenas (2004) wedi nodi bod digwyddiadau TOT (Tip-of-the-tongue occurences $)^{24}$ yn digwydd yn fwy aml ym mhrofiad siaradwyr dwyieithog nac ym mhrofiad siaradwyr uniaith. Mewn dwy astudiaeth, un ar oedolion dwyieithog yn y Sbaeneg a'r Saesneg, a'r llall ar oedolion dwyieithog yn y Tagalog a'r Saesneg, cymharwyd y siaradwyr dwyieithog hyn â'r siaradwyr uniaith cyfatebol.

Darganfuwyd bod digwyddiadau TOT ddwywaith yn fwy tebygol o ddigwydd yn achos siaradwyr dwyieithog (Gollan \& Acenas, 2004, "Results", TOT incidence, para. 1).

Mae tystiolaeth hefyd sy'n dangos bod siaradwyr dwyieithog yn arafach na siaradwyr uniaith o ran deall geiriau (Ransdell \& Fischler, 1987) a chynhyrchu geiriau (Gollan, Montoya, Fennema-Notestine, \& Morris, 2005; Ivanova \& Costa, 2008). Mewn astudiaeth ar 28 o fyfyrwyr uniaith Saesneg a 28 o fyfyrwyr dwyieithog, gyda'r Saesneg megis iaith gyntaf, cynhaliodd Ransdell a Fischler

\footnotetext{
${ }^{24}$ Dyma ddigwyddiadau lle mae oedi'n digwydd tra bod siaradwr ar ffin ddwyn gair i'r cof, er ei fod yn gwybod, neu'n teimlo fel ei fod yn gwybod, y gair a sut mae ei ddweud. Esbonia Gollan ac Acenas (2004, t. 246) TOCs yn fwy manwl fel "word retrieval failures characterized by a feeling of imminent recall and by a greater than chance probability of accurately reporting partial information about the target word (e.g., the first phoneme)."
} 
(1987) brofion er mwyn cymharu'r myfyrwyr dwyieithog a'r myfyrwyr uniaith mewn pedair tasg gof ar lafar. Er mai Saesneg yw eu hiaith gyntaf, darganfuwyd bod y myfyrwyr dwyieithog yn arafach o ran adnabod rhestr o eiriau haniaethol, a hefyd o ran penderfyniadau geirfaol ar gyfer geiriau haniaethol, diriaethol a ffugeiriau (pseudowords). Casglodd Ransdell a Fischler (1987, t. 400) fod caffael ail iaith yn dylanwadu ychydig ar brosesu yn yr iaith gyntaf, hyd yn oed os nad yw'r ail iaith yn cael ei gweithredu.

Mae Ivanova a Costa (2008) fodd bynnag wedi herio canfyddiadau Ransdell a Fischler (1987) gan nodi sawl gwendid methodolegol yn y ffordd y cynhaliwyd eu hastudiaeth. O ganlyniad, daw Ivanova a Costa (2008, t. 278) i'r casgliad nad oedd y dystiolaeth yn ddigonol er mwyn casglu bod dwyieithrwydd yn effeithio'n negyddol ar adalw eitemau geirfaol yn yr iaith gyntaf, neu'r iaith gryfaf. Er mwyn profi neu wrthbrofi canfyddiadau Ransdell a Fischler (1987) felly, cynhaliodd Ivanova a Costa brawf enwi lluniau trwy gyfrwng y Sbaeneg gydag un garfan o siaradwyr uniaith Sbaeneg a dwy garfan o siaradwyr dwyieithog, un o siaradwyr Sbaeneg iaith gyntaf a Chatalaneg ail iaith ac un arall o siaradwyr Catalaneg iaith gyntaf a Sbaeneg ail iaith. Darganfu Ivanova a Costa ganfyddiadau tebyg i ganfyddiadau blaenorol gan Gollan, Montoya, Fennema-Notestine a Morris (2005), a ddarganfu fod siaradwyr dwyieithog Saesneg-Sbaeneg yn adalw eitemau geirfaol yn y Saesneg yn arafach na siaradwyr uniaith Saesneg er gwaetha'r ffaith mai Saesneg oedd iaith gyntaf neu brif iaith y ddwy garfan. Mae astudiaeth Ivanova a Costa (2008, t. 284) hefyd yn casglu bod siaradwyr dwyieithog o dan anfantais o ran adalw a chynhyrchu eitemau geirfaol, nid yn unig yn eu hail iaith, ond hefyd yn eu hiaith gyntaf, neu iaith gryfaf. Er mwyn egluro pam mae siaradwyr dwyieithog yn perfformio'n waeth na siaradwyr uniaith mewn tasgau llafar fel hyn, mae dau esboniad wedi'u cynnig. Mae Gollan ac eraill (2005) a Ransdell a Fischler (1987) wedi dadlau mai oherwydd defnydd llai o'r ddwy iaith, o'i gymharu â defnydd siaradwyr uniaith o un iaith, y mae siaradwyr dwyieithog yn perfformio'n arafach yn y tasgau hyn. Gan nad yw'r siaradwr dwyieithog yn debygol o ddefnyddio un o'i ddwy iaith mor aml â siaradwr uniaith mewn un iaith, dadleua Gollan ac eraill a Ransdell a Fischler fod yna gysylltiadau geirfaol gwannach yn y ddwy iaith ac mae hyn yn effeithio ar ddwyn geiriau i gof yn y ddwy iaith. Wrth i'r siaradwr dwyieithog ailadrodd yr un profion dro ar ôl tro fodd bynnag, dangosodd Gollan ac eraill (2005, t. 1230) fod yr effeithiau amledd 
(frequency effects) hyn yn diflannu. I'r gwrthwyneb, fodd bynnag, mae astudiaeth Ivanova a Costa $(2008$, t. 285$)$ wedi cyflwyno tystiolaeth sy'n dangos nad yw effeithiau dwyieithrwydd yn diflannu, hyd yn oed ar ôl ailadrodd y profion. Dônt i'r casgliad canlynol: "In sum, we consider that, without further support, at this stage it appears more problematic than otherwise to embrace the assumption that the bilingualism effect disappears with repetition." (Ivanova a Costa, 2008, t. 285). Nid yw'r dystiolaeth o blaid yr hyn y mae Gollan ac eraill (2005) yn ei alw yn 'gysylltiadau gwannach' yn ddigon cadarn er mwyn esbonio'r anfantais hwn ymhlith siaradwyr dwyieithog.

Esboniad arall am berfformiad arafach siaradwyr dwyieithog mewn tasgau llafar yw bod caffael dwy iaith (neu ragor) yn achosi ymyrraeth wrth adalw geiriau o'r naill iaith neu'r llall oherwydd bod y ddwy iaith yn weithredol trwy'r amser. Dyma beth y mae Gollan ac eraill (2005) yn enwi'n 'ymyrraeth drawsieithyddol' (cross-language interference). Wrth i'r siaradwr dwyieithog fonitro'r sefyllfa ieithyddol, mae dwy iaith y siaradwr dwyieithog yn cael eu gweithredu ac mae'r gystadleuaeth rhwng y ddwy iaith weithredol hyn yn gorfodi'r siaradwr dwyieithog i reoli cyrchiad i un o'r ddwy iaith hyn (Marian \& Shook, 2012, t. 3). Oherwydd bod rhaid i'r siaradwr dwyieithog feddwl yn y ddwy iaith ar yr un pryd felly, mae'n bosibl fod hyn yn esbonio pam mae tasgau megis adalw geiriau ac enwi lluniau yn arafach yn achos siaradwyr dwyieithog. Mewn gair, ymddengys fod "the simple act of retrieving a common word is more effortful for bilinguals" (Bialystok ac eraill, 2012, t. 241). Cyn cloi'r bennod hon, rhaid tynnu sylw at rai astudiaethau ymchwil diweddar o Gymru a'u canfyddiadau ar y berthynas rhwng dwyieithrwydd â sgiliau uwchwybyddol (executive function skills). ${ }^{25}$ Mae nifer o astudiaethau (gweler, er enghraifft, Clare ac eraill, 2016; Gathercole ac eraill, 2010, 2014, 2016; Hindle ac eraill 2015; Rhys, 2013) wedi dangos yn gyson nad oes mantais ddwyieithog amlwg ar amrywiaeth o brofion sy'n mesur sgiliau uwchwybyddol. At ddiben yr is-bennod hon, canolbwyntir ar drafod canfyddiadau cymysg astudiaethau Gathercole ac eraill $(2010,2014)$ yn benodol.

\footnotetext{
${ }^{25}$ Diffinia Thomas a Webb-Davies (2017, t. 121) sgiliau uwchwybyddol yn "set o sgiliau gwybyddol sydd yn rheoli ein hymateb i wahanol bethau fel bod modd i ni gyflawni'r hyn yr ydym yn ei wneud." Dadleua Bialystok (1993, 1999, 2001a) y dylai fod gan siaradwyr fantais o ran tasgau sy'n mesur y sgiliau hyn oherwydd bod siaradwyr dwyieithog yn gorfod rheoli eu hieithoedd wrth iddynt gyfnewid o'r naill iaith i'r llall, gan atal eu sylw i un iaith wrth iddynt ddefnyddio'r llall.
} 
Yn eu hastudiaeth o blant dwyieithog (Cymraeg-Saesneg) a phlant uniaith (Saesneg) 7-8 a 13-15 oed o gefndiroedd ieithyddol gwahanol (cartrefi Cymraeg eu hiaith, cartrefi cymysg a chartrefi Saesneg eu hiaith), canfu Gathercole ac eraill (2010) ganfyddiadau cymysg o ran effaith dwyieithrwydd mewn tasgau tapio a Stroop. O ran y dasg tapio, canfuant mai'r plant ifanc dwyieithog o gartrefi Cymraeg eu hiaith a chartrefi Saesneg eu hiaith sy'n perfformio orau. Wrth ddadansoddi perfformiad y plant hŷn fodd bynnag, canfuant fod y fantais hon yn newid, gyda'r plant dwyieithog o gartrefi Cymraeg eu hiaith a chartrefi cymysg yn perfformio'n well na'r plant dwyieithog o gartrefi Saesneg eu hiaith a'r plant uniaith Saesneg. O ran y prawf Stroop, ni chanfuwyd gwahaniaethau sylweddol rhwng y grwpiau o blant yn y Gymraeg, a hynny, yn ôl Gathercole ac eraill (2010, t. 661), oherwydd bod pob un o'r grwpiau hyn yn ddwyieithog ac felly nad oes gan yr un ohonynt fantais uniaith Gymraeg. Canfuwyd canlyniadau llawer mwy cymhleth rhwng y cyfranogwyr yn y Saesneg. Er bod gan blant dwyieithog cytbwys ifanc (plant dwyieithog o gartrefi cymysg) fantais yn y prawf Stroop yn Saesneg, ymddengys nad oes gwahaniaeth sylweddol rhwng plant dwyieithog ac uniaith Saesneg sy’n hŷn. Daw Gathercole ac eraill (2010, t. 661) i'r casgliad fod y canfyddiadau yn llunio darlun cymysg a chymhleth iawn nad yw'n awgrymu mantais ddwyieithog ar y cyfan: "In sum, these results show a generally better performance among some bilinguals over monolinguals, but not all bilinguals, not in all conditions, and not irrespective of contributions from socio-economic, cognitive and linguistic factors."

Caiff y canfyddiadau hyn eu hatgyfnerthu gan ddwy astudiaeth arall o Gymru gan Gathercole ac eraill (2014). Yn eu hastudiaeth o berfformiad siaradwyr dwyieithog (Cymraeg eu hiaith gryfaf; dwyieithog cytbwys; Saesneg eu hiaith gryfaf) a siaradwyr uniaith Saesneg o ystod fawr o oedrannau (3-60 oed), ni chanfu Gathercole ac eraill (2014) dystiolaeth i gefnogi'r fantais ddwyieithog i siaradwyr dwyieithog cydamserol ac olynol mewn tasgau sortio cardiau, Simon a metaieithyddiaeth. Yn hytrach, canfuwyd bod siaradwyr sydd yn gryfach mewn un iaith yn tueddu i berfformio'n well mewn rhai agweddau ar y tasgau. Er hynny, mae Gathercole ac eraill yn cwestiynu effaith cyd-destun sosio-ieithyddol y gymuned dan sylw yn yr astudiaeth, sef Gogledd Cymru, ar y canfyddiadau hyn. Maent yn tynnu sylw at ganfyddiadau tebyg astudiaeth Duñabeitia ac eraill (2013) yng Ngwlad y Basg, lle na chanfu fantais i blant dwyieithog (Basgeg-Sbaeneg) o'u cymharu â phlant uniaith 
(Sbaeneg) mewn tasgau Stroop, gan amlygu nad yw'r astudiaethau ar gymunedau dwyieithog, lle mae'r ddwy iaith yn amlwg ym mywyd bob dydd y cyfranogwyr, wedi canfod mantais ddwyieithog o safbwynt sgiliau uwchwybyddol. Dadleua'r awduron fod gan siaradwyr dwyieithog cytbwys gysylltiadau cryf sy'n hwyluso'r cyfnewid o'r naill iaith i'r llall a bod hyn yn ymofyn llai o reoli gwybyddol ar ran y siaradwr dwyieithog cytbwys. O’u cymharu, mae gan siaradwyr dwyieithog sy'n llai rhugl mewn un iaith gysylltiadau gwannach rhwng yr ieithoedd, ac felly maent yn dadlau bod siaradwyr o'r fath yn gorfod rheoli ac atal eu hieithoedd gyda mwy o ymdrech wybyddol. Awgryma hyn mai siaradwyr dwyieithog penodol, hynny yw, siaradwyr sydd yn llai rhugl mewn un o'u hieithoedd ac sydd yn defnyddio eu hieithoedd ar gyfer swyddogaethau gwahanol o fewn y gymuned iaith, sydd yn dangos y fantais wybyddol hon. Oherwydd hynny, dadleua Gathercole ac eraill (2014) fod angen ymchwil bellach i astudio'r berthynas rhwng ffactorau sosioieithyddol â sgiliau gwybyddol siaradwyr dwyieithog. 


\section{Pennod 4: Y gymhariaeth ryngwladol: Tystiolaeth o Ganada, Gwlad y Basg a'r}

\section{Almaen}

\subsection{Trosolwg o addysg drochi a CLIL ar lwyfan rhyngwladol}

Oherwydd y gronfa gymharol fach o ymchwil ar addysg ddwyieithog a CAI sydd gennym yng Nghymru, edrychwyd ar wledydd eraill y byd sydd wedi cynnal ymchwil sylweddol yn y maes ar gyfer datblygu arfer da yng Nghymru. Fel y cyfeddyf Williams (2002, t. 5): "Nid oes gennym ein swmp ymchwil ni'n hunain am sefyllfa ddwyieithog Cymru eto, felly rhaid troi at ymchwilwyr gwledydd eraill." Mae ymchwil ryngwladol wedi bod yn ddylanwadol wrth ffurfio ymdrechion yng Nghymru i gyflwyno'r Gymraeg fel ail iaith yn llwyddiannus. Roedd hyn yn wir yng nghyd-destun Cynllun Trochi a Dysgu Dwys Bwrdd yr Iaith Gymraeg (2003-2008), a gafodd ei gomisiynu yn dilyn ymweliad Jane Davidson AC â Chanada er mwyn arsylwi'r arfer o ran cyflwyno'r Ffrangeg fel ail iaith i siaradwyr Saesneg eu hiaith. Roedd hyn hefyd yn wir yng nghyd-destun gwaith ymchwil C. Williams (2002), a dynnodd ar waith ymchwilwyr rhyngwladol.

Mae cyflwyno cwricwlwm addysg trwy gyfrwng yr ail iaith wedi bod yn ddull canolog mewn addysg ddwyieithog ers 1965 yn arbennig, ar ôl i arbrawf dylanwadol yn St. Lambert, Canada, gael ei gynnal gan Lambert a Tucker (1972). Erbyn hyn, mae dros 1,000 o astudiaethau wedi'u cynnal ar addysg drochi yn y Ffrangeg yng Nghanada (Baker, 2001, t. 208). Ers ei gweithredu yng Nghanada yn y 1960au, mae addysg drochi wedi lledaenu i nifer o wledydd eraill gan gynnwys Awstralia, Hwngari, Hong Kong, Singapore, Iwerddon, yr Alban, Seland Newydd, De Affrica, y Ffindir, a Chymru. Yn ogystal ag addysg drochi, mae dau brif gategori arall o addysg ddwyieithog y dylid eu crybwyll (Būdvytytě-Gudieně \& Toleikieně, 2008; Scott \& Beadle, 2014). Yr ail gategori o addysg ddwyieithog yw addysg foddi a ddatblygodd yn ddull poblogaidd yn ystod y 1960au a'r 1970au yn yr Unol Daleithiau i gyflwyno Saesneg yn fwy effeithiol i ddisgyblion nad oedd y Saesneg yn iaith gyntaf iddynt er mwyn iddynt ymuno ag addysg brif ffrwd. Y trydydd datblygiad ym maes cyfoes addysg ddwyieithog yw CLIL. Bathwyd y term CLIL yn ystod y 1990au a daeth CLIL yn boblogaidd wrth i'r Comisiwn Ewropeaidd ddechrau ariannu nifer o brosiectau CLIL ar draws Ewrop mewn ymateb i'r galw cynyddol am well addysg ail iaith / addysg iaith dramor fodern (Cenoz, Genesee, \& Gorter, 2014). Mae'n hollbwysig nodi bod nifer fawr o is-gategorïau ym maes 
addysg ddwyieithog. Er enghraifft, mae rhaglenni addysg ddwy-ffordd (two-way / dual-language programmes) (Baker, 2011; Baker \& Jones, 1998; García, 2009; Johnstone, 2002) ac addysg cynnal iaith (García, 2009), neu addysg iaith dreftadaeth fel y’i gelwir weithiau (Baker, 2011; Baker \& Jones, 1998), yn America wedi'u gweithredu yn fwy diweddar er mwyn cynnal iaith dreftadaeth mewnfudwyr, yn ogystal â throchi yn y Saesneg, tra hefyd yn trochi siaradwyr Saesneg eu hiaith yn yr iaith dramor a chynnal eu hiaith gyntaf, sef Saesneg. Ceir y fath raglen addysg ddwyieithog, er enghraifft, yn ysgol elfennol-ganol Wharton Dual Language Academy yn Huston, Tecsas. Yn ôl ei datganiad cenhadaeth: "Wharton Dual Language Academy's mission is to empower our students to become respectful, lifelong, bilingual and bi-literate inquirers who celebrate diversity as responsible citizens of the world." (Wharton Dual Language Academy, d.d.). Dyma un o'r rhaglenni yn yr Unol Daleithiau y gellir eu labelu'n wir addysg ddwyieithog, gan eu bod yn anelu at ddwyieithrwydd cynyddol, lle mae'r rhaglenni addysg foddi yn anelu at unieithrwydd yn y Saesneg (Būdvytytě-Gudieně \& Toleikieně, 2008, t. 92).

Yn yr adran hon, cymherir cyd-destunau addysg drochi a CLIL mewn tair cymuned wahanol sy'n rhannu nodweddion ag addysg drochi yng Nghymru. Oherwydd dylanwad addysg drochi yng Nghanada ar ffurfio rhaglenni addysg drochi a CLIL yn rhyngwladol, a'r ymchwil swmpus sydd bellach ar gael yn ei chylch, trafodir yn gyntaf y sefyllfa drochi honno. Troir wedyn i'r cyd-destun Ewropeaidd, wrth ganolbwyntio ar y sefyllfa addysg ddwyieithog yng Nghymuned Ymreolaethus Gwlad y Basg (CYGB o hyn ymlaen), gan mai dyma'r sefyllfa ieithyddol debycaf i Gymru (Gardner, 2002, "1.5. Conclusion", para. 1). Astudir hefyd y sefyllfa CLIL sydd wedi bod yn cael ei datblygu yn yr Almaen ers y 1960au, gan fod y model CLIL yn yr Almaen yn rhannu nifer o nodweddion â'r model carlam a gyflwynir yn Ysgol X (gweler Pennod 5).

\subsection{Sefyllfa'r Ffrangeg yn y gyfundrefn addysg yng Nghanada}

\subsubsection{Cyd-destun}

Er gwaethaf maint tiriogaeth Canada, nid yw ei phoblogaeth yn fawr. Mae gan Ganada boblogaeth o 35.9 miliwn (Statistics Canada, 2015). Yng Nghyfrifiad Canada 2011, nodwyd bod rhyw 5.8 miliwn o bobl, neu 17.5\% o'r boblogaeth, yn honni eu bod yn gallu cynnal sgwrs naill ai'n Saesneg neu'n Ffrangeg (Lepage \& 
Corbeil, 2013). O ran y nifer sy'n cofrestru ar raglenni addysg drochi, nodwyd bod dros 341,000 o blant mewn rhaglenni trochi erbyn 2010/2011. Dyma gynnydd sylweddol os cymherir y ffigur hwn â ffigyrau 1991/1992 a 1976/1977, lle nodwyd bod tua 267,000 a thua 23,000, yn eu tro, o ddisgyblion ar gofrestr rhaglenni addysg drochi (Lepage \& Corbeil, 2013, "Enrolment increasing in French immersion programs, but decreasing in regular FSL programs"). Er hynny, mae ysgolion trochi yng Nghanada yn fach eu nifer, gan gynnwys ond rhyw 6.8\% allan o'r 5 miliwn o ddisgyblion a gofrestrodd mewn ysgolion cynradd ac uwchradd yn 2010/2011 (Statistics Canada, 2013).

Yn debyg i Gymru felly, lleiafrif bach yw'r rhai sy'n medru'r ddwy iaith yn y wlad hon, ac er y gwelwyd cynnydd sylweddol yn nifer yr ysgolion trochi, bach iawn yw'r nifer o ysgolion yng Nghanada a Chymru sy'n cynnig addysg drochi yn yr ail iaith. Yng Nghanada, dechreuodd addysg drochi o ganlyniad i waith grŵp o rieni uniaith Saesneg dosbarth canol o St. Lambert, Montreal, yn ystod y 1960au a oedd am weld addysg Ffrangeg ail iaith fwy effeithiol i'w plant, gan nad oeddent yn fodlon ar y dulliau addysgu traddodiadol, megis drilio ac ailadrodd (Roy, 2008), a gyflwynir mewn rhaglenni Ffrangeg ail iaith traddodiadol mewn addysg cyfrwng Saesneg. Yn debyg i CAI yng Nghymru, mae'r rhaglen draddodiadol, sef 'Ffrangeg Craidd', yn cyflwyno'r Ffrangeg fel pwnc yn unig, gyda phwyslais ar ddysgu gramadeg. Er mai dyma'r rhaglen fwyaf tebyg i'r rhaglen CAI yng Nghymru (Davidson, 2001), rhaid nodi y cyflwynir y Ffrangeg yn y rhaglen hon am gyfnodau o 30-40 munud bob dydd (Canadian Council on Learning, 2007) gyda darpariaeth ar gael fel arfer o radd $4 \mathrm{i}$ radd 12 .

O'i chymaru â CAI, lle y mae'r oriau dysgu yn is o lawer ac yn amrywio'n fawr, dyma nifer uwch o lawer o oriau cyswllt. Fodd bynnag, er bod disgyblion wedi dysgu Ffrangeg, a hynny yn y ffordd draddodiadol, am hyd at 30 munud bob dydd, ni theimlai nifer o rieni fod y rhaglen draddodiadol hon yn ddigonol gan na fedrai disgyblion siarad yr iaith hyd yn oed ar ôl blynyddoedd o'i dysgu (Rebuffot \& Lyster, 1996, fel y dyfynnwyd yn Roy, 2008). O ganlyniad i waith y grŵp rhieni yn St. Lambert, cynhaliwyd y rhaglen addysg drochi gynnar gyntaf o dan arweiniad Wallace Lambert ym Mhrifysgol McGill yn 1965. Yn y rhaglen beilot hon, cafodd disgyblion eu trochi yn y Ffrangeg dros bedair blynedd, o'r feithrinfa hyd at radd 4 
yn yr ysgol elfennol (Lambert \& Tucker, 1972). Casglodd Tucker a d'Anglejan (1972) fod yr arbrawf wedi bod yn llwyddiannus o ran eu nodau:

...the experimental students appear to be able to read, write, speak, understand, and use English as well as youngsters instructed in English in the conventional manner. In addition and at no cost they can also read, write, speak and understand French in a way that English students who follow a traditional program of French as a second language never do. (Tucker \& d'Anglejan, 1972, t. 19)

Yn ogystal â'r canfyddiad bod disgyblion addysg drochi yn perfformio'n well na'u cyfoedion ar raglenni traddodiadol o ran Ffrangeg, a hynny heb effaith negyddol ar ddatblygiad eu hiaith gyntaf, darganfu Lambert a Tucker (1972) eu bod nhw hefyd yn perfformio'n well o ran eu sgiliau mathemateg a'u bod yn dangos agweddau cymdeithasol gwell at siaradwyr Ffrangeg iaith gyntaf o'u cymharu â'u cyfoedion. Datblygodd addysg drochi ar draws Canada, nid yn unig oherwydd anfodlonrwydd rhieni gyda'r rhaglenni Ffrangeg Craidd (Canadian Council on Learning, 2007) a'r llwyddiant a nodwyd yn y rhaglen beilot yn St. Lambert (Johnson \& Swain, 1997), ond hefyd oherwydd bod pobl Canada wedi dechrau sylweddoli gwerth cymdeithasol, gwleidyddol a gwerth economaidd siarad y Ffrangeg yng Nghanada (Johnson \& Swain, 1997). Gyda'r newidiadau hyn yn y cyd-destun cymdeithasol felly, mae'n debyg i addysg drochi ledaenu ar draws Canada mor gyflym ag y gwnaeth oherwydd rhoddai cyfle i blant ennill dwyieithrwydd cynyddol mewn dwy iaith fwyafrifol ac iddynt fri a statws mawr (Baker, 2001, t. 205).

Mae cyd-destun addysg ddwyieithog felly yn dra gwahanol i'r cyd-destun yng Nghymru. Yng Nghymru, nodir sefyllfa lle mae'r Gymraeg, yr iaith leiafrifol, yn bodoli ochr yn ochr â'r Saesneg, iaith fwyafrifol, nid yn unig yng Nghymru ond hefyd ym Mhrydain gyfan. Cynyddu'r nifer sy'n medru'r iaith leiafrifol megis ail iaith yw bwriad addysg drochi yng Nghymru. Yng Nghanada fodd bynnag, nodir sefyllfa lle mae dwy iaith fawr, ryngwladol yn bodoli ochr yn ochr â'i gilydd, ac felly nid cynnal a gwarchod iaith leiafrifol mo bwriad yr addysg drochi yma. Yn ardaloedd Ffrangeg eu hiaith megis Quebec, er mai'r Ffrangeg yw'r iaith fwyafrifol o ran nifer o siaradwyr, nid yw'r Saesneg dan fygythiad oherwydd ei phresenoldeb ar hyd a lled Canada ac yn rhyngwladol megis lingua franca. Bwriad gwreiddiol y rhaglen addysg drochi gyntaf yng Nghanada oedd grymuso'r lleiafrif uniaith Saesneg yn Quebec, ardal Ffrangeg ei hiaith, er mwyn iddynt elwa ar gyfleoedd a fyddai ar 
gael i siaradwyr Ffrangeg yn unig fel arall. Fel y gwelir yn achos addysgu Saesneg yn yr Almaen (gweler 4.4. Sefyllfa'r Saesneg mewn addysg ddwyieithog yn yr Almaen) prif werth dwyieithrwydd i'r siaradwyr Saesneg eu hiaith gyntaf yng Nghanada yw'r cyfleoedd gyrfaol posibl a all ddod yn sgil medru'r Ffrangeg.

\subsubsection{Canfyddiadau addysg drochi yng Nghanada}

Fel y nodwyd eisoes, erbyn hyn y mae dros 1,000 o astudiaethau wedi'u cynnal ar addysg drochi yng Nghanada. Ar sail y gronfa fawr o astudiaethau yma yn bennaf, cafodd sawl adolygiad ei gynnal yn ystod y 1980au (Genesee, 1983, 1984; Swain \& Lapkin, 1982) er mwyn dod i gasgliad ynglŷn ag effeithiolrwydd y rhaglenni addysg drochi. Awgrymodd yr adolygiadau hyn fod y rhaglen arbrofol wedi cyrraedd ei phrif nodau (Baker, 2011, t. 240), sef cynhyrchu disgyblion i) sydd â hyfedredd o ran siarad, darllen ac ysgrifennu Ffrangeg, ii) sy'n perfformio'n ddigonol yn y cwricwlwm gan gynnwys y Saesneg, iii) ac sy'n gwerthfawrogi diwylliant a thraddodiadau siaradwyr Ffrangeg iaith gyntaf a siaradwyr Saesneg iaith gyntaf (Baker, 2011, t. 239). Mae adolygiadau diweddarach yn ystod y 1990au hefyd wedi cadarnhau'r canfyddiadau hyn (Swain \& Johnson, 1997; Swain, 1997; Jones, 1997; Lauren, 1997; Arnau, 1997; Genesee \& Gándara, 1999). Ar sail yr adolygiadau hyn, gellir rhannu canlyniadau addysg drochi yng Nghanada i wahanol gategorïau, yn nhermau'r effaith ar ddysgu ail iaith, dysgu iaith gyntaf, pynciau cwricwlaidd eraill ac agweddau addysgol a chymdeithasol (Baker, 2011, tt. 265-268; Baker \& Jones, 1998, tt. 497-500).

\subsubsection{Yr ail iaith}

Un o'r agweddau sydd wedi'i hymchwilio fwyaf ym maes addysg drochi yw effaith ieithyddol addysg drochi ar ddatblygu'r ail iaith. Mae Swain a Johnson (1997) wedi cadarnhau fod hyfedredd yn yr ail iaith wedi'i brofi'n uwch ymhlith disgyblion rhaglenni trochi, os cymherir y disgyblion hyn â disgyblion sy'n dysgu'r ail iaith fel pwnc yn y ffordd draddodiadol. Cadarnheir hyn gan ganfyddiadau'r Carleton Board of Education (1996) yn Ottawa, wrth i'r ymchwil gasglu bod disgyblion rhaglenni trochi cynnar yn perfformio'n well yn gyson na disgyblion rhaglenni trochi canolig, a bod disgyblion rhaglenni trochi canolig yn perfformio'n well yn gyson na disgyblion rhaglenni trochi hwyr. Yn ogystal â hyn, casglwyd bod y disgyblion addysg drochi i gyd yn perfformio gryn dipyn yn well na'u cyfoedion a oedd yn dilyn Cwrs Ffrangeg 
Craidd (Carleton Board of Education, 1996; Johnstone, 2002). Er bod sgiliau goddefol, sef darllen a gwrando, disgyblion addysg drochi gynnar yn cyrraedd lefelau uchel erbyn 11 oed (Baker, 2011), nododd Swain a Johnson (1997) nad yw'r sgiliau gweithredol, sef siarad ac ysgrifennu, yn cyrraedd lefelau siaradwyr iaith gyntaf cymharol. Mae astudiaethau gan rai ysgolheigion hefyd yn awgrymu bod disgyblion rhaglenni addysg drochi Ffrangeg yn amlygu cyfyngiadau o ran eu gramadeg, yn arbennig o ran system ferfau'r Ffrangeg (Harley \& Swain, 1978), yn ogystal ag ynganiad (Hammerly, 1991). Mae'n bosibl mai diffyg cyswllt â'r iaith darged y tu hwnt i'r dosbarth yw un o'r rhesymau am hyn. Cred Baker (2011) fod angen cyswllt â'r iaith darged mewn cyd-destunau cymdeithasol y tu allan i'r ysgol ar ddisgyblion addysg drochi, os ydynt i ddefnyddio'r hyn y maent wedi ei ddysgu yn y rhaglen drochi. Pan gyflwynir rhaglenni addysg drochi mewn ardaloedd lle mai'r iaith gyntaf yw iaith fwyafrifol y gymuned, mae'n bosibl na chaiff disgyblion ail iaith yr un cyfleoedd â disgyblion iaith gyntaf i ddefnyddio ac ymarfer eu sgiliau cynhyrchiol yn yr ail iaith y tu hwnt i gyd-destun y dosbarth. Dyma bryder sy'n cael ei rannu yng nghyd-destun CAI, lle gellir dadlau nad oes digon o gyfleoedd cymdeithasol i ddisgyblion ail iaith ddefnyddio'r Gymraeg a ddysgir mewn ffordd bwrpasol (gweler, er enghraifft, Davies, 2013; Estyn, 2014b, 2015). Nid yw'n bosibl i addysg drochi sicrhau defnydd go iawn o'r ail iaith y tu hwnt i'r dosbarth ar ei phen ei hun. Caiff hyn ei gadarnhau gan astudiaeth gan Wesche (1993). Fel rhan o'r astudiaeth, gofynnodd Wesche i fyfyrwyr, a raddiodd o'r ysgol uwchradd ar ôl dilyn rhaglen addysg drochi, pa mor aml defnyddient y Ffrangeg. Darganfu Wesche fod y graddedigion yn defnyddio'r Ffrangeg yn anaml iawn, gyda 80\% o'r cyfranogwyr yn nodi nad oeddent yn defnyddio'r iaith yn eu bywydau ${ }^{26}$.

Wrth edrych ar addysg drochi Canada o safbwynt y dosbarth felly, gellir dadlau iddi fod yn llwyddiant, ond o safbwynt ennill hyfedredd yn yr ail iaith, hyfedredd cyfuwch a chystal â hyfedredd y siaradwr iaith gyntaf, mae'n bosibl dadlau nad yw addysg drochi yn llwyddiannus (Swain \& Johnson, 1997, t. 11). O'r safbwynt hwn, caiff siaradwyr ail iaith eu cymharu â siaradwyr iaith gyntaf o ran eu safon yn eu hail iaith. Enghraifft o hyn yw Hammerly (1991, t. 84), sy'n nodi mai'r “typical educated native speaker" yw'r “only valid standard” ar gyfer mesur a chymaru gwallau'r

\footnotetext{
${ }^{26}$ Cynigodd 80\% o'r cyfranogwyr yr ateb '2' ar gwestiwn sy'n cyflwyno graddfa 1-5 (gydag 1 yn golygu 'byth' a chyda 5 yn golygu 'trwy'r amser') (Wesche, 1993, t. 226).
} 
dysgwr ail iaith. Rhaid bod yn ofalus wrth gymharu dysgwyr ail iaith â siaradwyr iaith gyntaf o ran hyfedredd yn yr ail iaith fodd bynnag, yn arbennig wrth gymharu rhyngiaith (interlanguage) ${ }^{27}$ y dysgwr ail iaith ag iaith y siaradwr iaith gyntaf. Nid yw'r ddau fath hyn o iaith yn gymharus, mae'n debyg, oherwydd eu bod yn ddigon gwahanol i'w gilydd a bod gan ryngiaith y dysgwr ail iaith reolau systematig ei hun (Loewen \& Reinders, 2011, "Comparative fallacy", t. 33). Ar sail y fath ddamcaniaeth felly, sef ' $y$ camsyniad cymharu' (the comparative fallacy), ${ }^{28}$ nid yw ail iaith y dysgwr ag iaith y siaradwr iaith gyntaf yn gymharus oherwydd eu nodweddion gwahanol. Gwell felly yw cymharu disgyblion sydd heb ddatblygu hyfedredd yn yr ail iaith eto gyda'i gilydd a chyda dysgwyr tebyg, yn hytrach na'u cymharu â safonau afrealistig y siaradwr iaith gyntaf yn yr iaith honno.

\subsubsection{Iaith gyntaf}

O ran cyrhaeddiad yn yr iaith gyntaf, mae ymchwil yng Nghanada wedi awgrymu'n gyson nad yw'r ail iaith yn cael ei datblygu ar draul yr iaith gyntaf mewn rhaglenni addysg drochi (Johnstone, 2002). Er nad yw iaith gyntaf disgyblion sy'n dilyn rhaglen addysg drochi lawn gynnar yn datblygu mor gyson a chyflym ag iaith gyntaf disgyblion sy'n dilyn rhaglen addysg cyfrwng uniaith yn y pedair blynedd gyntaf, fel yr esbonia Baker a Jones (1998), nid yw hyn yn para:

After approximately six years of schooling, early total Immersion children have caught up with their monolingual peers in English language skills. By the end of elementary schooling, the early total Immersion experience has generally not affected first language speaking and writing development. (Baker \& Jones, 1998, t. 498).

Mae ymchwil yng Nghanada hefyd wedi cadarnhau nad oes effeithiau negyddol i'w cael ar yr iaith gyntaf ymhlith disgyblion ar y rhaglenni trochi rhannol cynnar na'r rhaglenni trochi hwyr (Genesee, 1983). I'r gwrthwyneb, mae tystiolaeth wedi dangos bod disgyblion rhaglenni trochi cynnar yn gallu perfformio'n well na disgyblion y brif ffrwd o ran cyrhaeddiad academaidd yn y Saesneg (Carleton Board of Education, 1996; Johnstone, 2002). Serch hynny, mae disgyblion rhaglenni trochi canolig a

\footnotetext{
${ }^{27}$ Term a fathwyd gan Selinker (1972) yw 'rhyngiaith'. Dyma'r system iaith ryngol sy'n cael ei chreu gan siaradwyr ail iaith wrth iddynt ddatblygu eu gwybodaeth yn yr ail iaith (Loewen \& Reinders, 2011 , t. 98). Gellir gweld rhyngiaith fel petai'n gam yn nysgu ail iaith sy'n digwydd 'hanner ffordd' rhwng unieithrwydd a hyfedredd mewn ail iaith (Baker, 2011, t. 302).

${ }^{28}$ Ymadrodd a fathwyd gan Bley-Vroman (1983) yw'r 'camsyniad cymharu'.
} 
hwyr yn tueddu i berfformio cystal (Carleton Board of Education, 1996; Johnstone, 2002).

\subsubsection{Pynciau cwricwlaidd eraill}

Mae ymchwil Canada hefyd wedi awgrymu nifer o ganfyddiadau o ran effaith addysg drochi ar gyrhaeddiad mewn pynciau cwricwlaidd eraill. Yn achos addysg drochi lawn gynnar, amlygodd ymchwil fod disgyblion yn perfformio cystal â'u cyfoedion mewn addysg brif ffrwd (Baker, 2011; Carleton Board of Education, 1996; Johnstone, 2002). Canfu Day a Shapson (1996), er enghraifft, na chaiff addysg drochi effaith negyddol ar ddysgu cynnwys, yn hytrach mae'n debyg bod disgyblion addysg drochi yn gallu perfformio'n well na'u cyfoedion o ran dysgu cynnwys, hyd yn oed pan gânt eu profi yn eu hiaith gyntaf. Fodd bynnag, mae'n bosibl fod disgyblion rhaglenni trochi hwyr (Baker, 2011; Johnstone, 2002) a disgyblion rhaglenni trochi cynnar rhannol (Baker, 2011) yn fwy araf na'u cyfoedion o ran eu datblygiad academaidd yn y pynciau sy'n fwy heriol o ran eu hieithwedd, megis Mathemateg, Gwyddoniaeth, Astudiaethau Cymdeithasol, Hanes a Daearyddiaeth. Mae Williams $(2002,2008)$ wedi nodi ei bryderon o ran cyflwyno pynciau sy'n heriol yn ieithyddol trwy gyfrwng ail iaith y disgyblion oherwydd nad yw eu sgiliau cyfathrebol wedi'u datblygu'n ddigonol er mwyn ymdopi â gofynion ieithyddol y pynciau hyn. Cynnig Williams $(2002,2008)$ felly bynciau ymarferol megis Celf, Addysg Gorfforol a Thechnoleg, gan mai dyma'r pynciau lleiaf heriol o ran eu hieithwedd.

\subsubsection{Agweddau cymdeithasol}

Yn ogystal ag effaith ar gyrhaeddiad academaidd, mae ymchwil yng Nghanada wedi awgrymu bod disgyblion ail iaith ar raglenni drochi, yn arbennig addysg drochi lawn gynnar (Baker, 2011), yn datblygu agweddau mwy cadarnhaol a goddefol at yr ail iaith, siaradwyr Ffrangeg iaith gyntaf a'u diwylliant. Credir bod disgyblion rhaglenni trochi yn cydymdeimlo'n fwy â siaradwyr iaith gyntaf yr iaith leiafrifol trwy eu cyswllt hwy â'r iaith. Mewn erthygl sy'n arolygu canfyddiadau ar addysg drochi yng Nghanada a'i heffaith gymdeithasol, daw Genesee a Gándara (1999) i’r casgliad canlynol:

Apparently participation in immersion resulted in the Immersion students perceiving less social distance between themselves and French Canadians, 
perhaps because, as bilinguals, they shared a salient characteristic of French Canadians or perhaps because of their increased experiences and familiarity with French Canadian culture in the immersion program. At the same time, immersion did not appear to have had any adverse effects on the Immersion students' identification with English Canadians.

(Genesee \& Gándara, 1999, t. 672).

Rhaid pwysleisio fodd bynnag fod cyd-destun a diben yr addysg ddwyieithog a ddarperir yn medru effeithio ar ei gallu i feithrin agweddau iach ymhlith disgyblion. Nododd Swain a Johnson (1997, tt. 10-11) nad nod addysg drochi bob tro yw mabwysiadu agweddau cadarnhaol at siaradwyr iaith gyntaf yr iaith darged, gan y gall siaradwyr ddysgu'r iaith darged er budd manteision cymdeithasol, academaidd ac economaidd. Cynnig Swain a Johnson (1997, t. 11) drochi yn y Saesneg yn Hong Kong fel enghraifft o hyn, gan nad prif ddiben addysgu Saesneg yn y cyd-destun hwnnw yw mabwysiadu agweddau cadarnhaol at siaradwyr iaith gyntaf Saesneg, yn hytrach, dysgir Saesneg yn y cyd-destun hwn yn bennaf er mwyn elwa ar y manteision cymdeithasol, academaidd ac economaidd a ddaw yn sgil cyfathrebu yn yr iaith honno.

\subsection{Sefyllfa'r Fasgeg yng Ngwlad y Basg}

\subsubsection{Cymhariaeth o'r cyd-destunau}

Er bod sefyllfa addysg ddwyieithog Gwlad y Basg yn unigryw yn ei ffordd ei hun, nodir ei bod yn rhannu nifer o nodweddion tebyg â sefyllfa addysg ddwyieithog yng Nghymru. Ar lefel sosio-ieithyddol, mae tebygrwydd amlwg rhwng Cymru a Gwlad y Basg ${ }^{29}$, o ran canran y boblogaeth gyfan, nifer y boblogaeth sy'n ddwyieithog, a bywiogrwydd ethno-ieithyddol (Gardner, 2008, t. 40). Mae gan Gymru boblogaeth o ryw 3.1 miliwn (Y Swyddfa Ystadegau Gwladol, 2012c, "Ffigurau allweddol", pwynt 1). Mae gan Wlad y Basg boblogaeth debyg iawn, gyda rhyw 2.2 miliwn o bobl yn CYGB (Eustat Municipal Inhabitant Statistics, 2014), tua 637,000 o bobl yn Navarre (Instituto Nacional de Estadística, 2015) a thua 290,000 o bobl yn byw yn y taleithiau sy'n cynrychioli gogledd Gwlad y Basg, neu Iparralde (Basque Cultural Institute, d.d.).

\footnotetext{
${ }^{29}$ Cyfeirir yma at y ddwy adran weinyddol rhanbarth yn Sbaen, sef Cymuned Ymreolaethus Gwlad y Basgiad a'r Navarre, yn hytrach na'r rhanbarth yn Ffrainc.
} 
O ran nifer y siaradwyr dwyieithog yn y gwledydd hyn, mae rhyw $27 \%$ o'r boblogaeth gyfan dros 16 oed yng Ngwlad y Basg yn honni eu bod yn ddwyieithog ${ }^{30}$ yn y Fasgeg ac un iaith arall (Ffrangeg neu Sbaeneg) (Llywodraeth Gwlad y Basg, 2013a, t. 15). Fodd bynnag, mae'n debyg fod gwir nifer y siaradwyr dwyieithog gryn dipyn yn uwch na hynny, gan mai plant a phobl ifanc yw'r garfan fwyaf dwys o siaradwyr dwyieithog oherwydd cyfraniad addysg (Llywodraeth Gwlad y Basg, 2013a, t. 11). Yng Nghymru, mae rhyw 15\% o'r boblogaeth dros 3 oed yn gallu siarad, darllen ac ysgrifennu yn Gymraeg (Swyddfa Ystadegau Gwladol, 2012c, t. 2). O ran y sefyllfa ieithyddol felly, mae'n debyg mai Gwlad y Basg yw'r fwyaf tebyg i Gymru. Fel y noda Gardner (2002, "1.5. Conclusion", para. 1): “As far as their language situation is concerned, they are more akin to the Welsh than, say, the Irish or the Scots or, looking further afield, Maori or American Native Indians in their respective territories.” Mae Swain a Johnson (1997, tt. 4-5) yn labelu sefyllfa addysg drochi Gwlad y Basg yn addysg drochi ar gyfer cyd-destun cynnal iaith, ac maent hefyd yn cyfeirio at bresenoldeb sefyllfa debyg yng Nghymru. Yn ôl Swain a Johnson (1997, t. 131), gellir gweld rhaglenni addysg drochi mewn gwledydd megis Gwlad y Basg yn ddull grymus ar gyfer cynnal ieithoedd lleiafrifol eu cymunedau a'u diwylliannau cysylltiedig er mwyn gwneud iawn am yr ormes a ddioddefodd yr ieithoedd hyn a'u diwylliannau cysylltiedig. Mae Lasagabaster (2001, t. 407) yntau'n nodi pwysigrwydd addysg yn y broses o wrthdroi shifft ieithyddol wrth iddo nodi: "As is the case in most Western countries with a minority language, education has been the main force when attempting to help the Basque language to survive." Gan rannu'r un teimlad â Lasagabaster (2001), dywed Gardner (2002, "4. Education (I): The Early Days", para. 1): “As in most western European language minorities, in the Basque Country too education has been both the motor for RLS [Reverse Language Shift] and the area where there has been greatest change." Gellid dadlau bod hyn hefyd yn wir yng nghyd-destun Cymru, lle mae addysg wedi chwarae rôl bwysig yn nhermau gwrthdroi'r shifft ieithyddol. Fel y noda Hopkins (2006), er enghraifft, mae ysgolion cyfrwng Cymraeg wedi chwarae rôl hollbwysig yn y frwydr i achub yr iaith.

\footnotetext{
${ }^{30} \mathrm{Ni}$ chyfeirir yma at siaradwyr sydd yn ddwyieithog goddefol. Mae rhyw 14.7\% o'r boblogaeth gyfan yn honni eu bod yn ddwyieithog goddefol (Llywodraeth Gwlad y Basg, 2013a, t. 15).
} 
Dylid nodi nad bwriad gwreiddiol y rhaglenni yng Ngwlad y Basg oedd trochi siaradwyr ail iaith. Yn wir, bwriad gwreiddiol y model cyfrwng Basgeg, neu Fodel D, yng Ngwlad y Basg, oedd cynnal siaradwyr treftadaeth y Fasgeg (Cenoz, 1998; Lyster, 2007). Erbyn hyn, gellir ystyried y fath raglenni "as both total immersion programs for native Spanish-speaking students and first language maintenance programs for native Basque speakers" (Cenoz, 1998, t. 177). Mae sefyllfa yn bodoli erbyn hyn yng Ngwlad y Basg lle mae cynnal y Fasgeg megis iaith dreftadaeth y siaradwyr iaith gyntaf yn digwydd ochr yn ochr â chynnal yr ieithoedd hyn fel ail iaith y siaradwyr Sbaeneg (neu iaith arall) eu hiaith gyntaf. Mae hyn hefyd yn wir yng nghyd-destun Cymru. Mae addysg ddwyieithog ac addysg cyfrwng Cymraeg ar hyn o bryd yn wynebu heriau o ran cyflwyno'r Gymraeg megis iaith dreftadaeth ac ail iaith hyd yn oed yn yr un gwersi mewn nifer o achosion (Lewis, 2008). (Am drafodaeth lawnach, gweler 2.5.1. Addysg drochi.)

\subsubsection{Sefyllfa'r Fasgeg yn y gyfundrefn addysg yng Ngwlad y Basg}

\subsubsection{Sefyllfa addysg ddwyieithog Gwlad y Basg}

Er nad oes cynifer o astudiaethau wedi'u cynnal ar addysg drochi yn y Fasgeg ag astudiaethau ar addysg drochi yng Nghanada, nodwyd yn 2001 gan Lasagabaster (2001) fod tua 50 astudiaeth wedi'u cynnal arni yn CYGB. Gan fod llawer o'r astudiaethau ar Fasgeg wedi'u cynnal yn y rhanbarth hwn, canolbwyntir yma ar ymchwil ar y gyfundrefn addysg yn CYGB sef yr unig ranbarth gweinyddol yng

Ngwlad y Basg lle mae Basgeg yn gyd-swyddogol â'r Sbaeneg trwy'r ardal gyfan. ${ }^{31}$ Dylid nodi bod y Fasgeg yn cael ei siarad a'i chyflwyno drwy addysg i raddau gwahanol yn y ddau ranbarth arall yn y diriogaeth gyfan a elwir yn 'Wlad y Basg', sef Navarre ac Iparralde. Mae erbyn hyn gronfa gynyddol o ymchwil ar y sefyllfa addysg drochi yn y cyd-destun hwn. Ers 1983, mae gan y tri model presennol yn CYGB gefnogaeth gyfreithiol, er y cyflwynwyd y modelau hyn rai blynyddoedd cyn dod yn gyfreithiol (Gardner, 2002, "4.4.2.1. Bilingual teaching models", para. 3). Mae'r modelau, sef Model A, Model B a Model D, yn amrywio o ran cyfrwng iaith yr addysgu, ac mae'n bosibl cymharu'r tri model hyn â'r tri phrif fodel a geir yng

\footnotetext{
${ }^{31}$ Mae'r Fasgeg yn gyd-swyddogol â'r Sbaeneg mewn rhai mannau o Navarre yn unig (Gardner, 2005, t. 9) ac nid yw'r Fasgeg yn iaith swyddogol o gwbl yn nhirogaeth Iparralde, gan nad oes gan ieithoedd rhanbarthol statws neu gydnabyddiaeth yn Ffrainc; dim ond y Ffrangeg a gydnabyddir (Stuijt \& Sanchez, 2007, t. 6).
} 
Nghymru. Addysg cyfrwng Sbaeneg gyda'r Fasgeg fel pwnc yw Model A. Anelir yn y model hwn at sicrhau bod gan ddisgyblion ddealltwriaeth dda o'r Fasgeg, paratoi disgyblion ar gyfer esbonio materion bob dydd yn y Fasgeg, cryfhau agweddau cadarnhaol tuag at y Fasgeg, a pharatoi disgyblion ar gyfer cymryd rhan mewn amgylcheddau Basgeg (Gardner, 2002, "Table 4: Definitions of Bilingual Teaching Models"). Gellir cymharu'r model hwn ag addysg cyfrwng Saesneg yng Nghymru lle cyflwynir y Gymraeg fel ail iaith yn bwnc yn unig hefyd. Er na chafodd ei weithredu, roedd cynllun gwreiddiol y model hwn am gynnwys rhai pynciau cyfrwng Basgeg ar ddiwedd blynyddoedd olaf addysg uwchradd orfodol. Pe bai'r model wedi dilyn y cynllun hwn, byddai gan y gyfundrefn addysg yng Ngwlad y Basg fodel addysg drochi rannol hwyr tebyg i'r un a gynigir yng Nghanada (Lasagabaster, 2007) a thebyg i'r model carlam a gynigir gan Ysgol X yng Nghymru (gweler 5.4. Cynllun Ysgol X: Y Cwrs Carlam).

Addysg ddwyieithog, gyda hanner o'r addysg yn cael ei chyflwyno trwy gyfrwng y Sbaeneg a'r hanner arall yn cael ei chyflwyno trwy gyfrwng y Fasgeg, yw diffiniad Model B. Nodau traddodiadol Model B oedd sicrhau bod gan ddisgyblion hyfedredd o ran cynhyrchu a deall y Fasgeg, a pharatoi disgyblion ar gyfer y posibilrwydd o drosglwyddo i addysg cyfrwng Basgeg (Gardner, 2002, "Table 4: Definitions of Bilingual Teaching Models"). Fel y noda Gardner (2008, t. 43), yr arfer ddiweddarach yn y model hwn yw tueddu i gynnwys elfen gryfach o addysg cyfrwng Basgeg, ac mae hyd yn oed rai ysgolion cynradd yn y model hwn sy'n cynnig bron pob pwnc, ac eithrio mathemateg a Sbaeneg, trwy gyfrwng y Fasgeg. Fel addysg ddwyieithog yng Nghymru, mae sefyllfa Model B felly yn gymhleth iawn gan fod nifer o amrywiadau posibl yn rhannu'r un label, 'addysg ddwyieithog'.

Addysg cyfrwng Basgeg gyda Sbaeneg fel pwnc yw Model D. Nodau ieithyddol gwreiddiol y model hwn oedd cryfhau hyfedredd yn y Fasgeg trwy ei chyflwyno'n iaith cyfrwng yr addysgu ac iaith sgwrsio, sicrhau y gall siaradwyr o'r cymunedau Basgeg ymdopi â phwysau sosio-ieithyddol arnynt oherwydd presenoldeb yr iaith fwyafrifol, sef y Sbaeneg, a sicrhau dealltwriaeth ddigonol o'r Sbaeneg (Gardner, 2002, "Table 4: Definitions of Bilingual Teaching Models"). Yn wahanol i gyddestun addysg drochi yng Nghanada, nid bwriad gwreiddiol addysg cyfrwng Basgeg yng Ngwlad y Basg oedd grymuso siaradwyr ail iaith i gael gwell gafael ar y Fasgeg. Anelwyd hi'n wreiddiol at gynnal siaradwyr brodorol y Fasgeg wrth gyflwyno 
Model D (Cenoz, 1998; Lyster, 2007). Fodd bynnag, mae nifer sylweddol o ddisgyblion o gefndiroedd ail iaith yn cofrestru mewn ysgolion Model D erbyn hyn, ac maent yn aml yn cynrychioli'r mwyafrif mewn ardaloedd Sbaeneg eu hiaith (Gardner, 2002, "4.4.2.1. Bilingual teaching models", para. 2). Mae gan CYGB sefyllfa debyg i'r hyn a welir yng Nghymru, lle mae dosbarthiadau cyfrwng Cymraeg yn gallu amrywio'n fawr rhwng cyflwyno'r iaith darged megis iaith dreftadaeth a chyflwyno'r iaith darged megis ail iaith.

Er gwaetha'r tebygrwydd rhwng y modelau yn CYGB a Chymru, yr hyn sydd yn drawiadol wahanol rhyngddynt yw bod rhyw $64.0 \%$ o ddisgyblion cynradd a $58.8 \%$ o ddisgyblion uwchradd CYGB yn mynychu ysgolion Model D ar gyfartaledd ${ }^{32}$ (Llywodraeth Gwlad y Basg, 2013b, t. 22). Cymharer hyn â'r nifer is o lawer yng Nghymru: $24.1 \%$ o ddisgyblion cynradd a $20.0 \%$ o ddisgyblion uwchradd Cymru yn mynychu ysgolion cyfrwng Cymraeg ${ }^{33}$ (LlC, 2017a, Tabl 2).

\subsubsection{Canfyddiadau ar addysg drochi yng Ngwlad y Basg}

\subsubsection{Yr ail iaith a'r drydedd iaith}

Mae ymchwil ar effeithiau addysg drochi ar gyrhaeddiad yn yr ail iaith yng Ngwlad y Basg wedi cadarnhau rhai o'r canfyddiadau a welwyd yng Nghanada. Mewn cyfres o astudiaethau cynhwysfawr ar addysg yng Ngwlad y Basg, sef EIFE: Influence of factors on the learning of Basque (1986, 1989, 1991), cymharwyd disgyblion y tri model addysg ar sail perfformiad mewn cyfres o is-brofion ${ }^{34}$ yn mesur gallu' $r$ disgyblion o ran gwrando a deall, mynegi ar lafar, darllen a deall, ac ysgrifennu yn y Fasgeg a'r Sbaeneg. O ganlyniad i'r astudiaethau hyn, ac eraill (gweler, er enghraifft, Lasagabaster, 2000), cynhyrchwyd cronfa bwysig o gasgliadau ar effaith addysg drochi ar gyrhaeddiad yn y Sbaeneg, yn ogystal â'r Fasgeg. Awgrymodd yr ymchwil fod gan ddisgyblion Model A afael wan iawn ar y Fasgeg ac mai hwn yw'r model

\footnotetext{
${ }^{32}$ Nodir bod y ffigyrau hyn yn cael eu seilio ar ddata a gasglwyd yng Ngradd 4 yn yr ysgol gynradd ac yng Ngradd 2 yr ysgol uwchradd.

${ }^{33}$ Yr ysgolion y mae LlC (2017a) yn cyfeirio atynt hwy wrth ddefnyddio'r ymadrodd 'ysgolion cyfrwng Cymraeg' yn Canlyniadau'r Cyfrifiad ysgolion 2017 yw Categorïau (i), (ii) a (iii) ysgolion cynradd, a Chategorïau (i) a (ii) ysgolion uwchradd (gweler 2.6.1. Diffinio'r ysgolion yn ôl eu darpariaeth cyfrwng Cymraeg.)

${ }^{34}$ Casglwyd data eraill hefyd yn yr astudiaethau hyn, ynglŷn â chefndir y disgyblion a chefndir yr ysgol a'r athrawon, er mwyn cymryd i ystyriaeth unrhyw newidion posibl eraill a all effeithio ar allu'r disgybl (Gabiña, Gorostidi, Iruretagoiena, Olaziregi, \& Sierra, 1986; Sierra \& Olaziredi, 1991; Sierra \& Olaziregi, 1989).
} 
gwannaf ar gyfer datblygu'r Fasgeg yn ail iaith (Gabiña ac eraill, 1986;

Lasagabaster, 2000; Sierra \& Olaziregi, 1989). At hyn, dangosodd Gabiña ac eraill (1986), Sierra ac Olaziregi (1991) a Lasagabaster (2000), fod Model B yn cynhyrchu siaradwyr sydd â medr uwch yn y Fasgeg o'u cymharu â disgyblion Model A, ond nid yw'r disgyblion hyn yn well na disgyblion Model D. Mae'r canfyddiadau hyn yn cyfateb i gasgliad Johnstone (2002) ar addysg drochi yng Nghanada, sef bod disgyblion addysg drochi gynnar yn perfformio'n well yn gyson na disgyblion addysg drochi ganolig, sydd yn eu tro'n perfformio'n well na disgyblion addysg drochi hwyr. Gellir hefyd gymharu'r canfyddiadau â rhai Gathercole a Thomas (2005) yng nghyd-destun addysg drochi yng Nghymru. (Am drafodaeth lawnach, gweler 2.2.2. Caffael ail iaith.) Awgryma'r dystiolaeth hon fod cydberthynas gadarnhaol rhwng cyswllt uwch â'r iaith darged a'i defnyddio megis iaith cyfrwng, a hyfedredd ieithyddol uwch yn yr iaith honno. Gan ddysgu trwy gyfrwng yr iaith darged, caiff y disgybl gyswllt uwch â'r iaith mewn ffordd ystyrlon, bwrpasol. Yn ddiweddarach, mae ysgolheigion wedi canolbwyntio ar effaith y gwahanol fodelau addysg ar gaffael y drydedd iaith, sef y Saesneg (Cenoz \& Valencia, 1994; Lasagabaster, 2000), ac yn fwy diweddar fyth, ar effaith dwyieithrwydd ar gaffael trydedd iaith (Cenoz, 2005, 2013). Mae'r astudiaethau hyn eto wedi awgrymu nad oes effaith negyddol ar yr iaith gyntaf nac ar yr ail iaith i'w chael wrth ddysgu trydedd iaith. Yn ogystal â datblygu'r ail iaith a dwyieithrwydd felly, mae'n debyg y gall addysg drochi baratoi disgyblion yn well ar gyfer dysgu trydedd iaith. Mewn astudiaeth gynharach, casglodd Cenoz a Valencia (1994) fod disgyblion dwyieithog sy'n dilyn addysg cyfrwng Basgeg, sef Model D, yn perfformio'n well yn Saesneg fel pwnc iaith dramor fodern na disgyblion uniaith Sbaeneg sy'n dilyn addysg cyfrwng Sbaeneg, sef Model A. Nodir bod astudiaethau eraill ar gymunedau ymreolaethus Valencia (Safont, 2005) a Chatalwnia (Sanz, 2000) hefyd wedi dangos bod dysgwyr dwyieithog yn y Gatalaneg a'r Sbaeneg yn perfformio'n well na'u cyfoedion uniaith Sbaeneg, o ran caffael hyfedredd cyffredinol a phragmatig yn y Saesneg. Ymddengys fod angen i ddysgwyr trydedd iaith gyrraedd y trothwy ieithyddol uchaf yn yr iaith gyntaf a'r ail iaith, fel y rhagdybiwyd gan Cummins (1976) a Toukomaa a Skutnabb-Kangas (1977), er mwyn iddynt elwa ar y manteision posibl o ddysgu'r drydedd iaith. Oherwydd hynny, dadleua Lasagabaster (2000, tt. 193-4) mai disgyblion Model D, hynny yw, y disgyblion sy'n cyrraedd lefelau 
cyfartal o hyfedredd eu dwy iaith, sef Basgeg a Sbaeneg, yw'r disgyblion sy'n medru manteisio ar eu dwyieithrwydd wrth ddysgu'r drydedd iaith, sef Saesneg.

\subsubsection{Iaith fwyafrifol}

Gan adlewyrchu'r canfyddiadau yng Nghanada, mae astudiaethau yng Ngwlad y Basg wedi casglu nad ydy addysg drochi rannol neu lawn yn yr iaith leiafrifol, fel a geir ym Modelau B a D, yn cael effaith negyddol ar gaffael yr iaith fwyafrifol, fel a geir ym Model D, ac mae hyn yn wir yng nghyd-destun disgyblion Sbaeneg iaith gyntaf yn ogystal â disgyblion Basgeg iaith gyntaf (Arzamendi \& Genesee, 1997, t. 165). I ddisgyblion Sbaeneg iaith gyntaf, lle ceir addysg drochi rannol yn y Fasgeg ym Model B neu addysg drochi lawn yn y Fasgeg ym Model D, golyga hyn nad ydy addysg yn yr ail iaith yn digwydd ar draul yr iaith gyntaf. Mae astudiaethau Sierra ac Olaziregi $(1991,1989)$ wedi cadarnhau hyn, wrth gasglu nad oes gwahaniaeth sylweddol rhwng sgiliau Sbaeneg disgyblion y tri model. Canfuwyd bod hyd yn oed siaradwyr Basgeg iaith gyntaf sy'n dilyn Model D a Model B yn dangos hyfedredd tebyg iawn i siaradwyr Sbaeneg eu hiaith sy'n dilyn Model A (Sierra \& Olaziredi, 1991; Sierra \& Olaziregi, 1989). Awgryma hyn nad yw'r iaith fwyafrifol yn dioddef wrth ddilyn addysg trwy gyfrwng yr iaith leiafrifol, ni waeth beth fo iaith gyntaf y dysgwr. Mae'n debyg iawn, oherwydd presenoldeb y Sbaeneg yn y gymuned gyfan y tu hwnt i'r ysgol, nad oes effaith ar hyfedredd ynddi hi (Arzamendi \& Genesee, 1997, t. 160; Cenoz, 2005, t. 50). Mae hyn hefyd yn wir yng nghyd-destun Cymru, oherwydd presenoldeb amlwg y Saesneg yng Nghymru ac ym Mhrydain gyfan. Yn yr un ffordd, nid yw'r Sbaeneg dan fygythiad o du'r Fasgeg, oherwydd statws a phresenoldeb y Sbaeneg trwy Sbaen gyfan ac yn rhyngwladol.

\subsubsection{Pynciau cwricwlaidd eraill}

Mewn astudiaethau gan Aierbe, Etxezarreta a Satrustegi (1974), Aierbe, Arregi, Etxeberria Balerdi ac Etxeberria-Sagastume (1989) a Sierra (1996), awgrymwyd nad oes gwahaniaeth sylweddol rhwng disgyblion yn y tri model o ran eu cyrhaeddiad yn y pynciau Mathemateg a'r Gwyddorau Cymdeithasol. Er bod Cenoz (2005, t. 50) o'r farn fod y canfyddiadau hyd yma yn CYGB yn cadarnhau'n gyffredinol yr hyn sydd wedi'i ganfod eisoes gan ysgolheigion yng nghyd-destun rhaglenni trochi yng Nghanada, mae angen ymchwil bellach ar effaith addysg y tri model gwahanol yng 
Ngwlad y Basg ar gyrhaeddiad mewn pynciau cwricwlaidd eraill, cyn y gellir dod i gasgliad pendant yn y cyd-destun Basgaidd.

\subsubsection{Agweddau cymdeithasol}

Fel y casglwyd gan astudiaethau yng Nghanada, mae astudiaethau yng Ngwlad y Basg hefyd wedi awgrymu bod gan addysg rôl arwyddocaol o ran ffurfio agweddau cadarnhaol tuag at yr iaith leiafrifol. Mewn astudiaeth o 441 disgybl ym mlwyddyn olaf eu haddysg orfodol mewn ysgolion Model A, B a D, canfu EtxeberríaSagastume (2006) fod agweddau'r disgyblion yn amrywio yn ôl y model y maent yn ei ddilyn. Yn achos Model A, mae gan y Fasgeg werth fel pwnc yn unig, lle yn achos Model D, ac i raddau llai yn achos Model B, mae gan y Fasgeg agweddau cadarnhaol tuag ati a gwerth symbolaidd ymhlith y disgyblion oherwydd y cyswllt rhwng y Fasgeg a hunaniaeth ddiwylliannol (Etxeberria-Sagastume, 2006, t. 132). Mae'r canfyddiad bod gan ddisgyblion Model D agweddau mwy cadarnhaol tuag at y Fasgeg wedi'i gefnogi gan astudiaethau eraill a ysgrifennwyd yn Sbaeneg (Larrañaga, 1995; Urrutia ac eraill, 1998; Urrutia, 2005, fel y dyfynnwyd yn Cenoz, 2009). Wedi ystyried yr astudiaethau ar agweddau a hunaniaeth sydd wedi'u cynnal yn CYGB, daw Cenoz (2009, t. 187) i'r casgliad mai un o'r canfyddiadau cyson o'r astudiaethau hyn yw bod disgyblion Model D, ni waeth beth fo eu hiaith gyntaf, yn dangos agweddau mwy cadarnhaol tuag at y Fasgeg.

Er pwysiced addysg drochi yn yr iaith leiafrifol wrth ffurfio agweddau cadarnhaol at hunaniaeth Fasgaidd disgyblion ail iaith, rhaid nodi pwysigrwydd y cyd-destunau y tu hwnt i'r ysgol. Wrth arolygu astudiaethau ar agweddau yn CYGB, mae Lasagabaster (2007, t. 73) yn amlygu mor bwysig yw'r ffactor sosio-ieithyddol a'i heffaith ar agweddau, wrth ddadlau po fwyaf y nifer sy'n siarad Basgeg mewn ardal, y mwyaf cadarnhaol yr agweddau tuag at Fasgeg yno. Mae hefyd yn bwysig ystyried y cyd-destun sosio-gwleidyddol yng Ngwlad y Basg a'i ddylanwad ar ffurfio agweddau hefyd. Ys dywed Cenoz (2009, 187): "Bilingual and multilingual schools are part of society and as such are affected by the socio-political context." Mae gan y Fasgeg werth a rôl sosio-dwylliannol a gwleidyddol is yn y cyd-destun ehangach, yn Sbaen gyfan, Ewrop, yr Unol Daleithiau a'r tu hwnt (Etxeberria-Sagastume, 2006). Oherwydd hynny, mae agweddau tuag at y Fasgeg yn debygol o ddioddef. Dyma pam ei bod yn bwysig i ysgolion geisio ffurfio agweddau iach. Yn ôl Etxeberria- 
Sagastume (2006), mae'r canfyddiadau'n awgrymu y gall ysgolion chwarae rôl allweddol o ran newid agweddau disgyblion.

\subsubsection{Casgliadau ar addysg drochi yng Nghanada a Gwlad y Basg}

Mae'r swmp mawr o ymchwil sydd wedi'i chynnal ar addysg drochi yn y cyd-destun Canadaidd, ac i raddau llai yn y cyd-destun Basgaidd, yn awgrymu nad oes effeithiau negyddol i'w canfod mewn perthynas â datblygu'r ail iaith, yr iaith gyntaf na chyrhaeddiad academaidd mewn pynciau cwricwlaidd. O bosibl, mae nifer o fanteision i'w cael, yn arbennig wrth ystyried rhaglenni trochi llawn cynnar, o ran mabwysiadu agweddau cymdeithasol cadarnhaol, datblygu sgiliau uwch o lawer yn yr ail iaith, mewn cymhariaeth, hynny yw, â disgyblion rhaglenni ail iaith traddodiadol, a hefyd sgiliau uwch yn gyffredinol yn yr iaith gyntaf.

Mae'r ymchwil hefyd wedi amlygu sawl cyfyngiad ar fanteision y fath addysg. Mae'n bosibl nad yw cywirdeb gramadegol disgyblion rhaglenni trochi cystal â siaradwyr iaith gyntaf cymharol. Er bod disgyblion y rhaglenni hyn yn cael nifer fawr o oriau cyswllt â'r iaith darged, nododd Hammerly (1988) ei bod yn bosibl i'r disgyblion hyn gynhyrchu gwallau gramadegol na fyddent yn bresennol yn iaith siaradwyr iaith gyntaf. Mae hefyd yn bosibl fod cyfyngiadau ar ddulliau ieithyddol y disgybl trochi a'i ddefnydd o gyweiriau addas (Baker, 2011). Fodd bynnag, rhaid bod yn ofalus wrth gymharu hyfedredd siaradwyr ail iaith â hyfedredd siaradwyr iaith gyntaf yn yr iaith darged, oherwydd nad oes gan ddisgyblion ail iaith yr un cyfleoedd i ymarfer a defnyddio'r iaith darged y tu hwnt i'r ysgol ac nid ydynt yn caffael yr iaith ar yr un adeg yn eu bywyd. Os cymherir hyfedredd disgyblion ail iaith ar raglenni trochi â hyfedredd disgyblion iaith gyntaf, mae'n debyg mai methiant yw addysg drochi (Swain \& Johnson, 1997, t. 11). Er nad yw addysg drochi bob tro’n creu siaradwyr ail iaith sydd yr un mor fedrus yn yr iaith darged ag y mae siaradwyr iaith gyntaf, mae ysgolheigion erbyn hyn yn cydnabod llwyddiant addysg drochi i ddatblygu hyfedredd disgyblion yn eu hail iaith y tu hwnt i'r hyn sy'n bosibl ar raglenni traddodiadol (Johnstone, 2007, t. 22).

Un o'r cyfyngiadau eraill yw nad yw addysg trwy gyfrwng yr iaith leiafrifol ar ei phen ei hun yn ddigonol i sicrhau agweddau sosio-ddiwylliannol a gwleidyddol cadarnhaol tuag at yr iaith leiafrifol. Fel y noda Lasagabaster $(2007$, t. 73$)$ yng 
nghyd-destun y Fasgeg, mae angen cyfraniadau sefydliadau cymdeithasol eraill hefyd er mwyn annog defnydd cynyddol o'r iaith leiafrifol honno.

Mewn arolygiadau a'u ffocws ar ddisgyblion ôl-radd o raglenni trochi yn y Ffrangeg, dangosodd Wesche (1993) nad yw'r disgyblion hyn yn defnyddio'r Ffrangeg yn aml ar ôl gadael yr ysgol. Mae perygl y bydd yr ail iaith yn dod yn iaith y dosbarth yn unig os na ddefnyddir hi mewn cyd-destunau cymdeithasol go iawn. Dyma bryder sydd wedi'i nodi gan Gruffudd (2000), er enghraifft, yng nghyd-destun y Gymraeg. Er bod disgyblion yn defnyddio'r Gymraeg yng nghyd-destun y dosbarth, nododd Gruffudd nad yw disgyblion o reidrwydd yn ei defnyddio hi y tu allan i'r cyd-destun hwn. Mae ymchwil gan D. Morris (2010) yn cefnogi'r honiad hwn. Yn ei hastudiaeth ar bobl ifanc ym maes addysg cyfrwng Cymraeg a dwyieithog a'i defnydd o'r Gymraeg mewn cyd-destunau cymdeithasol, darganfu hi fod natur ieithyddol y cartref yn ffactor allweddol o ran sicrhau cyfleoedd i bobl ifanc ddefnyddio'r Gymraeg yn gymdeithasol. Dangosodd Morris yn ei hymchwil fod y defnydd a wneir gan gyfranogwyr yr astudiaeth o'r Gymraeg yn is o lawer y tu allan i'r ysgol wrth siarad â phobl ifanc eraill, o’i gymharu â'r defnydd a wneir gan y cyfranogwyr o'r Gymraeg wrth siarad ag athrawon a disgyblion eraill yng nghyd-destun y dosbarth (Tabl 3, t. 87). Nododd D. Morris hefyd fod pobl ifanc o gartrefi di-Gymraeg yn defnyddio'r Gymraeg mewn rhwydweithiau cymdeithasol yn is o lawer o'u cymharu â phobl ifanc o gartrefi Cymraeg (Tabl 13, t. 94). Darganfu Hodges (2009) ganfyddiadau tebyg yn ei hastudiaeth hi ar ddefnyddio'r Gymraeg yn gymdeithasol, a hynny yn achos cyn-ddisgyblion Ysgol Gyfun Cwm Rhymni yn ne ddwyrain Cymru. Er bod yr ysgol yn cynhyrchu unigolion sy'n medru'r Gymraeg, nododd Hodges nad oes digon o gefnogaeth yn y gymuned a'r gweithle er mwyn i'r siaradwyr hyn normaleiddio ei defnydd. I'r rhai sy'n cael eu trochi yn y Gymraeg ac sy'n dod o gartrefi di-Gymraeg felly, heb sicrhau cyfleodd a rhwydweithiau i ddisgyblion addysg drochi ddefnyddio'r Gymraeg yn gymdeithasol, mae yna berygl y gall yr ail iaith ddod yn iaith y dosbarth yn unig. Er bod addysg drochi (a hyd yn oed addysg dreftadaeth) yn rhoi i ddisgyblion yr offer i gyfathrebu'n effeithiol yn yr ail iaith felly, nid oes sicrwydd y bydd disgyblion yn defnyddio'r offer hyn a ddysgwyd yn y gwersi mewn ffordd bwrpasol a chymdeithasol, os nad yw'r rhwydweithiau a chyfleoedd i ddefnyddio'r iaith leiafrifol yn eu lle. 
Cyfyngiad arall yng nghyd-destun yr ymchwil ar addysg drochi yng Ngwlad y Basg yw'r diffyg ystyriaeth a roddir i addysg drochi yn y sector addysg uwchradd, ynghyd â'r sectorau addysg bellach ac addysg uwch. Dyma bryder a godwyd gan Arzamendi a Genesee (1997), ac mae'n debyg fod angen ymchwil bellach yn y meysydd hyn o hyd. Mae'n bosibl fod hyn yn wir oherwydd newid diweddar o ran cyfundrefn addysg yn y wlad, ynghyd â shifft o ran ffocws ysgolheigion, o addysg drochi yn y Fasgeg i'r hyn a elwir yn raddol yn CLIL, lle cyflwynir addysg drwy gyfrwng y drydedd iaith, sef y Saesneg, yn ogystal â thrwy gyfrwng yr ail iaith, sef y Fasgeg. Er gwaetha'r cyfyngiadau hyn, mae'n amlwg fod gan addysg y potensial i greu siaradwyr newydd. Mae McLeod (2007), er enghraifft, wedi gwerthfawrogi effaith addysg ddwyieithog, yng Nghymru a Gwlad y Basg yn arbennig, ar greu siaradwyr newydd mewn ieithoedd dan fygythiad. I'r un perwyl, mae Newcombe (2007) hefyd wedi amlygu pwysigrwydd addysg, gan briodoli adnewyddu'r Gymraeg yn ail ran yr ugeinfed ganrif yn bennaf i rôl addysg:

Arguably, the most important influence in the fight to restore the Welsh language in the latter half of the 20th century has been education: the very institution that contributed so powerfully to its decline.

(Newcombe, 2007, t. 5)

\subsection{Sefyllfa'r Saesneg mewn addysg ddwyieithog yn yr Almaen}

Mae sefyllfa addysg ddwyieithog yn yr Almaen yn un gymhleth iawn, a hynny oherwydd yr amrywiaeth fawr sy'n bodoli rhwng yr un dalaith ar bymtheg o ran eu cyfundrefnau addysg (P. Burmeister, cyfathrebiad personol, 20 Tachwedd, 2015; Dieter Wolff, 2007). Amlwg serch hynny ar draws y rhan fwyaf o'r taleithiau yw bod rhaglenni CLIL ar gyfer ieithoedd megis Saesneg, Ffrangeg, ac i raddau llai, Eidaleg, Sbaeneg, Iseldireg, a Rwsieg yn bodoli erbyn y fan hon. Gellir olrhain gwreiddiau CLIL at ei darddiad yn 1963, ar ôl i Gytundeb Elysée rhwng Ffrainc a'r Almaen gael ei arwyddo er mwyn sefydlu perthynas newydd a chymodi'r ddwy wlad wedi'r Ail Ryfel Byd. Yn sgil y berthynas newydd hon, sefydlwyd rhaglenni a fyddai'n rhoi cyfle i ddisgyblion ar y ffin rhwng Ffrainc a'r Almaen astudio'n ddwyieithog. Ers hynny, mae poblogrwydd rhaglenni CLIL wedi tyfu ar draws yr Almaen, yn arbennig rhaglenni CLIL ar gyfer y Saesneg. Roedd CLIL yn y Saesneg wedi cynyddu'n sylweddol o ran eu nifer yn y 1980au pan agorwyd canghennau ar gyfer addysg ddwyieithog newydd mewn ysgolion gramadeg (Dieter Wolff, 2007, t. 94) ac 
ymhellach yn y 1990au wrth i'r Saesneg ddod yn fwyfwy dylanwadol megis iaith ryngwladol (Breidbach \& Viebrock, 2012, t. 5) mewn byd sy'n datblygu'n 'bentref byd-eang', yng nherminoleg McLuhan a Powers (1989).

Yng nghyd-destun y ddau achos arall a drafodwyd uchod, iaith frodorol y gymuned neu'r wlad yw'r iaith a gyflwynir drwy gyfrwng addysg drochi. Fodd bynnag, Saesneg yn aml yw'r ail iaith a gyflwynir yn addysg iaith dramor yr Almaen (Breidbach \& Viebrock, 2012), gan y daw y rhan fwyaf o ddisgyblion CLIL o gefndir lle nad yw'r Saesneg yn famiaith. Gwerth addysg ddwyieithog yn yr Almaen felly yw'r cyfle i ddatblygu dwyieithrwydd cynyddol plant mewn un neu fwy o'r ieithoedd Ewropeaidd eraill, a chredir y bydd dwyieithrwydd, neu amlieithrwydd, mewn ieithoedd Ewropeaidd eraill, neu 'hyfedredd Ewropeaidd', yn galluogi plant i elwa ar y manteision cyfathrebol, gyrfaol a rhyngbersonol posibl yn y dyfodol, ynghyd â datblygu hyfedredd ieithyddol cyffredinol (Wolff, d.d., t. 5). Yn y cyddestun hwn, dyma'r hyn y cyfeiria Breidbach a Viebrock $(2012$, t. 10) ato megis “intercultural learning”, neu 'ddysgu rhyngddiwylliannol'. Gellir nodi bod tebygrwydd rhwng cyd-destun addysgu Saesneg yn yr Almaen ac addysgu Ffrangeg yng Nghanada. Mae addysgu Saesneg a Ffrangeg yn y ddau gyd-destun yn digwydd at ddiben sicrhau dwyieithrwydd cynyddol disgyblion mewn dwy iaith fwyafrifol (gweler 2.3.5. Dwyieithrwydd cynyddol a dwyieithrwydd gostyngol) er mwyn iddynt elwa ar y manteision ehangach, yn y cyd-destun Ewropeaidd neu ryngwladol, a all ddod yn sgil bod yn ddwyieithog mewn dwy iaith ac iddynt fri mawr. Mae hyn yn gwrthdaro â chyd-destunau Cymru a Gwlad y Basg wrth gwrs, lle mae gan addysg drochi yn y Gymraeg a'r Fasgeg rôl wahanol, sef cynnal a chefnogi'r ieithoedd lleiafrifol hyn yn eu cymunedau ieithyddol.

Yn ôl arolwg diweddar gan Frühe Mehrsprachigkeit an Kitas und Schulen (FMKS) ${ }^{35}$ (2014) ar draws pob un o daleithiau'r Almaen, cyfrifwyd 287 o ysgolion cynradd dwyieithog sy'n cynnig o leiaf un iaith dramor megis iaith cyfrwng yr addysg yn ogystal â'r Almaeneg, ac o blith yr ysgolion hyn mae rhyw 44\% o'r ysgolion hyn yn cynnig Saesneg megis yr iaith gyfrwng honno. Fodd bynnag, nid oes ffigur swyddogol am faint o ysgolion uwchradd sy'n cynnig rhaglenni CLIL. Mae FMKS (cyfathrebiad personol, 20 Tachwedd, 2015) fodd bynnag wedi nodi ei bod yn debyg

\footnotetext{
${ }^{35}$ Sefydliad sy'n hyrwyddo'r arfer o drochi ieithyddol mewn ieithoedd tramor mewn canolfannau gofal dydd ac ysgolion er mwyn mabwysiadu amlieithrwydd yn yr Almaen, yw FMKS.
} 
fod tua 900 o ysgolion dwyieithog ${ }^{36}$ uwchradd yn bodoli erbyn hyn, gyda'r rhan fwyaf o'r rheini'n cynnig y Saesneg neu'r Ffrangeg fel iaith y rhaglen CLIL.

\subsubsection{Rhesymeg o ran canolbwyntio ar CLIL yn yr Almaen}

Er bod sefyllfa addysg ddwyieithog yr Almaen yn wahanol i'r un yng Nghymru, penderfynwyd canolbwyntio yma ar raglen ddwyieithog benodol sy'n bodoli yn y wlad hon oherwydd caiff nifer o'u nodweddion eu rhannu yn y rhaglen garlam a gyflwynir gan Ysgol X, sef un o'r ysgolion dan sylw yng ngwaith maes yr ymchwil hon. Gobaith canolbwyntio ar y rhaglen hon yw darganfod unrhyw dystiolaeth ynglŷn ag effeithiolrwydd rhaglen sy'n addysgu ail iaith trwy ei chyflwyno'n iaith gyfrwng ar gyfer pynciau cwricwlaidd ar wahân i'r pwnc iaith ei hun.

\subsubsection{Diffinio addysg drochi a CLIL yn yr Almaen}

Er bod addysg cyfrwng Saesneg yn yr Almaen yn debyg iawn mewn termau pedagogaidd i'r rhaglenni addysg drochi fwy traddodiadol a drafodwyd uchod, mae'n bosibl labelu'r fath addysg yn 'CLIL', gan fod CLIL erbyn hyn yn gysylltiedig i raddau helaeth ag addysgu Saesneg megis iaith dramor (Dalton-Puffer, Nikula, \& Smit, 2010; Whittaker \& Llinares, 2011). Fodd bynnag, am ei bod yn bosibl dehongli diffiniad CLIL mewn gwahanol ffyrdd (Cenoz ac eraill, 2014), ceir enghreifftiau lle mae CLIL yn debyg iawn ei ystyr i 'addysg drochi', a hynny'n ddibynnol ar faint o gynnwys yr addysg a geir drwy gyfrwng yr iaith dramor. Yn ôl diffiniad FMKS (2014, t. 1), mae rhaglenni addysg drochi yn yr Almaen yn cyflwyno o leiaf 50\% o'r addysg trwy gyfrwng yr iaith darged, lle mae rhaglenni CLIL yn cyflwyno hyd at 50\% o'r addysg trwy gyfrwng yr iaith darged. Yng nghyd-destun addysg Cymru, dyma'r hyn a elwir yn 'addysg ddwyieithog' ac 'addysg cyfrwng Cymraeg' yn eu tro (gweler 2.6.2. Addysg cyfrwng Cymraeg). Mae ysgolheigion eraill megis Breidbach a Viebrock $(2012$, t. 6) a Rumlich $(2014$, t. 81$)$ fodd bynnag yn mynegi mai math o addysg drochi hwyr yw CLIL yn nhermau strwythur, ac felly mae CLIL yn yr Almaen yn label newydd ar gyfer yr hyn a elwid yn flaenorol yn addysg drochi. (Am ddiffiniad o'r term, gweler 2.5.4. Content and Language

\footnotetext{
${ }^{36}$ Nodir bod ysgolion neu ganghennau ysgolion sy'n cynnig ffrwd CLIL yn aml yn cael eu galw'n 'ddwyieithog' yn y cyd-destun Almaenaidd (gweler, er enghraifft, Wolff, 2007), er nad ydynt yn cynnig mwy na 50\% o'r addysg trwy gyfrwng yr iaith darged, fel a wneir yng nghyd-destun addysg Cymru. 'Addysg ddwyieithog' oedd label yr ysgolion a oedd wedi cynnig addysg CLIL gynnar yn y 1960au (Breidbach \& Viebrock, 2012, t. 5), ac felly mae'n debyg mai dyma pam y defnyddir y label 'ysgolion dwyieithog' o hyd.
} 
Integrated Learning am ddiffinio'r term.) At ddiben yr is-adran hon, defnyddir y labeli 'addysg drochi rannol hwyr' a 'CLIL' yn gyfystyr, gan fod y llenyddiaeth yn defnyddio'r ddau derm hyn.

\subsubsection{Nodweddion CLIL traddodiadol}

Oherwydd y gall nodweddion y cyfundrefnau addysg amrywio'n fawr, nid yw'n bosibl llunio trosolwg cryno o bob model CLIL posibl yn yr Almaen (Wolff, 2007). O blith yr amryw raglenni addysg ddwyieithog sydd ar gael yn yr Almaen, canolbwyntir yma ar raglenni CLIL traddodiadol ${ }^{37}$ ar gyfer y Saesneg mewn addysg uwchradd. Fel y nodwyd gan Breidbach a Viebrock (2012), mae rhaglenni CLIL yn yr Almaen yn bennaf yn adlewyrchu strwythur addysg drochi rannol hwyr. Cyflwynir y Saesneg megis pwnc iaith dramor fodern yng ngradd 5 (sef 10 oed) a cheir tua 2 awr ychwanegol yr wythnos o addysg Saesneg ar y rhaglen CLIL, sef tua 7 awr yr wythnos, o'i chymharu â'r ddarpariaeth ar gyfer disgyblion y ffrwd draddodiadol. Mae'r cyfnod hwn o addysg iaith dramor ddwys yn paratoi'r disgyblion ar gyfer ymdopi â dilyn peth addysg cyfrwng Saesneg o radd 7 (sef 12 oed) ymlaen. Adeg gradd 7, cyflwynir y pwnc cwricwlaidd cyntaf trwy gyfrwng y Saesneg, Daearyddiaeth fel arfer, gydag un neu ddwy wers ychwanegol yr wythnos. Pan gyrhaeddir gradd 8, cyflwynir yr ail bwnc trwy gyfrwng y Saesneg, Hanes fel arfer, gyda dwy wers ychwanegol ar gyfer y pwnc. Fel y noda Wolff (2007), mae rhai rhaglenni CLIL ar draws yr Almaen yn cyflwyno trydydd pwnc trwy gyfrwng yr iaith dramor, ac mae hyn yn ychwanegu awr arall at y cwricwlwm.

Gellir cymharu nodweddion y model CLIL hwn â model y Cwrs Carlam a gynigir gan Ysgol X (gweler 5.4. Cynllun Ysgol X: Y Cwrs Carlam). Mae'r ddau fodel yn cyflwyno cyfnodau o ddysgu dwys i'r disgyblion cyn iddynt ymuno â'r rhaglen yn yr ysgol uwchradd, sef CLIL a'r Cwrs Carlam yn ôl eu trefn. Fel CLIL, cynnig model y Cwrs Carlam Ddaearyddiaeth a Hanes megis pynciau dwyieithog, ond at hyn ceir tri phwnc ychwanegol, sef Addysg Grefyddol, Addysg Gorfforol ac ABaCh. Mae pynciau megis Celf, Cerddoriaeth, Drama ac Addysg Gorfforol wedi'u hosgoi tan yn ddiweddar mewn rhaglenni CLIL yn yr Almaen (Breidbach \& Viebrock, 2012, t. 7), er y gellid eu galw'n bynciau llai heriol o ran eu hieithwedd (Williams, 2002, 2008). Noda Breidbach a Viebrock (2012) mai oherwydd gwreiddiau addysg ddwyieithog

${ }^{37}$ Dylid nodi bod dau fath arall o CLIL yn bodoli yn yr Almaen, sef CLIL modiwlar a CLIL ar gyfer cyd-destunau penodol (content-specific CLIL) (Wolff, 2007). 
yn yr Almaen, sef cymodi'r ddwy wlad Ffrainc a'r Almaen yn y 1960au, y rhoddwyd y flaenoriaeth i bynciau sy'n perthyn i'r Gwyddorau Cymdeithasol.

Mae'n bosibl dadlau fod y ddarpariaeth a geir ar y Cwrs Carlam yn Ysgol X yn fwy heriol na'r addysg a geir ar raglen CLIL, gan y ceir amrywiaeth fwy o bynciau trwy gyfrwng yr iaith darged. Un nodwedd debyg arall a gwendid, o bosibl, rhwng y ddau fodel hyn yw eu natur ddethol bosibl. Yn yr Almaen, cafodd rhaglenni CLIL cynnar eu gweithredu yn yr ysgolion uwchradd gramadeg, neu Gymnasium, yn unig gan mai'r disgyblion mwyaf dawnus, academaidd sy'n mynychu'r fath ysgol (Breidbach \& Viebrock, 2012; Burmeister \& Daniel, 2002). Er bod y mathau eraill o ysgolion uwchradd a chanddynt ofynion academaidd llai, sef Hauptschule a Realschule, wedi dechrau cynnig addysg CLIL erbyn hyn, mae'r rhan fwyaf o ddisgyblion, tua $45 \%$, yn mynychu ysgol Gymnasium (Breidbach \& Viebrock, 2012, t. 6).

Dyma wendid sy'n cael ei rannu gan fodel Ysgol X, sydd hefyd yn cynnig y Cwrs Carlam i ddisgyblion ar sail eu gallu academaidd (yn ogystal â meini prawf eraill) ym Mlwyddyn 6 (gweler 7.2.2.3. Darpariaeth ddwyieithog: Y Cwrs Carlam). Hawdd yw gweld sut y gall y naill fodel a'r llall gael eu galw'n elitaidd oherwydd y pryder nad yw'r rhaglenni hyn yn agored i ddysgwyr llai dawnus. Mae rhai ysgolheigion megis Mehisto (2007), Bruton (2013) a Paran (2013), er enghraifft, wedi mynegi eu pryder y gall rhaglenni CLIL denu nifer fawr o ddysgwyr disglair. Fel y mae Bruton (2013, t. 593) yn ei rybuddio: "rather than increasing the equality of opportunity, CLIL in certain contexts is subtly selecting students out..." Sut bynnag, nid pedagogeg CLIL sy'n gyfrifol am natur ddethol rhai rhaglenni CLIL (gweler 4.4.4. Canfyddiadau parthed CLIL traddodiadol), ond yn hytrach y ffordd y caiff y rhaglenni hyn eu gweithredu (Somers, 2018a). Yn ôl Somers (2018a, 2018b), dylai rhaglenni CLIL fod ar gael i wahanol fathau o ddysgwyr a rhaid sicrhau bod ysgolion a'u hathrawon yn derbyn digon o gefnogaeth o ran gweithredu rhaglenni CLIL er mwyn sicrhau nad ydynt yn ystyried gallu academaidd isel a hyfedredd ieithyddol isel yn yr iaith gyntaf a'r ail iaith yn ffactorau a all effeithio ar ansawdd rhaglenni CLIL. Ys dywed Somers (2018a, t. 8, pwyslais yr awdur): “... in contexts where the school administrators and teachers receive insufficient support and education, selecting high-achieving students becomes a way to safeguard the programme's success, which in turn may cause (or even aggravate) pedagogical deficiencies in the classroom...” Bydd hyn yn hollbwysig er mwyn sicrhau bod CLIL yn fodel 
cynhwysol o addysg, hynny yw, bod addysg CLIL ar gael i ddysgwyr sy'n tangyflawni, yn ogystal â’r dysgwyr sy’n gorgyflawni (Pérez-Cañado, 2016).

\subsubsection{Canfyddiadau parthed CLIL traddodiadol}

Mae astudiaethau ar CLIL yn yr Almaen wedi canolbwyntio'n arbennig ar fesur hyfedredd ieithyddol cyffredinol disgyblion CLIL yn yr iaith darged o'u cymharu â'u cyfoedion, ac effaith CLIL ar ddysgu cynnwys pynciau cwricwlaidd. Er bod rhai canfyddiadau ar effaith CLIL ar 'ddysgu rhyng-ddiwylliannol' disgyblion, nid yw'r canfyddiadau hyn wedi'u dadansoddi'n ddigonol erbyn hyn, a hynny, mae'n debyg, oherwydd diffyg cysondeb o ran diffinio'r cysyniad 'diwylliant' mewn ymchwil empirig (Breidbach \& Viebrock, 2012; Mehisto, 2013). Oherwydd hynny, nid yw'r dystiolaeth yn ddigon cadarn i'w hystyried yma, ac felly canolbwyntir yn benodol ar y canfyddiadau mewn perthynas â hyfedredd ieithyddol a dealltwriaeth o gynnwys pwnc-benodol.

\subsubsection{Saesneg megis iaith dramor fodern}

Mewn astudiaethau cynnar ar CLIL, canolbwyntiwyd yn arbennig ar sut roedd disgyblion ar raglen CLIL yn cymharu â'u cyfoedion ar raglen draddodiadol o gyflwyno iaith dramor fodern. Mewn astudiaeth hirdymor gan Bredenbröker (2000) yn ystod y 1990au, cymharwyd disgyblion CLIL â disgyblion y rhaglen draddodiadol mewn cyfres o brofion gramadeg, a darllen a deall, cyn iddynt ddechrau'r rhaglen, ar ôl blwyddyn, ac ar ôl dwy flynedd. Ar sail y canfyddiadau, casglodd Bredenbröker fod disgyblion CLIL yn perfformio'n sylweddol well o ran darllen a deall yn arbennig, ond hefyd o ran defnyddio strategaethau ar gyfer casglu gwybodaeth o rywbeth (inference), er enghraifft. Ni chasglwyd gwahaniaethau sylweddol ynglŷn â gramadeg rhwng y ddau grŵp, ond esbonia Breidbach a Viebrock (2012) mai oherwydd gwahaniaethau yn y ffordd y cyflwynir gramadeg yn y ddwy raglen yw'r rheswm am hyn:

Differences in the grammar test were less pronounced, which could be attributed to the fact that regular foreign language teaching is often more concerned with an explicit focus on grammar, whereas CLIL is more concerned with implicit grammatical knowledge, which is acquired in the process of exchanging subject-specific information. (Breidbach \& Viebrock, 2012, tt. 7-8) 
Mae hefyd yn bosibl fod disgyblion y rhaglen CLIL yn elwa ar ddatblygu geirfaoedd mwy, o'u cymharu â'u cyfoedion. Ys dywed Dalton-Puffer (2008, t. 144): “...through studying content subjects in the foreign language CLIL learners possess larger vocabularies of technical and semi-technical terms and possibly also of general academic language which gives them a clear advantage over their EFL-peers." Caiff y gred hon ei chefnogi gan Burmeister a Daniel (2002) yn eu hadolygiad cynhwysfawr o'r canfyddiadau ymchwil ar yr hyn y maent yn cyfeirio ato megis 'addysg drochi rannol hwyr', yn Schleswig-Holstein gan Brifysgol Kiel yn ystod y 1990au. Daw'r awduron i'r casgliad fod y dystiolaeth yn ddigonol i awgrymu bod geirfa disgyblion CLIL erbyn diwedd gradd 7 yn "considerably larger and more differentiated as to lexical fields, and more often than for the controls it derives from other sources, such as the IM classroom" (Burmeister \& Daniel, 2002, t. 511).

Mae astudiaethau tebyg eraill wedi cymharu disgyblion CLIL â disgyblion y rhaglen draddodiadol er mwyn darganfod gwahaniaethau hyfedredd ieithyddol cyffredinol. Er enghraifft, cymharodd Rumlich (2013) ddisgyblion CLIL â disgyblion rhaglen draddodiadol wrth ddefnyddio 'profion C' (C-tests) er mwyn profi a ydy hyfedredd ieithyddol cyffredinol darpar ddisgyblion y ffrwd CLIL yn uwch na'u cyfoedion ar y rhaglen draddodiadol, erbyn diwedd y cyfnod paratoi (gradd 6). Er bod canolbwynt yr astudiaeth yn gyfyng iawn wrth iddi ganolbwyntio ar y cyfnod paratoi lle ceir dwy wers ychwanegol yr wythnos cyn i'r disgyblion ymuno â'r rhaglen CLIL / rhaglen draddodiadol yn llwyr, mae canfyddiadau'r astudiaeth yn awgrymu bod darpar ddisgyblion CLIL yn perfformio gryn dipyn yn well o ran profion hyfedredd ieithyddol yn y Saesneg na'u cyfoedion ar y rhaglen draddodiadol yn yr un ysgol ac mewn ysgol arall nad yw'n cynnig CLIL. Mae'r canfyddiad hwn yn cefnogi canfyddiadau astudiaethau eraill yng Nghatalwnia (Sanz, 2000), Valencia (Safont, 2005), a Gwad y Basg (Cenoz \& Valencia, 1994; David Lasagabaster, 2000), sydd wedi awgrymu bod hyfedredd ieithyddol a hyfedredd pragmatig disgyblion dwyieithog yn y Saesneg yn well o'u cymharu â disgyblion uniaith cymharol. Casgl Rumlich (2013) fod canfyddiadau'r astudiaeth yn awgrymu y gwelir gwahaniaethau ieithyddol rhwng disgyblion, a hynny hyd yn oed cyn iddynt ddechrau'r rhaglen lawn, a hynny oherwydd sawl rheswm posibl. Awgryma Rumlich y gall y broses ddethol ac elitaidd o ddewis disgyblion ar gyfer CLIL, ynghyd ag ansawdd yr addysg a geir mewn rhaglenni CLIL, effeithio ar y canlyniadau. Mae athrawon yn y ffrwd 
CLIL yn debygol o fod yn well, gan fod yr athrawon hyn yn llawer mwy brwdfrydig dros addysgu'r Saesneg na'r athrawon yn y ffrwd draddodiadol, gan mai dyma'r ffrwd sydd â statws o fri yn yr ysgol (Rumlich, 2013, t. 197). Yn y pendraw, fodd bynnag, mae'n debyg mai dawn y disgyblion yn y ffrwd CLIL yw'r ffactor mwyaf sylweddol:

Under the same or similar conditions, some students benefit from teaching significantly more than others. In other words, it is bound to be the differences in individual student characteristics and their (language) learning dispositions that slow down or accelerate students' (language) learning process in both CLIL and non-CLIL classes. (Rumlich, 2013, t. 198)

Rhaid cofio felly mai deallusrwydd, gwybodaeth flaenorol o'r iaith, anogaeth i ddysgu'r iaith ac agweddau at yr iaith yw'r ffactorau allweddol sy'n pennu dawn a pharodrwydd disgyblion i gaffael ail iaith yn effeithiol, ac yn eu tro, mae'r rhain yn gallu effeithio ar ddatblygiad ieithyddol cyffredinol disgyblion CLIL. Nid yw'n bosibl felly priodoli addysg CLIL yn unig i sgorau uwch mewn profion hyfedredd ieithyddol cyffredinol ar sail canfyddiadau rhan gyntaf astudiaeth Rumlich (2013) yn unig, a bydd angen mwy o dystiolaeth o'r astudiaeth hirdymor hon am ddatblygiad disgyblion CLIL ymhellach ymlaen yn eu haddysg cyn cadarnhau hyn (Breidbach \& Viebrock, 2012, t. 8).

\subsubsection{Pynciau cwricwlaidd eraill}

O’u cymharu â'r canfyddiadau ar addysg drochi, mae canfyddiadau ymchwil ar CLIL a'r effaith ar ddeall cynnwys cwricwlaidd trwy gyfrwng yr iaith dramor yn tueddu i lunio darlun cymhleth, nad yw'n argyhoeddi, yn ôl Dalton-Puffer (2011, t. 188). Mae rhai canlyniadau'n cadarnhau 'damcaniaeth drothwy' Cummins (1976) a Toukomaa a Skutnabb-Kangas (1977), sy’n mynnu bod yn rhaid i ddisgyblion gyrraedd lefel isafswm o hyfedredd yn yr ail iaith. Fel arall, "interaction with the environment through that language, both in terms of input and output, is likely to be impoverished" (Cummins, 1979b, tt. 229-230). Mewn astudiaeth gan Zydatiß (2012), astudiwyd disgyblion CLIL a disgyblion y ffrwd draddodiadol er mwyn darganfod achosion o gydberthynas rhwng hyfedredd ieithyddol a pherfformiad mewn tasgau addysgol trwy gyfrwng y Saesneg, sef yr iaith dramor. Dangosodd Zydatiß fod yr achosion hyn o gydberthynas yn bodoli, ond bod angen i ddisgyblion gyrraedd lefel lleiafswm o hyfedredd yn yr iaith dramor, neu'r 'trothwy ieithyddol isaf' fel y'i 
hadweinir gan Cummins (1976), er mwyn iddynt allu ymdopi â gofynion ieithyddol dysgu cynnwys pynciol trwy gyfrwng yr iaith dramor:

I would maintain that adequate levels of academic discourse proficiency in German CLIL classrooms (of the extensive type realised by a 'bilingual stream') are dependent on rather high levels of linguistic competence (especially regarding vocabulary and grammar) and / or general proficiency in the working language (as mirrored in a C-test), if this academic proficiency is to be developed and used in a range of different subjects at secondary school level. (Zydatiß, 2012, t. 26)

Ar sail ei ganfyddiadau, ni chred Zydatiß y dylid lledaenu rhaglenni CLIL ar raddfa ehangach gan fod angen cyrraedd lefel uchel o hyfedredd ieithyddol, yn arbennig o ran gramadeg a geirfa, er mwyn cyrraedd y trothwy ieithyddol isaf, y lefel isaf o hyfedredd er mwyn deall mewnbwn yn yr iaith darged.

Mewn astudiaeth arall gan Bonnet (2004, fel y dyfynnwyd yn Dalton-Puffer, 2008), astudiwyd disgyblion CLIL a disgyblion ar raglen draddodiadol o ran eu gallu i ddefnyddio eu hiaith mewn cyd-destun pragmatig wrth iddynt ddatrys problemau mewn trafodaeth ar gemeg. Er bod nifer o achosion o newid cod i'r iaith gyntaf, sylwodd Bonnet na fyddai troi at y famiaith yn datrys y broblem bob tro. Awgryma hyn nad yw disgyblion CLIL o dan anfantais, ond yn hytrach mae disgyblion CLIL yn cymharu'n ffafriol â disgyblion y rhaglen draddodiadol o ran deall cysyniadau pwnc-benodol (Breidbach \& Viebrock, 2012). Mae'n bosibl fod y prosesu yn yr ail iaith yn gallu cyfrannu at ddysgu cysyniadau pwnc-benodol (Dalton-Puffer, 2008, 2011). Mae Vollmer, Heine, Troschke, Coetzee a Küttel (2006, fel y'u dyfynnwyd yn Dalton-Puffer, 2008, 2011) hefyd o'r farn nad yw'r anhawster ieithyddol hwn yn annog disgyblion i roi'r gorau i'r dasg. Yn hytrach, maent yn credu, gan fod angen i ddisgyblion CLIL ymdopi â chwblhau tasgau yn yr ail iaith trwy’r amser, fod ganddynt well rheolaeth ar rwystredigaeth sydd, o bosibl, yn arwain at adeiladu dealltwriaeth gryfach a gwell o gysyniadau cwricwlaidd.

Caiff canfyddiadau Bonnet (2004) a Vollmer ac eraill (2006) eu hategu ymhellach yn astudiaethau Wode (1999) a Koch (2005) sy'n cymharu dysgwyr CLIL a disgyblion y ffrwd draddodiadol. Yn hytrach na darganfod rhwystrau wrth ddysgu cynnwys pwnc-benodol, canfuwyd bod gan ddysgwyr CLIL wybodaeth gystal â'u cyfoedion yn y brif ffrwd mewn perthynas â Hanes a Daearyddiaeth (Wode, 1999), a gwybodaeth uwch na'u cyfoedion prif ffrwd mewn perthynas â Gwyddoniaeth 
(Koch, 2005). Er gwaetha'r rhwystrau naturiol a ddaw wrth ddysgu cynnwys trwy gyfrwng yr ail iaith, mae'n bosibl bod gan ddisgyblion CLIL y gallu i ymdopi â'r rhain ac mae hyn o bosibl yn arwain at adeiladu a chryfhau prosesu cysyniadau pwnc-benodol. Mae'r canfyddiadau hyn hefyd yn atgyfnerthu'r gred fod siaradwyr dwyieithog (cytbwys) yn elwa'n wybyddol a deallusol oherwydd eu bod yn llawer mwy hyblyg yn y ffordd y maent yn deall cysyniadau, o'u cymharu â'u cyfoedion uniaith. (Am drafodaeth lawnach, gweler Pennod 3.) Er hynny, rhaid bod yn ofalus wrth ddadansoddi'r canfyddiadau hyn oherwydd ni ellir ystyried y dysgwyr CLIL yn yr astudiaethau a astudiwyd yn siaradwyr dwyieithog cytbwys.

\subsubsection{Casgliadau ar CLIL yn yr Almaen a'r tu hwnt}

Mae ymchwil yn yr Almaen wedi cyfrannu at y gronfa gynyddol ym maes CLIL, gan awgrymu bod dysgwyr y rhaglen CLIL yn perfformio'n well yn yr ail iaith yn arbennig o ran geirfa (Burmeister \& Daniel, 2002), darllen a deall (Bredenbröker, 2000). Fodd bynnag, nid yw'r garfan hon o ddisgyblion yn perfformio'n sylweddol yn well na'u cyfoedion o ran gramadeg (Bredenbröker, 2000), mae'n debyg. Mae'r ymchwil hon hefyd yn cefnogi ar y cyfan y canfyddiadau cynnar ar addysg drochi a drafodwyd eisoes yn y traethawd hwn. Mae'r canfyddiadau ar ddysgu cynnwys pwnc-benodol trwy gyfrwng yr iaith dramor ar y cyfan wedi bod yn llai argyhoeddiadol, ac mae'n debyg fod hyn yn wir yn gyffredinol ar gyfer ymchwil ar CLIL, oherwydd diffyg profion safonol ar gyfer mesur cyrhaeddiad mewn pynciau CLIL megis Gwyddoniaeth a'r Astudiaethau Cymdeithasol (Dalton-Puffer, 2011, t. 188). Anodd felly yw i'r ysgolhaig gymharu canfyddiadau o'r naill astudiaeth a'r llall. Mae rhai canfyddiadau ar ddysgu cynnwys trwy gyfrwng yr iaith dramor yng nghyd-destun yr Almaen wedi bod yn gadarnhaol, gan awgrymu nad yw disgyblion CLIL o dan anfantais o'u cymharu â'u cyfoedion o ran deall cynnwys pwnc-benodol, ond yn hytrach mae'n debyg eu bod ar lefel gymharus â'u cyfoedion (Breidbach \& Viebrock, 2012). Mae'n bosibl fod hyn yn digwydd gan fod disgyblion CLIL, sy'n gorfod canolbwyntio ar gynnwys trwy'r amser er mwyn deall ar dasgau, yn dangos gwell rheolaeth o ran dygymod â rhwystredigaeth, a bod hyn yn arwain at well dealltwriaeth o gysyniadau (Vollmer ac eraill, 2006, fel y dyfynnwyd yn DaltonPuffer, 2008; 2011). Caiff y canfyddiadau hyn eu cefnogi gan astudiaethau eraill ar CLIL ar draws Ewrop sydd wedi awgrymu bod dysgwyr CLIL yn sicrhau cymhwysedd uwch yn yr ail iaith / iaith dramor (Pérez-Cañado \& Lancaster 2017; 
Pérez-Cañado 2018b), yn ogystal â gwybodaeth gystal mewn pynciau cwricwlaidd, megis Mathemateg a Gwyddoniaeth (Jäppinen, 2005), a hyd yn oed gwybodaeth uwch mewn Mathemateg (Surmont ac eraill, 2016; Ouazizi 2016) a'r Gwyddorau Naturiol (Pérez-Cañado 2018a), o’u cymharu â’u cyfoedion prif ffrwd.

Wrth reswm, dylid trin y canfyddiadau hyn gyda gofal. Mae cymharu disgyblion CLIL â disgyblion y brif ffrwd yn gallu cynhyrfu'r dyfroedd, yn arbennig yng nghyd-destun yr Almaen oherwydd yr elfen 'elitaidd' sy'n gysylltiedig â rhaglenni CLIL (Apsel, 2012). Nid yw CLIL yn orfodol yn yr Almaen, ac mae disgyblion CLIL yn cael eu dewis mewn proses ddethol, lle mae gradd gyfartaledd yn y Saesneg yn yr ysgol gynradd fel arfer yn ganolog i'r dewis hwn (Apsel, 2012, t. 50). Mae ymchwil Zydatiß (2012), er enghraifft, wedi awgrymu bod perfformiad academaidd a pherfformiad ieithyddol yn gyd-ddibynnol, fel yr oedd Cummins (1979b) wedi'i ddamcaniaethu. Y pryder a all godi wrth gymharu disgyblion CLIL â disgyblion eraill yw y gallai fod gan ddisgyblion CLIL, neu ddarpar ddisgyblion CLIL, fantais ar ddisgyblion eraill o'r cychwyn cyntaf, ac felly mae hyn yn ei wneud yn anodd cymharu'n empirig p'un ai'r mewnbwn a geir trwy'r rhaglen CLIL, ynteu ffactorau eraill, er enghraifft, o ran gallu gwybyddol ac ieithyddol, cymhelliant, neu ddangosiad i'r iaith cyn derbyn y ddarpariaeth Gymraeg ychwanegol, sy'n effeithio fwyaf ar y canfyddiadau hyn. Fel y mae Pérez-Cañado $(2012,2016)$ yn ei rybuddio, mae gan nifer o'r astudiaethau ar CLIL sydd wedi'u cynnal hyd yn hyn wendidau methodolegol. Er mwyn deall yn well sut effaith y caiff darpariaeth CLIL ar ddatblygu iaith gyntaf, ail iaith a dysgu cynnwys pynciol, noda Pérez-Cañado (2016, t. 19) 11 o newidynnau y dylid ceisio eu rheoli, sef: math o ysgol (e.e. ysgolion a gynhelir, ysgolion nas chynhelir); talaith; ardal (e.e. ardal wledig, ardal drefol); statws sosio-economaidd; cymhelliant; deallusrwydd geiriol; lefel o ddeallusrwydd o'r iaith darged; cyswllt â'r iaith darged o fewn ac y tu hwnt i'r ysgol; a cymhwysedd ieithyddol yr athrawon CLIL. Ys dywed hi:

"...what quantitative investigation there is rarely guarantees the homogeneity of the treatment and Control groups, factors in moderating vaiables, or performs statistical analyses to determine whether the gains observed are truly ascribable to CLIL practice ... Thus, further research is clearly called for in painting a comprehensive and empirically valid picture of where CLIL schemes stand in our continent." (Pérez-Cañado, 2012, t. 330) 
Mae astudiaethau ar CLIL mewn cyd-destunau eraill wedi cefnogi'n gryf yr honiad mai oherwydd cyswllt uwch â'r iaith darged yn bennaf y mae disgyblion CLIL, er enghraifft, yn ennill lefelau sylweddol uwch o eirfa oddefol (Agustín-Llach, \& Canga Alonso, 2016; Catalán \& de Zarobe, 2009; Sylven, 2010) a geirfa oddefol a chynhyrchiol (Merikivi \& Pietilä, 2014) yn yr iaith darged. Daw Ibarrola (2012) i gasgliad tebyg, gan briodoli effaith gadarnhaol y rhaglen CLIL ar gymhwysedd gramadegol y dysgwyr i'r ffaith bod CLIL yn darparu rhagor o gyswllt â'r iaith darged. Caiff yr honiad hwn ei gefnogi hefyd gan Gierlinger ac Wagner (2016) na chanfu gwahaniaeth sylweddol rhwng dysgwyr sydd wedi derbyn 60-80 awr ychwanegol o wersi CLIL a dysgwyr heb gael CLIL o ran datblygiad eu geirfaoedd goddefol. Ymddengys fod angen cyrraedd isafswm o oriau cyswllt ychwanegol er mwyn i ddysgwyr elwa ar fanteision y mewnbwn ychwanegol a geir trwy ddarpariaeth CLIL. Caiff yr honiad hwn ei atgyfnerthu yn adolygiad Muñoz (2015) o astudiaethau ar CLIL sy'n canolbwyntio ar effaith amser CLIL, ac yn fwy diweddar yn astudiaeth Artieda, Roquet a Nicolás-Conesa (2017), sy'n mynd cyn belled ag awgrymu bod angen i ddysgwyr dderbyn 300 awr neu ragor o wersi CLIL cyn y gellir canfod effaith y mewnbwn ychwanegol.

Er nad yw'n bosibl priodoli darpariaeth CLIL yn unig i'r canfyddiadau hyn, mae'r ymchwil hon yn awgrymu o leiaf fod cydberthynas rhwng y mewnbwn a geir a sgiliau ieithyddol uwch yn yr ail iaith. Fodd bynnag, er mwyn cadarnhau'r canfyddiadau hyn, bydd angen ymchwil bellach sy'n archwilio effeithlonrwydd CLIL ar gyfer mathau eraill o ddysgwyr (Breidbach \& Viebrock, 2012; Zydatiß, 2012), gan gynnwys dysgwyr sy'n tangyflawni (Apsel, 2012), yn ogystal ag effaith CLIL ar ddysgwyr dros gyfnod o amser drwy gynnal rhagor o astudiaethau hydredol (Pérez-Cañado, 2012, 2016). 


\section{Pennod 5: Y gymhariaeth genedlaethol: Cynlluniau trochi a dysgu dwys yng}

Nghymru

\subsection{Trosolwg o'r cynlluniau}

Mae'r ymchwil ar addysg drochi sydd bellach ar gael yn sylweddol ac mae wedi bod yn ddylanwadol ar ymdrechion addysgol rhyngwladol i gyflwyno'r ail iaith mewn ffordd bwrpasol a chyd-destunol. Erbyn hyn, mae addysg drochi yng Nghanada wedi dylanwadu ar addysg ddwyieithog yn Ewrop a thu hwnt, wrth i nifer o wledydd fabwysiadu'r hyn a wneid yng Nghanada i fod yn addas i'w cyd-destunau unigryw (Baker \& Jones, 1998, t. 501). Nid eithriad mo Cymru. Wrth reswm, nid yw addysg drochi fel cysyniad yn un newydd yng nghyd-destun Cymru, gan mai trochi yn y Gymraeg yw'r hyn a ddigwydd ym mhrofiad y mwyafrif o ddisgyblion mewn ysgolion cyfrwng Cymraeg a dwyieithog, yn arbennig yn y de-ddwyrain a'r gogledd ddwyrain (Williams, 2008, t. 20).

Bu profiadau addysg drochi yng Nghanada yn ddylanwadol ar gomisiynu Cynllun Peilot Trochi a Dysgu Dwys Bwrdd yr Iaith Gymraeg yn 2003, yn dilyn ymweliad Jane Davidson, y Gweinidog dros Addysg a Dysgu Gydol Oes ar y pryd, a’i dirprwyaeth â Newfoundland, Labrador a New Brunswick yng Nghanada i ddarganfod y ffeithiau ynglŷn ag addysg drochi yn y Ffrangeg a gwerthuso'r modelau yno a'r potensial i'w mabwysiadu yng Nghymru. Bu'r ymchwil ryngwladol ar addysg drochi hefyd yn ddylanwadol ar ddatblygu'r cynllun hwn ymhellach rhwng 2003 a 2008 wrth i'r ymgynghorydd arbenigol Cen Williams lunio papurau i Bwyllgor Llywio'r prosiect er mwyn dysgu gwersi oddi wrth y llenyddiaeth ryngwladol ar addysg drochi a dysgu drwy gynnwys. Er gwaethaf cyfraniad ymchwil ryngwladol a theorïau trochi ieithyddol at y dasg o roi siâp ar Gynllun Peilot Trochi a Dysgu Dwys Bwrdd yr Iaith Gymraeg, roedd nifer o ystyriaethau ymarferol a gafodd effaith ar ddatblygiad y Cynllun (Oldbell3 \& Dateb, 2008, t. 33). O ganlyniad i hyn, bu modelau terfynol y Cynllun Peilot Cenedlaethol hwn yn wahanol iawn i'r modelau Canadaidd a gafodd eu harsylwi a'u haddasu ar gyfer y cyd-destun Cymreig.

Yn ogystal â hyn, mae'n hollbwysig nodi bod un cynllun trochi arall wedi bodoli eisoes cyn i LlCC gomisiynu Bwrdd yr Iaith Gymraeg i weithredu'r cynllun peilot yn 2003. Rhwng 1994 a 1999, bu Cen Williams (2002) yn astudio cynnydd grŵp o 
ddisgyblion nad oeddent wedi derbyn addysg gynradd cyfrwng Cymraeg wrth iddynt gael eu trochi yn y Gymraeg ar Gynllun Trochi Hwyr Ysgol Maes Garmon. Ar y $\operatorname{pryd}^{38}$, dyma oedd yr "unig sefyllfa drochi 11-16 oed yng Nghymru lle ceir dosbarth cyfan yn cael iaith o'r newydd" (Williams, 2002, "Cyflwyniad", para. 6). Er mai bwriad gwaith Cen Williams oedd "casglu syniadau ar gyfer cyrsiau datblygiad staff trwy ymweld â'r sefyllfa real yn hytrach na dibynnu ar ymchwil a chanfyddiadau unigolion eraill” (Williams, 2008, t. 23), mae hefyd yn bosibl fod gan yr ymchwil hon gyfraniad ehangach at ddylanwadu ar bolisi cyhoeddus hefyd wrth gynnig "her i'r ysgolion hynny sydd y tu allan i'r sector ddwyieithog swyddogol i fabwysiadu elfennau o addysgu dwyieithog lle mae staffio'n caniatáu hynny (ac i ymestyn hynny i fod yn bolisi maes o law)" (Williams, 2008, t. 24). Gan fod ymchwil ddigonol ar y cynllun trochi hwyr a gynigir yn Ysgol Maes Garmon erbyn hyn, a chan nad oes ymchwil ar y cynlluniau diweddar sydd wedi ymddangos, trafodir Cynllun Trochi Hwyr Ysgol Maes Garmon yn unig yma.

Yn y ddau gynllun a enwir uchod, y nod yw creu pwynt mynediad hwyr er mwyn i ddisgyblion symud o'r sector cyfrwng Saesneg i'r sector cyfrwng Cymraeg yn 11 oed. Roedd sicrhau pwyntiau mynediad hwyr yn ystyriaeth amlwg yn Strategaeth Addysg Cyfrwng Cymraeg, lle nododd LlCC (2010, t. 8) y byddai'r strategaeth yn “darparu ar gyfer y posibilrwydd y bydd rhai dysgwyr yn cael mynediad i addysg cyfrwng Cymraeg ar gyfnod ar ôl tair oed”. Fodd bynnag, er pwysiced sicrhau pwyntiau mynediad hwyr i gynyddu nifer y disgyblion o'r sector Saesneg sy'n gallu trosglwyddo i addysg cyfrwng Cymraeg, ni cheir ystyriaeth yn y cynlluniau hyn am sut mae trawsnewid ysgolion cyfrwng Saesneg yn ysgolion dwyieithog a chyfrwng Cymraeg. Fodd bynnag, fel y cydnebydd L1C (2017b; 2017ch) yn ei strategaethau Cymraeg 2050 a'r Y Gymraeg mewn Addysg, rhaid i ysgolion bellach symud ar hyd y continwwm ieithyddol tuag at ddatblygu'n ysgolion dwyieithog a chyfrwng Cymraeg.

Yn y trydydd cynllun a drafodir fodd bynnag, sef y Cwrs Carlam yn 'Ysgol X', ${ }^{39}$ nod gwreiddiol y cynllun oedd "hyrwyddo, cefnogi a gwella perfformiad ieithyddol

\footnotetext{
${ }^{38}$ Erbyn hyn, mae nifer o ysgolion cyfrwng Cymraeg eraill yn cynnig cynlluniau trochi hwyr tebyg $\mathrm{i}$ ddisgyblion. Enghreifftiau o'r ysgolion hyn yw Ysgol Glan Clwyd, Ysgol Gyfun Gymraeg Bryn Tawe ac Ysgol y Creuddyn.

${ }^{39}$ Cadwyd enw'r ysgol yn gyfrinachol yn y traethawd gan i'r ymchwilydd benderfynu cynnal gwaith maes yn yr ysgol hon.
} 
disgyblion ail iaith Cyfnod Allweddol 3 drwy gynnig cyfle iddynt astudio rhai pynciau drwy gyfrwng y Gymraeg” (Guilfoyle, 2008, t. 32). Erbyn hyn, mae'r cynllun wedi esblygu ac mae gan Ysgol X enw o fod yn ysgol sy'n cynnig darpariaeth Gymraeg sy'n arwain y sector cyfrwng Saesneg (Estyn, 2012b, 2016). Dyma enghraifft unigryw felly o ysgol uwchradd cyfrwng Saesneg yng Nghymru sydd wrthi'n cynnig cynllun o'r fath sydd am wella cyrhaeddiad mewn CAI, yn hytrach na chynnig pwyntiau mynediad eraill i addysg cyfrwng Cymraeg. Er gwaethaf gwahaniaethau rhwng y tri chynllun, maent wedi cyfrannu at y gronfa gymharol fach o dystiolaeth ymchwil yng Nghymru, yn ogystal â'r gronfa gymharol fawr o dystiolaeth ymchwil ryngwladol, sydd wedi awgrymu'n gyson fod cyflwyno Cymraeg mewn ffordd fwy pwrpasol, cyd-destunol, a hynny trwy gyflwyno cynnwys trwy gyfrwng yr iaith ei hun, yn llwyddo i greu siaradwyr sydd â hyfedredd uwch yn eu hail iaith. Fel y noda Cen Williams (2008) wrth drafod canfyddiadau cyffredinol Cynllun Bwrdd yr Iaith Gymraeg:

Roedd mwyafrif llethol os nad y cyfan o'r egwyddorion a'r seiliau ymchwil a drafodwyd yng nghyd-destun addysg drochi Canada ac Unol Daleithiau'r America hefyd i'w gweld ar waith yma yng Nghymru. Nid yw ein dulliau na'n seiliau yn isradd i'r arweinwyr rhyng-genedlaethol yn y byd hwn. (Williams, 2008, t. 27)

\subsection{Cynllun Trochi Hwyr Ysgol Maes Garmon (1994-1999)}

\subsubsection{Cefndir y Cynllun Trochi Hwyr}

Cyn iddo gael ei benodi'n ymgynghorydd arbennig i Bwyllgor Llywio Cynllun Trochi a Dysgu Dwys Bwrdd yr Iaith Gymraeg rhwng 2003 a 2008 a fyddai'n gyfrifol am adrodd ar ganfyddiadau ymchwil ryngwladol a llunio deunyddiau hyfforddi i athrawon, bu Cen Williams yn gysylltiedig ag ymchwil astudio'r unig sefyllfa ar y pryd i gynnig trochi ieithyddol yn y Gymraeg i ddisgyblion nad oeddent wedi derbyn addysg gynradd cyfrwng Cymraeg. Canolbwynt yr ymchwil hon oedd datblygu deunyddiau a dulliau addysgu trochi ar lawr dosbarth yn Ysgol Maes Garmon. Yn ei astudiaeth Ennill Iaith (2002), astudiodd Williams garfan o ddisgyblion ${ }^{40}$ wrth iddynt ymuno â'r ysgol ym Mlwyddyn 7 ym mis Gorffennaf 1994

\footnotetext{
${ }^{40}$ Roedd yn rhaid i Williams (2008) ddilyn unigolion yn hytrach na dosbarth cyfan ym Mlynyddoedd
} $9-11$. 
hyd at gwblhau eu hastudiaethau gorfodol ym Mlwyddyn 11 ym mis Mawrth 1999. Dewiswyd yr ysgol hon ar sail nodweddion canlynol yr ysgol:

- record yr ysgol gyda dosbarthiadau dysgwyr tebyg yn y gorffennol;

- y ffordd y mae'r ysgol yn addasu'r cwricwlwm ym mlwyddyn 7 er mwyn cyflymu'r broses caffael ail iaith;

- cynllun yr adran Gymraeg ar gyfer blwyddyn 7;

- y ffordd y mae eu llwyddiant yn cael ei ragfynegi mewn theori;

- y ffaith bod yr athrawon yn ymwybodol o'u cyfrifoldeb i ddatblygu dwyieithrwydd y disgyblion;

- bod hwn yn fodel ar gyfer ysgolion eraill yng Nghymru.

(Williams, 2002, t. 5)

Bu gwaith Ennill Iaith yn ymestyniad o'i waith Ph.D., sef Arfarniad o Ddulliau Dysgu ac Addysgu yng Nghyd-destun Addysg Uwchradd Ddwyieithog (1994), lle yr arsylwodd Williams (1994) strategaethau addysgu dwyieithog ar lawr dosbarth mewn nifer o ysgolion uwchradd dwyieithog yng Ngwynedd er mwyn cyflwyno'r syniadaeth ar gyfer creu pecyn hyfforddiant mewn swydd ar gyfer athrawon uwchradd y sir a hefyd athrawon uwchradd Cymraeg drwy Gymru gyfan.

Seiliodd Williams astudiaeth Ennill Iaith ar Ddamcaniaeth y Trothwy Ieithyddol (Cummins, 1976; Toukomaa a Skutnabb-Kangas, 1977) a'r ddau allu ieithyddol a adwaenir gan Cummins (1979a) yn Sgiliau Cyfathrebol Rhyngbersonol Sylfaenol (Basic Interpersonal Communicative Skills (BICS o hyn ymlaen)) a Sgiliau Cyfathrebol Rhyngbersonol Sylfaenol (Cognitive Academic Language Proficiency (CALP o hyn ymlaen)). Mae'r ddamcaniaeth hon yn honni bod angen meistroli'r ddau allu ieithyddol hyn er mwyn cyrraedd hyfedredd llawn mewn iaith. Mae BICS yn gyd-destunol, ac nid yw'n heriol yn wybyddol. Mae CALP fodd bynnag yn llai dibynnol ar gyd-destun ac yn fwy heriol yn wybyddol. Cred Cen Williams (2002, t. 6) fod y ddamcaniaeth hon yn taflu goleuni ar pam nad yw dysgu Cymraeg fel petai'n bwnc iaith dramor, wrth ganolbwyntio ar ramadeg, wedi llwyddo. Os na chanolbwyntir ar ddatblygu CALP, lle ceir dealltwriaeth gyd-destunol o'r iaith, yn ogystal â datblygu BICS yn ddigonol, nid yw'n bosibl i ddisgyblion ail iaith feistroli'r ail iaith yn llawn. Wrth gyflwyno pynciau eraill trwy gyfrwng yr ail iaith, dadleuir nad yw'r disgyblion yn canolbwyntio ar ddysgu'r ail iaith ar lefel ymwybodol, meta-ieithyddol, yn hytrach maent yn canolbwyntio ar ddeall cynnwys 
y gwersi gan mai dyna'r nod (Williams, d.d., t. 29). Mae Williams (2002) yn ategu mai trwy addysgu mewn ffordd bwrpasol a chyd-destunol, a hynny trwy gyfrwng y Gymraeg, yn unig y ceir llwyddiant go iawn:

Mae profiadau'r gorffennol wedi dangos mai wrth ddefnyddio'r iaith yn gyfrwng y daeth y llwyddiant mwyaf dwys a pharhaol.

(Williams, 2002, t. 53).

Er pwysiced CALP, mae Williams fodd bynnag yn ildio i'r ffaith bod datblygu BICS yn ddigonol yn ogystal â'r CALP wrth ddatblygu siaradwyr dwyieithog yn hollbwysig. Wrth ystyried terfyniadau posibl ar y ddamcaniaeth, noda Williams (d.d.) mai:

Barn rhai ymchwilwyr bellach yw bod rhai disgyblion sy'n caffael ail iaith trwy ddulliau dysgu pynciol yn cyrraedd lefel uwch yn y CALP sef y dysg pynciol nag y maen nhw yn y BICS. Mae hyn yn groes i'r disgwyl a'r rheswm sy'n cael ei gynnig yw eu bod yn dueddol o droi i'r famiaith (Saesneg yn achos Cymru) ar gyfer cyfathrebu ar lefel is gyda'u cyfeillion a chydag athrawon. (Williams, d.d., t. 9).

Fel y mae Williams yn ei rybuddio, mae'n bosibl i ddisgyblion gyrraedd lefel uchel yn y CALP ond heb gyrraedd lefel uchel yn y BICS. Mae Baker a Jones (1998) hefyd wedi mynegi pryderon wrth ddosbarthu gallu ieithyddol siaradwyr yn unol â BICS a CALP. Noda Baker a Jones fod y fath ddosbarthu'n gallu camarwain o ran gwir hyfedredd siaradwyr yn eu hail iaith:

...the distinction paints a two stage idea. A child would seem to be in one category or another. Instead, children and adults move forward on multiple language dimensions with various sliding scales (e.g. listening, speaking, reading, writing) rather than one big jump. (Baker \& Jones, 1998, t. 94)

\subsubsection{Prif nodweddion y cynllun}

Mynega Williams (2002, t. 12) fod y newidiadau a wneir i'r cwricwlwm y mae Ysgol Maes Garmon yn ei gynnig i weddill y disgyblion ${ }^{41}$ yn galluogi'r disgyblion i feistroli'r agweddau cyfathrebol (BICS) tra hefyd yn defnyddio'r Gymraeg yn

\footnotetext{
${ }^{41}$ Cyfeiria Williams (2002, t. 7) at dair carfan ieithyddol o ddisgyblion sef 'Cymry' a gafodd eu haddysg gynradd trwy gyfrwng y Gymraeg ac sy'n dod o gartrefi Cymraeg, 'dysgwyr' a gafodd eu haddysg gynradd trwy gyfrwng y Gymraeg ond nad ydynt yn dod o gartrefi Cymraeg, a'r 'dysgwr' a gafodd eu haddysg gynradd trwy gyfrwng y Saesneg ac sydd yn dod o gartref Saesneg.
} 
bynciol mewn modd academaidd / gwybyddol (CALP) o'r cychwyn cyntaf. Gellir nodi'r newidiadau ym Mlwyddyn 7 fel a ganlyn:

- cwrs integredig gan gynnwys pynciau'r dyniaethau sef Hanes,

Daearyddiaeth a Drama, gyda'r Gymraeg am 9 awr yr wythnos. 5 gwers gan yr athro Cymraeg, 2 wers gan athro Daearyddiaeth, 2 wers gan yr athro Hanes.

- peidio â chynnig Ffrangeg ${ }^{42}$.

- peidio â rhoi hanner gwers côr i'r grŵp bob wythnos.

(Williams, 2002, tt. 10-11)

Erbyn Blwyddyn 9, caiff y garfan o ddisgyblion trochi eu diddymu wrth i'r disgyblion ymuno â'r disgyblion eraill ym mhob pwnc. Fel y gwelir uchod, mae'r cwrs integredig a gynigir ym Mlwyddyn 7 yn cynnwys peth addysg Gymraeg mewn pynciau na fyddai'r disgyblion wedi'u profi trwy gyfrwng y Gymraeg. Er mai prif nod athrawon y pynciau hyn oedd addysgu cynnwys y gwersi, noda Williams fod ganddynt gyfraniad pwysig megis athrawon iaith hefyd. Mae hyn yn amlwg yn isadrannau 3.2 a $3.3 \mathrm{yr}$ astudiaeth, wrth i Williams ddadansoddi strategaethau athrawon i hwyluso'r trochi tra hefyd yn cyflwyno eu pynciau eu hunain. Gan arsylwi gwers un o'r athrawon Hanes, noda Williams y canlynol o ran ei arddull addysgu:

- adolygu rhai geiriau

- gwahodd mwy o siarad gan y disgyblion

- ymestyn i ddisgyblaeth arall er mwyn rhoi cyfle iddyn nhw ddefnyddio geirfa y cawson nhw brofiad ohoni eisoes yn yr ysgol

- annog a chanmol gan roi'r argraff ei fod yn gwirioneddol fwynhau cyfraniadau'r dosbarth

- [bod yn] amyneddgar gan roi cyfle i un ferch gael dweud ei brawddeg er bod hynny'n cymryd cryn dipyn o amser

- Rhoi cyfle i nifer o blant gyfrannu er mwyn rhoi'r cyfle iddyn nhw ddefnyddio'r Gymraeg. (Williams, 2002, tt. 20-21)

\footnotetext{
${ }^{42} \mathrm{Ni}$ chaiff y Ffrangeg ei cholli fodd bynnag oherwydd caiff ei chyflwyno yn ystod cwrs o 10 awr ym mis Gorffennaf yn ogystal â thair gwers ym Mlwyddyn 8 .
} 
Mae Williams (2002, t. 21) yn nodi ymhellach y gwelir bod athrawon y dyniaethau sy'n rhan o'r cwrs integredig yn talu "sylw sensitif i'r elfen gyfathrebol (BICS)", ond eu bod yn “ymwybodol o ddatblygu'r iaith bynciol (CALP) ac o'u dyletswydd i addysgu'r pwnc." Mae'r strategaethau hyn yn y dosbarth yn dangos bod yr athro yn ymwybodol o lefel ieithyddol y disgyblion a'i fod yn mynd ati'n fwriadol i gyflwyno ei bwnc tra hefyd yn talu sylw i anghenion ieithyddol y dosbarth.

Yn ogystal ag iaith cyfrwng yr addysg a geir ar gynllun Ysgol Maes Garmon, pwysleisia Williams (2002, t. 20) hefyd bwysigrwydd cwrs allgyrsiol i lwyddiant y cynllun, wrth fynd cyn belled â dweud bod "Cwrs Glan-llyn yn drobwynt yn natblygiad ieithyddol mwyafrif y dosbarth...Dyma'r ysgogiad iddyn nhw ddechrau teimlo'n naturiol yn cyfathrebu drwy'r Gymraeg a daeth yr iaith yn iaith fwy gweithredol iddyn nhw." Ymddengys fod y cwrs hwn yn cynnig cyfle i ddatblygu sgiliau BICS ymhellach mewn cyd-destun cyfeillgar ac anffurfiol, sydd hefyd yn allweddol ar gyfer mabwysiadu agweddau cadarnhaol at ddefnyddio'r Gymraeg tu hwnt i'r dosbarth dysgu. Mae sicrhau'r cyswllt anffurfiol â'r Gymraeg wedi bod yn un o bryderon cyson Estyn dros y blynyddoedd ynglŷn â CAI yn arbennig (gweler, er enghraifft, Estyn, 2014, 2015). Mae Davies (2013), fel y gwelir yn argymhelliad 24 ei hadroddiad, hefyd wedi codi'r pryder hwn. Mae'n glir felly, yn ogystal ag ystyried sut mae cyflwyno'r Gymraeg yn ail iaith yn y sector cyfrwng Saesneg o safbwynt pedagogeg, ei bod yn hollbwysig ystyried y cyswllt anffurfiol â'r Gymraeg, a sut y gall ysgolion cyfrwng Saesneg rannu'r cyfrifoldeb o gyflwyno mwy o gyfleoedd i ddisgyblion glywed a siarad y Gymraeg y tu hwnt i gyd-destun y dosbarth dysgu, boed yn yr ysgol neu yn y gymuned. Er pwysiced datblygu sgiliau CALP, rhaid hefyd sicrhau sgiliau BICS disgyblion ail iaith. Fel arall, ni ellir disgwyl llwyddiant o ran creu siaradwyr sydd yn defnyddio'r iaith yn bwrpasol ac yn gyfathrebol mewn cyd-destunau bob dydd.

\subsubsection{Gwerthusiad o'r cynllun trochi hwyr}

Casgla Williams (2002) fod canfyddiadau'r astudiaeth ar Gynllun Trochi Hwyr Ysgol Maes Garmon yn dangos egwyddorion theori yn y maes ar waith mewn cyddestun ymarferol, yn enwedig gwaith Milk (1990), Christian ac eraill (1990) a Cummins (1980, 1991). Ar sail ei gasgliadau, cynnig Williams (2002) y tri argymell canlynol er mwyn cyflymu'r broses o ddatblygu disgyblion dwyieithog: 
- dylai pob ysgol fabwysiadu polisi sy'n gosod cyfrifoldeb ar adrannau am ddatblygu gwahanol agweddau ieithyddol sy'n digwydd yn naturiol yng ngwaith yr adran

- o fewn polisi cyfannol o'r fath dylai rôl yr adrannau iaith hefyd gael ei diffinio'n glir fel nad yw'r holl gyfrifoldeb a disgwyliadau yn disgyn arnyn nhw

- dylai pob adran o fewn yr ysgol fod yn glir ynglŷn â'i chyfrifoldeb ieithyddol ei hun. (Williams, 2002, t. 49)

Mae Williams (2008) o'r farn fod gan ysgolion cyfrwng Saesneg eu rôl arbennig eu hunain yn y broses o greu Cymru ddwyieithog, ac er mai cynyddu nifer y disgyblion o'r sector cyfrwng Saesneg a all gael mynediad at addysg cyfrwng Cymraeg yw bwriad y fath gynllun, cred y gallai ysgolion cyfrwng Saesneg gynnig peth addysg cyfrwng Cymraeg drwy ystyried cyflwyno'r Gymraeg fel iaith cyfrwng yn y pynciau canlynol, yn dibynnu ar afael y disgyblion ar y Gymraeg:

Cam 1: Pynciau ymarferol, e.e. Addysg Gorfforol, Technoleg, Celf: Dyma'r pynciau y gellid eu cyflwyno'n gyntaf i ddysgwyr lle y mae nifer ohonyn nhw'n weddol brin eu gafael ar yr ail iaith.

Cam 2: Pynciau fel Mathemateg a Gwyddoniaeth: Pynciau lle mae'r elfen ieithyddol yn cael ei chyflwyno mewn cyfnodau byrion sy'n arwain at wneud, cymhwyso a.y.y.b.

Cam 3: Pynciau dyniaethol gan gynnwys Addysg Grefyddol, Daearyddiaeth a Hanes: Pynciau sy'n mynnu meistrolaeth lefel uchel ar iaith y cyflwyno neu fe allai perfformiad yn y pwnc ddioddef.

(Williams, 2002, t. 50, 2008, tt. 28-29)

\subsection{Cynllun Peilot Trochi a Dysgu Dwys Bwrdd yr Iaith Gymraeg (2003 -2008)}

\subsubsection{Cyd-destun y Cynllun Trochi Canolig}

Cyn y Cynllun Peilot Trochi a Dysgu Dwys a lansiwyd yn 2003, roedd mynediad at addysg cyfrwng Cymraeg yn gyfyngedig ar ôl yr oed ysgol gorfodol, sef 5 oed. Wrth ystyried bod rhai rhieni wedi dewis addysg cyfrwng Saesneg i'w plant erbyn hyn, roedd diffyg pwyntiau mynediad hwyrach iddynt drosglwyddo eu plant $\mathrm{i}$ addysg cyfrwng Cymraeg (Davidson, 2001). Yn 2001, ymwelodd Jane Davidson AC (2001, t. 6), y Gweinidog dros Addysg a Dysgu Gydol Oes, a'i dirprwyaeth â

Newfoundland, Labrador a New Brunswick yng Nghanada yn 2001 er mwyn “archwilio'r amrywiaeth o fodelau trochi neu ddwys mewn ail iaith a ddefnyddir" yno a gwerthuso'r modelau Canadaidd a'r potensial i fabwysiadu ac addasu rhai 
ohonynt ar gyfer y cyd-destun Cymreig. O blith modelau gweithredol posibl a arsylwyd gan Davidson a'r ddirprwyaeth, sef Ffrangeg Craidd Llawnach, Ffrangeg Craidd Dwys a Throchi yn y Ffrangeg, casglodd Davidson a'i dirprwyaeth (2001, fel y dyfynnwyd yn Oldbell3 \& Dateb, 2008, t. 27) mewn adroddiad ar ôl eu hymweliad fod angen ystyried cynnal peilot ym Mlwyddyn 6 ar sail y model Ffrangeg Craidd Dwys. Ym marn Davidson (2001, t. 6), dyma oedd yr unig fodel a allai gynnig ail bwynt mynediad at addysg cyfrwng Cymraeg i ddisgyblion ail iaith a ddechreuodd eu haddysg yn y sector cyfrwng Saesneg o 5 oed ymlaen. Casglodd Davidson (2001) ymhellach mai bwriad y cynllun peilot fyddai galluogi disgyblion cynradd a addysgwyd yn Saesneg i wneud y canlynol:

- trosglwyddo i ysgol uwchradd cyfrwng Cymraeg;

- astudio mwy o bynciau nag y byddent wedi gwneud fel arall trwy gyfrwng y Gymraeg mewn ysgol ddwyieithog neu ysgol cyfrwng Saesneg gan fwyaf; neu

- magu digon o allu ieithyddol i fedru cymryd rhan mewn llawer mwy o weithgareddau addysgol a chymdeithasol Cymraeg yn yr ysgol a thu hwnt. (Davidson, 2001, t. 6)

Ar wahoddiad y Gweinidog dros Addysg a Dysgu Gydol Oes yng ngwanwyn 2002, sefydlwyd grŵp o arbenigwyr dan gadeiryddiaeth Bwrdd yr Iaith Gymraeg er mwyn arwain y prosiect yn ei gamau datblygu. Wedi trafod sawl model posibl i’w weithredu, gan gynnwys y rhai sydd wedi bodoli yng Nghymru eisoes megis y Canolfannau i Hwyrddyfodiaid a'r Cynllun Trochi Hwyr a astudiwyd yn fanwl gan Cen Williams (2002) rhwng 1994-1999, ynghyd â'r modelau Canadaidd a gafodd eu harsylwi gan Jane Davidson a'i dirprwyaeth yn 2001, argymhellodd y Grŵp y pedwar model cysyniadol canlynol i'w hystyried:

Model 1 - cwrs blwyddyn 6 (Mai - Mehefin) - 200 o oriau cyswllt â'r Gymraeg. Awgrymwyd peilota'r model hwn mewn dau fath o ysgol - y naill ysgol mewn ardal Gymraeg a'r llall mewn ardal Seisnig iawn.

Model 2 - Model gyda 3 opsiwn posibl ar gyfer dechrau

i) blwyddyn 3 neu 4 (Medi-Gorffennaf) - 350 o oriau cyswllt;

ii) blwyddyn 5 (Medi-Mai) - 300 o oriau cyswllt;

iii) blwyddyn 5 (Medi-Rhagfyr) - 300 o oriau cyswllt. 
Awgrymwyd peilota'r model hwn mewn ysgolion cynradd dwy ffrwd, mewn ysgolion cyfrwng Saesneg mewn ardaloedd Cymraeg a rhai dosbarthiadau arbennig mewn ysgolion cyfrwng Cymraeg.

Model 3 - Unedau trochi - tebyg i'r Canolfannau i Hwyrddyfodiaid - i'w gosod ar draws dwyrain Cymru.

Model 4 - Cwrs blwyddyn 6 (Medi - Rhagfyr) - 300 o oriau cyswllt (cyfieithiad o Oldbell3 \& Dateb, 2008, tt. 29-30)

Mewn ymateb i argymhellion y grŵp, penderfynwyd gan y Gweinidog Addysg ym mis Tachwedd 2002 y byddai Model 1, ac amrywiadau deilliadol o Fodel 2, yn cael eu gweithredu. Byddai Model 3 yn cael ei ohirio am y tro a byddai Model 4 yn cael ei wrthod ar y sail ei bod yn debyg o rwystro paratoadau ar gyfer TASau ${ }^{43}$ (Oldbell3 \& Dateb, 2008, t. 30). Ar ben hyn, gofynnwyd i Fwrdd yr Iaith Gymraeg a fyddent yn fodlon arwain y prosiect gyda chefnogaeth Pwyllgor Llywio a fyddai'n cynnwys cynrychiolwyr o Fwrdd yr Iaith Gymraeg, Adran Safonau a Pherfformiad LICC, Awdurdod Cymwysterau, Cwricwlwm ac Asesu Cymru (ACCAC), ac Estyn. Ar ôl ymgynghoriadau estynedig ag ysgolion, Awdurdodau Addysg Lleol, rhieni a disgyblion, dewiswyd y tri chlwstwr o ysgolion peilot cyntaf ar gyfer y cynllun. At y rhain, ymunodd un ysgol beilot ychwanegol ym mis Medi 2004, un ysgol beilot arall yn ystod haf 2005, a dau glwstwr arall o ysgolion hefyd yn 2005 â'r cynllun peilot gwreiddiol (Estyn, 2006a). Erbyn diwedd cam cyntaf y cynllun, roedd saith clwstwr o ysgolion wedi bod yn rhan o'r cynllun, gyda deuddeg peilot gwahanol yn gweithredu ar sail pum model gwahanol:

Model 1: Addysg drochi ym Mlwyddyn 6 (90\% o'r cwricwlwm trwy gyfrwng y Gymraeg). Model pum wythnos o hyd.

Model 2: Addysg drochi / dwys ym Mlwyddyn 6. Model pum - chwe wythnos o hyd. (Nid oedd hyn yn bosibl mewn nifer o ysgolion oherwydd cyfyngiadau staff (Estyn, 2006a, t. 13))

Model 3: Cwrs carlam Cymraeg ail iaith ar ddiwedd Blwyddyn 6 gyda rhan o'r prosiect yn digwydd yn yr haf (cyn i'r disgyblion ymuno ag ysgol uwchradd). Model pum wythnos o hyd.

\footnotetext{
${ }^{43} \mathrm{Ni}$ chynhelir TASau yng Nghymru erbyn hyn. Cafodd TASau eu disodli yng Nghymru yn 2004.
} 
Model 4: Addysg drochi barhaol o Flwyddyn 3 ymlaen. Pedair blynedd o hyd. (Dim ond un ysgol a ddilynodd y model yma).

Model 5: Addysg drochi ym Mlwyddyn 5 (chwe wythnos o hyd) gyda 20\% o'r cwricwlwm yn cael ei addysgu trwy gyfrwng y Gymraeg ym Mlwyddyn 6.

\subsubsection{Gwerthusiad cyntaf Cynllun Peilot Bwrdd yr Iaith Gymraeg (2003 - 2006)}

Yn unol â gofyniad LlCC i Fwrdd yr Iaith Gymraeg drefnu arolygiad allanol o'r cynllun peilot, cynhaliodd Estyn (2006a) arolygiad o flynyddoedd 2004-2005 a 2005-2006 y cynllun. Wrth gasglu cyflawniad cyffredinol disgyblion y gwahanol beilotau i gyd, nododd Estyn (2006a, t. 5) fod "mwyafrif llethol y disgyblion ar bob un o'r prosiectau peilot addysg drochi hyn wedi elwa'n sylweddol o ran eu medrau iaith a'u gwybodaeth a'u dealltwriaeth o'r Gymraeg”. Er hynny, nododd Estyn (2006a, t. 6) mai ym Model 1 y gwelwyd y datblygiad gorau ymhlith disgyblion yn y Gymraeg, wrth ychwanegu bellach fod Modelau 4 a 5 hefyd yn cynnig gwerth arian. Oherwydd rhwystrau wrth staffio yn ysgolion uwchradd Modelau 2 a 3, nid oedd yn bosibl cynnig cyfleoedd i ddisgyblion ddilyn pynciau trwy gyfrwng y Gymraeg yn yr ysgolion uwchradd ac felly ni fyddai'r modelau hyn yn debygol o lwyddo i gyflawni gweledigaeth Iaith Pawb (Estyn, 2006a, t. 7). Er nad yw'r argymhellion a gynigir gan Estyn i LlCC yn rhai manwl, mae'n amlwg fod yr arolygydd yn gefnogol i weld parhad y cynllun gyda chanolbwynt arbennig ar fynd ar drywydd y modelau sy'n cynnig y canfyddiadau mwyaf cadarnhaol. Argymhellodd Estyn i LlCC:

- barhau i gefnogi prosiectau peilot trochi ac addysgu Cymraeg yn ddwys ar lefelau cynradd ac uwchradd lle y mae cyfleoedd ar gyfer parhad;

- cefnogi'r modelau sy'n cynhyrchu'r canlyniadau gorau. (Estyn, 2006a, t. 9)

Mae Cen Williams (2008) wedi cynnig argymhellion llawer mwy manwl ar gyfer datblygiad y cynllun, yn arbennig o ran ymchwilio sut mae datblygu iaith disgyblion trochi'r cynllun yn effeithiol. O ran gweithredu'r modelau, credai Williams $(2008, \mathrm{t}$. 30) fod angen i reolwyr y cynllun peilot ddisodli Model 4 a rhoi Model 5 yn ei le. Yn ei farn ef, gan mai ond un ysgol oedd yn dilyn Model 4 erbyn hynny a chan fod Model 5 yn rhoi llai o faich ar ysgolion o ran staffio, byddai'n synhwyrol ystyried rhoi canolbwynt ar Fodel 5 yn hytrach. Efallai mai'r pwynt mwyaf allweddol a 
gododd Williams yw rôl ysgolion y sector cyfrwng Saesneg ar y cynllun, hynny yw, ysgolion Model 3. Lleisiai Williams farn amlwg am yr ysgolion hyn, wrth fynegi'r canlynol:

Ni ddylai'r hyn sy'n digwydd yng Nghynllun Trochi Bwrdd yr Iaith Gymraeg gael ei ymestyn i wneud iawn am fethiannau dysgu'r Gymraeg fel ail iaith yn ysgolion cyfrwng Saesneg Cymru. Mae angen Cynllun Peilot Ail Iaith i rymuso'r dysgu yn y sector hwnnw. (Williams, 2008, t. 28)

Ymddengys fod gan Williams syniad pendant o ran dyfodol y cynllun hwn erbyn hyn, ac nid yw'n credu bod gwerth i weithredu peilotau mewn ysgolion cyfrwng Saesneg gan na allant fel rheol gynnig darpariaeth Gymraeg ddigonol yn yr uwchradd ar ôl cyfnod y peilot. Mae Williams (cyfathrebiad personol, Hydref 12, 2015) wedi cadarnhau hyn yn ddiweddar wrth nodi iddo frwydro'n bersonol i newid cyfeiriad a nod hirdymor y cynllun gan ei fod yn ymwybodol o ddwy broblem wrth gynnwys ysgolion cyfrwng Saesneg ar y cynllun hwn. Yn gyntaf, mae'n bosibl y gallai ysgolion cyfrwng Saesneg a all gynnig peth dysgu drwy gyfrwng y Gymraeg ddwyn disgyblion oddi ar ysgolion uwchradd cyfrwng Cymraeg yr ardal. Yn ail, mae gan ysgolion cyfrwng Saesneg staff sydd angen hyfforddiant penodol ac arbenigol er mwyn iddynt fod yn gymwys i addysgu pynciau addysgol eraill yn effeithiol trwy gyfrwng y Gymraeg.

\subsubsection{Ail werthusiad Cynllun Peilot Bwrdd yr Iaith Gymraeg (2007 - 2008)}

Erbyn ail gam Cynllun Peilot y Bwrdd, roedd ei gyfeiriad a'i nod hirdymor wedi esblygu. Mewn adroddiad gan Wasanaethau Archwilio Mewnol Cynulliad Cenedlaethol Cymru (2006), galwyd ar y Pwyllgor Llywio i goethi'r cynllun:

... greater attention needs to be focused towards the consideration of the longterm goals of the project and the planning necessary for the achievement of these goals (Gwasanaethau Archwilio Mewnol Cynulliad Cenedlaethol Cymru, 2006, t. 2, fel y'i dyfynnwyd yn Oldbell3 \& Dateb, 2008, t. 36).

Yn sgil yr ymateb, byddai'r cynllun bellach yn tynnu'r cymorth ariannol i ysgolion cyfrwng Saesneg y cynllun yn ei ôl a chanolbwyntio ar gydweithio ag ysgolion cyfrwng Cymraeg yn unig. Mae'n amlwg erbyn hynny nad un o nodau'r cynllun erbyn diwedd ei oes oedd cynnal a datblygu disgyblion y sector cyfrwng Saesneg, ynteu, yng ngeiriau Iaith Pawb: 
[g]alluogi llawer mwy o ddisgyblion i symud i'r sector cyfrwng Cymraeg neu i astudio mwy o bynciau trwy gyfrwng y Gymraeg mewn lleoliadau eraill nag a fuasai'n bosibl fel arall. (LlCC, 2003, t. 41).

Mae Oldbell3 a Dateb (2008) wedi cadarnhau hyn, wrth nodi bod y prosiect yn arbennig wedi datblygu'n fwy clir o ran:

- cydnabod bod dysgu dwys ar ddiwedd CA2 yn rhan o daith hirach,

- targedu ysgolion a oedd o bwysigrwydd strategol yn hytrach nac ysgolion nad ydynt yn rhannu uchelgais y prosiect o ran cynnig cyfleoedd i ddisgyblion gael addysg trwy gyfrwng Cymraeg,

- tynnu cefnogaeth yn ôl o gynlluniau peilot nad oeddynt yn llwyddo i arwain disgyblion i ryw addysg gyfrwng Cymraeg,

- canolbwyntio'n arbennig ar y trothwy ieithyddol y dylid ei gyrraedd er mwyn elwa ar fanteision dwyieithrwydd.

Erbyn i'r ail werthusiad allanol o'r cynllun trochi cenedlaethol gael ei gyhoeddi gan Oldbell3 a Dateb (2008), roedd modelau'r cynllun wedi esblygu i raddau helaeth:

Model 1: Cyfnod dwys o Gymraeg ar ddiwedd Cyfnod Allweddol 2 (5-6 wythnos) gyda chefnogaeth dros ddwy flynedd trwy ddarpariaeth arbennig yn ystof Cyfnod Allweddol 3, cyn i'r disgyblion ymuno â disgyblion prif frwd ysgolion uwchradd cyfrwng Cymraeg.

Model 2: Cyfnod dwys o Gymraeg ar ddiwedd Cyfnod Allweddol 2 (5-6 wythnos) cyn trosglwyddo i ysgolion dwyieithog traddodiadol lle ceir rhai pynciau trwy gyfrwng y Gymraeg.

Model 3: Cyfnod o weithrediad yn ystod Blynyddoedd 3, 4 a 5 Cyfnod Allweddol 2, gyda darpariaeth ddilynol o fewn yr ysgol gynradd ond heb ddilyniant cynlluniedig i'r disgyblion drosglwyddo i ysgolion uwchradd cyfrwng y Gymraeg neu ddwyieithog.

(Cyfieithiad o Welsh Language Board, Mehefin, 2007, t. 7, fel y'i dyfynnwyd yn Oldbell3 a Dateb, 2008, tt. 34-35)

Yn yr ail adroddiad gwerthuso hwn, sef adroddiad terfynol y cynllun, rhoddwyd sylw manylach i ystyried datblygiad y prosiect a'i effeithiau erbyn hynny, a dod i gasgliadau ynglŷn â'r prosiect a'i werth yn ei gyfanrwydd. Yn gyffredinol, mae'r adroddiad yn canmol y prosiect cyfan, cyn belled â'i fod yn argymell i LlCC barhau â'r cynllun wrth ei ariannu dros gyfnod arall o dair blynedd, yn hytrach na'i ariannu 
fesul blwyddyn fel y gwnaeth yn 2007 a 2008, er mwyn hwyluso cynllunio hirdymor (Oldbell3 \& Dateb, 2008, t. 44) a chaniatáu mwy o amser i gasglu data am ddatblygiad ieithyddol a chynnydd academaidd disgyblion y cynllun (Oldbell3 \& Dateb, 2008, t. 137).

O ran cyfeiriad a nod hirdymor y cynllun, cytunodd Oldbell3 a Dateb (2008, t. 129) ag argymell Estyn (2006a) a phenderfyniad rheolwyr y prosiect i dynnu cefnogaeth yn ôl o'r peilotau yn yr ysgolion cyfrwng Saesneg wrth ddod i'r un casgliad nad yw uchelgais peilotau o'r fath yn cyd-fynd â gweledigaeth Iaith Pawb (2003) i agor mynediad at addysg gyfrwng Cymraeg. Er bod Oldbell3 a Dateb yn feirniadol o'r ffaith bod capasiti ysgolion y peilotiaid hyn yn gyfyngedig o ran gwella cyrhaeddiad disgyblion CAI, yn hytrach nag adeiladu ar eu darpariaeth Gymraeg, mae'n ddiddorol nad ydynt yn cynnig argymhellion ynglŷn â sut mae mynd ati i wireddu hyn. Mae'n amlwg felly fod y cyfle i argymell i LlCC gomisiynu cynllun arbennig ar wahân i CAI, fel yr awgrymwyd gan Cen Williams (2008), wedi cael ei golli yn yr adroddiad hwn.

Ar ôl i'r adroddiad gwerthuso olaf gael ei gyhoeddi, penderfynwyd na fyddai'r cynllun hwn yn cael ei adnewyddu o 2008 ymlaen. Mae LlC (2015, cyfathrebiad personol) wedi cadarnhau ei rhesymau dros y dewis hwn, wrth adrodd:

Erbyn hynny roedd digon o dystiolaeth yn bodoli ynglŷn â'r modelau mwyaf effeithiol, a'r trefniadau angenrheidiol ar gyfer eu gweithredu, a daeth y Llywodraeth i'r casgliad y gellid symud o gyfnod o beilotiaid wedi'u gweinyddu'n ganolog i gynlluniau lleol gan ysgolion ac awdurdodau lleol unigol. O ganlyniad, sicrhawyd bod arian ar gael o fewn Grant y Gymraeg mewn Addysg (Grant Gwella Addysg erbyn hyn) i alluogi ysgolion ac awdurdodau i barhau gyda chynlluniau trochi os dymunent. (LlC, 2015, cyfathrebiad personol).

\subsection{Cynllun Ysgol X: Y Cwrs Carlam}

\subsubsection{Cyd-destun y Cwrs Carlam}

Yng ngoleuni pryderon a godwyd yn ystod y flwyddyn academaidd 2000-2001 ynglŷn â chyrhaeddiad CAI yn CA3, cynhaliwyd trafodaeth rhwng Adran y Gymraeg Ysgol X a phenaethiaid ei hysgolion clwster ar sut mae gwella safonau CAI. Yn dilyn trafodaethau pellach rhwng staff Ysgol X, Adran Addysg y Fwrdeistref a Bwrdd yr Iaith Gymraeg, cafodd cynllun ei ddrafftio ar gyfer gwella safonau yn 
CA3, a danfonwyd copi o'r cynllun arfaethedig i Fwrdd Llywodraethwyr a holl staff yr ysgol. Nod y cynllun hwn oedd "hyrwyddo, cefnogi a gwella perfformiad ieithyddol disgyblion ail iaith Cyfnod Allweddol 3 drwy gynnig cyfle iddynt astudio rhai pynciau drwy gyfrwng y Gymraeg” (Guilfoyle, 2008, t. 32). Mae'r ddarpariaeth garlam hon, a gynigir i'r disgyblion "mwy galluog a dawnus o ysgolion cynradd cyfrwng Saesneg” (Estyn, 2012a, t. 1), yn rhoi cyfle i ddysgu trwy gyfrwng y Gymraeg mewn pynciau ar wahân i CAI, cyn symud ymlaen i astudio TGAU CAI a TAG Uwch Gyfrannol CAI yn gynharach na'r arfer mewn ysgolion cyfrwng Saesneg eraill. Elfen hollbwysig i'r cynllun hwn yw'r athrawon peripatetig sy'n cael eu cyflogi gan yr ysgol er mwyn "chwarae rôl sylweddol o ran gwella'r berthynas â chlwstwr o ysgolion cynradd sy'n bwydo'r ysgol gyfun” (Y Pwyllgor Mentor a Dysgu, 2010, t. 35). Trwy gydweithio'n agos â'r ysgolion clwster, mae'r athrawon teithiol hyn yn darparu adnoddau addysgu a chynnig cyngor arbenigol i staff yn y sector cynradd er mwyn paratoi'r disgyblion ar gyfer trosglwyddo i Ysgol X (Guilfoyle, 2008, t. 32). Cafodd y cynllun ei weithredu'n wreiddiol dros gyfnod o dair blynedd a noddwyd gan Adran Addysg yr Awdurdod Addysg Lleol, ond mae'r cynllun yn cael ei weithredu o hyd gan yr ysgol, er gwaethaf rhai newidiadau iddo.

\subsubsection{Datblygiad y Cynllun}

Ers ei weithredu'n wreiddiol yn 2001, mae cynllun Ysgol X wedi esblygu, a gwelir yn y tabl isod sut ddatblygodd y cynllun yn ystod y pum mlynedd gyntaf:

\begin{tabular}{|c|l|l|l|}
\hline Blwyddyn & \multicolumn{1}{|c|}{ Blynyddoedd Ysgol 7/8 } & Blwyddyn Ysgol 9 & \multicolumn{1}{c|}{$\begin{array}{c}\text { Blynyddoedd Ysgol } \\
10 / 11\end{array}$} \\
\hline 2001 & $\begin{array}{l}\text { Un dosbarth Cwrs Cymraeg } \\
\text { dwys yn astudio tri phwnc } \\
\text { yn ddwyieithog. }\end{array}$ & Tri phwnc yn ddwyieithog. & $\begin{array}{l}\text { TGAU Cymraeg Ail } \\
\text { Iaith. }\end{array}$ \\
\hline 2004 & $\begin{array}{l}\text { Dau ddosbarth yn astudio tri } \\
\text { phwnc yn ddwyieithog. }\end{array}$ & TGAU Cymraeg Ail Iaith. & $\begin{array}{l}\text { TAG UG Cymraeg Ail } \\
\text { Iaith. }\end{array}$ \\
\hline 2006 & $\begin{array}{l}\text { Dau ddosbarth yn astudio } \\
\text { pum pwnc yn ddwyieithog. }\end{array}$ & TGAU Cymraeg Ail Iaith. & $\begin{array}{l}\text { TAG UG Cymraeg Ail } \\
\text { Iaith. }\end{array}$ \\
\hline
\end{tabular}

Tabl 1. Datblygiad y Cwrs Carlam rhwng 2001 - 2006.

(Mae'r tabl hwn yn seiliedig ar y wybodaeth a gyflwynir yn Guilfoyle, 2008, t. 33) 
Erbyn hyn, mae Ysgol X yn cynnig Hanes, Daearyddiaeth, Addysg Grefyddol, Addysg Gorfforol ac Addysg Bersonol a Chymdeithasol (ABaCh o hyn ymlaen) yn ddwyieithog $^{44}$ i garfan o ddisgyblion Mwy Abl a Thalentog (MAT o hyn ymlaen) ${ }^{45}$ yn ystod Blynyddoedd 7, 8 a 9. Gellir dadlau mai amrywiad ar CLIL neu addysg drochi rannol hwyr yw'r hyn a geir y gwersi carlam. (Am drafodaethau llawnach, gweler 2.5.1. Addysg drochi a 2.5.4. Content and Language Integrated Language.) Honna Muñoz (2015) ac Ibarrola (2012) mai tua 12 oed yw'r cyfnod allweddol ar gyfer dechrau astudio trwy ddarpariaeth CLIL, a hynny ar sail y canfyddiad bod gan blant hŷn fantais o ran dysgu ymhlyg (implicit) ac echblyg (explicit) o'u cymharu â phlant iau (Muñoz, 2006; 2008). Diddorol felly yw canfod y caiff y Cwrs Carlam ei gynnig i ddisgyblion yn ystod y cyfnod allweddol hwn, sef 11-14 oed.

Yn ogystal â'r ddarpariaeth ddwyieithog hon, caiff disgyblion y Cwrs Carlam wersi CAI. Ar ôl astudio'r pynciau hyn yn ddwyieithog yn ystod CA3, mae cyfle i ddisgyblion y Cwrs Carlam sefyll eu harholiad TGAU CAI ar ddiwedd Blwyddyn 9. Golyga hyn y gall disgyblion y Cwrs Carlam ennill cymhwyster TGAU mewn Cymraeg ddwy flwyddyn yn gynt na'u cyfoedion yn y brif ffrwd cyfrwng Saesneg. Nid oes darpariaeth ddwyieithog i'r disgyblion hyn yn CA4, ac felly mae pob disgybl y Cwrs Carlam yn parhau â'u hastudiaethau TGAU trwy gyfrwng y Saesneg. Er hynny, mae'r disgyblion hyn yn parhau i astudio Cymraeg ar lefel Safon Uwch Gyfrannol ym Mlynyddoedd 10 ac 11 oherwydd rhaid i ddisgyblion astudio Cymraeg nes eu bod yn 16 oed. Golyga hyn y gall disgyblion y Cwrs Carlam ennill cymhwyster Safon Uwch Gyfrannol yn y Gymraeg flwyddyn yn gynt na'u cyfoedion yn y brif ffrwd cyfrwng Saesneg. Os bydd y disgybl yn penderfynu cwblhau'r Safon Uwch CAI gyflawn, mae cyfle i wneud hyn ym Mlwyddyn 13 yn y Chweched Dosbarth.

\subsubsection{Canlyniadau TGAU}

Cyn cloi'r is-bennod hon, cyflwynir yma ganlyniadau TGAU CAI disgyblion y Cwrs Carlam er mwyn amlygu'n well eu cyrhaeddiad yn yr ail iaith. Ar ôl i Ysgol X

\footnotetext{
${ }^{44}$ Beth a olygir gan y term 'dwyieithog' yma yw'r defnydd o'r Gymraeg a'r Saesneg yn y dosbarth fel cyfrwng dysgu ac addysgu.

${ }^{45}$ Diffinnir 'disgyblion MAT' gan LIC (2015a, t. 2) fel "dysgwyr sydd angen cyfleoedd mwy ymestynnol a chyfoethocach ar draws y cwricwlwm i ddatblygu eu galluoedd mewn un neu ragor o feysydd." Noder mai Ysgol X sydd wedi difinio'r disgyblion hyn yn rhai MAT, ac nid yr ymchwilydd.
} 
gytuno â chymryd rhan yn ail ran yr astudiaeth hon, sef yr ymchwil empirig (gweler Pennod 6), fe'i holwyd am ganlyniadau TGAU CAI diweddar disgyblion y Cwrs Carlam. Anfonodd Ysgol X y data ysgol gyfan ar gyfer y tair blynedd diwethaf (2013, 2014 a 2015) at yr ymchwilydd, gan amlygu pa rai a ddilynodd y Cwrs Carlam a pha rai a ddilynodd y prif ffrwd cyfrwng Saesneg (hynny yw, gwersi CAI Cwrs Llawn yn unig). ${ }^{46}$ Casglwyd data dienw ar gyfanswm o 261 o ddisgyblion sydd wedi ennill gradd mewn CAI. O blith y 261 o ddisgyblion hyn, amlygwyd bod 54 o'r disgyblion wedi dilyn y Cwrs Carlam, gyda'r gweddill yn dilyn CAI Cwrs Llawn yn unig.

Fel y gwelir yn Tabl 2 isod, mae pob un o'r disgyblion a ddilynodd y Cwrs Carlam yn ystod y tair blynedd diwethaf wedi cyflawni graddau A*-C mewn perthynas â CAI. O ran gweddill y disgyblion yn Ysgol $\mathrm{X}$ a enillodd radd mewn CAI, nodir bod $70.5 \%$ o'r disgyblion hyn wedi cyflawni graddau A*-C. Ar gyfartaledd, ymddengys fod $76.6 \%$ o holl ddisgyblion Ysgol X wedi cyflawni graddau A*-C mewn CAI yn ystod y cyfnod hwn. Mewn cymhariaeth â'r data cenedlaethol ar ganlyniadau CAI a gasglwyd gan CBAC (d.d.) yn ystod y cyfnod hwn, sy'n dangos bod 77.2\% o ddisgyblion wedi ennill gradd $\mathrm{A}^{*}$-C ar gyfartaledd, ymddengys fod leflau cyrhaeddiad Ysgol X wedi bod yn gyson â'r cyfartaledd cenedlaethol.

\footnotetext{
${ }^{46}$ Rhaid nodi nad oedd modd i'r ysgol amlygu i ba setiau CAI yr oedd gweddill y disgyblion hyn yn perthyn. Oherwydd hynny, nid oedd modd i'r ymchwilydd ddadansoddi'r data hyn ar lefel setiau.
} 


\begin{tabular}{|c|c|c|c|c|}
\hline \multirow{2}{*}{\multicolumn{2}{|c|}{ Ffin Gradd Cymraeg Ail Iaith }} & \multicolumn{3}{|c|}{ Cyfanswm } \\
\hline & & $\begin{array}{c}\text { CAI Cwrs Llawn } \\
\text { yn unig }\end{array}$ & $\begin{array}{l}\text { Cwrs } \\
\text { Carlam }\end{array}$ & $\begin{array}{l}\text { Cyfanswm } \\
\text { (Cronnus) }\end{array}$ \\
\hline \multirow[t]{2}{*}{$A^{*}-C$} & Nifer & 146 & 54 & 200 \\
\hline & $\%$ & $70.5 \%$ & $100.0 \%$ & $76.6 \%$ \\
\hline \multirow[t]{2}{*}{$\mathrm{D}-\mathrm{G}$} & Nifer & 57 & 0 & 257 \\
\hline & $\%$ & $27.5 \%$ & $0.0 \%$ & $98.5 \%$ \\
\hline \multirow[t]{2}{*}{$\mathrm{U}$} & Nifer & 4 & 0 & 261 \\
\hline & $\%$ & $1.9 \%$ & $0.0 \%$ & $100 \%$ \\
\hline & Cyfanswm & 207 & 54 & 261 \\
\hline
\end{tabular}

Tabl 2. $\quad$ Canlyniadau Cymraeg Ail Iaith Ysgol X 2013-2015.

\subsubsection{Trafodaeth ar y Cwrs Carlam}

Ar yr olwg gyntaf, ymddengys fod y Cwrs Carlam yn Ysgol X yn gynllun llwyddiannus o ran cefnogi datblygiad disgyblion MAT yn y Gymraeg. Fodd bynnag, rhwystr amlwg ar y Cwrs Carlam yw bod y ddarpariaeth ddwyieithog hon ar gael i ddisgyblion MAT, a hynny yn CA3 yn unig. Yn ei adroddiad thematig ar ddarpariaeth Ysgol X ar gyfer y Gymraeg, noda Estyn (2012c, tt. 1-2) fod “disgyblion mwy galluog a dawnus o ysgolion cynradd cyfrwng Saesneg yn cael cyfle i ddatblygu eu medrau ieithyddol drwy'r ddarpariaeth garlam." (Am drafodaeth lawnach, gweler 7.2.2. Darpariaeth ar gyfer y Gymraeg.) Mae'n debyg y caiff disgyblion y Cwrs Carlam eu dethol ar sail eu gallu wrth iddynt baratoi i symud o'r ysgol gynradd i'r ysgol uwchradd. Mae'n amlwg y bydd angen ymchwilio hyn ymhellach yn yr ymchwil empirig er mwyn dysgu rhagor am sut y mae Ysgol X yn mynd ati i ddethol disgyblion ar gyfer y Cwrs Carlam, y meini prawf y mae Ysgol X yn eu defnyddio i ddewis disgyblion, a phwy sy'n gyfrifol am y broses ddetholus hon. Hefyd, bydd angen ymchwilio ymhellach pam na chaiff darpariaeth y Cwrs Carlam ei hymestyn i ddisgyblion CA2, cyn i ddisgyblion ymuno â'r ysgol uwchradd, a disgyblion CA4 (ac o bosibl, CA5). Fodd bynnag, o ystyried mai amrywiad ar CLIL yw'r hyn a geir yn y Cwrs Carlam, mae'n bosibl fod oedran 
disgyblion wrth ddechrau'r Cwrs Carlam, sef tua 12, yn ddelfrydol ar gyfer dechrau dysgu trwy wersi CLIL (Ibarrola 2012; Fitzpatrick ac eraill, 2018; Muñoz, 2015).

Er gwaetha'r rhwystrau posibl a allai godi wrth gynnig darpariaeth ddwyieithog mewn ysgol cyfrwng Saesneg, mae canfyddiadau Estyn (2006b, 2012a, 2012c) wedi adrodd yn gyson ar lwyddiant yr ysgol o ran ei darpariaeth ac ethos dwyieithog a'i darpariaeth ar gyfer y Gymraeg. Yn ei adroddiad arolwg ar yr ysgol yn 2006, rhoddodd Estyn (2006b, t. 27) y radd uchaf posibl ar gyfer dwyieithrwydd, sef gradd 1, wrth nodi bod “datblygiad dwyieithrwydd yn nodwedd eithriadol o'r ysgol." Dyma'r tro cyntaf i unrhyw ysgol yng Nghymru gyflawni hyn (Guilfoyle, 2008, t. 32). Erbyn diwedd CA3, daeth Estyn (2006b, t. 40) i'r casgliad bod "disgyblion llwybr-carlam yn arddangos nodweddion eithriadol yn yr holl sgiliau ieithyddol." Oherwydd y gwaith a wneir gan yr athrawon teithiol, ymddengys fod gan ddisgyblion well dealltwriaeth wrth iddynt drosglwyddo i CA3, ac mae hyn yn paratoi'r disgyblion ar gyfer y ddarpariaeth a gynnig Ysgol X:

Addysgir disgyblion gan arbenigydd pwnc o'r ysgol yn ystod y ddwy flynedd olaf yn eu hysgol gynradd. Oherwydd eu dealltwriaeth o'r iaith wrth iddynt ddod i mewn, mae disgyblion yn gwbl gysurus â'r disgwyliadau uchel a osodir, gan mai dim ond y Gymraeg a siaredir gan yr athrawon ym mwyafrif y gwersi. Maent yn ymateb yn hyderus i'r holl weithgareddau a gyflwynir ar gyfer cysylltu sgiliau iaith. (Estyn, 2006b, t. 40).

Yn CA4, arsylwodd Estyn (2006b) ar safonau uchel unwaith eto, yn arbennig ymhlith disgyblion y Cwrs Carlam:

Mae disgyblion ar y cwrs llwybr-carlam yn cyflawni safonau eithriadol. Wrth astudio bwyta'n-iach yn B10, maent yn mynegi barn yn effeithiol, a chynnal deialogau yn hyderus o flaen eu cymheiriaid. Maent yn amrywio eu strwythurau brawddegol ac amserau'r ferf yn effeithiol iawn. (Estyn, 2006b, t. 41).

Mae unigrywiaeth y cynllun wedi'i nodi hefyd gan Hopkins (2006). Yn ei gyfrol Achub Ein Hiaith, noda Hopkins rôl hollbwysig ysgolion cyfrwng Saesneg wrth achub y Gymraeg a dadleua fod angen i bob ysgol Cymru fod yn ddwyieithog. Wedi arsylwi ar arfer Ysgol X, nododd Hopkins (2006, t. 21) fod y model hwn "wedi bod mor llwyddiannus fel y dylai Llywodraeth y Cynulliad yn awr ariannu ac ehangu'r fath brosiectau ledled Cymru fel rhan o'i [h]ymgyrch [sic] i Achub yr Iaith.” Nid dyma'r unig dro i'r model gael ei awgrymu fel enghraifft o arfer da. Yn ei adroddiad 
i LlCC, Dysgu ac addysgu Cymraeg fel ail iaith, cyfeiriodd Y Pwyllgor Menter a Dysgu (2010) sawl gwaith at arfer Ysgol X wrth lunio ei argymhellion. Cydnabuwyd bod gan athrawon peripatetig Ysgol X rôl hollbwysig yn ystod y Cyfnod Pontio, yn enwedig “o ran gwella'r berthynas â chlwstwr o ysgolion cynradd sy'n bwydo'r ysgol gyfun.” (Y Pwyllgor Menter a Dysgu, 2010, t. 35). Aeth y Pwyllgor (2010, t. 26) cyn belled ag argymell y dylai L1C gomisiynu ymchwil bellach "i'r arfer o gyflogi athrawon Cymraeg teithiol i helpu gyda'r broses bontio rhwng Cyfnod Allweddol 2 a 3 gyflogi athrawon peripatetig”, fel y gwna Ysgol X, er mwyn "archwilio cyfleoedd i rannu arfer gorau ag ysgolion cyfrwng Saesneg ledled Cymru.” Mae Hopkins (2006) hefyd wedi canmol Ysgol X o ran ei chynllun pontio. Cydnabu yntau'r posibilrwydd o ehangu'r cynllun pontio i gwmpasu ysgolion babanod a meithrin y dalgylch. Yn ei ôl ef, pe bai modd gwneud hyn, "byddai gwelliant nodedig yn rhuglder y disgyblion yn sicr o ddilyn" (Hopkins, 2006, t. 21).

Gan ategu'r safonau uchel a ganfuwyd gan Estyn yn 2006, mae adroddiad arolwg diweddar gan Estyn (2016a) eto wedi mynegi'r canlynol ynglŷn â darpariaeth Gymraeg:

Mae darpariaeth yr ysgol ar gyfer y Gymraeg a'r dimensiwn Cymreig yn arloesol ac yn arwain y sector. Mae'r ddarpariaeth helaeth yn cynnig cyfleoedd rhagorol i bron pob un o'r disgyblion, beth bynnag eu gallu, i ennill cymhwyster ffurfiol mewn Cymraeg mamiaith neu Gymraeg ail iaith. Mae darpariaeth 'Cwrs Carlam' yr ysgol yn galluogi disgyblion mwy abl a thalentog i wneud cynnydd cyflym wrth ddatblygu eu medrau Cymraeg. (Estyn, 2016a, t. 6).

Mewn adroddiad thematig gan Estyn (2012c) ar Ysgol X, sef Balchder yn y Gymraeg yn sicrhau llwyddiant, mae'r Arolygydd yn hyrwyddo'r ysgol fel enghraifft o arfer orau o ran ei darpariaeth Gymraeg a'i hethos Cymreig. Mynegodd Estyn (2012c) mai oherwydd cynllun yr ysgol y mae canlyniadau CA3 yn ystod y pum mlynedd cyn blwyddyn yr arolwg wedi bod yn y chwarter uchaf o'u cymharu â rhai ysgolion tebyg eraill sydd â'r hawl i Brydau Ysgol am Ddim. At hyn, adroddodd Estyn (2012c) ar ganlyniadau diweddar TGAU a TAG disgyblion ar y Cwrs Carlam. Wrth ystyried adroddiad Estyn a'r data TGAU CAI a gyflwynwyd uchod, ymddengys fod disgyblion yn llwyddo i ennill marciau uchel iawn, a hynny ddwy flynedd yn gynt na'r disgwyl ar lefel TGAU, a rhyw flwyddyn yn gynt na'r disgwyl ar lefel TAG. 
Er bod canlyniadau Ysgol X yn addawol ar yr olwg gyntaf, rhaid astudio mewnbwn yr ysgol yn fwy ofalus cyn y gellir dod i gasgliad mwy pendant am sut y mae'r ysgol yn cefnogi cyflwyno'r Gymraeg fel ail iaith yn llwyddiannus. Er bod y dystiolaeth ymchwil ryngwladol wedi awgrymu'n gryf mai trwy ddysgu cynnwys y ceir y llwyddiant gorau, rhaid trin llwyddiant Ysgol X yn ofalus gan nad oes eto dystiolaeth empirig i archwilio mewnbwn ac allbwn yr ysgol. Rhaid hefyd drin canlyniadau arholiadau CAI yn ofalus yn eu cyfanrwydd, wrth ystyried i ba raddau y mae'r cymwysterau hyn yn adlewyrchu gwir gaffaeliad disgyblion mewn perthynas â'r Gymraeg.

\subsection{Casgliadau ar y cynlluniau trochi a dysgu dwys}

O blith y tri chynllun a dafodwyd uchod, ymddengys mai ond cynllun Ysgol X sydd yn berthnasol i'r ymchwil hon, gan mai nod y ddau gynllun arall oedd cynnig ail bwynt mynediad i addysg cyfrwng Cymraeg o gyfeiriad addysg cyfrwng Saesneg. Mae'n debyg na fyddai cynnal peilotiaid trochi o'r fath yn ymarferol yn yr ardaloedd cymharol ddi-Gymraeg yng Nghymru ar hyn o bryd. Fel y mynegwyd gan Oldbell3 a Dateb (2008, tt. 51-52), mae baich ar ysgolion uwchradd cyfrwng Cymraeg yn yr ardaloedd hyn i gefnogi peilotau o'r fath, yn arbennig yn Ne-ddwyrain Cymru. Fodd bynnag, mae'n amlwg fod cyfle i weithredu peilotiaid ar wahân yn y sector cyfrwng Saesneg fel yr awgrymodd Williams (2008), er mwyn gwella safonau mewn perthynas â CAI. Ymddengys cynllun Ysgol X felly'n gynllun addas i'w ymchwilio yng nghyd-destun yr astudiaeth hon, sydd am archwilio sut orau y gellir cefnogi cyflwyno’r Gymraeg yn llwyddiannus mewn ysgolion cyfrwng Saesneg. 


\section{Pennod 6: Cynllun methodolegol yr ymchwil}

\subsection{Cyflwyniad i'r astudiaeth achos}

Penderfynwyd defnyddio'r astudiaeth achos fel methodoleg ymchwil i'r astudiaeth hon. Mae'r astudiaeth achos yn fethodoleg ymchwil sydd yn caniatáu i'r ymchwilydd astudio "instance" (Adelman ac eraill, 1980), "case" (Stake, 1995) neu "phenomenon" (Hitchcock \& Hughes, 1995) yn ei chyd-destun ei hun. Fel y mae Adelman, Kemmis a Jenkins (1980, t. 49) yn ei diffinio, yr astudiaeth achos yw "an umbrella term for a family of research methods having in common the decision to focus on enquiry around an instance." Ymhelaetha Hitchcock a Hughes (1995, t. 316) ar y diffiniad hwn gan nodi mai astudio "naturalistic everyday, cultural and interactional phenomena... in their own right and in their own territory" sydd dan sylw yn yr astudiaeth achos. I'r un perwyl, cyfeiria Cohen ac eraill $(2003$, t. 181) at yr astudiaeth achos fel astudiaeth ar "a unique example of real people in real situations."

Yr hyn sy'n amlwg o'r diffiniadau uchod yw nad dull ymchwil mo'r astudaeth achos, ond methodoleg ymchwil sy'n cwmpasu gwahanol fathau o ddulliau ymchwil at ddiben archwilio ffenomen benodol yn ei chyd-destun naturiol. Yng nghyd-destun ymchwil addysg, gall yr 'achos' hwn fod yn blentyn, dosbarth, ysgol neu gymuned (Cohen ac eraill, 2003, t. 181). Ni waeth beth fo'r ffenomen dan sylw fodd bynnag, yr hyn y mae'r ymchwilydd am ei gyflawni yw “[to] locate the 'story' of a certain aspect of social behaviour in a particular setting and the factors influencing the situation" fel bod modd amlygu a dadansoddi'r prif themâu, pynciau neu newidynau sy'n deillio o'r data (Hitchcock \& Hughes, 1995, t. 317).

Amlyga'r diffiniadau uchod fod yr astudiaeth achos yn caniatáu i'r ymchwilydd ddadansoddi ffenomen mewn modd holistaidd a chyd-destunol. Fel y noda Hitchcock a Hughes (1995, t. 319), ceir yn yr astudiaeth achos "focus and emphasis upon the specific, the clearly bounded and unique." Nid oes syndod felly fod tueddiad i ymchwilwyr ddefnyddio dulliau dadansoddi ansoddol mewn astudiaethau achos (Cohen ac eraill, 2003; Hitchcock \& Hughes, 1995), a hynny oherwydd y gall y dulliau hyn fynd ymhellach na dulliau meintiol gan gynnig darlun mwy cyflawn o'r ffenomen dan sylw (Nisbet a Watt, 1984). Er hynny, dylid nodi bod modd defnyddio 
dulliau dadansoddi meintiol mewn astudiaethau achos i gyd-fynd â dulliau dadansoddi ansoddol (Cohen ac eraill, 2005; Korzilius, 2012; Nisbet \& Watt, 1984).

Yng nghyd-destun ymchwil addysg, ymddengys yr astudiaeth achos yn fethodoleg ymchwil ddefnyddiol i'r ymchwilydd annibynnol sydd am astudio'n fanwl ysgolion a'u haelodau yn eu cyd-destun eu hunain (Bell, 1999) ac sydd yn wynebu cyfyngiadau o ran adnoddau ac amser (Nisbet \& Watt, 1984). Mae'n amlwg fod methodoleg yr astudiaeth achos yn addas os yw'r ymchwilydd am:

... study and portray the impact in a school of a particular curriculum, innovation, explore the experience of a staff development, trace the development of an idea through a number of social organizations, investigate the influence of a social and professional network, or portray a day in the life of a teacher, administrator or a pupil.” (Hitchcock \& Hughes 1995, t. 321)

\subsection{Mathau o astudiaeth achos}

Cyn mynd ati i gynnal astudiaeth achos, mae'n hollbwysig i'r ymchwilydd ystyried pa fath o astudiaeth achos y bydd ei angen. Yn ôl rhai ysgolheigion, mae'n bosibl nodi tri phrif fath o astudiaeth achos. Mae Yin (1984), er enghraifft, yn cynnig dosbarthu astudiaethau achos yn rhai archwiliadol, eglurhaol a disgrifiadol. Yn debyg iawn, cynnig Merriam (1988) gategoreiddio astudiaethau achos yn rhai disgrifiadol, deongliadol a gwerthusol. Mae Stenhouse (1985), fodd bynnag, yn awgrymu pedwar math o astudiaeth achos, sef ethnograffig, gwerthusol, addysgol ac ymchwil weithredol.

Fodd bynnag, fel y mae Adelman ac eraill (1980) yn ei amlygu, bydd y math o astudiaeth a ddetholir yn y pen draw yn dibynnu i raddau helaeth ar sut y bydd yr ymchwilydd yn mynd ati i gynllunio'r astudiaeth. Yn eu hôl hwy, gellir naill ai ddechrau gyda damcaniaeth a chwestiynau ymchwil penodol mewn golwg ac yna archwilio'r 'achos' er mwyn mynd i'r afael ag ateb y cwestiynau ymchwil hyn, ynteu ddechrau gyda'r 'achos' a'i archwilio yn ei rinwedd ei hun, gyda'r themâu a'r cwestiynau ymchwil yn codi wrth wneud. I'r un perwyl, mae Stake (1995) yn gwahaniaethu rhwng dau fath o astudiaeth achos yn ôl eu diben, sef yr astudiaeth achos gynhenid (intrinsic case study), lle astudir achos er mwyn dysgu mwy am yr achos dan sylw yn benodol, a'r astudiaeth achos gyfryngol (instrumental case study), lle astudir achos er mwyn ateb cwestiynau ymchwil penodol neu ddysgu mwy am achos arall. Fel y mae enw'r astudiaeth yn ei awgrymu, 'cyfrwng' a ddefnyddir er 
mwyn ateb cwestiynau ymchwil yw'r achos yn y math hwn o astudiaeth. At y rhain, ychwanega Stake (1995) un math arall o astudiaeth achos, sef yr astudiaeth achos gyfunol (collective case study). ${ }^{47}$ Yn y bôn, ceir i'r astudiaeth achos gyfunol gasgliad o astudiaethau achos unigol a gynhelir "er mwyn ennill darlun mwy manwl o'r dosbarth mae'r achosion yn perthyn iddo yn hytrach na bod yn gyfyng i un achos penodol" (Beard, 2016, t. 26). Gan enghreifftio'r math hwn o astudiaeth achos, noda Stake (1995):

... we may feel that we should choose several teachers to study rather than just one. Or we might choose to use schools as our cases and choose several schools. Each case study is instrumental to learning about the effects of the marking regulations but there will be important coordination between the individual studies. We may call the work collective case study.

(Stake, 1995, tt. 5-6)

Yr hyn sy'n bwysig wrth gynnal astudiaeth achos yw sicrhau bod y math o astudiaeth achos a ddewisir yn gweddu i anghenion yr astudiaeth dan sylw. Ond yn y bôn, os caiff astudiaethau achos eu cynnal yn "systematically and critically, if they are aimed at the improvement of education, if they are relatable, and if by publication of the findings they extend the boundaries of existing knowledge, then they are valid forms of educational research.” (Bassey, 1981, t. 86).

\subsection{Trosolwg o'r fethodoleg ymchwil}

Fel y nodwyd yn y Rhagair, cynrychiolodd dwy ysgol uwchradd cyfrwng Saesneg yn bennaf yn Ne Cymru, sef Ysgol X ac Ysgol Y, yr ysgolion cyfranogol, a chynrychiolodd tair carfan o ddisgyblion Cymraeg Ail Iaith ar draws y ddwy ysgol gyfranogol y disgyblion cyfranogol. Er bod gan yr ymchwilydd ddiddordeb penodol mewn un achos, sef y Cwrs Carlam a gynigir yn Ysgol X, dadleua nad astudiaeth achos gynhenid mo hon. Astudiaeth achos gyfunol (Stake, 1995) neu astudiaeth ddeongiadol (Merriam, 1988; Yin, 1994) yw hon gan fod mwy nag un 'achos' o dan sylw a chan fod yr ymchwilydd am ddysgu rhagor am sut y mae'r ddwy ysgol yn cefnogi cyflwyno'r Gymraeg fel ail iaith. At ddiben yr ymchwil hon, mabwysiadwyd diffiniad Stake (1995) o'r astudiaeth achos gyfunol i ddisgrifio'r astudiaeth hon, gan fod yr ymchwilydd am gynnal grŵp o astudiaethau er mwyn cynnig dadansoddiad ar draws achosion. Trwy ddadansoddi mwy nag un achos, bydd modd osgoi'r

${ }^{47}$ Defnyddir yma fathiadau Cymraeg Beard (2016) o derminoleg Stake (1995). 
feirniadaeth gyffredin o astudiaethau sy'n canolbwyntio ar un achos yn unig (Yin, 2006). Bydd hyn yn ei dro'n cryfhau natur ddibynadwy canfyddiadau'r astudiaeth.

\begin{tabular}{|c|c|}
\hline Methodoleg & $\begin{array}{l}\text { - Astudiaeth achos gyfunol (Stake, 1995) } \\
\text { - Dwy ysgol uwchradd cyfrwng Saesneg yn bennaf yn Ne Cymru. } \\
\text { - Tair carfan o ddisgyblion Cymraeg Ail Iaith ar draws y ddwy ysgol gyfranogol. }\end{array}$ \\
\hline Cynllun & $\begin{array}{l}\text { - Mewnbwn y ddwy ysgol gyfranogol } \\
\text { - Allbwn y tair carfan o ddisgyblion }\end{array}$ \\
\hline & $\begin{array}{l}\text { - Mewnbwn: Yr holiadur, y cyfweliadau lled-strwythuredig a'r arsylwadau } \\
\text { - Allbwn: Prawf llenwi bylchau, sgoriau darllen Saesneg a data Prydau Ysgol am Ddim }\end{array}$ \\
\hline $\begin{array}{l}\text { Dulliau } \\
\text { ladansoddi }\end{array}$ & $\begin{array}{l}\text { - Dadansoddi ansoddol: Yr holiadaur, y cyfweliadau a'r arsylwadau. } \\
\text { - Dadansoddi meintiol: Canfyddiadau'r prawf llenwi bylchau, sgoriau darllen Saesneg a } \\
\text { data Prydau Ysgol am Ddim }\end{array}$ \\
\hline
\end{tabular}

Ffigur 3. Cynllun yr ymchwil

Diben yr astudiaeth hon yw astudio'n fanwl sut y mae'r ysgolion cyfranogol yn cefnogi cyflwyno'r Gymraeg fel ail iaith yn llwyddiannus. Er mwyn gwneud hyn, rhennir yr astudiaeth yn ddwy ran, gyda'r naill ran yn canolbwyntio ar y mewnbwn a geir yn y ddwy ysgol gyfranogol a'r llall yn canolbwyntio ar allbwn tair carfarn o ddisgyblion (gweler Ffigur 4). Yn ogystal â hyn, bydd yr ymchwilydd yn mynd ati i werthuso'r prawf llenwi bylchau fel mesurydd hyfedredd ieithyddol cyffredinol disgyblion Cymraeg Ail Iaith yn ail ran yr astudiaeth. Caniatâ'r astudiaeth i'r ymchwilydd gyflwyno casgliadau ar y ddwy ysgol gyfranogol ac felly cynnig cyfraniad at ddealltwriaeth ym maes Cymraeg Ail Iaith. Gallai nifer o'r casgliadau hyn hefyd fod yn berthnasol i ysgolion cyfrwng Saesneg eraill yng Nghymru. Er hynny, nid diben yr astudiaeth achos yw cyffredinoli'r canfyddiadau i'r boblogaeth darged gyfan. (Am drafodaeth lawnach, gweler 6.10. Cyfyngiadau'r astudiaeth.) 
(i) Dadansoddi nodweddion y ddwy ysgol gyfranogol a'r mewnbwn y maent yn ei gynnig o safbwynt cefnogi cyflwyno'r Gymraeg fel ail iaith.

(ii) Cymharu'r canfyddiadau hyn â chanfyddiadau'r ymchwil ragarweiniol yn Pennod 1. (i) Archwilio allbwn y ddwy ysgol gyfranogol gan ddadansoddi proffiliau ieithyddol a sosio-economaidd tair carfan o ddisgyblion Cymraeg Ail Iaith ar draws yr ysgolion.

(ii) Gwerthuso'r prawf llenwi bylchau fel mesurydd hyfedredd ieithyddol cyffredinol disgyblion Cymraeg Ail Iaith.

Ffigur 4. Cynllun a nodau dwy ran yr astudiaeth.

\subsection{Ysgolion cyfranogol}

Fel y nodwyd yn yr ymchwil ragarweiniol, ymddengys y Cwrs Carlam yn Ysgol X yn gynllun addas i'w ymchwilio yng nghyd-destun yr ymchwil hon. Penderfynwyd astudio Ysgol X ac un ysgol uwchradd cyfrwng Saesneg gymharus arall, sef Ysgol Y, er mwyn dadansoddi nodweddion y ddwy ysgol gyfranogol a'r mewnbwn y maent yn ei gynnig o safbwynt cefnogi cyflwyno'r Gymraeg fel ail iaith. Er mwyn sicrhau bod y ddwy ysgol gyfranogol yn gymharus, fe'u cymharwyd o ran proffil sosioeconomaidd a sosio-ieithyddol eu disgyblion. (Am drafodaeth lawnach, gweler Pennod 7.) Cysylltodd yr ymchwilydd â'r ddwy ysgol trwy lythyr yn y lle cyntaf. Ar ôl llwyddo i gysylltu â'r ddwy ysgol, trefnodd yr ymchwilydd ymweliadau i gyfarfod â'r Prifathro a Phennaeth Adran y Gymraeg er mwyn trafod pwrpas a nodau'r ymchwil, yn ogystal â materion moesegol. Cytunodd y ddwy ysgol gyfranogi yn yr astudiaeth ar sail wirfoddodol.

Cyn mynd ati i gynnal y gwaith maes yn y ddwy ysgol, cwblhawyd adolygiad moesegol llai beichus o'r cynnig ymchwil llawn gan Bwyllgor Moeseg a Llywodraethu Ymchwil Coleg y Celfyddydau a'r Dyniaethau, Prifysgol Abertawe, er mwyn sicrhau bod yr ymchwil yn diwallu egwyddorion moesol allweddol. Gwnaed yn sicr bod gan yr ymchwilydd ganiatâd y Prif Athro a Phennaeth Adran y Gymraeg, fel porthgeidwaid, i gynnal y gwaith maes yn y ddwy ysgol. Yn ogystal â hyn, gwnaeth yr ymchwilydd gais i'r Y Gwasanaeth Datgelu a Gwahardd (DBS) i sicrhau dystysgrif i allu gweithio gyda phlant a lluniwyd ffurflen ganiatâd i'w chwblhau gan 
y disgyblion a'u rhiant / gwarcheidwad i sicrhau eu cysyniad gwybodus cyn cynnal y prawf llenwi bylchau (gweler Atodiad 1).

\subsection{Mewnbwn}

Nod cyntaf yr astudiaeth hon oedd dadansoddi nodweddion y ddwy ysgol gyfranogol a'r mewnbwn y maent yn ei gynnig o safbwynt cefnogi cyflwyno'r Gymraeg fel ail iaith a chymharu'r canfyddiadau hyn â chanfyddiadau'r ymchwil ragarweiniol yn Pennod 1. Penderfynwyd canolbwyntio ar y themâu a'r cwestiynau cysylltiedig canlynol, a ddeilliodd o'r ymchwil ragarweiniol yn Pennod 1, yn rhan gyntaf yr astudiaeth:

Darpariaeth i'r Gymraeg:

i. Beth yw'r llwybrau dysgu Cymraeg a gynigir gan yr ysgolion? Faint o amser a neilltuir i addysgu'r Gymraeg yn yr amserlen ar y llwybrau hyn?

ii. A ydy'r ysgol yn cynnig unrhyw bynciau trwy gyfrwng y Gymraeg neu'n ddwyieithog? Os felly, pa bynciau?

iii. Beth yw statws CAI yn yr ysgolion yn nhermau mesur perfformiad?

Ethos dwyieithog:

i. A oes gan yr ysgolion bolisi ar ddwyieithrwydd? Os felly, beth yw pwyntiau allweddol y polisi hwnnw?

ii. Sut mae'r ysgol yn gwireddu nodau'r polisi hwn yn ymarferol?

Hyfforddiant staff:

i. Pa hyfforddiant sydd ar gael i staff er mwyn datblygu methodoleg addysgu Cymraeg fel ail iaith / methodoleg addysgu dwyieithog / sgiliau cyffredinol yn y Gymraeg?

ii. Pa mor aml y mae'r staff yn cael cyfleoedd i dderbyn hyfforddiant? Ar ben hynny, pa mor aml y mae athrawon yr ysgol yn achub ar y cyfle i gael hyfforddiant? A oes unrhyw bryderon o ran cael mynediad at yr hyfforddiant hwn, ac os felly, beth yw'r pryderon hyn? 
Pontio rhwng y sectorau cynradd ac uwchradd:

i. Beth a wneir yn benodol yn yr ysgolion i hwyluso dilyniant disgyblion ail iaith yn y Gymraeg o'r sector cynradd i'r sector uwchradd?

Er mwyn casglu data ar y nodweddion uchod, penderfynwyd defnyddio'r dulliau ymchwil canlynol (a drafodir yn fanylach nes ymlaen yn y bennod hon):

i. Holiadur gyda Phennaeth Adran y Gymraeg

ii. Cyfweliad dilynol gyda Phennaeth Adran y Gymraeg

iii. Ymweliad i arsylwi ethos dwyieithog.

\subsection{Allbwn}

Ail nod yr astudiaeth oedd mesur allbwn y ddwy ysgol. Yn benodol, roedd yr ymchwilydd am ddadansoddi proffiliau ieithyddol a sosio-economaidd tair carfan o ddisgyblion Cymraeg ail iaith ar draws y ddwy ysgol gyfranogol. Penderfynwyd canolbwyntio'n benodol ar fesur y disgyblion o ran eu:

i. Hyfedredd ieithyddol cyffredinol yn y Gymraeg

ii. Gallu darllen yn y Saesneg

iii. Statws sosio-economaidd.

Er mwyn mesur y newidynnau hyn, penderfynwyd defnyddio'r dulliau ymchwil canlynol yn eu tro (a drafodir yn fanylach nes ymlaen yn y bennod hon):

i. Prawf llenwi bylchau

ii. Sgoriau darllen Saesneg y Prawf Darllen Cenedlaethol

iii. Data Prydau Ysgol am Ddim (PYDd o hyn ymlaen).

Detholwyd tair carfan o ddisgyblion ym Mlwyddyn 9, sef:

i. Disgyblion y Cwrs Carlam yn Ysgol X

ii. Disgyblion prif ffrwd sy'n astudio Cymraeg Ail Iaith (Set 1) fel pwnc yn unig yn Ysgol X

iii. Disgyblion prif ffrwd sy'n astudio Cymraeg Ail Iaith (Set 1) fel pwnc yn unig yn Ysgol Y. 
Dewiswyd disgyblion y Cwrs Carlam fel un o'r achosion i'w astudio am eu bod yn derbyn darpariaeth Gymraeg ychwanegol trwy astudio peth o'r cwricwlwm yn ddwyieithog, yn ogystal ag astudio Cymraeg fel pwnc. Trwy astudio disgyblion y Cwrs Carlam, anela'r ymchwilydd at ddysgu rhagor am yr achos ei hun (Stake, 1995; 2005). Fodd bynnag, anela'r ymchwilydd hefyd at ddysgu rhagor am allbwn y rhaglen CAI draddodiadol a geir yn y naill ysgol a'r llall a sut y mae hyn yn wahanol i allbwn y Cwrs Carlam. Yn ogystal ag astudio disgyblion y Cwrs Carlam felly, bwriedir astudio dwy garfan o ddisgyblion prif ffrwd sy'n astudio'r Gymraeg fel pwnc yn unig yn y ddwy ysgol gyfranogol. Rhaid pwysleisio nad cymharu disgyblion yn feintiol er mwyn gwerthuso effeithiolrwydd y Cwrs Carlam yw nod y rhan hon o'r astudiaeth. Nod y rhan hon o'r astudiaeth yw amlygu gwahaniaethau posibl rhwng y tair carfan o ddisgyblion o safbwynt eu proffiliau ieithyddol, at ddiben dysgu rhagor am allbwn y model traddodiadol (gwersi CAI yn unig) a'r cynllun amgen (darpariaeth ychwanegol y Cwrs Carlam), a'u proffiliau sosioeconomaidd, at ddiben darganfod statws sosio-economaidd y tair carfan o ddisgyblion.

\subsection{Cwestiynau ymchwil}

Fel y gwelwyd eisoes yn y bennod hon, daw nodau'r astudiaeth i'r amlwg wrth archwilio'r fethodoleg ymchwil. Er mwyn egluro diben yr astudiaeth, ffurfir y nodau hyn yn gwestiynau ymchwil fel a ganlyn:

1. Beth yw nodweddion y ddwy ysgol gyfranogol a'r mewnbwn y maent yn ei gynnig o safbwynt cefnogi cyflwyno'r Gymraeg fel ail iaith?

2. Beth yw proffiliau ieithyddol a sosio-economaidd tair carfan o ddysgwyr sydd yn derbyn gwahanol fewnbwn Cymraeg yn y ddwy ysgol gyfranogol?

3. A yw'r prawf llenwi bylchau yn brawf dibynadwy a dilys ar gyfer mesur hyfedredd ieithyddol cyffredinol dysgwyr ail iaith yn y Gymraeg?

\subsection{Dulliau dadansoddi}

Er nad oes un ffordd gywir o ddadansoddi data, rhaid i'r dadansoddi fod yn addas $\mathrm{i}$ ddiben yr ymchwil (Cohen ac eraill, 2011). Fel y mynna Cohen ac eraill (2011, t. 538), dylai pwrpas y dadansoddi bennu'r math o ddadansoddi y mae'r ymchwilydd 
am ei ddefnyddio. Gan mai disgrifio a chymharu nodweddion a mewnbynnau unigryw'r ddwy ysgol gyfranogol yw diben rhan gyntaf yr astudiaeth, penderfynwyd y byddai ymagwedd dadansoddol a dehongiadol ei naws yn fwy addas ar gyfer dadansoddi data'r holiadur, y cyfweliadau a'r arsylwadau. Ar gyfer ail ran yr astudiaeth, sef dadansoddi sgoriau'r prawf llenwi bylchau, y sgoriau darllen Saesneg a'r data PYDd, mabwysiadwyd ymagwedd meintiol gan ddefnyddio meddalwedd SPSS i ddadansoddi'r data yn ystadegol.

\subsection{Dulliau ymchwil}

\subsubsection{Yr holiadur a'r cyfweliad}

Er mwyn casglu gwybodaeth am unigolion, grwpiau neu sefydliadau (Cohen, Manion \& Morrison, 2011, t. 256), ynglŷn â ffeithiau (Dörnyei, 2003, t.8), nodweddion (Brown \& Rodgers, 2002, t. 142; de Vaus, 1996, t. 3), ymddygiadau (Dörnyei, 2003, t.8), agweddau (Dörnyei, 2003, t. 8; Brown \& Rodgers, 2002, t. 142), neu farnau (Brown \& Rodgers, 2002, t. 142), cynhelir arolygon. Mae gwahanol fathau o arolwg y gellir eu cynnal, megis yr arolwg ffeithiol, yr arolwg agweddol, yr arolwg cymdeithasol-seicolegol neu'r arolwg esboniadol (Ackroyd \& Hughes, 1981; Sutton, 2011). Cynhelir arolygon yn bennaf ar ffurf holiaduron, cyfweliadau neu arsylwadau. Fodd bynnag y mae'n bosibl defnyddio cyfuniad o ddulliau, megis holiadur a chyfweliad (Brown \& Rodgers, 2002, t. 142). Efallai mai'r dull mwyaf defnyddiol a phoblogaidd o gasglu gwybodaeth arolwg yw'r holiadur (Cohen ac eraill, 2011; Dörnyei, 2003).

Mae'r holiadur yn boblogaidd mewn ymchwil ail iaith gan ei fod yn hyblyg (Walliman, 2006, t. 88), mae'n hawdd ei lunio, mae ganddo amlbwrpas, ac mae'n bosibl casglu swmp mawr o wybodaeth arolwg yn gymharol gyflym (Dörnyei, 2003, t. 1). O'i gymharu â'r cyfweliad er enghraifft, gall yr holiadur fod yn fwy effeithlon i'r ymchwilydd, yn nhermau amser ac adnoddau ariannol (Dörnyei, 2003, t. 9; Walliman, 2006, t. 88). Mantais arall i'r holiadur yw ei bod yn bosibl i'r ymchwilydd ei gynnal heb fod yn bresennol ei hun (Cohen ac eraill, 2011, t. 377) - ffaith a all annog cyfranogwyr i ateb yn fwy gonest ac agored.

Er gwaetha'r manteision posibl, gall llunio ac adolygu holiaduron fod yn waith llafurus, ac ni all yr ymchwilydd archwilio atebion cyfranogwyr trwy ofyn 
cwestiynau dilynol, fel yr hyn sy'n bosibl trwy gyfweld, gan fod yn rhaid i gwestiynau'r holiadur fod yn syml a chlir (Walliman, 2006, t. 88). Dull o gasglu data ar ffurf lafar yw'r cyfweliad. Ceir yn y cyfweliad gyfnewid safbwyntiau rhwng y cyfwelydd a'r cyfwelai (Kvale, 1996, t. 2) ac mae'r rhyngweithiad cymdeithasol rhwng y ddau gyfranogwr yn eu galluogi i archwilio ystyr y cwestiynau a'r atebion a gynigir yn ystod y sgwrs (Brenner, Brown \& Canter, 1985, t. 3). Yn wahanol i'r holiadur felly, lle mae atebion y cyfranogwr yn dibynnu i raddau helaeth ar gyfarwyddiadau ysgrifenedig a strwythur y ffurflen, yn y cyfweliad y mae atebion y cyfranogwr yn dibynnu ar allu'r cyfwelydd i arwain y sgwrs yn llwyddiannus. Trwy gyfweld ag unigolion, ceir mewnwelediad i'w safbwyntiau, barnau, gwerthoedd, agweddau, dyheadau a'u profiadau am bwnc neu bynciau penodol (May, 2011, t. 141; Turner, 2010, t. 754). Mae'r cyfweliad yn arbennig o ddefnyddiol felly i'r ymchwilydd sydd am ddarganfod sut mae unigolion yn deall eu 'byd cymdeithasol' a'r rôl y maent yn chwarae ynddo (May, 2011, t. 157).

Yng nghyd-destun ymchwil addysgol, mae Cohen ac eraill (2011) yn awgrymu bod gan y cyfweliad dri diben posibl: fel y prif ddull o gasglu data, fel dull profi a darganfod rhagdybiaethau neu fel dull o gasglu data i'w ddefnyddio ar y cyd â dulliau eraill (t. 411). I'r un perwyl, mae Brown (2001), Dörnyei (2003) a Gillham (2000) wedi dadlau y gall holiaduron a chyfweliadau ategu ei gilydd gan fod yr ymagwedd hon yn galluogi'r ymchwilydd i archwilio data nid yn unig o safbwynt meintiol, ond hefyd o safbwynt ansoddol. Fel y mae Gillham (2000) yn ei awgrymu, gall data cyfweliad lled-strwythuredig roi i'r ymchwilydd well dealltwriaeth o'r data meintiol a gasglwyd trwy'r holiadur.

\subsubsection{Rhesymeg o ran defnyddio'r holiadur a'r cyfweliadau}

Ar ôl adolygu'r llenyddiaeth ar yr holiadur a'r cyfweliad fel dulliau casglu data, penderfynwyd llunio a chynnal holiadur, yn ogystal â chyfweliadau dilynol er mwyn casglu gwybodaeth gefndirol am y ddwy ysgol uwchradd cyfrwng Saesneg yn yr astudiaeth. ${ }^{48}$ Wrth ddefnyddio holiadur a chyfweliad ar y cyd, anelwyd at gasglu "a well-rounded collection of information" (Turner, 2010, t. 754). Ar ôl ailedrych ar y

\footnotetext{
${ }^{48}$ Roedd yr ymchwilydd hefyd am arsylwi disgyblion a chynnal grwpiau ffocws. Fodd bynnag, ar ôl trafod ag Ysgol X, daeth i'r amlwg nad oedd modd gwneud hyn oherwydd pwysau eraill ar yr ysgol ar y pryd (gweler 6.10. Cyfyngiadau'r astudiaeth).
} 
prif themâu a ganfuwyd yn ystod yr ymchwiliad i'r llenyddiaeth ar CAI, daeth y pedair thema ganlynol i'r golwg:
i. Darpariaeth ar gyfer y Gymraeg yn yr ysgol
ii. Ethos Cymraeg yr ysgol
iii. Hyfforddiant staff yr ysgol
iv. Pontio rhwng y sector cynradd a'r sector uwchradd.

Penderfynwyd y byddai'r ymchwil empirig yn mynd i'r afael â'r pedair ffactor hyn er mwyn galluogi i'r ymchwilydd archwilio'r ddwy ysgol o ran eu mewnbynnau a'u nodweddion. Yna, trafodir canfyddiadau'r gwaith maes ochr yn ochr â chanfyddiadau'r adolygiad llenyddol er mwyn taflu golau ar ymagwedd Ysgol X at gyflwyno'r Gymraeg yn y cyd-destun cyfrwng Saesneg.

Cynhaliwyd yr holiadur ynghyd â'r cyfweliadau â sampl fwriadus (purposive sample), sef Pennaeth Adran y Gymraeg ('Pennaeth y Gymraeg' o hyn ymlaen) y ddwy ysgol yn yr astudiaeth. Dewiswyd y sampl hon oherwydd mai "knowledgeable people" (Cohen ac eraill 2011, t. 157) yng nghyd-destun eu hysgolion yw Penaethiaid y Gymraeg a rhagwelwyd y byddai ganddynt wybodaeth drylwyr ynghylch y themâu uchod. Rheswm arall am ddewis y sampl hon oedd cyfrifoldeb Pennaeth y Gymraeg dros Adran y Gymraeg a'i rôl wrth hyrwyddo'r iaith a dwyieithrwydd trwy'r ysgol gyfan. Ar ôl casglu'r data sylfaenol ar ffurf yr holiadur a dadansoddi'r data hwn, lluniwyd cyfres o gwestiynau penodol i'w gofyn i'r ddau bennaeth yn y cyfweliadau dilynol. Diben y cwestiynau dilynol hyn oedd ysgogi manylder ac eglurder gan y cyfranogwyr, yn ogystal â dilysu'r hyn a ddarganfuwyd yn yr holiadur.

\subsubsection{Strwythur yr holiadur}

Ar ôl canfod themâu'r adolygiad llenyddol a dethol sampl addas ar gyfer yr astudiaeth, lluniwyd cyfres o gwestiynau ar gyfer yr holiadur. Fel y gwelir yn Atodiadau 2 a 3, trefnwyd cwestiynau'r holiadur o dan y pedair thema uchod. Er mwyn sicrhau y gallai'r cyfranogwr ateb o safbwynt ei 'ffrâm gyfeirio' ei hun (May, 2011, t. 136), defnyddiwyd cwestiynau penagored lle roedd hynny'n bosibl (33 allan o 43 cwestiwn posibl), yn ogystal â rhai cwestiynau caeedig (10 allan o 43 cwestiwn posibl) er mwyn casglu data meintiol ei naws. Paratowyd copi meddal o'r holiadur 
terfynol a'i ddanfon at Benaethiaid y Gymraeg dros e-bost. Cwblhawyd yr holiadur a'i ddychwelwyd i'r ymchwilydd, eto ar ffurf copi meddal dros e-bost.

\subsubsection{Strwythur y cyfweliadau}

Er mwyn caniatáu digon o ryddid i'r ymchwilydd i ofyn cwestiynau dilynol nad oeddent wedi'u paratoi ymlaen llaw, heb golli strwythur a dilyniant clir, penderfynwyd defnyddio'r dull lled-strwythuredig, fel y mae May (2011) yn ei awgrymu. Er mwyn llywio'r sgwrs, defnyddiwyd math penodol o gwestiynau penagored, sef cwestiynau twndis (funnel questions) (Cohen ac eraill, 2011), lle cyflwynir gosodiad neu gwestiwn cyffredinol yn gyntaf, ac yna cyflwynir cyfres o gwestiynau cysylltiedig â'r gosodiad neu'r cwestiwn gwreiddiol. Am fod y ddau gyfweliad yn anelu at gasglu data penodol am nodweddion y ddwy ysgol, nodir bod y cwestiynau yn y naill gyfweliad a'r llall yn benodol i'r ddwy ysgol, ac o ganlyniad, yn wahanol i'w gilydd (gweler Atodiadau 4 a 5). Serch hynny, nodir bod y cwestiynau yn dilyn yr un strwythur ac yn cynnwys yr un themâu â'r hyn a ddefnyddiwyd yn yr holiadur. (Am drafodaeth lawnach, gweler 6.9.1.1. Rhesymeg o ran defnyddio'r holiadur a'r cyfweliadau).

Cynhaliwyd y ddau gyfweliad ar safle'r ddwy ysgol mewn ystafell ddosbarth er mwyn lleihau aflonyddwch, megis sŵn plant yn chwarae, a allai amharu ar ansawdd y recordiad sain. Cyn dechrau'r cyfweliad, esboniwyd pwrpas a fformat y cyfweliad i'r cyfranogwyr. Cafwyd caniatâd ysgrifenedig gan y penaethiaid i'r cyfweliad gael ei recordio ar dâp cyn cynnal y cyfweliad, ac unwaith eto ar lafar ar ddechrau'r cyfweliad.

Ar ôl cynnal y ddau gyfweliad, cafodd y ddau recordiad sain eu trawsgrifio. Yn ôl Kvale (1996, t. 165), pwrpas trawsgrifio yw dehongli'r cyfweliad, a gafodd ei recordio'n wreiddiol ar lafar, mewn ffordd ysgrifenedig. O safbwynt Kvale, ni all trawsgrifiadau ysgrifenedig adlewyrchu'r sgwrs lafar wreiddiol a gofnodwyd yn berffaith oherwydd mai sgyrsiau heb gyd-destun ydynt hwy. Fel y mae Cohen ac eraill (2011, t. 426) yn ei esbonio: "Transcriptions are decontextualized, abstracted from time and space, from the dynamics of the situation, from the live form, and from the social, interactive, dynamic and fluid dimensions of their source; they are frozen." 
Gwell ystyried trawsgrifiadau felly fel "interpretive constructions" (Kvale, 1996, t. 165) o'r testun llafar gwreiddiol. Gan fod trawsgrifiad yn ddehongliad o'r hyn a drafodwyd yn y cyfweliad llafar, gellir ystyried y broses o drawsgrifio fel un o'r camau cyntaf yn y broses o ddadansoddi'r data (Kvale, 1996). Awgryma Kvale (1996) a Cohen ac eraill (2011) nad oes un dull cywir o drawsgrifio, ac felly dylai'r ymchwilydd ddewis ei ddull trawsgrifio gan ystyried faint o fanylder a fydd ei angen ynddo.

Ar adeg dadansoddi'r data, ni chafwyd hyd i unrhyw fodelau safonol ar gyfer trawsgrifio'r iaith Gymraeg a fyddai'n addas at ddiben y gwaith hwn. ${ }^{49}$ Oherwydd hynny, penderfynwyd llunio dull trawsgrifio wedi'i seilio ar rai o gonfensiynau dull trawsgrifio Jefferson (1984). Yn y dull trawsgrifio a ddefnyddiwyd, sylwir na cheir cymaint o fanylder â dull Jefferson (1984). Am mai diben y trawsgrifio oedd dadansoddi'r cynnwys, hynny yw, y wybodaeth a gyflwynodd y cyfranogwyr, yn hytrach na'r cyfathrebu, a'r ffordd y cyflwynodd y cyfranogwyr y wybodaeth ar lafar, penderfynwyd na fyddai'n addas trawsgrifio nodweddion ieithyddol megis goslef yn y llais, seibiau wrth siarad a chyflymder y siarad, na seiniau sy'n cynnig gwybodaeth gyd-destunol megis y gloch ginio yn canu, clirio gwddf a chwerthin.

\subsubsection{Arsylwi ethos dwyieithog}

Er anhawsed diffinio ethos, mae mesur ethos, yn arbennig ethos anffurfiol, yn anos fyth. Mae Allder (1993, t. 69) yn gwerthfawrogi hyn wrth ddisgrifio ethos fel "that illusive item which is so difficult to recognise, measure or improve." Cred Everard (1986), er bod astudio dogfennaeth ffurfiol yr ysgol, megis prosbectws yr ysgol, rheolau'r ysgol, a llawlyfr athrawon, yn ddigon i gael blas ar ethos ffurfiol yr ysgol, nad yw hyn yn ddigonol ar ei ben ei hun er mwyn creu darlun cyflawn. Mae'n bosibl, er enghraifft, y gallai'r ddogfennaeth hon gynnwys datganiadau sydd wedi'u dethol yn ofalus iawn er mwyn rhoi argraff benodol o'r ysgol: “...prospectuses are not always full of statements of how things are; they are more likely to be selective statements of how the head would like readers to think them to be." (Everard, 1986, t. 22). Dadleua Everard (1986, t. 22) fod ymweliadau ag ysgol yn ddull mwy

\footnotetext{
${ }^{49}$ Cafwyd hyd i gofensiynau trawsgrifio Cymraeg prosiect Corpws Siarad Bangor (Deuchar, M., Davies, P., Herring, J., Parafita Couto, M. \& Carter, D., 2014) a Chorpws Cenedlaethol Cymraeg Cyfoes (CorCenCC). Er hynny, nodir bod y confensiynau hyn yn fanwl iawn ac yn fwy addas ar gyfer astudiaethau sy'n canolbwyntio ar gofnodi nodweddion ieithyddol, yn hytrach na chynnwys sgyrsiau.
} 
dibynadwy o arsylwi ethos yr ysgol: "Spending a day in a school affords many opportunities to pick up the flavours of what a school stands for, what it is like and what sort of leadership the head gives." Fodd bynnag, mae ef yn mynd yn ei flaen $i$ rybuddio y gall ymweliadau â'r ysgol gamarwain yr ymchwilydd oherwydd ei bod yn bosibl y gallai'r ysgol gyflwyno ei hun mewn ffordd sy'n creu argraff benodol ohoni ei hun. Oherwydd hynny, mae Everard yn cydnabod gwerth holiaduron fel mesurydd ar gyfer cofnodi agweddau aelodau'r ysgol dan sylw. Dyma ddull casglu data sy'n cael ei ffafrio gan Freiberg (1998, 1999). Mae Freiberg (1998, tt. 23-24) yn cefnogi cynnal holiaduron gyda disgyblion gan y gall yr adborth a geir gan ddisgyblion ddangos sut y maent yn ymateb i'w hamgylchedd dysgu a chynnwys yr addysg.

Mae'n debyg na fydd y dulliau casglu data hyn at ei gilydd yn ddigonol i fodloni astudiaeth gyflawn a chynhwysol ar ethos. Mae Glover a Coleman (2005) hyd yn oed o'r farn nad yw ethos yn fesuradwy oherwydd bod ethos fel cysyniad yn oddrychol. Mae ethos anffurfiol yn dibynnu ar agweddau, ymddygiad ac arferion aelodau'r ysgol ac mae'r rhwydweithiau cymhleth mewn ysgol yn debyg o feithrin gwahanol agweddau, ymddygiadau ac arferion a allai wrthdaro ag ethos ffurfiol yr ysgol (Solvason, 2005, t. 89). Yn ogystal â hyn, mae'n bosibl y gallai agweddau, ymddygiadau ac arferion disgyblion wrthdaro ag ethos ffurfiol yr ysgol (Donnelly, 2000, t. 137). Hyd yn oed pe bai'r ysgol yn caniatáu i'r ymchwilydd arsylwi ethos anffurfiol yr ysgol, mae'n bosibl y byddai'r ysgol am ei phortreadu ei hun mewn ffordd nad yw efallai'n cyd-fynd â'r ethos go iawn ar lawr gwlad (Everard, 1986, tt. 23-24). Ymddengys felly fod gennym nifer o agweddau ar ethos yr ysgol ac mae hyn yn cymhlethu'r dasg o'i astudio wrth reswm.

Mae'r defnydd o'r term 'ethos' yn ymestyn i'r llenyddiaeth ym maes addysg yng Nghymru. Mae pwysigrwydd arsylwi ethos wedi'i gydnabod gan Estyn (d.d.) yn ei Fframwaith Arolygu Cyffredin, lle nodir 'ethos' fel un o agweddau'r arolygiad ysgol. Yn fwy penodol i arsylwi Cymraeg, mae Arweiniad atodol: Cymraeg (Estyn, 2013b) yn manylu i ryw raddau ar yr agweddau y dylai arolygwyr eu hystyried yn ystod eu hymweliadau. Ynglŷn ag ethos, mae'r ddogfen arweiniol hon yn gofyn i'r arolygydd ystyried y tri chwestiwn canlynol: 
- A oes gan yr ysgol ethos sy'n hyrwyddo'r Gymraeg a diwylliant Cymru ac agweddau cadarnhaol tuag atynt?

- A oes defnydd helaeth yn cael ei wneud o'r Gymraeg mewn arddangosfeydd ac arwyddion o amgylch yr ysgol?

- A yw'r ysgol yn annog disgyblion a staff i wneud defnydd cynyddol o'r Gymraeg, ar y lefel briodol, fel cyfrwng cyfathrebu anffurfiol? (Estyn, 2013b, t.5)

O'r cwestiynau uchod, gwelir bod ffocws byr iawn ar agweddau ethos, ac ni cheir yma feini prawf sy'n ddigonol i arwain yr arolygydd ynglŷn â sut y dylid mynd ati i ateb y cwestiynau hyn. Oherwydd natur niwlog y cwestiynau hyn a'r diffyg arweiniad a gynigir er mwyn eu hateb hwy, daw sawl cwestiwn i'r amlwg o safbwynt academaidd ynglŷn ag asesu:

i. Agweddau: Â phwy y dylai'r arolygydd siarad? Ai grwpiau o aelodau ysgol ynteu unigolion penodol? Faint o aelodau ysgol y dylid eu harsylwi er mwyn cael darlun teg o agweddau?

ii. Defnydd gweledol o'r Gymraeg: Beth a olygir gan 'ddefnydd helaeth' o'r Gymraeg? Faint o arddangosfeydd ac arwyddion y dylid eu harsylwi? Onid oes angen ystyried agweddau eraill megis dogfennaeth a pholisïau ffurfiol yr ysgol?

iii. Defnydd llafar o'r Gymraeg: Os yw'r ysgol yn annog disgyblion a staff i ddefnyddio'r Gymraeg fel cyfrwng anffurfiol, sut yn union y mae'r ysgol yn gwneud hyn yn ymarferol? Sut mae mesur 'defnydd cynyddol' o'r Gymraeg yn yr ysgol? Beth a olygir gan 'y lefel briodol'?

Fel y gwelir o'r cwestiynau posibl a restrir uchod, mae amwysedd ynglŷn â'r meini prawf y mae Estyn (2013b) yn eu cynnig. Fodd bynnag mae cynrychiolydd ar ran Estyn (cyfathrebiad personol, Chwefror 6, 2016), wedi nodi bod gan y corff arolygwyr ar eu timau arolygu'r arbenigedd yn y maes er mwyn dod i gasgliad cytbwys ynglŷn ag ethos dwyieithog ysgolion. Er mai cysyniad haniaethol yw ethos, cydnebydd y cynrychiolwr fod modd i arolygwyr Estyn ddod i gasgliad digon cytbwys am ethos dwyieithog trwy:

...drafod gyda staff a disgyblion am eu hagweddau tuag at y Gymraeg; ehangder ac ansawdd y ddarpariaeth ddwyieithog, er enghraifft lle'r Gymraeg 
yng nghwricwlwm yr ysgol; y defnydd a wneir ohoni er enghraifft mewn gwasanaethau a gwersi ac ar draws yr ysgol; a hefyd ystyried ffeithiau megis y ganran o ddisgyblion sy'n dilyn y cwrs llawn yn CA4 (Cymraeg ail iaith) o'i chymharu ag ysgolion tebyg.

(Cynrychiolydd Estyn, cyfathrebiad personol, Chwefror 6, 2016).

Felly cwestiynau sbardun yn unig yw'r rheini yn yr arweiniad atodol gan Estyn (2013b), ac nid ydynt yn rhestr wirio fanwl i'w chwblhau gan yr arolygwyr (cyfathrebiad personol, Chwefror 6, 2016). Mae arfarniadau Estyn o ethos dwyieithog mewn ysgolion felly yn ddibynnol i raddau helaeth ar brofiad ac arbenigedd eu harolygwyr, yn hytrach nac ar feini prawf penodol i'w dilyn. Mae'n bosibl cymharu'r drafferth a geir wrth fesur ethos dwyieithog â'r hyn a geir wrth fesur ethos Cristnogol. Er gwaethaf anhawster diffinio'r term, ceir defnydd amwys o'r term mewn cyd-destun Cristnogol gan Estyn. Mewn adroddiad diweddar ar un o ysgolion uwchradd yr Eglwys yng Nghymru, adroddodd Estyn (2014a, t. 9) fod gan yr ysgol hon "a very caring, Christian ethos" a bod hyn yn creu "an atmosphere of mutual support within a happy and disciplined environment." Yn debyg i Rutter ac eraill (1979), Allder (1993), a Solvason (2005), mae Estyn yn defnyddio'r termau 'ethos' ac 'awyrgylch' yn gyfystyr, ond heb gynnig arweiniad o ran beth a olygir gan y term neu sut mae mesur ethos Cristnogol.

Yn ogystal â'r diffyg arweinyddiaeth ar ran Estyn, rhaid nodi hefyd nad yw Mesur y Gymraeg (Cymru) (2011) yn gorfodi ysgolion y sector cynradd a'r sector uwchradd yng Nghymru i ddarparu a gweithredu strategaeth neu gynllun ar ddatblygu dwyieithrwydd yn yr ysgol. Mewn ysgolion lle nad oes strategaeth neu gynllun ffurfiol ar ddatblygu dwyieithrwydd yn ei lle, mae mesur ethos yn anos fyth gan nad oes gan yr arolygydd restr o egwyddorion neu ddisgwyliadau ffurfiol ar gyfer datblygu dwyieithrwydd yn yr ysgol y gellir cyfeirio atynt wrth arsylwi ethos ymarferol yn yr ysgol. Serch hynny, nodir bod nifer gynyddol o ysgolion cynradd cyfrwng Saesneg ar draws Cymru wrthi'n gweithredu Siarter Iaith Cymraeg Campus. ${ }^{50}$ Nod y cynllun hwn yw:

\footnotetext{
${ }^{50}$ Addasiad o'r Siarter Iaith Gymraeg yw Siarter Iaith Cymraeg Campus a chafodd ei ddatblygu gan gonsortiwm ERW. Datblygwyd y Siarter Iaith Gymraeg yn wreiddiol gan Gyngor Gwynedd ar gyfer ysgolion cynradd cyfrwng Cymraeg y sir "er mwyn darparu fframwaith clir i hyrwyddo a chynyddu defnydd plant a phobl ifanc o'r Gymraeg mewn cyd-destun cymdeithasol.” (LlC, 2016c, t. 9). Mae'r cynllun yn cael ei ehangu i gynnwys pob ysgol gynradd Gymraeg a dwyieithog ar draws Cymru maes o law (LlC, 2016c).
} 
... darparu fframwaith clir y gellir ei ddefnyddio i hyrwyddo a chynyddu'r defnydd o'r Gymraeg gan blant o fewn cyd-destun ysgol gyfan. Yn gryno, prif nod y Siarter yw hyrwyddo ethos Gymreig cadarn [sic] yn yr ysgolion ac i ddarparu ystod o weithgareddau cyfoethog sy'n galluogi'r plant i fwynhau dysgu'r Gymraeg. (ERW, 2016, t. 3)

Nid oes cyfrifoldeb cyfreithiol ar y sector addysg bellach i ddatblygu strategaeth ar ddwyieithrwydd ychwaith. Er hynny, cyhoeddwyd strategaeth o'r fath yn 2010 gan Golegau Cymru ${ }^{51}$ i'w gweithredu gan bob un o'r colegau a sefydliadau addysg bellach yng Nghymru sydd yn aelodau o Golegau Cymru. Yn y strategaeth hon, sef Strategaeth Genedlaethol ar Ddwyieithrwydd yn Addysg Bellach, mae Colegau Cymru (2010, t. 4-5) yn gwneud defnydd cydgyfnewidiol o'r termau 'ethos dwyieithog' ac 'ethos Cymraeg'. Daw pwysigrwydd datblygu ethos dwyieithog i'r amlwg wrth astudio'r adran 'Datblygu ethos Cymraeg' yn y strategaeth. Yn ôl y strategaeth, dylai pob coleg ymgorffori'r egwyddorion canlynol wrth weithredu ei strategaeth ar ddwyieithrwydd:

- Dylai pob arwydd yn y coleg fod yn ddwyieithog

- Dylai staff y rheng flaen allu cwrdd a chyfarch (o leiaf) yn Gymraeg ac yn Saesneg.

- Dylai'r cyfarchiad Cymraeg ddod gyntaf. Dylid gwneud pob ymdrech i sicrhau fod gan unedau sy'n wynebu'r cwsmer o leiaf un person sy'n gallu sgwrsio'n rhugl yn Gymraeg.

- Dylai galwadau ffôn i mewn gael eu hateb yn Gymraeg gyntaf, gyda phob aelod staff ffôn yn meddu ar sgiliau iaith Gymraeg "cwrdd a chyfarch" sylfaenol.

- Dylai dogfennaeth (noder rhestr) fod ar gael yn ddwyieithog. Dylai'r fersiwn Gymraeg fod mor hawdd cael gafael arni â'r fersiwn Saesneg.

- Dylai gwefannau a deunyddiau marchnata esbonio sut y mae'r coleg yn hyrwyddo ethos dwyieithog (h.y. nid dim ond datgan fod ganddo Gynllun Iaith Gymraeg) a dylid nodi'n glir mewn prosbectysau pa gyrsiau/elfennau o gyrsiau sydd ar gael yn ddwyieithog neu drwy gyfrwng y Gymraeg.

- Digwyddiadau ffurfiol a chymdeithasol sy'n cefnogi ac yn hyrwyddo dwyieithrwydd. (Colegau Cymru, 2010, t. 5)

${ }^{51}$ Corff cenedlaethol sy'n cynrychioli pob un o'r 15 o golegau a sefydliadau addysg bellach yng Nghymru yw Colegau Cymru. 


\subsubsection{Rhesymeg o ran arsylwi ethos dwyieithog}

Er mai ethos dwyieithog yw un o'r themâu y cesglir gwybodaeth amdanynt yn yr holiadur a'r cyfweliadau, credwyd y byddai'n hanfodol casglu data ychwanegol ynglŷn â'r thema hon er mwyn gallu dadansoddi a chymharu agweddau ffurfiol ar ethos yn y ddwy ysgol yn fanylach. Er mwyn gwneud hyn, penderfynwyd llunio meini prawf a fyddai'n sylfaen i arsylwi ethos dwyieithog yn yr ysgolion yn yr astudiaeth. ${ }^{52}$ Ar ôl arolygu'r llenyddiaeth ar ethos, yn arbennig sut mae 'mesur' ethos dwyieithog mewn addysg, teimlwyd y byddai egwyddorion strategaeth Colegau Cymru (2010) ar ddwyieithrwydd yn cynnig meini prawf cryfach a manylach ar gyfer arsylwi ethos dwyieithog mewn ysgolion uwchradd, o'u cymharu â'r arweiniad y mae Estyn (2013b) yn ei gynnig, a chan fod Siarter Iaith Cymraeg Campus wedi'i ddatblygu ar gyfer ysgolion cynradd yn benodol, ni theimlwyd y byddai meini prawf y cynllun hwn fod yn addas wrth fesur ethos dwyieithog mewn ysgolion uwchradd. Ar sail yr egwyddorion hyn a restrir gan Golegau Cymru (2010, t. 5), lluniwyd meini prawf (gweler Atodiad 6).

\subsubsection{Y prawf llenwi bylchau}

Ar ôl ymddangos am y tro cyntaf yng ngwaith W. L. Taylor (1953), astudiwyd y dull llenwi bylchau (cloze procedure) yn wreiddiol er mwyn canfod ei effeithiolrwydd ar gyfer mesur natur ddarllenadwy deunyddiau darllen plant ysgol yn yr Unol Daleithiau (Brown, 2002). Erbyn y 1960au a'r 1970au, roedd ymchwilwyr wedi dechrau defnyddio'r dull llenwi bylchau nid yn unig ar gyfer mesur gallu darllen siaradwyr Saesneg iaith gyntaf, ond ar gyfer mesur hyfedredd ieithyddol cyffredinol (overall measure of language proficiency) siaradwyr Saesneg fel ail iaith (Brown, 2002). Er y cydnabyddir bod y rhan fwyaf o'r llenyddiaeth dan sylw yn deillio o gyddestunau addysgu Saesneg fel ail iaith / iaith dramor, mae'r dull llenwi bylchau wedi'i ddefnyddio mewn cyd-destunau eraill. Er enghraifft, mae Tremblay a Garrison (2010) a Tremblay (2011) yn defnyddio'r prawf llenwi bylchau wrth fesur hyfedredd ieithyddol cyffredinol siaradwyr Ffrangeg ail iaith. Mae Gellert ac Elbro (2013) yn defnyddio'r prawf llenwi bylchau hefyd er mwyn mesur darllen a deall oedolion Daneg iaith gyntaf. Mae Ulusoy (2008) yntau wedi dangos bod gwahanol

\footnotetext{
${ }^{52}$ Oherwydd pryderon Ysgol X am bwysau ychwanegol posibl ar y disgyblion a oedd ar y pryd yn wynebu asesiadau ac arolygiad gan Estyn, nid oedd yn bosibl arsylwi sut y caiff ethos dwyieithog yr ysgolion ei wireddu ar lefel anffurfiol, er enghraifft, trwy gynnal grwpiau ffocws gyda disgyblion.
} 
fathau o'r prawf llenwi bylchau yn addas ar gyfer mesur natur ddarllenadwy testunau yn ogystal â gallu darllen disgyblion gradd 6 (12 oed) yn y Dyrceg. Mae'n debyg y gallai'r prawf llenwi bylchau hefyd fod yn ddull ymchwil defnyddiol hyd yn oed ar gyfer ieithoedd nad ydynt yn defnyddio system yr wyddor (Douglas, 1994; Yamashita, 1994). Yn y papur ymchwil gan Tremblay a Garrison (2010), cynhaliwyd arolygiad o'r dulliau asesu hyfedredd ieithyddol mewn ymchwil ar siaradwyr ail iaith rhwng 2000-2008. ${ }^{53}$ Darganfu Tremblay a Garrison (2010, Tabl 1, t. 74) fod rhyw $30 \%$ o'r astudiaethau, lle defnyddiwyd profion annibynnol i fesur hyfedredd siaradwyr ail iaith, wedi defnyddio'r prawf llenwi bylchau.

\subsubsection{Ffurfiau gwahanol y prawf llenwi bylchau}

Yn syml, ymarfer lle mae'r cyfranogwr yn ceisio adfer geiriau sydd 'ar goll' mewn brawddeg/au ysgrifenedig yw'r dull llenwi bylchau. Caiff y geiriau eu dileu yn fwriadol a thrwy lenwi'r bylchau hyn sydd yn y testun, mae'r cyfranogwr yn cyfannu'r testun. Wrth ddefnyddio'r dull llenwi bylchau, mae'n bosibl llunio prawf sy'n profi cyfranogwyr o ran eu gallu i adfer geiriau mewn testun, sef y prawf llenwi bylchau (cloze test). Oherwydd ei natur olygadwy, mae amryw o strwythurau posibl y gellir eu gweithredu wrth lunio prawf llenwi bylchau. Mae Hadley a Naaykens (1997), er enghraifft, wedi nodi bod pum math o brawf llenwi bylchau posibl, sy'n amrywio o ran eu strwythur, sef y dull dileu geiriau mewn patrwm sefydlog (fixedrate deletion), y dull dileu geiriau ar sail resymegol (selective / rational deletion), y prawf llenwi bylchau amlddewis (multipe-choice cloze), y prawf llenwi bylchau trwy ganfod a chywiro (elide cloze), a'r prawf ' $\mathrm{C}$ ' $(C$-test). Yn y prawf llenwi bylchau sy'n dileu geiriau mewn patrwm sefydlog, dilëir pob n ${ }^{\text {fed }}$ gair o'r testun, fel arfer pob $5^{\text {ed }}$ neu $7^{\text {fed }}$ gair (Bilki, 2011, t. 16), ond mae'n bosibl dileu mwy neu lai o eiriau na hynny. Yn y prawf llenwi bylchau sy’n dileu geiriau ar sail resymegol, dilëir geiriau penodol er mwyn mireinio anhawster darllen y prawf, yn ogystal â mesur gwybodaeth ramadegol a geirfaol penodol y cyfranogwyr (Hadley \& Naaykens, 1997, t. 113). Mae tystiolaeth gan Brown (2002) wedi awgrymu nad yw'r dull dileu geiriau mewn patrwm sefydlog yn ddibynadwy o ran gwahaniaethu rhwng cyfranogwyr. Wrth ddefnyddio'r un testun mewn pum prawf llenwi bylchau, defnyddiwyd pum dull dileu gwahanol (mewn patrymau sefydlog) er mwyn dileu

\footnotetext{
${ }^{53}$ Arolygodd Tremblay a Garrison (2010) y cylchgronau canlynol yn eu papur: French Language Studies, Second Language Research a Studies in Second Language Acquisition.
} 
geiriau gwahanol ym mhob prawf. Darganfu Brown fod y dulliau dileu geiriau mewn patrwm sefydlog yn amrywio o ran eu canlyniadau oherwydd caiff geiriau gwahanol eu dileu yn achos pob un. Gan ddibynnu ar ba eiriau sydd wedi'u dileu a lefelau hyfedredd y cyfranogwyr, mae'n bosibl felly i'r naill brawf a'r llall amrywio o ran eu hanhawster. Oherwydd hynny, dadleua Brown nad yw'r dull hwn o ddileu geiriau yn ddibynadwy o ran ei natur i wahaniaethu rhwng eitemau, ac felly rhaid llunio prawf sy'n dileu geiriau ar sail resymegol os yw'r prawf i dargedu eitemau sy'n debyg o wahaniaethu:

The results of this study would seem to indicate that the every nth word strategy is far too inefficient for responsible use in decision-making. Instead, we should probably use what we now know about the way some cloze items discriminate (and most others do not) to refine the strategies we use to tailor cloze tests that are efficient. (Brown, 2002, t. 110)

Yn y dull amlddewis, yn debyg i'r ddau ddull dileu cyntaf, dilëir geiriau naill ai ar sail resymegol ynteu ar sail patrwm sefydlog a chynigir dewis o eitemau posibl i lenwi'r bylchau. Awgryma Tremblay (2011) y gall y dull hwn fod yn fwy addas i brofi siaradwyr ail iaith sydd â hyfedredd is (yn eu hail iaith). Mae ymchwil gan Brown (1980) ac Ulusoy (2008) yn cefnogi hyn wrth ddangos bod cyfranogwyr yn perfformio'n well mewn profion llenwi bylchau lle cynigir eitemau amlddewis, o’u cymharu â phrofion llenwi bylchau lle mae'r eitemau'n benagored. Yn y dull canfod, gellir dileu eitemau o'r testun a rhoi eitemau gwahanol nad ydynt yn gywir yn eu lle, neu ychwanegu eitemau diangen i mewn i'r testun. Yn achos y ddau arfer, rhaid i'r cyfranogwr ganfod y geiriau anghywir a'u cywiro neu eu croesi hwy allan o'r testun. Yn y prawf ' $C$ ', yn hytrach na dileu geiriau cyfan o'r testun, dilëir ail ran bob yn ail air. Wrth wneud hyn, caiff y cyfranogwyr eu herio i raddau mwy na'r prawf llenwi bylchau traddodiadol gan fod mwy o eitemau i'w hadfer mewn testun. Ymddengys o'r llenyddiaeth nad oes swmp mawr o ymchwil ar y ddau ddull olaf hyn ac mae'r ffocws yn bennaf ar effeithiolrwydd y tri dull cyntaf. Mae Tremblay (2011) yn cadarnhau hyn yn achos y prawf ' $\mathrm{C}$ ', wrth ei drin fel prawf gwahanol i'r prawf llenwi bylchau sydd heb gymaint o ymchwil iddo ef. Yn ôl Tremblay (2011, t. 346): “The present study favors cloze tests over C-tests, simply because further research exists on the validity, reliability, and discriminability of cloze tests..." 


\subsubsection{Dulliau sgorio'r prawf llenwi bylchau}

Yn ogystal â'r amryw strwythurau posibl y gellir eu defnyddio wrth lunio prawf llenwi bylchau, mae'n bosibl defnyddio nifer o ddulliau sgorio. Mae dewis pa ddull sgorio yw'r mwyaf effeithiol wedi bod yn bwnc trafod ymhlith ysgolheigion (e.e. Brown, 1980; J. W. J. Oller, 1972). Mae tri phrif ddull sgorio sef i) derbyn yr union air a oedd yn y testun gwreiddiol yn unig, ii) derbyn unrhyw air sy'n gwneud synnwyr yn gyd-destunol, neu iii) dewis gair o blith rhestr amlddewis o eitemau. Mae hefyd yn bosibl defnyddio'r dull sgorio clozentropy, a gynigwyd gyntaf gan Darnell (1968), lle caiff atebion y cyfranogwyr ail iaith eu pwyso a'u mesur yn logarithmig yn unol ag amledd atebion cywir siaradwyr iaith gyntaf a gafodd eu profi mewn prawf peilot. Er bod ysgolheigion wedi dangos bod y dull sgorio hwn yn un dilys a dibynadwy (Brown, 1980; Darnell, 1968), mae Brown (1980, Tabl VIII, t. 316) yn nodi nad yw clozentropy mor ymarferol â dulliau sgorio eraill oherwydd bod y system sgorio hon mor gymhleth.

Mewn astudiaeth gan Oller (1972), sgoriwyd tri phrawf llenwi bylchau gwahanol wrth ddefnyddio pum dull sgorio gwahanol a chymharwyd sgoriau'r profion â sgoriau arholiad lleoli (placement examination) Prifysgol Califfornia Los Angeles. Darganfu Oller fod y dull sgorio sy'n derbyn unrhyw air sy'n gywir yn gyd-destunol yn cydberthyn yn fwy agos â sgoriau'r arholiad lleoli. Cafodd y canfyddiad hwn ei gefnogi hefyd gan Brown (1980). Gan ddadansoddi dilysrwydd ${ }^{54}$ a dibynadwyedd $^{55}$ y dulliau sgorio posibl ar gyfer profion llenwi bylchau, dengys Brown, ni waeth pa ddull sgorio a ddefnyddir, fod pob un o'r profion yn ddilys a gall pob un wahaniaethu rhwng gwahanol lefelau ieithyddol dysgwyr ail iaith. Fodd bynnag, daw Brown i'r casgliad mai derbyn unrhyw air sy'n gywir yn gyd-destunol yw'r dull sgorio mwyaf dibynadwy a chred Brown fod y dull hwn yn fwy teg i siaradwyr ail iaith na fyddent yn gallu dyfalu'r union air fel arall:

Perhaps the best reason of all for taking the trouble to use the AC [acceptable word] method is that it appears much fairer than the EX [exact word] method to the students themselves. There is something inherently repugnant about counting an answer wrong, which is actually correct, simply because the

\footnotetext{
${ }^{54}$ Pa mor dda y mae'r prawf yn mesur yr hyn y mae'r prawf yn honni ei fod yn ei fesur yw ystyr 'dilysrwydd' yma (Brown, 1980, tt. 311-312).

${ }^{55}$ Pa mor gyson yw canlyniadau'r prawf yw ystyr 'dibynadwyedd' yma (Brown, 1980, t. 311).
} 
author of the original passage did not choose to use that word; yet, precisely that often happens when the EX method is used. (Brown, 1980, t. 316)

\subsubsection{Rhai o gyfyngiadau'r prawf llenwi bylchau}

Er bod hwn yn brawf sydd wedi'i ddefnyddio'n aml mewn ymchwil, ymddengys nad oes cytundeb ymhlith ysgolheigion o ran pa agweddau ieithyddol penodol a gaiff eu mesur gan y prawf llenwi bylchau (Tremblay, 2011; Tremblay \& Garrison, 2010). Mae rhai astudiaethau wedi honni bod y prawf yn gallu mesur sgiliau ieithyddol lefel isel yn unig (Alderson, 1979; Shanahan, Kamil, \& Tobin, 1982). Er enghraifft, mewn cyfres o dri arbrawf fe astudiodd Shanahan, Kamil a Tobin (1982) pa fath o effaith y mae defnyddio gwybodaeth a gesglir ar draws brawddegau (intersentential information) yn ei gael ar berfformiad mewn prawf llenwi bylchau. Trwy gymharu profion llenwi bylchau lle cafodd y testun ei adael yn union fel y mae, â phrofion llenwi bylchau lle cafodd y testun ei anhrefnu, darganfu'r awduron na chafodd yr anhrefnu effaith sylweddol ar allu'r cyfranogwyr i berfformio yn y prawf llenwi bylchau ac felly daethant i'r casgliad nad oes cydberthyniad rhwng defnyddio gwybodaeth a gesglir ar draws brawddegau a pherfformiad mewn profion llenwi bylchau.

Yn groes i'r casgliad hwn, mae rhai ysgolheigion wedi dadlau bod y prawf yn fwy integredig ac yn gallu mesur cymhwysedd disgwrs lefel uchel (Bachman, 1982; Hanania \& Shikhani, 1986; Jonz, 1990; Laesch \& Kleeck, 1987). Mae Bachman (1982) er enghraifft wedi dangos bod y prawf llenwi bylchau, sy'n defnyddio dull dileu geiriau ar sail resymegol, yn mynnu gwybodaeth ar draws cymalau a brawddegau, yn ogystal â gwybodaeth ar lefel cymalau a brawddegau. Wrth ddileu geiriau ar sail resymegol, ceisiodd Bachman sicrhau bod y prawf yn cynnwys eitemau cydlynol (cohesive items) a fyddai'n dangos a yw'r prawf yn gorfodi'r cyfranogwyr i ddefnyddio gwybodaeth ar draws cymalau a brawddegau wrth lenwi'r prawf. Mae canfyddiadau Bachman yn awgrymu bod y prawf llenwi bylchau sy'n dileu geiriau ar sail resymegol yn brawf integredig sy'n mynnu sgiliau ieithyddol uwch oherwydd bod y prawf yn gorfodi'r cyfranogwyr i chwilio am wybodaeth ar draws cymalau a brawddegau, yn ogystal â mesul cymal a brawddeg, er mwyn llenwi'r bylchau'n gywir. 
Caiff y safbwynt hwn ei gadarnhau gan Jonz (1990). Mae Jonz o'r farn fod y dull llenwi bylchau yn herio prosesau megis adnabod geiriau ac adeiladu cydsyniadau, ac felly mae'r prawf yn mynnu sgiliau ieithyddol uwch o reidrwydd. Fel y noda: "The cloze procedure, it is asserted, challenges universal processing mechanism... at all levels from word recognition through concept building; therefore, responding to cloze tests must necessarily involve a great deal of higher-order language processing." (Jonz, 1990, tt. 62-63). Mae Laesch a Kleeck (1987) hefyd yn cadarnhau bod y prawf llenwi bylchau yn addas ar gyfer gwahaniaethu rhwng gwahanol lefelau hyfedredd ieithyddol. Astudiodd Laesch a Kleeck 30 o ddisgyblion dwyieithog (Sbaeneg-Saesneg) gradd 3 (8-9 mlwydd oed) a gafodd eu labelu'n 'rhugl' yn y Saesneg ar sail Language Assessment Scales (LAS). Gan weithredu prawf llenwi bylchau, darganfu'r ymchwilwyr fod y disgyblion, er y gellid eu hystyried yn 'rhugl', yn gwahaniaethu o ran eu sgoriau ar y prawf llenwi bylchau, gyda'r rhai a berfformiodd orau yn y prawf yn defnyddio cliwiau ieithyddol a chyddestunol yn well na'u cyfoedion wrth ateb y prawf llenwi bylchau. Cred Laesch a Kleeck (1987, t. 184) fod y perfformwyr gorau yn "less bound to the decoding of grammar and had greater cognitive availability to process larger chunks of information and search for meaning."

Ymddengys o'r llenyddiaeth fod angen i'r cyfranogwr fedru defnyddio nifer o sgiliau ieithyddol mewn ffordd integredig, er mwyn cwblhau prawf llenwi bylchau. Ymddengys hefyd fod y rhai sy'n perfformio orau mewn prawf llenwi bylchau yn gallu gwneud gwell defnydd o'r testun a'r wybodaeth rhwng cymalau / brawddegau ac ynddynt hwy hefyd. Mae Laesch a Kleeck (1987) yn mynd cyn belled ag awgrymu fod hyn yn profi y gall y prawf llenwi bylchau fesur sgiliau mwy academaidd. Gan fod perfformwyr gorau'r prawf llenwi bylchau yn gallu prosesu darnau hwy o destun, yn hytrach na dehongli'r testun fesul gair neu frawddeg fel y perfformwyr gwannach, dadleua Laesch a Kleeck fod hyn yn dangos hyfedredd mwy academaidd, sef CALP.

Rhaid nodi na all y fath brawf fesur sgiliau penodol, megis sgiliau llafar a chlywedol, a gall hyn fod yn gyfyngiad mawr ar unrhyw ymchwil sy'n ceisio astudio'r sgiliau hyn yn benodol (Tremblay, 2011, t. 363; Tremblay \& Garrison, 2010, t. 84). Dadleua Tremblay a Garrison (2010, t. 84), a Tremblay (2011, t. 362), fod angen i brofion llenwi bylchau gael eu defnyddio ar y cyd â meini prawf eraill, megis cefndiroedd 
ieithyddol y cyfranogwyr ac asesiadau llafar i fesur sgiliau llafar a chlywedol, er mwyn cryfhau dilysrwydd y prawf llenwi bylchau. Er i ymchwil Bilki (2011) ddangos bod cydberthynas rhwng sgoriau cyfweliadau llafar a sgoriau profion llenwi bylchau, nododd ei bod yn well defnyddio'r prawf llenwi bylchau i gefnogi profion penodol sy'n mesur sgiliau cynhyrchu yn hytrach na'i ddefnyddio i gymryd lle'r profion penodol hyn oherwydd diffyg tystiolaeth ac ymchwil bellach i'r maes:

Although strong correlation was found between writing/speaking tests and cloze tests, the numbers indicate that the cloze tests might not be adequate measuring tools for the productive skills on their own. This suggests a supportive use of cloze tests. The differing correlations also suggested a careful use of such tests. In conclusion, cloze tests can be valuable tools, if used correctly, to help assess speaking and writing skills. (Bilki, 2011, t. 76)

Mae McKamey (2006) yntau wedi ymgymryd â safbwynt gofalus tebyg i Bilki (2011). Dadleua McKamey fod yn rhaid darganfod pa sgiliau ieithyddol penodol y mae'r prawf llenwi bylchau yn eu mesur, ac i ba raddau, os ydyw i gael ei ddefnyddio fel prawf dilys ar gyfer mesur hyfedredd ieithyddol cyffredinol. Wrth astudio sgiliau ieithyddol penodol gramadeg, darllen a geirfa, ceisiodd McKamey ddangos i ba raddau y mae'r sgiliau hyn yn cyfrannu at berfformiad mewn prawf llenwi bylchau. Dangoswyd bod y sgiliau ieithyddol hyn yn cyfrannu'n gyfartal at berfformiad mewn prawf llenwi bylchau ac felly fod y prawf yn "fair measurement of language ability” (McKamey, 2006, t. 149). Fodd bynnag, cred McKamey fod defnyddio'r prawf llenwi bylchau ar gyfer mesur sgiliau penodol yn agored i ddadleuon pellach o hyd a rhybuddia hi ymchwilwyr rhag llunio prawf o'r fath heb ystyried natur y prawf a nodau penodol eu hymchwil hwy.

\subsubsection{Y prawf llenwi bylchau fel mesurydd hyfedredd ieithyddol cyffredinol}

Er gwaetha'r anghytuno o ran pa agweddau ieithyddol yn union y mae'r prawf llenwi bylchau yn eu mesur, mae'n debyg fod ganddo botensial fel mesurydd hyfedredd ieithyddol cyffredinol, oherwydd ei fod yn mynnu defnydd o sgiliau ieithyddol mewn ffordd integredig. Mewn sawl astudiaeth, dangoswyd bod profion llenwi bylchau yn cydberthyn â phrofion sy'n mesur gallu darllen a deall (Benjama \& Palanukulwong, 2015; Hinofotis, 1980; McKamey, 2006; J. W. J. Oller \& Conrad, 1971), gwrando ac ysgrifennu (J. W. J. Oller \& Conrad, 1971), gwrando a deall (Hinofotis, 1980), gramadeg (Benjama \& Palanukulwong, 2015; Hinofotis, 1980; 
McKamey, 2006; J. W. J. Oller \& Conrad, 1971), a geirfa (Benjama \&

Palanukulwong, 2015; Hinofotis, 1980; McKamey, 2006; J. W. J. Oller \& Conrad, 1971). Oherwydd canfyddiadau o'r fath, cred ysgolheigion fel Oller a Conrad (1971)

a Hinofotis (1980) fod y prawf llenwi bylchau yn fesurydd hyfedredd ieithyddol cyffredinol, yn hytrach na mesurydd darllen a deall yn unig (McKamey, 2006, t. 117).

Mae effeithiolrwydd y prawf fel mesurydd hyfedredd ieithyddol cyffredinol hefyd wedi'i gadarnhau gan nifer o astudiaethau sydd wedi dangos bod sgoriau ar y prawf llenwi bylchau yn cydberthyn yn agos â sgoriau ar brofion ieithyddol safonol eraill, megis TOEFL (Test of English as a Foreign Language) (Darnell, 1968; Fotos, 1991), prawf lleoli Prifysgol Califfornia Los Angeles (University of California at Los Angeles ESL Placement Examination) (J. W. J. Oller, 1972), a phrawf mynediad Saesneg Prifysgol Americanaidd Beirut (English Entrance Examination of the American University of Beirut) (Stubbs \& Tucker, 1974). Yn yr astudiaeth olaf, astudiodd Fotos (1991) y prawf llenwi bylchau a'i gydberthynas â sgoriau TOEFL a sgoriau traethodau Saesneg ymhlith myfyrwyr mewn addysg uwch yn Siapan.

Pwrpas yr astudiaeth oedd darganfod a yw'n bosibl defnyddio'r prawf llenwi bylchau megis "alternative measure of integrative language ability" (Fotos, 1991, t.322). Gan ddilyn strwythur Hanania a Shikhani (1986), dewiswyd tri thestun ysgrifenedig a oedd yn addas i lefel ganolradd siaradwyr Saesneg fel iaith dramor. Darganfu Fotos fod gan y prawf llenwi bylchau gydberthynas agos â sgoriau yn y traethodau a'r prawf TOEFL, a bod y gorgyffwrdd a ganfuwyd rhwng y tri ohonynt yn awgrymu eu bod yn ddilys ar gyfer mesur hyfedredd ieithyddol yn y Saesneg:

Not only did the three tests correlate highly with each other, but the results of multiple regression analysis also indicated that the combination of any two measures significantly increased the predicting of the third. The existence of a substantial overlap among the cloze test, the essay, and the TOEFL indicates their construct validity as measurements of English language proficiency. (Fotos, 1991, t. 331)

Casglodd Fotos (1991, t. 331) fod ei chanfyddiadau yn cefnogi'r honiad y gall y prawf llenwi bylchau gael ei ddefnyddio megis "[a] measure of integrative language proficiency" ac awgrymodd fod y sgiliau y mae'r prawf yn eu mesur yn dibynnu ar lefel hyfedredd y cyfranogwyr. Er enghraifft, os llunnir prawf ar gyfer siaradwyr 
sydd â hyfedredd sylfaenol yn yr ail iaith, mae'n bosibl y gallai'r prawf fesur sgiliau sylfaenol yn unig. Ond os llunnir prawf ar gyfer siaradwyr sydd â hyfedredd uwch, fel y mae Fotos yn ei wneud, mae'n bosibl y gallai'r prawf fesur sgiliau ieithyddol uwch. Mae Sasaki (2000) wedi amlygu pwysigrwydd dewis testun addas ar gyfer y prawf, wrth ddarganfod bod testun sy'n anghyfarwydd yn ddiwylliannol yn gallu amharu ar allu i berfformio mewn prawf llenwi bylchau. Mae Tremblay a Garrison (2010) hefyd yn cydnabod pwysigrwydd dewis testun addas yn eu hastudiaeth o siaradwyr Ffrangeg ail iaith. Yma, dewiswyd testun sydd yn "accessible in content to a population of college-level students" (Tremblay a Garrison, 2010, t. 77). Os yw'r prawf am wahaniaethu rhwng cyfranogwyr sydd â lefelau tebyg o hyfedredd, ymddengys fod rhaid i'r testun fod yn addas ac yn deg i bawb sy'n cyfrannu. Yn y pen draw, mae dilysrwydd y prawf fel mesurydd dibynadwy ar gyfer hyfedredd ieithyddol cyffredinol yn dibynnu i raddau helaeth ar ba mor ofalus y caiff y prawf ei baratoi, ei dreialu a'i ddilysu (Fotos, 1991, t. 334; Hanania \& Shikhani, 1986, t. 108).

\subsubsection{Manteision ymarferol defnyddio'r prawf llenwi bylchau}

Yn ogystal â'i fudd fel prawf sydd â'r potensial i fesur hyfedredd ieithyddol cyffredinol siaradwyr ail iaith, yn hytrach na mesur sgiliau wedi'u targedu'n benodol, mae'n debyg fod y prawf llenwi bylchau wedi bod yn boblogaidd fel dull ymchwil oherwydd ei ymarferoldeb. Mae Brown (1980), er enghraifft, wedi dangos bod y prawf llenwi bylchau yn ddull ymchwil ymarferol o safbwynt ei lunio, ei weithredu a'i sgorio, ni waeth pa ddull sgorio a ddefnyddir. Mae Stubbs a Tucker (1974) hefyd wedi cefnogi'r safbwynt hwn. Disgrifiant y prawf llenwi bylchau fel "[a] powerful and economical measure of English-language proficiency for nonnative speakers as well as a useful diagnostic tool for the classroom teacher." (Stubbs \& Tucker, 1974, t. 241). Yn fwy diweddar o lawer, mae Tremblay (2011), a hefyd Tremblay a Garrison (2010), wedi mynegi bod y prawf llenwi bylchau yn ymarferol megis dull ymchwil i siaradwyr ail iaith yn arbennig os nad yw profion safonol ar gael iddynt. Ymddengys hefyd y gall y prawf llenwi bylchau fod yn fwy ymarferol na phrofion safonol oherwydd ei fod yn cymryd llai o amser i gwblhau'r prawf llenwi bylchau a'i fod yn fwy hyblyg o ran ei ffurf (Tremblay, 2011, t. 346; Tremblay \& Garrison, 2010, t. 75). 


\subsubsection{Rhesymeg o ran defnyddio'r prawf llenwi bylchau}

Dewiswyd y prawf llenwi bylchau ar gyfer y gwaith maes hwn am bedwar rheswm penodol, sef:

i. Diffyg profion safonol sydd yn addas ar gyfer mesur gallu ieithyddol disgyblion ail iaith yn benodol yn y Gymraeg ${ }^{56}$

ii. Addasrwydd y prawf fel mesurydd hyfedredd ieithyddol cyffredinol siaradwyr ail iaith, yn hytrach na sgiliau ieithyddol penodol

iii. Ymarferoldeb llunio, gweithredu a sgorio'r prawf

iv. Cyngor cyn-ymchwilydd profiadol i Sefydliad Cenedlaethol er Ymchwil i Addysg (SCYA o hyn ymlaen) ${ }^{57}$

Fel y nodwyd eisoes, penderfynodd yr ymchwilydd lunio prawf llenwi bylchau gwreiddiol (gweler Atodiad 7) er mwyn mynd i'r afael â chwestiynau ymchwil 2 a 3:

2. Beth yw proffiliau ieithyddol a sosio-economaidd tair carfan o ddysgwyr sydd yn derbyn gwahanol fewnbwn Cymraeg yn y ddwy ysgol gyfranogol?

3. A yw'r prawf llenwi bylchau yn brawf dibynadwy a dilys ar gyfer mesur hyfedredd ieithyddol cyffredinol dysgwyr ail iaith yn y Gymraeg?

\subsubsection{Ffurf y prawf}

Yn unol ag arfer Tremblay a Garrison (2010), penderfynwyd llunio prawf llenwi bylchau sy'n dileu geiriau ar sail resymegol, hynny yw, dileu nifer debyg o'r gwahanol fathau o eiriau cynnwys (content words) a geiriau swyddogaethol (function words). Pwrpas dileu geiriau yn y ffordd hon yw sicrhau bod y prawf yn profi gwahanol fathau o eiriau posibl yn hytrach na chanolbwyntio ar eitemau ieithyddol penodol megis berfau, ansoddeiriau ayyb.

\footnotetext{
${ }^{56}$ Er yr ystyriwyd Profion Darllen Cenedlaethol a Profion Darllen Cymru Gyfan, penderfynwyd na fyddent yn addas ar gyfer asesu hyfedredd darllen Cymraeg disgyblion Cymraeg Ail Iaith oherwydd bod y profion hyn wedi'u llunio at ddiben mesur gallu darllen disgyblion mewn ffrydiau / lleoliadau cyfrwng Cymraeg.

${ }^{57}$ Ar ffurf e-byst a chyfarfod â'r arbenigwr, trafodwyd y profion safonol posibl (gweler y troed nodyn uchod) y gellid eu defnyddio ar gyfer y gwaith maes, yn ogystal â'r posibilrwydd o lunio a gweithredu prawf llenwi bylchau gwreiddiol. Ar ôl trafod yr anhawster ynglŷn â defnyddio'r profion hyn, trafodwyd â'r arbenigwr y posiblrwydd o lunio prawf llenwi bylchau gwreiddiol a chynigodd ef gyngor ynglŷn â sut y mae llunio, gweithredu a marcio profion llenwi bylchau.
} 
Er bod yr ymchwil yn awgrymu bod cynnig eitemau amlddewis yn gwneud y prawf llenwi bylchau yn haws (Brown, 1980), ac felly bod hyn yn fwy addas i gynulleidfa darged sydd â lefelau hyfedredd is yn yr ail iaith (Tremblay, 2011), ni chynigiwyd eitemau amlddewis yn y prawf llenwi bylchau hwn am ddau reswm penodol. Yn gyntaf, penderfynwyd na fyddai prawf amlddewis yn ymarferol o safbwynt ei lunio (rhaid creu cronfa o 4-5 gair tebyg ar gyfer pob eitem) ac o safbwynt ei wedd (mae'n anodd sicrhau bod prawf amlddewis yn gryno ond ar yr un pryd yn hawdd ei ddarllen a'i ddefnyddio gan y cyfranogwyr). Yn ail, cadwyd y bylchau'n benagored er mwyn gwneud y prawf yn anos, sy'n bwysig os yw'r prawf i wahaniaethu rhwng siaradwyr ail iaith sydd â lefelau hyfedredd uwch (Tremblay \& Garrison, 2010). Er na fydd hyn yn hollbwysig ar gyfer y prawf peilot (gan y bydd y disgyblion yn debyg o ran eu gallu), bydd yn bwysig wrth wahaniaethu rhwng disgyblion y Cwrs Carlam a disgyblion y ddau grŵp rheoli. Yn ogystal â dileu eitemau sy'n mynnu gwybodaeth o fewn y frawddeg (28 eitem), penderfynwyd dileu eitemau sy'n mynnu gwybodaeth ar draws brawddegau (11 eitem), a gwybodaeth o fannau eraill yn y testun (4 eitem) i geisio gorfodi'r cyfranogwyr i ddefnyddio sgiliau ieithyddol uwch er mwyn llenwi rhai o'r bylchau. Penderfynwyd rhannu'r prawf yn ddwy ran, sef 'Rhan A' a 'Rhan B', er mwyn ceisio sicrhau bod anhawster y prawf yn dwysáu wrth i'r cyfranogwr fynd yn ei flaen trwy'r prawf.

\subsubsection{Dull sgorio'r prawf}

Ar sail canfyddiadau'r llenyddiaeth, sy'n awgrymu mai derbyn unrhyw air sy'n gwneud synnwyr yn gyd-destunol yw'r dull mwyaf dibynadwy, penderfynwyd defnyddio'r dull sgorio hwn. Gan mai hyfedredd ieithyddol sylfaenol ${ }^{58}$ a fydd gan gyfranogwyr y prawf llenwi bylchau, ni fydd angen cynnal rhagbrawf gyda disgyblion iaith gyntaf er mwyn casglu rhestr o eitemau posibl, fel a wneid gan Tremblay a Garrison (2010). Yn hytrach, penderfyna'r ymchwilydd a yw'r eitemau a gynigir gan y cyfranogwyr yn gywir yng nghyd-destun y prawf a osodir. Os bydd yr eitem yn gywir, rhoddir 1 marc. Os bydd yn anghywir, ni roddir marc. Os bydd unrhyw amheuaeth yn codi o ran dilysrwydd eitemau penodol, oherwydd llawysgrifen wael neu sillafu aneglur, ni cheir marc ar gyfer yr eitem. Eto, gan mai

\footnotetext{
${ }^{58}$ Cyfeirir at gyfranogwyr yr astudiaeth fel dysgwyr ail iaith 'sylfaenol' ar y sail: i) yr ychydig o flynyddoedd y maent wedi derbyn addysg yn y Gymraeg erbyn hyn; a ii) cyswllt cyfyngedig y dysgwyr â'r Gymraeg, ac felly eu cyfleoedd i ddefnyddio'r iaith, y tu hwnt i'r dosbarth.
} 
hyfedredd ieithyddol sylfaenol a fydd gan gyfranogwyr y prawf, penderfynwyd caniatáu eitemau sydd wedi'u camsillafu os bydd y gair y mae'r cyfranogwr yn ceisio ei ddefnyddio yn glir i'r ymchwilydd.

\subsubsection{Testun y prawf}

Un o'r themâu sy'n codi'n gyson yn y llenyddiaeth yw arwyddocâd dewis testun addas i'r rhai sy'n sefyll y prawf. Fel y nodwyd eisoes, mae Sasaki (2000) wedi dangos y gall testun sy'n anghyfarwydd i'r cyfranogwr o ran ei gyd-destun diwylliannol amharu ar allu'r cyfranogwr i berfformio mewn prawf llenwi bylchau. Os yw'r prawf i fesur gallu ieithyddol am fod yn deg ac addas, rhaid dewis testun sy’n gyfarwydd i gyfranogwyr y prawf:

... if the intended purpose includes measuring examinees' linguistic ability ... culturally unfamiliar texts may present a serious threat to adequate test score interpretation. In such a case, test developers should choose texts that are familiar enough for the intended examinees to fully use their knowledge (Sasaki, 2000, t. 108).

Ar ôl ystyried nifer o destunau addas posibl ${ }^{59}$ a chynnal cyfarfod anffurfiol â chynymchwilydd profiadol i SCYA, penderfynwyd y byddai llunio testun gwreiddiol ${ }^{60}$ yn fwy addas oherwydd gellir rheoli geirfa a phatrymau ieithyddol, yn ogystal â dewis pwnc a chyd-destun sydd o fewn cyrraedd disgyblion CAI ym Mlwyddyn Ysgol 9. Ar ôl ystyried y pynciau posibl a fyddai o fewn cyrraedd y cyfranogwyr ${ }^{61}$, dewiswyd 'chwaraeon' gan fod hon yn thema sy'n codi yn y pwnc 'hobïau' ym Mlwyddyn 7 ac ym mhynciau 'hamdden' a ‘bwyta'n iach a chadw'n heini' ym Mlwyddyn 8/9.

\subsubsection{Sgoriau darllen Saesneg}

Yn ogystal â defnyddio sgoriau'r prawf llenwi bylchau at ddiben gyflwyno proffiliau ieithyddol y tair carfan o ddisgyblion, penderfynwyd defnyddio sgoriau Saesneg y

\footnotetext{
${ }^{59}$ Ystyriwyd cylchgronau megis iaw! a Lingo Newydd; yn ogystal â blog a luniwyd gan Adran Gymraeg mewn ysgol uwchradd cyfrwng Saesneg ar gyfer eu disgyblion CA3 a 4.

${ }^{60}$ Cafodd testun y prawf terfynol ei seilio ar batrymau ieithyddol a geirfa mewn llyfr iaith ar gyfer disgyblion Cymraeg Ail Iaith ym mlwyddyn ysgol 9, sef Llyfr Iaith Blwyddyn 9. Darparwyd y llyfr iaith hwn ar gyfer yr ymchwilydd gan bennaeth adran Gymraeg mewn ysgol uwchradd cyfrwng Saesneg nad oedd yn rhan o'r prawf terfynol.

${ }^{61}$ Yn CA3, nid oes pynciau sefydlog a gaiff eu haddysgu'n orfodol. Fodd bynnag, ar wefan Cymraegailiaith (ar gael ar: http://www.cymraegailiaith.com) ceir trosolwg o'r pynciau posibl a all gael eu haddysgu ym Mlynyddoedd 7, 8 a 9.
} 
Prawf Darllen Cenedlaethol (canlyniadau Blwyddyn 9) fel mesurydd o allu ieithyddol y disgyblion yn yr iaith gyntaf, sef Saesneg. Penderfynwyd dadansoddi sgoriau Saesneg y Prawf Darllen Cenedlaethol yn benodol am mai profion safonol yw'r rhain ac felly bydd gan bob disgybl sgôr darllen safonedig y gellir ei defnyddio wrth lunio proffiliau ieithyddol ar gyfer y tri dosbarth. Fel y nodwyd eisoes, nid oedd prawf safonol tebyg, a fyddai'n addas ar gyfer mesur gallu ieithyddol y disgyblion hyn yn y Gymraeg, ar gael i'r ymchwilydd adeg cynnal yr astudiaeth hon. Gan mai prawf dienw oedd y prawf llenwi bylchau, nodir hefyd nad oedd modd paru sgoriau'r prawf hwn â sgoriau'r Prawf Darllen fesul cyfranogwr. Oherwydd hynny, bydd y dadansoddiad yn cyflwyno data'r prawf llenwi bylchau a'r data PYDd fesul dosbarth.

\subsubsection{Prydau Ysgol am Ddim}

Defnyddio data PYDd yw un o'r ffyrdd mwyaf cyffredin o fesur amddifadedd cymdeithasol (LlC, 2016a). Fel y mae LlC yn ei nodi, "pan fo nifer y disgyblion sy'n cael prydau ysgol am ddim yn cynyddu", fel arfer y "mae lefelau perfformiad yn gostwng" (L1C, 2016a, Cwestiynau cyffredin, "Pam defnyddio Prydau Ysgol Am Ddim?"). Fodd bynnag, cydnabyddir nad "cydberthynas bendant" yw hon, a bod rhai ysgolion yn perfformio'n well o'u cymharu ag ysgolion sydd â lefelau uwch na thebyg o PYDd (L1C, 2016a, Cwestiynau cyffredin, "Pam defnyddio Prydau Ysgol Am Ddim?"). Serch hynny, mae LlC yn defnyddio data PYDd fel dangosydd o amddifadedd cymdeithasol o fewn ysgolion ar draws Cymru. Defnyddir y data hwn hefyd wrth rannu ysgolion yn deuluoedd o ysgolion. Diben gosod ysgolion yn deuluoedd o ysgolion yw sicrhau eu bod yn "wynebu'r un lefel o her yn gyffredinol" ag ysgolion eraill o fewn yr un teulu (L1C, 2016a, Cwestiynau cyffredin, "Pam defnyddio Prydau Ysgol Am Ddim?”).

Yn dilyn arfer LIC (2016a), penderfynwyd defnyddio data PYDd y tri dosbarth a gymerodd ran yn y prawf llenwi bylchau fel dangosydd o'u hamddifadedd cymdeithasol wrth lunio proffiliau sosio-economaidd ar gyfer y tair carfan o ddisgyblion. Cydnabyddir y byddai mesur o gefndir sosio-economaidd rhieni'r plant, er enghraifft, lefelau addysgol y rhieni, wedi bod yn well fesur o'u lefelau sosioeconomaidd, gan mai dyma un o'r mesuryddion mwyaf dibynadwy ar gyfer rhagfynegi cyflawniad academaidd a chyrhaeddiad addysgol disgyblion (Reardon, 
2011). Er hynny, nodir nad oedd modd cyrchu data o'r fath oherwydd cyfyngiadau'r gwaith maes. (Am drafodaeth lawnach, gweler 6.10. Cyfyngiadau'r astudiaeth.) Eto, gan mai prawf dienw oedd y prawf llenwi bylchau, nodir nad oedd modd paru data'r prawf llenwi bylchau â'r data PYDd fesul cyfranogwr. Oherwydd hynny, bydd y dadansoddiad yn cyflwyno data'r prawf llenwi bylchau a'r data PYDd fesul dosbarth.

\subsection{Cyfyngiadau'r astudiaeth}

Er gwaethaf manteision defnyddio methodoleg yr astudiaeth achos i'r ymchwil hon a nodwyd eisoes, rhaid hefyd dalu sylw i'r cyfyngiadau posibl. Efallai mai prif gyfyngiad y fethodoleg hon yw nad yw'n caniatáu i'r ymchwilydd greu cymhariaeth uniongyrchol ar draws y tair carfan o ddisgyblion. Pe bai modd cymharu'r dysgwyr, gallai'r ymchwilydd fod wedi gwerthuso effeithiolrwydd y Cwrs Carlam fel model addysg ddwyieithog. Er bod yr ymchwilydd am werthuso'r Cwrs Carlam trwy gynnal ymchwil arbrofol, daeth i'r amlwg wrth drafod y gwaith maes gyda'r ddwy ysgol na fyddai modd gwneud hyn yn yr astudiaeth hon, a hynny yn bennaf oherwydd pwysau eraill (ar Ysgol X yn benodol) ar y pryd, megis arolygiad Estyn ac arholiadau. Teimlai'r ysgol y byddai'n well pe gallai'r ymchwilydd gynnal gwaith maes a fyddai'n golygu'r ymyrraeth leiaf posibl ar y disgyblion a'r dysgu o ddydd i ddydd. Oherwydd hynny, nid oedd modd mesur hyfedredd ieithyddol cyffredinol y dysgwyr yn y Gymraeg cyn, yn ystod, ac ar ôl yr ymyrraeth (hynny yw, y ddarpariaeth Gymraeg ychwanegol a geir trwy astudio'r Cwrs Carlam).

Nodir nad oedd modd i'r ymchwilydd gynnal ymchwil ac iddi ffocws penodol ar aelodau eraill o gymuned yr ysgol, megis y disgyblion, eu rhieni, a'r staff addysgu (e.e. trwy gynnal grwpiau ffocws). Byddai ffocws o'r fath wedi bod yn fuddiol, er enghraifft, er mwyn archwilio cymhelliant disgyblion y tair carfan o ran dysgu Cymraeg, yn ogystal â chymhelliant athrawon sy'n addysgu'r disgyblion hyn, ac er mwyn cychu data ar lefelau addysgol rhieni'r disgyblion cyfranogol. Yn ogystal â hyn, nodir oedd gan yr ymchwilydd fynediad at fesuriadau o allu an-ieithyddol y disgyblion cyfranogol. Cynabyddir y byddai mesuriadau o'r fath wedi bod yn ddefnyddiol er mwyn amlygu sgiliau cyffredinol y gwahanol ddosbarthiadau. Er hynny, wrth ystyried honiad Zydatiß (2012) bod cydberthynas rhwng gallu academaidd a gallu ieithyddol, gellid dadlau bod sgoriau darllen Saesneg y 
disgyblion cyfranogol yn ddangosydd o'u gallu cyffredinol, yn ogystal â'u gallu yn yr iaith gyntaf.

Cyfyngiad arall ar yr astudiaeth achos yw nad yw'r math hwn o ymchwil yn anelu at brofi achosiaeth. Dyma un o brif gryfderau ymchwil arbrofol, er enghraifft, lle mae'r ymchwilydd yn rheoli a thrin newidynnau er mwyn canfod y berthynas rhwng newidynnau a'u heffaith ar y ffenomen dan sylw (Cohen ac eraill, 2003). Er bod modd i'r ymchwilydd ddadansoddi'r berthynas rhwng newidynnau mewn modd ansoddol a holistaidd yn yr astudiaeth achos, nid oes modd i'r ymchwilydd ddangos yn feintiol i ba raddau y mae'r gwahanol newidynnau yn effeithio ar y ffenonmen dan sylw. I'r un perwyl, rhaid nodi hefyd nad diben yr astudiaeth achos yw cyffredinoli canfyddiadau i'r boblogaeth darged, sef pob ysgol uwchradd cyfrwng Saesneg yng Nghymru. Fel y mae Cohen ac eraill (2003) yn nodi, canolbwyntia'r astudiaeth achos ar gyflwyno casgliadau ar achosion penodol y gellir dysgu ganddynt, yn hytrach na chyflwyno canfyddiadau a all gael eu cyffredinoli i bob sefyllfa debyg. I'r un perwyl, pwysleisia Bassey (1981, t. 85) fod 'relatability' canfyddiadau'r astudiaeth achos, hynny yw, i ba raddau y mae'r canfyddiadau yn "sufficient and appropriate for a teacher working in a similar situation to relate his decision making to that described in the case-study", yn bwysicach na gallu'r canfyddiadau gael eu cyffredinoli i'r boblogaeth darged. A dywed Stake (2005, t. 460): "Case studies are of value for refining theory and suggesting complexities for further investigation, as well as helping to establish the limits of generalizability." Nod yr astudiaeth achos hon felly yw taflu golau ar y Cwrs Carlam a'r ffordd y mae'r ddwy ysgol gyfranogol yn mynd ati i gefnogi cyflwyno'r Gymraeg yn llwyddiannus, gan hefyd awgrymu cyfeiriadau newydd i ymchwil bellach. 


\section{Pennod 7: Canlyniadau i: Yr holiadur, y cyfweliadau a'r arsylwadau ar ethos}

\subsection{Ysgol Y: Yr holiadur a'r cyfweliad}

\subsubsection{Gwybodaeth sosio-economaidd a sosio-ieithyddol}

Ysgol uwchradd cyfrwng Saesneg yn bennaf yn ne Cymru yw Ysgol Y (L1C, 2016a). Lleolir yr ysgol mewn cymuned gymharol ddi-Gymraeg, lle mae 7.9\% o boblogaeth y ward etholiad yn nodi eu bod yn gallu siarad Cymraeg ${ }^{62}$ ac mae $15.3 \%$ o boblogaeth yr awdurdod unedol ehangach yn nodi eu bod yn gallu siarad Cymraeg (Stats Cymru, 2013; Swyddfa Ystadegau Gwladol, 2012ch). Mae gan yr ysgol hon ryw 770 o ddisgyblion ar ei chofrestr (LlC, 2016a), sy'n cael eu tynnu o ystod o gefndiroedd sosio-economaidd (Estyn, 2013a). Nifer bresennol y rhai sydd â hawl i PYDd yn yr ysgol hon yw 24.1\% (LlC, 2016a). ${ }^{63}$

\subsubsection{Darpariaeth ar gyfer y Gymraeg}

Fel y nodwyd eisoes, ysgol cyfrwng Saesneg yn bennaf yw Ysgol Y, a chyflwynir pob pwnc cwricwlaidd trwy gyfrwng yr iaith Saesneg. Mae'r ysgol yn cynnig darpariaeth ar gyfer Cymraeg ar ffurf gwersi $\mathrm{CAI}^{64}$ yn unig, lle cyflwynir yr iaith yn y ffordd draddodiadol, fel pwnc cwricwlaidd mewn addysg brif ffrwd (gweler 2.5.3. Addysg iaith dramor fodern ac addysg ail iaith). Neilltuir tair awr y pythefnos $\mathrm{i}$ addysgu CAI yn CA3 (tua 57 awr y flwyddyn), pum awr y pythefnos yn CA4 (tua 97 awr y flwyddyn), ac wyth awr y pythefnos yn CA5 (tua 156 awr y flwyddyn). Erbyn diwedd eu haddysg orfodol yn 16 oed, bydd disgyblion yr ysgol wedi derbyn tua 365 o oriau dysgu Cymraeg fel pwnc ail iaith.

O ran y cyrsiau Cymraeg yn CA4, nodwyd mai'r Cwrs Llawn TGAU CAI yn unig a gynigir. Yn CA5, nodwyd y cynigir y cwrs TAG Safon Uwch Gyfrannol CAI (Safon UG CAI o hyn ymlaen) a'r cwrs TAG Safon Uwch Cymraeg (Safon Uwch CAI o

\footnotetext{
${ }^{62}$ Rhaid nodi bod nifer o ddisgyblion yn y dalgylch yn dod o ysgolion cynradd mewn pedair ward etholiad arall y tu allan i ward etholiad yr ysgol lle mae nifer y siaradwyr Cymraeg yn amrywio rhwng $7.9 \%$ hyd at $20.3 \%$. Ar gyfartaledd, mae $10.9 \%$ o boblogaeth y wardiau etholiad yn nalgylch yr ysgol yn gallu siarad Cymraeg.

${ }^{63}$ Seilir y ffigur hwn ar gyfartaledd PYDd dros y tair blynedd diwethaf.

${ }^{64}$ Defnyddir yr ymadrodd 'Cymraeg Ail Iaith' yma yn y cyd-destun addysgol, yn hytrach na chyddestun ieithyddol y disgybl. Mae'n bosibl i ddisgyblion, sydd wedi derbyn addysg gynradd drwy gyfrwng y Gymraeg, astudio'r pwnc Cymraeg Ail Iaith. Nid yw'r Gymraeg o reidrwydd yn ail iaith i bob disgybl, yn nhermau caffael iaith (gweler 2.6.5. Disgybl Cymraeg Ail Iaith.)
} 
hyn ymlaen). Mynegodd Pennaeth y Gymraeg ei balchder fod Prifathro'r ysgol wedi bod yn fodlon cynnig y Cwrs Llawn yn unig ar lefel TGAU. Roedd hithau o'r farn fod cynnig y Cwrs Llawn yn unig yn rhoi mwy o statws i'r pwnc, ac yn sgil hynny, mae'r disgyblion yn ei drin fel pwnc o werth. Gan nad yw'r ysgol yn cynnig y Cwrs Byr, gofynnwyd i Bennaeth y Gymraeg sut mae'r adran yn sicrhau y gall y disgyblion gwannaf ymdopi â gofynion y Cwrs Llawn. Esboniodd fod yr adran yn canolbwyntio ar agweddau haen sylfaenol y cwrs, megis "mynegi barn, yr amser gorffennol" (Atodiad 8, para. 10), er mwyn i'r disgyblion hyn allu cyrraedd gradd C yn y Gymraeg. Ychwanegodd hithau fod yr amser a neilltuir i addysgu'r Gymraeg (5 awr y pythefnos), ynghyd â maint bychan y setiau (rhwng 10 - 12 o ddisgyblion yn y setiau gwaelod), yn caniatáu i'r athrawon sicrhau y gall y disgyblion gwannaf dderbyn digon o fewnbwn er mwyn paratoi ar gyfer gofynion y Cwrs Llawn. Oherwydd statws CAI fel pwnc sylfaenol o fewn y cwricwlwm cenedlaethol, yn hytrach na phwnc craidd, ni chaiff ysgolion uwchradd Cymru eu cymharu â'i gilydd gan LlC o ran perfformiad yn y pwnc hwn, yn nhermau mesur perfformiad. Serch hynny, nododd Pennaeth y Gymraeg y caiff Cymraeg ei thrin fel petai'n un o'r pynciau craidd o fewn yr ysgol. Priodolwyd hyn i gefnogaeth y Prifathro, a'r Dirprwy sy'n gyn-bennaeth Adran y Gymraeg, i’r iaith Gymraeg ac Adran y Gymraeg yn yr ysgol. Oherwydd ymrwymiad y tîm uwch-reoli i drin y Gymraeg fel pwnc craidd, caiff pwysigrwydd y pwnc ei bwysleisio i'r disgyblion gan y staff. Teimlodd Pennaeth y Gymraeg fod y disgyblion ar y cyfan felly yn "derbyn" statws a phwysigrwydd y pwnc: “Ac rydyn ni i gyd yn ei ddrilio fe i mewn iddyn nhw. Pynciau craidd yn yr ysgol ydy Gwyddoniaeth, Mathemateg, Saesneg, Cymraeg ac Addysg Grefyddol. Ac maen nhw'n ei dderbyn e wedyn.” (Atodiad 8, para. 20). Fel y nodwyd eisoes, ni cheir darpariaeth ddwyieithog na chyfrwng Cymraeg yn Ysgol Y. Serch hynny, holwyd Pennaeth y Gymraeg am gapasiti’r ysgol i gynnig peth addysg ddwyieithog. Nododd yn syth mai un o'r prif ystyriaethau yw diffyg cymhwyster gan athrawon yr ysgol i addysgu Cymraeg a thrwy gyfrwng yr iaith honno. Er bod gan rai athrawon pwnc nad oes ganddynt gymhwyster addysgu Cymraeg y gallu i addysgu'r iaith hyd at lefel CA3, mae'n amlwg fod diffyg hyfforddiant yn golygu nad oes ganddynt y cymhwyster na'r hyder i gyflwyno gwersi Cymraeg na gwersi trwy gyfrwng y Gymraeg. Ychwanegodd mai ystyriaeth bwysig arall wrth weithredu addysg ddwyieithog yw sicrhau bod gan athrawon nid 
yn unig y gallu i addysgu trwy gyfrwng y Gymraeg ond yr awydd i wneud hefyd. Fel y mynegodd Pennaeth y Gymraeg, rhaid sicrhau bod yr athrawon hynny'n 'fodlon' addysgu trwy gyfrwng yr iaith Gymraeg.

Thema arall a godwyd wrth drafod y posibilrwydd o gynnig addysg ddwyieithog yn yr ysgol oedd prinder arian ar gyfer hyfforddi athrawon i addysgu'n ddwyieithog. Roedd Pennaeth y Gymraeg o'r farn y byddai angen sicrhau bod arian yn ei le pe bai'r ysgol yn cynnig hyfforddiant o'r fath i'w hathrawon:

PA: Wel hefyd mae'n dod i lawr i'r arian. So os mae rhyw fath o scheme, neu rywbeth sy'n gallu cynnig arian i'r ysgol, ac wedyn gofyn os mae'n bosibl i wneud rhyw fath o hyfforddiant fel hwnna, efallai bydd e'n bosibl. Dw i ddim yn siŵr. (Atodiad 8, para. 26)

Nodir ansicrwydd hefyd yn ei sylwadau parthed ymateb y Prifathro i gynnig y fath ddarpariaeth yn yr ysgol, fel y gwelir yn y dyfyniad canlynol: "Dw i ddim yn siŵr os mae'r prifathro yn mynd i fod yn hapus os rydyn ni'n cynnig - ac mae'n dod i lawr i'r plant." (Atodiad 8, para. 22). Er nad yw Pennaeth y Gymraeg yn mynd yn ei blaen i esbonio'r rhesymau dros yr ansicrwydd hwn, mae'n amlwg ei fod yn deillio o'r ffaith nad yw cynnig addysg ddwyieithog erioed wedi bod yn bwnc trafod o ddifrif yn yr ysgol: "Dydyn ni ddim rîlî wedi meddwl am hynna i fod yn onest gyda ti..." (Atodiad 8, para. 22). Pwynt arall i'w nodi yw bod Pennaeth y Gymraeg yn cyfeirio yma at y ffaith nad oes digon o ddisgyblion o 'gefndir Cymraeg' er mwyn i'r ysgol gyfiawnhau cynnig dosbarth dwyieithog. Mae'r cyfeiriad yma at ddisgyblion CIG o bosibl yn dangos bod Pennaeth y Gymraeg heb ystyried y posibilrwydd o gynnig rhaglen ddwyieithog i ddisgyblion ail iaith, ac mae'r dyfyniad uchod yn cefnogi hyn.

\subsubsection{Ethos dwyieithog}

Yng nghwestiwn 1, rhan 2 o'r holiadur, holwyd Pennaeth y Gymraeg a oes gan Ysgol Y bolisi swyddogol, ar bapur, ar gyfer datblygu a hyrwyddo dwyieithrwydd yn yr ysgol. Cadarnhaodd hi nad oes polisi o'r fath yn ei le. Fodd bynnag, nododd ei bod hi'n newydd i rôl Pennaeth Adran y Gymraeg ac mae hi am gydweithio â'r Prifathro, y Dirprwy Brifathro a'r disgyblion er mwyn llunio polisi ar ddwyieithrwydd, er lles y disgyblion a'u rheini. 
Er nad oes polisi swyddogol ar ddwyieithrwydd yn ei le, dywedodd Pennaeth y Gymraeg fod yr ysgol yn sicrhau y caiff dwyieithrwydd ei hyrwyddo yn feunyddiol yn bennaf ar ffurf "posteri o gwmpas yr ysgol" i helpu athrawon i ysgrifennu'r dyddiad a nod y wers, ac yn ystod y gwasanaethau sy'n “cynnwys dwyieithrwydd fel gweddïau a themâu." (Atodiad 2, Rhan 2).

Ar wahân i'r gweithgareddau beunyddiol hyn, ychwanegodd hi fod gan ddisgyblion $\mathrm{CIG}^{65}$ yn y Chweched Dosbarth rôl allweddol wrth hyrwyddo ethos dwyieithog yr ysgol. Yn ddiweddar, cafwyd trafodaeth ar y posibilrwydd o sefydlu 'Clwb Cymraeg' ar gyfer y disgyblion CIG, yn y gobaith y byddai disgyblion ail iaith yn ymuno nes ymlaen er mwyn cael eu mentora gan y disgyblion CIG. Rhaid nodi nad oedd clwb o'r fath wedi'i sefydlu ar adeg cynnal y gwaith maes.

Ymddengys hefyd fod rhai o'r disgyblion CIG eisoes wedi bod yn cefnogi'r adran gan gynorthwyo'r athrawon Cymraeg mewn dosbarthiadau CAI. Ymhelaethodd Pennaeth y Gymraeg ynghylch cymorth y disgyblion hyn yn y Chweched Dosbarth wrth i'r sgwrs symud yn ei blaen i drafod hyfforddiant yr ysgol ar gyfer defnyddio'r Gymraeg yn achlysurol. Ychwanegodd hi fod presenoldeb y disgyblion hyn yn y dosbarth CAI nid yn unig yn cynorthwyo'r athro sydd â dosbarth cyfan o ddisgyblion i'w haddysgu, ond hefyd yn sicrhau y gall y disgyblion ail iaith weld a chlywed disgyblion yn defnyddio'r Gymraeg:

PA: Hefyd, dw i'n credu bod y plant yn leicio gweld pobl o'r Chweched yn dod i mewn sy'n gallu siarad Cymraeg yn rhugl. Fel dywedais i, maen nhw'n edrych fel eu bod nhw'n cŵl ac maen nhw'n moyn siarad Cymraeg wedyn achos bod rhywun arall yn gallu ei wneud e. (Atodiad 8, para. 41)

\subsubsection{Hyfforddiant staff yr ysgol}

Er mwyn darganfod a oes modd i staff ddatblygu eu sgiliau dwyieithog a'u methodoleg addysgu cyfrwng Cymraeg / dwyieithog trwy hyfforddiant, holwyd cyfres o gwestiynau ynghylch y thema hon yn yr holiadur. Cadarnhaodd Pennaeth y Gymraeg fod yr ysgol yn cynnig hyfforddiant i'w staff er mwyn iddynt ddatblygu sgiliau dwyieithog. Fodd bynnag, datgelwyd mai gweithgareddau beunyddiol, yn

${ }^{65}$ Cyfeirir yma at ystyr addysgol y term, yn hytrach na'r iaith a gafodd ei chaffael yn gyntaf gan y disgybl. Dyma ddisgybl, er enghraifft, sydd wedi derbyn addysg gynradd cyfrwng Cymraeg ac sydd wedi trosglwyddo i'r ysgol uwchradd cyfrwng Saesneg (gweler 2.6.3. Disgybl Cymraeg Iaith Gyntaf.) 
hytrach na hyfforddiant ffurfiol, yw'r gweithgareddau y mae Pennaeth y Gymraeg yn cyfeirio atynt yn y fan hon. Nododd yn benodol fod y "staff yn hapus i gwrdd a chyfarch yn Gymraeg” a bod "llawer yn trio defnyddio'r iaith Gymraeg bob dydd yn eu gwersi neu yn y coridor." (Atodiad 2, Rhan 3). Nododd hefyd fod cyfle i staff ymarfer eu Cymraeg yn ystod cyfarfodydd. Yn ogystal â hyn, roedd Pennaeth y Gymraeg yn ystyried y posteri sy'n cael eu harddangos yn y dosbarth yn rhan o'r hyfforddiant i ddatblygu sgiliau dwyieithog y staff, gan eu bod yn eu "helpu nhw [y staff addysgu] gyda'r dyddiad, nod y wers" ac ati (Atodiad 2, Rhan 3). Ar ôl trafod hyn ymhellach yn ystod y cyfweliad, daeth i'r amlwg nad oes hyfforddiant ffurfiol yn cael ei ddarparu gan yr ysgol.

Cadarnhaodd Pennaeth y Gymraeg nad yw'r ysgol yn cynnig hyfforddiant i staff er mwyn datblygu eu methodoleg addysgu trwy gyfrwng y Gymraeg. Nododd Pennaeth y Gymraeg na fyddai digon o amser i’w drefnu, er y byddent yn ei groesawu. Cododd 'amser' ac 'arian' fel themâu eto yn y cyfweliad wrth drafod y posibilrwydd o gynnig cwrs hyfforddi gan yr ysgol:

PA: Mae'n rîlî anodd achos i fi, mae'n bwysig iawn os rydyn ni'n gallu cynnig gwersi yn Gymraeg ac yn Saesneg, so maen nhw'n gallu cael y dewis wedyn. Ond jest yr amser i'w drefnu, cael yr athrawon i mewn i'w wneud, a'r arian dw i'n credu. Dyna beth mae'n dod i lawr iddo. (Atodiad 8, para. 53)

Er i Bennaeth y Gymraeg ddangos ei diddordeb mewn cynnig cwrs hyfforddi ar ôl ysgol, ymddengys na allai'r ysgol ariannu cwrs hyfforddi wedi'i drefnu gan gorff allanol oherwydd prinder arian yn yr adran. Fel yr esbonia hi eto, arian yw un o'r prif rwystrau rhag cynnig hyfforddiant ffurfiol:

PA: Yn anffodus mae lot yn dod i lawr i'r arian. Dyna beth yw e rîlî, os rydyn ni'n - os rydyn ni'n dweud beth yw'r broblem, arian yw'r broblem yn anffodus. Rydyn ni'n cael trafferth i gael yr arian i wneud stwff yn yr ysgol ac yn yr adran Gymraeg. (Atodiad 8, para. 57)

Efallai mai'r prif rwystr i gynnig hyfforddiant ffurfiol ar gyfer datblygu sgiliau dwyieithog ac ar gyfer hyrwyddo dwyieithrwydd trwy'r ysgol yn gyffredinol yw categori ieithyddol yr ysgol. Fel yr esbonia Pennaeth y Gymraeg (Atodiad 8, para. 53) yn y cyfweliad, gan mai ysgol cyfrwng Saesneg yn bennaf yw Ysgol Y, yr iaith 
“Saesneg sy'n dod yn gyntaf yn anffodus." Er gwaethaf ymdrechion gan Adran y Gymraeg i hyrwyddo'r Gymraeg a dwyieithrwydd, ymddengys mai'r Saesneg sy'n cael ei blaenoriaethu oherwydd categori ieithyddol yr ysgol. Fel ysgol cyfrwng Saesneg yn bennaf, mae'n naturiol disgwyl mai Saesneg fydd "iaith yr ysgol o ddydd i ddydd" (L1CC, 2007b, t. 14) yn Ysgol Y.

\subsubsection{Pontio rhwng y sector cynradd a'r sector uwchradd}

Gan mai ysgol uwchradd gymharol fechan yw Ysgol Y, tair ysgol gynradd benodedig yn unig sy'n ei bwydo o fewn ei chlwstwr. Er hynny, nododd Pennaeth y Gymraeg y derbynia'r ysgol ddisgyblion o dair ysgol gynradd cyfrwng Saesneg arall yn yr ardal leol yn flynyddol. Cadarnhaodd hefyd mewn ymateb i gwestiwn 2 nad oes un o'r ysgolion cynradd o fewn y clwstwr yn ysgol cyfrwng Cymraeg.

Holwyd Pennaeth y Gymraeg beth y mae'r ysgol yn ei wneud yn benodol i hwyluso trosglwyddiad y disgyblion CAI o'r sector cynradd i'r sector uwchradd. Nododd fod cyswllt arbennig o gryf rhwng Ysgol Y ag ysgol gynradd agosaf ei chlwstwr. Fel rhan o fenter a drefnwyd gan Ysgol Y ar y cyd â'r ysgol gynradd hon y llynedd, roedd disgyblion yn astudio Cymraeg yn y Chweched Dosbarth yn cefnogi'r ysgol gynradd hon unwaith yr wythnos, gan gynorthwyo athrawon yn y dosbarth. Er nad yw'n glir pa mor rheolaidd y mae'r cymorth yn cael ei gynnig bellach, ymddengys fod Prifathrawes yr ysgol gynradd yn dal i gysylltu â Phennaeth Adran y Gymraeg yn Ysgol Y o bryd i'w gilydd er mwyn sicrhau help y disgyblion. Cred Pennaeth y Gymraeg fod y fenter hon wedi bod o gymorth, yn arbennig i'r athrawon cynradd yn yr ysgol gynradd agosaf sy'n ddihyder wrth addysgu Cymraeg fel pwnc. Ymddengys hefyd nad oes gan athrawon cynradd y clwstwr ddigon o hyder o ran pennu lefelau cyrhaeddiad yn y Gymraeg. Mynegwyd hyn wrth Bennaeth y Gymraeg y llynedd mewn cyfarfodydd cymedroli ar gyfer CAI. Yr oedd Pennaeth y Gymraeg hefyd o'r farn fod y fenter hon wedi creu argraff dda ar ddisgyblion ifanc yr ysgol gynradd, sy'n mwynhau gweithgareddau Cymraeg disgyblion y Chweched Dosbarth. Yn ogystal â chynorthwyo athrawon yr ysgol gynradd sy'n ddihyder wrth addysgu Cymraeg a chreu argraff dda ar ddisgyblion ifanc y cynradd, mae'n amlwg fod y fenter hon rhwng Ysgol Y a'r ysgol gynradd leol hefyd wedi galluogi disgyblion y Chweched Dosbarth i ymarfer eu Cymraeg ac ennill profiad gwaith wrth addysgu 
hefyd sy'n hollbwysig, fel y nododd Pennaeth y Gymraeg, wrth lunio cais UCAS i astudio yn y brifysgol.

\subsection{Ysgol X: Yr holiadur a'r cyfweliad}

\subsubsection{Gwybodaeth sosio-economaidd a sosio-ieithyddol}

Ysgol uwchradd cyfrwng Saesneg yn bennaf ond â defnydd sylweddol o'r Gymraeg yw Ysgol X (LlC, 2016a). Lleolir yr ysgol mewn cymuned gymharol ddi-Gymraeg yn ne Cymru, lle mae $14.5 \%$ o'r boblogaeth yn nodi eu bod yn gallu siarad Cymraeg $^{66}$ ac mae $12.3 \%$ o boblogaeth yr awdurdod unedol ehangach yn nodi eu bod yn gallu siarad Cymraeg (Stats Cymru, 2013; Y Swyddfa Ystadegau Gwladol, $2012 \mathrm{ch}$ ). Serch hynny, nodir y gall 10\% o'r disgyblion siarad Cymraeg yn rhugl, gyda $8 \%$ yn siarad Cymraeg gartref, a gall 39\% siarad Cymraeg, ond nid yn rhugl (Estyn, 2016). Mae gan Ysgol X 1,609 o ddisgyblion ar ei chofrestr (L1C, 2016a). Nifer bresennol y rhai sydd â'r hawl i Brydau Ysgol Am Ddim (PYDd) yn yr ysgol hon yw $22.9 \%$ (LlC, 2016a). ${ }^{67}$

\subsubsection{Darpariaeth ar gyfer y Gymraeg}

Fel y nodwyd eisoes, ysgol uwchradd cyfrwng Saesneg yn bennaf ond â defnydd sylweddol o'r Gymraeg yw Ysgol X. Fel rheol, cynnig ysgolion yn y categori hwn rhwng 20 - 49\% o'r cwricwlwm drwy gyfrwng y Gymraeg ond fel rheol, disgwylir i bob pwnc gael ei gyflwyno trwy gyfrwng y Saesneg hefyd (L1CC, 2007, t. 13). Yn achos Ysgol X, ceir darpariaeth cyfrwng Saesneg, darpariaeth cyfrwng Cymraeg a darpariaeth ddwyieithog.

\subsubsection{Darpariaeth cyfrwng Saesneg: Y ffrwd Gymraeg Ail Iaith}

Yn debyg i'r disgyblion yn Ysgol Y (gweler 7.1.2. Darpariaeth ar gyfer y Gymraeg) mae'r rhan fwyaf o ddisgyblion yn Ysgol X yn derbyn eu haddysg drwy gyfrwng $\mathrm{y}$ Saesneg yn unig. Caiff y disgyblion hyn addysg orfodol yn y Gymraeg hyd at 16 oed, a hynny ar ffurf gwersi CAI, lle cyflwynir yr iaith fel pwnc cwricwlaidd yn unig. O

\footnotetext{
${ }^{66}$ Rhaid nodi bod nifer o ddisgyblion yn y dalgylch yn dod o ysgolion cynradd mewn tair ward etholiad arall y tu allan i ward etholiad yr ysgol lle mae nifer y siaradwyr Cymraeg yn amrywio rhwng $12.1 \%$ hyd at $15.6 \%$. Ar gyfartaledd, mae $13.6 \%$ o boblogaeth y wardiau etholiad yn nalgylch yr ysgol yn gallu siarad Cymraeg.

${ }^{67}$ Seilir y ffigur hwn ar gyfartaledd PYDd dros y tair blynedd diwethaf.
} 
ran cyrsiau Cymraeg a gynigir ar gyfer disgyblion yn y ffrwd hon, nodwyd bod pob disgybl mewn addysg brif ffrwd yn CA4 yn astudio'r TGAU Cwrs Llawn CAI. Ni chyflwynir yn yr ysgol hon y Cwrs Byr. Yr unig eithriad i astudio ar y Cwrs Llawn yw'r dosbarth ar gyfer disgyblion sydd ag Anghenion Addysgol Arbennig (AAA o hyn ymlaen) ac un dosbarth arall sy'n cynnwys tuag ugain o ddisgyblion gwannaf y flwyddyn ysgol. Yn y ddau ddosbarth hyn, cyflwynir NVQ Lefel 1 Darllen (Cymraeg). Er bod rhaid trin CAI fel pwnc sylfaenol yn nhermau mesur perfformiad mewn ysgolion cyfrwng Saesneg, dywedodd Pennaeth y Gymraeg Ysgol X y caiff y pwnc statws fel pwnc craidd o fewn yr ysgol:

PA: O fewn yr ysgol baswn i, a'r prifathro, yn dadlau ein bod ni'n cael statws pwnc craidd. Hynny yw, mae pob disgybl yn gwybod mai'r pynciau craidd fel petai, neu'r pynciau mae'n rhaid iddyn nhw eu gwneud, ydy Cymraeg, Gwyddoniaeth, Saesneg a Mathemateg. (Atodiad 9, para. 14)

Yn debyg i Bennaeth Adran y Gymraeg Ysgol Y, mae Pennaeth Adran y Gymraeg Ysgol X yn mynd yn ei blaen i briodoli cynnig y Cwrs Llawn i'r rhan fwyaf o'r disgyblion i 'statws craidd' y pwnc yn yr ysgol. Yn ôl Pennaeth y Gymraeg, mae cynnig y Cwrs Llawn, ac nid y Cwrs Byr, yn sicrhau y caiff y pwnc ei ystyried yn bwnc pwysig ymhlith y disgyblion. Yn CA5, cynigir Safon UG CAI a Safon Uwch CAI i ddisgyblion yn y ffrwd hon. O ran oriau dysgu CAI, neilltuir pedair awr y pythefnos yn CA3 a 4 (tua 76 awr y flwyddyn) ac wyth awr y pythefnos yn CA5 (tua 156 awr y flwyddyn). Erbyn diwedd eu haddysg orfodol, bydd disgyblion y ffrwd hon wedi derbyn tua 380 o oriau dysgu Cymraeg fel pwnc ail iaith. Yr unig eithriad i hyn yw'r dosbarthiadau ar gyfer y disgyblion gwannaf a'r disgyblion AAA, lle neilltuir dwy awr o addysg y pythefnos.

Pan ofynnwyd i Bennaeth Adran y Gymraeg a oes rheswm pam y mae'r ysgol yn cynnig y cwrs NVQ yn hytrach na'r Cwrs Byr, esboniodd fod yr ysgol o'r farn y gall y Cwrs Byr fod yn rhy heriol i'r disgyblion gwannaf, sy'n ddihyder yn arbennig wrth ddefnyddio'r Gymraeg ar lafar ac ysgrifenedig. Penderfynodd yr ysgol y byddai cyflwyno'r cwrs NVQ Lefel 1 Darllen yn fwy addas ar gyfer y garfan hon o ddisgyblion. Teimlodd fod y disgyblion hyn yn fwy hyderus o ran eu sgiliau darllen o'u cymharu â'u sgiliau eraill. Oherwydd hynny, penderfynwyd cyflwyno'r uned ar ddarllen, yn hytrach nag unedau eraill yr NVQ sy'n asesu'r tair sgìl arall. Mae'n 
amlwg fod cynnig y ddarpariaeth hon yn sicrhau y gall y disgyblion gwannaf hyn ennill cymhwyster yn y Gymraeg. Fodd bynnag, o safbwynt datblygu hyfedredd yn y Gymraeg, gellir dadlau bod y disgyblion hyn dan anfantais o'u cymharu â'u cyfoedion sy'n dilyn y Cwrs Llawn oherwydd nad oes ganddynt yr un cyfle i ddatblygu'r tair sgìl arall.

Er nad yw'r adran yn cyflwyno unedau eraill yr NVQ, nododd Pennaeth y Gymraeg fod nifer yr oriau a neilltuir i'r dosbarthiadau'r NVQ ar fin cael eu cynyddu, ac o ganlyniad y mae'r adran yn ystyried cyflwyno uned ychwanegol, o bosibl NVQ Lefel 1 Gwrando, ar gyfer y disgyblion gwannaf, ac eithrio'r disgyblion AAA, unwaith i unedau'r cwrs NVQ gael eu cadarnhau gan fwrdd arholi OCR. Ers cynnal y gwaith maes fodd bynnag, mae OCR (2016, 25 Medi) wedi cadarnhau'n swyddogol na fydd yn cynnig y gyfres o gymwysterau NVQ ar gyfer yr ieithoedd Ffrangeg, Almaeneg, Sbaeneg, a Chymraeg o 31 Rhagfyr 2016 ymlaen.

\subsubsection{Darpariaeth cyfrwng Cymraeg: Y ffrwd Gymraeg Iaith Gyntaf}

Fel y nodir nes ymlaen yn y traethawd (gweler 7.2.5. Pontio rhwng y sector cynradd a'r sector uwchradd) mae Ysgol X yn derbyn nifer o ddisgyblion y tu allan i'w chlwstwr bob blwyddyn. O blith yr ysgolion hyn, nodir bod tair a ddiffinnir yn ysgolion cynradd cyfrwng Cymraeg. Oherwydd hyn, nododd Pennaeth y Gymraeg fod y ffrwd CIG wedi cael ei sefydlu yn 2009 er mwyn rhoi cyfle i'r disgyblion hyn barhau ag ychydig o’u pynciau drwy gyfrwng y Gymraeg.

Ar hyn o bryd, gyflwynir yn y ffrwd hon wyth pwnc cwricwlaidd yn CA3 drwy gyfrwng y Gymraeg (gan gynnwys y Gymraeg). Cyflwynir gweddill y pynciau drwy gyfrwng y Saesneg yn CA3 a 4. Y pynciau cyfrwng Cymraeg a gyflwynir yw CIG, Hanes, Daearyddiaeth, Addysg Grefyddol, Addysg Gorfforol, Drama, Coginio ac ABaCh. Mae cyfle i'r disgyblion hyn astudio'r cwrs TGAU CIG yn CA4, a Safon Uwch CIG yn CA5. Nodir bod pob disgybl yn y ffrwd hon yn parhau â'u hastudiaethau TGAU eraill trwy gyfrwng y Saesneg yn CA4. Oherwydd hynny, nid oes cyfle i ddisgyblion y ffrwd hon sefyll arholiadau TGAU trwy gyfrwng y Gymraeg. Er hynny, mae'n bosibl i ddisgyblion astudio Hanes trwy'r Gymraeg yn CA4 yn dibynnu ar y niferoedd sydd yn dewis astudio'r pwnc trwy'r Gymraeg. Rhaid nodi hefyd mai disgyblion CIG yn unig sy'n gymwys ar gyfer y ffrwd cyfrwng Cymraeg ac nad yw disgyblion CAI yn gallu astudio'r pynciau uchod drwy gyfrwng 
y Gymraeg. Eglurwyd yn y cyfweliad y defnyddir y label ‘Cymraeg Iaith Gyntaf’ yn ei ystyr addysgol, yn hytrach na chyfeirio at yr iaith a gafodd ei chaffael yn gyntaf gan y disgybl neu'r iaith a ddefnyddir yn y cartref. Fel y nodwyd eisoes (gweler 7.2.1. Gwybodaeth sosio-economaidd a sosio-ieithyddol) mae nifer fechan o'r disgyblion yn siarad Cymraeg gartref (8\% o ddisgyblion ar y gofrestr) ${ }^{68} \mathrm{Wrth}$ ystyried y ddarpariaeth a gynigir yn y ffrwd hon, gellir dadlau mai dyma amrywiad ar raglen CLIL, neu raglen addysg drochi rannol (gweler 2.5.4. Content and Language Integrated Learning).

\subsubsection{Darpariaeth ddwyieithog: Y Cwrs Carlam}

Cadarnhaodd Pennaeth y Gymraeg yn yr holiadur a'r cyfweliad fod y wybodaeth am y Cwrs Carlam a gyflwynwyd eisoes yn Pennod 5 yn gywir. Er mwyn osgoi ailadrodd, ni cheir yn yr adran hon amlinelliad o'r cynllun a'i brif nodweddion. Yn hytrach, trafodir yma y wybodaeth newydd a gasglwyd yn yr holiadur a'r cyfweliad ynghylch cynllun y Cwrs Carlam yn Ysgol X.

Nodwyd yn Pennod 5 fod y Cwrs Carlam ar gael i ddisgyblion MAT yn unig. Yn y cyfweliad dilynol, esboniodd Pennaeth y Gymraeg sut y dewisir disgyblion cymwys ar gyfer y Cwrs Carlam. Ymddengys y caiff disgyblion eu dethol ar sail y meini prawf canlynol:

- Gallu academaidd - Lefelau disgyblion ym Mlwyddyn 6 yn y pynciau Saesneg, Cymraeg (Ail Iaith), Mathemateg a Gwyddoniaeth,

- Gallu gwybyddol - Canlyniadau'r disgyblion ym Mlwyddyn 6 yn y Profion Gallu Gwybyddol, neu Cognitive Abilities Tests (CATs), a

- Cymhelliant yn y Gymraeg - arsylwadau gan yr athrawon pontio o’r disgyblion cynradd.

Ar ôl casglu'r data hyn oddi wrth ysgolion cynradd y clwstwr, caiff y chwe deg uchaf o ddisgyblion yn y flwyddyn ysgol eu canfod a'u cofnodi mewn rhestr. Dyma'r hyn y mae'r ysgol yn ei alw'n 'Band P' a chaiff pob un o'r disgyblion hyn eu "gwthio" ym mhob pwnc ar draws y cwricwlwm. O fewn y band hwn, dewisir pedwar deg o ddisgyblion. Ni ddewisir y pedwar deg uchaf yn y band hwn o

\footnotetext{
${ }^{68}$ Ystyr 'disgyblion Cymraeg Iaith Gyntaf' yma yw disgyblion sydd wedi derbyn addysg gynradd yn y sector cyfrwng Cymraeg ac sydd bellach yn astudio yn y sector uwchradd cyfrwng Saesneg (gweler 2.6.3. Disgybl Cymraeg Iaith Gyntaf.)
} 
reidrwydd, ond yn hytrach dewisir disgyblion ar sail 'ymdrech' ac 'agwedd' tuag at y Gymraeg yn yr ysgol gynradd, yn ogystal â'u lefelau yn y Gymraeg. Mae'n debyg mai cymhelliant yw'r hyn y mae'r Pennaeth yn cyfeirio ato yma, ond nid yw'n glir sut mae'r ysgol yn mynd ati i fesur hyn. Os yw'r broses ddethol hon i fod yn gwbl wrthrychol, rhaid sicrhau y caiff cymhelliant disgyblion yn y Gymraeg ei fesur mewn ffordd wrthrychol. Ymddengys y byddai rhestr wirio ar gyfer mesur cymhelliant disgyblion yn gwella'r maen prawf hwn ym mhroses ddethol y Cwrs Carlam.

Fel y nodwyd eisoes yn y traethawd hwn (gweler 5.4. Cynllun Ysgol X: Y Cwrs Carlam) wrth wraidd y cynllun yn Ysgol X y mae'r athrawon peripatetig a gaiff eu cyflogi i gydweithio ag ysgolion cynradd y clwstwr. Nododd Pennaeth y Gymraeg yn y cyfweliad fod yr athrawon hyn yn chwarae rôl allweddol yn y broses o ddethol disgyblion cymwys ar gyfer y Cwrs Carlam a hynny, mae'n ymddangos, oherwydd eu hymwneud agos â'r ysgolion cynradd. Yn ogystal â'u rôl yn y broses o ddethol disgyblion ar gyfer y Cwrs Carlam, mae'r athrawon pontio hyn yn gyfrifol am roi lefelau ar gyfer y Gymraeg yn ysgolion cynradd y clwstwr ac am addysgu Cymraeg ym Mlynyddoedd 5 a 6 a chynorthwyo athrawon i addysgu Cymraeg ym Mlynyddoedd 2 a 3 yn yr ysgolion clwstwr. Ar ben hyn, nodir bod yr athrawon hyn yn gyfrifol am addysgu ambell ddosbarth Blwyddyn 7 (gweler 7.2.5. Pontio rhwng y sector cynradd a'r sector uwchradd). Ar ôl dethol y disgyblion cymwys, danfonir llythyron adref at rieni a gwarcheidwaid y disgyblion ar ddiwedd Blwyddyn 6 yn eu gwahodd i gyfarfod er mwyn trafod cynnig y Cwrs Carlam i'w plant. Nododd Pennaeth y Gymraeg fod cyfle i rieni a gwarcheidwaid y disgyblion ofyn cwestiynau ynglŷn â'r cwrs er mwyn tawelu eu meddyliau. Er hynny, ychwanegodd fod y gymuned bellach yn ymwybodol o'r cwrs a'r ddarpariaeth a geir yn y Cwrs Carlam oherwydd bod y rhaglen hon wedi'i chydlynu ers pymtheng mlynedd bellach. Ar yr amod y bydd y plentyn a'i riant neu warcheidwad yn fodlon, caiff ffurflen ei harwyddo er mwyn rhoi sêl bendith i'r plentyn dderbyn darpariaeth y Cwrs Carlam.

Cadarnhaodd Pennaeth y Gymraeg nad oes modd i ddisgyblion nad ydynt wedi'u dethol ar gyfer y Cwrs Carlam elwa o'r ddarpariaeth hon, a hynny oherwydd y ffordd y caiff y system amserlennu ei threfnu. Ymddengys nad oes digon o ryddid yn yr amserlen er mwyn i'r ysgol allu cynnig y ddarpariaeth hon i ystod ehangach o ddisgyblion. Er na all yr ysgol gynnig y ddarpariaeth garlam i bob disgybl, pwysleisiodd Pennaeth y Gymraeg (Atodiad 9, para. 30) nad yw disgyblion y brif 
ffrwd cyfrwng Saesneg ar eu colled oherwydd cânt ddarpariaeth sydd yn "o safon uchel iawn" ac sydd yn eu galluogi i ennill y canlyniadau gorau posibl mewn CAI. Nodir yma fod y gred hon yn cyd-fynd â'r egwyddor a osodir yn Nod 2 polisi Ysgol $X$ ar ddwyieithrwydd (gweler Atodiad 9), sef y dylai'r ysgol sicrhau y caiff dysgwyr bob cyfle i lwyddo trwy gyfrwng y Saesneg a'r Gymraeg. Caiff y gred hon ei hailadrodd gan Bennaeth y Gymraeg ar ddiwedd y cyfweliad, wrth iddi gynnig ei sylwadau terfynol:

PA: $\quad . .$. mae llwyddiant pob disgybl yn yr ysgol hon yn bwysig i ni. Chimod, un o bethau'r ysgol ydy, "a community school committed to excellence”. Hynny yw, bod pob disgybl yn troi lan at y gât ac yn cael yr addysg orau bosibl yn yr ysgol. Felly os ydyn nhw ar y Cwrs Carlam neu beidio, rydyn ni'n teimlo mai'r un safon o addysgu maen nhw'n ei chael yn y dosbarth, ar lawr y dosbarth, yn eu gwersi Cymraeg Ail Iaith. Ond yn y Cwrs Carlam - maen nhw'n cael eu gwthio ychydig yn gyflymach, maen nhw'n cael eu gwthio'n fwy er mwyn cyrraedd y nod, sef yr arholiad ar diwedd Blwyddyn 9 a Blwyddyn 11 wrth gwrs. Ond, dyna'n darpariaeth ni ar gyfer y disgyblion mwyaf abl a thalentog, fel petai. (Atodiad 9, para. 83)

Ymddengys o'r sylwadau uchod, ac ar ôl sgwrsio â Phennaeth y Gymraeg cyn y cyfweliad, mai blaenoriaeth yr ysgol yw safon yr addysg a geir yn y dosbarth, o ran dysgu cynnwys cwricwlaidd, yn hytrach na chyfrwng yr addysg. Pwysleisiwyd hyn gan Bennaeth y Gymraeg (Atodiad 9, para. 46) yn gynharach yn y cyfweliad, gan nodi mai'r pwnc sy'n “dod yn gyntaf” a bod y ddarpariaeth Gymraeg ychwanegol a geir yn y dosbarth carlam yn “cyfoethogi'r dysgu."

Gofynnwyd i Bennaeth yr Adran pa fath o gwestiynau sy'n arfer cael ei godi yn ystod y cyfarfodydd â rhieni er mwyn cyflwyno'r Cwrs Carlam. Ymddengys mai'r cwestiwn amlaf a'r cwestiwn sy'n codi'r gofid mwyaf ymhlith rhieni yw a fydd eu plentyn yn gallu ymdopi â phwysau'r gwaith gan y bydd angen deall cynnwys cwricwlaidd y pynciau trwy gyfrwng y Gymraeg a'r Saesneg. Er mwyn tawelu meddwl, mae'r Adran yn esbonio i rieni na chaiff disgyblion eu hasesu'n ffurfiol yn y Gymraeg a chaiff y rhan fwyafrif o'r cwricwlwm y mae'r disgyblion yn ei ddilyn ei chyflwyno a'i hasesu yn yr iaith Saesneg.

Er nad astudio arferion addysgol athrawon yn y dosbarth Carlam oedd dan sylw yn y gwaith maes, teimlwyd ei bod yn bwysig holi am sut y caiff y ddwy iaith eu defnyddio'n ymarferol yn y dosbarth er mwyn cael gwell darlun o'r ddarpariaeth 
ddwyieithog a gynigir. Wrth reswm, mae cyflwyno cynnwys y pwnc yn effeithiol ac effeithlon wrth hefyd ddefnyddio digon o Gymraeg er mwyn cyfoethogi'r dysgu yn sicr o fynnu defnydd gofalus o'r ddwy iaith ar ran yr athro ar lawr y dosbarth dwyieithog. Cytunodd Pennaeth y Gymraeg fod defnyddio'r ddwy iaith yn y dosbarth yn broses gymhleth a thactegol ac esboniodd fod y defnydd o'r Gymraeg yn y dosbarthiadau hyn yn gorfod amrywio yn unol â lefel y disgyblion. Ymddengys fod y defnydd o'r Gymraeg yn y dosbarth yn cynyddu'n raddol o Flwyddyn 7 i Flwyddyn 9:

PA: $\quad$... mae'n rhaid bod yn ofalus nad ydyn nhw'n cael gormod o ofn, fel petai. Felly rydyn ni'n dechrau'n araf bach. Erbyn Blwyddyn 9 wedyn, byddwn i'n meddwl bod iaith y dosbarth, mwy neu lai, yn hollol Gymraeg o ran cyfarwyddiadau. "Trowch y dudalen", "ysgrifennwch", "darllenwch". Chimod, geirfa - "Beth yw eich barn chi am Jac y Llarpiwr?", "pwy oedd ar fai?". Byddwn ni wedi cyflwyno geirfa, patrymau, fel eu bod nhw'n ddigon hyderus i allu ateb ar lafar ac yn ysgrifenedig gyda'r patrymau hynny maen nhw'n eu gwybod. (Atodiad 9, para. 44)

Cadarnhawyd hefyd nad oes methodoleg benodol y mae'r athrawon pynciol yn ei defnyddio wrth gyflwyno eu gwersi'n ddwyieithog. Teimlodd Pennaeth y Gymraeg (Atodiad 9, para. 46) fod y defnydd o'r ddwy iaith yn y dosbarth yn "rhywbeth sy'n dod o brofiad" ac "sy'n datblygu'n organig." Er hynny, nododd hithau y caiff athrawon pynciol y Cwrs Carlam lyfryn ac ynddo batrymau ieithyddol y mae'r disgyblion yn eu defnyddio yn y gwersi Cymraeg. Ymddengys fod y llyfryn yn helpu i arwain yr athrawon hynny nad ydynt wedi hyfforddi fel athrawon Cymraeg.

Cadarnhaodd Pennaeth y Gymraeg na chaiff disgyblion y Cwrs Carlam eu hasesu'n ffurfiol yn y Gymraeg. Er hynny, caiff un dasg ysgrifenedig yn Gymraeg nas asesir ei chlustnodi bob hanner tymor er mwyn monitro cynnydd y disgyblion yn y Gymraeg. Yn ogystal â hyn, nododd Pennaeth y Gymraeg y caiff y tasgau hyn eu llunio er mwyn pontio rhwng y gwersi Cymraeg a'r pynciau dwyieithog, er mwyn sicrhau y gall y disgyblion drosglwyddo'r patrymau ieithyddol a'r eirfa y maent yn eu defnyddio yn y gwersi Cymraeg i’r pynciau eraill.

Er mwyn cael gwybod am gynaliadwyedd y Cwrs Carlam, a gallu'r ysgol i ddatblygu'r ddarpariaeth Gymraeg a dwyieithog ymhellach, holwyd sut y bydd yr ysgol yn cynllunio er mwyn cynnal yr athrawon sy'n medru addysgu trwy gyfrwng y 
Gymraeg neu'n ddwyieithog, yn ogystal â chyflogi athrawon newydd. Cadarnhaodd Pennaeth y Gymraeg nad oes polisi staffio yn ei le. Golyga hyn na fyddai staff newydd sy'n medru addysgu trwy gyfrwng y Gymraeg neu'n ddwyieithog yn cael eu blaenoriaethu o reidrwydd wrth gyflogi i lenwi lle staff sy'n addysgu'r Cwrs Carlam a'r ffrwd Gymraeg Iaith Gyntaf a allai adael yr ysgol yn y dyfodol. O ran cynllunio i sicrhau twf darpariaeth Gymraeg yn yr ysgol, nododd Pennaeth y Gymraeg fod gan yr ysgol fwriad i allu cynnig rhagor o bynciau trwy gyfrwng y Gymraeg ac yn ddwyieithog, ond byddai hynny'n digwydd yn y pynciau lle mae arbenigedd yn ei le yn barod. Ymddengys nad yw Ysgol X yn cyflogi staff ar sail eu gallu ieithyddol, yn hytrach y mae'n “cyflogi'r athrawon gorau i'r pynciau fel sydd angen.” (Atodiad 3, Rhan 1).

\subsubsection{Ethos dwyieithog}

Holwyd Pennaeth y Gymraeg a oes gan Ysgol X bolisi swyddogol, ar bapur, ar gyfer datblygu, hyrwyddo a hybu dwyieithrwydd yn yr ysgol. Cadarnhaodd hi fod polisi swyddogol yn ei le a chyflwynodd gopi o'r polisi hwn ar yr un pryd â'r holiadur wedi'i lenwi (gweler Atodiad 10). Yn y polisi hwn, ceir wyth nod ac un deg wyth amcan sy'n gosod yn ffurfiol sut y bwriedir hyrwyddo a hybu dwyieithrwydd a darpariaeth cyfrwng Cymraeg yn yr ysgol. Nodyn i dynnu sylw ato yma yw nad oedd fersiwn Gymraeg neu ddwyieithog o'r polisi hwn ar gael ar adeg casglu data oddi wrth yr ysgol.

O ran gweithgareddau beunyddiol i hyrwyddo dwyieithrwydd, nodir mai defnyddio'r Gymraeg yn achlysurol yw un o'r prif themâu sy'n codi. Er enghraifft, mynna'r polisi (Atodiad 10, Amcan 5) y dylid "encourage pupils to develop their ability to speak Welsh in a range of contexts and situations beyond familiar contexts and the classroom." I'r un perwyl, mae Amcan 7 y polisi yn mynnu y dylid "encourage all staff to make use of incidental Welsh when talking to pupils and provide support for them to do so." Thema arall sy'n codi o ran hyrwyddo dwyieithrwydd yn feunyddiol yw'r defnydd o "some written Welsh in all books other than English and MFL" a nodir yn Amcan 8, a Chymraeg llafar mewn cyfarfodydd boreol a nodir yn Amcan 9. Nododd Pennaeth y Gymraeg (Atodiad 3, Rhan 2) mewn ymateb i'r holiadur mai prif nod y polisi hwn yw Nod 1, sef "[d]atblygu ac ymestyn dwyieithrwydd fel rhan annatod o addysgu." Bwriedir cyflawni hyn, fel y mae Amcan 1 y polisi (Atodiad 10) 
yn ei nodi, wrth ddatblygu ac ymestyn y ddarpariaeth ddwyieithog a Chymraeg Iaith Gyntaf a geir yn yr ysgol. At hynny, mae Amcan 18 yn nodi y bydd yr ysgol yn cyflogi staff newydd er mwyn datblygu'r ddarpariaeth ddwyieithog o fewn yr ysgol ac o fewn yr ysgolion bwydo. Fodd bynnag, fel y nodwyd eisoes, nid oes gan Ysgol $\mathrm{X}$ bolisi staffio yn ei le er mwyn sicrhau y caiff staff sydd â sgiliau addysgu trwy gyfrwng y Gymraeg ac yn ddwyieithog eu blaenoriaethu wrth gyflogi staff newydd (gweler 7.2.2.3. Darpariaeth ddwyieithog: Y Cwrs Carlam). Yn hytrach, fel y mae Pennaeth y Gymraeg (Atodiad 3, Rhan 1) yn ei gadarnhau yn yr holiadur, mae'r ysgol yn "cyflogi'r athrawon gorau i'r pynciau fel sydd angen" ac mae'n ymestyn y ddarpariaeth i'r pynciau lle mae arbenigedd yno yn barod. Er hynny, nodir bod yr honiadau hyn yn cyd-fynd â Nod 7 y polisi (Atodiad 10), sef "[t]o make efficient use of sufficiently qualified staff, able to teach bilingually."

\subsubsection{Hyfforddiant staff yr ysgol}

Holwyd cyfres o gwestiynau ynghylch y cyfleoedd hyfforddi sydd ar gael i staff yr ysgol er mwyn datblygu eu sgiliau dwyieithog a'u methodoleg addysgu cyfrwng Cymraeg / dwyieithog. Cadarnhaodd Pennaeth y Gymraeg fod yr ysgol yn cynnig cwrs hyfforddi i'w staff er mwyn datblygu eu sgiliau dwyieithog cyffredinol. Nododd Pennaeth y Gymraeg (Atodiad 3, Rhan 3) mai prif ddiben yr hyfforddiant hwn yw "datblygu sgiliau sylfaenol defnyddio Cymraeg yn achlysurol o fewn y dosbarth.” Esboniodd hi y caiff y cwrs hwn, o'r enw 'Cymraeg yn y gweithle', ei gynnal o dan arweiniad un o'r prifysgolion lleol am awr yr wythnos ar ôl y diwrnod ysgol, dros gyfnod o ddeg wythnos. Cafodd y cwrs hyfforddi hwn ei gyflwyno yn Ysgol X am y tro cyntaf ym mlwyddyn academaidd 2011-2012 ac eto y flwyddyn olynol, yn 2012-2013. Oherwydd sefydlogrwydd y staff ar y pryd, penderfynwyd na fyddai'r adran yn cynnig yr hyfforddiant am y ddwy flynedd nesaf, sef blynyddoedd academaidd 2013-2014 a 2014-2015. Er hynny, cadarnhaodd Pennaeth y Gymraeg y bydd y cwrs yn cael ei gynnal eto yn ystod 2015-2016 ar gyfer staff newydd a staff sydd am ailddysgu neu adolygu eu sgiliau dwyieithog. Ar adeg casglu'r data, nododd Pennaeth y Gymraeg fod tua phum aelod o'r staff wedi cwblhau cwrs uwch y cynllun hyfforddi ac wedi cwblhau'r asesiad ar ddiwedd y cynllun. Ar gyfer y cwrs hyfforddi eleni, cadarnhaodd mai rhwng 20 - 25 o'r staff sydd wedi dangos diddordeb yn y cwrs hyd yn hyn. Fodd bynnag, cydnabu y gall trefnu'r cwrs ar ddiwrnod penodol fod yn drafferthus oherwydd ymroddiadau a chyfrifoldebau eraill y staff. 
Holwyd Pennaeth y Gymraeg yn y cyfweliad a yw'r cwrs hyfforddiant hwn ar gael i staff eraill yr ysgol. Er mai prif ddiben yr hyfforddiant yw "datblygu sgiliau sylfaenol defnyddio Cymraeg yn achlysurol o fewn y dosbarth", yn ôl Pennaeth y Gymraeg (Atodiad 3, Rhan 3), daeth i'r amlwg yn y cyfweliad y caiff yr hyfforddiant hwn ei anelu'n fwy cyffredinol at ddatblygu sgiliau dwyieithog unrhyw aelod o staff drwy'r ysgol gyfan, ac nid staff addysgu yn unig. Nodir bod cynnig hyfforddiant o'r fath wrth wraidd polisi swyddogol yr ysgol ar ddwyieithrwydd, yn benodol Nod 8 . Mae Nod 8 yn mynnu y dylai'r ysgol ddarparu cyfleoedd i'r holl staff ddatblygu sgiliau dwyieithog. Nodir hefyd mai'r ysgol ei hun sy'n ariannu'r hyfforddiant allanol hwn. Ni chafodd cost yr hyfforddiant ei datgelu yn yr holiadur na'r cyfweliad ond ymddengys ei bod yn gost sylweddol i'r ysgol. Er gwaetha'r gost, teimla'r ysgol fod angen hyfforddiant o'r fath ar ei staff.

O ran datblygu methodoleg addysgu trwy gyfrwng y Gymraeg a dwyieithog, cadarnhaodd Pennaeth y Gymraeg fod hyfforddiant yn ei le. Fodd bynnag, ymddengys mai hyfforddiant mewnol, yn hytrach na hyfforddiant allanol, sydd dan sylw. Nododd Pennaeth y Gymraeg y caiff darpar athrawon y ffrwd Gymraeg Iaith Gyntaf a'r Cwrs Carlam hyfforddiant mewnol yn gyntaf, a hynny ar ffurf arsylwi dosbarthiadau yn y pynciau hynny lle mae'r Gymraeg yn cael ei defnyddio fel cyfrwng addysgu. Sylwir yma mai'r athrawon sy'n hyfedr yn y Gymraeg yn unig a gaiff eu hystyried ar gyfer addysgu yn y ffrydiau hyn gan yr adran. Yn ogystal â'r hyfforddiant mewnol ac allanol y mae'r ysgol yn ei gynnig, nododd Pennaeth y Gymraeg fod nifer o'r staff, nad ydynt yn addysgu'r ffrwd Gymraeg Iaith Gyntaf a'r Cwrs Carlam, wedi mynychu cyrsiau Cymraeg i Oedolion o’u gwirfodd yn y gorffennol. Roedd hi o'r farn fod y profiad hwn wedi codi hyder o ran defnyddio'r Gymraeg ymhlith yr athrawon hynny.

\subsubsection{Pontio rhwng y sector cynradd a'r sector uwchradd}

O’i chymharu ag Ysgol Y, ysgol uwchradd gymharol fawr yw Ysgol X. Oherwydd ei maint, mae gan yr ysgol hon saith ysgol gynradd fwydo benodedig o fewn ei chlwstwr. Yn ogystal â'r ysgolion hyn, nododd Pennaeth y Gymraeg mewn ymateb i gwestiwn 1, rhan 4 yr holiadur y derbynia'r ysgol ddisgyblion o tua ugain ysgol gynradd arall y tu allan i'r clwstwr yn flynyddol. Ymhelaethodd hi ar hyn yn y cyfweliad (Atodiad 9, para. 65) gan nodi mai "rhwng rhyw ddeugain a hanner cant" 
o ddisgyblion sy'n dod o'r tu allan i'r clwstwr bob blwyddyn. O blith yr ugain ysgol gynradd ychwanegol hyn, nodwyd bod tair ysgol a ddiffinnir yn ysgolion cynradd cyfrwng Cymraeg. Mae'n ymddangos fod Ysgol X yn boblogaidd nid yn unig ymhlith rhieni sydd â phlant yn ysgolion cynradd cyfrwng Saesneg yr ardal, ond hefyd ymhlith rhai rhieni sydd â phlant yn yr ysgolion cynradd cyfrwng Cymraeg yn ogystal (gweler 7.2.2. Darpariaeth ar gyfer y Gymraeg).

Holwyd Pennaeth y Gymraeg am yr hyn y mae'r ysgol yn ei wneud yn benodol i hwyluso trosglwyddiad disgyblion o'r sector cynradd cyfrwng Cymraeg i'r sector uwchradd cyfrwng Saesneg. Cyfeiriodd eto at y ddarpariaeth Gymraeg Iaith Gyntaf a nodwyd eisoes yn yr holiadur. Ychwanegodd hi (Atodiad 3, Rhan 4) fod athrawon sy'n siarad Cymraeg yn rhugl yn addysgu'r dosbarthiadau ar gyfer Mathemateg a Gwyddoniaeth “er mwyn helpu nhw i drosglwyddo o astudio'r pynciau hynny yn Gymraeg yn y cynradd i Saesneg yn yr uwchradd." ${ }^{69}$ Er mwyn cefnogi'r dysgu ymhob pwnc, nododd Pennaeth y Gymraeg y caiff pob disgybl y ffrwd hon lyfryn o eirfa a thermau allweddol.

Yng nghwestiwn 5, holwyd Pennaeth y Gymraeg beth y mae'r ysgol yn ei wneud yn benodol i hwyluso trosglwyddiad disgyblion CAI o'r sector cynradd i'r sector uwchradd. Cyfeiriodd eto at yr athrawon peripatetig y mae'r ysgol yn eu cyflogi, sy'n addysgu nifer o ddosbarthiadau CAI ym Mlwyddyn 7, yn ogystal ag addysgu CAI ym Mlynyddoedd 5 a 6. Roedd Pennaeth y Gymraeg o'r farn fod gwaith yr athrawon hyn wrth bontio'r addysg rhwng y ddau sector yn sicrhau trosglwyddiad hwylus ar gyfer y disgyblion hyn. Ymddengys mai rôl yr athrawon pontio hyn yw sicrhau gwell cysondeb o ran addysgu a lefelu CAI, yn ogystal â monitro safonau yn fwy cyffredinol, rhwng ysgolion cynradd y clwstwr a'r ysgol uwchradd.

Nodwyd hefyd y trefnir dau 'ddiwrnod trochi' ddwywaith y flwyddyn ar gyfer disgyblion sydd newydd drosglwyddo i'r ysgol uwchradd. Cynhelir y diwrnodau hyn unwaith ar ddechrau'r flwyddyn academaidd, tua mis Tachwedd, ac unwaith eto ym mis Gorffennaf. Wrth gynnal y diwrnodau hyn ddwywaith y flwyddyn, gall yr ysgol rannu disgyblion y flwyddyn ysgol yn ddwy er mwyn sicrhau y caiff pob disgybl y cyfle i gymryd rhan. Eglurodd Pennaeth y Gymraeg yn y cyfweliad mai natur y

\footnotetext{
${ }^{69}$ Sylwer na chaiff y pynciau Mathemateg a Gwyddoniaeth eu cyflwyno yn y Gymraeg yn y ffrwd Gymraeg Iaith Gyntaf (gweler 7.2.2.2. Darpariaeth cyfrwng Cymraeg: Y ffrwd Gymraeg Iaith Gyntaf.)
} 
diwrnodau hyn yw rhoi cyfle i ddisgyblion ddefnyddio'r Gymraeg y tu hwnt i'r dosbarth iaith:

PA: $\quad$... Felly ar y diwrnod ym mis Medi - Hydref - maen nhw'n gwneud pethau fel coginio, dawnsio, drama, chwaraeon. Aethon ni orienteering eleni, roedd y tywydd yn dda. Felly jest gweithgareddau fel hynny lle maen nhw'n defnyddio'r Gymraeg mewn sefyllfa allgyrsiol, mewn ffordd ar wahân i'r dosbarth. (Atodiad 9, para. 72)

Yn ogystal â'r ddau ddiwrnod allgyrsiol hyn, trefnir diwrnod trochi ychwanegol ar gyfer y rhai sy'n dod o'r tu allan i'r clwstwr. Yn wahanol i'r ddau uchod, canolbwyntir ar gyflwyno patrymau ieithyddol Cymraeg ar ddechrau'r diwrnod trochi hwn. Ar ôl bore o wersi Cymraeg, cynhelir gweithgareddau allgyrsiol yn y prynhawn. Pwrpas cyflwyno'r diwrnod trochi hwn i'r disgyblion hyn sy'n dod o'r tu allan i'r clwstwr yw darganfod a "ydyn nhw wedi cyrraedd yr un safon ieithyddol â gweddill y disgyblion yn y clwstwr.” (Atodiad 9, para. 75). Sylwer nad yw'r athrawon pontio yn cydweithio â'r ysgolion cynradd eraill nad ydynt yn rhan o'r clwstwr. Nid yw'r athrawon hyn felly yn gyfarwydd â safon ieithyddol y disgyblion hyn. Dyma gyfle nid yn unig i'r ysgol gysoni safonau CAI a pharatoi disgyblion ar gyfer trosglwyddo o'r naill sector addysg i'r llall, ond hefyd i'r disgyblion ymarfer eu Cymraeg yn achlysurol mewn cyd-destun anffurfiol. Nodir bod y gweithgareddau hyn yn cyd-fynd yn benodol ag Amcanion 5, 6 a 7 polisi'r ysgol ar ddwyieithrwydd, sef:

5. To encourage pupils to develop their ability to speak Welsh in a range of contexts and situations beyond familiar contexts and the classroom.

6. To encourage all pupils to participate in extracurricular activities which offer the opportunity to make use of English and Welsh.

7. To encourage all staff to make use of incidental Welsh when talking to pupils and provide support for them to do so. (Atodiad 10)

O ganlyniad, gellir ystyried y diwrnodau allgyrsiol a throchi hyn fel gweithgareddau sy'n cyfrannu at ethos dwyieithog yr ysgol, yn ogystal â threfniadau'r ysgol o ran pontio rhwng y sector cynradd a'r sector uwchradd. Fodd bynnag, bydd angen ymchwil bellach i archwilio cynnwys y diwrnodau trochi hyn ac i werthuso eu heffeithiolrwydd o ran gwella dilyniant ieithyddol disgyblion rhwng y cynradd a'r uwchradd. 


\subsection{Arsylwadau ar ethos}

\subsubsection{Ysgogi sgwrs yn Gymraeg}

Trefnwyd ymweliadau â'r ddwy ysgol ar y cyd â Phennaeth Adran y Gymraeg a chynhaliwyd yr ymweliadau yn ystod diwrnodau ysgol. Ar ddiwrnod yr ymweliadau, aeth yr ymchwilydd at y dderbynfa er mwyn ysgogi sgwrs yn Gymraeg ag aelod o'r staff rheng flaen. Gwelir yn y tabl isod y data a gasglwyd o'r naill ysgol a'r llall.

\begin{tabular}{|l|l|l|l|}
\hline Ysgol & $\begin{array}{c}\text { A wnaeth aelod o'r staff } \\
\text { rheng flaen gwrdd a } \\
\text { chyfarch yr ymchwilydd } \\
\text { yn Gymraeg? }\end{array}$ & $\begin{array}{c}\text { A oedd yr aelod o'r staff } \\
\text { rheng flaen yn gallu } \\
\text { cynnal sgwrs yn } \\
\text { Gymraeg? }\end{array}$ & $\begin{array}{c}\text { A gyfeiriodd yr } \\
\text { ymchwilydd at aelod arall } \\
\text { o'r staff sy'n gallu siarad } \\
\text { Cymraeg? }\end{array}$ \\
\hline Ysgol Y & Naddo. & Nac oedd. & Naddo. \\
\hline Ysgol X & Do. & $\begin{array}{l}\text { Nac oedd. Esboniodd yr } \\
\text { aelod o'r staff rheng flaen } \\
\text { ei bod wrthi'n dysgu } \\
\text { Cymraeg. }\end{array}$ & Naddo. \\
\hline
\end{tabular}

Tabl 3. Ysgogi sgwrs yn Gymraeg yn Ysgol Y ac Ysgol X.

\subsubsection{Casglu dogfennaeth a theithio o amgylch yr ysgolion}

Ar ôl arwyddo i mewn i Ysgol Y a chyfarfod â Phennaeth Adran y Gymraeg, cafodd yr ymchwilydd ei wahodd i fynychu'r gwasanaeth boreol yng nghapel yr ysgol. Er i'r gwasanaeth gael ei gynnal trwy gyfrwng y Saesneg, adroddwyd Gweddi'r Arglwydd yn Gymraeg gan yr holl staff yn y gwasanaeth. Cafodd pawb yn y gwasanaeth gopi o'r gweddïau a'r emynau a adroddir yn ystod y gwasanaeth hwn, gan gynnwys Gweddi'r Arglwydd yn Gymraeg. Ar ôl y gwasanaeth, aeth Pennaeth y Gymraeg â'r ymchwilydd i'w dosbarth cofrestru, lle cafodd Gweddi'r Arglwydd ei hadrodd yn Gymraeg ar ddechrau'r wers gan y dosbarth cyfan. Er bod y geiriad ar gael ar waliau'r dosbarth, nodwyd y gallai pob disgybl yn y dosbarth hwn adrodd y weddi ar eu cof. Ar ôl y dosbarth cofrestru, aeth Pennaeth y Gymraeg â'r ymchwilydd i gasglu copi papur o'r prosbectysau diweddaraf. Wrth deithio o amgylch yr ysgol, tynnodd Pennaeth y Gymraeg sylw at enghreifftiau o Gymraeg ar arwyddion ac arddangosfeydd. Ar adeg yr ymweliad, nid oedd copi o brosbectws diweddaraf (2016) yr ysgol gyfan ar gael yn Gymraeg nac yn Saesneg, a chafwyd copi o'r hen brosbectws Saesneg yn ei le. Fodd bynnag, cafodd yr ymchwilydd ei gyfeirio at 
wefan Ysgol Y lle y gellir lawrlwytho copi electronig o'r prosbectws Saesneg yn unig. Yn ogystal â'r prosbectysau, casglwyd copïau o'r ddau gylchlythyr diweddaraf o'r dderbynfa. Dim ond copïau Saesneg o'r cylchlythyrau oedd ar gael adeg casglu'r ddogfennaeth. Ar ôl casglu'r ddogfennaeth, gadawodd Pennaeth Adran y Gymraeg yr ymchwilydd i gynnal ei arsylwad o'r arwyddion o amgylch yr ysgol.

Ar ôl arwyddo i mewn i Ysgol X, casglodd yr ymchwilydd gopïau o'r ddau gylchlythyr diweddaraf a gafodd eu harddangos ar fwrdd yn y dderbynfa. Cyfarfu'r ymchwilydd â Phennaeth y Gymraeg, cyn mynd am dro o amgylch yr ysgol. Wrth deithio o amgylch yr ysgol, tynnodd Pennaeth y Gymraeg sylw at enghreifftiau o arwyddion ac arddangosfeydd Cymraeg a dwyieithog. Fodd bynnag, datganodd Pennaeth y Gymraeg y gellid gwneud mwy fyth i gysoni'r defnydd o'r ddwy iaith ar arwyddion answyddogol ar draws yr ysgol ac ar arddangosfeydd ymhob rhan o'r ysgol. Arsylwyd dwy sgwrs fach yn Gymraeg rhwng Pennaeth y Gymraeg a rhai disgyblion yn ystod y daith o amgylch yr ysgol. Daeth i'r golwg mai disgyblion a ddilynodd y Cwrs Carlam oedd y rhain. Ar ôl y daith, cyfeiriodd Pennaeth y Gymraeg yr ymchwilydd at wefan yr ysgol er mwyn cael gafael ar gopi o'r prosbectysau gan nad oedd copïau papur ar gael adeg yr ymweliad. Gadawodd i'r ymchwilydd gynnal ei arsylwad o'r arwyddion o amgylch yr ysgol.

\subsubsection{Arwyddion dwyieithog}

O blith yr ugain arwydd a gofnodwyd yn Ysgol Y, nodwyd bod y rhan fwyaf o'r rhain yn ymddangos yn Saesneg yn unig (12 allan o 20 arwydd). Darganfuwyd un arwydd ac arno ond ychydig o Gymraeg, wrth gât yr ysgol. Ar yr arwydd hwn, sylwyd bod enw'r ysgol yn Gymraeg yn ymddangos o dan y testun Saesneg a bod y testun Cymraeg yn ymddangos yn llai o ran maint ei ffont o'i gymharu â'r testun Saesneg. Sylwyd hefyd, ar yr arwydd hwn, nad yw arwyddair yr ysgol yn ymddangos yn Gymraeg. Gan nad yw'r arwydd hwn yn bodloni egwyddorion Canllawiau Dylunio Dwyieithog Comisiynydd y Gymraeg (2014, t. 18) nid ystyriwyd yr arwydd hwn yn un dwyieithog.

Fodd bynnag, nodwyd bod y defnydd o'r ddwy iaith yn gyfartal o ran "ffont, fformat, lliw, maint, eglurder, amlygrwydd ac ansawdd" (Comisiynydd y Gymraeg, 2014, t. 18) ar gyfer nifer o'r arwyddion a gofnodwyd yn Ysgol Y (7 allan o 20 arwydd). Sylwyd hefyd ar yr arwyddion hyn y caiff y Gymraeg ei blaenoriaethu wrth i'r testun 
Cymraeg ymddangos uwchben y testun Saesneg bob tro. O ganlyniad, penderfynwyd bod yr arwyddion yn bodloni egwyddorion y Comisiynydd (2014) a chofnodwyd hwy yn arwyddion dwyieithog. Yn ogystal â'r saith arwydd dwyieithog, cofnodwyd un arwydd a oedd yn ymddangos yn Gymraeg yn unig. Er nad yw'r testun ar yr arwydd yn ymddangos yn y ddwy iaith, gellir ystyried yr arwydd hwn yn un sy'n hyrwyddo'r iaith Gymraeg yn yr ysgol, ac felly ethos dwyieithog yr ysgol, oherwydd caiff y Gymraeg ei normaleiddio yn y cyd-destun hwn. Fel y gwelir yn y tabl isod, mae llai na hanner o'r arwyddion a gofnodwyd yn Ysgol Y yn arwyddion y gellir eu hystyried naill ai'n ddwyieithog neu'n uniaith Gymraeg (8 allan o 20 arwydd).

Mewn cymhariaeth â hyn, nodwyd bod y rhan fwyaf o'r arwyddion a gofnodwyd yn Ysgol X yn ymddangos yn ddwyieithog (18 allan o 20 arwydd). Roedd y ddau arwydd arall yn uniaith Saesneg, y naill yn agos i gât yr ysgol a'r llall yn y brif dderbynfa. Yn achos yr arwyddion a ystyriwyd yn ddwyieithog, nodwyd bod patrwm cyffredin yn codi, sef cyflwyno'r testun Saesneg yn gyntaf ac wedyn defnyddio'r testun Cymraeg naill ai ar ôl neu o dan y testun Saesneg, yn aml mewn ffont italig (11 allan o'r 18 arwydd dwyieithog). Nodwyd bod hyn yn arfer digwydd pan fyddai'r testun Saesneg a'r testun Cymraeg yn agos iawn at ei gilydd. Ymddengys felly y defnyddir ffont italig at ddiben gwahaniaethu gwybodaeth yn y ddwy iaith a sicrhau bod gwedd y ddwy iaith yr un mor glir â'i gilydd lle'r bo'r ddwy iaith ochr yn ochr.

Er hynny, nodwyd ambell enghraifft lle nad oes defnydd o ffont italig i wahaniaethu rhwng y Gymraeg a'r Saesneg, hyd yn oed pan yw'r ddwy iaith ochr yn ochr â'i gilydd (7 allan o'r 18 arwydd dwyieithog). Wrth ystyried mai yn Saesneg yr ymddengys y testun yn gyntaf ar yr arwyddion hyn, teimlwyd bod modd gwella gallu'r arwyddion i wahaniaethu rhwng y ddwy iaith trwy ddefnyddio ffont italig, fel a wnaed yn achos yr arwyddion dwyieithog eraill. Sylwyd hefyd ar y defnydd anghyson o enw Cymraeg tref yr ysgol ar yr arwyddion (9 allan o'r 18 arwydd dwyieithog). Ymddengys fod hyn yn digwydd er mwyn osgoi ailadrodd yn y ddwy iaith ac ni theimlai'r ymchwilydd ei bod yn amharu ar wedd dwyieithog yr arwyddion hyn.

Er gwaetha'r anghysondebau a nodwyd uchod, teimlai'r ymchwilydd fod yr arwyddion hyn yn trin y ddwy iaith yn gyfartal o ran eu gwedd, yn arbennig yn 
nhermau ffont, fformat, lliw a maint (Comisiynydd y Gymraeg, 2014, t. 18), er bod modd gwella 'amlygrwydd' y ddwy iaith ar yr arwyddion hyn. Penderfynwyd felly fod yr arwyddion dwyieithog naill ai'n bodloni holl egwyddorion y Comisiynydd (2014), neu ran helaeth ohonynt, ac o ganlyniad cofnodwyd y rhain yn arwyddion dwyieithog gan yr ymchwilydd.

\begin{tabular}{|l|l|l|l|}
\hline \multicolumn{1}{|c|}{ Ysgol } & \multicolumn{1}{|c|}{$\begin{array}{c}\text { Arwyddion uniaith } \\
\text { Saesneg }\end{array}$} & Arwyddion dwyieithog & \multicolumn{1}{c|}{$\begin{array}{c}\text { Arwyddion uniaith } \\
\text { Gymraeg }\end{array}$} \\
\hline Ysgol Y & 12 & 7 & 1 \\
\hline Ysgol X & 2 & 18 & 0 \\
\hline
\end{tabular}

Tabl 4. Nifer yr arwyddion uniaith Saesneg, dwyieithog ac uniaith Gymraeg a ganfuwyd yn Ysgol Y ac Ysgol X.

\subsubsection{Galwadau ffôn i’r ysgolion}

Ar ôl ymweld â'r ddwy ysgol, galwodd yr ymchwilydd yr ysgolion ar fore diwrnod ysgol pan oeddent ar agor er mwyn ysgogi sgwrs yn Gymraeg ag aelod o'r staff rheng flaen dros y ffôn. Gwelir yn y tabl isod y data a gofnodwyd ar ôl galw'r ddwy ysgol. 


\begin{tabular}{|l|l|l|l|l|}
\hline Ysgol & $\begin{array}{c}\text { A atebodd yr aelod } \\
\text { o'r staff rheng flaen } \\
\text { gyda chyfarchiad } \\
\text { dwyieithog syml? }\end{array}$ & $\begin{array}{c}\text { Pa iaith a gafodd ei } \\
\text { blaenoriaethu wrth } \\
\text { ateb y ffôn, Cymraeg } \\
\text { neu Saesneg? }\end{array}$ & $\begin{array}{c}\text { A oedd yr aelod o'r } \\
\text { staff yn gallu cynnal } \\
\text { sgwrs yn Gymraeg } \\
\text { dros y ffôn? }\end{array}$ & $\begin{array}{c}\text { A gyfeiriodd yr } \\
\text { ymchwilydd at aelod } \\
\text { arall o'r staff sydd yn } \\
\text { gallu siarad } \\
\text { Cymraeg? }\end{array}$ \\
\hline Ysgol Y & Naddo. & Saesneg. & $\begin{array}{l}\text { Nac oedd. } \\
\text { Cadarnhaodd yr } \\
\text { aelod o'r staff nad yw } \\
\text { hi'n deall Cymraeg a } \\
\text { gofynnodd i'r } \\
\text { ymchwilydd siarad } \\
\text { yn Saesneg. }\end{array}$ & Naddo. \\
\hline Ysgol X & Do. & Cymraeg. & $\begin{array}{l}\text { Nac oedd. Esboniodd } \\
\text { yr aelod o'r staff ei } \\
\text { bod yn dysgu } \\
\text { Cymraeg. }\end{array}$ & Naddo. \\
\hline
\end{tabular}

Tabl 5. Galwadau ffôn i Ysgol Y ac Ysgol X pan oeddent ar agor.

Galwodd yr ymchwilydd y ddwy ysgol unwaith eto ar adeg pan oeddent ar gau, er mwyn cofnodi neges y peiriant ateb. Gwelir yn y tabl isod y data a gofnodwyd ar ôl galw’r ysgolion.

\begin{tabular}{|l|l|l|}
\hline \multicolumn{1}{|c|}{ Ysgol } & $\begin{array}{r}\text { A gynigodd peiriant ateb yr ysgol neges } \\
\text { ddwyieithog? }\end{array}$ & $\begin{array}{c}\text { Pa iaith a gafodd ei blaenoriaethu ar } \\
\text { neges peiriant yr ysgol? }\end{array}$ \\
\hline Ysgol Y & Naddo. & Nid oedd neges peiriant ateb ar gael. \\
\hline Ysgol X & Do. & Cymraeg. \\
\hline
\end{tabular}

Tabl 6. Galwadau ffôn i Ysgol Y ac Ysgol X pan oeddent ar gau.

\subsubsection{Ymweld â gwefannau'r ysgolion}

Er mwyn darganfod sut mae'r ddwy ysgol yn hyrwyddo a marchnata'r Gymraeg a'i hethos dwyieithog ar-lein, ymwelodd yr ymchwilydd â'u gwefannau. Nodwyd wrth agor gwefan Ysgol Y nad yw'r dudalen flaen yn cynnig dewis rhwng fersiwn Cymraeg neu fersiwn Saesneg o'r wefan. Yn ogystal â hynny, nodwyd nad oes dolen ar gael ar un o dudalennau'r wefan er mwyn i'r ymwelydd newid o'r naill iaith i'r llall. O ganlyniad, ymddangosodd holl gynnwys y wefan yn uniaith Saesneg yn unig. 
Nid ystyriwyd gwefan Ysgol Y yn un ddwyieithog oherwydd na chafwyd defnydd o'r Gymraeg o gwbl arni. Gan nad oedd y wefan yn ddwyieithog, nid oedd yn bosibl barnu i ba raddau y mae hi'n bodloni egwyddorion y Comisiynydd (2014).

Er mwyn canfod unrhyw dudalennau sy'n hyrwyddo'r Gymraeg a dwyieithrwydd, porwyd drwy'r wefan gan ddefnyddio'r dolennau mewnol. Yn ogystal â hyn, defnyddiwyd blwch chwilio'r wefan er mwyn chwilio am unrhyw ganlyniadau yn gysylltiedig â'r allweddeiriau a restrir yn y meini prawf (Atodiad 6) sef 'Cymraeg', 'Welsh', 'dwyieithrwydd', 'bilingualism', 'dwyieithog', 'bilingual' ac 'ethos'. Ar ôl pori trwy ddolennau'r wefan a chwilio am yr allweddeiriau yn y blwch chwilio, ni chafwyd hyd i unrhyw dudalennau sy'n gysylltiedig â'r allweddeiriau hyn. Nodir hefyd na chafwyd sôn am y gweithgareddau y mae Pennaeth y Gymraeg yn eu nodi yn yr holiadur a'r cyfweliad sy'n hyrwyddo'r iaith Gymraeg a dwyieithrwydd trwy'r ysgol gyfan (gweler 7.1.3. Ethos dwyieithog).

Wrth fynd i mewn i wefan Ysgol X, nodwyd nad yw'r dudalen flaen yn cynnig dewis rhwng fersiwn Cymraeg neu fersiwn Saesneg o'r wefan. Nodwyd hefyd nad oes dolen ar gael ar un o dudalennau'r wefan er mwyn i'r ymwelydd newid o'r naill iaith i'r llall. Golyga hyn fod cynnwys y wefan gyfan yn uniaith Saesneg yn unig. Er hynny, nodwyd bod logo ac arwyddair yr ysgol yn ymddangos yn fach ar ben pob tudalen yn y wefan yn y ddwy iaith a bod y Gymraeg a'r Saesneg yn gyfartal o ran ffont, fformat, lliw a maint (Comisiynydd y Gymraeg, 2014, t. 18). Fodd bynnag, teimlwyd bod modd gwahaniaethu'n well rhwng y ddwy iaith yma, er enghraifft trwy ddefnyddio ffont italig, er mwyn gwella 'amlygrwydd' y ddwy iaith yn arwyddair ac ar logo'r ysgol. Nid ystyriwyd y wefan hon yn un ddwyieithog gan na chafwyd defnydd cyfartal o'r ddwy iaith ar draws y wefan i gyd. O ganlyniad, ni fodlonodd y wefan yr egwyddorion a bennir yn Canllawiau Dylunio Dwyieithog (Comisiynydd y Gymraeg, 2014).

Ar adeg ymweld â gwefan Ysgol X, nid oedd blwch chwilio ar gael er mwyn chwilio am yr allweddeiriau a restrir yn y meini prawf (gweler Atodiad 6). Er mwyn canfod unrhyw dudalennau sy'n hyrwyddo'r Gymraeg a dwyieithrwydd felly, porwyd trwy'r wefan gan glicio ar bob dolen fewnol. Wrth bori trwy dudalennau'r wefan, nodwyd mai go brin oedd y sôn am sut mae'r ysgol yn hyrwyddo dwyieithrwydd. Mewn gwirionedd, yr unig enghraifft lle cafodd dwyieithrwydd ei hybu yn benodol oedd blognod sy'n adrodd am y canlyniadau diweddar yn CA3. Nodir yma yn y blognod 
hwn fod ymrwymiad yr ysgol i ddatblygu sgiliau dwyieithog disgyblion wedi galluogi disgyblion ar y Cwrs Carlam i ennill gradd A*-C yn y Gymraeg ddwy flynedd yn gynt na'r arfer. Yn ogystal â hyn, nodwyd bod gwefan Ysgol X yn sôn am y Cwrs Carlam wrth drafod y ddarpariaeth a gynnig yr ysgol ar gyfer disgyblion MAT. Fodd bynnag, nid aed sôn am y ddarpariaeth Gymraeg Iaith Gyntaf a gynnig Ysgol X, er bod y ddarpariaeth hon yn rhan allweddol o bolisi'r ysgol ar ddwyieithrwydd (gweler Atodiad 10).

\subsubsection{Dogfennaeth yr ysgolion}

\subsection{Prosbectysau'r ysgol}

Yn ogystal â'r prosbectws ar gyfer yr ysgol gyfan, nodir bod gan y ddwy ysgol brosbectws ar wahân ar gyfer y Chweched Dosbarth. Penderfynwyd dadansoddi'r ddau brosbectws hefyd er mwyn meithrin gwell dealltwriaeth o ethos yr ysgol gyfan. Wrth ddarllen trwy ddau brosbectws Ysgol Y, nodwyd na cheir defnydd o'r iaith Gymraeg yn eu testunau ac ymddengys holl gynnwys y ddau brosbectws yn uniaith Saesneg yn unig. O ganlyniad, nodir mai ond fersiwn Saesneg o'r prosbectysau diweddaraf a oedd ar gael yn yr ysgol hon. Yn mhrosbectws yr ysgol gyfan, ceir trosolwg o'r cwricwlwm, gwerthoedd yr ysgol a'i pholisïau. Ym mhrosbectws y Chweched Dosbarth, canolbwyntir ar y cyrsiau ôl-16 a gynigir gan Ysgol Y, gydag ychydig o wybodaeth gefndirol am yr ysgol.

Un o'r prif themâu sy'n codi yn y ddau brosbectws yw ethos Cristnogol Ysgol Y. Mae cyflwyniad y prifathro i brosbectws yr ysgol gyfan yn gosod hyn yn glir. Nodir bod safonau Cristnogol yr ysgol yn mynnu "the highest standards of expectation and commitment, recognising the distinct human dignity of every one of our students and doing all within out power to unlock his or her potential." Caiff yr ethos hwn ei bwysleisio eto pan drafodir ymrwymiad yr ysgol i 'werthoedd Cristnogol.' Ymddengys fod y gwerthoedd hyn wrth wraidd ethos yr ysgol, ac fel y mae prosbectws y Chweched Dosbarth yn ei nodi, mae'r gwerthoedd hyn wrth wraidd pob penderfyniad yn yr ysgol: “... our commitment to our faith is central to each decision that we make and everything that we do." Er ymddengys fod gan Ysgol Y ymrwymiad clir a ffurfiol i ddatblygu ei hethos Cristnogol, ni cheir yn y prosbectysau'r un ymrwymiad i ddatblygu ei hethos dwyieithog. Yn y ddau brosbectws, ni sonnir am sut y caiff y Gymraeg a dwyieithrwydd eu hyrwyddo a'u 
datblygu trwy'r ysgol yn achlysurol, nac yn ffurfiol ar draws y cwricwlwm. Ni cheir sôn ychwaith am y gweithgareddau y mae Pennaeth Adran y Gymraeg yn eu nodi yn yr holiadur a'r cyfweliad.

Er hynny, nodir bod prosbectws y Chweched Dosbarth yn cadarnhau pwynt a godwyd gan Bennaeth Adran y Gymraeg yn yr holiadur a'r cyfweliad dilynol. Nododd Pennaeth y Gymraeg fod rhai o ddisgyblion y Chweched Dosbarth yn cynorthwyo athrawon mewn gwersi CAI. Pwysleisir yn y prosbectws fod yr ysgol yn ystyried disgyblion y Chweched Dosbarth fel "leaders of the school who work alongside staff" a bod gan y rhan fwyaf ohonynt rôl fugeiliol, gan gynnwys cynorthwyo addysgu yn yr ysgol. Nodir hefyd fod prosbectws y Chweched Dosbarth yn hyrwyddo cyfleoedd gyrfaol y gall disgyblion fanteisio arnynt yn sgil astudio'r Gymraeg ar Lefel A, wrth nodi bod sgiliau Cymraeg naill ai'n hanfodol neu'n ddymunol ar gyfer ystod o sectorau, gan gynnwys addysg, y cyfryngau, yr heddlu, iechyd, gwasanaethau gofal, llywodraeth a thwristiaeth.

Wrth ddarllen trwy ddau brosbectws Ysgol X, nodwyd bod rhai enghreifftiau lle defnyddir Cymraeg yn ogystal â Saesneg, yn benodol wrth gyflwyno teitl y dogfennau, logo ac arwyddair yr ysgol, penawdau a rhai o'r is-benawdau trwy gydol y prosbectysau. Yn yr enghreifftiau hyn lle defnyddir y ddwy iaith, sylwyd y caiff y testun Saesneg ei flaenoriaethu fel arfer, wrth i'r testun Cymraeg ymddangos ar ôl y testun Saesneg. Nodwyd hefyd y caiff llythyr croeso'r Prifathro yng nghyflwyniad prosbectws yr ysgol gyfan ei gynnig yn ddwyieithog, ond rhaid nodi mai'r testun Saesneg sy'n cael y flaenoriaeth. Yn yr enghreifftiau lle defnyddir y Gymraeg a'r Saesneg yn y dogfennau hyn, nodwyd bod y ddwy iaith yn ymddangos yn gyfartal o ran ffont, fformat, lliw a maint (Comisiynydd y Gymraeg, 2014, t. 18). Gellid dadlau bod defnyddio Cymraeg yn y dogfennau Saesneg hyn i ryw raddau yn normaleiddio'r Gymraeg a dwyieithrwydd a bod hyn yn ei dro yn hyrwyddo ethos dwyieithog yr ysgol. Er hynny, teimlai'r ymchwilydd nad yw'r defnydd o'r Gymraeg yn ddigon cyson drwy'r ddau brosbectws. Sylwyd ar enghreifftiau, ym mhrosbectws y Chweched Dosbarth yn benodol, lle ceir penawdau ac is-benawdau yn y Saesneg yn unig. Rhaid nodi hefyd y caiff y rhan fwyaf o'r cynnwys yn y ddau destun hyn ei chyflwyno'n uniaith Saesneg. Oherwydd y defnydd anghyson o'r Gymraeg, nid ystyriwyd y ddau brosbectws yn ddogfennau dwyieithog. 
Mewn cymhariaeth â phrosbectws ysgol gyfan Ysgol Y, mae prosbectws ysgol gyfan Ysgol X yn fanylach o lawer a cheir ynddo wybodaeth drylwyr am wahanol agweddau ar yr ysgol, megis y cwricwlwm, polisïau'r ysgol a'r ddarpariaeth a gynigir i’w disgyblion. Yn wahanol i'r prosbectws ysgol gyfan yn Ysgol Y, ceir ym mhrosbectws ysgol gyfan Ysgol X sylw penodol at yr iaith Gymraeg a dwyieithrwydd a sut y mae'r ysgol yn eu hyrwyddo. Yn ei lythyr croeso i'r ysgol yng nghyflwyniad y prosbectws, er enghraifft, sonia'r Prifathro yn benodol am yr iaith Gymraeg a dwyieithrwydd. Yn ogystal ag amlinellu'r ddarpariaeth cyfrwng Cymraeg a dwyieithog a gynnig Ysgol X, mae'r Prifathro yn mynd yn ei flaen i honni bod darpariaeth yr ysgol ar gyfer y Gymraeg wedi'i chydnabod gan Estyn fel un sy'n arwain y sector.

Nes ymlaen yn y prosbectws hwn, ceir trafodaeth fwy cyflawn o'r 'defnydd o'r iaith Gymraeg' a 'dwyieithrwydd' yn yr ysgol, o dan is-benawdau o'r un enwau. O dan yr is-benawdau hyn, ceir manylion pellach am ddarpariaeth yr ysgol ar gyfer y Gymraeg, yn benodol y Cwrs Carlam a'r ffrwd cyfrwng Cymraeg a gynnig yr ysgol. Nodir hefyd fod sylw eto at gydnabyddiaeth yr ysgol mewn arolygiadau Estyn yn 2012 a 2016 am 'arwain y sector' o ran ei darpariaeth ar gyfer hyrwyddo'r iaith. Priodola'r ysgol y llwyddiant hwn wrth hyrwyddo'r Gymraeg i agweddau 'ymrwymedig' a 'brwdfrydig' Adran y Gymraeg a'r disgyblion at yr iaith. Pwysleisir hefyd bwysigrwydd datblygu dilyniant yn y Gymraeg o'r sector cynradd i'r sector uwchradd, gan roi gwybod am gynllun yr ysgol i gyflogi athrawon peripatetig i weithio ar y cyd â'r ysgolion cynradd sy'n bwydo'r ysgol.

Sonnir hefyd yma am y cyfleodd y mae'r ysgol yn eu cynnig i'w disgyblion er mwyn datblygu eu sgiliau Cymraeg. Rhestrir nifer o sefydliadau y mae'r ysgol yn gweithio mewn partneriaeth â nhw, megis Yr Urdd, Menter Iaith a'r prifysgolion lleol, er mwyn i ddisgyblion gael cyfleoedd i ddefnyddio'r iaith mewn gweithgareddau ffurfiol ac anffurfiol a mwynhau defnyddio'r iaith. At hyn ychwanegir bod yr ysgol yn cynnig 'ystod' o weithgareddau allgyrsiol, megis ei gwibdaith i Langrannog, er mwyn i ddisgyblion ddefnyddio'r iaith y tu hwnt i'r dosbarth.

Nodwedd arall i sylwi arno ym mhrosbectws yr ysgol gyfan yw'r ffordd yr ymdrinnir â'r Gymraeg fel pwnc craidd. Er bod gan y Gymraeg statws fel pwnc sylfaenol yn unig yn nhermau mesur perfformiad ysgolion, fe'i rhestrir yn un o'r pynciau craidd 
wrth gyflwyno'r cwricwlwm a gynigir. Cafodd hyn ei ategu hefyd gan Bennaeth y Gymraeg yn y cyfweliad dilynol.

Ym mhrosbectws y Chweched Dosbarth, ni chaiff dwyieithrwydd na'r iaith Gymraeg eu hyrwyddo'n benodol. Fodd bynnag, ceir trafodaeth ar y cyfleoedd gyrfaol y gall disgyblion fanteisio arnynt ar ôl astudio Cymraeg fel Lefel A. Yn ogystal â rhestru'r cyfleoedd gyrfaol hyn, ceir yma ddefnydd diddorol o iaith rethregol er mwyn hybu CIG a CAI fel pynciau Lefel A i ddarpar ddisgyblion:

The possibilities are endless! Do you want to be a part of it?

Remember Welsh is not a subject - it's a way of life! Cymraeg - y ffordd ymlaen!

So what about you? Do you want to study Welsh in form 6 ?

Felly, beth amdani? Ydych chi am astudio'r Gymraeg yn y Chweched Dosbarth?

Yma, ceisir hybu gwerth y Gymraeg o safbwynt gyrfaol, fel ffordd o ddod ymlaen yn y byd, yn hytrach na phwnc yn unig. Gellir dadlau felly fod yr iaith berswadiol yma yn atgyfnerthu ethos dwyieithog ffurfiol yr ysgol, trwy hyrwyddo gwerth y Gymraeg a dwyieithrwydd.

\subsection{Cylchlythyrau'r ysgol}

Yn y ddau gylchlythyr a gasglwyd o Ysgol Y, nodir na cheir defnydd o'r iaith Gymraeg yn eu testunau hwy ac ymddengys holl gynnwys y dogfennau yn uniaith Saesneg. Gan nad oedd y testunau hyn yn ddwyieithog, nid oedd yn bosibl barnu i ba raddau y maent yn bodloni egwyddorion Comisiynydd y Gymraeg (2014). Wrth ddarllen trwy'r cylchlythyrau, canolbwyntir ar nifer o weithgareddau a gwibdeithiau allgyrsiol a gynhaliwyd gan yr ysgol yn ystod y tymor. Fodd bynnag, nodwyd na cheir sôn am weithgareddau allgyrsiol, gwibdeithiau neu lwyddiannau disgyblion lle roedd y Gymraeg wrth eu gwraidd yn y testunau hyn. O ganlyniad, ni chaiff dwyieithrwydd ei farchnata na'i hyrwyddo'n ffurfiol yn y testunau hyn dan sylw. Yng nghylchlythyr Tachwedd 2015 Ysgol X, nodwyd na cheir defnydd sylweddol o'r Gymraeg yn y testun. Yr unig enghreifftiau a gofnodwyd lle defnyddir Cymraeg yn y testun hwn oedd logo'r ysgol ar dudalen flaen y cylchlythyr a'r ymadrodd " $\mathrm{Da}$ iawn pawb!" ar ddiwedd un o golofnau byrion y cylchlythyr. Golyga hyn y caiff y 
rhan fwyaf o'r testun hwn ei chyflwyno yn Saesneg yn unig. Yng nghylchlythyr Mawrth 2016 fodd bynnag, nodwyd y ceir defnydd o'r Gymraeg a'r Saesneg mewn un o'r colofnau byrion sy'n crybwyll gwibdaith ddiweddar disgyblion i wersyll Llangrannog. Nodwyd bod y testun Cymraeg yn ymddangos yn gyntaf mewn lliw melyn tywyll, a'r testun Saesneg yn ei ddilyn mewn lliw gwyn. Mae blaenoriaethu'r testun Cymraeg a defnyddio lliwiau gwahanol ar gyfer y ddwy iaith yn tynnu sylw'r darllenydd at y testun Cymraeg. Er y gellid gwella darllenadwyedd y ddwy iaith yn gyffredinol yn y ddau gylchlythyr, oherwydd y gwrthdaro rhwng lliwiau'r testunau, sef melyn tywyll a gwyn, a'r lliwiau cefndirol a ddefnyddiwyd o dan y ddau destun, sef glas tywyll a choch, teimlwyd bod y testun Cymraeg a'r testun Saesneg yn y golofn yn gyfartal o ran ffont, maint ac amlygrwydd (Comisiynydd y Gymraeg, 2014, t. 18). Ar wahân i'r enghraifft hon, nodwyd na cheid defnydd o'r Gymraeg yn y ddogfen hon.

\subsection{Trafodaeth a chasgliadau}

\subsubsection{Darpariaeth ar gyfer y Gymraeg}

O ystyried canfyddiadau Lewis (2010a) parthed nifer yr oriau a neilltuir i'r Gymraeg mewn addysg cyfrwng Saesneg yn Pennod 1, sylwir bod nifer yr oriau y mae'r ddwy ysgol yn eu neilltuo i wersi CAI yn nodweddiadol o'r hyn a geir mewn ysgolion uwchradd cyfrwng Saesneg eraill. Yr unig eithriad i hyn yw'r disgyblion gwannaf yn CA4 yn y ddwy ysgol. Caiff disgyblion gwannaf Ysgol Y gynifer o oriau dysgu CAI â'r setiau uwch yn yr ysgol, sef pum awr y pythefnos, o'u cymharu â disgyblion gwannaf Ysgol X, sydd yn derbyn dwy awr y pythefnos yn unig drwy'r cwrs NVQ Lefel 1 Darllen Cymraeg.

Er bod y ddwy ysgol uwchradd yn neilltuo'r un nifer o oriau i addysgu Cymraeg i'r disgyblion sy'n astudio Cwrs Llawn TGAU CAI, mae darpariaeth ddwyieithog y Cwrs Carlam yn CA3 yn golygu y caiff y disgyblion yn y ffrwd hon ragor o gyswllt â'r iaith o'u cymharu â'u cyfoedion sydd yn derbyn gwersi CAI traddodiadol yn unig. Yn ogystal ag addysgu'r iaith yn ffurfiol fel pwnc cwricwlaidd, lle canolbwyntir ar ffurf iaith, ceir defnydd o'r Gymraeg fel cyfrwng addysgu yn y dosbarth carlam. Trwy ddefnyddio'r patrymau a gyflwynir yn y gwersi CAI ar draws pynciau cwricwlaidd eraill, caiff disgyblion y dosbarth carlam ragor o gyfleoedd i ddefnyddio'r Gymraeg yn ystyrlon ac yn bwrpasol. 
Yn ystod y gwaith maes, danfonodd Pennaeth y Gymraeg yn Ysgol X gopi o amserlen nodweddiadol un o'r disgyblion Cwrs Carlam presennol er mwyn i'r ymchwilydd gael gwell dealltwriaeth o'r system amserlennu. Yn ogystal â'r pedair awr o wersi a neilltuir i CAI yn yr amserlen bythefnosol (2 awr o wersi bob wythnos) neilltuir deg awr o wersi i'r pynciau dwyieithog (5 awr o wersi bob wythnos). Fodd bynnag, nid oedd yn bosibl cymharu'n fanwl gywir y ffrydiau cyfrwng Saesneg â'r ffrwd ddwyieithog o ran nifer yr oriau cyswllt â'r Gymraeg oherwydd gall y defnydd a wneir o'r Gymraeg yn y dosbarth carlam amrywio'n fawr gan ddibynnu ar flwyddyn ysgol y disgyblion ac anhawster y cynnwys y mae'r athrawon yn ei gyflwyno (gweler 7.2.2.3. Darpariaeth ddwyieithog: Y Cwrs Carlam). Rhaid nodi hefyd na cheir defnydd o'r Gymraeg fel cyfrwng addysgu mewn gwersi CAI traddodiadol. Serch hynny, mae'n amlwg fod defnyddio'r Gymraeg fel cyfrwng addysgu yn y pum pwnc dwyieithog, yn ogystal â'r gwersi CAI, yn golygu y caiff disgyblion yn y ffrwd ddwyieithog fwy o gyswllt â'r Gymraeg o fewn yr amserlen bythefnosol (14 awr y pythefnos) na'u cyfoedion yn y ffrwd cyfrwng Saesneg yn Ysgol X (4 awr y pythefnos) ac Ysgol Y (3 awr y pythefnos) yn CA3. Fodd bynnag, rhaid pwysleisio nad oes gwahaniaeth rhwng y ffrydiau hyn erbyn CA4 oherwydd bod pob disgybl, yn Ysgol Y ac Ysgol X, yn astudio eu pynciau TGAU trwy gyfrwng y Saesneg.

Er bod disgyblion y Cwrs Carlam yn derbyn nifer sylweddol fwy o oriau mewn cyswllt ffurfiol â'r Gymraeg o'u cymharu â'u cyfoedion yn y ffrwd cyfrwng Saesneg, rhaid nodi mai ond carfan benodol o ddisgyblion sy'n gymwys ar gyfer astudio ar gyfer y Cwrs Carlam. Yn Pennod 4 canfuwyd bod rhai ysgolheigion yn ystyried rhaglenni CLIL yn yr Almaen fel rhai 'elitaidd' oherwydd caiff disgyblion y rhaglenni hyn eu dethol ar sail eu perfformiad academaidd (Apsel, 2012; Zydatiß, 2012). Yn aml iawn, bydd gradd gyfartaledd ar gyfer Saesneg yn yr ysgol gynradd wrth wraidd y penderfyniad hwn (Apsel, 2012, t. 50). Canfuwyd yn ystod y cyfweliad dilynol y caiff darpar ddisgyblion y Cwrs Carlam eu dethol mewn ffordd debyg iawn, ar sail y meini prawf canlynol:

- Lefelau disgyblion ym Mlwyddyn 6 yn y pynciau Saesneg, Cymraeg (Ail Iaith), Mathemateg a Gwyddoniaeth,

- Canlyniadau'r disgyblion ym Mlwyddyn 6 yn y Profion Gallu Gwybyddol (CATs), a 
- Cymhelliant y disgyblion wrth addysgu Cymraeg.

Wrth ystyried y rhestr uchod, ymddengys fod perfformiad academaidd hefyd yn ffactor allweddol yn y broses o ddethol disgyblion ar gyfer y Cwrs Carlam. O ganlyniad, gellid dadlau bod disgyblion llai galluog o dan anfantais, o'u cymharu â disgyblion MAT er enghraifft, am eu bod yn llai tebygol o gael eu hystyried ar gyfer darpariaeth ddetholus y Cwrs Carlam. Yn y cyfweliad, cyfeiriodd Pennaeth y Gymraeg (Atodiad 9, para. 30) at ddisgyblion nad ydynt yn cael eu dethol ar gyfer y Cwrs Carlam ond a allai “ymdopi gyda'r cwrs" a'i ofynion. Fodd bynnag, mewn tystiolaeth anecdotaidd gan un o athrawon y Cwrs Carlam, codwyd pryder ynglŷn ag ymarferoldeb estyn darpariaeth y cwrs hwn i ddisgyblion llai galluog. Nododd fod disgyblion yn y Cwrs Carlam yn ddisgybledig iawn a bod eu parodrwydd a'u gallu i ganolbwyntio a gweithio heb gael eu hysgogi yn y dosbarth yn caniatáu i'r athrawes, neu athro, gynnig y ddarpariaeth Gymraeg ychwanegol. Gan y gall disgyblaeth fod yn her yn nosbarthiadau'r setiau isaf, roedd hi'n amheus am barodrwydd a gallu rhai disgyblion yn y dosbarthiadau hyn i ganolbwyntio ar y ddarpariaeth Gymraeg ychwanegol, yn ogystal â dysgu'r cynnwys cwricwlaidd. Mae'r dystiolaeth anecdotaidd hon yn cefnogi canfyddiad yr holiadur a'r cyfweliad, sef y caiff safon yr addysg ei blaenoriaethu dros y ddarpariaeth Gymraeg ychwanegol yn Ysgol X (gweler 7.2.2.3. Darpariaeth ddwyieithog: Y Cwrs Carlam).

Cadarnhaodd Pennaeth y Gymraeg nad yw'n bosibl i'r ysgol estyn y ddarpariaeth hon i garfannau eraill o ddisgyblion ar hyn o bryd oherwydd nad oes rhyddid o fewn yr amserlen bresennol i allu cynnig mwy o wersi dwyieithog. O ganlyniad, dim ond y 40 disgybl sy'n perfformio orau o ran y meini prawf uchod a gaiff eu hystyried ar gyfer y Cwrs Carlam. Er hynny, mynnodd Pennaeth y Gymraeg y caiff pob disgybl yr un safon o addysg yn y gwersi Cymraeg, ni waeth pa ffrwd mae'r disgybl yn ei dilyn. Mae canlyniadau TGAU CAI diweddar disgyblion Ysgol X (gweler 5.4.3. Canlyniadau TGAU) ac adroddiadau arolygiad Estyn (2006b, 2012a, 2016a) yn cadarnhau bod darpariaeth ar gyfer y Gymraeg yn gryfder yn yr ysgol. Er hynny, nid yw'n amlwg ar hyn o bryd sut y bydd Ysgol $\mathrm{X}$ yn mynd ati i ddatblygu ac ymestyn dwyieithrwydd fel rhan annatod o addysgu ar draws yr ysgol, fel y nodwyd ym mholisi'r ysgol ar ddwyieithrwydd, heb neilltuo mwy o amser yn yr amserlen i sicrhau y gall ystod ehangach o ddisgyblion elwa o'r ddarpariaeth ddwyieithog hon. Mae'n debyg hefyd na fydd modd datblygu'r ddarpariaeth ddwyieithog yn sylweddol 
heb ystyried gweithredu polisi staffio a fydd yn rhoi blaenoriaeth i gyflogi athrawon sy'n medru addysgu trwy gyfrwng y Gymraeg (gweler 7.2.4. Hyfforddiant staff yr ysgolion). Er bod darpariaeth ar gyfer y Gymraeg yn gryfder yn Ysgol X felly, credir bod cyfle i'r ysgol fynd i'r afael â'r rhwystrau a drafodwyd uchod er mwyn gallu ehangu ei chapasiti i gynnig y ddarpariaeth ddwyieithog i garfannau eraill o ddisgyblion CAI a sicrhau bod y Cwrs Carlam yn fodel mwy cynhwysol.

Daeth i'r amlwg yn yr holiadur fod Ysgol X yn denu disgyblion o tua ugain o ysgolion cynradd y tu allan i'w chlwstwr, gan gynnwys tair ysgol gynradd cyfrwng Cymraeg leol. Efallai mai'r brif ffactor y gellir ei phriodoli i boblogrwydd Ysgol X yw darpariaeth a safon addysg yr ysgol. Mae arolygiadau gan Estyn (2006b, 2012a, 2016a), er enghraifft, wedi adrodd yn gyson fod perfformiad cyffredinol yr ysgol yn gryf. Mae perfformiad yr ysgol o safbwynt canran gymedrig y disgyblion a gyflawnodd drothwy lefel $2^{70}$ dros y chwe blynedd ddiwethaf, sef $91.5 \%$ (L1C, 2016a), hefyd yn cadarnhau bod ei safonau diweddar wedi bod yn uchel. Mewn gwirionedd, allan o'r 11 o ysgolion uwchradd yn ei theulu ysgol, Ysgol X oedd yn ail o safbwynt ei pherfformiad yn 2016, yn ôl y data a ddarparwyd i'r ymchwilydd gan gynrychiolydd ar ran LIC (cyfathrebiad personol, Mawrth 31, 2016).

Ffactor arall y gellir ei phriodoli i boblogrwydd Ysgol X yn ei dalgylch yw ei darpariaeth helaeth yn y Gymraeg. Mae adroddiadau arolygiad Estyn (2006b, 2012a, 2016a) unwaith eto'n datgelu bod darpariaeth Ysgol X i'r Gymraeg yn gryfder yn yr ysgol. Yn benodol, mae'r adroddiadau hyn yn amlygu'r ystod eang o gyrsiau Cymraeg y mae hi'n eu darparu, ei darpariaeth ar gyfer hyrwyddo ethos Cymraeg, a'i darpariaeth beripatetig ar gyfer codi safonau yn y Gymraeg o fewn y clwstwr (Estyn, 2006b, 2012a, 2016a). Clywyd hefyd yn anecdotaidd bod rhai rhieni yn penderfynu danfon eu plant i Ysgol X ar ôl iddynt gwblhau eu haddysg gynradd yn yr ysgol cyfrwng Cymraeg. Er na all canfyddiadau'r gwaith maes gadarnhau'r dystiolaeth anecdotaidd hon, mae'n bosibl dadlau fod darpariaeth helaeth Ysgol X i'r Gymraeg, o'i chymharu â'r ddarpariaeth Gymraeg a geir mewn ysgolion uwchradd eraill y dalgylch, yn gwneud yr ysgol yn deniadol i rieni sydd â phlant yn y sector cynradd cyfrwng Cymraeg. Os felly, gellid dadlau bod darpariaeth Ysgol X i’r Gymraeg yn denu disgyblion o'r sector hwnnw.

\footnotetext{
${ }^{70}$ Dyma gyfanswm y cymwysterau sy'n cyfateb i bum TGAU gradd A* i C.
} 
Mae pryderon am oblygiadau darpariaeth Gymraeg Ysgol X ar gyfer y sector cyfrwng Cymraeg eisoes wedi'u mynegi gan Cennard Davies (2010). Yn ei adroddiad i'r Pwyllgor Menter a Dysgu (2010), mynegodd Davies ei bryder fod y disgyblion hyn sy'n trosglwyddo o'r ysgol gynradd cyfrwng Cymraeg i Ysgol X o dan anfantais o'u cymharu â'u cyfoedion sy'n trosglwyddo i'r ysgol uwchradd cyfrwng Cymraeg. Roedd Davies o'r farn fod y disgyblion hyn ar eu colled gan nad ydynt yn cael eu hymestyn yn ieithyddol trwy astudio ystod eang o bynciau trwy gyfrwng y Gymraeg a chymdeithasu yn Gymraeg drwy'r diwrnod ysgol. Mynegwyd pryder tebyg o ran cynllunio dilyniant ieithyddol disgyblion yn y sector cyfrwng Cymraeg hefyd yn yr adroddiad Un Iaith i Bawb (2013). Yn ôl yr adroddiad, mae disgyblion sy'n trosglwyddo o un cyfrwng addysgu i'r llall yn ystod y cyfnod pontio ar eu colled am "n[a]d yw mwyafrif yr unigolion hyn yn cael digon o gyfle i adeiladu ar eu sgiliau Cymraeg a'u datblygu ymhellach" (Davies, 2013, t. 28). Bydd yn hanfodol ymchwilio'r effaith bosibl y gallai'r model hwn ei chael ar addysg cyfrwng Cymraeg ar lefel leol a sut mae cryfhau cynllunio'r dilyniant ieithyddol rhwng yr ysgol gynradd cyfrwng Cymraeg a'r ysgol uwchradd cyfrwng Cymraeg er mwyn lleihau nifer y disgyblion sy'n trosglwyddo o'r naill gyfrwng addysgu i'r llall.

Er gwaetha'r goblygiadau posibl hyn a nodir uchod, ymddengys fod y Cwrs Carlam yn fodel llwyddiannus o safbwynt ei darpariaeth i'r Gymraeg ar gyfer disgyblion ail iaith. Mae'r oriau cyswllt ychwanegol â'r Gymraeg a geir yn y model hwn yn sicrhau y caiff disgyblion ragor o gyfleoedd i ymarfer yr hyn y maent yn ei ddysgu'n ffurfiol yn y gwersi CAI, ac i ddefnyddio'r iaith Gymraeg fel cyfrwng dysgu yn y dosbarth. Mae'r canfyddiadau ymchwil ryngwladol yn Pennod 4 wedi dangos eisoes fod manteision posibl i'r rhai sy'n astudio rhannau o'r cwricwlwm drwy gyfrwng yr ail iaith mewn rhaglenni CLIL. Am fod ei ddarpariaeth yn cynnig cyfle i ddysgwyr astudio elfennau o'r cwricwlwm drwy gyfrwng eu hail iaith, ymddengys y Cwrs Carlam yn enghraifft wreiddiol o CLIL ar waith yn y cyd-destun CAI yng Nghymru.

Fel yr esboniwyd eisoes, nid diben yr ymchwil hon yw astudio methodoleg addysgu'r athrawon yn y naill ysgol a'r llall. Serch hynny, canfuwyd yn ystod y gwaith maes nad yw athrawon y Cwrs Carlam yn dilyn methodoleg/au addysgu penodol wrth gyflwyno pynciau cwricwlaidd yn ddwyieithog. Yn ôl Pennaeth y Gymraeg, mae'r athrawon hyn yn tynnu ar eu profiad o addysgu ac arsylwi addysgu dwyieithog yn yr ysgol. Mae Adran y Gymraeg hefyd yn paratoi llawlyfr o'r 
gramadeg a'r eirfa a gyflwynir yn y gwersi CAI er mwyn i'r athrawon eu hymarfer yn y dosbarth carlam. Cydnabyddir bod angen ymchwil bellach i werthuso'r fethodoleg/au addysgu y mae athrawon yn eu defnyddio rhwng Blwyddyn 7 a Blwyddyn 9 i ganfod y dulliau addysgu dwyieithog sydd eu hangen er mwyn cyflwyno pynciau cwricwlaidd yn rhannol ddwyieithog yn llwyddiannus mewn ysgolion cyfrwng Saesneg eraill.

\subsubsection{Ethos dwyieithog}

Canfuwyd yn Pennod 1 fod datblygu ethos dwyieithog cryf trwy'r ysgol gyfan yn ganolog i sicrhau y caiff y Gymraeg ei chyflwyno'n llwyddiannus fel ail iaith mewn addysg. Yn yr holiadur a'r cyfweliad dilynol a gynhaliwyd â Phennaeth y Gymraeg yn Ysgol Y, canfuwyd bod yr ysgol yn hyrwyddo dwyieithrwydd ar ffurf gweithgareddau beunyddiol yn bennaf. Yn ogystal â gweithgareddau beunyddiol, pwysleisiodd Pennaeth y Gymraeg fod gan ddisgyblion y Chweched Dosbarth rôl allweddol wrth hyrwyddo'r iaith yn yr ysgol. Nododd fod disgyblion y Chweched Dosbarth wedi cynorthwyo athrawon yn y dosbarth trwy ddatblygu sgiliau Cymraeg disgyblion iau yn yr ysgol, yn ogystal â disgyblion yn yr ysgol gynradd leol. Nodwyd hefyd bod gan grŵp o ddisgyblion CIG awydd sefydlu 'Clwb Cymraeg'. I'r un perwyl, nododd Pennaeth y Gymraeg yn Ysgol X fod yr ysgol yn hyrwyddo defnyddio'r Gymraeg yn achlysurol o amgylch yr ysgol. Un enghraifft a nododd Pennaeth y Gymraeg yn ystod y cyfweliad oedd y diwrnodau trochi allgyrsiol a drefnir gan Adran y Gymraeg. Mae'r diwrnodau hyn yn cynnig cyfle i ddisgyblion Blwyddyn 7 ddefnyddio'r Gymraeg mewn cyd-destun anffurfiol, hynny yw, cyddestunau y tu hwnt i'r dosbarth lle y gellir canolbwyntio ar gyfathrebu yn naturiol yn yr iaith darged, yn hytrach na ffurf iaith fel a wneir yn y dosbarth iaith (gweler 2.2. Caffael a dysgu iaith).

Efallai mai un o brif gryfderau Ysgol X o ran datblygu ethos dwyieithog yw'r ffordd y mae'r ysgol yn hyrwyddo'r iaith Gymraeg yn ffurfiol. Er bod gan Ysgol Y awydd dros lunio polisi ffurfiol ar ddwyieithrwydd, nid oedd polisi o'r fath yn ei le adeg casglu'r data. Yn Ysgol X, gweithredir polisi ffurfiol ar ddwyieithrwydd a darpariaeth i'r Gymraeg. Fel y gwelir yn y polisi hwn (gweler Atodiad 10) ceir manylder o ran sut y mae'r ysgol yn mynd ati i hyrwyddo a datblygu'r iaith Gymraeg a dwyieithrwydd, yn ogystal â'r ddarpariaeth Gymraeg Iaith Gyntaf a darpariaeth y 
Cwrs Carlam. Er pwysiced gweithgareddau a mentrau anffurfiol o ddydd i ddydd, dadleuir bod ffurfioli'r hyn a wneir i hyrwyddo'r Gymraeg a dwyieithrwydd yn feunyddiol drwy bolisi nid yn unig yn dangos ymrwymiad cadarn yr ysgol i ddatblygu'r ethos dwyieithog, ond hefyd yn sicrhau mai'r ysgol gyfan sy'n atebol am ddatblygu'r ethos dwyieithog ac nid Adran y Gymraeg yn unig. Wrth weithredu polisi ar ddwyieithrwydd, caiff pwysigrwydd yr iaith Gymraeg ei ddatgan yn ffurfiol ac yn swyddogol gan yr ysgol. Dadleuir y byddai aelodau'r ysgol, sef ei staff, ei disgyblion a'u rhieni, yn fwy tebygol o gydnabod a gwerthfawrogi dwyieithrwydd fel rhan annatod o fywyd ysgol, pe bai polisi ffurfiol yn ei le i bwysleisio hyn. Heb bolisi ffurfiol yn ei le i bwysleisio pwysigrwydd yr iaith Gymraeg a dwyieithrwydd, y perygl yw na fydd aelodau'r ysgol yn cydweithio ar lefel anffurfiol er mwyn datblygu'r Gymraeg fel iaith bob dydd ar draws yr ysgol.

Yn ogystal â'r wybodaeth a gasglwyd gan y ddwy ysgol ar ffurf yr holiadur a'r cyfweliad ynglŷn ag ethos dwyieithog y ddwy ysgol, arsylwyd ethos yr ysgolion trwy ddefnyddio amryw o ddulliau o gasglu data. Er na allai'r aelodau o staff rheng flaen yn y ddwy ysgol y siaradwyd â nhw yn ystod yr ymweliad gynnig gwasanaeth dwyieithog, nid oes modd casglu a oedd pob aelod o'r staff rheng flaen yn medru cwrdd, cyfarch a chynnal sgwrs yn Gymraeg. Rhaid nodi hefyd bod defnyddio'r Gymraeg a'r Saesneg yn gyfartal wrth ateb y ffôn ac yn neges y peiriant ateb, fel yn achos Ysgol X, yn sicrhau y caiff dwyieithrwydd ei normaleiddio i ryw raddau. Dadleuir bod defnyddio'r Gymraeg yn y ffordd hon, fel iaith bob dydd yn yr ysgol, yn fan cychwyn pwysig iawn i ysgolion cyfrwng Saesneg mewn ardaloedd cymharol ddi-Gymraeg os ydynt am hyrwyddo eu hethos dwyieithog mewn ffordd swyddogol.

Gellid dadlau bod gweithredu arwyddion dwyieithog yn sicrhau y caiff dwyieithrwydd ei hyrwyddo'n weledol trwy'r ysgol gyfan. Mae Colegau Cymru (2010, t. 5) wedi amlygu pwysigrwydd gweithredu arwyddion dwyieithog i ddatblygu ethos dwyieithog yng nghyd-destun colegau, wrth fynnu "[d]ylai pob arwydd yn y coleg fod yn ddwyieithog." Ymddengys Ysgol X yn enghraifft dda o ysgol sy'n hyrwyddo dwyieithrwydd yn weithredol ar ei harwyddion swyddogol. Er hynny, cydnabu Pennaeth y Gymraeg ei hun y gellir gwneud mwy i gysoni'r defnydd cyson o'r ddwy iaith ar arwyddion answyddogol, megis baneri a phosteri, ac ar arddangosfeydd ar draws yr ysgol. Mae'n amlwg fod cyfle i ysgolion cyfrwng Saesneg gysoni'r defnydd o'r ddwy iaith ar eu harwyddion swyddogol ac 
answyddogol, os ydynt am sicrhau bod cymaint â phosibl o'u harwyddion yn ymddangos yn y naill iaith a'r llall. Gallai gosod ymrwymiad clir i ddatblygu arwyddion dwyieithog ar ffurf nod strategol mewn polisi fod yn ffordd i ysgolion gyflawni hyn yn y dyfodol.

Fel adnodd sydd yn marchnata'r coleg, neu ysgol yng nghyd-destun y gwaith hwn, dylai'r wefan esbonio sut y caiff ethos dwyieithog ei hyrwyddo yn weithredol (Colegau Cymru, 2010, t. 5). Fodd bynnag, canfuwyd nad yw gwefannau'r naill ysgol na'r llall yn esbonio sut y caiff dwyieithrwydd eu hyrwyddo ar lefel anffurfiol. Nodir hefyd na cheir fersiwn dwyieithog o'r ddwy wefan ac felly cyflwynir holl gynnwys y gwefannau yn Saesneg yn unig. Fel un o'r prif adnoddau marchnata sydd ar gael i ysgolion, dadleuir bod gwefan ysgol yn hynod o bwysig o safbwynt hyrwyddo ethos. Yn ogystal â disgrifio'r hyn a wneir o ddydd i ddydd yn yr ysgol i hyrwyddo dwyieithrwydd, hynny yw, ethos anffurfiol yr ysgol, gall gwefan hyrwyddo ei hethos ffurfiol, er enghraifft trwy gyflwyno polisïau'r ysgol a datganiadau swyddogol gan y Prifathro. Gall gwefan ysgol fod yn adnodd defnyddiol sy'n dangos sut mae ethos anffurfiol yr ysgol yn cael ei wireddu yn ethos a pholisi ffurfiol yr ysgol. Ymddengys o'r canfyddiadau fod cyfle i gryfhau ethos dwyieithog ysgolion, a hynny trwy ddatblygu gwefannau ysgol dwyieithog a marchnata'n well yr hyn y mae aelodau ysgol yn ei wneud i hyrwyddo'r iaith Gymraeg a dwyieithrwydd ar lefel anffurfiol, megis gweithgareddau allgyrsiol.

Dylai dogfennaeth ffurfiol “fod ar gael yn ddwyieithog” gyda'r fersiwn Cymraeg “fod mor hawdd cael gafael arno â'r fersiwn Saesneg”, yn ôl Colegau Cymru (2010, t. 5). Fel gwefan ysgol, dylai dogfennaeth ysgol hefyd esbonio sut y caiff yr ethos dwyieithog ei hyrwyddo. Er nad yw cynnwys dogfennaeth ffurfiol Ysgol X ar gael yn y naill iaith a'r llall, neu'n ddwyieithog, gellid dadlau bod y defnydd helaeth o'r Gymraeg yn y ddogfennaeth Saesneg, yn benodol y prosbectysau, yn atgyfnerthu ethos dwyieithog yr ysgol gan ei fod yn normaleiddio'r iaith Gymraeg. Er hynny, teimlir y dylai cysoni'r defnydd o'r ddwy iaith mewn dogfennaeth swyddogol fod yn ystyriaeth bwysig i Ysgol X wrth symud ymlaen i ddatblygu ethos dwyieithog yr ysgol yn y tymor hir. Mae cyfle i ysgolion cyfrwng Saesneg eraill sicrhau bod dwyieithrwydd yn weledol mewn dogfennaeth swyddogol, a hynny trwy wneud defnydd mwy o'r Gymraeg. 
Ar sail canfyddiadau'r gwaith maes, dadleuir bod ymagwedd Ysgol X at ddatblygu ei hethos dwyieithog yn fwy effeithiol a chyson o'i chymharu ag ymagwedd Ysgol Y. Mae canfyddiadau'r gwaith maes yn eu cyfanrwydd hefyd yn cadarnhau'r hyn y sylwyd arno gan Y Pwyllgor Mentor a Dysgu (2010), sef bod Ysgol X yn fodel o arfer da ar gyfer datblygu ethos dwyieithog. Caiff ethos dwyieithog yr ysgol ei hyrwyddo nid yn unig trwy arferion a gweithgareddau aelodau'r ysgol bob dydd, ond hefyd drwy hyrwyddo dwyieithrwydd yn ffurfiol. Dadleuir bod ymagwedd Ysgol X at hyrwyddo'r iaith Gymraeg a dwyieithrwydd yn fwy cyflawn am fod ei hethos yn cwmpasu agweddau ffurfiol, megis gweithredu polisi ffurfiol ar ddwyieithrwydd a threfnu hyfforddiant allanol i ddatblygu sgiliau dwyieithog staff, ac agweddau anffurfiol, megis gweithgareddau allgyrsiol. Fel y mae Donelly (2000) yn ei gydnabod, mae sicrhau bod ethos anffurfiol aelodau'r ysgol yn cyd-fynd ag ethos ffurfiol yr ysgol yn allweddol er mwyn datblygu ethos cyson ar gyfer yr ysgol gyfan. Yn syml, ymddengys y caiff yr hyn a wna aelodau Ysgol X wrth hyrwyddo'r Gymraeg a dwyieithrwydd o ddydd i ddydd, sef ei hethos anffurfiol, ei wireddu i raddau helaeth yn yr hyn a fynegir yn ei pholisi ffurfiol, sef ei hethos ffurfiol.

\subsubsection{Hyfforddiant staff yr ysgolion}

Canfuwyd yn Pennod 1 mai un o'r prif ffactorau sy'n rhwystro ysgolion cyfrwng Saesneg rhag cyflwyno'r Gymraeg yn llwyddiannus yn y sector cyfrwng Saesneg yw'r diffygion o ran darparu'r hyfforddiant priodol i athrawon yn y sector.

Cadarnhawyd yn yr holiadur a'r cyfweliad dilynol nad yw Ysgol Y yn cynnig hyfforddiant ffurfiol, boed hynny'n allanol neu'n fewnol, i ddatblygu methodoleg addysgu dwyieithog eu staff. Y rheswm mwyaf amlwg am hyn yw nad yw'r ysgol yn cynnig, neu'n ystyried cynnig, darpariaeth cyfrwng Cymraeg neu ddwyieithog. Er bod nifer o athrawon pynciol yn yr ysgol a allai addysgu Cymraeg fel pwnc hyd at CA3, teimlai Pennaeth y Gymraeg na fyddai ganddynt yr hyder na'r sgiliau er mwyn cyflwyno eu pynciau nhw eu hunain trwy gyfrwng y Gymraeg. Priodolodd Pennaeth y Gymraeg gyfyngiadau ariannol ac amser yn benodol i ddiffyg hyfforddiant ffurfiol yn yr ysgol. Oherwydd hyn, ymddengys nad oes gan Ysgol Y y capasiti ar hyn o bryd i allu cynnig elfennau o'r cwricwlwm trwy gyfrwng y Gymraeg neu'n ddwyieithog. 
Yn Ysgol X, cadarnhaodd Pennaeth y Gymraeg nad oes hyfforddiant allanol ar gael i ddatblygu methodoleg addysgu dwyieithog eu staff. Fodd bynnag, cadarnhaodd fod nifer o'r staff yn brofiadol wrth addysgu'n ddwyieithog ac mae'r athrawon hyn yn hyfforddi athrawon eraill sy'n ymuno â thîm addysgu'r Cwrs Carlam. Caiff yr hyfforddiant mewnol hwn ei gyflwyno ar ffurf gwersi enghreifftiol, lle mae darpar athrawon y Cwrs Carlam yn arsylwi'r athrawon profiadol yn addysgu eu pynciau drwy gyfrwng y ddwy iaith. Yn ôl Pennaeth y Gymraeg yn y cyfweliad, mae addysgu dwyieithog effeithiol yn dod o brofiad yn bennaf ac nid oes methodoleg/au penodol y mae'r athrawon yn ei defnyddio wrth gyflwyno gwersi dwyieithog. Fodd bynnag, er pwysiced arsylwi gwersi dwyieithog, hanfodol yw sicrhau y caiff athrawon pynciol, yn ogystal ag athrawon Cymraeg, y wybodaeth ddiweddaraf ynghylch methodoleg addysgu dwyieithog a sut y maent yn gallu seilio eu harferion addysgu ar y wybodaeth hon. Mae'n bwysig y caiff athrawon y sector cyfrwng Saesneg y cyfle i fynychu cyrsiau hyfforddi ffurfiol sy'n seiliedig ar ganfyddiadau'r ymchwil ddiweddaraf, yn ogystal â chael cyfle i dderbyn hyfforddiant ymarferol, fel y mae Ysgol X yn ei gynnig. Ymddengys fod Ysgol X mewn sefyllfa gryfach o lawer o'i chymharu ag Ysgol Y, am ei bod hi'n gallu manteisio ar ddefnyddio ei staff profiadol er mwyn cynnig hyfforddiant addysgu dwyieithog i'w hathrawon eraill. Er hynny, nodir na ellir gwerthuso effeithiolrwydd yr hyfforddiant y mae Ysgol X yn ei gynnig, na'i ddeilliannau, yn yr ymchwil hon. Teimlir hefyd fod angen hyfforddiant ffurfiol ar athrawon Ysgol X er mwyn cyd-fynd â'r hyfforddiant mewnol anffurfiol y maent yn ei gael trwy'r ysgol.

Fel y canfuwyd yn Pennod 1, mae bwlch yn yr hyfforddiant y mae'r Cynllun Sabothol yn ei ddarparu ar hyn o bryd o ran datblygu methodoleg addysgu dwyieithog athrawon pynciol yn y sector cyfrwng Saesneg. Heb sicrhau bod hyfforddiant iaith Gymraeg a methodoleg addysgu dwyieithog yn eu lle er mwyn cynyddu nifer yr athrawon yng ngweithlu'r sector cyfrwng Saesneg sydd yn medru defnyddio'r iaith yn achlysurol ac addysgu'n ddwyieithog, ymddengys na fydd yn bosibl efelychu'r ddarpariaeth ddwyieithog y mae Ysgol X yn ei chynnig ar raddfa genedlaethol.

O ran datblygu sgiliau dwyieithog cyffredinol, cadarnhawyd nad yw Ysgol Y yn cynnig hyfforddiant i'w staff ddatblygu eu sgiliau dwyieithog yn y gweithle. Fodd bynnag, oherwydd y cyfyngiadau ariannol a wynebir gan Ysgol Y, ac Adran y 
Gymraeg yn benodol, ymddengys nad yw cynnig hyfforddiant allanol o'r fath yn bosibl ar hyn o bryd heb sicrhau cyllid. Yn Ysgol X, cadarnhawyd ei bod yn cynnig hyfforddiant ffurfiol allanol i'w holl staff. Yn wahanol i Adran y Gymraeg yn Ysgol Y felly, mae Ysgol X mewn sefyllfa lle mae hi'n gallu ariannu cwrs hyfforddiant allanol. Er hynny, cydnabu Pennaeth y Gymraeg fod arian yn un o'r prif rwystrau y mae'r ysgol yn eu hwynebu a bod cost yr hyfforddiant y mae'r ysgol yn ei gynnig yn sylweddol.

Yn ogystal â'r her 'arian', rhaid tynnu sylw at her arall a ddeilliodd o'r cyfweliadau dilynol yn y naill ysgol a'r 1lall, sef 'amser'. Yn Ysgol Y, nododd Pennaeth y Gymraeg ei bod yn anodd neilltuo'r amser i drefnu a chynnal hyfforddiant yn ystod y diwrnod ysgol, yn bennaf oherwydd am mai ond dau o'r staff addysgu sy'n gweithio'n llawn amser yn Adran y Gymraeg, gan gynnwys y Pennaeth ei hun. Er hynny, cyfaddefodd ei bod yn agored i'r posibilrwydd o drefnu hyfforddiant mewnol o'r fath ar ôl ysgol, fel y gwna Ysgol X. Yn ôl Pennaeth y Gymraeg yn Ysgol X fodd bynnag, gall trefnu hyfforddiant ar ôl ysgol hyd yn oed fod yn drafferthus oherwydd ymroddiadau a chyfrifoldebau eraill y staff. Er bod sicrhau cyllid yn ystyriaeth hollbwysig wrth drefnu hyfforddiant ffurfiol, ymddengys felly fod cynllunio'r hyfforddiant o amgylch ymroddiadau a chyfrifoldebau'r staff yn ystyriaeth arall ar gyfer ysgolion. Dyma bryder sydd eisoes wedi'i nodi yn astudiaeth Lewis (2010a) o'r sector cyfrwng Saesneg. Yn ei astudiaeth, canfuwyd bod 66\% o'r athrawon CAI a holwyd o'r farn nad ydynt yn cael digon o gyfleoedd i fynychu cyrsiau Datblygiad Proffesiynol Parhaus er mwyn eu cefnogi i godi safonau CAI (W.G. Lewis, 2010a, '6.2 Secondary'). Mae'n amlwg felly fod yn rhaid i ysgolion gynllunio'n ofalus er mwyn caniatáu i'w staff allu manteisio ar yr holl gyrsiau hyfforddi sydd ar gael iddynt.

Cyn cloi'r drafodaeth ar hyfforddiant addysgu dwyieithog, rhaid tynnu sylw yma at bolisi staffio Ysgol X. Fel y nodwyd eisoes (gweler 7.2.2.3. Darpariaeth ddwyieithog: Y Cwrs Carlam), y pwnc sy'n dod yn gyntaf yn y dosbarth carlam, ac nid y ddarpariaeth Gymraeg ychwanegol. Diben y ddarpariaeth Gymraeg ychwanegol hon a geir yn y dosbarth carlam felly yw cyfoethogi'r addysg. Fel y nododd Pennaeth y Gymraeg, ni chaiff athrawon eu cyflogi ar sail eu gallu i addysgu drwy gyfrwng y naill iaith a'r llall. O ganlyniad i hyn, nodir bod cyfyng-gyngor yn codi. Er bod Ysgol X am ddatblygu ei darpariaeth Gymraeg, mae'r pwyslais ar 
sicrhau'r 'addysg orau bosibl' yn golygu y daw'r Saesneg yn gyntaf fel cyfrwng addysgu am mai dyma iaith gyntaf y rhan fwyaf o'r disgyblion. Er pwysiced yr hyfforddiant mewnol y mae Ysgol X yn ei gynnig felly, anodd yw rhagweld datblygiad darpariaeth Gymraeg yr ysgol yn y tymor hir heb bolisi staffio yn ei le i gydnabod addysgu dwyieithog fel un o'r blaenoriaethau wrth gyflogi athrawon. Bydd hyn yn hollbwysig os bydd Ysgol $\mathrm{X}$ am gynnal y pynciau a gyflwynir yn ddwyieithog ar hyn o bryd, ac yn bwysicach fyth os bydd yr ysgol am ehangu'r ddarpariaeth ddwyieithog hon i rannau eraill o'r cwricwlwm a charfannau eraill o ddisgyblion yn y dyfodol. Bydd sicrhau hir oes i'r Cwrs Carlam yn dibynnu i raddau helaeth ar allu'r ysgol i gynnal a chynyddu nifer y staff a all addysgu trwy gyfrwng y naill iaith a'r llall.

Ymddengys o'r canfyddiadau bod Ysgol X mewn sefyllfa gryfach o'i chymharu ag Ysgol Y o safbwynt ei darpariaeth ar gyfer datblygu sgiliau dwyieithog y staff. Gan fod yr hyfforddiant hwn ar gael i'r staff nad ydynt yn addysgu, yn ogystal â'r staff addysgu, mae'n bosibl i unrhyw aelod o'r staff fanteisio ar wella eu sgiliau dwyieithog ar gyfer y gweithle. Fodd bynnag, rhaid nodi nad yw'r staff yn gorfod mynychu'r hyfforddiant hwn. Y rhai sydd yn mynychu'r cwrs hyfforddi hwn yw'r staff sydd yn rhydd ar gyfer y diwrnodau pan drefnir yr hyfforddiant. Cadarnhaodd Pennaeth y Gymraeg yn y cyfweliad fod tua 20 i 25 o staff wedi dangos diddordeb yn y cwrs a gynhelir yn 2016/17. O ystyried bod nifer o'r staff naill ai'n siaradwyr rhugl yn y Gymraeg neu'n ddysgwyr sydd eisoes wedi mynychu'r cwrs o'r blaen, cytunir â Phennaeth y Gymraeg mai dyma nifer gadarnhaol o fynychwyr.

\subsubsection{Pontio rhwng y sector cynradd a'r sector uwchradd}

Er bod llawer o glystyrau o ysgolion cyfrwng Saesneg yn cydweithio'n effeithiol yn ystod y cyfnod pontio er mwyn cynllunio dilyniant ieithyddol disgyblion yn y Gymraeg o'r naill sector i'r llall, canfuwyd yn Pennod 1 nad yw hyn yn wir ymhob achos. Yn y cyfweliad â Phennaeth y Gymraeg yn Ysgol Y, cadarnhaodd ei bod yn cyfarfod ag ysgolion cynradd y clwstwr unwaith neu ddwywaith y tymor er mwyn cydweithio ar gynllunio'r Gymraeg. Fodd bynnag, teimlodd hi mai 'amser' yw'r prif rwystr rhag cyfarfod â'r ysgolion cynradd hyn yn fwy rheolaidd. Ar adeg casglu'r data, dim ond dau aelod o'r staff, gan gynnwys y Pennaeth ei hun, oedd yn gweithio'n llawn amser yn yr adran. Oherwydd diffyg staff felly, ymddengys nad 
oedd yn bosibl i Bennaeth yr adran drefnu ymweld â'r ysgolion bwydo yn fwy rheolaidd heb amharu ar yr addysgu yn ei hadran ei hun. Daeth i'r amlwg fod Ysgol Y wedi cydweithio'n agos â'r ysgolion cynradd y llynedd pan gynhaliwyd cyfres o gyfarfodydd cymedroli ar gyfer CAI. Yn ystod y cyfarfodydd hyn, mynegodd nifer o ysgolion bwydo wrth Bennaeth y Gymraeg yn Ysgol Y fod eu staff yn ddihyder o ran pennu lefelau asesiad i'r Gymraeg a bod angen iddi fynd i mewn i'r ysgolion hynny er mwyn rhoi cymorth a chefnogaeth i'r athrawon. Sylwer yma fod y canfyddiad hwn yn ategu'r hyn a ddarganfuwyd yn Pennod 1, sef y diffyg hyder a'r arbenigedd ymysg athrawon cynradd wrth addysgu Cymraeg a'i defnyddio'n achlysurol.

Ar wahân i'r cyfarfodydd hyn rhwng Pennaeth y Gymraeg â'r ysgolion cynradd, esboniwyd bod menter wedi'i datblygu ar y cyd â'r ysgol gynradd agosaf. Er nad oedd yn amlwg a yw'r fenter yn cael ei threfnu yn rheolaidd o hyd, mae'n ymddangos y gallai'r fenter hon fod yn fanteisiol mewn sawl ffordd. Yn ogystal â chynorthwyo'r athrawon cynradd nad ydynt yn hyderus wrth addysgu Cymraeg, gallai'r fenter hon fod yn fanteisiol o safbwynt cysoni safonau Cymraeg y disgyblion cynradd cyn iddynt gyrraedd yr uwchradd. Mantais arall yw bod y fenter hon yn rhoi cyfle i ddisgyblion y Chweched Dosbarth ennill profiad o addysgu ac ymarfer eu Cymraeg nhw eu hunain.

Efallai mai un o'r agweddau mwyaf nodweddiadol ar y cynllun pontio sydd ar waith yn Ysgol X yw'r ddarpariaeth beripatetig y mae'r ysgol yn ei chynnig. Rôl yr athrawon peripatetig yw cydweithio ag ysgolion cynradd y clwstwr trwy:

- $\quad$ addysgu gwersi Cymraeg ym Mlynyddoedd 5 a 6, yn ogystal ag ambell wers ym Mlwyddyn 7;

- cynorthwyo athrawon cynradd i addysgu Cymraeg ym Mlynyddoedd 2 a 3 drwy greu adnoddau dysgu;

- rhoi lefelau asesiad a monitro cynnydd disgyblion yn y Gymraeg; a

- rhannu data ac adborth am ddisgyblion â'r ysgol uwchradd er mwyn hwyluso eu trosglwyddiad o'r naill sector i'r llall.

O ystyried y cyfrifoldebau uchod, mae'n amlwg fod gan yr athrawon peripatetig rôl ganolog yng nghynllun pontio Ysgol X. Nodir hefyd fod gwaith yr athrawon peripatetig hyn yn adlewyrchu'r un math o gymorth y mae Athrawon Bro yn ei gynnig i ysgolion cynradd cyfrwng Saesneg ar draws Cymru. Canfuwyd yn Pennod 1 
fod cyfran sylweddol o ysgolion cynradd cyfrwng Saesneg yn dal i ddibynnu i raddau helaeth ar gymorth yr Athrawon Bro. Darganfu Lewis (2010a, para. 9.1.) yn ei astudiaeth, er enghraifft, fod 96\% o'r prifathrawon ysgolion cynradd a holwyd yn credu na fyddai'n bosibl cyflwyno CAI heb gymorth yr Athrawon Bro. Mewn adroddiad ar ran LlC, sef Arolwg o'r gwasanaeth cymorth iaith Gymraeg $i$ ysgolion, mynegwyd bod "angen i Awdurdodau Lleol symud disgwyliadau ysgolion, fel eu bod yn cymryd cyfrifoldeb dros eu capasiti iaith eu hunain, yn hytrach na dibynnu ar Athrawon Bro i gau bylchau yn sgiliau ieithyddol eu staff" (Burrowes ac eraill, 2011, t. 86). Mae cynllun pontio Ysgol X yn ymddangos yn enghraifft o arfer da am fod y ddarpariaeth beripatetig yn caniatáu i'r ysgol ysgwyddo rhywfaint o'r cyfrifoldeb sydd ar yr ysgolion bwydo am ddilyniant ieithyddol disgyblion yn y Gymraeg yn ystod y Cyfnod Pontio. Rhaid pwysleisio hefyd fod y cynllun hwn yn fanteisiol nid yn unig i'r ysgolion cynradd yn y clwstwr, sydd angen arbenigedd addysgu CAI yr ysgol uwchradd, ond i'r ysgol uwchradd ei hun, sydd am gysoni a chodi safonau disgyblion yn y Gymraeg cyn iddynt ymuno â'r uwchradd ym Mlwyddyn 7.

Fodd bynnag, cyn cloi'r drafodaeth hon, rhaid tynnu sylw at gostau cyflogi athrawon o'r fath i'r ysgol uwchradd. Canfuwyd yn y gwaith maes mai Ysgol X sydd yn cyflogi'r athrawon hyn, ac er na ddatgelwyd union gost y cynllun i'r ymchwilydd, mae'n debyg bod cyflogi dwy athrawes beripatetig yn fuddsoddiad sylweddol ar ran yr ysgol. Mae'r canfyddiadau yn Ysgol Y hefyd yn awgrymu na allai'r ysgol hon gynnig darpariaeth bontio debyg i Ysgol X heb sicrhau cyllid i gyflogi athrawon peripatetig, oherwydd nad oes ganddi'r adnoddau staff na'r adnoddau cyllidol. Pe byddid yn efelychu'r cynllun pontio yn Ysgol X mewn clystyrau eraill o ysgolion ar draws Cymru, mae'n amlwg y byddai angen ymchwil bellach er mwyn gwerthuso deilliannau'r ddarpariaeth beripatetig, ac ystyried sut y gellid ariannu athrawon o'r fath ar raddfa genedlaethol.

Agwedd bwysig arall ar y cynllun pontio yn Ysgol X yw'r diwrnodau trochi allgyrsiol a drefnir i ddisgyblion Blwyddyn 7 sydd newydd drosglwyddo o'r ysgolion cynradd. Yn ogystal â bod yn rhan allweddol o nod y cynllun i wella safonau Cymraeg yn ystod y Cyfnod Pontio, nodir bod y diwrnodau allgyrsiol hyn yn cyfrannu at ddatblygu ethos dwyieithog yr ysgol yn fwy cyffredinol (gweler 7.2.3. Ethos dwyieithog). Yn ychwanegol at y diwrnodau allgyrsiol hyn, caiff diwrnod trochi ei drefnu ar gyfer y disgyblion CAI sy'n dod y tu allan i glwstwr Ysgol X. 
Mae'r diwrnod trochi hwn yn canolbwyntio ar gysoni safonau Cymraeg y disgyblion hyn â safonau'r disgyblion a ymunodd â'r ysgol o'r clwstwr, a fyddai wedi derbyn mewnbwn Cymraeg ychwanegol yr athrawon peripatetig.

Cesglir yma fod canfyddiadau'r gwaith maes yn cefnogi'r hyn a ganfuwyd am effeithiolrwydd cynllun pontio Ysgol X yn Pennod 5. Er y cydnebydd yr ymchwilydd fod modd ehangu darpariaeth y cynllun i ysgolion eraill yn nalgylch Ysgol X, fel yr awgrymodd Hopkins (2006), nid yw'n amlwg o'r canfyddiadau ymchwil a oes gan Ysgol X yr awydd a'r adnoddau i gyflogi athrawon peripatetig ychwanegol yn y dyfodol. Serch hynny, ymddengys cynllun pontio Ysgol X yn gynllun effeithiol sydd yn arwain y sector. Dyma gynllun y gallai Awdurdodau Lleol, a'u hysgolion, ar draws Cymru ei ystyried fel enghraifft o arfer da wrth iddynt wella trefniadau pontio'r Gymraeg rhwng y sector gynradd a'r sector uwchradd. 
Pennod 8: Canlyniadau ii: Y prawf llenwi bylchau, y prawf darllen Saesneg a'r data Prydau Ysgol am Ddim

\subsection{Y prawf llenwi bylchau}

\subsubsection{Dull}

Cynhaliwyd y prawf llenwi bylchau gyda 74 o ddisgyblion Cymraeg ail iaith ym Mlwyddyn 9 (13 - 14 oed) yn y ddwy ysgol gyfranogol. Fel y gwelir yn Tabl 7 isod, mae 46 o'r cyfranogwyr yn deillio o ddwy ffrwd wahanol yn Ysgol X, sef y brif ffrwd cyfrwng Saesneg (26 o gyfranogwyr, Dosbarth A) a'r ffrwd rannol ddwyieithog (20 o gyfranogwyr, Dosbarth B). Mae gweddill y cyfranogwyr yn deillio o Ysgol Y, sydd â ffrwd cyfrwng Saesneg yn unig (28 o gyfranogwyr, Dosbarth C).

\begin{tabular}{|l|l|l|}
\hline \multicolumn{1}{|c|}{ Dosbarth } & Amledd & Canran \\
\hline $\begin{array}{l}\text { Dosbarth A: } \\
\text { CAI Set 1 Ysgol X }\end{array}$ & 26 & 35.1 \\
Dosbarth B: & 20 & 27.0 \\
Cwrs Carlam Ysgol X & & \\
Dosbarth C: & 28 & 37.8 \\
CAI Set 1 Ysgol Y & 74 & 100.0 \\
Cyfanswm & 74 & \\
\hline
\end{tabular}

Tabl 7. Nifer y cyfranogwyr fesul dosbarth

\subsubsection{Dadansoddi sgoriau'r prawf}

\subsubsection{Gwasgariad sgoriau cyfanswm yr holl gyfranogwyr}

Yn Atodiad 11, gwelir histogram sy’n dangos amledd sgoriau cyfanswm yr holl gyfranogwyr fel canrannau. Ymddengys fod gwasgariad sgoriau cyfanswm y papurau yn weddol ganolog (cymedr $=39.69$; cyfeiliornad safonol o'r cymedr $=$ 2.28 ) ond nodir bod sgiwedd positif bychan (sgiwedd $=0.912$; cyfeiliornad safonol $=$ 0.279). Ymddengys fod cwrtosis o fewn yr amrediad gwallau (cwrtosis = -.087; cyfeiliornad safonol $=.552$ ). Er mwyn darganfod a yw cyflwr y data yn normal, 
gweithredwyd prawf Shapiro-Wilk. Dangosodd prawf Shapiro-Wilk fod gwasgariad y sgoriau yn gwyro'n sylweddol oddi wrth y normal ( $p=>0.01)$. Mae'r plot Q-Q yn Atodiad 11 hefyd yn cefnogi'r canfyddiad hwn.

\subsubsection{Gwasgariad sgoriau cyfanswm fesul dosbarth}

Er mwyn darganfod a yw gwasgariad sgoriau'r tri dosbarth ar wahân yn normal, lluniwyd histogramau a phlotiau Q-Q ar gyfer y tri dosbarth (gweler Atodiadau 12, 13, ac 14). Wrth astudio'r histogramau hyn, ymddengys nad yw sgoriau'r tri dosbarth yn gwyro'n sylweddol oddi wrth y normal disgwyliedig. Wrth ddadansoddi sgiwedd sgoriau'r tri dosbarth ar wahân yn Tabl 8 isod, sylwir bod sgiwedd positif bychan yn achos sgoriau Dosbarth A (sgiwedd = .721) ond bod gwasgariad sgoriau Dosbarth B a Dosbarth C yn fwy agos at y normal (sgiwedd Grŵp B = .180; sgiwedd Grŵp C = .181). Awgryma hyn fod disgyblion Dosbarth A ar gyfartaledd yn fwy tebygol o ennill sgôr yn hanner is gwasgariad sgoriau eu grŵp. Wrth arsylwi cwrtosis y tri dosbarth ar wahân, eto yn Tabl 8, nodir bod sgoriau Dosbarth B yn cael eu gwasgaru'n weddol gul (cwrtosis = .679) o'u cymharu â sgoriau Dosbarth A $($ cwrtosis $=-.518)$ a Dosbarth C (cwrtosis $=-.466)$ sydd yn cael eu gwasgaru'n fwy eang. Er mwyn cadarnhau cyflwr gwasgariad sgoriau'r tri dosbarth, gweithredwyd prawf Shapiro-Wilk ar gyfer pob un. Y tro hwn, dangosodd y prawf fod y sgoriau yn cael eu gwasgaru'n normal yn achos Dosbarth B ( $p=.163)$ a Dosbarth C $(p=.760)$. Yn achos Dosbarth A fodd bynnag, mae'r prawf yn awgrymu bod y sgoriau yn gwyro'n sylweddol oddi wrth y normal $(p=.032)$. Mae'r plotiau Q-Q yn Atodiadau 12,13 a 14 hefyd yn cefnogi'r canfyddiadau hyn. 


\begin{tabular}{|l|l|l|l|l|l|}
\hline \multicolumn{1}{|c|}{ Dosbarth } & Amrediad & Sgiwedd & $\begin{array}{c}\text { Cyfeiliornad } \\
\text { Safonol o'r } \\
\text { Sgiwedd }\end{array}$ & Cwrtosis & $\begin{array}{c}\text { Cyfeiliornad } \\
\text { Safonol o'r } \\
\text { Cwrtosis }\end{array}$ \\
\hline $\begin{array}{l}\text { Dosbarth A: } \\
\text { CAI Set 1 Ysgol X }\end{array}$ & $18.6-60.5$ & .721 & .456 & -.518 & .887 \\
$\begin{array}{l}\text { Dosbarth B: } \\
\text { Cwrs Carlam Ysgol X }\end{array}$ & $39.5-95.3$ & .180 & .512 & .679 & .992 \\
$\begin{array}{l}\text { Dosbarth C: } \\
\text { CAI Set 1 Ysgol Y }\end{array}$ & $14.0-41.9$ & .181 & .441 & -.466 & .858 \\
\hline
\end{tabular}

Tabl 8. Amrediad a gwasgariad sgoriau cyfanswm y tri dosbarth.

\subsubsection{Sgoriau cymedrig fesul dosbarth}

Fel y nodwyd eisoes (gweler 6.9.3.7. Ffurf y prawf) rhannwyd y prawf yn ddwy ran er mwyn ceisio creu un rhan haws ('Rhan A') ac un rhan anos ('Rhan B'). Fel y disgwylir felly, mae sgôr gymedrig Rhan A yr holl gyfranogwyr $($ cymedr $=48.5)$ ychydig yn uwch o'i chymharu â sgôr gymedrig Rhan B (cymedr = 35.5). Sylwir yn Tabl 9 isod fod hyn yn wir hefyd yn achos pob dosbarth, wrth ddadansoddi sgoriau cymedrig Rhan A a B fesul dosbarth. Sgôr gyfanswm gymedrig yr holl gyfranogwyr oedd $39.7 \%$. Y sgôr gyfanswm uchafswm a gofnodwyd oedd $95.3 \%$ (a enillwyd gan Rif Papur 45) a'r sgôr gyfanswm isafswm a gofnodwyd oedd 14.0\% (a enillwyd gan Rif Papurau 50 a 73). 


\begin{tabular}{|c|c|c|c|c|}
\hline & Dosbarth & $\begin{array}{c}\text { Sgôr Rhan A } \\
(\%)\end{array}$ & $\begin{array}{c}\text { Sgôr Rhan B } \\
(\%)\end{array}$ & $\begin{array}{c}\text { Sgôr Gyfanswm } \\
(\%)\end{array}$ \\
\hline Dosbarth A: & Cymedr & 43.1 & 28.4 & 33.2 \\
\hline CAI Set 1 & Gwyriad Safonol & 17.8 & 10.5 & 12.1 \\
\hline Ysgol X & Cyfeiliornad Safonol o'r Cymedr & 3.5 & 2.1 & 2.4 \\
\hline Dosbarth B: & Cymedr & 72.9 & 62.6 & 65.9 \\
\hline Cwrs Carlam & Gwyriad Safonol & 13.2 & 16.4 & 13.8 \\
\hline Ysgol X & Cyfeiliornad Safonol o'r Cymedr & 3.0 & 3.7 & 3.1 \\
\hline Dosbarth C: & Cymedr & 36.0 & 22.7 & 27.0 \\
\hline CAI Set 1 & Gwyriad Safonol & 9.2 & 8.5 & 7.2 \\
\hline Ysgol Y & Cyfeiliornad Safonol o'r Cymedr & 1.7 & 1.6 & 1.4 \\
\hline \multirow[t]{3}{*}{ Cyfanswm } & Cymedr & 48.5 & 35.5 & 39.7 \\
\hline & Gwyriad Safonol & 20.5 & 20.4 & 19.6 \\
\hline & Cyfeiliornad Safonol o'r Cymedr & 2.4 & 2.4 & 2.3 \\
\hline
\end{tabular}

Tabl 9. Sgoriau cymedrig Rhan A, Rhan B a chyfanswm fesul dosbarth

\subsubsection{Dadansoddi geiriau cynnwys a geiriau swyddogaethol}

Dadansoddwyd sgoriau cyfanswm y tri dosbarth ymhellach wrth eu rhannu fesul geiriau cynnwys a geiriau swyddogaethol. Yn Tabl 10, gwelir sgoriau cymedrig y tri dosbarth yn achos y geiriau cynnwys a'r geiriau swyddogaethol ar wahân. Nodir bod sgoriau geiriau cynnwys a geiriau swyddogaethol yn amrywio'n fawr, yn arbennig yn achos Dosbarth A (amrediad Geiriau Cynnwys $=42.3$; amrediad Geiriau Swyddogaethol $=47.0)$ a Dosbarth B (amrediad Geiriau Cynnwys $=50$; amrediad Geiriau Swyddogaethol =70.6). Ymddengys o Dabl 9 fod pob un o'r tri dosbarth wedi perfformio'n well ar gyfartaledd gydag eitemau sy'n cymell gair cynnwys nag eitemau sy'n cymell gair swyddogaethol. Dyma ganfyddiad sy'n groes i ganfyddiad Tremblay a Garrison (2010). Yn eu prawf cyfannu hwy, canfu Tremblay a Garrison mai eitemau sy'n cymell geiriau cynnwys oedd yr eitemau anhawsaf ar gyfer pob grŵp yn eu hastudiaeth. 


\begin{tabular}{|c|c|c|c|c|c|c|}
\hline \multirow[b]{2}{*}{ Dosbarth } & \multicolumn{3}{|c|}{ Geiriau Cynnwys $(k=26)$} & \multicolumn{3}{|c|}{ Geiriau Swyddogaethol $(k=17)$} \\
\hline & Cymedr (\%) & $\begin{array}{c}\text { Cyfeiliornad } \\
\text { Safonol o'r } \\
\text { Cymedr }\end{array}$ & Amrediad & Cymedr $(\%)$ & $\begin{array}{c}\text { Cyfeiliornad } \\
\text { Safonol o'r } \\
\text { Cymedr }\end{array}$ & Amrediad \\
\hline Dosbarth A: CAI Set 1 & & & & & & \\
\hline Ysgol X $(n=26)$ & 34.8 & 2.3 & $19.2-61.5$ & 30.8 & 2.8 & $11.8-58.8$ \\
\hline Dosbarth B: Cwrs & & & & & & \\
\hline Carlam Ysgol X $(n=20)$ & 66.7 & 2.9 & $46.2-96.2$ & 64.7 & 3.9 & $23.5-94.1$ \\
\hline $\begin{array}{l}\text { Dosbarth C: CAI Set } 1 \\
\text { Ysgol Y }(n=28)\end{array}$ & 29.5 & 1.5 & $15.4-46.2$ & 23.1 & 1.6 & $5.9-41.2$ \\
\hline
\end{tabular}

Tabl 10. Sgoriau cymedrig y tri dosbarth ar gyfer Geiriau Cynnwys a Geiriau Swyddogaethol.

\subsubsection{Dadansoddi'r eitemau fesul mathau o air}

Er mwyn dadansoddi ymhellach sgoriau'r tri dosbarth o ran geiriau cynnwys a geiriau swyddogaethol, rhannwyd sgoriau geiriau cynnwys a geiriau swyddogaethol fesul mathau o air ar gyfer y tri grŵp (enwau, ansoddeiriau, berfau, berfenwau, adferfau, rhagenwau, arddodiaid, cysyllteiriau, a'r fannod). Gwelir yn Tabl 11 a Tabl 12 sgoriau'r tri dosbarth fesul mathau o air. Yn achos y geiriau cynnwys, sylwir mai'r eitemau lle cafodd ansoddair ei ddileu yw'r eitemau a gafodd eu hateb yn gywir fwyaf (cymedr = 60.8) a'r eitemau lle cafodd enw ei ddileu yw'r eitemau a gafodd eu hateb yn gywir leiaf. Yn achos y geiriau swyddogaethol, gwelir mai'r eitemau lle cafodd cysylltair ei ddileu yw'r eitemau a gafodd eu hateb yn gywir fwyaf (cymedr $=46.8)$ a'r eitemau lle cafodd y fannod ei dileu yw'r eitemau a gafodd eu hateb yn gywir leiaf $($ cymedr $=16.9)$. 


\begin{tabular}{|c|c|c|c|c|c|c|}
\hline Dosbartl & & $\begin{array}{c}\text { Sgôr Enwau } \\
(k=5)\end{array}$ & $\begin{array}{c}\text { Sgôr } \\
\text { Ansoddeiriau } \\
(k=5)\end{array}$ & $\begin{array}{c}\text { Sgôr Berfau } \\
\quad(k=6)\end{array}$ & $\begin{array}{c}\text { Sgôr } \\
\text { Berfenwau } \\
(k=5)\end{array}$ & $\begin{array}{c}\text { Sgôr } \\
\text { Adferfau }(k= \\
5)\end{array}$ \\
\hline $\begin{array}{l}\text { Dosbarth A: } \\
\text { CAI Set } 1 \text { Ysgol X }\end{array}$ & $\begin{array}{l}\text { Cymedr } \\
\text { Amrediad }\end{array}$ & $\begin{array}{l}7.7 \\
40.0\end{array}$ & $\begin{array}{l}57.7 \\
80.0\end{array}$ & $\begin{array}{l}28.2 \\
66.7\end{array}$ & $\begin{array}{l}41.5 \\
80.0\end{array}$ & $\begin{array}{l}40.0 \\
80.0\end{array}$ \\
\hline $\begin{array}{l}\text { Dosbarth B: } \\
\text { Cwrs Carlam } \\
\text { Ysgol X }\end{array}$ & $\begin{array}{l}\text { Cymedr } \\
\text { Amrediad }\end{array}$ & $\begin{array}{l}73.0 \\
60.0\end{array}$ & $\begin{array}{l}70.0 \\
60.0\end{array}$ & $\begin{array}{l}55.0 \\
66.7\end{array}$ & $\begin{array}{l}64.0 \\
60.0\end{array}$ & $\begin{array}{l}74.0 \\
60.0\end{array}$ \\
\hline $\begin{array}{l}\text { Dosbarth C: } \\
\text { CAI Set } 1 \text { Ysgol Y }\end{array}$ & $\begin{array}{l}\text { Cymedr } \\
\text { Amrediad }\end{array}$ & $\begin{array}{l}4.3 \\
40.0\end{array}$ & $\begin{array}{l}57.1 \\
80.0\end{array}$ & $\begin{array}{l}14.3 \\
33.3\end{array}$ & $\begin{array}{l}32.9 \\
80.0\end{array}$ & $\begin{array}{l}42.1 \\
80.0\end{array}$ \\
\hline Prawf cyfan & $\begin{array}{l}\text { Cymedr } \\
\text { Amrediad }\end{array}$ & $\begin{array}{l}24.1 \\
100.0\end{array}$ & $\begin{array}{l}60.8 \\
80.0\end{array}$ & $\begin{array}{l}30.2 \\
83.3\end{array}$ & $\begin{array}{l}44.3 \\
100.0\end{array}$ & $\begin{array}{l}50.0 \\
100.0\end{array}$ \\
\hline
\end{tabular}

Tabl 11. Sgoriau cymedrig geiriau cynnwys y tri dosbarth fesul mathau o air.

\begin{tabular}{|c|c|c|c|c|c|}
\hline Dosbartl & & $\begin{array}{c}\text { Sgôr } \\
\text { Rhagenwau } \\
(k=5)\end{array}$ & $\begin{array}{c}\text { Sgôr } \\
\text { Arddodiaid } \\
(k=5)\end{array}$ & $\begin{array}{c}\text { Sgôr } \\
\text { Cysyllteiriau } \\
(k=5)\end{array}$ & $\begin{array}{c}\text { Sgôr y Fannod } \\
\qquad(k=2)\end{array}$ \\
\hline $\begin{array}{l}\text { Dosbarth A: } \\
\text { CAI Set } 1 \text { Ysgol X }\end{array}$ & $\begin{array}{l}\text { Cymedr } \\
\text { Amrediad }\end{array}$ & $\begin{array}{l}36.9 \\
80.0\end{array}$ & $\begin{array}{l}20.8 \\
80.0\end{array}$ & $\begin{array}{l}44.6 \\
80.0\end{array}$ & $\begin{array}{l}5.8 \\
50.0\end{array}$ \\
\hline $\begin{array}{l}\text { Dosbarth B: } \\
\text { Cwrs Carlam Ysgol X }\end{array}$ & $\begin{array}{l}\text { Cymedr } \\
\text { Amrediad }\end{array}$ & $\begin{array}{l}72.0 \\
40.0\end{array}$ & $\begin{array}{l}55.0 \\
100.0\end{array}$ & $\begin{array}{l}74.0 \\
80.0\end{array}$ & $\begin{array}{l}47.5 \\
100.0\end{array}$ \\
\hline $\begin{array}{l}\text { Dosbarth C: } \\
\text { CAI Set } 1 \text { Ysgol Y }\end{array}$ & $\begin{array}{l}\text { Cymedr } \\
\text { Amrediad }\end{array}$ & $\begin{array}{l}36.4 \\
80.0\end{array}$ & $\begin{array}{l}10.7 \\
40.0\end{array}$ & $\begin{array}{l}29.3 \\
60.0\end{array}$ & $\begin{array}{l}5.4 \\
50.0\end{array}$ \\
\hline Prawf cyfan & $\begin{array}{l}\text { Cymedr } \\
\text { Amrediad }\end{array}$ & $\begin{array}{l}46.2 \\
100.0\end{array}$ & $\begin{array}{l}26.2 \\
100.0\end{array}$ & $\begin{array}{l}46.8 \\
100.0\end{array}$ & $\begin{array}{l}16.9 \\
100.0\end{array}$ \\
\hline
\end{tabular}

Tabl 12. Sgoriau cymedrig geiriau swyddogaethol y tri dosbarth fesul mathau o air. 


\subsection{Enwau}

Rhannwyd yr eitemau sy'n cymell enwau ac archwiliwyd yr eitemau hyn yn fanylach er mwyn canfod unrhyw nodweddion pwysig. Gwelir yn Tabl 13 isod ddadansoddiad o'r eitemau hyn. Yn achos eitem 1, nodwyd sawl enghraifft lle cynnig y cyfranogwyr y ddau ddosbarth rheoli 'hwyl' neu 'pryd' er mwyn cyfannu'r frawddeg fel a ganlyn:

1. Bob wythnos, mae llawer o hwyl yn mynd i'r clwb.

Ymddengys y fath atebion nad yw'r cyfranogwr wedi darllen a deall y frawddeg yn ei chyfanrwydd, ond mae'n deall y gall yr enwau 'hwyl' ddod ar ôl 'llawer o' er mwyn creu'r frawddeg 'mae llawer o hwyl'. Gwelir yr un fath o atebion ar gyfer eitem 34 hefyd, lle mae'n bosibl fod y cyfranogwyr yn cadw at batrwm cyfarwydd:

34. Gwelais i lawer o hwyl - ond dim tad!

Nodir mai dau gyfranogwr yn unig sy'n deillo o Ddosbarth $\mathrm{C}$ ac sydd wedi ateb eitem 34 yn gywir. Enwau eraill o ddiddordeb yw'r rheiny yn eitemau 32, 36 ac i raddau llai, 15. Yn achos eitemau 32 a 36, nodir nad oes un cyfranogwr yn Dosbarth A na Dosbarth C sydd wedi ateb yr eitemau hyn yn gywir. Wrth archwilio atebion yr eitemau hyn, ymddengys nad yw'r rhan fwyaf o'r cyfranogwyr yn gallu defnyddio'r wybodaeth o fewn y testun er mwyn llenwi'r eitemau'n gywir a nodir bod nifer o enghreifftiau lle nad yw'r cyfranogwr yn cynnig unrhyw ateb ar gyfer yr eitemau hyn. Gellir casglu felly fod eitemau sy'n cymell enwau yn arbennig o anodd i'r cyfranogwyr sydd â lefelau is o hyfedredd ieithyddol cyffredinol. 


\begin{tabular}{|c|c|c|c|c|c|c|}
\hline \multicolumn{2}{|c|}{ Dosbarth } & $\begin{array}{l}\text { Eitem 1: } \\
\text { 'blant' }\end{array}$ & $\begin{array}{l}\text { Eitem 15: } \\
\text { 'gêm' }\end{array}$ & $\begin{array}{l}\text { Eitem 32: } \\
\text { 'clwb' }\end{array}$ & $\begin{array}{l}\text { Eitem 34: } \\
\text { 'bobl' }\end{array}$ & $\begin{array}{l}\text { Eitem 36: } \\
\text { 'esgidiau' }\end{array}$ \\
\hline \multirow[t]{2}{*}{$\begin{array}{l}\text { Dosbarth A: } \\
\text { CAI Set } 1 \text { Ysgol X }\end{array}$} & $\begin{array}{l}\text { Nifer } \\
\text { \% o'r dosbarth }\end{array}$ & $\begin{array}{l}4 \\
15.4\end{array}$ & $\begin{array}{l}6 \\
23.1\end{array}$ & $\begin{array}{l}0 \\
0\end{array}$ & $\begin{array}{l}0 \\
0\end{array}$ & $\begin{array}{l}0 \\
0\end{array}$ \\
\hline & \% o'r cyfanswm & 5.4 & 8.1 & 0 & 0 & 0 \\
\hline \multirow{2}{*}{$\begin{array}{l}\text { Dosbarth B: } \\
\text { Cwrs Carlam } \\
\text { Ysgol X }\end{array}$} & $\begin{array}{l}\text { Nifer } \\
\text { \% o'r dosbarth }\end{array}$ & $\begin{array}{l}20 \\
100.0\end{array}$ & $\begin{array}{l}17 \\
85.0\end{array}$ & $\begin{array}{l}9 \\
45.0\end{array}$ & $\begin{array}{l}15 \\
75.0\end{array}$ & $\begin{array}{l}12 \\
60.0\end{array}$ \\
\hline & \% o'r cyfanswm & 27.0 & 23.0 & 12.2 & 20.3 & 16.2 \\
\hline \multirow[t]{2}{*}{$\begin{array}{l}\text { Dosbarth C: } \\
\text { CAI Set } 1 \text { Ysgol Y }\end{array}$} & $\begin{array}{l}\text { Nifer } \\
\text { \% o'r dosbarth }\end{array}$ & $\begin{array}{l}3 \\
10.7\end{array}$ & $\begin{array}{l}1 \\
3.6\end{array}$ & $\begin{array}{l}0 \\
0\end{array}$ & $\begin{array}{l}2 \\
10.0\end{array}$ & $\begin{array}{l}0 \\
0\end{array}$ \\
\hline & \% o'r cyfanswm & 4.1 & 1.4 & 0 & 2.7 & 0 \\
\hline Cyfanswm & $\begin{array}{l}\text { Nifer } \\
\%\end{array}$ & $\begin{array}{l}27 \\
36.5\end{array}$ & $\begin{array}{l}24 \\
32.4\end{array}$ & $\begin{array}{l}9 \\
12.2\end{array}$ & $\begin{array}{l}17 \\
23.0\end{array}$ & $\begin{array}{l}12 \\
16.2\end{array}$ \\
\hline
\end{tabular}

Tabl 13. Nifer a chanran y cyfranogwyr a atebodd yr eitemau sy'n cymell enw yn gywir fesul dosbarth

\subsection{Ansoddeiriau}

Yn Tabl 14 nodir bod sgoriau'r tri dosbarth ar eitemau sy'n cymell ansoddair yn gyson yn dda. Mae'n bosibl i'r cyfranogwyr berfformio orau o ran yr eitemau hyn oherwydd natur benagored ansoddeiriau. Er enghraifft, mae eitem 3 yn gofyn i'r cyfranogwr gynnig ansoddair yn y frawddeg ganlynol:

3. Mae Siân, Tom a Harri yn hoffi mynd i'r clwb achos mae'n

Yn yr enghraifft uchod, mae'n bosibl cynnig sawl gair addas ar wahân i'r un a ddilëwyd, sef 'hwyl'. ${ }^{71}$ Cynigwyd sawl ansoddair gwahanol gan y cyfranogwyr, gan gynnwys: 'gwych', 'anhygoel', 'ardderchog', 'adloniadol', 'cyffrous', 'ffantastig', a 'bendigedig'. Gweler o'r rhestr hon o atebion cywir posibl fod amrywiaeth o ansoddeiriau posibl y gellir eu cynnig ar gyfer yr eitem hon. Mae hyn yn wir yn

\footnotetext{
${ }^{71}$ Er mai enw yw 'hwyl', fe'i defnyddiwyd gan ei fod yn cyflawni swyddogaeth ansoddair yn y cyddestun hwn.
} 
achos eitemau 5, 23 a 30 hefyd, sydd ag atebion gweddol agored. Beth sy'n ddiddorol am eitem 26 fodd bynnag yw bod angen ansoddair penodol, megis 'sâl' neu 'tost', sy'n cyd-fynd â chyd-destun y darn. Mae eitem 11 yn Rhan A hefyd yn cynnig ciw cyd-destunol ar gyfer eitem 26 yn Rhan B:

11. Ond fydd Harri ddim yn mynd ddydd Sul achos mae pen tost gyda fe.

26. Roedd Harri a Tom yn teimlo'n __âl _.

Ymddengys felly fod yr amrywiaeth a geir yn achos rhai eitemau sy'n cymell ansoddair yn gallu lleihau anhawster yr eitemau hyn a dyma pam y perfformiodd y cyfranogwyr yn dda ar y cyfan o ran yr eitemau hyn. Os nad oes amrywiaeth o atebion posibl fodd bynnag, mae'n debyg fod angen i'r cyfranogwr ddibynnu ar ddeall y cyd-destun wrth ddefnyddio gwybodaeth ar draws brawddegau ac ar draws y testun. 


\begin{tabular}{|c|c|c|c|c|c|c|}
\hline \multicolumn{2}{|c|}{ Dosbarth } & $\begin{array}{l}\text { Eitem 3: } \\
\text { 'hwyl' }\end{array}$ & $\begin{array}{l}\text { Eitem 5: } \\
\text { 'talentog', }\end{array}$ & $\begin{array}{l}\text { Eitem 23: } \\
\text { 'gyffrous' }\end{array}$ & $\begin{array}{l}\text { Eitem 26: } \\
\text { 'dost' }\end{array}$ & $\begin{array}{c}\text { Eitem 30: } \\
\text { 'hapus' }\end{array}$ \\
\hline $\begin{array}{l}\text { Dosbarth A: } \\
\text { CAI Set } 1 \text { Ysgol X }\end{array}$ & $\begin{array}{l}\text { Nifer } \\
\text { \% o'r dosbarth } \\
\text { \% o'r } \\
\text { cyfanswm }\end{array}$ & $\begin{array}{l}25 \\
96.2 \\
33.8\end{array}$ & $\begin{array}{l}20 \\
76.9 \\
27.0\end{array}$ & $\begin{array}{l}4 \\
15.4 \\
5.4\end{array}$ & $\begin{array}{l}7 \\
26.9 \\
9.5\end{array}$ & $\begin{array}{l}19 \\
73.1 \\
25.7\end{array}$ \\
\hline $\begin{array}{l}\text { Dosbarth B: } \\
\text { Cwrs Carlam Ysgol } \\
\text { X }\end{array}$ & $\begin{array}{l}\text { Nifer } \\
\text { \% o'r dosbarth } \\
\text { \% o'r } \\
\text { cyfanswm }\end{array}$ & $\begin{array}{l}19 \\
95.0 \\
\\
25.7\end{array}$ & $\begin{array}{l}16 \\
80.0 \\
21.6\end{array}$ & $\begin{array}{l}8 \\
40.0 \\
10.8\end{array}$ & $\begin{array}{l}9 \\
45.0 \\
12.2\end{array}$ & $\begin{array}{l}18 \\
90.0 \\
24.3\end{array}$ \\
\hline $\begin{array}{l}\text { Dosbarth C: } \\
\text { CAI Set } 1 \text { Ysgol Y }\end{array}$ & $\begin{array}{l}\text { Nifer } \\
\text { \% o'r dosbarth } \\
\text { \% o'r } \\
\text { cyfanswm }\end{array}$ & $\begin{array}{l}27 \\
96.4 \\
36.5\end{array}$ & $\begin{array}{l}21 \\
75.0 \\
28.4\end{array}$ & $\begin{array}{l}5 \\
17.9 \\
6.8\end{array}$ & $\begin{array}{l}4 \\
14.3 \\
5.4\end{array}$ & $\begin{array}{l}23 \\
82.1 \\
31.1\end{array}$ \\
\hline Cyfanswm & $\begin{array}{l}\text { Nifer } \\
\%\end{array}$ & $\begin{array}{l}71 \\
95.9\end{array}$ & $\begin{array}{l}57 \\
77.0\end{array}$ & $\begin{array}{l}17 \\
23.0\end{array}$ & $\begin{array}{l}20 \\
27.0\end{array}$ & $\begin{array}{l}60 \\
81.1\end{array}$ \\
\hline
\end{tabular}

Tabl 14. Nifer a chanran y cyfranogwyr a atebodd yr eitemau sy'n cymell ansoddair yn gywir fesul dosbarth

\subsection{Berfau}

O ran eitemau sy'n cymell berf, nodir bod sgoriau'r tri dosbarth ar eitemau hyn yn gyson yn isel. Wrth sylwi ar Dabl 14, nodir dwy enghraifft o ferfau a barodd drafferth yn benodol, sef 'gwelais' a 'mwynheais' yn eitemau 24 a 42:

24. Yn y clwb, i fy ffrind Siân.

42. Wel, i'r penwythnos yn fawr iawn!

Er i'r terfyniad person cyntaf amser gorffennol 'i' gael ei leoli ar ôl y bylchau hyn fel ciw cyd-destunol er mwyn cynorthwyo'r cyfranogwr, mae'n amlwg nad yw'r ciw hwn o gymorth ac felly mae'r eitemau hyn yn rhy anodd i'r rhan fwyaf o'r cyfranogwyr. Yn Tabl 15, nodir mai pum cyfranogwr yn unig a atebodd eitem 24 yn 
gywir a dau gyfranogwr yn unig a atebodd eitem 42 yn gywir. Nodir hefyd fod nifer fawr o gyfranogwyr, yn arbennig yn achos Dosbarth A a Dosbarth C, a adawodd eitem 42 yn wag. Enghraifft arall o ferf a barodd drafferth oedd eitem 7:

7. Enw ffrind Siân Jade.

Er bod yr eitem hon yn cymell y cyfranogwr i gyfannu'r frawddeg gypladol hon gan gynnig 'ydy', cofnodwyd nifer fawr o bapurau lle cynigodd y cyfranogwr gysylltair megis 'a/ac' a 'gyda' yn hytrach. Wrth weld dau enw priod fel 'Siân' a 'Jade' gyda bwlch rhyngddynt, mae'n bosibl y credai rhai cyfranogwyr fod angen y cysylltair 'a/ac' er mwyn cwblhau'r rhestr o enwau. Mae cynnig y cysylltair 'gyda' hefyd yn ddiddorol ac mae'n bosibl fod rhai cyfranogwyr wedi'i gynnig oherwydd eu bod wedi camddeall y frawddeg fel petai'n dweud 'mae Siân yn ffrindiau gyda Jade'. Er na ellir cynnig rhesymau penodol dros yr atebion anghywir yma, mae'n amlwg fod rhai disgyblion yn fwy cyfarwydd â phatrwm y frawddeg gypladol nac eraill.

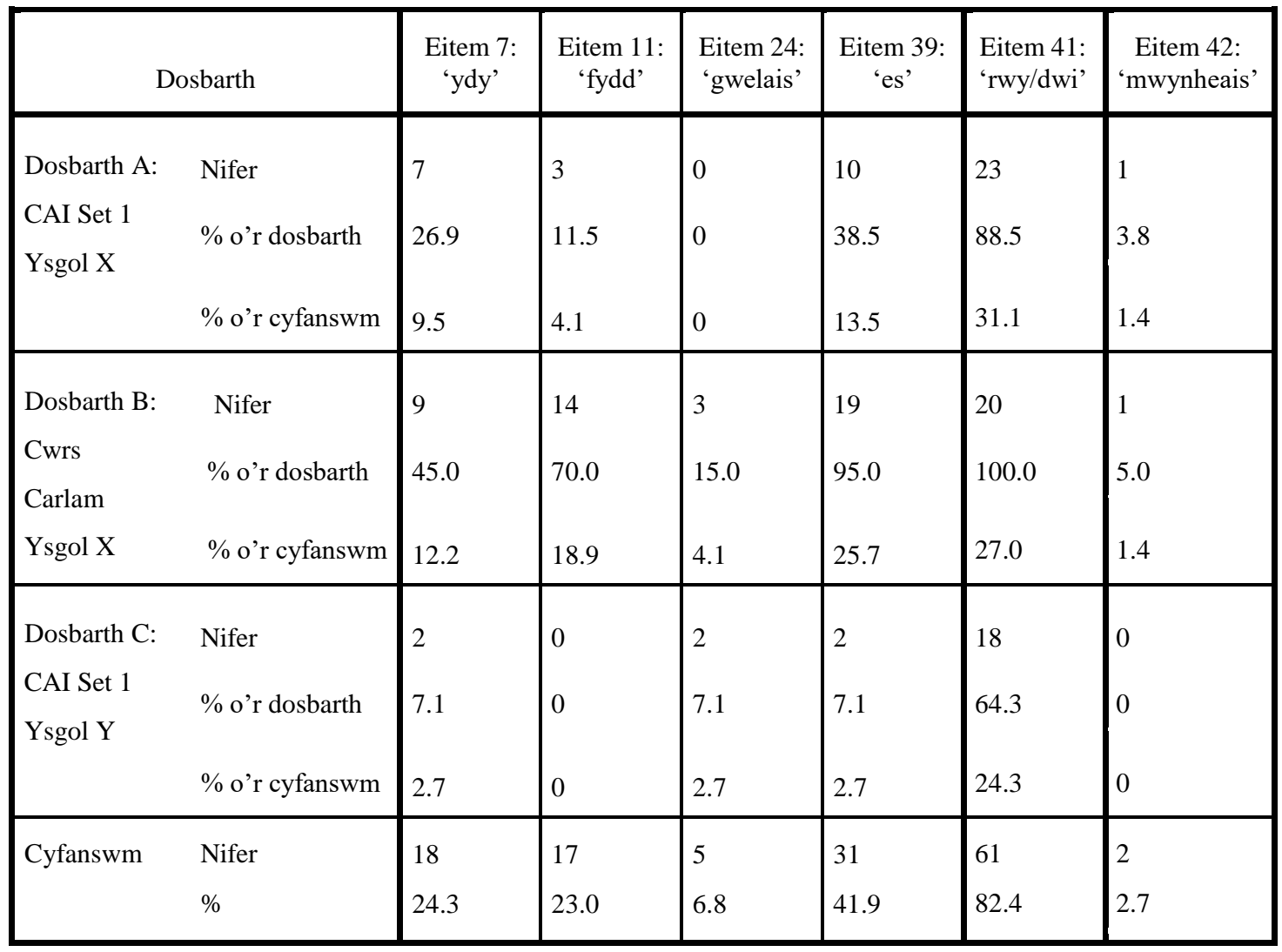

Tabl 15. Nifer a chanran y cyfranogwyr a atebodd yr eitemau sy' $n$ cymell berf yn gywir fesul dosbarth 


\subsection{Berfenwau}

Ynglŷn â pherfformiad ar eitemau sy'n cymell berfenw, nodir bod amrywiaeth rhwng y cyfranogwyr ym mhob un o'r tri dosbarth. Ni ddisgwylid canfod amrywiaeth sylweddol rhwng yr eitmau hyn oherwydd bod y berfenwau a ddewiswyd yn rhai y disgwylir i'r cyfranogwyr i gyd fod yn gyfarwydd â hwy, sef 'hoffi', 'gwylio', 'gallu', 'meddwl' a 'mynd'. Serch hynny, nodir mai eitemau 18 a 21, lle cafwyd y berfenwau 'meddwl' a 'mynd' eu dileu yn wreiddiol, yw dwy o'r eitemau anhawsaf ar y prawf:

18. 'Dw i'n bod dy esgidiau di yn y gegin' atebodd mam.

21. Fel arfer, dw i'n i’r gêm gyda fy ffrindiau.

Er bod y patrymau 'dw i'n meddwl bod' a 'dw i'n mynd i' yn yr enghreifftiau uchod yn rhai sylfaenol y byddai'r cyfranogwyr wedi eu defnyddio erbyn hyn, mae'n bosibl bod defnyddio'r patrymau hyn mewn cyd-destun gwahanol yn mynnu sgiliau uwch. Awgryma hyn fod deall y testun o amgylch yr eitem yn hanfodol os yw'r cyfranogwr i gyfannu'r bwlch yn gywir. 


\begin{tabular}{|c|c|c|c|c|c|c|}
\hline \multicolumn{2}{|c|}{ Dosbarth } & $\begin{array}{c}\text { Eitem 2: } \\
\text { 'hoffi' }\end{array}$ & $\begin{array}{c}\text { Eitem 12: } \\
\text { 'gwylio' }\end{array}$ & $\begin{array}{c}\text { Eitem 16: } \\
\text { 'gallu' }\end{array}$ & $\begin{array}{l}\text { Eitem 18: } \\
\text { 'meddwl' }\end{array}$ & $\begin{array}{c}\text { Eitem 21: } \\
\text { 'mynd' }\end{array}$ \\
\hline $\begin{array}{l}\text { Dosbarth A: } \\
\text { CAI Set } 1 \\
\text { Ysgol X }\end{array}$ & $\begin{array}{l}\text { Nifer } \\
\text { \% o'r dosbarth } \\
\text { \% o'r cyfanswm }\end{array}$ & $\begin{array}{l}10 \\
38.5 \\
13.5\end{array}$ & $\begin{array}{l}24 \\
92.3 \\
32.4\end{array}$ & $\begin{array}{l}17 \\
65.4 \\
23.0\end{array}$ & $\begin{array}{l}3 \\
11.5 \\
4.1\end{array}$ & $\begin{array}{l}0 \\
0.0 \\
0.0\end{array}$ \\
\hline $\begin{array}{l}\text { Dosbarth B: } \\
\text { Cwrs Carlam } \\
\text { Ysgol X }\end{array}$ & $\begin{array}{l}\text { Nifer } \\
\text { \% o'r dosbarth } \\
\text { \% o'r cyfanswm }\end{array}$ & $\begin{array}{l}17 \\
85.0 \\
23.0\end{array}$ & $\begin{array}{l}19 \\
95.0 \\
25.7\end{array}$ & $\begin{array}{l}16 \\
80.0 \\
21.6\end{array}$ & $\begin{array}{l}6 \\
30.0 \\
8.1\end{array}$ & $\begin{array}{l}6 \\
30.0 \\
8.1\end{array}$ \\
\hline $\begin{array}{l}\text { Dosbarth C: } \\
\text { CAI Set } 1 \\
\text { Ysgol Y }\end{array}$ & $\begin{array}{l}\text { Nifer } \\
\text { \% o'r dosbarth } \\
\text { \% o'r cyfanswm }\end{array}$ & $\begin{array}{l}4 \\
14.3 \\
5.4\end{array}$ & $\begin{array}{l}18 \\
64.3 \\
24.3\end{array}$ & $\begin{array}{l}19 \\
67.9 \\
25.7\end{array}$ & $\begin{array}{l}0 \\
0.0 \\
0.0\end{array}$ & $\begin{array}{l}5 \\
17.9 \\
6.8\end{array}$ \\
\hline Cyfanswm & $\begin{array}{l}\text { Nifer } \\
\%\end{array}$ & $\begin{array}{l}31 \\
41.9\end{array}$ & $\begin{array}{l}61 \\
82.4\end{array}$ & $\begin{array}{l}52 \\
70.3\end{array}$ & $\begin{array}{l}9 \\
12.2\end{array}$ & $\begin{array}{l}11 \\
14.9\end{array}$ \\
\hline
\end{tabular}

Tabl 16. Nifer a chanran y cyfranogwyr a atebodd yr eitemau sy'n cymell berfenw yn gywir fesul dosbarth

\subsection{Adferfau}

Yn Tabl 17, gwelir dadansoddiad o sgoriau'r tri dosbarth ar eitemau sy'n cymell adferf. Sylwir mai dwy o'r eitemau a gafodd eu hateb yn gywir fwyaf oedd eitemau 8 a 33:

8. nos Wener, mae Siân a Jade yn mynd i'r clwb.

33. Roeddwn i'n nerfus

Wrth archwilio'r atebion a gynigwyd ar gyfer eitem 8, ymddengys fod defnyddio ymadrodd adferfol fel 'bob nos Wener' neu 'ar nos Wener' ar ddechrau brawddeg yn batrwm cyfarwydd i'r cyfranogwyr. Mae'n ymddangos bod 'bob nos Wener' yn batrwm arbennig o gyfarwydd gan mai dyma'r ateb a gynigiwyd fwyaf gan gyfranogwyr ar gyfer yr eitem hon. Ar gyfer eitem 33, ni chynigiodd neb yr adferf gwreiddiol a ddilëwyd o'r testun, sef 'hefyd'. Yn achos pob un o'r atebion cywir, cynigiwyd yr adferf 'iawn' yn ei le. Eto, mae'n debyg mai'r ymadrodd 'nerfus iawn' 
sy'n fwy cyfarwydd i'r cyfranogwyr ac nid yw'n ddigon clir o'r cyd-destun mai 'hefyd' sydd wedi'i ddileu. Nodir dwy enghraifft arall lle mae'n bosibl nad yw'r cyddestun yn rhoi digon o giw i'r cyfranogwr er mwyn deall mai adferf sydd ei angen:

6. , mae Siân a'i ffrind yn chwarae rygbi.

28. Mae gêm fawr gyda ni

O blith y 5 eitem sy'n cymell adferf, mae'n amlwg mai un enghraifft yn arbennig sydd wedi gwahaniaethu'n dda, sef 'adref' yn eitem 13:

13. Bydd pawb arall yn mynd i wylio'r teledu.

Nodir bod pob un o'r cyfranogwyr a atebodd eitem 13 yn gywir yn deillio o Dosbarth B. Ymddengys felly nad yw 'adref' yn adferf y mae cyfranogwyr y ddau grŵp arall yn gyfarwydd ag ef erbyn hyn.

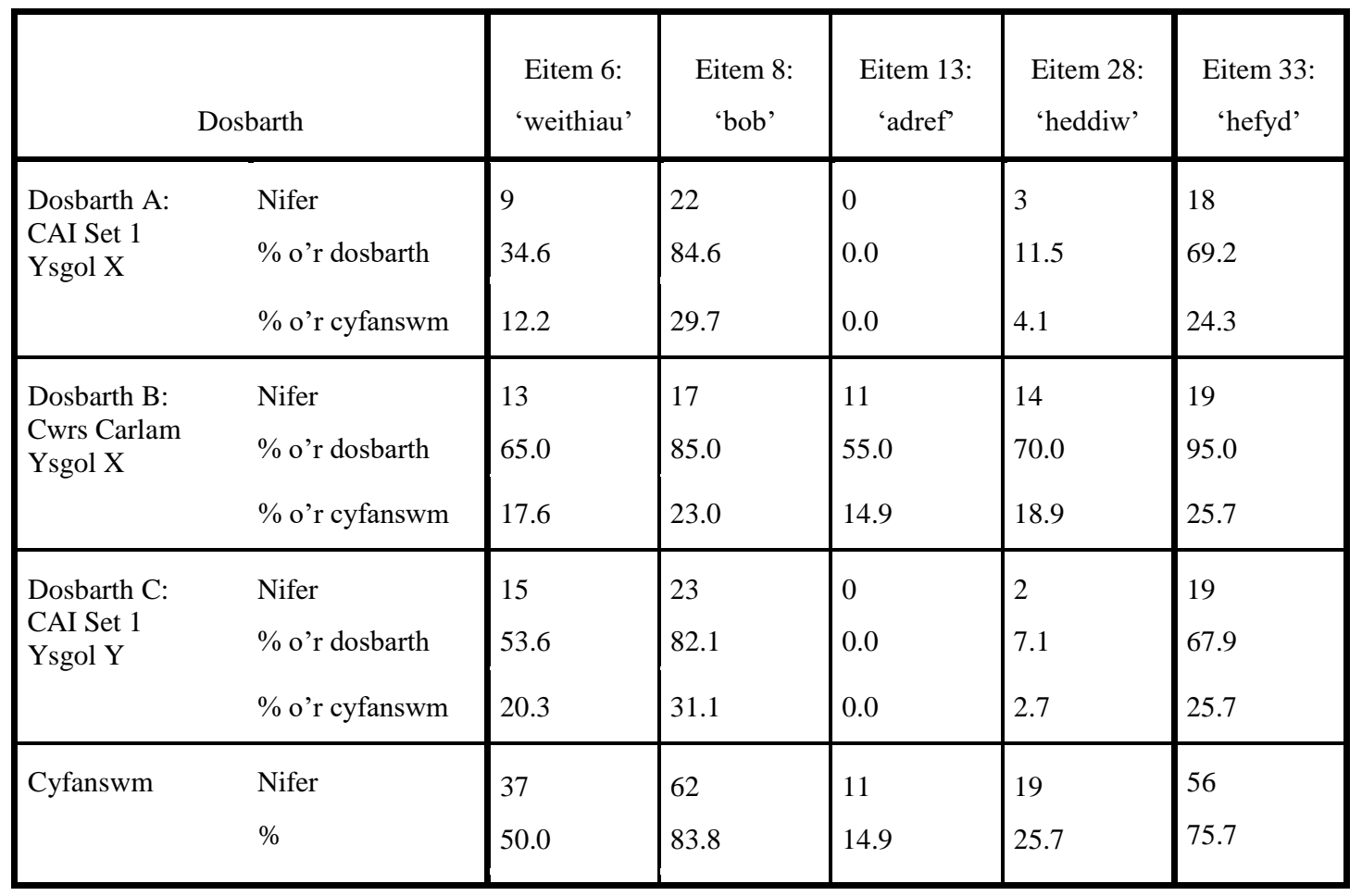

Tabl 17. Nifer a chanran y cyfranogwyr a atebodd yr eitemau sy'n cymell adferf yn gywir fesul dosbarth 


\subsection{Rhagenwau}

Wrth archwilio'r atebion i'r eitemau sy'n cymell rhagenwau, ymddengys fod rhai cyfranogwyr yn fwy cyfarwydd â rhai rhagenwau ac felly maent yn gallu defnyddio'r ciwiau cyd-destunol yn well na'u cyfoedion. Enghraifft o hyn yw eitem 17 a gafodd ei hateb yn gywir gan saith cyfranogwr yn unig, gyda chwech o'r cyfranogwyr hyn yn deillio o Dosbarth B:

17. Gofynnais i fy mam, 'Mam, ble mae esgidiau i?'.

Er bod ciw cyd-destunol yn y frawddeg, sef y rhagenw personol ategol 'i', ymddengys o'r atebion na all y rhan fwyaf o'r cyfranogwyr ddefnyddio'r ciw cyddestunol hwn er mwyn ateb yr eitem hon yn gywir. Serch hynny, mae'n amlwg y gall y cyfranogwyr gynnig y rhagenwau personol eraill yn gywir mewn enghreifftiau eraill, fel y gwelir yn achos eitemau 9, 27 a 31:

9. Maen 'n mynd i'r clwb gyda ffrindiau.

27. Gofynnais i i Siân 'Sut wyt Siân?'

31. Ond heddiw, roedd 'n edrych yn nerfus.

Gan fod ciw cyd-destunol ar gyfer pob un o'r eitemau uchod, gan gynnwys eitem 17, mae'n bosibl fod rhai patrymau brawddegol, fel y gwelir yn eitemau 9, 27 a 31, yn fwy cyfarwydd na phatrymau brawddegol eraill, fel y gwelir yn eitemau 17 a 35 . 


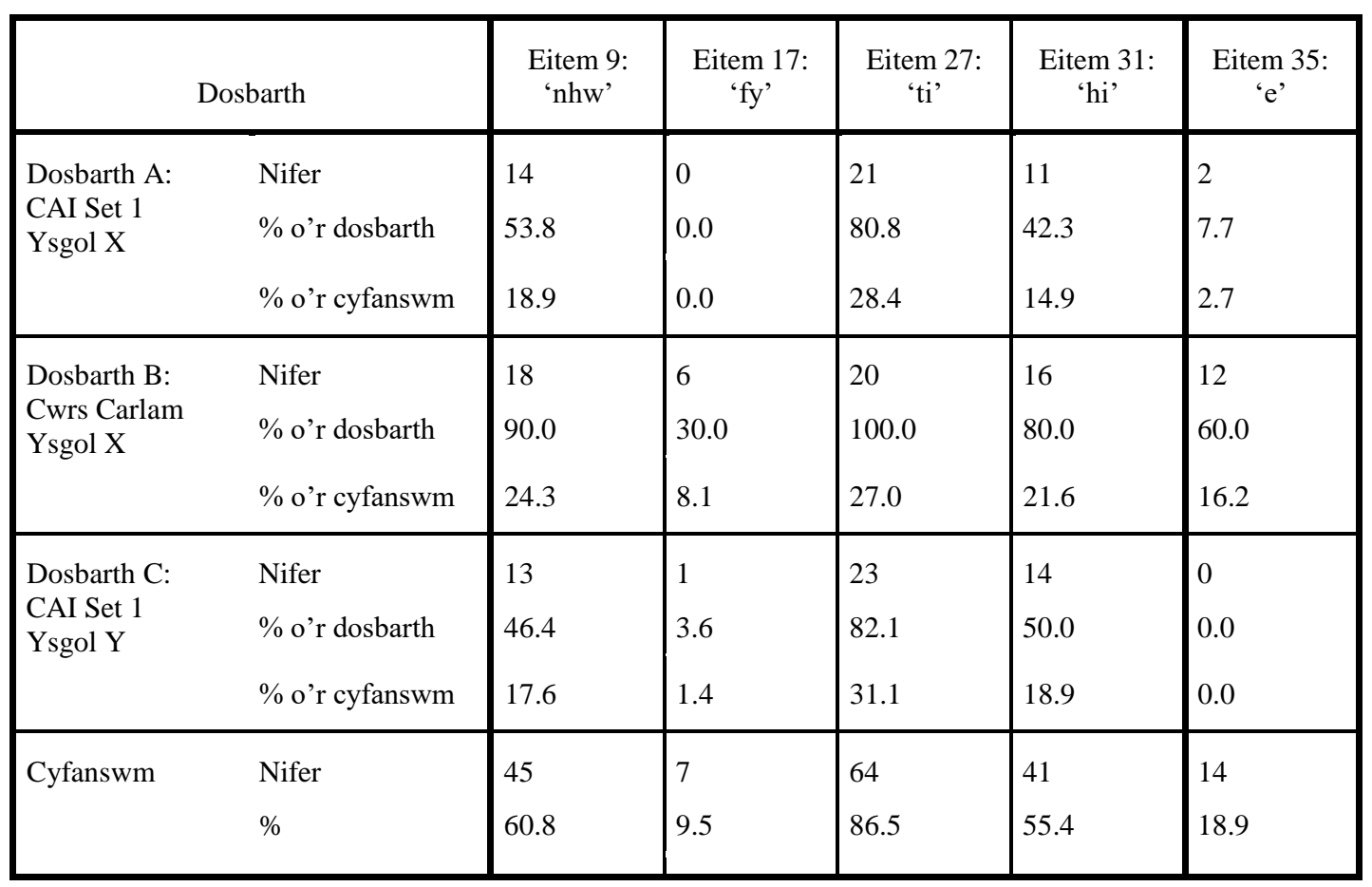

Tabl 18. Nifer a chanran y cyfranogwyr a atebodd yr eitemau sy'n cymell rhagenw yn gywir fesul dosbarth

\subsection{Arddodiaid}

Yn Tabl 19, nodir bod sgoriau ar gyfer arddodiaid yn isel iawn ar y cyfan, o’u cymharu â'r sgoriau ar gyfer y mathau eraill o eiriau swyddogaethol. O blith y 5 eitem ansoddair, nodir bod un enghraifft lle y mae angen deall yr idiom yn y frawddeg er mwyn ateb yr eitem gyda'r arddodiad cywir, sef eitem 38:

38. Enillon ni'r gêm ac roeddwn i fy modd!

O blith y $31.1 \%$ o'r cyfranogwyr a atebodd yr eitem hon yn gywir, nodir bod y rhan fwyaf o'r cyfranogwyr hyn yn deillo o Dosbarth B, sef 69.6\%. Awgryma hyn fod cyfranogwyr y dosbarth carlam yn fwy cyfarwydd â'r idiom hwn yn eitem 38 na'u cyfoedion yn y ddau grŵp arall.

Efallai mai'r arddodiad a barodd y drafferth fwyaf i'r cyfranogwyr fodd bynnag oedd 'heb' yn eitem 37:

37. Wps! Dw i ddim yn gallu chwarae rygbi esgidiau!! 
Er bod y cyd-destun yn awgrymu nad oes gan gymeriad y stori, Jade, ei hesgidiau, mae'n bosibl nad oedd y rhan fwyaf o'r cyfranogwyr yn gyfarwydd â'r arddodiad cywir 'heb' er mwyn cwblhau'r eitem hon yn gywir. Pum cyfranogwr (cymedr = 6.9) yn unig a atebodd yr eitem yn gywir, gyda phob un o'r cyfranogwyr hyn yn deillo o Dosbarth B. Fodd bynnag, nodir sawl enghraifft lle mae'n ymddangos fod y cyfranogwr yn deall y frawddeg a'r hyn sydd ei angen ond nid yw'n gallu cynnig y gair priodol. Mae'r cyfranogwyr hyn felly yn rhoi un o'r enghreifftiau canlynol yn ei le: 'dim' a 'gyda dim'. Er bod yr ail enghraifft yn gwneud synnwyr yng nghyddestun y frawddeg, mae'r ateb hwn yn torri rheol, sef bod angen cynnig un gair yn unig ar gyfer pob eitem, ac o ganlyniad marciwyd yr ateb hwn yn anghywir. Ar wahân i'r nifer fechan o gyfranogwyr a atebodd eitem 37 yn gywir $($ cymedr $=6.8)$ ymddengys nad oedd y rhan fwyaf o'r cyfranogwyr eraill yn gallu deall y frawddeg neu'r cyd-destun ac o ganlyniad fe gynigiasant atebion anghywir megis 'glas', 'gwyrdd', 'yn' a 'gyda'.

Wrth ddadansoddi eitemau 4, 10 a 40 yn benodol, mae'n amlwg fod rhai arddodiaid yn fwy cyfarwydd i'r tri dosbarth nag eraill. Dengys y sgoriau yn Tabl 19 y gall canfod rhai arddodiaid fod yn drafferthus iawn, ac mae'n bosibl fod cyfranogwyr Dosbarth B yn fwy cyfarwydd â'r arddodiaid hyn na'u cyfoedion yn Dosbarth A a C, yn arbennig yn achos eitemau 37 a 40. 


\begin{tabular}{|c|c|c|c|c|c|c|}
\hline & barth & $\begin{array}{c}\text { Eitem 4: } \\
\text { 'ar' }\end{array}$ & $\begin{array}{c}\text { Eitem 10: } \\
\text { 'i' }\end{array}$ & $\begin{array}{l}\text { Eitem 37: } \\
\text { 'heb' }\end{array}$ & $\begin{array}{c}\text { Eitem 38: } \\
\text { 'wrth' }\end{array}$ & $\begin{array}{c}\text { Eitem 40: } \\
\text { 'o' }\end{array}$ \\
\hline $\begin{array}{l}\text { Dosbarth A: } \\
\text { CAI Set } 1 \\
\text { Ysgol X }\end{array}$ & $\begin{array}{l}\text { Nifer } \\
\text { \% o'r dosbarth } \\
\text { \% o'r cyfanswm }\end{array}$ & $\begin{array}{l}11 \\
42.3 \\
14.9\end{array}$ & $\begin{array}{l}6 \\
23.1 \\
8.1\end{array}$ & $\begin{array}{l}0 \\
0 \\
0\end{array}$ & $\begin{array}{l}5 \\
19.2 \\
6.8\end{array}$ & $\begin{array}{l}5 \\
19.2 \\
6.8\end{array}$ \\
\hline $\begin{array}{l}\text { Dosbarth B: } \\
\text { Cwrs Carlam } \\
\text { Ysgol X }\end{array}$ & $\begin{array}{l}\text { Nifer } \\
\text { \% o'r dosbarth } \\
\text { \% o'r cyfanswm }\end{array}$ & $\begin{array}{l}9 \\
45.0 \\
12.2\end{array}$ & $\begin{array}{l}11 \\
55.0 \\
14.9\end{array}$ & $\begin{array}{l}5 \\
25.0 \\
6.8\end{array}$ & $\begin{array}{l}16 \\
80.0 \\
21.6\end{array}$ & $\begin{array}{l}14 \\
70.0 \\
18.9\end{array}$ \\
\hline $\begin{array}{l}\text { Dosbarth C: } \\
\text { CAI Set 1 } \\
\text { Ysgol Y }\end{array}$ & $\begin{array}{l}\text { Nifer } \\
\text { \% o'r dosbarth } \\
\text { \% o'r cyfanswm }\end{array}$ & $\begin{array}{l}12 \\
42.9 \\
16.2\end{array}$ & $\begin{array}{l}1 \\
3.6 \\
1.4\end{array}$ & $\begin{array}{l}0 \\
0 \\
0\end{array}$ & $\begin{array}{l}2 \\
7.1 \\
2.7\end{array}$ & $\begin{array}{l}0 \\
0 \\
0\end{array}$ \\
\hline Cyfanswm & $\begin{array}{l}\text { Nifer } \\
\%\end{array}$ & $\begin{array}{l}32 \\
43.3\end{array}$ & $\begin{array}{l}18 \\
24.4\end{array}$ & $\begin{array}{l}5 \\
6.8\end{array}$ & $\begin{array}{l}23 \\
31.1\end{array}$ & $\begin{array}{l}19 \\
25.7\end{array}$ \\
\hline
\end{tabular}

Tabl 19. Nifer a chanran y cyfranogwyr a atebodd yr eitemau sy'n cymell arddodiad yn gywir fesul dosbarth

\subsection{Cysyllteiriau}

Wrth rannu'r sgoriau ar gyfer eitemau sy'n cymell cysyllteiriau fesul eitem a dosbarth, fel y gwnaed yn Tabl 20, nodir bod dwy eitem benodol a barodd gryn drafferth, sef eitemau 22 a 29:

22. heddiw, roedd dad eisiau dod gyda fi.

29. Wyt ti'n gyffrous 'n nerfus?

Er y bydd cyfranogwyr yn gyfarwydd â'r cysylltair 'ond', ymddengys fod canfod y cysylltair hwn ar gyfer eitem 22 yn anodd oherwydd y cyd-destun. Mae ateb eitem 22 yn gywir yn dibynnu, ymddengys, ar allu'r cyfranogwr i ddeall a defnyddio'r ciw cyd-destunol 'fel arfer' ar ddechrau eitem 21:

21. Fel arfer, dw i'n _ mynd i'r gêm gyda fy ffrindiau.

22. Ond heddiw, roedd dad eisiau dod gyda fi.

Mae'r ciw cyd-destunol hwn yn y frawddeg flaenorol yn awgrymu y bydd newid i'r drefn arferol yn y frawddeg ddilynol. Dyma enghraifft lle mae defnyddio 
gwybodaeth ar draws brawddegau yn hanfodol os yw'r cyfranogwr am ateb yr eitem yn gywir.

Yn achos eitem 29, gwelir bod defnyddio gwybodaeth o fewn y frawddeg yn allweddol ar gyfer ateb yr eitem hon yn gywir. Er bod yr ansoddeiriau cyferbyniol ‘cyffrous' a 'nerfus' yn cynnig ciw cyd-destunol i'r cyfranogwyr, mae'n amlwg o sgoriau'r tri dosbarth nad yw'r eitem yn un hawdd i'w hateb yn gywir. Nodir enghreifftiau lle mae'r cyfranogwr yn cynnig rhagenwau personol megis 'hi', 'i', 'e', a 'nhw', neu'r ferf 'mae', ac mae fel petai'r 'yn' traethiadol talfyredig sydd yn dilyn y bwlch yn camarwain y cyfranogwyr hyn at gynnig yr atebion anghywir hyn. Mae atebion o'r fath yn awgrymu nad yw'r disgyblion hyn yn gyfarwydd â defnyddio'r cysylltair 'neu' erbyn hyn.

Un o'r canfyddiadau mwyaf diddorol oedd sgoriau'r tri dosbarth ar gyfer eitem 19, sy'n cymell y cysylltair 'achos':

19. Roedd yr esgidiau yn bwysig roedd gyda fi gêm rygbi fawr yn y prynhawn.

Er y bydd yr holl gyfranogwyr yn gyfarwydd â defnyddio'r cysylltair 'achos' ar gyfer mynegi barn, sylwir yn Tabl 20 fod sgoriau'r tri dosbarth yn gwahaniaethu'n fawr. Wrth archwilio atebion y cyfranogwyr, gwelir bod rhai cyfranogwyr yn deall bod angen cysylltair ar gyfer yr eitem hon, ac felly maent yn cynnig cysyllteiriau megis 'ond' a farciwyd yn anghywir, ac 'a/ac' a farciwyd yn gywir. Nodir hefyd enghreifftiau lle cynigiwyd yr adferf 'iawn' gan ambell gyfranogwr er mwyn pwysleisio pwysigrwydd yr esgidiau ar gyfer y gêm rygbi. Er nad oes gwahannod megis atalnod neu gysylltnod yn dilyn y bwlch er mwyn rhannu'r frawddeg, marciwyd 'iawn' yn gywir beth bynnag oherwydd ei fod yn gwneud synnwyr yn y cyd-destun hwn.

Ar gyfer eitem 25, sylwir hefyd fod enghreifftiau lle cynigiwyd atebion gwahanol i'r cysylltair gwreiddiol a ddilëwyd ac fe'u marciwyd yn gywir:

25. Ond, welais i ddim Harri Tom!

Er mai'r cysylltair 'na' sydd ei angen oherwydd y cymal negyddol, nodir bod y rhan fwyaf o'r cyfranogwyr naill ai yn cynnig 'a' ynteu 'neu' ar gyfer yr eitem hon. Gan 
nad oedd un cyfranogwr a gynigodd y cysylltair cywir 'na', a chan ei bod yn amlwg nad oedd y cyfranogwyr yn gyfarwydd â defnyddio'r cysylltair hwn mewn cymal negyddol, penderfynwyd rhoi marc i gyfranogwyr a gynigodd y cysyllteiriau hyn.

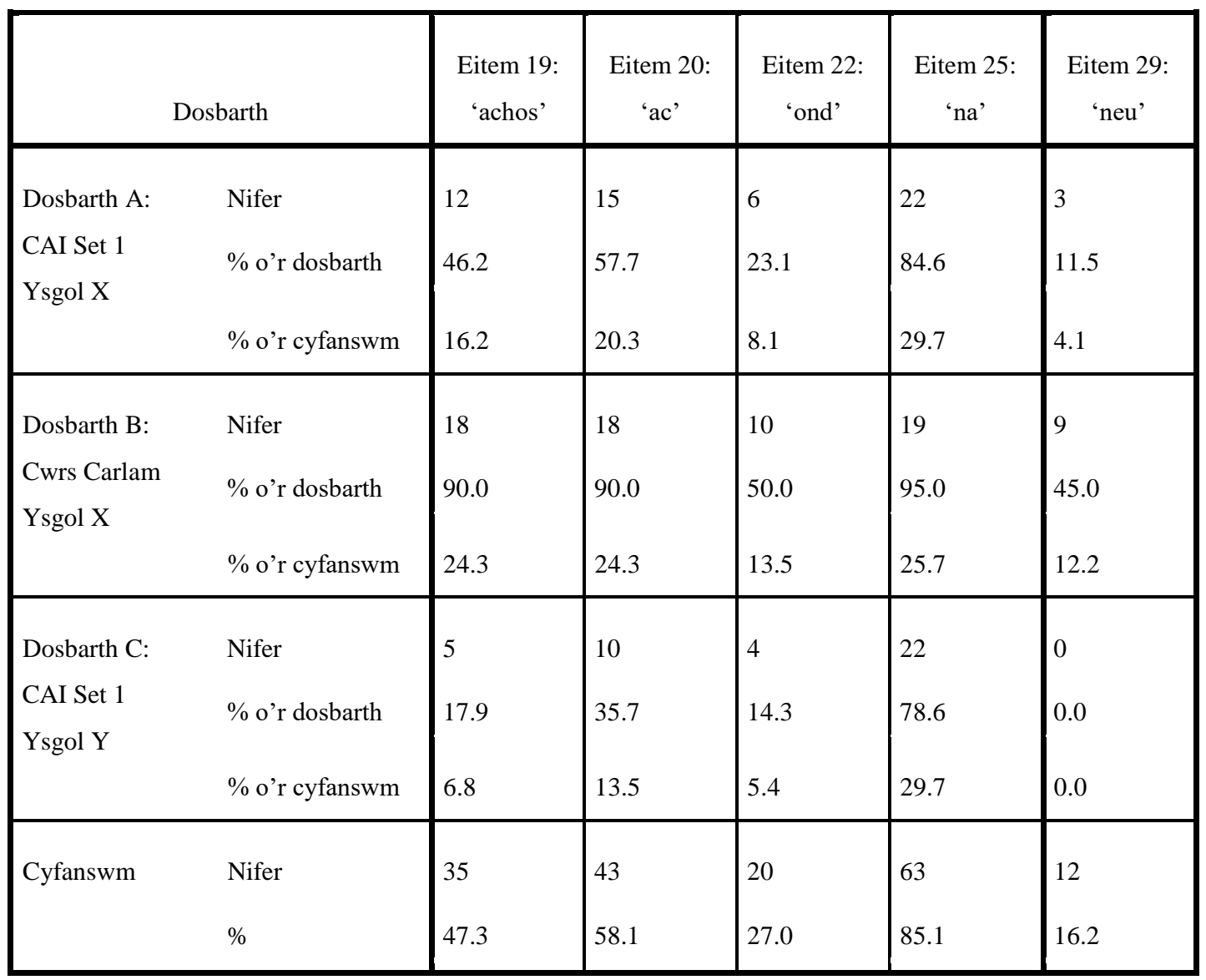

Tabl 20. Nifer a chanran y cyfranogwyr a atebodd yr eitemau sy'n cymell cysylltair yn gywir fesul dosbarth

\subsection{Y fannod}

Er mai dwy eitem yn unig sy'n mynnu'r fannod, sef eitem 14 ac eitem 43, nodir bod y sgoriau cymedrig ar gyfer yr eitmau hyn yn isel iawn ar y cyfan. Nid syndod felly yw sylwi bod yr eitemau hyn yn rhan o draean uchaf yr eitemau mwyaf anodd yn y prawf (gweler Atodiad 15).

Ar gyfer eitemau 14 a 43, nodir bod angen canfod y fannod er mwyn cwblhau'r brawddegau cypladol canlynol:

14. 'Cariad at Chwaraeon' ydy'r clwb gorau yn byd!

43. Ond dydd Sul oedd diwrnod gorau! 
Er y credid na fyddai nifer o'r cyfranogwyr yn gyfarwydd iawn â phatrwm y frawddeg gypladol, penderfynwyd cynnwys dwy enghraifft er mwyn gwahaniaethu rhwng lefelau hyfedredd ieithyddol uwch y cyfranogwyr yn y dosbarthiadau. Fel y disgwylir, mae'r ddwy eitem hyn wedi peri cryn drafferth i'r cyfranogwyr i gyd.

\begin{tabular}{|c|c|c|c|}
\hline \multicolumn{2}{|c|}{ Dosbarth } & Eitem 14: 'y’ & Eitem 43: 'y' \\
\hline Dosbarth A: & Nifer & 2 & 1 \\
\hline CAI Set 1 & $\%$ o'r dosbarth & 7.7 & 3.8 \\
\hline Ysgol X & \% o'r cyfanswm & 2.7 & 1.4 \\
\hline Dosbarth B: & Nifer & 11 & 8 \\
\hline Cwrs Carlam & $\%$ o'r dosbarth & 55.0 & 40.0 \\
\hline Ysgol X & \% o'r cyfanswm & 14.9 & 10.8 \\
\hline Dosbarth C: & Nifer & 2 & 1 \\
\hline CAI Set 1 & \% o'r dosbarth & 7.1 & 3.6 \\
\hline Ysgol Y & \% o'r cyfanswm & 2.7 & 1.4 \\
\hline Cyfanswm & Nifer & 15 & 10 \\
\hline & $\%$ & 20.3 & 13.5 \\
\hline
\end{tabular}

Tabl 21. Nifer a chanran y cyfranogwyr a atebodd yr eitemau sy'n cymell y fannod yn gywir fesul dosbarth

\subsubsection{Dadansoddi dibynadwyedd a dilysrwydd y prawf}

\subsubsection{Cydberthyniad sgoriau'r prawf}

Er mwyn dangos a oes unrhyw gydberthyniad rhwng sgoriau Rhan A a Rhan B, lluniwyd plot gwasgariad. Ymddengys fod y plot gwasgariad, a welir yn Atodiad 16, yn dangos cydberthyniad positif rhwng sgoriau dwy ran y prawf. Fodd bynnag, wrth ddadansoddi'r llinell atchwel a'r llinell Loess, ymddengys fod y cydberthyniad yn gwyro ychydig oddi wrth linoledd $\left(\mathrm{R}^{2}\right.$ llinol = .679). Lluniwyd dau blot gwasgariad arall hefyd er mwyn darganfod unrhyw gydberthyniad rhwng Sgôr Rhan A a Sgôr Gyfanswm (gweler Atodiad 17), a Sgôr Rhan B a Sgôr Gyfanswm (gweler Atodiad 18). Wrth astudio'r llinellau atchwel a llinellau Loess yn Atodiadau 18 a 19, gellir nodi cydberthyniadau llinol rhwng Sgôr Rhan A a Sgôr Gyfanswm $\left(\mathrm{R}^{2}\right.$ llinol = .842), a Sgôr Rhan B a Sgôr Gyfanswm $\left(\mathrm{R}^{2}\right.$ llinol = .963). 
Gan na chaiff y newidynnau eu gwasgaru'n normal a chan nad oes cydberthynas llinol rhwng pob newidyn, ${ }^{72}$ penderfynwyd rhedeg prawf Spearman ${ }^{73}$ yn lle prawf Pearson er mwyn cadarnhau a ydy'r cydberthyniadau rhwng y newidynnau'n bwysig o safbwynt ystadegol. Gellir gweld canlyniadau'r prawf Spearman yn Tabl 22 isod. Dangosodd y prawf Spearman fod y cydberthyniad rhwng sgoriau'r cyfranogwyr yn Rhan A a Rhan B y prawf llenwi bylchau yn gryf ac yn ystadegol-bwysig $\left(r_{s}=.785\right.$; $p=<.001)$. O ran Sgôr Rhan A a Sgôr Gyfanswm, nodir eto fod y cydberthyniad rhwng y ddwy sgôr yn gryf ac yn ystadegol-bwysig $\left(r_{s}=.895 ; p=<.001\right)$. Fodd bynnag, nodir bod y cydberthyniad rhwng Sgôr Rhan B a Sgôr Gyfanswm yn gryfach $\left(r_{s}=.970 ; p=<.001\right)$, mewn cymhariaeth â'r cydberthyniad rhwng Sgôr Rhan A a Sgôr Gyfanswm. Ymddengys felly fod Sgôr Rhan B yn ddangosydd gwell na Sgôr Rhan A ar gyfer rhagfynegi Sgôr Gyfanswm. Nid yw'r canfyddiad hwn yn annisgwyl fodd bynnag oherwydd bod eitemau Rhan B yn cynrychioli'r gyfran fwyaf o'r papur, sef 67\% (29 eitem allan o 43). Mae Rhan B felly yn cynnig rhagor o gyfleoedd i'r cyfranogwr ennill marciau na Rhan A, sy'n cynrychioli 33\% o'r papur yn unig (14 eitem allan o 43). Yn ogystal â hyn, anelwyd at sicrhau bod anhawster yr eitemau yn dwysáu wrth fynd ymlaen trwy'r prawf. Nid oes syndod felly mai'r dosbarth a berfformiodd orau yn Rhan B, sef Dosbarth B, yw'r rhai a berfformiodd orau hefyd yn y prawf cyfan. Mae'r canfyddiad hwn yn awgrymu bod y prawf llenwi bylchau yn llwyddo i herio'r cyfranogwyr fwyfwy wrth fynd ymlaen trwy'r papur.

\footnotetext{
72 Dyma ddwy o'r pedair rhagdyb y dylid eu bodloni cyn cynnal prawf cydberthyniad parametrig Pearson (Larson-Hall, 2010, t. 160)

${ }^{73}$ Dyma un o'r profion amharametrig y gellir eu defnyddio yn lle prawf Pearson os na ellir bodloni'r rhagdybiau.
} 


\begin{tabular}{|c|c|c|c|c|c|}
\hline & & & Sgôr Rhan A & Sgôr Rhan B & Sgôr Gyfanswm \\
\hline \multirow{9}{*}{$\begin{array}{l}\text { Spearman's } \\
\text { rho }\end{array}$} & \multirow{3}{*}{ Sgôr Rhan A } & Cyfernod Cydberthyniad & 1.000 & $.785^{* *}$ & $.895^{* *}$ \\
\hline & & Sig. (2-tailed) & & .000 & .000 \\
\hline & & Nifer & 74 & 74 & 74 \\
\hline & \multirow[t]{3}{*}{ Sgôr Rhan B } & Cyfernod Cydberthyniad & $.785^{* *}$ & 1.000 & $.970^{* *}$ \\
\hline & & Sig. (2-tailed) & .000 & & .000 \\
\hline & & Nifer & 74 & 74 & 74 \\
\hline & \multirow{3}{*}{$\begin{array}{l}\text { Sgôr Gyfanswm } \\
\text { (Canran) }\end{array}$} & Cyfernod Cydberthyniad & $.895^{* *}$ & $.970^{* *}$ & 1.000 \\
\hline & & Sig. (2-tailed) & .000 & .000 & \\
\hline & & Nifer & 74 & 74 & 74 \\
\hline
\end{tabular}

Tabl 22. Cydberthyniad Spearman ar gyfer Sgôr Rhan A, Sgôr Rhan B a Sgôr Gyfanswm.

\subsubsection{Anhawster yr eitemau unigol}

Er mwyn cadarnhau a yw'r prawf yn llwyddo i herio'r cyfranogwyr fwyfwy wrth fynd ymlaen trwy'r papur, lluniwyd siart bar sy'n rhestru eitemau yn ôl eu hanhawster, gyda'r eitemau anhawsaf ar ben y siart bar a'r eitemau hawsaf ar y gwaelod (gweler Atodiad 15). Nodir bod amrywiaeth fawr o ran anhawster yr eitemau. Wrth ddadansoddi'r siart bar ymhellach, nodir bod y traean uchaf o'r eitemau, sef y 14 eitem gyntaf, yn cynnwys 3 eitem o Ran A ac 11 eitem o Ran B. Dengys hyn fod y rhan fwyaf o'r eitemau anhawsaf yn perthyn i'r rhan anhawsaf, yn ôl y disgwyl. Fodd bynnag, diddorol yw nodi bod 9 o'r 14 eitem hawsaf yn perthyn i Ran B, ac mae 5 eitem yn perthyn i Ran A. Er bod nifer o'r eitemau anhawsaf yn ymddangos yn Rhan B, nodir bod nifer o eitemau haws yn ymddangos yn Rhan B hefyd. Yn ogystal â hyn, nodir bod enghreifftiau o eitemau yn Rhan A sy'n anos na rhai eitemau yn Rhan B. Er gwaetha'r ffaith nad yw anhawster yr eitemau yn dwysáu o eitem i eitem mor gyson ag y disgwyliwyd, mae'r canfyddiadau yn Atodiad 15 yn dangos bod amrywiaeth fawr o ran anhawster yr eitemau unigol.

\subsubsection{Cysondeb fewnol eitemau'r prawf}

Er mwyn mesur dibynadwyedd y prawf, penderfynwyd dadansoddi cysondeb fewnol eitemau'r prawf. Wrth ddadansoddi holl eitemau'r prawf yn y set ddata gyfan (43 eitem, 74 cyfranogwr) nodir bod gan y prawf gyfernod cydberthyniad (correlation 
co-efficient) uchel iawn, sef .920 (gweler Tabl 23 isod). Awgryma hyn fod eitemau'r prawf yn cydberthyn yn agos iawn ${ }^{74}$ pan ystyrir y prawf yn ei gyfanrwydd. Fodd bynnag, os ystyrir yr eitemau fesul grŵp, fel y gwneir hefyd yn Tabl 23, ymddengys fod y cyfernodau cydberthyniad ar gyfer Dosbarth A $(\alpha=.813)$ a Dosbarth B ( $\alpha=$ $.816)$ ychydig yn is na chyfernod cydberthyniad yr holl brawf $(\alpha=.920)$, a bod cyfernod cydberthyniad Dosbarth $\mathrm{C}(\alpha=.538)$ yn is fyth. Gan nad yw cyfernod cydberthyniad Grŵp C yn bodloni'r gofyniad lleiaf ar gyfer eitemau y gellir eu hystyried fel rhai sy'n cydberthyn, sef .7, ymddengys nad yw'r prawf hwn yn gwahaniaethu'n dda rhwng cyfranogwyr yn y dosbarth hwn.

Fodd bynnag, wrth ddadansoddi'r sgoriau'r papurau fesul dosbarth eto, nodir bod nifer sylweddol o eitemau nad ydynt wedi'u llenwi gan rai cyfranogwyr yn Dosbarth C. O blith y papurau lle mae un eitem na rhagor wedi'i hepgor gan gyfranogwyr yn Dosbarth C (16 papur allan o 28), nodir mai'r nifer gymedrig o eitemau sy'n cael eu hepgor yw 13.5. O blith y papurau lle mae un eitem neu ragor wedi'i hepgor gan gyfranogwyr yn Dosbarth A (20 papur allan o 26) a Dosbarth B (4 papur allan o 20) nodir mai'r nifer gymedrig o eitemau sy'n cael eu hepgor yw 8.3 a 8.8, yn ôl eu trefn. Mae'n debyg mai'r nifer uchel o fylchau sydd heb eu llenwi ym mhapurau Dosbarth C sy'n gyfrifol am darganfod cyfernod cydberthyniad is yn achos y grŵp hwn.

\begin{tabular}{|c|c|c|c|c|}
\hline \multirow[b]{2}{*}{ Dosbarth } & \multirow{2}{*}{$\begin{array}{c}\text { Cyfernod } \\
\text { Cydberthyniad } \\
\text { Crombach's alpha } \\
(\alpha)\end{array}$} & \multicolumn{2}{|c|}{ Sgôr Ganrannol Gymedrig (\%) } & \multirow[b]{2}{*}{$\begin{array}{l}\text { Cyfeirnod } \\
\text { Didoli }\end{array}$} \\
\hline & & Y Traean Uchaf & Y Traean Isaf & \\
\hline $\begin{array}{l}\text { Dosbarth Carlam Ysgol X } \\
(n=20)\end{array}$ & .816 & 79.0 & 53.2 & 25.8 \\
\hline $\begin{array}{l}\text { Dosbarth Set } 1 \text { Ysgol X } \\
(n=26)\end{array}$ & .813 & 47.3 & 21.4 & 25.9 \\
\hline $\begin{array}{l}\text { Dosbarth Set } 1 \text { Ysgol Y } \\
(n=28)\end{array}$ & .538 & 35.4 & 19.4 & 16.0 \\
\hline Prawf Cyfan & .92 & 63.6 & 21.7 & 42.0 \\
\hline
\end{tabular}

Tabl 23. Dadansoddi cysondeb mewnol yr eitemau a gallu'r eitemau $i$ wahaniaethu

\footnotetext{
${ }^{74}$ Noda Lowie a Seton $(2013$, t. 58) fod cyfernodau cydberthyniad sy'n uwch na .7 yn awgrymu bod gan eitemau'r prawf gydberthyniad uchel.
} 


\subsubsection{Gallu gwahaniaethu eitemau unigol y prawf}

Er mwyn darganfod a ydy eitemau unigol y prawf yn gwahaniaethu'n ddigonol rhwng lefelau hyfedredd gwahanol y cyfranogwyr, cyfrifwyd cyfeirnodau didoli (discrimination indices) ar gyfer pob eitem (gweler Atodiad 19). Er mwyn darganfod cyfeirnodau didoli pob eitem, cyfrifwyd sgoriau cymedrig traean uchaf $(n=25)$ a thraean isaf $(n=25)$ y cyfranogwyr ar gyfer pob eitem a thynnwyd sgoriau cymedrig y traean isaf oddi wrth sgoriau cymedrig y traean uchaf (Brown, 2003; Tremblay \& Garrison, 2010). O blith y 43 eitem ar y prawf, ymddengys mai ond 3 eitem nad yw'n gwahaniaethu yn ddigonol $(<20 \%$ o wahaniaeth) ac felly mae'n ymddangos fod y rhan fwyaf o'r eitemau yn gwahaniaethu'n ddigonol. ${ }^{75}$ Wrth ddadansoddi'r cyfeirnodau didoli eto yn Atodiad 19, sylwir eto fod Rhan B yn anos na Rhan A gan fod 11 eitem yn Rhan B lle nad oes neb yn nhraean isaf y cyfranogwyr wedi'u hateb yn gywir ${ }^{76}$ o'u cymharu â 3 eitem yn Rhan A. ${ }^{77} \mathrm{O}$ ystyried yr eitemau lle na chafodd cyfranogwyr yn y traean isaf sgôr gymedrig o 20\% neu fwy, gellir nodi 15 eitem arall sydd wedi peri trafferth, gyda sgoriau cymedrig llai na $20 \%{ }^{78}$ Sylwir hefyd mai 2 eitem yn unig sydd wedi peri trafferth i gyfranogwyr yn y traean uchaf. ${ }^{79}$ Mae'r canfyddiadau hyn yn atgyfnerthu'r casgliad y byddai'r prawf hwn yn fwy addas i'r rhai sydd â hyfedredd ieithyddol uwch na'r rhai sydd â hyfedredd ieithyddol is. Er ei bod yn bosibl symleiddio'r prawf hwn er mwyn ei wneud yn fwy addas i’r siaradwyr sydd â hyfedredd is, er enghraifft, trwy gynnig eitemau amlddewis, mae cadw eitemau'r prawf yn benagored yn sicrhau bod y prawf yn anos, sy'n bwysig os yw'r prawf i wahaniaethu rhwng y siaradwyr sydd â lefelau hyfedredd ieithyddol uwch. (Am drafodaeth lawnach, gweler 6.9.3.7. Ffurf y Prawf.)

\subsubsection{Trafodaeth o'r canlyniadau}

O ystyried canlyniadau'r prawf yn eu cyfanrwydd, ymddengys fod y tri dosbarth wedi perfformio yn ôl y disgwyl. Canfuwyd bod sgôr gyfanswm Dosbarth B (cymedr =65.9) yn llawer uwch o'i chymharu â sgoriau cyfanswm cymedrig Dosbarth A

\footnotetext{
${ }^{75}$ Defnyddir yma ddealltwriaeth Tremblay a Garrison (2010) a Tremblay (2011) o wahaniaethu annigonol fel cyfeirnod didoli sy'n llai na $20 \%$.

${ }^{76}$ Gweler eitemau 15, 17, 28, 29, 32, 34, 35, 36, 37, 38, 42

77 Gweler eitemau 10, 11, 13

${ }^{78}$ Gweler eitemau 1, 2, 6, 7, 14, 18, 19, 21, 22, 23, 24, 26, 39, 40, 43

${ }^{79}$ Gweler eitemau 24, 42
} 
$($ cymedr $=33.2)$ a Dosbarth C $($ cymedr $=27.0)$. Canfuwyd hefyd fod sgoriau Rhan A (cymedr $=72.9)$ a Rhan B (cymedr $=62.6)$ Dosbarth B gryn uwch o'u cymharu â sgoriau Dosbarth A (sgôr gymedrig Rhan A = 43.1; sgôr gymedrig Rhan B = 28.4) a Dosbarth C (sgôr gymedrig Rhan A = 36.0; sgôr gymedrig Rhan B = 22.7). Wrth archwilio sgoriau'r tair carfan fesul geiriau cynnwys a geiriau swyddogaethol, a fesul eitem, canfuwyd eto mai Dosbarth B a berfformiodd orau. Ymddengys o'r canfyddiadau hyn fod disgyblion MAT y Cwrs Carlam ar y blaen o ran eu hyfedredd ieithyddol cyffredinol yn y Gymraeg, o'u cymharu â'u cyfoedion prif ffrwd cyfrwng Saesneg yn yr un ysgol (Ysgol X) ac mewn un ysgol gymharus arall (Ysgol Y). Gellir hefyd gasglu bod gan y ddwy garfan o ddisgyblion CAI lefelau tebyg o hyfedredd ieithyddol cyffredinol yn y Gymraeg, gan eu bod wedi perfformio'n gymharol yn y prawf.

\subsubsection{Casgliadau ar y prawf llenwi bylchau fel mesurydd hyfedredd ieithyddol cyffredinol yn y Gymraeg}

\subsubsection{Dibynadwyedd y prawf}

Ar sail y canlyniadau a gasglwyd, gellir dadlau bod y prawf llenwi bylchau dan sylw yn llwyddo i fesur gwahanol lefelau hyfedredd ieithyddol cyffredinol disgyblion Cymraeg ail iaith. Dangoswyd bod y prawf yn ddibynadwy oherwydd bod eitemau'r prawf yn cydberthyn yn agos iawn pan ystyrir y set ddata yn ei chyfanrwydd ( $\alpha=$ .920). Yn ogystal â hyn, ymddengys fod y prawf llenwi bylchau yn addas ar gyfer gwahaniaethu rhwng disgyblion sydd â lefelau gwahanol o hyfedredd ieithyddol yn y Gymraeg. Fodd bynnag, ymddengys nad yw eitemau'r prawf yn gwahaniaethu'n dda rhwng disgyblion ar lefel y dosbarth, yn arbennig yn achos Dosbarth $\mathrm{C}(\alpha=.538)$. Gwelir canfyddiad tebyg yn astudiaeth Tremblay a Garrison (2010) a Tremblay (2011) a gasglodd nad yw'r prawf llenwi bylchau yn eu hastudiaeth hwy yn gwahaniaethu'n dda rhwng cyfranogwyr ar lefel y dosbarth ychwaith. Er bod lefelau hyfedredd y disgyblion yn gallu amrywio ar lefel y dosbarth, sy'n amlwg wrth sylwi ar amrediad y sgoriau cymedrig cyfanswm fesul dosbarth, mae'n bosibl nad yw'r prawf llenwi bylchau yn ddefnyddiol fel mesurydd hyfedredd iaith ar lefel y dosbarth os yw'r lefelau hyfedredd ieithyddol yn debyg i'w gilydd. Cred Tremblay (2011) mai gwell fyddai defnyddio'r prawf llenwi bylchau ar gyfer gwahaniaethu rhwng lefelau hyfedredd haenedig yn hytrach na lefelau hyfedredd tebyg: 
These last results suggest that the cloze test does not discriminate very well among L2 learners within classroom levels. The test is thus more appropriate for discriminating among coarsely grained proficiency levels, but it could also be used for ensuring that participants within a single classroom form a homogeneous group with respect to their proficiency in French.

(Tremblay, 2011, t. 356)

O'r safbwynt hwn, ymddengys fod y prawf llenwi bylchau dan sylw yn ddibynadwy a dilys gan ei fod yn gwahaniaethu'n dda rhwng disgyblion y Cwrs Carlam, sef Dosbarth B, a disgyblion y ddau grŵp rheoli, sef Dosbarth B a Dosbarth C. Dangoswyd hefyd fod anhawster yr eitemau yn amrywio'n fawr. Mae'n bosibl fod amrywiaeth o'r fath yn esbonio pam y gall profion llenwi bylchau wahaniaethu'n dda rhwng siaradwyr ail iaith sydd â lefelau gwahanol o hyfedredd ieithyddol (Tremblay \& Garrison, 2010). At hyn, dangosodd y cyfeirnodau didoli ar gyfer pob eitem, a gyflwynir yn Atodiad 19, fod gan y rhan fwyaf o'r eitemau yn y prawf y gallu i wahaniaethu'n ddigonol rhwng traean uchaf a thraen isaf y cyfranogwyr.

\subsubsection{Dilysrwydd y prawf}

Mae Brown (1980) yn nodi y gellir sicrhau dilysrwydd prawf llenwi bylchau wrth ystyried i ba raddau y mae'r prawf yn samplo cynnwys y pwnc. Mae Park (2011) yn ymhelaethu ar hyn wrth gynnig pedair ffactor y dylid eu rheoli, sef y deunyddiau, y dull dileu geiriau, nodweddion yr eitemau, a'r dull sgorio. Wrth ystyried dilysrwydd y prawf o'r safbwynt hwn, credir bod y prawf hwn yn ddilys gan fod y pedair ffactor hyn wedi'u rheoli'n effeithiol.

Fel y mae Brown (1984) yn ei ddadlau, mae'n bosibl mai dewis darn addas ar gyfer prawf llenwi bylchau yw'r ffactor bwysicaf. Yn hytrach na dewis sampl o destun addas fel sylfaen ar gyfer testun y prawf, penderfynwyd creu testun gwreiddiol er mwyn rheoli'r eirfa a'r patrymau ieithyddol, ynghyd â phwnc a chyd-destun y testun (gweler 6.9.3. Y prawf llenwi bylchau). Seiliwyd y testun ar lyfr iaith cyfredol a ddarparwyd gan Bennaeth Adran y Gymraeg mewn ysgol uwchradd cyfrwng Saesneg, yn ogystal ag awgrymiadau gan athrawon CAI. Cyfeiriwyd hefyd at y wefan www.cymraegailiaith.com wrth benderfynu ar gyd-destun y darn yn arbennig. Wrth seilio'r darn gwreiddiol ar y deunyddiau a'r adnoddau hyn, a'i adolygu ar ôl cynnal peilot, roedd hi'n bosibl samplo cynnwys CAI ym mlwyddyn 9 a'i osod o fewn thema gyfarwydd i bawb, sef chwaraeon. 
Yn ogystal â chreu darn gwreiddiol, defnyddiwyd dull dileu geiriau ar sail resymegol, er mwyn dileu nifer debyg o'r gwahanol fathau o eiriau cynnwys a geiriau swyddogaethol. Wrth ddileu geiriau yn y dull hwn, roedd hi'n bosibl sicrhau bod y prawf yn profi gwahanol fathau o eiriau posibl. Credir bod hyn yn bwysig os yw'r prawf am brofi hyfedredd ieithyddol cyffredinol y cyfranogwyr, yn hytrach na phrofi gramadeg penodol y cyfranogwyr wrth ganolbwyntio ar eitemau ieithyddol penodol megis berfau, enwau neu arddodiaid. Wrth adolygu'r prawf ar ôl cynnal peilot, roedd hi'n bosibl mireinio eitemau'r prawf ymhellach a chael gwared â nifer o eitemau nad oeddent yn gwahaniaethu. Fodd bynnag, rhaid nodi bod ambell eitem a gafodd eu dewis er mwyn gwahaniaethu rhwng y cyfranogwyr gorau yn benodol (gweler, er enghraifft, eitemau 13, 14, 43). Sicrhau cydbwysedd rhwng dileu geiriau sydd o fewn cyrraedd pawb ac ambell air sydd o fewn cyrraedd y perfformwyr gorau oedd un o'r heriau mwyaf wrth lunio prawf sy'n gwahaniaethu'n ddigonol rhwng disgyblion gwahanol lwybrau dysgu Cymraeg. Serch hynny, mae'r canfyddiad bod y rhan fwyaf o'r eitemau'n gwahaniaethu'n ddigonol yn atgyfnerthu'r honiad bod y prawf hwn yn samplo cynnwys priodol.

Er mwyn sicrhau bod y prawf yn cyd-fynd â lefel hyfedredd ieithyddol y cyfranogwyr, penderfynwyd defnyddio dull sgorio sy'n caniatáu unrhyw air sy’n gwneud synnwyr yn y cyd-destun. Er bod defnyddio'r dull sgorio hwn yn haws, ymddengys ei fod yn fwy addas a theg i siaradwyr ail iaith sydd â hyfedredd ieithyddol isaf na siaradwyr iaith gyntaf (Brown, 1980). Pe bai'r ymchwilydd yn ail farcio'r prawf wrth ddefnyddio'r dull sgorio sy'n caniatáu'r gair gwreiddiol yn unig, ymddengys o'r atebion a gynigwyd y byddai'r cyfranogwyr wedi perfformio gryn dipyn yn waeth. Credir bod y rheolaeth ar lunio'r darn gwreiddiol, dewis y geiriau i'w dileu, cadw eitemau'n benagored a defnyddio dull sgorio addas wedi sicrhau bod y prawf nid yn unig yn samplo'r cynnwys priodol, ond hefyd yn cyflwyno eitemau sydd yn gwahaniaethu rhwng lefelau hyfedredd ieithyddol y cyfranogwyr.

\subsubsection{Cyfyngiadau'r prawf}

Er gwaethaf canlyniadau trawiadol y prawf, rhaid bod yn ofalus wrth eu dehongli. Yn gyntaf, er gwaethaf pob ymdrech i sicrhau bod yr eitemau'n benagored, rhaid nodi nad yw'n bosibl gwneud hyn ar gyfer pob eitem. Yn achos ychydig iawn o'r eitemau, gellir cynnig un ateb cywir yn unig. Mae hyn yn arbennig o wir yn achos yr 
eitemau sy'n mynnu rhagenw, lle na ellir ateb yr eitem wrth gynnig unrhyw ragenw arall ar wahân i'r un y mae'r cyd-destun yn ei fynnu. Enghraifft o hyn yw eitem 9:

9. Maen _ nhw 'n mynd i'r clwb gyda ffrindiau.

Ynglŷn â'r sgoriau fesul dosbarth, rhaid nodi bod amrediad sgoriau'r tri dosbarth yn gorgyffwrdd â'i gilydd (gweler Tabl 8). Golyga hyn fod ambell gyfranogwr yn y ddau ddosbarth rheoli wedi perfformio'n well ar y prawf cyfan na rhai o'r cyfranogwyr yn y dosbarth carlam. Mae amrediad sgoriau'r tri dosbarth yn awgrymu y gall hyfedredd ieithyddol disgyblion amrywio hyd yn oed ar lefel y dosbarth. O ganlyniad, nid yw'n bosibl felly gasglu bod gan bob un o ddisgyblion y dosbarth carlam hyfedredd ieithyddol uwch na'u cyfoedion yn y ddau ddosbarth rheoli. Rhaid hefyd fod yn ofalus wrth ddehongli sgoriau eitemau fesul mathau o air gan ei bod yn bosibl cynnig math arall o air i'r hyn sydd ar goll ar gyfer rhai eitemau. Enghraifft o'r fath eitem yw eitem 28:

28. 'Mae gêm fawr gyda ni Er mai'r adferf 'heddiw' sydd wedi'i ddileu yn eitem 28, nodir bod enghreifftiau o gyfranogwyr sy'n cynnig enw, megis 'Siân' neu 'ffrind', yn ei le er mwyn cyfannu'r bwlch yn gywir. Pe bai'r ymchwilydd am ddadansoddi cryfderau a gwendidau'r cyfranogwyr wrth ganfod mathau penodol o eiriau, byddai'n rhaid felly reoli hyn a dehongli sgoriau ar gyfer eitemau o'r fath gyda gofal.

Er gwaetha'r dystiolaeth uchod sy'n awgrymu bod y prawf yn ddibynadwy a dilys fel mesurydd hyfedredd ieithyddol cyffredinol, cydnabyddir hefyd fod rhai cyfyngiadau ar yr hyn y gellir ei gasglu o'r prawf. Fel y nodwyd yn Pennod 6, cymerwyd gofal i sicrhau bod rhai eitemau yn mynnu gwybodaeth o fewn y frawddeg, ar draws brawddegau, ac o fannau eraill yn y testun. Er y gellid dadlau bod rhai o'r eitemau yn mynnu sgiliau ieithyddol uwch (15 eitem allan o 43), rhaid pwysleisio bod y rhan fwyaf o'r eitemau ar y prawf yn mynnu gwybodaeth o fewn brawddegau yn unig (28 eitem allan o 43). O'r herwydd, gellir casglu bod y prawf llenwi bylchau hwn yn mesur sgiliau ieithyddol is yn y Gymraeg yn bennaf, ac ni fydd yn addas ar gyfer grwpiau gwahanol heb ei addasu. Fodd bynnag, am mai diben y prawf oedd cymharu disgyblion sydd â hyfedredd ieithyddol sylfaenol yn y Gymraeg (gweler 6.9.3.8. Dull 
sgorio'r prawf), ymddengys fod y prawf yn ddilys ar gyfer yr ymchwil hon am ei bod gwahaniaethu'n dda rhwng lefelau ieithyddol y tri dosbarth o ddisgyblion.

Mae'n amlwg nad yw'r prawf yn mesur sgiliau llafar a chlywedol y cyfranogwyr. Ar gyfer ymchwil sy'n ceisio astudio sgiliau penodol fel y rhain, dyma gyfyngiad mawr (Tremblay, 2011; Tremblay \& Garrison, 2010). Pe bai modd cynnal cyfweliadau llafar a gwrando â'r cyfranogwyr, byddai cymhariaeth o sgoriau'r cyfweliadau hyn a sgoriau'r prawf llenwi bylchau, fel y gwnaed gan Bilki (2011), wedi atgyfnerthu dilysrwydd y prawf a chynnig gwell dealltwriaeth o hyfedredd ieithyddol cyffredinol y cyfranogwyr. Heb ymchwil bellach a chymariaethau rhwng sgoriau profion llenwi bylchau â sgoriau profion safonol sy'n mesur sgiliau ieithyddol penodol yn y Gymraeg, ni allai'r ymchwilydd ddod i gasgliad pendant ynghylch yr union sgiliau ieithyddol y mae'r prawf hwn yn eu mesur.

\subsection{Y prawf darllen Saesneg}

\subsubsection{Dull}

Casglwyd sgoriau darllen Saesneg y tair carfan o ddisgyblion oddi wrth yr ysgolion cyfranogol. Ar ôl casglu'r data, fe'u mewnbynnwyd i'r meddalwedd SPSS a chafodd y data eu grwpio fesul dosbarth, fel y gwelir yn Tabl 24. Fel y nodwyd yn Pennod 6, nid oedd modd paru sgoriau darllen Saesneg â sgoriau'r prawf llenwi bylchau fesul cyfranogwr am mai prawf dienw oedd y prawf llenwi bylchau. Oherwydd hynny, ceir dadansoddiad o'r data fesul dosbarth. Casglwyd data ar gyfer cyfanswm o 83 o ddisgyblion. O blith y disgyblion hyn, nodir bod 29 yn deillio o Dosbarth A, 21 yn deillio o Dosbarth B a 33 yn deillio o Dosbarth C. 


\begin{tabular}{|l|l|l|}
\hline \multicolumn{1}{|c|}{ Dosbarth } & \multicolumn{1}{|c|}{ Amledd } & Canran y disgyblion \\
\hline $\begin{array}{l}\text { Dosbarth A: } \\
\text { CAI Set 1 Ysgol X }\end{array}$ & 29 & 34.9 \\
Dosbarth B: & 21 & 25.3 \\
Cwrs Carlam Ysgol X & 33 & 39.8 \\
Dosbarth C: & & \\
CAI Set 1 Ysgol Y & 83 & 100.0 \\
\hline
\end{tabular}

Tabl 24. Nifer a chanran y disgyblion cyfranogol.

\subsubsection{Dadansoddi sgoriau darllen Saesneg}

\subsubsection{Gwasgariad y sgoriau fesul dosbarth}

Yn Tabl 25, gwelir dadansoddiad o sgoriau cymedrig y tair carfan o ddisgyblion yn y prawf darllen Saesneg. O ran Dosbarth A, nodir bod gwasgariad sgoriau'r dosbarth yn ganolog $($ cymedr $=98.69$; cyfeiliornad safonol o'r cymedr $=1.244)$ ond nodir bod sgiwedd positif bychan ( giwedd $=0.105$; cyfeiliornad safonol $=0.434$ ). Ymddengys hefyd fod cwrtosis y sgoriau o fewn yr amrediad gwallau (cwrtosis = .228; cyfeiliornad safonol $=.845)$. Er mwyn cadarnhau a yw gwasgariad y sgoriau'n normal, cynhaliwyd prawf Shapiro-Wilk. Dangosodd y prawf fod y data yn cael eu gwasgaru'n normal ( $p=$.648). Wrth archwilio'r histogram a'r plot Q-Q a gyflwynir yn Atodiad 20, ceir tystiolaeth bellach i gadarnhau bod y sgoriau darllen Dosbarth A yn cael eu gwasgaru'n normal.

O ran Dosbarth B, nodir bod gwasgariad eu sgoriau darllen yn weddol ganolog $($ cymedr $=115.43$; cyfeiliornad safonol o'r cymedr $=2.167)$ a nodir bod sgiwedd positif $($ sgiwedd $=1.271$; cyfeiliornad safonol $=0.501)$. Er bod cwrtosis y sgoriau yn bositif, mae hyn o fewn yr amrediad gwallau (cwrtosis = 1.650; cyfeiliornad safonol $=.972$ ). Dangosodd prawf Shapiro-Wilk fodd bynnag nad yw'r sgoriau yn cael eu gwasgaru'n normal ( $p=<0.05$ ). Yn Atodiad 21, gwelir bod y histogram a'r plot QQ yn cadarnhau bod sgoriau darllen y dosbarth yn gwyro'n sylweddol oddi wrth y normal. 
O ran Dosbarth C, gwelir bod gwasgariad y sgoriau darllen yn ganolog (cymedr $=$ 101.79; cyfeiliornad safonol o'r cymedr $=2.141)$ a bod sgiwedd positif bychan $($ sgiwedd $=.262$; cyfeiliornad safonol $=.409)$. Er bod cwrtosis y sgoriau yn negyddol, nodir bod hyn o fewn yr amrediad gwallau (cwrtosis = -.792; cyfeiliornad safonol = .798). Dangosodd prawf Shapiro-Wilk fod y sgoriau yn gwyro'n sylweddol oddi wrth y normal $(p=.206)$. Mae'r histogram a'r plot Q-Q yn Atodiad 22 hefyd yn cadarnhau'r canfyddiad hwn.

\begin{tabular}{|l|l|l|l|l|l|l|l|}
\hline \multicolumn{1}{|c|}{ Dosbarth } & Cymedr (\%) & $\begin{array}{c}\text { Cyfeiliornad } \\
\text { Safonol o'r } \\
\text { Cymedr }\end{array}$ & Amrediad & Sgiwedd & $\begin{array}{c}\text { Cyfeiliornad } \\
\text { Safonol o'r } \\
\text { Sgiwedd }\end{array}$ & Cwrtosis & $\begin{array}{c}\text { Cyfeiliornad } \\
\text { Safonol o'r } \\
\text { Cwrtosis }\end{array}$ \\
\hline $\begin{array}{l}\text { Dosbarth A: } \\
\text { CAI Set 1 } \\
\text { Ysgol X }\end{array}$ & 98.69 & 1.244 & $86-114$ & .105 & .434 & .228 & .845 \\
$\begin{array}{l}\text { Dosbarth B: } \\
\text { Cwrs Carlam } \\
\text { Ysgol X }\end{array}$ & 115.43 & 2.167 & $100-140$ & 1.271 & .501 & 1.650 & .972 \\
$\begin{array}{l}\text { Dosbarth C: } \\
\text { CAI Set 1 } \\
\text { Ysgol Y }\end{array}$ & 101.79 & 2.141 & $78-123$ & .262 & .409 & -.792 & .798 \\
\hline
\end{tabular}

Tabl 25. Sgoriau darllen Saesneg fesul dosbarth.

\subsubsection{Trafodaeth o'r canlyniadau}

Mae Tabl 25 uchod yn datgelu mai dosbarth y Cwrs Carlam (Dosbarth B) a berfformiodd orau ar gyfartaledd yn y prawf darllen Saesneg (cymedr $=115.4$ ), a bod y ddau ddosbarth arall (Dosbarth A Dosbarth C) wedi perfformio'n debyg iawn i'w gilydd (cymedr Dosbarth A = 98.7; cymedr Dosbarth C = 101.8). Serch hynny, rhaid nodi bod gwahaniaeth nodweddiadol rhwng y ddau ddosbarth rheoli o ran amrediad eu sgoriau (amrediad Dosbarth A = 28; amrediad Dosbarth $\mathrm{C}=45$ ). Nodir hefyd fod amrediad uchel o sgoriau yn Dosbarth B (amrediad $=40$ ). Gan fod disgyblion y ddau ddosbarth arall yn ddosbarthiadau CAI Set 1, rhagdybiwyd y byddai amrediad sgoriau'r ddau ddosbarth yn debyg i'w gilydd. Fodd bynnag, mae'r canfyddiadau uchod yn dangos bod lefelau darllen yn y Saesneg yn amrywio'n fwy yn Dosbarth C o'u cymharu â Dosbarth A. Mae'r amrediad uchel o ran sgoriau darllen Saesneg yn achos dosbarth y Cwrs Carlam, sef Dosbarth B, yn awgrymu nad yw'r sgoriau hyn yr 
un mor bwysig â'r ffactorau eraill a ystyria Ysgol X wrth ddethol disgyblion ar gyfer y Cwrs Carlam.

\subsection{Data Prydau Ysgol am Ddim}

Yn Tabl 26, gwelir hawliau'r disgyblion i PYDd fesul dosbarth. Nodir mai ond chwe disgybl sydd â'r hawl i PYDd (7.2\% o'r set ddata gyflawn), gan gynnwys dau ddisgybl yn Dosbarth B (9.5\% o'r dosbarth cyfan) a phedwar disgybl yn Dosbarth C (12.1\% o'r dosbarth cyfan). Awgryma'r canfyddiadau hyn felly nad oes un o'r tri dosbarth a gymerodd ran yn y prawf llenwi bylchau o dan unrhyw anfantais sosioeconomaidd amlwg.

\begin{tabular}{|c|c|c|c|c|c|}
\hline \multirow{2}{*}{\multicolumn{2}{|c|}{ Hawl i Brydau Ysgol am Ddim }} & \multicolumn{3}{|c|}{ Dosbarth } & \multirow[b]{2}{*}{ Cyfanswm } \\
\hline & & $\begin{array}{c}\text { Dosbarth A: } \\
\text { CAI Set } 1 \\
\text { Ysgol X }\end{array}$ & $\begin{array}{c}\text { Dosbarth B: } \\
\text { Cwrs Carlam } \\
\text { Ysgol X }\end{array}$ & $\begin{array}{c}\text { Dosbarth C: } \\
\text { CAI Set } 1 \\
\text { Ysgol Y }\end{array}$ & \\
\hline \multirow[t]{2}{*}{ Oes } & Nifer & 0 & 2 & 4 & 6 \\
\hline & $\%$ o fewn Darpariaeth & $0.0 \%$ & $9.5 \%$ & $12.1 \%$ & $7.2 \%$ \\
\hline \multirow[t]{2}{*}{ Nac oes } & Nifer & 29 & 19 & 29 & 77 \\
\hline & $\%$ o fewn Darpariaeth & $100.0 \%$ & $90.5 \%$ & $87.9 \%$ & $92.8 \%$ \\
\hline \multicolumn{2}{|c|}{ Cyfanswm } & 29 & 21 & 33 & 83 \\
\hline \multicolumn{2}{|l|}{$\%$} & $100.0 \%$ & $100.0 \%$ & $100.0 \%$ & $100.0 \%$ \\
\hline
\end{tabular}

Tabl 26. Hawl i Brydau Ysgol am Ddim fesul dosbarth.

\subsection{Trafodaeth a chasgliadau}

Fel y nodwyd yn Pennod 6, penderfynwyd dadansoddi sgoriau'r prawf llenwi bylchau a'r prawf darllen Saesneg er mwyn cyflwyno proffiliau ieithyddol ar gyfer y tair carfan o ddisgyblion ar draws Ysgol X ac Ysgol Y. Trwy gyflwyno proffiliau ieithyddol ar gyfer y tair carfan o ddisgyblion, anelir at ddysgu rhagor am sgiliau ieithyddol disgyblion y rhaglen CAI draddodiadol a'r Cwrs Carlam yn y Gymraeg a'r Saesneg. Yn ogystal â dadansoddi proffiliau ieithyddol, penderfynwyd hefyd ddadansoddi data PYDd y disgyblion cyfranogol er mwyn amlygu cefndiroedd sosioeconomaidd y tair carfan o ddisgyblion. Yn Tabl 27 isod, crynhoir proffiliau ieithyddol a sosio-economaidd y disgyblion cyfranogol fesul dosbarth. 


\begin{tabular}{|c|c|c|c|}
\hline Dosbarth & $\begin{array}{c}\text { Prawf llenwi bylchau } \\
\text { (\%) }\end{array}$ & $\begin{array}{l}\text { Prawf darllen Saesneg } \\
\qquad \%)\end{array}$ & $\begin{array}{c}\text { Hawl i PYDd } \\
(\%)\end{array}$ \\
\hline $\begin{array}{l}\text { Dosbarth A: } \\
\text { CAI Set } 1 \text { Ysgol X }\end{array}$ & 33.2 & 98.7 & 0.0 \\
\hline $\begin{array}{l}\text { Dosbarth B: } \\
\text { Cwrs Carlam Ysgol X }\end{array}$ & 65.9 & 115.4 & 9.5 \\
\hline $\begin{array}{l}\text { Dosbarth C: } \\
\text { CAI Set } 1 \text { Ysgol Y }\end{array}$ & 27.0 & 101.8 & 12.1 \\
\hline
\end{tabular}

Tabl 27. Trosolwg o broffiliau ieithyddol a sosio-economaidd y disgyblion cyfranogol fesul dosbarth

\subsubsection{Proffiliau ieithyddol y disgyblion cyfranogol}

O ystyried canlyniadau'r prawf cyfannu a'r prawf darllen Saesneg yn eu cyfanrwydd, ymddengys fod y tri dosbarth wedi perfformio yn ôl y disgwyl. O ran y prawf llenwi bylchau, canfuwyd bod sgôr gyfanswm disgyblion y Cwrs Carlam $($ cymedr $=65.9)$ yn llawer uwch o'i chymharu â sgoriau cyfanswm cymedrig y ddau ddosbarth CAI Set 1 (Dosbarth A, cymedr = 33.2; Dosbarth C, cymedr = 27.0). Canfuwyd hefyd fod sgoriau Rhan A (cymedr $=72.9)$ a Rhan B (cymedr $=62.6)$ y dosbarth carlam gryn uwch o'u cymharu â sgoriau Dosbarth A (sgôr gymedrig Rhan A =43.1; sgôr gymedrig Rhan B = 28.4) a Dosbarth C (sgôr gymedrig Rhan A = 36.0; sgôr gymedrig Rhan B = 22.7). Wrth archwilio sgoriau'r tri dosbarth fesul geiriau cynnwys a geiriau swyddogaethol, a fesul eitem, canfuwyd eto mai Dosbarth B a berfformiodd orau. O ran y prawf darllen Saesneg, ymddengys eto fod y tri dosbarth wedi perfformio yn ôl y disgwyl, gyda disgyblion y Cwrs Carlam yn sicrhau sgôr gyfanswm gymedrig ychydig yn uwch (cymedr $=115.43$ ) o'u cymharu â'r ddau ddosbarth CAI Set 1 yn Ysgol X (cymedr = 98.69) ac Ysgol Y (101.79).

Dengys y canfyddiadau hyn yn feintiol fod y disgyblion sydd yn derbyn y Cwrs Carlam ar y blaen o ran eu sgiliau ieithyddol, yn y Gymraeg ac yn y Saesneg, o'u cymharu â'r disgyblion CAI yn yr un ysgol, sef Ysgol X, ac mewn un ysgol 
gymharus arall, sef Ysgol Y. Dengys y canfyddiadau hyn hefyd fod gan ddisgyblion y ddau ddosbarth CAI traddodiadol sgiliau ieithyddol tebyg. Nid oes modd i'r astudiaeth hon brofi i ba raddau mai oherwydd darpariaeth ychwanegol y Cwrs Carlam, ynteu oherwydd ffactorau eraill, megis y math o ddysgwyr sy'n cael astudio'r cwrs a'u cymhelliant, y mae gan ddysgwyr y ffrwd hon sgiliau ieithyddol uwch yn y ddwy iaith. (Am drafodaeth lawnach ar 'achosaeth', gweler Pennod 6.) Er hynny, gellir dadlau bod y canfyddiadau hyn yn cyd-fynd â'r honiadau a drafodwyd yn Pennod 1 a 4, hynny yw, po fwyaf yw'r mewnbwn a geir, y mwyaf yw'r allbwn. Fel y nodwyd yn Pennod 7, mae disgyblion y Cwrs Carlam yn derbyn 10 awr ychwanegol o gyswllt â'r iaith Gymraeg y flwyddyn, o'u cymharu â'u cyfoedion prif ffrwd cyfrwng Saesneg, a hynny trwy astudio pum pwnc yn ddwyieithog. Gellid dadlau bod astudio pum pwnc yn ddwyieithog, yn ogystal ag astudio'r Gymraeg fel pwnc, yn cynnig rhagor o gyfleoedd i'r dysgwyr hyn ddysgu'r iaith yn echblyg (hynny yw, dysgu gyda ffocws ar ffurf iaith) ac ymhlyg (hynny yw, dysgu heb ffocws penodol ar ffurf iaith). (Am drafodaeth lawnach ar hyn, gweler 9.4.2.1. Proffiliau ieithyddol a sosio-ieithyddol.)

\subsubsection{Proffiliau sosio-economaidd y disgyblion cyfranogol}

Daeth i'r amlwg wrth ddadansoddi data PYDd y tri dosbarth na cheir tystiolaeth i ddangos bod gan ddisgyblion y Cwrs Carlam a gyfrannodd at yr astudiaeth hon statws sosio-economaidd uwch o'u cymharu â'u cyfoedion prif ffrwd cyfrwng Saesneg yn Ysgol X ac Ysgol Y. Wrth ddadansoddi'r data PYDd, canfuwyd mai ychydig iawn o ddisgyblion oedd yn gymwys i gael PYDd, sef dau ddisgybl yn Dosbarth B a phedwar disgybl yn Dosbarth C. Gan nad oedd yn bosibl paru data PYDd y disgyblion â'u sgoriau prawf llenwi bylchau priodol, nid oedd modd cadarnhau a oes cydberthynas ystadegol rhwng y ddau newidyn hyn ar lefel yr unigolyn. Fodd bynnag, wrth ystyried bod dau o'r disgyblion hyn yn deillio o'r dosbarth a berfformiodd orau yn y prawf llenwi bylchau, sef y dosbarth carlam, ymddengys nad oes cydberthynas amlwg rhwng statws sosio-economaidd y tri dosbarth a'u perfformiad yn y ddau brawf.

Cydnabyddir mai sampl fechan sydd dan sylw yn yr astudiaeth hon. Byddai dadansoddiad o statws sosio-economaidd disgyblion eraill sydd wedi dilyn y Cwrs Carlam yn y blynyddoedd diwethaf wedi datgelu rhagor am gefndiroedd sosio- 
economaidd y disgyblion hyn. Fel y nodwyd yn Pennod 6, cydnabyddir hefyd y byddai mesur o statws sosio-economaidd rhieni'r plant wedi bod yn well fesur o lefelau sosio-economaidd y disgyblion cyfranogol. Gan ystyried nad oes modd profi achosiaeth yn yr astudiaeth hon, nodir hefyd na all yr ymchwilydd ddod i gasgliad pendant am y gydberthynas rhwng statws sosio-economaidd a pherfformiad yn y prawf llenwi bylchau a'r prawf darllen Saesneg. 


\section{Pennod 9: Casgliadau}

\subsection{Y cyd-destun polisi ac arfer}

Daeth i'r amlwg wrth adolygu'r llenyddiaeth yn Penodau 1 a 2 fod gagendor yn bodoli o hyd rhwng yr hyn a fynegir mewn polisi, sef gweledigaeth LlC (2017b) ar gyfer cyrraedd miliwn o siaradwyr Cymraeg erbyn 2050, a'r hyn a wneir mewn arfer ar hyn o bryd yn yr ysgolion cyfrwng Saesneg yng Nghymru. Os yw LlC am gyflawni'r uchelgais a gyflwynir yn Cymraeg 2050, rhaid sicrhau bod cyfundrefn addysg yn ei lle sydd yn creu siaradwyr newydd yn y Gymraeg, yn ogystal â chynnal siaradwyr rhugl. Er y bydd gan y sector cyfrwng Cymraeg rôl hanfodol o ran creu a chynnal siaradwyr Cymraeg, rhaid cydnabod bod “cyfraniad pwysig i'w wneud gan y sector cyfrwng Saesneg" hefyd wrth wireddu'r nod (L1C, 2017, t. 38). O ystyried bod $67 \%$ o ysgolion cynradd a $76 \%$ o ysgolion uwchradd Cymru yn ysgolion cyfrwng Saesneg yn bennaf (Stats Cymru, 2017b), ${ }^{80}$ daw i'r amlwg y bydd angen rhoi pwyslais penodol ar rôl y sector cyfrwng Saesneg wrth greu siaradwyr newydd yn y Gymraeg. Fodd bynnag, gan nad yw'r model traddodiadol ar gyfer cyflwyno'r Gymraeg fel ail iaith mewn ysgolion cyfrwng Saesneg yn llwyddo, ar ei ben ei hun, i ddatblygu disgyblion yn siaradwyr Cymraeg erbyn diwedd eu haddysg orfodol, rhaid ystyried sut arall y gall ysgolion cyfrwng Saesneg gefnogi cyflwyno'r Gymraeg yn llwyddiannus.

\subsection{Yr ymchwil ryngwladol: Addysg drochi a CLIL}

At ei gilydd, dengys ymchwil ryngwladol fod dysgu iaith yn fwy effeithiol pan fydd yn cael ei gyfuno â dysgu cynnwys (Harris \& Ó Duibhir, 2011). Yn fwy penodol, mae canfyddiadau'r ymchwil ryngwladol a drafodwyd yn awgrymu bod disgyblion sy'n dilyn y rhaglen CLIL yn perfformio'n well na'u cyfoedion mewn rhaglen draddodiadol o ran eu sgiliau darllen a deall yn yr iaith darged a hefyd o ran maint eu geirfa yn yr iaith darged. Fodd bynnag, ymddengys o'r dystiolaeth a adolygwyd nad yw disgyblion CLIL yn perfformio'n sylweddol well na'u cyfoedion rhaglen draddodiadol, er enghraifft, o ran gramadeg. Gwelwyd tystiolaeth bellach o'r llenyddiaeth ar addysg drochi i gefnogi defnyddio'r iaith darged fel cyfrwng addysgu

\footnotetext{
${ }^{80}$ Mae'r ffigyrau hyn yn cynnwys ysgolion cynradd ac uwchradd sydd wedi'u categoreiddio naill ai'n 'ysgolion cyfrwng Saesneg yn bennaf ond â defnydd sylweddol o'r Gymraeg', neu'n 'ysgolion cyfrwng Saesneg yn bennaf'. (Am ddiffiniadau o'r categorïau hyn, gweler 2.6.1. Diffinio ysgolion yn ôl eu darpariaeth cyfrwng Cymraeg.)
} 
er mwyn datblygu'r ail iaith ymhellach na'r hyn sy'n bosibl trwy'r rhaglen draddodiadol. Yn fwy eang, mae'r canfyddiadau hyn ar addysg ddwyieithog yn cydfynd â'r canfyddiadau ymchwil ar ddwyieithrwydd a drafodwyd yn Pennod 3, sy'n awgrymu bod nifer o fanteision ieithyddol ac anieithyddol i fod yn ddwyieithog.

Nid yw'n glir o'r ymchwil ryngwladol eto i ba raddau mai oherwydd cyrchddull CLIL yn benodol (hynny yw, pedagogeg y cyrchddull), ynteu oherwydd yr amser ychwanegol a geir mewn cyswllt â'r iaith darged trwy'r gwersi CLIL, y mae rhaglenni CLIL (gyda gwersi ail iaith traddodiadol) i'w gweld yn fwy effeithiol na gwersi ail iaith traddodiadol yn unig. Er hynny, cafwyd hyd i dystiolaeth sy'n awgrymu mai po fwyaf yw'r mewnbwn yn yr iaith darged, y mwyaf yw'r allbwn. Mae'n amlwg mai un o brif fanteision CLIL yw'r ffaith ei fod yn gallu sicrhau nifer uwch o oriau cyswllt a'r iaith, a bod hyn yn ei dro yn cynyddu'r cyfleoedd i ddysgwyr ddysgu'r iaith yn echblyg ac ymhlyg trwy ddefnyddio'r iaith darged fel cyfrwng dysgu. Mae'n debyg, fodd bynnag, fod isafswm o oriau cyswllt ychwanegol, efallai 300 awr neu ragor (Artieda ac eraill, 2017; Muñoz, 2015), dros gyfnod estynedig y mae eu hangen ar ddysgwyr CLIL er mwyn iddynt elwa ar y mewnbwn ychwanegol hwn. Fel y casgl Fitzpatrick ac eraill (2018, t. 61): "Dangosir bod CLIL fwyaf effeithiol pan fydd wedi'i gyfuno â mwy o gysylltiad â'r iaith a phan fydd hyn yn digwydd yn gyson am gyfnod hwy."

Themâu ymchwil eraill a nodwyd yn yr ymchwil ryngwladol oedd effaith dysgu elfennau cwricwlaidd trwy gyfrwng yr iaith darged ar ddatblygiad iaith gyntaf y disgyblion a'u cyrhaeddiad academaidd mewn pynciau a astudiwyd drwy gyfwng yr iaith darged. Unwaith eto, mae'r dystiolaeth a ganfuwyd yn awgrymu nad yw disgyblion addysg drochi rannol hwyr o dan anfantais o ran datblygiad yr iaith gyntaf, o'u cymharu â'u cyfoedion prif ffrwd sy'n astudio trwy gyfrwng yr iaith gyntaf. Ymddengys fod presenoldeb yr iaith gyntaf fel iaith fwyafrifol y tu hwnt i'r dosbarth yn y gymuned yn sicrhau na chaiff addysg trwy gyfrwng yr ail iaith effaith negyddol ar hyfedredd disgyblion CLIL yn yr iaith gyntaf. O ran effaith addysg drochi hwyr ar gyrhaeddiad academaidd, cafwyd hyd i dystiolaeth sy'n dangos bod disgyblion y rhaglen hon yn fwy araf ar y cychwyn o ran eu datblygiad academaidd yn y pynciau sy'n fwy heriol eu hiaith, megis y Dyniaethau a'r Gwyddorau, os caiff y pynciau hyn eu cyflwyno yn yr iaith darged. Mae'r llenyddiaeth ar raglenni CLIL hefyd yn tueddu i lunio darlun cymhleth o ran effaith CLIL ar gyrhaeddiad 
academaidd yn gyffredinol. Bydd angen ymchwil bellach er mwyn archwilio effaith rhaglenni CLIL ar ddisgyblion o lefelau academaidd gwahanol (Breidbach \& Viebrock, 2012; Zydatiß, 2012), gan gynnwys y rhai sy’n tangyflawni (Apsel, 2012) a'r rhai sydd yn mewnfudo i Gymru gyda Chymraeg a Saesneg fel ieithoedd ychwanegol.

\subsection{Yr ymchwil genedlaethol: Cynllun Ysgol X}

Wrth ymchwilio'r gwahanol gynlluniau trochi a dysgu dwys sydd wedi'u treialu yng Nghymru yn Pennod 5, daeth yr ymchwilydd o hyd i gynllun unigryw sydd ar waith mewn ysgol uwchradd cyfrwng Saesneg yn Ne Cymru. Wrth adolygu'r llenyddiaeth ryngwladol ar raglenni addysg drochi a CLIL yn Pennod 4, amlygwyd y ffaith fod y Cwrs Carlam yn Ysgol X yn rhannu nifer o nodweddion â'r rhaglenni hyn, yn enwedig y rhaglenni CLIL yn yr Almaen. Yn wahanol i'r cynlluniau trochi a dysgu dwys eraill a adolygwyd yng nghyd-destun Cymru, sydd wedi anelu at greu pwyntiau mynediad hwyr i addysg cyfrwng Cymraeg, mae Ysgol X wedi mynd ati'n rhagweithiol i wella safonau CAI o fewn y sector cyfrwng Saesneg. Wrth ddatblygu'r Gymraeg fel cyfrwng addysgu ac iaith bob dydd o fewn yr ysgol, caiff disgyblion Ysgol X y cyfle i ddefnyddio'r Gymraeg mewn modd cyfathrebol ar draws yr ysgol. Mae darpariaeth ychwanegol i ddisgyblion MAT hefyd yn sicrhau bod modd i'r disgyblion hyn ddefnyddio'r Gymraeg fel cyfrwng dysgu ar draws rhannau helaeth o'r cwricwlwm, yn ogystal â'i hastudio fel pwnc trwy wersi CAI traddodiadol. Ar sail canfyddiadau'r adolygiad llenyddiaeth, penderfynwyd cynnal ymchwil empirig i archwilio'r cynllun ar waith yn Ysgol X, a'i darpariaeth garlam yn benodol, ac yn fwy eang, astudio sut y mae Ysgol X ac un ysgol gymharus, sef Ysgol Y, yn cefnogi cyflwyno'r Gymraeg fel ail iaith yn eu cyd-destunau eu hunain.

\subsection{Prif ganfyddiadau'r ymchwil empirig a'u cyfraniad at y llenyddiaeth}

Mae gan yr ymchwil hon ganfyddiad pwysig i'r llenyddiaeth ym maes CAI, ynghyd â'r llenyddiaeth ym meysydd dysgu a chaffael ail iaith. Yn fwy penodol, nodir bod yr ymchwil hon yn cyfrannu at y llenyddiaeth ryngwladol ar CLIL ac addysg drochi hwyr, a phrofi ac asesu ail iaith. Yn ogystal â chrynhoi'r prif ganfyddiadau ymchwil felly, trafodir yma sut y mae'r ymchwil hon yn cyfrannu at y meysydd hyn. 


\subsubsection{Mewnbwn}

Wrth ddadansoddi'r holiadur, y cyfweliadau a'r arsylwadau yn Pennod 7, trafodwyd canfyddiadau'r ymchwil empirig ochr yn ochr â chanfyddiadau yn Pennod 1 er mwyn archwilio sut y mae'r ddwy ysgol gyfranogol yn cefnogi cyflwyno'r Gymraeg fel ail iaith. Amlygwyd yn y dadansoddiad y gellir priodoli'r ffactorau canlynol yn benodol i lwyddiant Ysgol X o ran cyflwyno'r Gymraeg yn llwyddiannus yn yr ysgol:

i. Ymagwedd effeithiol a chyson at ddatblygu ei hethos dwyieithog. Mae Ysgol $\mathrm{X}$ yn hyrwyddo a hybu defnyddio'r Gymraeg nid yn unig fel cyfrwng addysgu, ond hefyd fel iaith bob dydd yn yr ysgol. Mae'n amlwg fod yr ymagwedd tuag at ddatblygu ethos dwyieithog yn dod o'r uwch dîm rheoli sy'n rhoi statws craidd i'r Gymraeg fel disgyblaeth ac iaith bob dydd yn yr ysgol.

ii. Nifer sylweddol o staff sydd yn medru siarad ac addysgu yn Gymraeg. Mae Ysgol X yn gallu manteisio ar y staff hyn er mwyn addysgu elfennau o'r cwricwlwm trwy gyfrwng y Gymraeg.

iii. Adnoddau ariannol. Daeth i'r amlwg wrth ymchwilio i Ysgol X ei bod yn gallu cyflogi dwy athrawes beripatetig a chynnig darpariaeth hyfforddiant iaith Gymraeg er mwyn datblygu sgiliau dwyieithog y staff.

iv. Trefniadau pontio effeithiol ar gyfer y Gymraeg. Mae cynllun pontio Ysgol X rhwng CA2 a 3 wedi gwella a chysoni safonau disgyblion cynradd y clwstwr mewn Cymraeg erbyn iddynt gyrraedd Blwyddyn 7.

Mae canfyddiadau'r ymchwil hon yn cadarnhau'r hyn y sylwyd arno yn yr ymchwil ragarweiniol ar Ysgol X yn Pennod 5. Mae Ysgol X wedi'i chanmol yn benodol am fod yn fodel o arfer da o ran datblygu ethos dwyieithog (Estyn, 2006b, 2012a, 2016a, Y Pwyllgor Menter a Dysgu, 2010), ac am effeithiolrwydd ei chynllun pontio o ran gwella safonau CAI (Estyn, 2006b, 2012a, 2016a, Hopkins, 2006; Y Pwyllgor Menter a Dysgu, 2010). Yn ogystal â hyn, mae'r ymchwil gyfredol yn cynnig tystiolaeth sy'n gefnogi'r canfyddiadau ar arfer ym maes CAI yn Pennod 2. Efallai mai'r prif ganfyddiad y mae'r ymchwil yn ei gefnogi yw'r angen am fuddsoddiad sylweddol mewn hyfforddiant i athrawon (Davies, 2013; Y Pwyllgor Menter a Dysgu, 2010; C. Williams, 2008). Heb sicrhau bod yr hyfforddiant priodol ar gael i 
athrawon, mae'n anodd rhagweld sut y gall ysgolion cyfrwng Saesneg lwyddo i integreiddio'r Gymraeg yn fwyfwy ar draws y cwricwlwm newydd.

At ei gilydd, dengys canfyddiadau'r ymchwil hon fod ymgorffori ymagwedd holistaidd at ddatblygu'r Gymraeg, nid yn unig fel cyfrwng dysgu ar draws y cwricwlwm, ond hefyd fel iaith fyw a ddefnyddir bob dydd ar draws yr ysgol gyfan yn hollbwysig er mwyn cefnogi cyflwyno'r Gymraeg fel ail iaith yn y sector cyfrwng Saesneg. Rhaid cofio mai rhan o gynlluniau ehangach Ysgol X yw'r Cwrs Carlam, ac felly mae'n hollbwysig ystyried yr agweddau eraill ar gyflwyno Cymraeg ar wahân i'r ddarpariaeth ddwyieithog a gynigir trwy'r Cwrs Carlam. Byddai hyn yn arbennig o allweddol pe ba ysgolion a'u chymunedau ehangachi brynu i mewn i CLIL. Fel y crynhoa Fitzpatrick ac eraill (2018):

... byddai unrhyw symudiad tuag at ddatblygu CLIL yng nghyd-destun Cymru yn ei gwneud hi'n ofynnol trafod â chymuned ehangach yr ysgol i sicrhau bod cefnogaeth i ddatblygiadau o'r math hwn, gan gynnwys sylweddoli y byddai goblygiadau ar gyfer hyfforddiant/datblygiad athrawon. (Fitzpatrick ac eraill, 2018, t. 61)

\subsubsection{Allbwn}

\subsubsection{Proffiliau ieithyddol a sosio-economaidd}

Yn y dadansoddiad a wnaed o sgoriau'r tri dosbarth yn y prawf llenwi bylchau a'r prawf darllen Saesneg yn Pennod 8, canfuwyd bod gan ddisgyblion y ddau ddosbarth CAI Set 1 yn Ysgol X ac Ysgol Y broffiliau ieithyddol cymharus. Yn y prawf llenwi bylchau, cyflawnodd y ddau ddosbarth sgoriau cyfanswm cymedrig o $33.2 \%$ a $27.0 \%$, yn ôl eu trefn. Yn y prawf darllen Saesneg, cyflawnodd y ddau dosbarth hyn sgoriau cymedrig o 98.7 a 101.8, yn ôl eu trefn. O’u cymharu â hyn, canfuwyd bod gan ddisgyblion y Cwrs Carlam broffil ieithyddol gwahanol. Cyflawnodd disgyblion y Cwrs Carlam sgôr gyfanswm gymedrig ddwywaith yn fwy yn y prawf llenwi bylchau, sef $65.9 \%$, a sgôr gymedrig ychydig yn uwch yn y prawf darllen Saesneg, sef 115.4. Ar ôl dadansoddi sgoriau'r prawf llenwi bylchau ymhellach, fesul rhan prawf a math o air, cadarnhawyd hefyd mai dosbarth y Cwrs Carlam a berfformiodd orau o'r tri dosbarth yn yr astudiaeth, wrth i'r ddau ddosbarth CAI traddodiadol berfformio'n weddol gymharus ar y gwahanol agweddau ar y prawf. Dengys y canfyddiadau hyn yn feintiol fod gan ddisgyblion y Cwrs Carlam sgiliau ieithyddol 
uwch yn y Gymraeg a'r Saesneg o'u cymharu â'u cyfoedion sydd ond yn astudio CAI fel pwnc yn y ffordd draddodiadol.

Nid oes tystiolaeth yn yr astudiaeth hon i ddangos i ba raddau mai oherwydd darpariaeth ychwanegol y Cwrs Carlam, ynteu oherwydd ffactorau eraill sy'n effeithio ar gaffael ail iaith yn effeithiol, megis deallusrwydd y dysgwyr, eu gwybodaeth flaenorol o'r Gymraeg, a'u cymhelliant o ran dysgu'r Gymraeg, y mae gan ddysgwyr y Cwrs Carlam sgiliau ieithyddol uwch yn y Gymraeg. Fel y trafodwyd yn Pennod 6, nid diben yr astudiaeth hon oedd gwerthuso'r Cwrs Carlam, ond amlygu'r proffiliau ieithyddol gwahanol rhwng dysgwyr y Cwrs Carlam a dysgwyr CAI traddodiadol. Oherwydd hynny, nid oedd modd rheoli'r gwahanol newidynnau a dadansoddi eu heffaith ar y canlyniadau hyn.

Fodd bynnag, o ystyried y gwahaniaethau sylweddol a amlygwyd rhwng y tair carfan o ddysgwyr o ran eu proffiliau ieithyddol, a'u hyfedredd ieithyddol cyffredinol yn y Gymraeg yn benodol, dadleuir bod tystiolaeth yn yr astudiaeth hon sy'n cyd-fynd â'r honiad mai po fwyaf yw'r mewnbwn a geir, y mwyaf yw'r allbwn. Fel y canfuwyd yn Pennod 7, caiff disgyblion y Cwrs Carlam 14 awr o gyswllt â'r Gymraeg yr wythnos, trwy ei hastudio fel pwnc a'i defnyddio fel cyfrwng dysgu cynnwys pynciol, o'u cymharu â'u cyfoedion prif ffrwd cyfrwng Saesneg sydd ond yn astudio'r Gymraeg fel pwnc hyd at 4 awr yr wythnos. Golyga hyn y caiff disgyblion y Cwrs Carlam 380 awr ychwanegol o oriau cyswllt â'r iaith y flwyddyn, sydd hefyd yn cyd-fynd â honiad Muñoz (2015) ac Artieda ac eraill (2017) fod angen o leiaf 300 o oriau cyswllt ychwanegol er mwyn i ddysgwyr elwa ar fanteision y mewnbwn ychwanegol hwn.

Yn y dadansoddiad a wnaed o ddata PYDd yn Pennod 8, ni chanfuwyd tystiolaeth i ddangos bod gan ddosbarth y Cwrs Carlam statws sosio-economaidd uwch o'u cymharu â'u cyfoedion prif ffrwd cyfrwng Saesneg yn Ysgol X ac Ysgol Y. O'r chwe chyfranogwr a oedd yn gymwys i dderbyn PYDd, canfuwyd bod dau o'r rheini yn dod o'r dosbarth carlam, a bod pedwar yn dod o'r dosbarth CAI Set 1 yn Ysgol Y. Er y cydnabyddir mai sampl fechan iawn oedd dan sylw yn y dadansoddiad hwn ac nad oedd modd paru sgoriau'r ddau brawf â'r data PYDd ar lefel yr unigolyn, mae'r canfyddiad hwn o leiaf yn awgrymu nad oes cydberthynas amlwg rhwng proffiliau ieithyddol a sosio-economaidd y tair carfan o ddysgwyr ar lefel ddosbarth. Fodd 
bynnag, bydd angen ymchwil bellach i allu cadarnhau'n ystadegol p'un a oes cydberthnas rhwng proffiliau sosio-economaidd a phroffiliau ieithyddol neu beidio.

\subsubsection{Y prawf llenwi bylchau fel mesurydd hyfedredd ieithyddol cyffredinol yn y Gymraeg}

Ar sail canlyniadau'r prawf llenwi bylchau a gyflwynwyd yn Pennod 8, casglwyd bod y prawf hwn yn un dibynadwy a dilys. Dangoswyd, er enghraifft, fod eitemau'r prawf yn cydberthyn â'i gilydd yn agos iawn pan ystyrir y set ddata yn ei chyfanrwydd $(\alpha=.920)$. Ar ben hyn, dangoswyd bod y prawf llenwi bylchau yn ddibynadwy ac yn ddilys fel prawf hyfedredd ieithyddol cyffredinol am ei fod yn gallu gwahaniaethu'n dda rhwng lefelau gwahanol o ddisgyblion, a hynny oherwydd bod anhawster yr eitemau'n amrywio'n ddigonol. Wrth seilio testun y prawf llenwi bylchau a luniwyd ar ddeunyddiau megis llyfryn iaith Blwyddyn 9, ac wrth ymgynghori ag athrawon CAI ac arbenigwr profion darllen Cymraeg, roedd yn bosibl dylunio prawf dilys sydd yn samplo cynnwys y pwnc CAI, gan gynnwys geirfa, patrymau ieithyddol a themâu / pynciau cyfarwydd i ddisgyblion CAI. Yn ogystal â hyn, nodir bod y dull sgorio a ddefnyddiwyd, lle caniateir unrhyw air sy'n gwneud synnwyr yn y cyd-destun, yn un dilys ac addas am iddo sicrhau bod anhawster y prawf yn cyd-fynd â lefelau hyfedredd ieithyddol y cyfranogwyr.

At ei gilydd, ymddengys fod y prawf llenwi bylchau yn ddull ymchwil addas, dibynadwy a dilys ar gyfer mesur gwahanol lefelau hyfedredd ieithyddol cyffredinol dysgwyr ail iaith yn y Gymraeg. Fodd bynnag, gan nad oedd yn bosibl cymharu sgoriau'r profion llenwi bylchau â sgoriau mewn profion safonol sy'n mesur sgiliau ieithyddol penodol yn y Gymraeg, ni ellir casglu o'r dadansoddiad pa gyfraniad sydd gan y sgiliau ieithyddol gwahanol at gwblhau'r prawf cyfrannu. Rhaid cydnabod hefyd nad yw'r prawf llenwi bylchau yn ymddangos yn fesurydd dibynadwy ar gyfer y sgiliau llafar a gwrando yn benodol, a dyma gyfyngiad amlwg ar y prawf hwn. Fodd bynnag, ceir o'r canfyddiadau ymchwil dystiolaeth sy'n cefnogi'r honiad fod y prawf llenwi bylchau yn un integredig (Bachman, 1982; Hanania \& Shikhani, 1986; Jonz, 1990; Laesch \& Kleeck, 1987). Mae'r prawf llenwi bylchau hefyd yn ymddangos yn ddibynadwy a dilys am ei fod yn gallu gwahaniaethu rhwng dysgwyr ail iaith sydd â lefelau gwahanol o hyfedredd ieithyddol (Tremblay, 2011; Tremblay 
\& Garrison, 2010), os yw'r prawf wedi'i gynllunio'n fedrus (Fotos, 1991; Hanania \& Shikhani, 1986; Sasaki, 2000; Tremblay \& Garrison, 2010).

\subsubsection{Casgliadau ar y Cwrs Carlam}

Yng nghyd-destun yr astudiaeth hon, ymddengys y Cwrs Carlam yn gynllun unigryw sy'n llwyddo i sicrhau bod disgyblion MAT yn ennill lefelau uchel o hyfedredd ieithyddol cyffredinol yn y Gymraeg. Trwy astudio gwersi CLIL, yn ogystal â gwersi ail iaith traddodiadol, mae disgyblion y Cwrs Carlam yn derbyn rhagor o gyswllt â'r Gymraeg na'r hyn y mae disgyblion CAI traddodiadol yn ei dderbyn, a hynny heb amharu ar nifer yr oriau dysgu a neilltuir i gyflwyno pynciau eraill o fewn y cwricwlwm. Mae'r Cwrs Carlam yn enghraifft o CLIL ar waith yn y cyd-destun cyfrwng Saesneg yng Nghymru. Oherwydd hynny, cred yr ymchwilydd y dylai LlC gynnal gwerthusiad cyflawn o'r Cwrs Carlam yn Ysgol X er mwyn archwilio effeithiolrwydd CLIL, o'i gymharu'n uniongyrchol â'r rhaglen CAI gyfredol. Ymddengys CLIL yn gyrchddull hyblyg y gellir ystyried ei efelychu mewn ysgolion cyfrwng Saesneg ar lefel genedlaethol maes o law er mwyn sicrhau rhagor o gyswllt â'r iaith Gymraeg (Fitzpatrick ac eraill, 2018; Somers, 2018b), heb amharu ar nifer yr oriau dysgu a neilltuir yn yr amserlen i gyflwyno pynciau eraill o fewn y cwricwlwm newydd. Os mai nod LlC yw sicrhau bod gan ddysgwyr ail iaith "sgiliau ffwythiannol effeithiol" yn y Gymraeg erbyn iddynt adael addysg orfodol, fel yr awgrymwyd mewn adroddiad ymchwil diweddar (Fitzpatrick ac eraill, 2018, t. 7), mae'n debyg y gall CLIL fod yn ffordd bosibl o ddatblygu cymhwysedd ffwythiannol (Muñoz, 2002; Pérez-Cañado, 2012). Er hynny, bydd angen cynnal ymchwil bellach i astudio effaith gwersi CLIL ar ddisgyblion o lefelau academaidd gwahanol (Breidbach \& Viebrock, 2012; Zydatiß, 2012), gan gynnwys y rhai sy’n tangyflawni (Apsel, 2012), cyn y gellir argymell efelychu'r cyrchddull CLIL ar lefel genedlaethol yng Nghymru. Bydd yn rhaid i LlC hefyd sicrhau bod y disgwyliadau ar gyfer y sector cyfrwng Saesneg yn glir, er mwyn sicrhau bod CLIL, fel cyrchddull addysgu ail iaith, yn addas ar gyfer y nod. Ar hyn o bryd, nid yw'n gwbl glir beth fydd y disgwyliadau ar gyfer dysgwyr CAI. 


\subsection{Goblygiadau a heriau}

Wedi trafod prif ganfyddiadau'r ymchwil hon a'u cyfraniad at y llenyddiaeth, trafodir yma oblygiadau'r ymchwil i bolisi ac arfer o ran addysgu'r Gymraeg fel ail iaith. Yn benodol, trafodir yr heriau y mae ysgolion cyfrwng Saesneg yn eu hwynebu o ran symud yn nes at ddatblygu'n ysgolion dwyieithog, effaith posibl efelychu CLIL ar addysg cyfrwng Cymraeg leol, yn ogystal â'r goblygiadau ynghylch datblygu un continwwm Cymraeg i'w weithredu mewn ysgolion. Bydd y drafodaeth hon, ynghyd â'r prif ganfyddiadau ymchwil a grynhowyd uchod, yn ffurfio seilwaith i'r argymhellion a gyflwynir ar ddiwedd y bennod hon.

\subsubsection{Y sector cyfrwng Saesneg}

Fel y trafodwyd yn y cyflwyniad i'r bennod hon, nid yw'r gyfundrefn bresennol ar gyfer cyflwyno'r Gymraeg fel ail iaith yn ddigonol i gyflawni uchelgais Cymraeg 2050, sef sicrhau bod o leiaf 70 y cant o ddysgwyr yn medru siarad Cymraeg erbyn iddynt adael yr ysgol. Fel y mae'r straetegaeth newydd ei hun yn ei nodi, rhaid "gweddnewid sut rydym yn addysgu Cymraeg i bob dysgwr" (L1C, 2017b, t. 12), os am gyflawni'r nod uchelgeisiol hwn. Wrth gofio bod y newidiadau hyn i'r ffordd y cyflwynir y Gymraeg fel ail iaith yn digwydd yng nghyd-destun ehangach datblygu cwricwlwm newydd i Gymru (gweler 9.5.3. Cwricwlwm i Gymru a'r continwwm Cymraeg am drafodaeth lawnach), rhaid cydnabod yr heriau y mae'r sector hwn yn eu hwynebu.

Efallai mai'r her fwyaf y mae'r sector yn ei hwynebu, fel yr amlygwyd yn Pennod 2, yw'r diffyg ymarferwyr sydd â'r sgiliau priodol i addysgu Cymraeg fel ail iaith ac i addysgu cynnwys cwricwlaidd yn ddwyieithog. Cydnebydd yr ymchwilydd y bydd angen rhoi blaenoriaeth benodol i ddarparu hyfforddiant methodoleg addysgu Cymraeg ac addysgu dwyieithog i athrawon newydd, trwy Hyfforddiant Cychwynnol, ac i athrawon cyfredol, trwy Hyfforddiant Mewn Swydd, yng Nghymru. Bydd angen buddsoddiad sylweddol mewn hyfforddiant $\mathrm{i}$ athrawon yn y sector hwn, a hynny ar frys, os yw ysgolion cyfrwng Saesneg i ymateb yn briodol i'r galw ar iddynt symud yn bellach ar hyd y continwwm ieithyddol.

Her arall y mae ysgolion cyfrwng Saesneg yn ei hwynebu yw'r diffyg statws a roddir i'r Gymraeg, nid yn unig fel pwnc craidd yn y cwricwlwm, ond hefyd fel iaith achlysurol. Wrth ystyried bwriad LIC (2017b) i ddileu'r pwnc CAI a rhoi yn ei le un 
continwwm Cymraeg, credir y bydd yn angenrheidiol i ysgolion cyfrwng Saesneg gryfhau statws y Gymraeg, ar lefel ffurfiol ac anffurfiol, trwy ddatblygu ethos gwirioneddol ddwyieithog. Heb os, proses hirdymor fydd datblygu ethos dwyieithog anffurfiol ysgolion cyfrwng Saesneg yn yr ardaloedd cymharol ddi-Gymraeg. Fodd bynnag, os yw ysgolion cyfrwng Saesneg i symud yn bellach ar hyd y continwwm ieithyddol tuag at addysg ddwyieithog a chyfrwng Cymraeg, bydd yn rhaid iddynt o leiaf weithredu polisi ffurfiol ar ddwyieithrwydd. Byddai hyn yn hollbwysig i sicrhau y caiff y Gymraeg ei thrin fel pwnc craidd a bod dwyieithrwydd yn cael ei normaleiddio o ddydd i ddydd. Rhaid hefyd i LlC ystyried yn ofalus sut y byddai'n trin y Gymraeg yn nhermau mesur perfformiad, pe bai un continwwm Cymraeg ym mhob ysgol, er mwyn sicrhau y caiff deilliannau mewn Cymraeg eu cymharu'n deg ar draws y sector cyfrwng Saesneg a'r sector cyfrwng Cymraeg.

Rhaid hefyd i ysgolion cynradd ac uwchradd ystyried sut y mae cydweithio'n fwy effeithiol er mwyn datblygu dilyniant ieithyddol dysgwyr o'r naill sector i'r llall. O ystyried y canfyddiad fod prinder arbenigedd a hyder o ran addysgu ac asesu'r Gymraeg fel ail iaith ymhlith athrawon mewn ysgolion cynradd cyfrwng Saesneg, yn arbennig yn yr ardaloedd cymharol ddi-Gymraeg, mae'n amlwg y bydd y cydweithredu hwn rhwng y ddau sector yn hanfodol er mwyn gwella safonau Cymraeg a sicrhau dilyniant ieithyddol effeithiol. Cydnabyddir y byddai disgwyl i ysgolion uwchradd ysgwyddo rhywfaint o'r cyfrifoldeb am addysgu'r Gymraeg yn effeithiol yn y sector hwnnw a phontio rhwng y naill sector a'r llall yn y tymor byr, wrth i'r continwwm Cymraeg gael ei dreialu a'i ddatblygu. Fodd bynnag, byddai'n rhaid i ysgolion cynradd gymryd mwy o gyfrifoldeb am addysgu'r Gymraeg ac addysgu trwy gyfrwng y Gymraeg maes o law wrth i sgiliau'r gweithlu ymateb i'r anghenion hyfforddi angenrheidiol a nodwyd uchod. Os yw ysgolion cyfrwng Saesneg i gyflwyno'r continwwm Cymraeg yn ei gyfanrwydd yn llwyddiannus felly, bydd y cydweithredu rhwng ysgolion cynradd ac uwchradd yn y sector cyfrwng Saesneg yn hollbwysig.

\subsubsection{Y sector cyfrwng Cymraeg}

Yn ogystal â'r goblygiadau i'r sector cyfrwng Saesneg wrth symud ysgolion ar hyd y continwwm ieithyddol, rhaid hefyd gydnabod y gall fod goblygiadau i'r sector cyfrwng Cymraeg. Nodwyd yn Pennod 2 fod cryn bryder am ddysgwyr sy'n trosglwyddo o'r sector cyfrwng Cymraeg i'r sector cyfrwng Saesneg ac effaith hynny 
ar eu datblygiad ieithyddol yn y Gymraeg. Nodwyd pryder tebyg am y cynllun yn Ysgol X yn yr ymchwil ddisgrifiadol yn Pennod 5. Yn ôl Davies (2010), mae'r disgyblion hynny sy'n trosglwyddo o'r ysgolion cynradd Cymraeg i Ysgol X o dan anfantais o'u cymharu â'u cyfoedion sy'n parhau i astudio yn y sector cyfrwng Cymraeg oherwydd na chânt eu hymestyn yn ieithyddol trwy astudio ystod eang o bynciau yn Gymraeg a chymdeithasu yn yr iaith drwy'r diwrnod ysgol.

Cydnabyddir bod y pryder hwn yn un dilys a rhaid rhoi ystyriaeth lawn i sut mae gwella dilyniant ieithyddol yn y sector cyfrwng Cymraeg er mwyn lleihau nifer y dysgwyr sydd yn trosglwyddo i'r sector cyfrwng Saesneg. Yn wir, mae gwella dilyniant ieithyddol dysgwyr o'r naill Gyfnod Allweddol i'r llall wedi bod yn thema gyson trwy gydol oes y Strategaeth Addysg Cyfrwng Cymraeg (2010), hyd nes i'r strategaeth ddod i ben yn 2015. Fodd bynnag, rhaid pwysleisio nad diben datblygu addysg cyfrwng Cymraeg o fewn ysgolion uwchradd cyfrwng Saesneg yw tynnu dysgwyr oddi wrth y sector cyfrwng Cymraeg. Yn hytrach, diben datblygu addysg cyfrwng Cymraeg yn y sector cyfrwng Saesneg yw sicrhau gwell addysg yn y Gymraeg i ddysgwyr y sector hwn. Fodd bynnag cydnabyddir bod gan rieni'r hawl i symud eu plant o'r naill gyfrwng i'r llall. Oherwydd hynny, rhaid sicrhau y gall pob dysgwr ddatblygu ei sgiliau Cymraeg, hyd yn oed os ydynt yn trosglwyddo o'r cyfrwng Cymraeg i'r cyfrwng Saesneg (Davies, 2013, t. 28). Ym marn yr ymchwilydd, gwell fyddai datblygu capasiti ysgolion cyfrwng Saesneg i gynnig o leiaf peth addysg Gymraeg a chreu awyrgylch dwyieithog i'r disgyblion hynny sydd wedi penderfynu trosglwyddo o addysg cyfrwng Cymraeg. Byddai hyn yn sicrhau y gallai'r disgyblion hynny symud yn bellach ar hyd y continwwm dwyieithog nag sy'n bosibl wrth astudio'r pwnc CAI yn unig, fel a ddigwydd yn y rhan fwyaf o ysgolion cyfrwng Saesneg ar hyn o bryd. Er pwysiced cynllunio dilyniant dysgwyr yn y sector cyfrwng Cymraeg, ni ellir gwrthod gwell darpariaeth Gymraeg i'r mwyafrif o ddysgwyr mewn ysgolion cyfrwng Saesneg. Serch hynny, cydnebydd yr ymchwilydd y bydd yn rhaid i'r sectorau cyfrwng Saesneg a chyfrwng Cymraeg gydweithio'n agosach â'i gilydd, â'u cymunedau ysgol, yn enwedig wrth i'r continwwm Cymraeg a Maes Dysgu a Phrofiad 'Ieithoedd, Llythrennedd a Chyfathrebu y cwricwlwm newydd gael eu sefydlu, er mwyn lleihau nifer y dysgwyr sydd yn symud o'r naill gyfrwng dysgu i'r llall. 


\subsubsection{Cwricwlwm i Gymru a'r continwwn Cymraeg}

Adeg cwblhau'r traethawd ymchwil hwn, cafodd Canllaw $i$ Gwricwlwm Drafft $i$ Gymru 2022 (2019a), ynghyd â'i ddogfennau cysylltiedig, megis Cwricwlwm Drafft $i$ Gymru 2022: Ieithoedd, Llythrennedd a Chyfathrebu (2019b), eu cyhoeddi gan Y Gweinidog Addysg, Kirsty Williams, a chychwynodd ymgynghoriad cenedlaethol ar y cwricwlwm drafft. Yn y dogfennau hyn, cyflwynir y manylion cyntaf am strwythur ac elfennau'r cwricwlwm newydd. Bydd y cwricwlwm newydd, sydd wrthi'n cael ei ddatblygu gan LIC mewn cydweithrediad ag athrawon ac ymarferwyr yr Ysgolion Arloesol $^{81}$ a rhanddeiliaid eraill, yn cael ei weithredu erbyn 2022. Nodir y bydd y cwricwlwm newydd yn cael ei drefnu o amgylch chwe Maes Dysgu a Phrofiad, ${ }^{82}$ gyda Chymraeg, Saesneg ac ieithoedd rhyngwladol yn cael eu cynllunio o dan 'Ieithoedd, Llythrennedd a Chyfathrebu'. Nodir hefyd y bydd y cwricwlwm newydd yn dileu'r Cyfnodau Allweddol gan gyflwyno yn eu lle continwwm dysgu ar gyfer y pedwar Maes Dysgu a Phrofiad a Chamau Cynnydd i fesur dysgwyr wrth iddynt symud ar hyd y continwwm dysgu hwn.

Yng nghyd-destun addysgu'r Gymraeg fel ail iaith, gellir dadlau bod cyhoeddi'r cwricwlwm drafft yn gam filltir pwysig iawn. Cynebydd L1C (2019a, t.13) mai “[u]n o brif nodweddion pedwar diben y cwricwlwm yw datblygu dysgwyr uchelgeisiol, galluog sy'n gallu cyfathrebu'n effeithiol mewn gwahanol ffurfiau a lleoliadau, drwy'r Gymraeg a'r Saesneg." Yn fwy penodol, nodir bod galw ar bob ysgol i ddatblygu'r Gymraeg trwy ei hintegreiddio'n drawsbynciol yn y cwricwlwm newydd. Fel y pwysleisia L1C (2019a, t. 13): "Dylai lleoliadau ac ysgolion gefnogi datblygiad sgiliau dysgwyr yn y Gymraeg ar draws y cwricwlwm cyfan." Cydnebydd LlC bellach nad pwnc yn unig yw'r Gymraeg ac y dylai pob athro fod yn gyfrifol am ddatblygu llythrennedd deuol disgyblion yn y Gymraeg a'r Saesneg ar draws y cwricwlwm cyfan. Mae'r datblygiadau hyn i gyd yn atgyfnerthu'r ddadl dros ymchwilio i CLIL a'i defnyddio er mwyn sicrhau rhagor o gyswllt â'r Gymraeg yng nghyd-destun dysgu ac addygsu CAI.

\footnotetext{
${ }^{81}$ Rhwydwaith o ysgolion sydd yn arwain ar ddylunio a datblygu'r gwahanol elfennau ar y cwricwlwm newydd yw'r Ysgolion Arloesi. Diddorol yw nodi bod dwy ysgol gyfranogol yr ymchwil empirig wedi cyfrannu at waith y rhwydwaith hwn.

${ }^{82} \mathrm{Y}$ chwe Maes Dysgu a Phrofiad a gyflwynir yn y cwricwlwm drafft yw'r Celfyddydau Mynegiannol; y Dyniaethau; Gwyddoniaeth a Thechnoleg; Iechyd a Lles; Ieithoedd, Llythrennedd a Chyfathrebu; a Mathemateg a Rhifedd.
} 
Er ei bod yn debyg bellach mai "fframwaith cynnydd mewn ieithoedd" sy'n “[d]echrau gyda dim iaith neu heb fawr ddim iaith a gweithio tuag at ruglder" (L1C, 2019b, t.10) yw'r continwwm arfaethedig a drafodwyd yn Pennod 2, rhaid nodi bod nifer o faterion y bydd yn rhaid i LIC eu hystyried cyn cyflwyno'r cwricwlwm newydd terfynol. Er enghraifft, sut y caiff perfformiad dysgwyr Cymraeg mewn lleoliadau cyfrwng Saesneg a chyfrwng Cymraeg ei fesur yn erbyn un continwwm? Beth fydd y disgwyliadau ar gyfer dysgwyr yn y naill sector a'r llall? Sut y bydd y continwwm yn cael ei ddatblygu fel sail ar gyfer asesu ieithoedd modern eraill yn y dyfodol? A fydd disgwyl i ysgolion ddatblygu llythrennedd triphlyg maes o law? Sut y bydd un cymhwyster newydd yn y Gymraeg yn cael ei alinio â'r Maes Dysgu a Phrofiad Ieithoedd, Llythrennedd a Chyfathrebu? A fydd alinio'r cymhwyster mewn Cymraeg, ynghyd â'r ITM, â Fframwaith Cyfeirio Ewropeaidd Cyffredin "yn helpu i ddarparu sylfaen ar gyfer continwwm”? (Cymwysterau Cymru, d.d., t. 8). Mae'n amlwg nad yw'r cysyniad o 'gontinwwm' na'r goblygiadau ar gyfer asesu ar hyd continwwm eto'n gwbl glir. Ond hyd nes y bydd y continwwm Cymraeg yn cael ei ddiffinio'n fwy clir, ni fydd modd dylunio cymhwyster Cymraeg newydd na'i ofynion (Cymwysterau Cymru, d.d., t. 2).

Rhaid i LlC hefyd ddiffinio'r hyn a olyga wrth 'siaradwyr Cymraeg' mewn perthynas â'i strategaeth Cymraeg 2050: Miliwn o siaradwyr mewn perthynas ag addysg. Ai disgyblion sydd â lefel benodol o hyfedredd yn y Gymraeg yw ystyr ‘siaradwyr Cymraeg' yma, ynteu ddisgyblion sy'n defnyddio'r Gymraeg yn gyson? Heb egluro ystyr 'siaradwyr Cymraeg', anodd iawn fydd cynllunio'r continwwm Cymraeg, y cymhwyster Cymraeg newydd a'r disgwyliadau ieithyddol priodol ac ymarferol i ddisgyblion y sector cyfrwng Saesneg a'r sector cyfrwng Cymraeg. Ymddengys fod nifer o ystyriaethau y mae angen eu harchwilio'n llawnach o hyd. Serch hynny, mae'r diwygiad cyfredol o'r cwricwlwm yng Nghymru yn cynnig cyfle i LlC fynd i'r afael â'r ystyriaethau hyn a chynorthwyo ysgolion cyfrwng Saesneg wrth iddynt fynd ati i wella'r ffordd y maent yn cyflwyno'r Gymraeg ac ieithoedd eraill yn y dyfodol. 


\section{Pennod 10: Argymhellion}

Daw o'r ymchwil gyfredol nifer o ganfyddiadau a goblygiadau pwysig i feysydd arfer CAI, polisi addysgu'r Gymraeg yng Nghymru, a meysydd addysg drochi a CLIL. Yn ogystal â hyn, nodir bod gan yr ymchwil hon gyfraniad at faes profi ac asesu ail iaith. Ar sail y canfyddiadau a'r ystyriaethau a drafodwyd uchod, cyflwynir yma argymhellion yr ymchwilydd ynglŷn â chyfeiriadau i bolisi ac arfer o ran addysgu'r Gymraeg fel ail iaith, ynghyd â'i argymhellion ar gyfer ymchwil bellach.

\subsection{Cyfeiriadau at bolisi ac arfer}

\subsubsection{Gwella darpariaeth CAI mewn addysg cyfrwng Saesneg}

- Wrth i LlC fynd ati i ddatblygu'r cwricwlwm newydd, a Maes Dysgu a Phrofiad 'Ieithoedd, Llythrennedd a Chyfathrebu' yn benodol, cynghorir iddi roi ystyriaeth i sut y gellir gwella darpariaeth CAI mewn ysgolion cyfrwng Saesneg trwy gynnydu'r oriau cyswllt â'r Gymraeg y mae dysgwyr CAI yn eu derbyn. Mae canfyddiadau'r astudiaeth hon, ynghyd â chanfyddiadau ymchwil gan eraill mewn cyd-destunau addysg drochi a CLIL, wedi cynnig tystiolaeth gref o blaid cyflwyno cynnwys cwricwlaidd trwy gyfrwng yr ail iaith. Mae ymchwil ar fewnbwn ieithyddol mewn perthynas â chaffael ail iaith hefyd wedi dangos bod amlder y mewnbwn yn yr iaith darged yn cyd-fynd â pha mor gyflym y mae'r dysgwr yn ei chaffael.

- Un ffordd o gynyddu'r cyswllt â'r iaith Gymraeg mewn lleoliadau cyfrwng Saesneg, fel yr amlygwyd yn yr ymchwil hon, yw CLIL. Ymddengys CLIL yn gyrchddull hyblyg ac effeithiol y gellid ystyried ei efelychu mewn ysgolion cyfrwng Saesneg ar lefel genedlaethol maes o law er mwyn sicrhau rhagor o gyswllt â'r Gymraeg, heb amharu ar yr oriau dysgu a neilltuir i gyflwyno pynciau eraill. Er hynny, rhaid cydnabod y bydd angen cynnal ymchwil bellach ar effaith CLIL yng nghyd-destun CAI yng Nghymru (gweler 10.2. Yr angen am ymchwil bellach). Os yw LlC am i ysgolion cyfrwng Saesneg symud tuag at ddefnyddio maes o law, bydd rhaid rhoi ystyriaeth benodol i'r goblygiadau posibl ar gyfer hyfforddiant a datblygiad proffesiynol athrawon y sector cyfrwng Saesneg yn benodol (gweler 10.1.3. Gwella'r cyfleoedd hyfforddi i staff addysgu a gweinyddu'r sector cyfrwng Saesneg). 
- Un o'r prif ddatblygiadau ym maes CAI yw'r datblygiad o un continwwm dysgu Cymraeg i bawb, fel rhan o Faes Dysgu a Phrofiad 'Ieithoedd, Llythrennedd a Chyfathrebu' y cwricwlwm newydd. Fel y trafodwyd yn Pennod 2, ymddengys fod peth ansicrwydd o ran ystyr a diffiniad y term 'continnwm', yn sicr yng nghyd-destun y cwricwlwm newydd i Gymru. Er ei bod yn debyg mai fframwaith cynnydd yw ystyr y term, dylai LlC gynnig diffiniad clir o'r hyn a olygir ganddo yng nghyd-destun y cwricwlwm newydd.

- Yn ogystal â hyn, dylid ystyried sut y mae alinio'r continwwm Cymraeg arfaethedig ag un cymhwyster Cymraeg i bawb. Fel y nodwyd yn Pennod 9, heb ddiffiniad clir o'r hyn a olygir gan 'gontinwwm', anodd fydd dylunio'r cymhwyster newydd a'i ofynion. Dylai LIC hefyd ystyried sut y bydd yn mesur a chymharu perfformiad mewn Cymraeg ar draws ysgolion cyfrwng Saesneg a chyfrwng Cymraeg. Ym marn yr ymchwilydd, bydd yn rhaid i LlC bennu disgwyliadau clir ar gyfer y ddau sector o ran deilliannau cyflawniad mewn perthynas â'r Gymraeg. Cynghorir LlC i ystyried alinio asesu perfformiad mewn Cymraeg a'r ITM â, er enghraifft, lefelau Fframwaith Cyfeirio Ewropeaidd Cyffredin er mwyn cysoni asesu ieithoedd yng Nghymru â safonau Ewropeaidd. Gallai hyn yn ei dro roi gwell syniad i gyflogwyr yng Nghymru o broffil ieithyddol pobl ifanc erbyn iddynt adael ysgol ac ymuno â'r byd gwaith.

- Er mwyn cyd-fynd â'r datblygiadau o ran y continwwm a'r cwricwlwm newydd, cynghorir LlC i ddatblygu set o brofion darllen Cymraeg cenedlaethol newydd a fyddai'n addas ar gyfer mesur dilyniant ieithyddol dysgwyr y sector cyfrwng Saesneg a'r sector cyfrwng Cymraeg hefyd. Canfuwyd yn Pennod 7 nad oes profion darllen Cymraeg ar gael ar hyn o bryd i ddysgwyr y sector cyfrwng Saesneg. Golyga hyn na chaiff y dysgwyr hyn eu hasesu'n ffurfiol yn y Gymraeg tan ddiwedd Blwyddyn 6, a hynny gan athrawon nad ydynt yn arbenigwyr CAI gan amlaf (gweler Pennod 1). Os yw LlC am sefydlu un continwwm dysgu'r Gymraeg, cred yr ymchwilydd y bydd angen datblygu profion Cymraeg newydd i'w defnyddio er mwyn mapio dilyniant ieithyddol dysgwyr ail iaith o'r cynradd i'r uwchradd. 


\subsubsection{Datblygu ethos dwyieithog o fewn ysgolion cyfrwng Saesneg}

- Yn ogystal â mynd i'r afael â gwella darpariaeth CAI, mae'n hollbwysig ystyried sut y gellir cefnogi cyflwyno'r Gymraeg mewn ffyrdd gwahanol yn yr ysgol, hynny yw, trwy ddatblygu ethos dwyieithog cryf, gwella'r cyfleoedd hyfforddi i staff a chryfau'r terfniadau pontio rhwng y cynradd a'r uwchradd. Cynghorir LlC i ystyried sut y gall gefnogi ysgolion i ymgorffori ymagwedd holistaidd at ddatblygu'r Gymraeg nid yn unig fel cyfrwng dysgu ar draws y cwricwlwm ond hefyd fel iaith fyw a ddefnyddir yn rheolaidd ar draws yr ysgol gyfan. Yn hyn o beth, braf yw gweld bod egwyddorion y Siarter Iaith yn cyd-fynd â chanllawiau Cwricwlwm i Gymru (LlC, 2019a, 2019b). Cynghorir LlC, mewn cydweithrediad â'r pedwar consortiwm addysg, i ystyried ymestyn gwaith Siarter Iaith Cymraeg Campus i bob ysgol cynradd cyfrwng Saesneg yng Nghymru maes o law.

- Cynghorir ysgolion cyfrwng Saesneg a'r consortia addysg hefyd i gydweithio er mwyn cynllunio a gweithredu strategaethau clir ar gyfer datblygu ethos dwyieithog o fewn yr ysgol. Yng nghyd-destun yr ymchwil hon, diffinnir 'ethos dwyieithog' fel agweddau, ymddygiadau ac arferion ffurfiol ac anffurfiol aelodau'r ysgol tuag at ddwyieithrwydd (gweler Pennod 3). Ar hyn o bryd, nid yw Mesur y Gymraeg (2011) yn gorfodi ysgolion cynradd ac uwchradd yng Nghymru i baratoi strategaeth o'r fath. Fodd bynnag, bydd rhaid gweithredu polisi ar ddwyieithrwydd ymhob ysgol cyfrwng Saesneg os yw ysgolion i ymgorffori ymagwedd holistaidd at ddatblygu'r Gymraeg yn rhagweithiol ar draws yr ysgol gyfan, fel yr argymhellir uchod. Yn hyn o beth, cynghorir ysgolion ac Awdurdodau Lleol i gyfeirio at strategaeth Colegau Cymru (2010) ar ddwyieithrwydd, fel enghraifft o arfer da.

- Cynghorir Estyn i fynd ati i ddatblygu canllaw ac iddo feini prawf manwl ar gyfer arolygu ethos dwyieithog ysgolion cyfrwng Saesneg. Er mai ethos dwyieithog yw un o'r themâu mewn arolygiadau ysgolion, canfuwyd yn Pennod 7 nad yw Estyn yn defnyddio meini prawf mesuradwy ar gyfer arolygu ethos dwyieithog. Yn hytrach, ymddengys fod yr Arolygydd yn dibynnu i raddau helaeth ar arbenigedd a phrofiad eu harolygwyr wrth ddod i gasgliad am ethos yr ysgol (Cynrychiolydd ar ran Estyn, cyfathrebiad personol, Chwefror 6, 2016). Fel yr Arolygydd dros Addysg a Hyfforddi yng Nghymru, dylai fod gan Estyn rôl ragweithiol wrth ddwyn ysgolion cyfrwng Saesneg i gyfrif o safbwynt cynllunio 
ethos dwyieithog, yn ogystal â chynllunio darpariaeth cyfrwng Cymraeg. Bydd y gwaith hwn yn dod yn fwyfwy pwysig yng nghyd-destun integreiddio'r Gymraeg ar draws y cwricwlwm newydd. Cynghorir Estyn i ystyried egwyddorion strategaeth genedlaethol Colegau Cymru (2010) fel man cychwyn wrth ddatblygu meini prawf ar gyfer mesur ethos dwyieithog mewn ysgolion.

- Yn ogystal â datblygu ethos dwyieithog cadarn o fewn ysgolion cyfrwng Saesneg, dylai ysgolion hefyd ystyried sut y mae manteisio ar y cyfleoedd o fewn y gymuned leol i ddefnyddio'r Gymraeg. Fel y canfuwyd yn Pennod 1, mae cyfle i ysgolion sicrhau y caiff y Gymraeg ei throsglwyddo o'r dosbarth i'r gymuned trwy gydweithio, er enghraifft, â chanolfannau Cymraeg. Gallai hyn sicrhau bod disgyblion yn ymgysylltu â'r iaith Gymraeg fel iaith byw y tu hwnt i'r dosbarth ffurfiol.

\subsubsection{Gwella'r cyfleoedd hyfforddi i staff addysgu a gweinyddu'r sector cyfrwng Saesneg}

- Wrth ystyried y nod uchelgeisiol o gyrraedd miliwn o siaradwyr Cymraeg erbyn 2050, rhaid i LlC sicrhau digonedd o gyfleoedd hyfforddi iaith Gymraeg ac addysgu dwyieithog i staff y sector cyfrwng Saesneg. Mae'r Cynllun Sabothol Cenedlaethol, a ariennir gan y Grant Gwella Addysg, yn cynnig nifer o gyrsiau ar lefel genedlaethol trwy sefydliadau Prifysgol Bangor, Prifysgol y Drindod Dewi Sant a Phrifysgol Caerdydd. Fodd bynnag, er bod LlC yn ystyried cynnig hyfforddiant methodoleg addysgu dwyieithog i athrawon pwnc uwchradd yn y sector cyfrwng Saesneg yn y dyfodol (Cynrychiolydd ar ran y Cynllun Sabothol, cyfathrebiad personol, Mawrth 22, 2017), canfuwyd na chaiff cwrs hyfforddi o'r fath ei ddarparu i garfan hon o ymarferwyr ar hyn o bryd. Os yw LlC am gyflawni nod mor uchelgeisiol erbyn 2050, rhaid sicrhau bod gan y gyfundrefn weithlu sydd â'r sgiliau priodol i allu addysgu trwy gyfrwng a defnyddio'r iaith yn rheolaidd ar draws yr ysgol gyfan. Cynghorir LlC felly i estyn darpariaeth y Cynllun Sabothol i gynnwys cwrs Hyfforddi Mewn Swydd ar gyfer datblygu methodoleg addysgu dwyieithog i athrawon pynciol uwchradd yn y sector cyfrwng Saesneg yn benodol.

- Yn ogystal â sicrhau bod digonedd o gyfleoedd Hyfforddiant Mewn Swydd, dylai LlC hefyd barhau i gefnogi darpar athrawon i ystyried hyfforddi fel athrawon yn 
y Gymraeg ac fel athrawon cyfrwng Cymraeg. Wrth ystyried uchelgais LlC (2017ch) parthed cynyddu nifer yr athrawon uwchradd sy'n gallu addysgu'r Gymraeg o 500 i 1,200 erbyn 2050, a nifer yr athrawon uwchradd sy'n gallu addysgu trwy gyfrwng y Gymraeg o 1,800 i 4,200 erbyn 2050, bydd yn angenrheidiol i'r llywodraeth flaenoriaethu hyfforddiant athrawon, a hynny fel mater o frys.

\subsubsection{Cryfhau trefniadau pontio'r Gymraeg}

- Dylai ysgolion uwchradd cyfrwng Saesneg fynd ati i gydweithio'n agosach ac yn fwy effeithiol â'u hysgolion bwydo yn y clwstwr er mwyn cryfhau trefniadau pontio'r Gymraeg fel ail iaith rhwng CA2 a 3. Er y cafwyd hyd i dystiolaeth sy'n awgrymu bod nifer o ysgolion yn cydweithio'n effeithiol, ymddengys nad yw hyn yn wir yn y rhan fwyaf o achosion (gweler Pennod 1). Ar sail canfyddiadau'r ymchwil empirig (Pennod 7) a'r ymchwil ragarweiniol (Pennod 1), cynghorir Awdurdodau Lleol a'u hysgolion i ystyried cynllun pontio Ysgol X fel enghraifft o arfer sy'n arwain y sector ar gyfer cynllunio'r Gymraeg yn effeithiol wrth iddynt wella trefniadau pontio'r Gymraeg rhwng yr ysgol gynradd a'r ysgol uwchradd.

- Os yw ysgolion cyfrwng Saesneg i symud tuag at CLIL, bydd yn rhaid sicrhau na chaiff hyn effaith andwyol ar ddatblygiad addysg drochi llawn ac addysg gynnal iaith yn y sector cyfrwng Cymraeg ar lefel leol. Er y cydnabyddir bod gan rieni'r hawl i symud eu plant o'r naill gyfrwng i'r llall, dylai Awdurdodau Lleol (a’u hysgolion priodol) barhau i gydweithio i wella dilyniant ieithyddol yn y Gymraeg, yn enwedig rhwng yr ysgol gynradd a'r ysgol uwchradd, er mwyn lleihau nifer y dysgwyr sydd yn symud o'r cyfrwng Cymraeg i'r cyfrwng Saesneg.

\subsection{Yr angen am ymchwil bellach}

\subsubsection{Gwerthuso Cwrs Carlam a rhaglenni CLIL tebyg yng nghyd-destun CAI} yng Nghymru

- Dylai LIC gomisiynu ymchwil bellach i astudio effaith darpariaeth CLIL ar gyrhaeddiad academaidd a hyfedredd ieithyddol disgyblion sydd â lefelau 
gwahanol o allu, gan gynnwys y rhai sy'n tangyflawni a'r rhai sydd yn mewnfudo i Gymru ac yn dysgu Cymraeg a Saesneg fel ieithoedd ychwanegol. Bydd yr ymchwil hon yn arbennig o bwysig os yw LlC am symud tuag at fodel cymhwysol o CLIL yng Nghymru (Somers, 2018a).Yn yr ymchwil gyfredol, ceir tystiolaeth sy'n cefnogi defnyddio'r Gymraeg ar draws y cwricwlwm fel cyfrwng dysgu ac addysgu. Cafwyd tystiolaeth bellach i gefnogi defnyddio CLIL mewn ysgolion cyfrwng Saesneg mewn adroddiad diweddar a gomisiynwyd gan LlC, sef Asesiad Cyflym o'r Dystiolaeth: Ymagweddau a Dulliau Addysgu Ail Iaith Effeithiol (Fitzpatrick ac eraill, 2018). Fodd bynnag, cydnabydd yr ymchwilydd y byddai angen cynnal ymchwil bellach ar CLIL yng nghyd-destun CAI yng Nghymru, cyn y gallai LlC fynd ati i weithredu darpariaeth o'r fath ar lefel genedlaethol.

- Dylai LlC ystyried gomisiynu cynllun peilot newydd sydd â ffocws ar gyflwyno CLIL mewn lleoliadau cyfrwng Saesneg. Fel enghraifft o CLIL sydd ar waith yng nghyd-destun Cymru, cynghorir LIC i gomisiynu ymchwil bellach i werthuso effeithiolrwydd y Cwrs Carlam, fel rhan o'r cynllun peilot arfaethedig hwn. Wrth ystyried faint o gynllunio gofalus y bydd angen ei wneud ymlaen llaw er mwyn i ysgolion cyfrwng Saesneg allu datblygu eu capasiti i gynnig addysg trwy gyfrwng y Gymraeg maes o law, cynghorir LlC i gomisiynu'r ymchwil hon fel mater o flaenoriaeth.

\subsubsection{Gwerthuso'r dulliau addysgu dwyieithog a ddefnyddir gan athrawon yn y Cwrs Carlam yn Ysgol X}

- Fel rhan o'r cynllun peilot a nodwyd uchod, dylai LlC ystyried gwerthuso'r dulliau addysgu dwyieithog a ddefnyddir gan athrawon y Cwrs Carlam yn Ysgol X. Daeth i'r amlwg yn yr ymchwil gyfredol nad yw athrawon y Cwrs Carlam yn defnyddio dulliau addysgu dwyieithog penodol wrth gyflwyno cynnwys pynciol, ond yn hytrach y mae'r athrawon hyn yn dibynnu i raddau helaeth ar eu profiad yn unig (gweler Pennod 7). Er nad drwg o beth yw defnyddio profiad er mwyn llywio'r dulliau addysgu a ddefnyddir yn y dosbarth, cydnabyddir y byddai'n rhaid gwerthuso dulliau addysgu dwyieithog yr athrawon hyn trwy arsylwi eu harferion addysgol yn y dosbarth er mwyn deall yn well beth sydd yn digwydd ar lawr dosbarth. Cynghorir LIC hefyd i ystyried arsylwi arfer da athrawon mewn 
unrhyw ysgolion sydd â thraddodiad cryf o gyflwyno elfennau o'r cwricwlwm trwy gyfrwng y Gymraeg neu'n ddwyieithog i ddysgwyr o gefndiroedd ail iaith.

\subsubsection{Gwerthuso deilliannau darpariaeth beripatetig Ysgol X}

- Dylai LlC ystyried comisiynu ymchwil bellach i werthuso deilliannau darpariaeth beripatetig Ysgol $\mathrm{X}$ a dichonoldeb ariannu'r cynllun peripatetig ar lefel genedlaethol. Casglwyd yn Pennod 7 fod trefniadau Ysgol X ar gyfer pontio'r Gymraeg o'r ysgol gynradd i'r ysgol uwchradd yn ymddangos yn arbennig o gadarn a bod cyfle i ysgolion cyfrwng Saesneg eraill wella eu trefniadau pontio trwy ymgorffori cynlluniau tebyg. Cydnabyddir nad oedd mesur deilliannau darpariaeth beripatetig Ysgol X yn benodol o fewn cwmpas yr ymchwil hon. Fodd bynnag, byddai gwerthusiad o ddarpariaeth beripatetig Ysgol X yn cynnig tystiolaeth bellach i gadarnhau effeithiolrwydd cynllun pontio Ysgol X, yn ogystal â dichonoldeb ariannu cynllun peripatetig ar lefel genedlaethol er mwyn ymateb i'r heriau a amlygwyd yn Pennod 1 y mae'r sector cyfrwng Saesneg yn eu hwynebu o ran pontio'r Gymraeg o'r ysgol gynradd i'r ysgol uwchradd.

\subsubsection{Gwerthuso ethos dwyieithog anffurfiol Ysgol X}

- Dylai LIC ystyried comisiynu ymchwil bellach i werthuso ethos dwyieithog anffurfiol mewn ysgolion. Canfuwyd yn Pennod 2 fod datblygu ethos dwyieithog cryf yn hanfodol i gyflwyno'r Gymraeg yn llwyddiannus mewn ysgolion cyfrwng Saesneg. Yn yr ymchwil hon, canolbwyntiwyd yn benodol ar ddadansoddi ethos dwyieithog ffurfiol, megis arwyddion, polisïau, dogfennaeth swyddogol a marchnata'r ysgol, oherwydd terfyniadau ar yr ymchwil. Fodd bynnag, byddai gwerthusiad o ethos dwyieithog anffurfiol mewn ysgolion megis Ysgol X, gan gynnwys arferion, credoau, ac ymddygiadau aelodau'r ysgol o ddydd i ddydd, yn cryfhau canfyddiadau'r ymchwil hon. 


\section{Atodiadau}

Atodiad 1. Ffurflen ganiatâd i'w chwblhau gan y disgybl a'r rhiant / gwarcheidwad

\section{CONSENT FORM}

\section{TO BE COMPLETED BY YOUNG PERSON AND PARENT/GUARDIAN}

PART A TO BE COMPLETED BY THE YOUNG PERSON.

I agree to take part in a cloze test (reading test) for the purpose of a Ph.D. research project on Welsh Second Language education.

I have read and understood the accompanying information. I know what the study is about and the part I will be involved in. I understand that any data gathered from the cloze test will be used in the final work published by the researcher. I understand that participation in the reading test is voluntary and I can decide not to complete the reading test at any time.

Signature:

Age:

\section{PART B TO BE COMPLETED BY THE PARENT/GUARDIAN}

I have read and understood the accompanying information leaflet and give permission for the child (named above) to participate in the research.

Relationship to the above young person:

Signature: 
As part of a Ph.D. research project studying how best Welsh Second Language can be presented in English-medium schools, the researcher will be administering reading tests in two English medium schools in Wales in order to measure students general language competence in Welsh.

Having carried out pilot tests, the researcher is now holding part of his final testing with pupils in [Name of School]. The results of these tests will be anonymous, but they will appear in the final dissertation of the researcher, as well as any linked works that he publishes. 


\section{FFURFLEN GANIATÂD}

\section{I'W CHWBLHAU GAN Y DISGYBL A'R RHIANT / GWARCHEIDWAD}

\section{RHAN A: I'W CHWBLHAU GAN Y DISGYBL}

Rwy'n cytuno i gymryd rhan yn y prawf cloze (prawf darllen) at ddiben prosiect ymchwil Ph.D. ar addysg Gymraeg Ail Iaith.

Rwy wedi darllen a deall y wybodaeth ynghlwm wrth y ffurflen hon. Rwy'n deall pwrpas yr astudiaeth a'r rhan y bydda i'n ei chwarae. Rwy'n deall y bydd unrhyw ddata sy'n deillio o'r prawf yn cael ei ddefnyddio yng ngwaith terfynol yr ymchwilydd, yn ogystal ag unrhyw weithiau cysylltiedig. Rwy'n deall bod cwblhau'r prawf yn wirfoddol a galla i benderfynu peidio â chwblhau'r prawf ar unrhyw adeg.

Llofnod:

Oed:

\section{RHAN B: I'W CHWBLHAU GAN Y RHIANT / GWARCHEIDWAD}

Rwy wedi darllen a deall y wybodaeth ynghlwm wrth y ffurflen hon ac rwy'n caniatáu i'r plentyn a nodir uchod gymryd rhan yn yr ymchwil.

Perthynas â'r plentyn uchod:

Llofnod: 


\section{GWYBODAETH AM YR YMCHWIL}

Fel rhan o brosiect Ph.D. sy'n ymchwilio i sut orau y gall y Gymraeg fel ail iaith gael ei chyflwyno mewn ysgolion cyfrwng Saesneg, bydd yr ymchwilydd yn cynnal prawf llenwi bylchau (cloze) mewn dwy ysgol cyfrwng Saesneg yng Nghymru er mwyn mesur hyfedredd ieithyddol cyffredinol disgyblion yn y Gymraeg.

Wedi treialu'r prawf, bydd yr ymchwilydd nawr yn cynnal rhan o'r prawf gyda disgyblion yn [Enw'r Ysgol]. Bydd canlyniadau'r prawf yn ddienw ond fe fydd y canlyniadau yn ymddangos yn nhraethawd terfynol yr ymchwilydd, yn ogystal ag unrhyw weithiau cysylltiedig y mae'n eu cyhoeddi. 
Atodiad 2. Holiadur Ysgol Y

\section{Datganiad:}

Pwrpas yr holiadur hwn ydy casglu data gan Bennaeth Adran y Gymraeg yn yr ysgol dan sylw ynglŷn â'r meysydd canlynol: darpariaeth ar gyfer y Gymraeg yn yr ysgol, ethos Cymraeg/dwyieithog yr ysgol, hyfforddiant staff yr ysgol ynglŷn â dysgu ac addysgu Cymraeg, a phontio rhwng y sector cynradd a'r sector uwchradd. Caiff yr astudiaeth hon ei chynnal gan Alexander Lovell, ymgeisydd Ph.D. yn Academi Hywel Teifi, Prifysgol Abertawe. A fyddech mor garedig â llenwi'r holiadur hwn gan roi eich barn chi eich hun. Ni ddefnyddir y data a gesglir yma at unrhyw ddibenion eraill ar wahân i bwrpas gwaith maes y prosiect ymchwil a gynhelir gan yr ymchwilydd. Cofiwch fod cymryd rhan wrth lenwi'r holiadur hwn yn gwbl wirfoddol. Diolch yn fawr iawn i chi am eich amser.

\section{Rhan 1: Darpariaeth ar gyfer y Gymraeg yn yr Ysgol}

Rhowch ateb mor gyflawn â phosibl ar gyfer pob cwestiwn, os gwelwch yn dda.

\section{Beth ydy'r llwybrau dysgu Cymraeg sy'n cael eu cynnig gan yr ysgol?}

Cyfnod Allweddol 3 Cymraeg Ail Iaith, Cyfnod Allweddol 4 Cymraeg Ail Iaith (TGAU Cwrs Llawn) Lefel AS/A Cymraeg Ail Iaith

\section{Pa gymwysterau y mae'n bosibl eu hennill wrth ddilyn y llwybrau dysgu} Cymraeg hyn?

TGAU Cymraeg Ail Iaith (Cwrs Llawn) Lefel AS/A Cymraeg Ail Iaith 
3. Faint o amser yn yr amserlen a neilltuir i addysgu'r Gymraeg yn achos pob un o'r llwybrau dysgu hyn?

Cyf.Allweddol $3=3$ awr bob pythefnos, Cyf.Allweddol $4=5$ awr bob pythefnos, Lefel AS/A = 8 awr bob pythefnos

\section{Beth ydy statws Cymraeg Ail Iaith yn yr ysgol yn nhermau Mesur}

Perfformiad?

Pwnc sylfaenol

囚 Pwnc craidd

5. Ydy'r ysgol yn cynnig unrhyw bynciau / modiwlau sy'n cael eu cyflwyno trwy gyfrwng y Gymraeg yn unig?

Ydy

囚 Nac ydy (Ewch i gwestiwn 9)

6. Pa bynciau / modiwlau trwy gyfrwng y Gymraeg sy'n cael eu cynnig gan yr

ysgol?

7. Pa ddisgyblion sydd yn gymwys ar gyfer y pynciau / modiwlau cyfrwng

\section{Cymraeg?}


8. Ym mha flynyddoedd ysgol y mae disgyblion yn astudio'r pynciau / modiwlau cyfrwng Cymraeg hyn?

9. Ydy'r ysgol yn cynnig unrhyw bynciau / modiwlau yn ddwyieithog (lle gwneir defnydd o'r Gymraeg a'r Saesneg wrth gyflwyno cynnwys y pwnc / modiwl yn y dosbarth)?

Ydy

囚 Nac ydy $\quad$ (Ewch i Ran 2: Ethos Dwyieithog yr Ysgol)

10. Os ydy, pa bynciau / modiwlau dwyieithog sy'n cael eu cynnig gan yr ysgol?

11. Ym mha flynyddoedd ysgol y mae disgyblion yn astudio'r pynciau / modiwlau dwyieithog hyn?

12. Pa ddisgyblion sydd yn gymwys ar gyfer astudio'r pynciau / modiwlau

dwyieithog? 
13. Ar gyfartaledd, faint o ddefnydd a wneir gan athrawon o'r Gymraeg yn y dosbarth wrth addysgu cynnwys y pwnc / modiwl yn ddwyieithog?

Llai na $50 \%$

$50 \%$ neu fwy

Arall

14. Trwy gyfrwng pa iaith / ieithoedd y gall y disgyblion hyn sefyll arholiadau TGAU wedi astudio'r pwnc / modiwl yn ddwyieithog?

Saesneg

Cymraeg

15. Beth ydy polisi staffio'r ysgol o safbwynt cyflogi a chynnal athrawon sy'n medru addysgu trwy gyfrwng y Gymraeg / yn ddwyieithog?

16. Oes gan yr ysgol fwriad i allu cynnig pynciau newydd trwy gyfrwng y Gymraeg / yn ddwyieithog?
$\square$ Oes
(Ewch i gwestiwn 18)

Nac oes

17. Os nac oes, oes modd cynnig rhesymau am hynny?

(Ewch i Ran 2: Ethos Dwyieithog yr Ysgol) 
18. Os oes, sut bydd yr ysgol yn cynllunio i sicrhau bod digon o athrawon cymwys i gynnig pynciau newydd trwy gyfrwng y Gymraeg / yn ddwyieithog?

\section{Rhan 2: Ethos Dwyieithog yr Ysgol}

Rhowch ateb mor gyflawn â phosibl ar gyfer pob cwestiwn, os gwelwch yn dda.

1. Oes gan yr ysgol bolisi / strategaeth ffurfiol (ar bapur) ar gyfer datblygu, hyrwyddo a hybu dwyieithrwydd yn yr ysgol?

$\square$ Oes $\quad$ (Ewch i gwestiwn 3)

凶Nac oes

2. Os nad oes, sut y mae'r ysgol yn sicrhau y caiff dwyieithrwydd ei ddatblygu, ei hyrwyddo a'i hybu trwy'r ysgol gyfan o ddydd i ddydd?

Posteri o gwmpas yr ysgol, yn yr ystafelloedd ac ar y drysau. Gwasanaethau yn cynnwys dwyieithrwydd fel gweddïau a themâu.

(Ewch i Ran 3: Hyfforddiant staff yn y Gymraeg)

3. Beth ydy prif amcanion y polisi hwnnw / strategaeth honno? 
4. Sut caiff egwyddorion y polisi ffurfiol hwnnw / y strategaeth ffurfiol honno eu gwireddu'n ymarferol o ddydd i ddydd yn yr ysgol?

\section{Rhan 3: Hyfforddiant staff yn y Gymraeg}

Rhowch ateb mor gyflawn â phosibl ar gyfer pob cwestiwn, os gwelwch yn dda.

1. Oes hyfforddiant ar gael i staff yr ysgol er mwyn iddynt ddatblygu sgiliau dwyieithog cyffredinol ar gyfer y gweithle?

囚 Oes (Ewch i gwestiwn 4)

Nac Oes

2. Os nad oes, oes modd cynnig unrhyw resymau am hynny?

3. Yn eich barn chi, fyddai'r fath hyfforddiant yn cael ei groesawu gan staff yr ysgol? Oes modd cynnig unrhyw resymau am gredu hynny? 
(Ewch i gwestiwn 9)

4. Beth ydy natur yr hyfforddiant hwn? Hynny yw, sut mae'r hyfforddiant hwn yn paratoi staff i weithio'n ddwyieithog yn y gweithle? e.e. addysgu sut mae 'cwrdd a chyfarch' yn Gymraeg

Mae'r staff yn hapus i gwrdd a chyfarch yn Gymraeg, defnyddio Cymraeg yn y dosbarth posteri i helpu nhw gyda'r dyddiad, nod y wers a.y.y.b

Yn ystod cyfarfodydd mae cyfle i ymarfer Cymraeg - staff yn yr adran yn cefnogi pobl.

\section{Pa gorff / gyrff sy'n gyfrifol am ddarparu'r hyfforddiant hwn?}

Adran y Gymraeg

6. Ai'r ysgol sy'n ariannu'r hyfforddiant hwn, neu gorff arall / cyrff eraill?

7. Pa mor aml ydy'r fath hyfforddiant yn cael ei gynnig i staff?

Mae'r staff yn ymwybodol bod yr adran yn barod i gefnogi nhw unrhyw amser

8. Pa mor aml, yn eich barn chi, ydy'r staff yn achub ar y cyfleoedd hyfforddi

Mae llawer yn trio defnyddio'r iaith Gymraeg bob dydd yn eu gwersi neu yn y coridor hyn? 
9. Oes hyfforddiant ar gael i athrawon yr ysgol ar gyfer datblygu methodoleg addysgu cyfrwng Cymraeg / dwyieithog?

$\square$ Oes $\quad$ (Ewch i gwestiwn 12)

凶Nac oes

10. Os nad oes, oes modd cynnig unrhyw resymau am hynny?

11. Yn eich barn chi, fyddai'r fath hyfforddiant yn cael ei groesawu gan athrawon yr ysgol? Oes modd cynnig unrhyw resymau am gredu hynny?

Fi'n credu bydd y staff yn groesawu'r hyfforddiant ond jest yr amser i wneud e (Ewch i Ran 4: Pontio Rhwng y Sector Cynradd a'r Sector Uwchradd)

12. Beth ydy natur yr hyfforddiant hwn? Hynny yw, sut mae'r hyfforddiant hwn yn datblygu methodoleg addysgu cyfrwng Cymraeg / dwyieithog

athrawon?

13. Pa gorff / gyrff sy'n gyfrifol am ddarparu'r hyfforddiant hwn?

14. Ai'r ysgol sy'n ariannu'r hyfforddiant hwn, neu gorff arall / cyrff eraill? 
15. Pa mor aml ydy'r fath hyfforddiant ar gael i'r staff?

16. Pa mor aml, yn eich barn chi, ydy'r staff addysgu yn achub ar y cyfleoedd hyfforddi hyn?

Rhan 4: Pontio Rhwng y Sector Cynradd a'r Sector Uwchradd

Rhowch ateb mor gyflawn â phosibl ar gyfer pob cwestiwn, os gwelwch yn dda.

1. Pa ysgolion cynradd yn y dalgylch sy'n bwydo'r ysgol uwchradd hon?

Ysgol Gynradd [Enw ysgol] a [Enw ysgol], Ysgol Gynradd [Enw ysgol]

Rhai disgyblion yn dod o Ysgol Gynradd [Enw ysgol], [Enw ysgol] ac [Enw ysgol]

2. O blith yr ysgolion cynradd bwydo hyn, oes unrhyw ysgolion sy'n cael eu diffinio'n ysgolion cyfrwng Cymraeg / ysgolion dwyieithog?

$\square$ Oes

囚 Nac oes (Ewch i gwestiwn 5) 
3. Pa ysgolion yw'r rhai sy'n cael eu diffinio'n ysgolion cynradd cyfrwng Cymraeg / ysgolion dwyieithog?

4. Beth mae'r ysgol yn ei wneud yn benodol i ddarparu ar gyfer anghenion ieithyddol y disgyblion hyn yn y Gymraeg wrth iddynt drosglwyddo o'r sector cynradd i'r sector uwchradd?

5. Beth mae'r ysgol yn ei wneud yn benodol i hwyluso trosglwyddiad disgyblion Cymraeg Ail Iaith o'r sector cynradd i'r sector uwchradd?

Mae'r disgyblion Cymraeg yn y 6ed dosbarth yn helpu Ysgol Gynradd [Enw ysgol] bob wythnos gyda Chymraeg. Hefyd mae cyfleoedd i gwrdd â'r staff er mwyn trafod dysgu Cymraeg yn yr Ysgol Gynradd - yn ddiweddar roedd cyfarfod 'Cluster Moderation' er mwyn trafod gwaith Cyfnod Allweddol 2 \& 3 - cyfle i drafod lefelau.

Diwedd.

Diolch yn fawr iawn am eich amser. 
Atodiad 3. Holiadur Ysgol X

\section{Datganiad:}

Pwrpas yr holiadur hwn ydy casglu data gan Bennaeth Adran y Gymraeg yn yr ysgol dan sylw ynglŷn â'r meysydd canlynol: darpariaeth ar gyfer y Gymraeg yn yr ysgol, ethos Cymraeg/dwyieithog yr ysgol, hyfforddiant staff yr ysgol ynglynn â dysgu ac addysgu Cymraeg, a phontio rhwng y sector cynradd a'r sector uwchradd. Caiff yr astudiaeth hon ei chynnal gan Alexander Lovell, ymgeisydd Ph.D. yn Academi Hywel Teifi, Prifysgol Abertawe. A fyddech mor garedig â llenwi'r holiadur hwn gan roi eich barn chi eich hun. Ni ddefnyddir y data a gesglir yma at unrhyw ddibenion eraill ar wahân i bwrpas gwaith maes y prosiect ymchwil a gynhelir gan yr ymchwilydd. Cofiwch fod cymryd rhan wrth lenwi'r holiadur hwn yn gwbl wirfoddol. Diolch yn fawr iawn i chi am eich amser.

\section{Rhan 1: Darpariaeth ar gyfer y Gymraeg yn yr Ysgol}

Rhowch ateb mor gyflawn â phosibl ar gyfer pob cwestiwn, os gwelwch yn dda.

\section{Beth ydy'r llwybrau dysgu Cymraeg sy'n cael eu cynnig gan yr ysgol?}

Blwddyn 8 a 9 Cwrs Carlam yn astudio'r cwrs TGAU Llawn Ail-Iaith ac eistedd yr arholiad ar ddiwedd blwyddyn 9.

Blwyddyn 10 ac 11 cwrs Carlam yn astudio'r cwrs AS dros dwy flynedd ac yn eistedd yr arholiad ar ddiwedd Blwyddyn 11.

Pawb yn astudio'r Cwrs Llawn TGAU Llawn Ail-Iaith yn CA4 heblaw tua 35/40 o ddisgyblion gwanaf. Maen nhw'n astudio NVQ Lefel 1 Darllen. Ac mae'r disgyblion Iaith Gyntaf yn astudio TGAU Cymraeg Iaith Gyntaf yn CA4.

Cyrsiau Uwch Gyfrannol a Safon Uwch ar gael i ddisgyblion i'w astudio yn CA5 - Iaith Gyntaf ac Ail Iaith (er nid oes neb yn astudio Iaith Gyntaf ar hyn o bryd) 
2. Pa gymwysterau y mae'n bosibl eu hennill wrth ddilyn y llwybrau dysgu Cymraeg hyn?

Gweler uchod.

3. Faint o amser yn yr amserlen a neilltuir i addysgu'r Gymraeg yn achos pob un o'r llwybrau dysgu hyn?

Mae'r pawb yn cael 2 awr yr wythnos yn CA3 a CA4 gyda 4 awr yr wythnos yn CA5.

Eithriad - Mae'r dosbarthiadau AAA yn CA3 a CA4 yn cael awr yr wythnos yn ogystal a'r dosbarth NVQ.

\section{Beth ydy statws Cymraeg Ail Iaith yn yr ysgol yn nhermau Mesur}

\section{Perfformiad?}

囚 Pwnc sylfaenol

Pwnc craidd

5. Ydy'r ysgol yn cynnig unrhyw bynciau / modiwlau sy'n cael eu cyflwyno trwy gyfrwng y Gymraeg yn unig?

囚 Ydy

Nac ydy (Ewchi gwestiwn 9)

6. Pa bynciau / modiwlau trwy gyfrwng y Gymraeg sy'n cael eu cynnig gan yr

Mae'r pynciau canlynol yn cael eu haddsygu drwy gyfrwng y Gymraeg yn CA3

Hanes (opsiwn i'w hastudio trwy gyfrwng y Gymraeg yn CA4 yn dibynnu ar niferoedd)

Daearyddiaeth

Addysg Grefyddol

Addysg Gorfforol

Drama

Coginio

$\mathrm{ABCH}$ 
ysgol?

7. Pa ddisgyblion sydd yn gymwys ar gyfer y pynciau / modiwlau cyfrwng

Disgyblion Iaith Gyntaf yr ysgol.

\section{Cymraeg?}

8. Ym mha flynyddoedd ysgol y mae disgyblion yn astudio'r pynciau / modiwlau cyfrwng Cymraeg hyn?

CA3

9. Ydy'r ysgol yn cynnig unrhyw bynciau / modiwlau yn ddwyieithog (lle gwneir defnydd o'r Gymraeg a'r Saesneg wrth gyflwyno cynnwys y pwnc / modiwl yn y dosbarth)?

囚 Ydy

Nac ydy $\quad$ (Ewch i Ran 2: Ethos Dwyieithog yr Ysgol)

10. Os ydy, pa bynciau / modiwlau dwyieithog sy'n cael eu cynnig gan yr ysgol?

Mae'r pynciau canlynol yn cael eu haddsygu'n ddwyieithog yn CA3 i ddisgyblion y Cwrs Carlam.

Hanes (opsiwn i'w hastudio trwy gyfrwng y Gymraeg yn CA4 yn dibynnu ar niferoedd) Daearyddiaeth Addysg Grefyddol Addysg Gorfforol $\mathrm{ABCH}$ 
11. Ym mha flynyddoedd ysgol y mae disgyblion yn astudio'r pynciau / modiwlau dwyieithog hyn?

CA3

12. Pa ddisgyblion sydd yn gymwys ar gyfer astudio'r pynciau / modiwlau

Disgyblion Cwrs Carlam

dwyieithog?

13. Ar gyfartaledd, faint o ddefnydd a wneir gan athrawon o'r Gymraeg yn y dosbarth wrth addysgu cynnwys y pwnc / modiwl yn ddwyieithog?

Llai na $50 \%$

囚 $50 \%$ neu fwy

Arall

14. Trwy gyfrwng pa iaith / ieithoedd y gall y disgyblion hyn sefyll arholiadau TGAU wedi astudio'r pwnc / modiwl yn ddwyieithog?

凶aesneg

Cymraeg

15. Beth ydy polisi staffio'r ysgol o safbwynt cyflogi a chynnal athrawon sy'n medru addysgu trwy gyfrwng y Gymraeg / yn ddwyieithog?

Does dim polisi - mae'r ysgol yn cyflogi'r athrawon gorau i'r pynciau fel sydd angen. 
16. Oes gan yr ysgol fwriad i allu cynnig pynciau newydd trwy gyfrwng y Gymraeg / yn ddwyieithog?

囚 Oes

(Ewch i gwestiwn 18)

Nac oes

17. Os nac oes, oes modd cynnig rhesymau am hynny?

(Ewch i Ran 2: Ethos Dwyieithog yr Ysgol)

18. Os oes, sut bydd yr ysgol yn cynllunio i sicrhau bod digon o athrawon cymwys i gynnig pynciau newydd trwy gyfrwng y Gymraeg / yn ddwyieithog?

Ond yn dewis pynciau lle mae'r arbenigedd gyda ni yn barod.

\section{Rhan 2: Ethos Dwyieithog yr Ysgol}

Rhowch ateb mor gyflawn â phosibl ar gyfer pob cwestiwn, os gwelwch yn dda. 
1. Oes gan yr ysgol bolisi / strategaeth ffurfiol (ar bapur) ar gyfer datblygu, hyrwyddo a hybu dwyieithrwydd yn yr ysgol?

囚 Oes (Ewch i gwestiwn 3)

Nac oes

2. Os nad oes, sut y mae'r ysgol yn sicrhau y caiff dwyieithrwydd ei ddatblygu, ei hyrwyddo a'i hybu trwy'r ysgol gyfan o ddydd i ddydd?

(Ewch i Ran 3: Hyfforddiant staff yn y Gymraeg)

3. Beth ydy prif amcanion y polisi hwnnw / strategaeth honno?

I ddatblygu ac ymestyn dwyieithrwydd fel rhan annatod o addysgu.

4. Sut caiff egwyddorion y polisi ffurfiol hwnnw / y strategaeth ffurfiol honno eu gwireddu'n ymarferol o ddydd i ddydd yn yr ysgol?

Gweler y polisi 


\section{Rhan 3: Hyfforddiant staff yn y Gymraeg}

Rhowch ateb mor gyflawn â phosibl ar gyfer pob cwestiwn, os gwelwch yn dda.

1. Oes hyfforddiant ar gael i staff yr ysgol er mwyn iddynt ddatblygu sgiliau dwyieithog cyffredinol ar gyfer y gweithle?

囚 Oes $\quad($ Ewch $i$ gwestiwn 4)

Nac Oes

2. Os nad oes, oes modd cynnig unrhyw resymau am hynny?

3. Yn eich barn chi, fyddai'r fath hyfforddiant yn cael ei groesawu gan staff yr ysgol? Oes modd cynnig unrhyw resymau am gredu hynny?

(Ewch i gwestiwn 9)

4. Beth ydy natur yr hyfforddiant hwn? Hynny yw, sut mae'r hyfforddiant hwn yn paratoi staff i weithio'n ddwyieithog yn y gweithle? e.e. addysgu sut mae 'cwrdd a chyfarch' yn Gymraeg

Yn 2011/2012 cynhaliwyd gwersi staff ar ôl ysgol gan [Enw prifysgol] o dan arweiniad [Enw'r cydlynydd]. Mae cynllun newydd eleni ar gyfer gwersi o'r fath hefyd.

Mae'r mwyafrif o'r staff yn ystod y cyfnodau yma wedi neu'n mynd i fynychu'r cwrs 10 wythnos am awr yr wythnos er mwyn datblygu sgiliau sylfaenol defnyddio Cymraeg achlysurol o fewn y dosbarth.

Mae tua 5 aelod o'r staff wedi cwblhau cwrs uwch ar ôl ysgol a chwblhau asesiad ar ddiwedd y cyfnod.

Mae'r athrawon hynny sy'n addysgu'r pynciau Cymraeg/Dwyieithog yn rhugl yn y Gymraeg. 


\section{Pa gorff / gyrff sy'n gyfrifol am ddarparu'r hyfforddiant hwn?}

Mae gwersi'r staff ar ôl ysgol wedi cael eu cynnal gan [Enw prifysgol] o dan arweiniad [Enw'r cydlynydd]

6. Ai'r ysgol sy'n ariannu'r hyfforddiant hwn, neu gorff arall / cyrff eraill? Yr ysgol

7. Pa mor aml ydy'r fath hyfforddiant yn cael ei gynnig i staff?

Cynhaliwyd cyrsiau yn 2011/2012 ac maen nhw'n cael eu cynnal eto eleni.

8. Pa mor aml, yn eich barn chi, ydy'r staff yn achub ar y cyfleoedd hyfforddi

Bob tro rydyn ni wedi ei gynnig mae'r athrawon wedi dangos diddordeb. 2011-2012, 2012-2013, 2015-2016.

hyn?

9. Oes hyfforddiant ar gael i athrawon yr ysgol ar gyfer datblygu methodoleg addysgu cyfrwng Cymraeg / dwyieithog?

囚 Oes (Ewch i gwestiwn 12)

$\square$ Nac oes

10. Os nad oes, oes modd cynnig unrhyw resymau am hynny? 
11. Yn eich barn chi, fyddai'r fath hyfforddiant yn cael ei groesawu gan athrawon yr ysgol? Oes modd cynnig unrhyw resymau am gredu hynny?

(Ewch i Ran 4: Pontio Rhwng y Sector Cynradd a'r Sector Uwchradd)

12. Beth ydy natur yr hyfforddiant hwn? Hynny yw, sut mae'r hyfforddiant hwn yn datblygu methodoleg addysgu cyfrwng Cymraeg / dwyieithog

Mae'r athrwon wedi arsylwi'r adran Gymraeg yn addysgu Cymraeg a phynciau eraill megis Hanes. Daear ac Addysg Grefyddol.

athrawon?

13. Pa gorff / gyrff sy'n gyfrifol am ddarparu'r hyfforddiant hwn?

Yn fewnol o fewn yr ysgol.

14. Ai'r ysgol sy'n ariannu'r hyfforddiant hwn, neu gorff arall / cyrff eraill? Yr ysgol

15. Pa mor aml ydy'r fath hyfforddiant ar gael i'r staff?

Cwrs yn rhedeg bob wythnos am 10 wythnos. Gweler ateb 8.

16. Pa mor aml, yn eich barn chi, ydy'r staff addysgu yn achub ar y cyfleoedd hyfforddi hyn?

Gweler yr ateb blaenorol 


\section{Rhan 4: Pontio Rhwng y Sector Cynradd a'r Sector Uwchradd}

Rhowch ateb mor gyflawn â phosibl ar gyfer pob cwestiwn, os gwelwch yn dda.

\section{Pa ysgolion cynradd yn y dalgylch sy'n bwydo'r ysgol uwchradd hon?}

Dyma'r ysgolion yn y dalgylch sy'n bwydo'r ysgol sy'n cael 2 athrawes o'r ysgol hon yn addysgu Cymraeg i Flwyddyn 5 a 6 am awr yr wythnos.

Ysgol Gynradd [Enw ysgol]

Ysgol Gynradd [Enw ysgol]

Ysgol Gynradd [Enw ysgol]

Ysgol Gynradd [Enw ysgol]

Ysgol Gynradd [Enw ysgol]

Ysgol Gynradd [Enw ysgol]

Ysgol Gynradd [Enw ysgol]

Mae rhai disgyblion o'r ysgolion Cymraeg canlynol hefyd yn mynychu'r ysgol

Ysgol Gynradd Gymraeg [Enw ysgol]

Ysgol Gynradd Gymraeg [Enw ysgol]

Ysgol Gynradd Gymraeg [Enw ysgol]

Oherwydd poblogrwydd yr ysgol rydyn ni'n derbyn disgyblion o tua 20 ysgol arall y tu hwnt i'r clwstwr yn flynyddol.

2. O blith yr ysgolion cynradd bwydo hyn, oes unrhyw ysgolion sy'n cael eu diffinio'n ysgolion cyfrwng Cymraeg / ysgolion dwyieithog? 
凶 Oes

Nac oes

(Ewch i gwestiwn 5)

\section{Pa ysgolion yw'r rhai sy'n cael eu diffinio'n ysgolion cynradd cyfrwng}

\section{Cymraeg / ysgolion dwyieithog?}

Mae rhai disgyblion o'r ysgolion Cymraeg canlynol hefyd yn mynychu'r ysgol

Ysgol Gynradd Gymraeg [Enw ysgol]

Ysgol Gynradd Gymraeg [Enw ysgol]

Ysgol Gynradd Gymraeg [Enw ysgol]

\section{Beth mae'r ysgol yn ei wneud yn benodol i ddarparu ar gyfer anghenion ieithyddol y disgyblion hyn yn y Gymraeg wrth iddynt drosglwyddo o'r sector cynradd i'r sector uwchradd?}

Gweler uchod am y pynciau a ddarparir drwy gyfrwng y Gymraeg. Yn ogystal â hyn mae athrawon sy'n gallu siarad Cymraeg yn addysgu'r dosbarth ar gyfer Mathemateg a Gwyddoniaeth er mwyn helpu nhw i drosglwyddo o astudio'r pynciau hynny yn Gymraeg yn y cynradd i Saesneg yn yr uwchradd.

Mae gan bob disgybl bwcled o eiriau/termau allweddol ar gyfer pob pwnc er mwyn eu cefnogi.

5. Beth mae'r ysgol yn ei wneud yn benodol i hwyluso trosglwyddiad disgyblion Cymraeg Ail Iaith o'r sector cynradd i'r sector uwchradd?

Mae 2 athrawes yn addsygu yn y cynradd ac maen nhw hefyd yn addysgu nifer o'r dosbarthiadau ym Mlwyddyn 7 - mae hyn felly yn hwyluso'r trosglwyddiad.

Yn ogystal â hyn mae 2 diwrnod trochi - un ym mis Medi ac un arall ym mis Gorffennaf ac un ychwanegol ar gyfer y disgyblion hynny sy'n dod i'r ysgol o du hwnt i'r clwstwr. 
Diwedd.

Diolch yn fawr iawn am eich amser. 
Atodiad 4. Canllaw i'r cyfweliad ag Ysgol Y

Darpariaeth ar gyfer y Gymraeg yn yr Ysgol:

1. Rwy'n deall bod yr ysgol yn cyflwyno Cymraeg Ail Iaith Cwrs Llawn yn unig yng Nghyfnod Allweddol 4.

a) Oes rheswm penodol pam fod yr ysgol yn cynnig y Cwrs Llawn yn unig, ac nid y Cwrs Byr yn ogystal?

b) Sut mae'r ysgol yn paratoi'r disgyblion gwannaf am ofynion y Cwrs Llawn?

c) Ar gyfer y disgyblion gwannaf, ydy'r ysgol erioed wedi ystyried cynnig cwrs gwahanol, e.e. NVQ Lefel 1? Os felly, oes rheswm penodol am beidio â'i gynnig?

2. Rwyt ti'n nodi yn yr holiadur bod gan Gymraeg Ail Iaith statws fel pwnc craidd yn yr ysgol.

a) Ers pryd mae'r ysgol wedi trin Cymraeg Ail Iaith fel pwnc craidd, yn hytrach na phwnc sylfaenol?

b) Beth oedd rhesymeg yr ysgol am newid statws y pwnc?

3. Mae'r ysgol yn cynnig darpariaeth cyfrwng Saesneg yn unig i'w disgyblion, ac felly mae'n bosibl ei labelu'n 'ysgol cyfrwng Saesneg yn bennaf' neu 'ysgol Saesneg ei hiaith'.

a) Oes gan yr ysgol y capasiti i gynnig ychydig o bynciau yn ddwyieithog?

Hynny yw, defnyddio arbenigedd staff cyfredol?

b) Os oes, beth yw'r prif rwystrau wrth gynnig darpariaeth ddwyieithog?

Ethos Dwyieithog yr Ysgol:

4. Rwyt ti'n amlygu yn yr holiadur caiff dwyieithrwydd ei hyrwyddo trwy'r ysgol gyfan, ar ffurf posteri a gwasanaethau yn bennaf, ond hefyd trwy ddefnyddio'r Gymraeg yn achlysurol yn y dosbarth ac ymhlith y staff. Mae nifer o ysgolion Saesneg bellach yn gweithredu polisi ar ddwyieithrwydd er mwyn ymrwymo i ddatblygu ethos dwyieithog. 
a) Oes rheswm penodol pam nad oes gan yr ysgol bolisi ar ddwyieithrwydd? Hyfforddiant staff yn y Gymraeg:

5. O ran datblygu sgiliau dwyieithog cyffredinol, rwyt ti wedi nodi bod staff yr adran Gymraeg yn fodlon cefnogi'r staff eraill a bod cyfleoedd i ddefnyddio'r Gymraeg yn achlysurol trwy'r ysgol.

a) Ond ar wahân i hynny, oes unrhyw hyfforddiant ffurfiol ar gael? Hynny yw, cyrsiau mewnol / allanol?

b) Os oes, beth yw natur yr hyfforddiant hwn?

c) Os nac oes, oes modd cynnig rheswm am hynny?

6. Rwy ti hefyd yn cadarnhau yn yr holiadur nad yw'r ysgol yn cynnig hyfforddiant ar gyfer datblygu methodoleg addysgu'n ddwyieithog. Ei di ymlaen wedi hynny i nodi byddai'r staff yn croesawu'r hyfforddiant ond does dim digon o amser.

a) Oes modd i ti fanylu ar y diffyg amser rwyt ti'n ei nodi yma?

b) Ydy'r ysgol erioed wedi ystyried cynnig cyrsiau hyfforddi o'r fath ar ôl ysgol?

Pontio Rhwng y Sector Cynradd a'r Sector Uwchradd:

7. O ran sicrhau trosglwyddiad hwylus disgyblion Cymraeg ail iaith o'r sector cynradd i'r sector uwchradd, rwy ti'n amlygu bod disgyblion y Chweched Dosbarth yn helpu'r adran bob wythnos "gyda Chymraeg”.

a) Sut mae'r disgyblion hyn yn helpu'r adran Gymraeg? Beth yw natur yr help yma?

8. Mae'r holiadur hefyd yn nodi bod "cyfleoedd i gwrdd â'r staff” yn yr ysgolion cynradd er mwyn trafod addysgu Cymraeg yng Nghyfnodau Allweddol 2 a 3, sef y Cyfnod Pontio.

a) Pa mor aml ydy staff yr adran yn cael cyfle i gwrdd â'r ysgolion cynradd hyn?

b) Beth yw natur y cyfarfodydd hyn? Hynny yw, beth yw'r prif bethau sy'n cael eu trafod yn ystod y cyfarfodydd hyn? 


\section{Atodiad 5. Canllaw i'r cyfweliad ag Ysgol X}

Darpariaeth ar gyfer y Gymraeg yn yr Ysgol:

1. Rydych chi'n nodi yn yr holiadur bod y 35/40 gwannaf o ddisgyblion yn astudio NVQ Lefel 1 Darllen, yn hytrach na Chwrs Byr Cymraeg Ail Iaith, yng Nghyfnod Allweddol 4.

a) Oes rheswm, neu resymau, am i'r ysgol gynnig y cwrs hwn yn lle Cwrs Byr Cymraeg Ail Iaith?

b) Rwy'n deall nad yw'r ysgol yn cynnig unedau eraill yr NVQ Lefel 1 Cymraeg, fel Siarad, Deall Iaith Lafar ac Ysgrifennu, oes rheswm am hynny?

c) Oes rheswm am i'r ysgol gynnig yr uned ar ddarllen yn benodol, yn hytrach na'r unedau lefel 1 eraill?

2. Rwy'n deall bod yr ysgol yn trin Cymraeg Ail Iaith yn bwnc sylfaenol. Mae'n rhaid i ysgolion cyfrwng Saesneg o leiaf drin Cymraeg Ail Iaith yn bwnc sylfaenol, ond mae modd i ysgolion cyfrwng Saesneg ei drin yn bwnc craidd yn nhermau Mesur Perfformiad.

a) Wrth ystyried bod yr ysgol yn cynnig Cymraeg Iaith Gyntaf, sydd â statws fel pwnc craidd, oes rheswm pam nad yw Cymraeg Ail Iaith yn rhannu'r un statws?

3. Rydych chi wedi cadarnhau bod yr ysgol yn cynnig darpariaeth cyfrwng Cymraeg i “ddisgyblion iaith gyntaf” yng Nghyfnod Allweddol 3 wrth gyflwyno saith pwnc drwy gyfrwng Cymraeg.

a) Beth ydych chi'n ei olygu yma wrth y term 'disgybl iaith gyntaf'? Ai disgybl sydd wedi mynychu ysgol gynradd Gymraeg yw hwn? Neu ddisgybl sy'n dod o gartref Cymraeg ei iaith?

b) Ydy'r pwnc Cymraeg Iaith Gyntaf yn cael ei gyflwyno trwy gyfrwng y Gymraeg?

4. Rydych chi hefyd wedi cadarnhau bod yr ysgol yn cynnig darpariaeth ddwyieithog i ddisgyblion y Cwrs Carlam yn ystod Nghyfnod Allweddol 3 trwy gyflwyno pum pwnc yn ddwyieithog.

a) Ydy'r pwnc Cymraeg Ail Iaith yn cael ei gyflwyno'n ddwyieithog hefyd? 
b) Sut mae'r ysgol yn dethol y disgyblion 'cymwys' ar gyfer y Cwrs Carlam cyn iddyn nhw drosglwyddo i'r ysgol ym mlwyddyn 7 ?

c) Oes modd i ddisgyblion nad ydyn nhw wedi'u dethol gael mynd at ddarpariaeth y Cwrs Carlam?

5. Rwy'n deall mai proses gymhleth yw addysgu'n ddwyieithog ac mae athrawon yn gorfod defnyddio'r ddwy iaith yn dactegol yn y dosbarth er mwyn sicrhau gall disgyblion ymdopi â dysgu cynnwys yn y ddwy iaith. Yn yr holiadur, rydych chi'n nodi bod athrawon yn defnyddio'r Gymraeg fel iaith gyfrwng am '50\% neu fwy' o'r addysgu yn y dosbarth carlam.

a) Er mwyn eglurder, faint o ddefnydd mae'r athrawon yn ei wneud o'r Gymraeg wrth addysgu fyddech chi'n dweud, ai 50\% neu'n fwy?

b) Pa ddulliau addysgu mae'r athrawon eu defnyddio er mwyn cyflawni hyn?

c) Pa mor aml ydy disgyblion yn cwblhau tasgau ac asesiadau yn Gymraeg ar gyfartaledd?

Hyfforddiant staff yn y Gymraeg:

6. Rydych chi'n cadarnhau yn yr holiadur bod hyfforddiant wedi bod ar gael i staff ers y flwyddyn academaidd 2011-2012 er mwyn iddyn nhw "[d]datblygu sgiliau sylfaenol defnyddio Cymraeg achlysurol o fewn y dosbarth".

a) Ai'r staff addysgu yn unig sy'n gallu manteisio ar yr hyfforddiant hwn, neu unrhyw aelod o'r staff?

b) Oedd yr hyfforddiant hwn ar gael cyn 2011-2012? Os nac oedd, pam?

c) Rwy'n deall nad oedd yr hyfforddiant ar gael yn ystod y blynyddoedd academaidd 2013-2014 a 2014-2015. Oes rheswm am hynny?

ch) Faint o'r staff yn mynychu'r cwrs hyfforddi eleni?

7. Mae'r athrawon sy'n addysgu'n ddwyieithog a thrwy gyfrwng y Gymraeg hefyd yn derbyn hyfforddiant mewnol ar gyfer datblygu eu methodoleg addysgu wrth arsylwi gwersi athrawon eraill yr ysgol. 
a) Ar wahân i'r hyfforddiant mewnol hwn, oes disgwyliad, neu angen, bod gan yr athrawon hyn hyfforddiant neu brofiad wrth addysgu'n ddwyieithog / trwy gyfrwng y Gymraeg yn flaenorol cyn addysgu'n ddwyieithog / trwy gyfrwng y Gymraeg yn yr ysgol?

Pontio Rhwng y Sector Cynradd a'r Sector Uwchradd:

8. Rydych chi wedi manylu ychydig ar sut mae'r ysgol yn darparu ar gyfer anghenion ieithyddol y disgyblion ar y Cwrs Carlam a'r disgyblion sy'n cael eu haddysgu trwy gyfrwng y Gymraeg. Mae polisi ar ddwyieithrwydd yn ychwanegu bod "trefnu cyfarfodydd" ar gyfer y disgyblion hyn a'u rhieni yn un o amcanion eraill yr ysgol.

a) Beth yw natur y cyfarfodydd hyn?

b) Pa mor aml ydy'r cyfarfodydd hyn yn cael eu trefnu?

9. O ran sicrhau trosglwyddiad hwylus disgyblion Cymraeg ail iaith o'r sector cynradd i'r sector uwchradd, rydych chi'n amlygu bod yr ysgol yn cynnig dau ddiwrnod trochi, un ym mis Medi ac un arall ym mis Gorffennaf, yn ogystal ag un diwrnod trochi ychwanegol ar gyfer y rhai sy'n dod i'r ysgol o du hwnt i'r clwstwr.

a) Beth yw natur y diwrnodau trochi hyn?

b) Pa ddisgyblion sy'n gallu mynychu'r diwrnodau trochi? Ai disgyblion y Cwrs Carlam yn unig, neu unrhyw ddisgybl ar y llwybr Cymraeg ail iaith? 
Atodiad 6. Meini prawf ar gyfer arsylwi ethos dwyieithog ffurfiol y ddwy ysgol

1. Ymweliadau â'r ysgolion $i$ arsylwi ethos

a. Arwyddion swyddogol dwyieithog o amgylch yr ysgol:

i. Cofnodi ar bapur yr ugain arwydd swyddogol ${ }^{83}$ gyntaf y daw'r ymchwilydd o hyd iddynt, wrth nodi unrhyw arwyddion nad ydynt yn cynnig y Gymraeg. Penderfynwyd cofnodi’r ugain arwydd swyddogol gyntaf, yn hytrach na phob arwydd, oherwydd ymarferoldeb. Nodir hefyd na chofnodir arwyddion answyddogol, megis baneri, posteri ac arddangosfeydd gwaith, a hynny oherwydd rhagdybir y byddai'r arwyddion swyddogol yn fwy tebygol o ddangos ethos ffurfiol y ddwy ysgol.

ii. Cofnodi arwyddion ar y waliau a'r drysau allanol a mewnol, wrth gerdded o fynedfa'r ysgol hyd at y dderbynfa, ac o'r dderbynfa i'r ffreutur. Penderfynwyd cofnodi arwyddion yn yr ardaloedd hyn yn yr ysgol oherwydd mai dyma rai o'r ardaloedd mwyaf prysur lle y byddid yn disgwyl i ddisgyblion, staff ac ymwelwyr fel ei gilydd ymweld â hwy / galw heibio iddynt. O ganlyniad, rhagdybir mai'r arwyddion hyn fyddai'r rhai mwyaf tebyg o fod yn ddwyieithog oherwydd natur weladwy'r arwyddion yn yr ardaloedd hyn.

iii. Cyfrif faint o'r arwyddion a ganfuwyd sy'n ymddangos yn ddwyieithog. Er mwyn penderfynu a ellir ystyried arwydd yn ddwyieithog, cyfeirir at Ganllawiau Dylunio Comisiynydd y Gymraeg (2014). Nid oes "rheolau cyffredinol" wrth ddylunio arwyddion dwyieithog (Comisiynydd y Gymraeg, 2014, t. 18). Er hynny, “[r] haid peidio â thrin y Gymraeg yn llai ffafriol na'r

\footnotetext{
${ }^{83}$ Seilir ystyr 'arwydd' ar ddiffiniad Oxford Dictionaries Online (d.d.), sef hysbyseb a arddangosir yn gyhoeddus sy'n cyflwyno gwybodaeth neu gyfarwyddiadau ar ffurf ysgrifenedig neu ar ffurf symbolau. Yng nghyd-destun y gwaith ymchwil, ystyr 'arwyddion swyddogol' yw arwyddion sy'n cyfeirio ymwelwyr, staff a disgyblion at lefydd o amgylch yr ysgol, sef arwyddbyst; cyflwyno gwybodaeth bwysig, er enghraifft enwau'r adeiladau; ynteu rybuddio o rywbeth, er enghraifft amodau parcio. Fel arfer, disgwylir i'r arwyddion hyn ddilyn dyluniad swyddogol a osodwyd gan yr ysgol. Ystyr 'arwyddion anffurfiol' yng nghyd-destun y gwaith yw arwyddion megis arddangosfeydd gwaith, posteri a baneri nad ydynt yn cyflawni swyddogaeth yr arwyddion swyddogol a nodwyd uchod.
} 
Saesneg o ran ffont, fformat, lliw, maint, eglurder, amlygrwydd nac ansawdd" (Comisiynydd y Gymraeg, 2014, t. 8) wrth ddylunio arwyddion dwyieithog. Os na theimlir bod y ddwy iaith yn gyfartal o ran y nodweddion hyn, nid ystyrir hwy yn ddwyieithog.

b. Mynd i'r dderbynfa a chychwyn sgwrs wyneb yn wyneb ag aelod o'r staff rheng flaen er mwyn darganfod:

i. $\quad$ A all yr aelod o'r staff rheng flaen o leiaf ' $g$ wrdd a chyfarch' yn Gymraeg wyneb yn wyneb?

ii. A oedd yr aelod o'r staff rheng flaen yn gallu cynnal sgwrs yn Gymraeg?

iii. Os nac oedd yr aelod o'r staff yn gallu cynnal sgwrs yn Gymraeg, a gyfeiriodd yr ymchwilydd at aelod arall o'r staff sydd yn gallu?

Er na ragwelir y bydd yr aelod o'r staff rheng flaen yn rhugl yn Gymraeg, dylai'r aelod o'r staff o leiaf fod yn gallu cyfarch yn Gymraeg a chyfeirio'r ymwelydd at rywun sydd yn gallu siarad Cymraeg. Cynigir cyfarchiad syml yn Gymraeg fel 'bore da' neu 'shwmae'. Os ceir cyfarchiad yn Gymraeg, parheir y sgwrs yn Gymraeg. Os ceir ateb yn Saesneg, gofynnir i'r aelod o'r staff a ydy'n siarad Cymraeg. Os na all yr aelod o'r staff siarad Cymraeg, parheir y sgwrs yn Saesneg. Cymerir nodiadau gwaith maes ar bapur yn syth ar ôl cynnal y sgwrs i'w dadansoddi ar ôl cwblhau'r ymweliad.

\section{Galwadau ffôn i'r ysgol}

a. Galw'r ysgol pan fyddai ar agor er mwyn darganfod:

i. $\quad$ A ydy'r aelod o'r staff rheng flaen yn ateb y ffôn gyda chyfarchiad dwyieithog syml? 
ii. Pa iaith gafodd ei blaenoriaethu wrth ateb y ffôn, y Gymraeg neu'r Saesneg?

iii. A oedd yr aelod o'r staff yn gallu cynnal sgwrs yn Gymraeg dros y ffôn?

iv. Os nac oedd yr aelod o'r staff yn gallu cynnal sgwrs yn Gymraeg, a gyfeiriodd yr ymchwilydd at aelod arall o'r staff sydd yn gallu?

Os na fydd yr aelod o'r staff yn gallu deall y Gymraeg, parheir y sgwrs yn Saesneg. Cymerir nodiadau gwaith maes ar bapur yn syth ar ôl galw'r ysgol.

b. Galw'r ysgol pan fyddai ar gau er mwyn darganfod:

i. A ydy peiriant ateb yr ysgol yn cynnig neges ddwyieithog?

ii. A ydy'r neges yn blaenoriaethu'r Gymraeg?

Cofnodir ar bapur neges y peiriant ateb yn syth ar ôl galw'r ysgol.

3. Gwefannau'r ysgolion

a. Ymweld â gwefannau'r ddwy ysgol er mwyn darganfod:

i. A oes fersiwn Cymraeg, yn ogystal â fersiwn Saesneg, o wefan yr ysgol? Os oes, i ba raddau y mae'r wefan yn cyd-fynd ag egwyddorion Canllawiau Dylunio Dwyieithog Comisiynydd y Gymraeg (2014, t. 30)?

ii. A ydy'r ysgol yn hyrwyddo'r Gymraeg a dwyieithrwydd yn weithredol ar ei gwefan? Er mwyn darganfod hyn, porir trwy'r wefan wrth ddefnyddio dolennau mewnol y wefan. Os bydd ar gael, defnyddir hefyd flwch chwilio'r wefan er mwyn canfod y canlyniadau a geir ar gyfer yr allweddeiriau 'Cymraeg', 'Welsh', 'dwyieithrwydd', 'bilingualism', 'dwyieithog', 'bilingual' ac 'ethos'.

4. Dogfennaeth swyddogol yr ysgolion: 
a. Casglu dau fath o ddogfen swyddogol, ar bapur lle bo hynny'n bosibl, sef

i) prosbectysau diweddaraf yr ysgol a'i Chweched Dosbarth a ii) dau gylchlythyr diweddaraf yr ysgol, er mwyn darganfod:

i. A ydy'r dogfennau ar gael yn y naill iaith a'r llall neu'n ddwyieithog?

ii. Os ydy, a ydy cael gafael ar y fersiwn Cymraeg / dwyieithog yr un mor hawdd?

iii. Os ydy, a ydy'r dogfennau yn cyd-fynd ag egwyddorion Canllawiau Dylunio Dwyieithog Comisiynydd y Gymraeg (2014)?

iv. A ydy'r dogfennau'n hyrwyddo dwyieithrwydd yr ysgol?

Dewiswyd y prosbectws a chylchlythyrau i'w dadansoddi yn benodol oherwydd mai natur y dogfennau hyn yw marchnata a hyrwyddo ethos ffurfiol yr ysgol. Os yw'r ysgolion yn marchnata a hyrwyddo eu hethos dwyieithog yn ffurfiol, yn y dogfennau hyn y byddid yn disgwyl i'r ysgolion wneud.

5. Casglu gwybodaeth ynglŷn â digwyddiadau a gweithgareddau'r ysgol i hyrwyddo dwyieithrwydd yn ffurfiol ac yn anffurfiol:

a. Holi Pennaeth Adran y Gymraeg yn y ddwy ysgol yn yr astudiaeth am y digwyddiadau a gweithgareddau i hyrwyddo dwyieithrwydd ffurfiol ac anffurfiol trwy'r ysgol, ar ffurf:

i. Holiadur, a

ii. Cyfweliadau dilynol. 


\section{Prawf Darllen Blwyddyn 9}

\section{a) Y clwb chwaraeon}

Ym mhob brawddeg isod mae bwlch. Ysgrifennwch un gair Cymraeg yn y bwlch i wneud brawddeg synhwyrol. Mae'r ddwy frawddeg gynta' wedi'u gwneud yn barod. There is a gap in each sentence below. Write one Welsh word in the gap so that the sentence makes sense. The first two sentences have been done already.

Mae clwb chwaraeon newydd yng Nghaerdydd.

Enw'r clwb

1. Bob wythnos, mae llawer o yn mynd i'r clwb.

2. Mae pawb yn y clwb yn chwaraeon.

3. Mae Siân, Tom a Harri yn hoffi mynd i'r clwb achos mae'n

4. Maen nhw'n dwli chwarae hoci.

5. Mae Siân, Tom a Harri yn chwaraewyr hoci iawn.

6. , mae Siân a’i ffrind yn chwarae rygbi.

7. Enw ffrind Siân Jade.

8. nos Wener, mae Siân a Jade yn mynd i'r clwb.

9. Maen 'n mynd i'r clwb gyda ffrindiau.

10. Ar ddydd Sul, bydd Tom a Siân yn mynd i'r clwb wylio gêm rygbi Jade.

11. Ond Harri ddim yn mynd ddydd Sul achos mae pen tost gyda fe. 
12. Ar ôl y gêm, bydd Siân a Jade yn ffilm comedi newydd yn y sinema.

13. Bydd pawb arall yn mynd i wylio'r teledu.

14. 'Cariad at Chwaraeon' ydy'r clwb gorau yn byd!

\section{b) Y gêm rygbi fawr}

Dyma flog Jade. Mae sawl bwlch yn y blog yma. Llanwch bob bwlch trwy ysgrifennu gair Cymraeg sy'n gwneud synnwyr yno. Mae'r ddwy frawddeg gynta' wedi'u gwneud yn barod.

This is Jade's blog. There are several gaps in this blog. Fill each gap by writing a Welsh word which makes sense in the gap. The first two sentences have been done already.

Shwmae bawb, Jade sydd yma! Dyma flog newydd . Dw i'n ysgrifennu blog newydd achos hoffwn i siarad _a am yêm rygbi dros y penwythnos!

Penwythnos bendigedig! Dydd Sul, chwaraeais i rygbi yn y clwb chwaraeon newydd. Ond yn y bore, doeddwn i ddim yn ffeindio fy esgidiau rygbi! O na! Gofynnais i fy mam, 'Mam, ble mae esgidiau i ?',

'Dw i'n bod dy esgidiau di yn y gegin' atebodd mam. Roedd yr esgidiau yn bwysig roedd gyda fi gêm rygbi fawr yn y prynhawn. Roeddwn i'n gyffrous iawn yn nerfus iawn hefyd!

Fel arfer, dw i'n i'r gêm gyda fy ffrindiau. heddiw, roedd tad eisiau dod gyda fi. Roedd e'n am y gêm fawr! 
Yn y clwb, i fy ffrind Siân. Ond, welais i ddim Harri

Tom! Roedd Harri a Tom yn teimlo'n

Gofynnais i i Siân 'Sut wyt Siân? Mae gêm fawr gyda ni

! Wyt ti'n gyffrous 'n nerfus?'. Fel arfer mae Siân yn iawn achos mae hi'n mwynhau chwarae rygbi. Ond heddiw, roedd 'n edrych yn nerfus. Roedd llawer o bobl wedi dod i'r i wylio'r gêm!

Roeddwn i'n nerfus ! Gwelais i lawer o - ond dim tad!

Ble oedd wedi mynd?! Yna, gwelais i dad yn rhedeg ata i gyda fy i ! Wps! Dw i ddim yn gallu chwarae rygbi

esgidiau!!

Enillon ni'r gêm ac roeddwn i fy modd! Ar ôl y gêm,

i'r sinema i wylio ffilm comedi newydd. Roedd digon hiwmor yn y ffilm! i'n hoffi ffilmiau comedi achos maen nhw'n ddoniol.

Wel, i'r penwythnos yn fawr iawn! Ond dydd Sul oedd diwrnod gorau! Diwedd perffaith i benwythnos arbennig!

Diolch yn fawr iawn am gwblhau'r prawf.

Thank you very much for completing the test. 
Atodiad 8. Trawsgrifiad o'r cyfweliad â Phennaeth Adran y Gymraeg yn Ysgol Y

\begin{tabular}{|l|l|l|}
\hline Symbol & Enw & Pwrpas \\
\hline testun & Ffont italig & $\begin{array}{l}\text { Dangos pryd mae'r siaradwr yn } \\
\text { newid cod i'r Saesneg. }\end{array}$ \\
\hline$=$ & Hafalnod & $\begin{array}{l}\text { Dangos pryd mae'r naill } \\
\text { siaradwr yn cymryd llyw'r sgwrs } \\
\text { oddi wrth y llall yn sydyn. }\end{array}$ \\
\hline- & Cysylltnod & $\begin{array}{l}\text { Dangos toriad sydyn yn llif y } \\
\text { llefaru. }\end{array}$ \\
\hline testun ] & Bachau petryal & $\begin{array}{l}\text { Dangos pryd mae'r cyfranogwr } \\
\text { a'r ymchwilydd yn llefaru ar yr } \\
\text { un pryd â'i gilydd. }\end{array}$ \\
\hline$\{$ testun $\}$ & Cromfachau cyrliog $)$ & $\begin{array}{l}\text { Gair neu ymadrodd nad yw'n } \\
\text { glir ac sydd yn codi amheuaeth. } \\
\text { Cynigir yma ddyfaliad. }\end{array}$ \\
\hline
\end{tabular}

Seilir y dull trawsgrifio hwn yn rhannol ar ddull Jefferson, G. (1984). Transcription Notation. Yn J.M. Atkinson \& J. Heritage (Gol.), Structures of social action. Caergrawnt: Gwasg Prifysgol Caergrawnt.

\footnotetext{
Allwedd:

$\mathrm{Y}=$ Yr ymchwilydd

$\mathrm{P}=$ Pennaeth Adran Gymraeg yr ysgol
} 
1 YY: Dyna ni. Yn gyntaf felly, diolch yn fawr iawn am gytuno gwneud hyn heddiw. Ydy yn well gyda ti 'ti' neu 'chi' yn y [cyfweliad. Ti? Dim problem ] o

2 PA:

[Ti mae'n iawn, dweud ti, ie. ]

3 YY: gwbl. Fel dw i wedi esbonio yn gynharach, pwrpas y cyfweliad yma yw manylu, egluro, ambell beth sydd wedi codi o'r holiadur wnest ti rai misoedd yn ôl. Felly, bydda i’n gofyn cwpwl o gwestiynau dilynol.

$4 \quad$ PA: Iawn.

5 YY: Bydd y cwestiynau yn dilyn patrwm yr holiadur. Felly bydda i'n dechrau gyda darpariaeth ar gyfer y Gymraeg, wedyn ethos, wedyn hyfforddiant ac wedyn pontio rhwng y cynradd a'r uwchradd. Rwyt ti wedi dweud yn barod dy fod di'n hapus, dy fod di'n fodlon i fi recordio. Dal yn hapus?

$6 \quad$ PA: Iawn, dw i'n hapus gyda hynna.

7 YY: Gwych, grêt. Ac eto, mae'n bosibl bydda i'n cymryd ambell nodyn er mwyn atgoffa i. Ga i ddechrau, os galla i felly, gyda darpariaeth. Dwi'n deall bod yr ysgol yn ysgol Saesneg ac yn yr adran yma, rydych chi'n cyflwyno'r Gymraeg fel ail iaith. Dim ond y Cwrs Llawn sydd ar gael yma. Oes unrhyw reswm penodol am beidio â chynnig y cwrs byr, a dim ond y Cwrs Llawn, o gwbl?

$8 \quad$ PA: Wel rydyn ni'n rîlî lwcus fan hyn bod y prif athro yn rîlî ein helpu ni ac yn cefnogi'r adran Gymraeg ac mae'n hapus i jest cynnig y Cwrs Llawn. A rydyn ni'n lwcus nawr, dim ond y Cwrs Llawn bydd ar gael nawr o mis Medi. Hefyd dw i'n credu bod y disgyblion wedyn yn cymryd Cymraeg fel pwnc pwysig iawn os mae'n Cwrs Llawn. A hefyd rydyn ni'n cael cefnogaeth wedyn wrth y rhieni os maen nhw'n gweld, (on'd yfe), fel cwrs gradd ac mae'n Cwrs Llawn, maen nhw'n hapus wedyn i cefnogi'r plant ac yn hapus i’w cefnogi nhw yn yr ysgol gyda'r Cwrs Llawn. 
9 YY: Mae'r cwestiwn yn dod felly, ac mae hyn wedi codi gyda sawl ysgol rydw i wedi ymweld â nhw ac maen nhw'n dweud bod yna broblemau gyda'r Cwrs Llawn o ran addysgu'r rhai gwannaf. Felly sut mae'r adran yn paratoi ar gyfer hyn ac yn sicrhau eu bod nhw'n gallu ymdopi? Os bydd yna broblemau wrth gwrs. Sut mae'r ysgol yn paratoi'r disgyblion gwannaf ar gyfer y Cwrs Llawn?

10 PA: Rydyn ni'n lwcus gyda'r amser rydyn ni'n ei gael i ddysgu'r Cwrs Llawn fan hyn. Rydyn ni'n cael pum awr bob pythefnos ar gyfer y sets uwch a'r set sylfaenol, felly mae amser gyda ni i gefnogi'r plant sylfaenol a'r plant sy'n cael trafferth gyda'r Cwrs Llawn. Hefyd maen nhw mewn sets, so rydyn ni'n gallu canolbwyntio ar y plant sy'n gwneud yr haen sylfaenol - canolbwyntio ar y cwestiynau sy'n codi dim ond yn y papur sylfaenol. Rydyn ni'n tynnu'r themâu haen uwch mas, fel problemau pobl ifanc neu amgylchedd sy'n themâu ar gyfer yr haen uwch, ac wedyn canolbwyntio ar bethau mwy syml gyda'r sylfaenol. Pethau fel mynegi barn, yr amser gorffennol, sy'n eu helpu nhw wedyn i gael y gradd C yn y Gymraeg. Mae'n helpu bod y sets yn eithaf bach rîlî, dim ond deg neu un deg dau sydd yn y sets gwaelod. Felly mae plant yn y sets yna yn cael mwy o gefnogaeth.

11 YY: Achos bod y dosbarth [yn llai? ] iawn, OK. Pwy sy'n penderfynu pa themâu

12 PA: [Yn llai ie.]

13 YY: sy’n (addas)? Ydy hi’n amlwg (pa themâu sy’n addas), neu?

14 PA: Dw i'n credu ei fod e yn. Dw i wedi bod yn dysgu fan hyn nawr am wyth mlynedd a dw i'n hapus gyda'r ffordd y mae'r themâu yn codi bob blwyddyn. Rwyt ti'n gallu gweld pa fath o bethau maen nhw'n edrych (amdanyn nhw) ar gyfer y sylfaenol ac ar gyfer yr uwch. Ar gyfer sylfaenol, os maen nhw'n gallu mynegi barn a defnyddio'r amser gorffennol, dyna i gyd maen nhw'n edrych amdano. A dyna beth dw i'n canolbwyntio (arnyn nhw) ar gyfer y dosbarthiadau haen sylfaenol. 
15 YY: Gwych, grêt. O ran cwestiwn pedwar, ynglŷn â darpariaeth eto, rwyt ti'n nodi yn yr holiadur bod gan Gymraeg Ail Iaith statws fel pwnc craidd. Beth oedd y rhesymeg y tu ôl i hynny? Pam fod yr ysgol yn ei thrin (yn bwnc craidd) yn hytrach na phwnc sylfaenol [fel, timod, ie. Oes rheswm penodol?] =

16 PA

[Fel lot o ysgolion yn, ie. ] ] = Dw i wedi bod yma nawr am wyth mlynedd, ac yn ystod yr wyth mlynedd dw i wedi bod yma mae wedi bod yn bwnc craidd. Dydw i ddim yn hollol siŵr pam maen nhw wedi penderfynu hwnna. Dwi'n credu ei fod yn mynd nôl i'r prif athro a'r dirprwy - oedd hi'n arfer bod yn bennaeth yr adran Gymraeg - ac mae hi'n rîlî gefnogol (o'r) adran. So dw i’n credu mae'n dod oddi wrthi hi efallai achos mae hi mor gefnogol o'r iaith Gymraeg. A rydyn ni'n cael canlyniadau da iawn. Mae prifathro newydd gyda ni, dechreuodd e ddwy fynydd yn ôl, ac mae e'n hapus i gario ymlaen i wneud e fel pwnc craidd. So rydyn ni'n cael Saesneg, Mathemateg, Gwyddoniaeth dwbl, Addysg Grefyddol yn bynciau craidd fan hyn, a Chymraeg Ail Iaith.

17 YY: A sut mae hynna'n effeithio ar fesur perfformiad rhwng yr ysgolion? Ydy'n cael effaith?

18 PA: Na dim rîlî. Rydyn ni'n cael bron yr un canlyniadau â Mathemateg, Saesneg a Gwyddoniaeth. Eleni, rydyn ni wedi cael chwe deg, bron chwe deg naw y cant, A* i C ym Mlwyddyn 11 yn y Cwrs Llawn. So roeddwn i'n rîlî hapus gyda hwnna. Roedd Mathemateg, Gwyddoniaeth a Saesneg wedi cael saith deg, saith deg un y cant, so rydyn ni bron ar yr un lefel â Mathemateg a Saesneg.

19 YY: So, dyw e ddim yn creu problem o ran $=$

20 PA: $=\mathrm{Na}$, achos dyw'r plant byth wedi dilyn y cwrs byr a dydyn nhw ddim yn gwybod dim byd arall. So maen nhw jest yn derbyn bod Cymraeg yn bwnc craidd ac mae pawb yn ei wneud e. Dydyn nhw ddim rîlî yn gofyn cwestiynau. Ddim yn siŵr sut mae hwnna'n digwydd. Rwyt ti’n cael cwpwl sy'n cwyno ac sy'n dweud 'pam rydyn ni'n gwneud Cymraeg', blah blah blah. Ond, achos maen nhw'n cael yr oriau wedyn, maen nhw'n ei weld e fel Mathemateg, 
Cymraeg, a Saesneg. Ac rydyn ni i gyd yn ei ddrilio fe i mewn iddyn nhw. Pynciau craidd yn yr ysgol ydy Gwyddoniaeth, Mathemateg, Saesneg, Cymraeg ac Addysg Grefyddol. Ac maen nhw'n ei dderbyn e wedyn.

21 YY: OK, diolch. Dw i'n credu ein bod ni wedi ateb y cwestiwn yr ail gwestiwn o ran hynny, yr is-gwestiwn. O ran cwestiwn naw, eto o dan darpariaeth. Fel soniais i, mae'n bosibl labelu'r ysgol yma yn ysgol Saesneg wrth gwrs achos dyna brif iaith yr ysgol o ddydd i ddydd. Ond tybed, oes gan yr ysgol y capasiti i gynnig ychydig o bynciau trwy gyfrwng y Gymraeg a'r Saesneg, felly yn ddwyieithog? Ydy hynna'n bosibl, rwyt ti'n feddwl?

22 PA: Wel mae'n dod i lawr i'r athrawon sy'n gallu dysgu Cymraeg. Mae cwpwl yn yr ysgol fan hyn sy’n gallu dysgu Cymraeg. Does dim gradd yn y Gymraeg gyda nhw ond mae'n nhw'n gallu dysgu Cyfnod Allweddol 3. Dw i ddim yn siŵr os bydden nhw'n teimlo'n ddigon hyderus i ddysgu trwy gyfrwng y Gymraeg. A dyna beth yw'r broblem, dw i'n credu, yw'r athrawon i ddysgu trwy gyfrwng y Gymraeg, achos yn yr Adran Gymraeg, dim ond fi ac un arall sy'n llawn amser. So mae'n dod i lawr i'r athrawon, dw i'n teimlo. Dw i ddim yn siŵr os mae'r prifathro yn mynd i fod yn hapus os rydyn ni'n cynnig - ac mae'n dod i lawr i'r plant. Mae cwpwl yn dod o gefndir Cymraeg, ond does dim lot i gael dosbarth. So dw i ddim yn hollol siŵr. Dydyn ni ddim rîlî wedi meddwl am hynna i fod yn onest gyda ti, ond =

23 YY: $=\mathrm{Y}$ prif rwystr felly, baset ti'n ddweud, yw bod yna ddim digon o [hyfforddiant ] i'r athrawon [yna.]

24 PA:

[Hyfforddiant]

[ie. ]

25 YY: Ydy'n bosibl i'r ysgol gynnig rhywbeth fel hwnna, neu - wel, rwyt ti wedi dweud yn barod nad yw'r ysgol wedi ystyried addysg o'r fath. So wyt ti'n meddwl bod - ydy hynny'n bosibl trwy'r ysgol, o ran hyfforddiant?

26 PA: Wel hefyd mae'n dod i lawr i'r arian. So os mae rhyw fath o scheme, neu rywbeth sy'n gallu cynnig arian i'r ysgol, ac wedyn gofyn os mae'n bosibl i wneud 
rhyw fath o hyfforddiant fel hwnna, efallai bydd e'n bosibl. Dw i ddim yn siŵr. Ond, dw i'n credu ei bod hi'n dod i lawr i'r athrawon sy'n gallu, ac yn fodlon i'w wneud e, os mae'r arian ar gael i'w wneud.

27 YY: Diolch. Os ga i, hoffwn i symud ymlaen i ethos dwyieithog yr ysgol. A dim ond un cwestiwn sydd gyda fi mewn gwirionedd. Rwyt ti'n amlygu yn yr holiadur bod dwyieithrwydd yn cael ei hyrwyddo trwy'r ysgol gyfan, a hynny'n bennaf ar ffurf posteri, gwasanaethau a phethau fel hynny. Ond ar wahân i'r pethau mewnol, pethau o ddydd i ddydd rydych chi'n eu gwneud fel adran ac fel ysgol gyfan, oes unrhyw hyfforddiant o gwbl ar gyfer defnyddio'r Gymraeg yn achlysurol yn y dosbarth?

28 PA: Rydyn ni yn trio cefnogi'r plant sydd wedi dod o gefndir Cymraeg, ac maen nhw wedi dangos diddordeb i helpu gyda dwyieithrwydd yn yr ysgol. Maen nhw'n sôn ar hyn o bryd am wneud rhyw fath o clwb Cymraeg ar gyfer y rhai sy'n iaith gyntaf, ac wedyn gobeithio bydd y disgyblion ail iaith yn dod i mewn ac maen nhw'n gallu gwneud rhyw fath o-peer mentoring neu rywbeth fel hynny - gyda nhw a'u helpu nhw trwy'r gwersi Cymraeg. Rydyn ni'n cael cwpwl o ddisgyblion o \{Enw ysgol uwchradd Gymraeg\} sy'n ymuno â'r Chweched Dosbarth, ac maen nhw'n dod i mewn ac maen nhw'n mynd o gwmpas y dosbarthiadau i helpu disgyblion ail iaith, a threfnu tripiau a phethau felly. Ond hefyd, dw i'n credu bod - achos mae'n bwnc craidd, mae'r disgyblion yn eithaf - wel maen nhw'n teimlo'n falch o'r iaith Gymraeg yn yr ysgol fan hyn, ac maen nhw'n hapus i gefnogi'r iaith a hysbysebu'r iaith Gymraeg a'r pwysigrwydd o ddysgu'r yr iaith.

29 YY: Felly, nid y staff yn unig sy'n hyrwyddo'r iaith, [rwyt ti'n dweud] bod y

30 PA: [Na, disgyblion. ]

31 YY: disgyblion [hefyd] - Ydyn nhw'n tueddu i ddefnyddio'r Gymraeg lot o 32 PA: [Ie. ] 
33 YY: gwmpas yr ysgol? Wyt ti'n clywed lot (o Gymraeg)?

34 PA: Y rhai sydd wedi ymuno â'r Chweched, so wedi dod o [Enw ysgol uwchradd Gymraeg\}. Roedd un bachgen gyda fi y bore yma. Daeth i mewn i'r wers, Blwyddyn 10. Achos mae'r plant yn ei adnabod, maen nhw'n meddwl wedyn ei fod e'n gallu siarad Cymraeg yn rhugl ac mae hwnna'n edrych yn cŵl. Ond mae rhai nawr wedi dweud wrthyf i 'dw i rîlî moyn siarad Cymraeg yn rhugl, fel [enw'r disgybl]'. So maen nhw'n ei weld e wedyn, fel rhywbeth sy'n edrych yn cŵl, achos mae'r disgyblion - maen nhw'n edrych lan iddo. (Mae'n) creu rhyw fath o statws gyda'r iaith Gymraeg, ei bod hi'n cŵl ac yn dda os wyt ti yn gallu siarad Cymraeg yn rhugl.

35 YY: Iawn. OK. Gwna i ddod nôl at y pwynt yna, achos mae cwestiwn gen i sy’n dilyn hynny. Ond jest cyn hynny - rwyt ti'n nodi, yn yr holiadur eto, nad oes polisi ffurfiol ar gyfer [dwyieithrwydd]. Dw i'n gwybod bod mwy, erbyn hyn, o

37 PA: $\quad[\mathrm{Na} . \quad]$

38 YY: ysgolion Saesneg sy'n gweithredu polisi o'r fath. Oes unrhyw reswm penodol, yn dy feddwl di, pam nad yw'r ysgol yn =

39 PA:

$=$ Dw i ddim yn hollol siŵr ar hyn o bryd achos dw i'n newydd i'r swydd fel pennaeth yr adran Gymraeg. Dw i ddim yn hollol siŵr pam does dim polisi ar gael. Dw i'n moyn gwneud rhywbeth ac dw i'n hapus i weithio gyda'r dirprwy a'r prifathro i wneud rhyw fath o bolisi ar ddwyieithrwydd yn yr ysgol. A hefyd cael y plant - os mae'n nhw'n gallu fy helpu i a siarad am ryw fath o bolisi gyda'n gilydd a - gobeithio bydd e ar gael wedyn ar gyfer y rhieni, a'r plant, ac maen nhw'n gallu edrych trwyddo. Ac unrhyw sylwadau sydd gyda nhw hefyd, rydyn ni'n gallu eu nodi nhw i lawr a chreu rhyw fath o bolisi gyda'n gilydd, yn lle jest fi yn ei wneud e. Bydd hwnna'n edrych yn dda, os rydyn ni'n gallu gweithio gyda'n gilydd. 
40 YY: Grêt. Dw i'n credu bod hyn yn ein harwain ni'n ôl at hyfforddiant. Roedden ni'n sôn yn gynharach am ddefnyddio'r Gymraeg yn achlysurol, sut mae'r staff yn gwneud hyn. Ac dw i'n sî̂r dy fod di'n dweud yn yr holiadur bod y disgyblion, rwyt ti wedi bod yn cyfeirio atyn nhw yma, bod y disgyblion - yn arbennig yn y

Chweched dosbarth - maen nhw'n helpu'r adran. Dw i'n siŵr ei fod e'n dweud yn yr holiadur "helpu gyda Chymraeg”. Felly sut mae'r disgyblion hyn yn helpu'r adran? Beth maen nhw'n ei wneud yn benodol i helpu'r adran?

41 PA: Wel maen nhw'n dod i mewn i wersi, enwedig gwersi TGAU, ac maen nhw'n fy helpu i lot achos mae rhaid i fi mynd o gwmpas a helpu disgyblion, fel unigolion, a rhoi cefnogaeth iddyn nhw. So os oes rhywun arall yn y dosbarth hefyd sy'n gallu gwneud hynny gyda fi, mae'n help fawr. Hefyd, dw i'n credu bod y plant yn leicio gweld pobl o'r Chweched yn dod i mewn sy'n gallu siarad Cymraeg yn rhugl. Fel dywedais i, maen nhw'n edrych fel eu bod nhw'n cŵl ac maen nhw'n moyn siarad Cymraeg wedyn achos bod rhywun arall yn gallu ei wneud e. Rydyn ni'n edrych hefyd am wneud rhyw fath o clwb Cymraeg ar gyfer y rhai sy'n iaith gyntaf, a sut rydyn ni'n gallu defnyddio'r disgyblion wedyn i fynd i mewn i fwy o wersi Cymraeg, neu wneud gwasanaethau yn Gymraeg, neu ar ddiwylliant yn Gymraeg, pethau fel hynny. Rydyn ni'n cael cwpwl nawr o'r ysgol gynradd Gymraeg yn yr ardal fan hyn i \{Ysgol Y\}, oherwydd y daith i \{Enw ysgol uwchradd Gymraeg\} o $\{$ Enw ardal Ysgol Y\} yn hir iawn.

42 YY: Dw i'n siŵr yn yr holiadur, dyw e ddim yn dweud bod ysgolion cynradd Cymraeg yn rhan o'r [ clwstwr ond mae yna rai sy'n dod ] tu fas i'r -

$43 \quad \mathrm{PA}$ [Na 'dyn nhw ddim. Mae rhai'n dod, ie ]

44 YY: OK diolch.

45 PA: A dw i'n lwcus hefyd, dw i'n diwtor i Flwyddyn 7 ac mae rhai rhieni wedi gofyn os dw i'n gallu cael eu plant yn fy nosbarth i - os dw i'n gallu siarad Cymraeg gyda nhw, os maen nhw'n dod o ysgol gynradd Gymraeg. Mae hwnna'n helpu lot hefyd ac mae rhieni'n rîlî leicio hwnna, achos dw i'n gallu siarad Cymraeg gyda nhw 
trwy'r dydd. Dydyn nhw ddim yn colli'r iaith achos maen nhw'n dod i'r ysgol gyfun Saesneg - So mae hynna wedi dechrau'r flwyddyn 'ma =

ti'n feddwl, gyda disgyblion sy'n dod o'r sector Cymraeg - cyfrwng Cymraeg - ac sy'n mynd i mewn i'r sector cyfrwng Saesneg? Ydy hynny, yn dy feddwl di, yn broblem? Rwyt ti wedi cyfeirio ati yn fanna dy fod di'n ceisio sicrhau bod y disgyblion iaith gyntaf - Cymraeg Iaith Gyntaf - yn cael digon o fewnbwn, digon o gyfle i siarad Cymraeg. Ydy'n broblem, rwyt ti'n feddwl, yn gyffredinol gyda ysgolion ac yn yr ysgol yma?

47 PA: Beth dw i'n teimlo weithiau yw bod y rhieni gyda phlant iaith gyntaf, maen nhw'n credu - achos maen nhw'n dod i'r ysgol gyfun Saesneg, maen nhw'n gallu gwneud y TGAU yn gynnar. A dw i'n ffeindio bod yr iaith sydd gyda nhw yn gallu bod yn wân, weithiau. Mae'n dibynnu ar y disgybl. Mae'r iaith yn gallu bod yn wân achos dydyn nhw ddim - yn yr ysgol gynradd, maen nhw wedi pigo lan mwy o'r iaith lafar yn lle yr iaith ffurfiol rydyn ni'n ei defnyddio yn y cwrs ail iaith. Ac maen nhw'n cael trafferth pan maen nhw mewn gwersi. Weithiau maen nhw'n dadlau gyda fi taw nhw sy'n gywir a taw fi sy'n anghywir. Ond dw i'n trio dweud wrthyn nhw rydyn ni'n dysgu trwy'r iaith ffurfiol - ffurfiol iawn - yn lle, pethau iaith lafar ac iaith anffurfiol. Rydyn ni'n trio rhoi gwaith arall iddyn nhw. Pethau fel atalnodi a phriflythrennau yn achosi problemau gyda nhw achos maen nhw - dydyn nhw ddim wedi dysgu lot o'r iaith Saesneg yn yr ysgol gynradd Gymraeg - mwy o'r iaith Gymraeg wrth gwrs. A maen nhw'n colli pethau wedyn, fel atalnodi a phriflythrennau yn y ddwy iaith, dw i'n credu. Ond roeddwn i'n cael lot o rieni yn dweud wrthyf i 'pam nad ydyn nhw'n gallu sefyll y TGAU yn gynnar?'. Wel wrth gwrs, maen nhw'n gallu ei sefyll yn gynnar, ond bydden nhw'n cael B neu A a dylen nhw gael A* weithiau, dw i'n meddwl. So dw i'n eu gwthio nhw i ddal ati tan Flwyddyn Deg neu Un ar ddeg nes fy mod i'n teimlo eu bod nhw'n barod i'w wneud e, ac maen nhw'n gallu defnyddio'r iaith ffurfiol, sy'n gywir ar gyfer y papur ail iaith. 
48 YY: Diddorol iawn. Diolch. Iawn, dw i'n credu ein bod ni wedi - do, iawn. So, does dim hyfforddiant penodol felly o ran - rhywbeth mwy ymarferol, rhywbeth sy'n digwydd o ddydd i ddydd [o ran defnyddio'r] Gymraeg yn achlysurol. Iawn.

49 PA:

[ Wrth gwrs, ie. ]

50 YY: Rwyt ti hefyd yn cadarnhau yn yr holiadur, does dim - rwyt ti wedi dweud hyn yn barod dw i'n gwybod - does dim byd ar gael o ran hyfforddiant methodoleg addysgu trwy gyfrwng y Gymraeg. A rwyt ti wedi dweud yn barod, er dy fod di'n gallu siarad Cymraeg, dyw hynny ddim o reidrwydd yn golygu dy fod di'n gallu dysgu trwy gyfrwng [yr iaith] honno. Ydy hynna'n rhywbeth, rwyt ti'n feddwl, sydd

51 PA: [ Ie. $]$

52 YY: ei angen yn fwy mewn ysgolion Saesneg yn gyffredinol? Neu hyd yn oed yn yr ysgol hon, efallai, achos bod rhai disgyblion sy'n rhugl yn y Gymraeg?

53 PA: Y peth yw achos mae'n ysgol Saesneg, Saesneg sy'n dod yn gyntaf yn anffodus. Mae'n rîlî anodd achos i fi, mae'n bwysig iawn os rydyn ni'n gallu cynnig gwersi yn Gymraeg ac yn Saesneg, so maen nhw'n gallu cael y dewis wedyn. Ond jest yr amser i'w drefnu, cael yr athrawon i mewn i'w wneud, a'r arian dw i'n credu. Dyna beth mae'n dod i lawr iddo.

54 YY: $\quad$ Ie. Dim digon o amser yn ystod y diwrnod gwaith fel petai, neu'n gyffredinol, ar ôl ysgol, achos =

$55 \quad$ PA: $\quad$ = Efallai ein bod ni'n gallu cynnig rhywbeth ar ôl ysgol, byddai hwnna'n rhywbeth rydyn ni'n gallu meddwl am =

$56 \quad \mathrm{YY}$ :

$=$ Dw i'n meddwl bod

cyrsiau - rhaid i'r ysgol talu - trwy \{Enw Prifysgol $\}$. Dw i ddim yn sicr o ran eu prisiau ond fel arfer, dw i'n credu beth sy'n digwydd yw - maen nhw'n gallu bod yn 
hyblyg - maen nhw'n cynnig y cyrsiau ar ôl ysgol ac mae hyd y cwrs yn dibynnu ar faint o fewnbwn a faint o hyfforddiant rydych chi'n ei angen. Wyt ti'n meddwl bod hwnna, efallai, yn dibynnu ar y [arian] =

$57 \quad$ PA:

[Ie. ] = Yn anffodus mae lot yn dod i lawr i'r arian.

Dyna beth yw e rîlî, os rydyn ni'n - os rydyn ni'n dweud beth yw'r broblem, arian yw'r broblem yn anffodus. Rydyn ni'n cael trafferth i gael yr arian i wneud stwff yn yr ysgol ac yn yr adran Gymraeg. Dim ond fi ac un arall sydd fan hyn, ac mae amserlenni llawn gyda ni. Mae cwpwl o athrawon eraill yn dysgu tipyn bach yng Nghyfnod Allweddol 3, Cymraeg. Ond arian yw'r broblem dw i'n credu. Dyna beth yw hi.

58 YY: Diolch. Iawn, a'r cwestiynau olaf. Dw i'n credu - o ran pontio rhwng y sector cynradd a'r sector uwchradd - dw i'n credu fy mod i wedi gofyn y cwestiwn cyntaf yn barod. Hynny yw, sut mae'r disgyblion yn y Chweched yn helpu - rydyn ni wedi cyfeirio at hynny - ond un peth arall sy'n codi yn yr holiadur yw'r cyfarfodydd, rwyt ti wedi cyfeirio atyn nhw yn yr holiadur, gyda'r ysgolion cynradd yn y dalgylch. Felly beth yw natur y cyfarfodydd hyn? Hynny yw, beth sy'n cael ei drafod yn ystod y cyfarfodydd hyn?

59 PA: Wel mae ysgol gynradd \{Enw ysgol $\}$ jest i lawr yr heol, so rydyn ni'n eithaf agos at yr ysgol honna. Llynedd, roedden ni wedi trefnu i'r disgyblion Lefel A, AS, fynd i mewn i'r ysgol gynradd i helpu gyda Chymraeg achos roedd yr athrawon yn yr ysgol gynradd - roedden nhw'n teimlo - doedd dim digon o hyder gyda nhw i wneud stwff yn y Gymraeg. Dw i ddim yn siŵr faint o hyfforddiant maen nhw'n ei gael i addysgu Cymraeg yn yr ysgol gynradd, ond roedden nhw'n rîlî hapus - ac maen nhw'n teimlo'n rîlî lwcus i gael y Chweched i mewn i helpu. Hefyd, roedd y plant bach - roedden nhw'n rîlî leicio hynny hefyd. I weld y disgyblion o'r ysgol gyfun yn mynd i mewn a gwneud stwff Cymraeg gyda nhw. Hefyd y llynedd, roedd cluster moderation gyda ni ar Gymraeg Ail Iaith ac roedden nhw'n poeni tipyn bach am lefelau, achos doedden nhw ddim yn teimlo'n digon hyderus i roi lefelau ar gyfer Cymraeg. So, es i mewn i'w helpu nhw ac i fod yn onest, maen nhw'n gwneud lot o waith Cymraeg yn yr ysgol gynradd. Ddim yn siŵr am yr ysgolion cynradd eraill, fel 
\{Enw ysgol $\}$ a $\{$ Enw ysgol $\}$, ond yn $\{$ Enw ysgol $\}$, maen nhw'n gwneud lot o waith yn y Gymraeg. Maen nhw'n darllen llyfrau yn Gymraeg, maen nhw'n gwneud lot o waith darllen a deall, ysgrifennu. Ces i sioc i fod yn onest achos dydw i byth wedi bod mewn ysgol gynradd i weld faint o Gymraeg maen nhw'n ei gwneud a chwarae teg, maen nhw'n gwneud gwaith da iawn.

60 YY: Pa mor aml ydy'r cyfarfodydd hyn yn arfer cael eu cynnal?

$61 \quad$ PA: Llynedd, lot, ar gyfer y cluster moderation. Ond nawr, efallai un neu dwywaith y tymor rydw i'n gallu mynd i mewn. Ond jest yr amser. Mae'n dod lawr i'r amser a mynd i mewn, [ cwrdd lan, ] fi sy'n mynd. Dw i'n gallu mynd i

62 YY: [Ife ti sy'n mynd?]

63 PA: mewn heddiw, neu e-bostio nhw, a siarad gyda'r brifathrawes i ofyn iddi hi beth mae hi'n ei ddisgwyl. Mae hi jest yn fy e-bostio i ddweud, os mae'r disgyblion yn gallu dod i mewn i wneud stwff, ar y stori fer maen nhw'n ei gwneud, neu jest pethau syml (fel) lliwiau a rhifau. Ac mae hi’n rîlî rîlî hapus i gael y gefnogaeth oddi wrth yr ysgol gyfun.

64 YY: Mae diffyg hyder felly ymhlith yr [ athrawon yn yr ysgol gynradd? ]

65 PA: [Athrawon yn yr ysgol gynradd, oes.]

66 YY: Beth am eu gafael ar y Gymraeg [hefyd] =

67 PA: $\quad[\mathrm{Ie}]=,\mathrm{dw}$ i'n credu bod gyda nhw athrawes bro sy'n mynd i mewn. Dw i ddim yn siŵr os mae hi'n mynd i mewn bob tymor, neu bob wythnos, dw i ddim yn hollol siŵr am hwnna. Ond, mae'n dod i lawr i'r amser i gael rhywun fel hwnna i ddod i ddysgu'r plant, ym mhob blwyddyn, a'u helpu nhw gyda Chymraeg. Felly y llynedd, ro'n ni wedi cael y Chweched i fynd i mewn bob prynhawn dydd Mercher. Mae'n helpu'r Chweched gyda'r iaith Gymraeg 
ac mae'n edrych yn dda ar eu cais UCAS ar gyfer y brifysgol wedyn. Os maen nhw'n gwneud rhyw fath o teacher aid, mae'n edrych yn dda ar eu cais nhw.

68 YY: Gwych. Dw i'n credu mai dyna'r cyfan sydd gen i o ran y cwestiynau, gwna' i jest tsiecio yn gyflym os ga i. O ie, un cwestiwn bach dw i wedi ei golli, nôl at y disgyblion gwannaf. Rwyt ti wedi sôn am y ffaith eich bod chi'n dewis y themâu er mwyn sicrhau eu bod nhw'n cyrraedd y 'C', neu beth bynnag. Dw i'n ymwybodol bod cyrsiau eraill, ar wahân i’r Cwrs Byr, mae cwrs NVQ hefyd. Ydy'r ysgol erioed wedi ystyried $=$

69 PA: $\quad=$ Roedden ni'n arfer ei wneud e. Cwpwl o flynyddoedd yn ôl nawr, ond mae'r NVQ yn mynd hefyd. Dim ond y Cwrs Llawn sy'n dod i mewn nawr. Dyna pam dydyn ni ddim wedi cario ymlaen gyda fe. Ie, doeddwn i ddim wedi ei ddysgu. So dw i ddim yn rîlî yn gyfarwydd â'r cwrs. Ond roedd yr hen bennaeth adran - roedd hi'n ei ddysgu a dywedodd ei fod jest lot well gwneud yr haen sylfaenol gyda nhw. Yr un fath o stwff roedden nhw'n ei wneud, ond roedd e'n lot well wedyn iddyn nhw gael gradd yn lle rhyw fath o lefel neu rywbeth, fel maen nhw'n ei gael. Roedd e'n lot well i'r disgyblion gael gradd. Ond dw i erioed wedi'i ddysgu, so dw i ddim yn gyfarwydd â'r cwrs.

70 YY: Iawn. OK, diolch. Dw i ddim yn credu bod unrhyw beth arall. Diolch yn fawr am dy amser di. Oes unrhyw sylwadau sydd gyda ti, cyn i ni orffen?

71 PA: Na, dw i ddim yn credu. [Rydyn ni] wedi - na $=$

72 YY: $\quad[\mathrm{Na}$ ? $] \quad$ = Wel [diolch yn fawr] iawn

73 PA:

[diolch yn fawr]

74 YY: eto am dy amser.

75 PA: Dim problem. Da bo’. 
76 YY: Da bo'. 
Atodiad 9. Trawsgrifiad o'r cyfweliad â Phennaeth Adran y Gymraeg Ysgol X.

\begin{tabular}{|l|l|l|}
\hline Symbol & Enw & Pwrpas \\
\hline testun & Ffont italig & $\begin{array}{l}\text { Dangos pryd mae'r siaradwr yn } \\
\text { newid cod i'r Saesneg. }\end{array}$ \\
\hline$=$ & Hafalnod & $\begin{array}{l}\text { Dangos pryd mae'r naill siaradwr } \\
\text { yn cymryd llyw'r sgwrs oddi wrth } \\
\text { y llall yn sydyn. }\end{array}$ \\
\hline- & Cysylltnod & $\begin{array}{l}\text { Dangos toriad sydyn yn llif y } \\
\text { llefaru. }\end{array}$ \\
\hline [ testun ] & Bachau petryal & $\begin{array}{l}\text { Dangos pryd mae'r cyfranogwr a'r } \\
\text { ymchwilydd yn llefaru ar yr un } \\
\text { pryd â'i gilydd. }\end{array}$ \\
\hline ( testun $)$ & Cromfachau sengl & $\begin{array}{l}\text { Gair neu ymadrodd nad yw'n glir } \\
\text { ac sydd yn codi amheuaeth. } \\
\text { Cynigir yma ddyfaliad. }\end{array}$ \\
\hline$\{$ testun $\}$ & Cromfachau cyrliog & $\begin{array}{l}\text { Gwybodaeth sensitif wedi'i } \\
\text { hanonymeiddio }\end{array}$ \\
\hline & &
\end{tabular}

Seilir y dull trawsgrifio hwn ar ddull Jefferson, G. (1984). Transcription Notation. Yn J.M.

Atkinson \& J. Heritage (Gol.), Structures of social action. Caergrawnt: Gwasg Prifysgol Caergrawnt.

Allwedd:

$\mathrm{Y}=$ Yr ymchwilydd

$\mathrm{P}=$ Pennaeth Adran Gymraeg yr ysgol 
1 YY: Helô. Diolch yn fawr am yr amser heddiw, ac am gytuno cynnal y cyfweliad. Cyn i ni ddechrau, ydy'n well 'da chi ‘chi' neu ['ti' - yn bendant?] Iawn, dim

2 PA:

[O ti plîs, ie. ] ]

3 YY: problem. OK, fel dw i wedi'i esbonio yn gynharach, pwrpas y cyfweliad yw egluro a manylu ar ambell beth sy'n codi o'r holiadur gwnest ti ei lenwi rai misoedd yn ôl. Bydd y cyfweliad yn dilyn patrwm yr holiadur, hynny yw, y themâu darpariaeth, hyfforddiant, ethos ac ati. Fydda i ddim yn gofyn gwestiynau ynglŷn ag ethos, dim ond darpariaeth, hyfforddiant a phontio rhwng y sectorau heddiw. Os ga' i ddechrau felly gyda darpariaeth - rwyt ti'n nodi yn yr holiadur, mewn ymateb i gwestiwn un dw i'n credu, nad yw'r ysgol yn cynnig y Cwrs Byr. A dw i'n deall bod yr ysgol yn cynnig yr NVQ Darllen Lefel 1 ar gyfer y tri deg pump - pedwar deg disgybl gwannaf. Oes rheswm, neu resymau efallai, am i'r ysgol gynnig y cwrs hwn yn lle'r Cwrs Byr?

4 PA: O ran hynny, mae'r Cwrs Byr - nid yw e o reidrwydd yn haws na'r Cwrs Llawn, mae e jest hanner y Cwrs Llawn. Dw i'n gwybod mai'r hanner symlaf o'r cwrs ydy e, ond o ran eu teimladau personol ni, a'r ysgol, teimlwyd ei fod e'n dal yn rhy heriol ac yn rhy anodd ar gyfer y disgyblion, yn enwedig gan fod yna elfen gref o lafar ac ysgrifennu ynddyn nhw. Roeddwn i'n sylweddoli gyda'r plant gwannaf, un o'u prif bethau nhw ydy diffyg hyder ar lafar yn bendant a doeddwn i ddim eisiau mynd i'r sefyllfa ble roedden nhw'n dweud dim ar dâp, neu ond yn gallu ymateb i rai pytiau bychain, ac yn ysgrifenedig hefyd. Gan ein bod ni'n ei ddarparu ar gyfer y plant sydd ag anghenion arbennig, wrth gwrs ysgrifennu hefyd yn mynd i fod yn broblem. O ran sgiliau llythrennedd y disgyblion felly, penderfynwyd ar y darllen gan fod gyda'r disgyblion sydd gyda ni'r eirfa Gymraeg ac y sgìl darllen oedd y peth roedden nhw'n teimlo'n gyffyrddus ynddo, ac yn teimlo'n fwy hyderus ynddo. Felly penderfynwyd hynny ar gyfer y dosbarth anghenion arbennig i gyd, a hefyd rhyw ugain o'r dysgwyr gwannaf o'r holl flwyddyn ysgol. 'Dych chi'n siarad, o ran blwyddyn ysgol fan hyn, am rhwng dau gant pedwar deg a dau gant chwe deg o ddisgyblion. Felly, fel rydych chi'n ei weld, y mwyafrif - dros dau gant - yn gwneud 
y Cwrs Llawn. Wel, dros dri gant yn mynd i wneud y Cwrs Llawn TGAU, gyda nifer fechan yn gwneud yr NVQ Darllen.

$5 \quad$ YY: $\quad$ Reit, OK. A'r rheswm - wrth gwrs, rwyt ti wedi esbonio fan hyn - y rheswm am wneud y Darllen Lefel 1 yn hytrach na'r Cwrs Byr - achos mae yna unedau [ eraill - oes, oes ] =

6 PA: $\quad[$ Mae pedair sgil, oes $]=\mathrm{ie}-$ mae pedair sgil yn cael $[\mathrm{eu}$ hasesu asesu $]=$

$7 \quad \mathrm{YY}:$ defnyddio'r uned honno achos bod cryfder gyda nhw o ran y darllen ac o ran yr eirfa, ie?

$8 \quad$ PA: $\quad$ Ie.

$9 \quad$ YY: $\quad$ Reit, diolch yn fawr $=$

10 PA: $\quad$ = A byddwn ni - ar hyn o bryd rydyn ni'n edrych ar achos mae nifer yr oriau mae'r dosbarth hwnnw'n cael yn ehangu, felly byddwn ni'n edrych ar y sgìl arall, efallai gwrando. Ac wedyn y sgiliau eraill, o bosibl. Nid o reidrwydd gyda'r anghenion arbennig, ond efallai gyda'r ugain nesaf. Rydyn ni'n edrych mwy ar gyfer hynny, ond gyda'r NVQ yn newid y flwyddyn nesaf yn 2017, rydyn ni'n mynd i aros a gweld beth yw'r newidiadau cyn symud ymlaen $=$

11 YY:

$$
=\text { Reit, }
$$

achos clywais i fod yr NVQ yn newid - doeddwn i ddim yn sî̂r yw'n dod i ben neu so mae e yn newid?

12 PA: Mae e'n mynd i barhau a dw i wedi cael cadarnhad o hynny gan y bwrdd arholi OCR ond dydyn nhw ddim yn sicr eto o fewn pa wedd bydd y cymhwyster ond bydd y cymhwyster yn bodoli. 
13 YY: Gwych, diolch am hynny. Rwyt ti wedi ateb y trydydd is-gwestiwn sydd gyda fi fan hyn. Gwna' i symud ymlaen felly. Mae'r ysgol yn trin Cymraeg Ail Iaith fel pwnc sylfaenol ac mae'n rhaid i ysgolion Saesneg o leiaf ei drin fel pwnc sylfaenol, wrth gwrs. Ond mae modd i ysgolion drin Cymraeg Ail Iaith fel pwnc craidd. Wrth ystyried bod yr ysgol yn cynnig Cymraeg Iaith Gyntaf, a darpariaeth ddwyieithog - hynny yw'r Cwrs Carlam - oes unrhyw reswm neu resymau penodol pam nad yw'r ysgol yn trin y Gymraeg fel pwnc craidd yn nhermau mesur perfformiad?

14 PA: O fewn yr ysgol baswn i, a'r prifathro, yn dadlau ei bod hi'n cael statws fel pwnc craidd. Hynny yw, mae pob disgybl yn gwybod mai'r pynciau craidd fel petai, neu'r pynciau mae'n rhaid iddyn nhw eu gwneud, ydy Cymraeg, Gwyddoniaeth, Saesneg a Mathemateg. O ran mesur hynny, yn nhermau mesur llwyddiant ac yn y blaen, dyna'r llywodraeth wrth gwrs sy'n defnyddio'r llinynnau mesur hynny fel, yr enghraifft Lefel 1 a Lefel 2. A dydy Cymraeg Ail Iaith ddim yn cyfrif tuag at rheiny o ran y llinynnau mesur hynny. Wrth gwrs, mae'r canlyniadau yn cael eu defnyddio ar gyfer Lefel 1 achos mae hynny'n - dyna'r pum cymhwyster A* i C. Ac wrth gwrs mae Cymraeg yn cyfrif tuag (ati hi) ond dydy hi ddim yn cyfrif tuag at y pynciau craidd fel Mathemateg, Gwyddoniaeth a Saesneg o ran y llywodraeth. Ond o fewn yr ysgol, baswn i'n dweud ein bod ni'n ei thrin fel pwnc craidd achos mae pawb yn gwneud y Cwrs Llawn, heblaw am y dosbarth NVQ.

15 YY: OK, diolch. Rydyn ni wedi dweud hyn yn barod efallai - wrth gwrs, mae'r ysgol yn cynnig darpariaeth cyfrwng Cymraeg i ddisgyblion iaith gyntaf, dw i'n dyfynnu fan hyn, hynny yw'r ddarpariaeth cyfrwng Cymraeg yng Nghyfnod Allweddol 3. Mae yna saith pwnc, dw i'n credu, trwy gyfrwng y Gymraeg - tua saith pwnc?

16 PA: Ie, ar gyfer y disgyblion iaith gyntaf mae yna saith pwnc iddyn nhw. Ac wedyn ar gyfer y cyrsiau carlam, pump pwnc sydd gyda nhw, ie =

17 YY:

$$
=\text { Un peth }- \text { un }
$$

cwestiwn sy'n codi o ran - achos dw i ddim yn canolbwyntio ar y rhai iaith gyntaf 
ond mae dau gwestiwn gyda fi. Er mwyn jest egluro beth yn union rydyn ni'n ei olygu fan hyn wrth y term disgybl iaith gyntaf. Efallai ei fod e'n cyfeirio at yr ysgol maen nhw wedi ei [mynychu] pan oedden nhw'n iau yn yr ysgol [gynradd] Gymraeg

18

PA: $\quad[$ Ie. $]$

[ Ie. ]

19 YY: neu'r iaith maen nhw'n ei siarad yn y cartref. So beth rydyn ni'n =

20 PA: $=$ Fan hyn

- pa ysgol gynradd maen nhw wedi'i ei mynychu. Felly os maen nhw wedi mynychu ysgolion cynradd Cymraeg yr ardal, $\{$ Enw ysgol $\}$ neu $\{$ Enw ysgol $\}$, yna dyna'r disgyblion sy'n mynd mewn i'r cwrs iaith gyntaf gyda ni. Felly nid o reidrwydd eu bod nhw'n siarad Cymraeg yn y cartref. Byddwn i'n meddwl mai nifer fechan iawn fyddai gydag un rhiant sy'n siarad Cymraeg yn y cartref. Mae'n ddibynnu ar yr ysgol gynradd gwnaethon nhw ei mynychu.

21 YY: Reit, OK. A'r ail gwestiwn sydd gyda fi yw - mae yna saith (pwnc) cyfrwng Cymraeg, ond ydy Cymraeg Iaith Gyntaf yn cael ei gyflwyno trwy gyfrwng y Gymraeg [ hefyd, neu - felly, ] mewn ffordd [mae wyth pwnc] =

22 PA: [Ie, ydy, ie wrth gwrs.] [Wyth oes, ie. ] = Sori, wnes i ddim ystyried - ie felly saith gyda'r [Gymraeg] $=$

23 YY: $\quad[$ Gyda -$]=$ Reit, OK. A'r un cwestiwn efallai gyda'r Cwrs Carlam $=$

24 PA: = Ie, byddai'n bump pwnc ychwanegol i'r Gymraeg.

25 YY: [Cymraeg] Ail Iaith yn [cael] ei gyflwyno trwy gyfrwng [dwyieithog?]

26 PA: [ Ie. ] [ Ie.] [ Ydy, ie. ] 
27 YY: OK, gwych diolch. Mae hynny'n ateb tri chwestiwn yn weddol glou. So mae'r ysgol yn cynnig y Cwrs Carlam. Felly sut mae'r ysgol yn dethol y disgyblion ar gyfer y Cwrs Carlam? Hynny yw, beth yw'r meini prawf, os licet ti =

ddigon teg. Beth sy'n digwydd ydy - wrth gwrs, mae'r ysgolion cynradd yn rhoi data i ni o ran lefelau'r disgyblion yn y pynciau craidd i gyd, sef y Saesneg, Cymraeg Ail Iaith, Mathemateg a Gwyddoniaeth. O ran hynny hefyd, mae'r athrawon Cymraeg pontio sy' gyda ni wedi bod yn rhan o'r broses gyda lefelu Cymraeg Ail Iaith. Yn ogystal â hynny wedyn, mae pob disgybl ym Mlwyddyn 6 yn gwneud profion CATs. Wedyn mae'r data gyda'r lefelau yn cael eu defnyddio er mwyn rancio'r disgyblion o fewn top chwe deg y flwyddyn ysgol i gyd, ac o fewn y top chwe deg 'na - maen nhw'n cael eu galw'n Band $P$ ar draws yr ysgol - felly o'r dechrau cyntaf mae'r top chwe deg 'na yn cael eu gwthio ym mhob pwnc. Rydyn ni wedyn yn dewis ein top pedwar deg ni o'r top chwe deg felly, wrth edrych ar eu lefelau Cymraeg nhw, ac wrth ystyried eu hymdrech a'u hagwedd nhw tuag at y Gymraeg yn yr ysgolion cynradd, gan ddefnyddio'r athrawon pontio.

29 YY: Reit. Dw i jest yn meddwl efallai byddai disgybl am gael ymuno â'r gwersi dwyieithog. Ydy'n bosibl o gwbl i ddisgyblion nad ydyn nhw wedi cael eu dethol cael mynd at y ddarpariaeth $=$

PA: $=\mathrm{Na}$, yn anffodus. Oherwydd ffordd mae'r ysgol wedi $-\mathrm{y}$ ffordd mae'r amserlen wedi cael ei chreu, mae e ond yn agored i'r disgyblion hynny sy'n cyrraedd y nod ar gyfer top chwe deg y flwyddyn ysgol. Beth rydyn ni'n dweud - er bod hynny efallai yn siom i rai disgyblion ac efallai hefyd bod yna ddisgyblion eraill a fyddai'n fwy na'n gallu ymdopi gyda'r cwrs - beth rydyn ni'n ei ddweud ydy, wrth gwrs bod y ddarpariaeth maen nhw'n cael yn y dosbarthiadau arferol, normal (beth bynnag) 'dych chi eisiau eu galw nhw, yn dal o safon uchel iawn ac byddan nhw'n dal yn gallu cyrraedd y brig gyda'u canlyniadau ar ddiwedd Blwyddyn 11 fel pawb arall yn eu blwyddyn ysgol nhw. Ond wrth gwrs oherwydd y ffordd mae'r system yn cael ei hamserlennu, dyna'r unig ffordd maen nhw'n gallu cael darpariaeth y Cwrs Carlam, ond yn anffodus, na. 
31 YY: Reit, ac os maen nhw'n cael eu dethol, oes modd i'r rhieni neu'r plant ddweud na, dw i ddim am gael - beth sy'n digwydd wedyn?

32 PA: Reit, wel mae'r top - mae'r pedwar deg rydyn ni'n eu dewis yn derbyn llythyron adref ar ddiwedd Blwyddyn 6. Maen nhw'n cael gwahoddiad i ddod am gyfarfod gyda ni - fi, ail yr adran a'r pennaeth cynorthwyol sy'n gyfrifol am bontio. Maen nhw'n dod i fyny ac rydyn ni'n gwneud cyflwyniad yn esbonio yn syml beth ydy e - er erbyn hyn, ar ôl pymtheg mlynedd mae'r rhieni yn deall beth yw e ac mae'r gymuned yn deall beth yw e - ac wedyn mae gyda nhw gyfle i gofyn cwestiynau, (unrhyw beth) sy'n peri pryder iddyn nhw, ac os 'dyn ni'n gallu ateb hynny a'u bod nhw'n hapus, maen nhw'n dewis bod y plant yn dod i'r Cwrs Carlam. Ac wedyn maen nhw'n arwyddo rhyw fath o - nid cytundeb - ond arwyddo eu bod nhw'n hapus i'r disgyblion fynychu'r cwrs.

33 YY: Iawn, OK. Pa fath o gwestiynau fyddai'n codi gan y rhieni, a'r plant efallai, sy'n meddwl am fynd am y cwrs?

34 PA: Y cwestiynau sy'n peri'r gofid mwyaf ydy a fydd y gwaith - yn y pynciau eraill - yn Gymraeg ac felly fyddan nhw ddim yn gallu helpu nhw gyda'r gwaith cartref. Y cwestiwn arall ydy, pe bai'r pwysau yn ormod iddyn nhw, a fyddai hawl iddyn nhw cael eu tynnu oddi ar y cwrs. Dw i'n ceisio meddwl am rywbeth arall. Dyna'r cwestiynau fel arfer i fod yn onest. Os 'dyn nhw'n gallu ymdopi gyda'r pwysau, os 'dyn nhw'n gallu ymdopi gyda'r gwaith, a fydd gwaith yn y pynciau eraill yn ormod o Gymraeg fel eu bod nhw ddim yn gallu helpu gyda gwaith cartref. Ac yn ail, bod e'n peri iddyn nhw fethu deall y cynnwys ac felly i fethu llwyddo yn y pynciau hynny. Ond unwaith ein bod ni wedi esbonio mai iaith darged y dosbarth yw'r Gymraeg yn y pynciau eraill ac byddai yna dasgau Cymraeg dim ond unwaith bob hanner tymor - tasgau yn y dosbarth ydyn nhw - ac felly byddai'r mwyafrif o'r cwricwlwm maen nhw'n ei ddilyn trwy gyfrwng y Saesneg ac felly fydd e ddim yn effeithio ar eu lefelau nhw yn y pynciau hynny. 
35 YY: Ac rwyt ti wedi ateb [cwestiwn arall sydd] gyda fi, sef tasgau asesiadau -

36 PA:

[ Y cwestiwn nesaf. ]

$37 \quad$ YY: felly unwaith y tymor ddwedaist ti?

38 PA: Dydyn nhw ddim yn asesiadau o reidrwydd. Mae'r asesiadau - maen nhw'n dilyn yn gymwys yr un cwricwlwm â phawb arall yn y flwyddyn ysgol wrth gwrs maen nhw'n cael eu gwthio ychydig yn fwy achos dyna ddisgyblion mwyaf abl a thalentog y flwyddyn. Serch hynny, fel rhan o'r Cwrs Carlam, rydyn ni wedi clustnodi un dasg - nid asesiad - jest un dasg bob hanner tymor (fel eu bod nhw'n gallu) ysgrifennu yn Gymraeg. Pa mor anodd ydy hyn yn amrywio - yn dibynnu ar y flwyddyn ysgol. Felly ym Mlwyddyn 7, byddai'n dasg labelu neu ddefnyddio geirfa yn unig. Erbyn Blwyddyn 8, bydden nhw'n ateb mewn brawddegau (cyfan), efallai mynegi barn neu rywbeth. Ac wedyn ym Mlwyddyn 9, byddwn i’n gofyn iddyn nhw ymateb i ryw baragraff yn mynegi barn neu rywbeth a rhoi rhesymau. Oherwydd gyda disgyblion Blwyddyn 9 - dw i'n dysgu Hanes - maen nhw newydd ysgrifennu disgrifiad o Lundain yn 1888 achos ein bod ni'n astudio Jac y Llarpiwr. Tasgau bach fel honna sy'n jest defnyddio'r sgìl - a Blwyddyn 8 hefyd yn ysgrifennu - maen nhw'n astudio gwyliau yn y Gymraeg. Maen nhw'n astudio gwyliau mewn Daearyddiaeth felly maen nhw'n ysgrifennu cerdyn post mewn Daearyddiaeth er mwyn defnyddio'r Gymraeg. Felly rydyn ni’n ceisio creu tasgau pontio fel petai pethau sy'n cydredeg gyda'r Gymraeg a'u bod nhw'n gallu eu defnyddio nhw yn y pynciau eraill wedyn.

39 YY: Reit diolch. OK fydda i ddim yn gofyn y cwestiwn - nes fy mod i'n dod ato - af i ymlaen wedyn i'r cwestiwn nesaf, ac mae'n mynd nôl at sut mae'r athrawon yn defnyddio'r Gymraeg yn y dosbarth. Dw i'n siŵr bod defnyddio'r ddwy iaith yn broses gymhleth [ iawn ] ac mae'n rhaid defnyddio'r ddwy iaith yn dactegol, er

PA: $\quad[$ Ydy. $]$ 
41 YY: mwyn sicrhau bod - gall y disgyblion ddeall y cynnwys - a hefyd eu bod nhw'n deall y Gymraeg, wrth gwrs. Wedi siarad gyda ti sawl gwaith am hyn, mae'n amlwg i fi mai'r pwnc sy'n dod yn gyntaf, ac wedyn maen nhw'n dysgu geirfa Cymraeg a sgiliau yn y Gymraeg - mae hynny'n dod yn ail. Mae'n rhaid iddyn nhw [ddysgu'r] cynnwys. OK, dw i'n iawn [ i gredu hynny. ]

$42 \quad$ PA: [Ydy, ie.] [Ydych, ie yn bendant.]

43 YY: Ond jest i gael syniad o sut mae'n gweithio, timod, ar lawr y dosbarth fel petai, dw i wedi gofyn yn yr holiadur faint o ddefnydd mae'r athrawon yn ei wneud o'r Gymraeg, pum deg y cant neu'n fwy. Ydy'n bosibl i ti fanylu ychydig ar hynny?

$44 \quad$ PA: Wrth gwrs, mae'n gorfod amrywio o Flwyddyn 7 i Flwyddyn 9. Chimod, ym Mlwyddyn 7 maen nhw newydd ddod yma o'r ysgol gynradd ond maen nhw yn gyfarwydd â'r addysgu achos mae'r ysgol gynradd yn defnyddio tipyn o'r iaith darged o'u cwmpas nhw. Serch hynny, mae'n rhaid bod yn ofalus nad ydyn nhw'n cael gormod o ofn, fel petai. Felly rydyn ni'n dechrau'n araf bach. Erbyn Blwyddyn 9 wedyn, byddwn i'n meddwl bod iaith y dosbarth, mwy neu lai, yn hollol Gymraeg o ran cyfarwyddiadau. “Trowch y dudalen”, “ysgrifennwch”, “darllenwch”. Chimod, geirfa - "Beth yw eich barn chi am Jac y Llarpiwr?”, "pwy oedd ar fai?”. Byddwn ni wedi cyflwyno geirfa, patrymau, fel eu bod nhw'n ddigon hyderus i allu ateb ar lafar ac yn ysgrifenedig gyda'r patrymau hynny maen nhw'n eu gwybod. Felly mae pob athro - er bod ein bod ni fel adran yn gyfrifol am ddysgu nifer o'r pynciau erbyn hyn, ni sy’n gyfrifol am ddysgu Hanes i gyd, Blwyddyn 7 i Flwyddyn 9 yn y Cwrs Carlam. Mae un aelod o'r adran yn gyfrifol am dysgu Daearyddiaeth i hanner y dosbarthiadau fel petai, ac wedyn mae gyda ni un athrawes sy'n gyfrifol am hanner y dosbarthiadau yn Addysg Grefyddol. Felly wrth gwrs, rydyn ni'n deall pa batrymau rydyn ni wedi'u cyflwyno i'r disgyblion. Gyda'r athrawon nad ydyn nhw'n athrawon Cymraeg, rydyn ni wedi paratoi bwcled gyda'r patrymau ieithyddol mae'r disgyblion yn eu gwybod a phryd maen nhw'n cael eu cyflwyno. Felly maen nhw'n deall wedyn pa phatrymau i'w defnyddio a sut wedyn i adeiladu ar hynny. Achos wrth gwrs mae'n haws i ni, yr athrawon Cymraeg, achos rydyn ni'n gwybod y patrymau sydd gyda nhw. Ond, gyda 
rhywun arall mae yn broses o gorfod edrych ar y fwcled honno ac wedyn paratoi ar ei gyfer e i fod yn onest.

45 YY: Diolch. Mae hyn mewn ffordd yn ein arwain ni wedyn i mewn i'r thema nesaf. Ond cyn i fi - dw i'n credu fy mod wedi gofyn pob cwestiwn - ie, cyn i ni symud ymlaen at hyfforddiant, achos dyna un o'r cwestiynau roeddwn i'n mynd i'w gofyn - hynny yw, oes yna ganllaw fel petai ac roeddet ti'n cyfeirio fanna at ryw fath o fwcled maen nhw'n dilyn o ran patrymau - felly cyn i fi symud ymlaen at hyfforddiant, oes dulliau addysgu penodol mae'r athrawon yn eu defnyddio? Achos $\mathrm{dw}$ i wedi bod yn darllen am addysg dwyieithog mewn gwledydd eraill ac mae yna lot o waith ar bedagogeg a sut mae cyflwyno'r ddwy iaith yn effeithiol er mwyn sicrhau eu bod nhw'n deall y cynnwys. Oes methodoleg neu ddulliau addysgu arbennig maen nhw'n eu dilyn?

46 PA: Fyddwn i ddim yn dweud ei bod yn wahanol i'r ffordd maen nhw'n cyflwyno eu pwnc yn arferol, achos fel rydyn ni wedi'i ddweud, y peth pwysicaf yw bod y pwnc yn dod yn gyntaf, felly maen nhw'n athrawon arbenigol yn eu pynciau nhw. Felly wrth gwrs y pwnc fydd yn dod yn gyntaf, gyda'r Gymraeg yn ail i hynny, ond yn cyfoethogi'r dysgu ac yn cyfoethogi'r hyn sy'n cael ei gyflwyno yn y Gymraeg. Maen nhw wedi cael y bwcled, er enghraifft, ac maen nhw'n arsylwi gwersi gyda ni yn yr adran fel eu bod nhw'n gallu gweld arfer dda. Ond sut ydyn ni'n ei wneud e - mae yn broses. Dyw e ddim yn rhywbeth sy'n gallu digwydd - un diwrnod dych chi'n gallu, y diwrnod nesaf eich bod chi'n gallu dysgu'n ddwyieithog. Mae yn rhywbeth sy'n dod o brofiad ac yn rhywbeth sy'n datblygu'n organig, fyddwn i'n dweud fel rhan o'r dysgu naturiol. Felly fyddwn i ddim yn meddwl bod yna ryw fethodoleg glir efallai, jest addysgu da, neu addysgu ardderchog i fod yn deg. Gyda'r Gymraeg yn cael ei defnyddio'n naturiol o fewn y dosbarth.

47 YY: Diolch. Awn ni ymlaen felly at y cwestiynau ynglŷn â hyfforddiant, os yw'n iawn gyda ti. Felly hyfforddiant staff yn y Gymraeg. Rwyt ti wedi nodi fanna o ran yr hyfforddiant mewnol maen nhw'n ei gael, ynglŷn ag arsylwi gwersi ac ati ac wrth gwrs, mae hynna'n hollbwysig, ond dw i'n jest yn tybio oes unrhyw beth ar 
wahân i hynny? Dw i'n gwybod bod hyfforddiant gyda \{Enw prifysgol $\}$ ac maen nhw'n dod mewn. Oes patrwm [o ran yr hyfforddiant] =

PA: $\quad[\mathrm{Na}$, beth - ] ] = mae hwnna ar wahân, mwy neu lai, i'r Cwrs Carlam. Gyda'r Cwrs Carlam wrth gwrs, athrawon sy'n hyderus yn y Gymraeg - hynny yw, bod y Gymraeg, mwy neu lai, yn iaith gyntaf iddyn nhw neu eu bod nhw'n ddysgwyr da iawn - dyna'r unig athrawon fyddai'n cael eu ystyried er mwyn addysgu'r Cwrs Carlam. Wedyn o ran clywed y Gymraeg ar draws yr ysgol felly, dyna rywbeth ar wahân, sef dwyieithrwydd yr ysgol gyfan. Yna, dyna ble mae'r hyfforddiant hwn gyda \{Enw prifysgol $\}$ wedi cael ei defnyddio er mwyn hybu'r defnydd o Gymraeg achlysurol ar draws yr ysgol. Felly ymhob pwnc, ble bynnag maen nhw ar draws yr ysgol, dylen nhw fod yn clywed y Gymraeg. Ac er mwyn galluogi hynny, dyna'r hyfforddiant rydyn ni wedi ei gael - rydyn ni wedi bod yn ei gynnig. Gwnaethon ei gynnig, dw i'n credu, am ddwy flynedd yn olynol ac wedyn wrth gwrs, roedd y staff yn eithaf statig, fel petai. Doedd yna ddim lot o newid staff. Felly fe gawson ni doriad o ddwy flynedd ac rydyn ni wedi bod yn ail ddechrau nawr gyda'r staff newydd sydd wedi dod i mewn - dechrau'r broses eto. Ac wedyn, y rhai sydd eisiau rhyw fath o refresher, maen nhw'n gallu mynd nôl hefyd i wneud y cwrs. Felly cwrs deg wythnos o hyd yw e, wedyn awr yr wythnos er mwyn hybu defnyddio'r Gymraeg yn achlysurol.

49 YY: Reit, a dyw'r brifysgol ddim yn cynnig unrhyw beth ynglŷn â datblygu methodoleg felly, [ dim byd fel] =

50 PA:

$[$ Nac ydyn, na $]=$ dim ond y defnydd o'r Gymraeg $=$

51 YY: = y Gymraeg yn achlysurol, iawn OK. Hyd y gwn i, does dim byd amlwg o ran hyfforddiant ar gael i ysgolion, er mwyn eu hannog i addysgu'n ddwyieithog. Oes unrhyw beth =

52 PA: $=\mathrm{I}$ fod $\mathrm{yn}$

deg, dw i ddim yn ymwybodol bod yna - dim byd - alla i ddim dweud gant y cant nad yw e'n bodoli, ond dw i ddim yn ymwybodol bod yna unrhyw beth ar gael. Dw i'n 
gwybod yn y sector cynradd - dw i'n tybio ei bod e achos wrth gwrs mae yna lot mwy o alw am y dwyieithrwydd hynny - ar draws pynciau yn y cynradd, mae yna lot mwy o bwyslais ar hynny gan y llywodraeth. Ond ar gyfer Cyfnod Allweddol 3, neu'n uwch, dw i ddim yn ymwybodol bod yna gwrs er mwyn helpu gyda'r fethodoleg hynny =

53 YY: = yn $\{$ Enw ardal y brifysgol $\}$ dw i'n credu eu bod nhw'n gwneud lot gyda'r sector cynradd dysgu'n ddwyieithog.

$54 \quad$ PA: Oes, dw i'n gwybod bod yna athrawon - mae gyda ni fyfyrwyr sydd wedi mynd i \{Enw prifysgol \} i wneud addysg gynradd - ond dw i'n gwybod eu bod nhw'n cael eu rhoi i mewn i dair ffrwd fanna, sef y siaradwyr Cymraeg hyderus, iaith gyntaf, yn mynd i mewn i un ffrwd, wedyn mae yna ddysgwyr da mewn ffrwd arall ac wedyn mae yna'r athrawon sy'n fwy neu lai yn Saeson, neu chimod, heb unrhyw Gymraeg yn mynd i mewn i ffrwd arall. Ac wedyn mae'r athrawon yn y canol yn cael mwy o hyfforddiant - eu hyfforddi nhw i fod yn fwy o athrawon dwyieithog. Mae un o'n cyn-ddisgyblion ni, i fod yn onest, yn \{Enw ysgol gynradd $\}$. Digwydd bod wedi bod trwy'r Cwrs Carlam gyda ni hefyd. Ac aeth e i mewn i'r ffrwd yna yn y brifysgol. A rydych chi'n sylweddoli - mae dysgu Blwyddyn 6 nawr yn $\{$ Enw ysgol $\}$ - dw i'n sylweddoli bod y disgyblion yn gryf iawn, yndw. Ydy i fod yn onest. Felly, mae'n neis i weld ffrwyth eich gwaith caled dod nôl ambell flwyddyn, on'd yw e? Ond heblaw am hynny, o ran unrhyw gwrs hyfforddiant, dw i ddim yn ymwybodol bod yna un ar gael.

55 YY: Reit, diolch. Yn ôl at hyfforddiant, defnyddio'r Gymraeg yn achlysurol, ydy'r hyfforddiant ar gael dim ond ar gyfer y staff addysgu, neu unrhyw [aelod o staff? Reit, OK ] =

56 PA: [Unrhyw aelod o staff - ] = y staff cefnogi, y staff yn y swyddfa - mae yn agored i unrhyw un sydd gydag awr ar ôl ysgol. Achos hefyd, mae gyda ni beth sy'n cael ei alw'n - nid is bennaeth blwyddyn - beth sy'n cael ei alw'n GLSOs, sef Guide and Learning Support Officers. Dydyn nhw ddim yn athrawon, maen nhw'n unigolion sy'n cael eu defnyddio er mwyn cefnogi'r penaethiaid blwyddyn gyda'r gwaith papur, 
gwaith bugeiliol ac yn y blaen, gyda'r disgyblion. Ond maen nhw hefyd wedi dilyn y cyrsiau Cymraeg achlysurol.

57 YY: Grêt. Ydy'r ysgol yn cynnig - felly maen nhw'n cynnig yr hyfforddiant eto eleni?

$58 \quad$ PA: Ydyn, ydyn.

$59 \quad$ YY: $\quad$ A faint sy'n - faint fydd yn $=$

60 PA: $\quad=$ I fod yn onest, dw i ddim yn sicr achos wrth gwrs - mae yna, dw i'n credu bod yna ryw ugain sydd wedi dangos diddordeb - ond wrth gwrs, unwaith eich bod chi'n dewis noson - ac os nad yw'r noson yna'n gyfleus i bawb - mae yn mynd yn anodd. Felly dw i ddim yn sicr ble rydyn ni o ran niferoedd cadarn ar hyn o bryd. Ond mae yna ryw ugain i bump ar hugain sydd wedi dangos diddordeb mewn gwella’u Cymraeg. Ond gan ystyried mae'n ysgol â thua - dros gant o staff - mae'n dal yn nifer dda iawn gan ystyried hefyd bod yna nifer uchel o siaradwyr Cymraeg iaith gyntaf i'w cael yma, a bod nifer wedi bod trwy'r system yn barod, wrth gwrs. Ac mae nifer o'r staff, i fod yn deg, hefyd wedi gwneud cyrsiau Cymraeg i Oedolion yn y gorffennol. Nid efallai o reidrwydd y safon uchaf, ond wedi mynychu'r cyrsiau ac maen nhw'n hyderus i ddefnyddio'r Gymraeg yn barod.

$61 \quad$ YY: Grêt. Ac un cwestiwn arall sydd gyda fi ynglŷn â hyfforddiant, ynglŷn â defnyddio'r Gymraeg yn achlysurol. Oedd yr hyfforddiant ar gael cyn 2011/2012?

62 PA: Yn 2011 ddechreuodd hwnna. Doedd gyda ni ddim byd swyddogol yn ei le, na. Roedd yna - nid sesiynau - hyfforddiant yn ystod efallai cyfarfodydd hyfforddi mewn swydd, chimod, diwrnod hyfforddi mewn swydd. Dw i'n credu - oedden ni wedi gwneud sesiynau - roedd yna bobl wedi derbyn taflenni gyda geirfa. Roedden ni'n e-bostio posteri ac yn y blaen (er mwyn) rhoi ar y wal, ond doedden ni ddim yn ymwybodol bod yna unrhyw beth arall ar gael nes i ni chwilio, i fod yn onest. Ac wrth gwrs mae popeth yn dod i lawr i arian, on'd yw e. Felly, mae'n gostus. Felly, gwnaethon ni chwilio am beth oedd o'n cwmpas ni a sylweddoli bod ei angen arnon ni, a dyna pryd gwnaethon ni chwilio am wahanol asiantaethau wedyn, i fod yn deg. 
63 YY: Reit, diolch yn fawr. Rydyn ni wedi ateb y cwestiwn nesaf, sy'n wych. Os bydd yn iawn felly, gwna' i symud ymlaen at y cwestiynau olaf ynglŷn â'r thema pontio rhwng y sector cynradd a'r sector uwchradd. Rwyt ti wedi manylu ychydig ar sut mae'r ysgol yn darparu ar gyfer anghenion ieithyddol disgyblion, ar gyfer y Cwrs Carlam a hefyd ar gyfer y rhai iaith gyntaf sy'n cael eu haddysgu drwy gyfrwng y Gymraeg. Mae polisi'r ysgol ar ddwyieithrwydd hefyd yn nodi mai trefnu cyfarfodydd yw un o amcanion yr ysgol. Felly beth yw natur y cyfarfodydd hyn hynny yw, beth sy'n cael ei drafod yn ystod y cyfarfodydd hyn?

64 PA: Gan fod gyda ni ddwy athrawes pontio sydd yn addysgu awr yr wythnos i Flwyddyn 6, ac awr yr wythnos i Flwyddyn 5, ym mhob ysgol gynradd - hynny yw, pob ysgol gynradd Saesneg yr ardal - dydyn ni ddim ag unrhyw gysylltiadau fel hwnna gyda'r ysgolion cyfrwng Cymraeg. Gan mai \{Enw ysgol $\}$ wrth gwrs yw'r ysgol uwchradd Gymraeg, nhw fyddai'n gwneud y system bontio gyda'r ysgolion hynny. Gyda'r rhai wedyn - os ydyn nhw'n dewis dod atyn ni, byddan nhw'n dod i fynychu'r diwrnodau gyda ni wedyn, yn hytrach na derbyn y gwaith pontio rydyn ni'n cyflwyno. O ran hynny, gan eu bod nhw'n fwy brofiadol, a chan eu bod nhw wedi bod yn ei wneud ers blynyddoedd maith bellach, mae'r perthynas rhyngddyn nhw a'r athrawon yn un gryf iawn. Felly maen nhw'n cyfathrebu am lefelau, disgwyliadau a chyrhaeddiad y disgyblion yn gyson tra eu bod nhw i lawr yn yr ysgolion. Wrth gwrs, unwaith bob tymor, dw i'n credu fy mod i'n gywir, byddan nhw'n cyfarfod naill ai gyda Blwyddyn 6, athrawon Blwyddyn 6, neu gyda chydlynwyr y Gymraeg yr ysgolion hynny. Trafod lefelau a chyrhaeddiad yw'r peth pwysicaf ar hyn o bryd, i fod yn onest, gyda Blwyddyn 6. Ar ôl i'r llywodraeth gyflwyno systemau newydd y llynedd, mae'n rhaid cael proffil disgybl ar gyfer gwahanol lefelau. Felly maen nhw'n gweithio ar y cyd wedyn gyda'r ysgolion hynny er mwyn creu'r proffiliau ar gyfer y disgyblion. A'r drafodaeth wedyn ydy cysoni gwaith a chymedroli.

65 YY: Iawn. Felly mae yna gyswllt cryf, baset ti'n ddweud, rhwng yr ysgol yma a'r ysgolion sydd o fewn [ y clwstwr? ]

66 PA:

[Yn bendant, ie.] 
67 YY: Wrth gwrs, maen nhw'n trosglwyddo wedyn i'r ysgol yma. A dw i'n sî̂r bod y polisi yn manylu ar sut maen nhw'n trefnu cyfarfodydd ar gyfer y rhieni a'r disgyblion er mwyn sicrhau - beth yw'r cyfarfodydd hynny?

68 PA: O ran y cyfarfod, wel rydyn ni wrth gwrs yn cyfarfod gyda'r rhieni ar gyfer y Cwrs Carlam. Mae'r ysgol wedyn yn cynnal cyfarfod ar gyfer Blwyddyn 6 i gyd, er mwyn trafod disgwyliadau'r ysgol, o ran polisïau'r ysgol, beth ydy rheolau'r ysgol, gwisg ysgol ac yn y blaen. Felly dydyn ni ddim ynghlwm â'r cyfarfod hynny. Ac wedyn ar gyfer y noson agored ar gyfer Blwyddyn 6, Cymraeg yw un o'r pynciau sy'n cyflwyno ar y noson hynny, ac felly mae'r rhieni yn cael cyfle i gwrdd â ni, cael gwybod ychydig am yr adran a gofyn unrhyw gwestiynau sydd gyda nhw wedyn.

69 YY: Iawn, so dyw'r adran ddim o reidrwydd yn trefnu cyfarfodydd ychwanegol [ i'r disgyblion - reit, OK. ]

$70 \quad$ PA: $\quad[\mathrm{Na}-$ nid ar gyfer y disgyblion - nid ar wahân. $]$

71 YY: Diolch yn fawr. Dw i'n credu bod hyn yn dod â ni at y cwestiynau olaf sicrhau trosglwyddiad hwylus disgyblion ail iaith o'r sector cynradd i'r sector uwchradd. Rwyt ti'n nodi yn yr holiadur bod yr ysgol - bod yr adran - yn cynnig dau ddiwrnod trochi, un ym mis Medi ac un ym mis Gorffennaf. Beth yw natur y diwrnodau trochi hyn?

72 PA: Natur y diwrnod trochi, mwy neu lai, yw rhoi cyfle i ddisgyblion defnyddio'r Gymraeg tu allan i'r dosbarth, i fod yn onest. Cael hwyl wrth ddefnyddio'r Gymraeg a gwneud gweithgareddau hwylus. Felly ar y diwrnod ym mis Medi - Hydref - maen nhw'n gwneud pethau fel coginio, dawnsio, drama, chwaraeon. Aethon ni orienteering eleni, roedd y tywydd yn dda. Felly jest gweithgareddau fel hynny lle maen nhw'n defnyddio'r Gymraeg mewn sefyllfa allgyrsiol, mewn ffordd ar wahân i'r dosbarth.

73 YY: A does dim pwyslais - dim canolbwynt - felly ar batrymau, mae [ jest yn digwydd yn naturiol? ] Reit, OK.

74 PA: [Na, digwydd yn naturiol. Ydy.] 
75 PA: Ac wedyn ym mis Gorffennaf, rydyn ni'n mynd i \{Enw amgueddfa $\}$ am y dydd ac mae yna gwis, hela trysor ac rydyn ni'n defnyddio - mae cliwiau yn Gymraeg ac yn y blaen felly. Rydyn ni wedi teimlo bod - achos ein bod ni yn yr ysgolion cynradd yn wythnosol - bod dim angen, chimod, maen nhw wedi cael eu cyflwyno i'r patrymau. Felly mae'r diwrnodau yma jest fel rhywbeth hollol wahanol iddyn nhw. O ran y disgyblion sy'n dod atyn ni tu allan i'r clwstwr - achos mae nifer fawr yn gwneud hynny nawr, rhwng rhyw ddeugain a hanner cant yn dod bob blwyddyn o'r ysgolion tu allan i'r clwstwr - rydyn ni'n cael diwrnod ar wahân iddyn nhw er mwyn cyflwyno patrymau, er mwyn i ni gael gweld a ydyn nhw wedi cyrraedd yr un safon ieithyddol â gweddill y disgyblion yn y clwstwr. Felly maen nhw'n dod mewn am bore o wersi, ac wedyn gweithgareddau yn y brynhawn.

76 YY: Reit a dyna'r rhai sy'n dod [ tu allan - reit, OK. ] =

$77 \quad$ PA: $[\mathrm{Tu}$ allan i'r clwstwr, ie $]=$ felly mae yna ddarpariaeth ychwanegol - wahanol iddyn nhw achos wrth gwrs, dydyn ni ddim yn ymwybodol o pa fath o ddarpariaeth ar gyfer y Gymraeg maen nhw wedi ei chael [ yn y gynradd ] =

78 YY: [Achos, wrth gwrs] = dyw'r ddwy athrawes [ddim yn gweithio gyda'r]

79 PA: [ Na, dydyn nhw ddim. ]

80 YY: $\quad$ ysgolion hynny - felly pwy sy'n gymwys - pwy sy'n gallu mynd ar y - dim ond y disgyblion sy'n mynd i wneud y Cwrs Carlam, neu [neu unrhyw?] =

$81 \quad$ PA:

[ Na unrhyw ] $=$ pob

disgybl y tu allan i'r clwstwr yn gallu dod i'r diwrnodau ychwanegol hynny. O ran y diwrnodau ym mis Hydref a mis Gorffennaf wrth gwrs, achos - chimod - rydyn ni'n siarad am ddau gant a hanner o ddisgyblion. Dydyn ni ddim yn gallu eu cael nhw i gyd lan ar yr un diwrnod, felly beth ydyn ni'n ceisio ei wneud ydy cael rhai ym mis Hydref a rhai gwahanol ym mis Gorffennaf. Ac unrhyw un - dydyn nhw ddim yn ddisgyblion Cwrs Carlam - maen nhw'n ddisgyblion sy'n mwynhau'r Gymraeg, sy'n dangos agwedd bositif tuag at y Gymraeg, ac yn gwneud yn dda yn Gymraeg. Mae'r broses o ddewis y disgyblion - mae'n cael ei benderfynu gan yr athrawon pontio a'r 
athrawon ysgol. Ond rydyn ni'n ceisio cael gwahanol disgyblion yn y hydref a'r haf achos mae'r gweithgareddau rydyn ni'n eu cynnal yn golygu nad ydyn ni'n gallu cael y ddau gant a hanner i gyd i fyny ar yr un diwrnod.

82 YY: Reit, dw i'n gweld. Dyna i gyd sydd gyda fi, dw i'n credu. Felly, oes unrhyw sylwadau, unrhyw gwestiynau, sydd gyda [ti cyn i ni orffen? ]=

83 PA:

$[\mathrm{Na}, \operatorname{dim}$ gwestiynau $]=$ sai'n credu. $\mathrm{Yr}$

unig sylwadau dw i eisiau eu gwneud - wrth gwrs, mae pob disgybl - mae llwyddiant pob disgybl yn yr ysgol hon yn bwysig i ni. Chimod, un o bethau'r ysgol ydy, " $a$ community school committed to excellence". Hynny yw, bod pob disgybl yn troi lan at y gât ac yn cael yr addysg orau bosibl yn yr ysgol. Felly os ydyn nhw ar y Cwrs Carlam neu beidio, rydyn ni'n teimlo mai'r un safon o addysgu maen nhw'n ei chael yn y dosbarth, ar lawr y dosbarth, yn eu gwersi Cymraeg Ail Iaith. Ond yn y Cwrs Carlam - maen nhw'n cael eu gwthio ychydig yn gyflymach, maen nhw'n cael eu gwthio'n fwy er mwyn cyrraedd y nod, sef yr arholiad ar diwedd Blwyddyn 9 a Blwyddyn 11 wrth gwrs. Ond, dyna'n darpariaeth ni ar gyfer y disgyblion mwy abl a thalentog, fel petai. Mae safon yr addysgu, byddwn i'n dweud, yn cystal ac mae pob disgybl yn cael yr un hawl i'r addysg â phob disgybl arall, byddwn i'n ei ddweud.

84 YY: Diolch yn fawr iawn [eto am ] yr amser. Da bo' =

85 PA

[Croeso.]

$=$ Diolch yn fawr.

86 YY: Diolch. 


\section{[Logo'r ysgol]}

Policy for Bilingualism and First Language Welsh

This policy was agreed by the Governing Body in January 2012 and implemented in January 2012. It will be reviewed in November 2016.

Signed ................................ Chair of Governors

Signed Headteacher

AIMS

1. To develop and extend the scope of bilingualism as an integral part of the teaching programme.

2. To ensure that learners are given the opportunities to achieve through the mediums of English and Welsh.

3. To use teaching methods which develop pupils' competence in English and Welsh.

4. To ensure that pupils and parents are aware of the advantages of developing their abilities in English and Welsh.

5. To ensure that leaders, all staff, governors and other stakeholders are familiar with how the school promotes bilingualism and Welsh first language amongst all of its pupils. 
6. To allow the school to evaluate its bilingual and Welsh first language provision and teaching methods and monitor pupil learning effectively.

7. To make efficient use of sufficiently qualified staff, able to teach bilingually.

8. To provide all staff with the opportunities to develop bilingual skills.

\section{OBJECTIVES}

1. To develop and extend the provision of bilingual and fist language Welsh teaching at the school.

2. To monitor standards of teaching and learning to ensure that learners make good progress in English and Welsh.

3. To provide pupils with opportunities to improve standards in the communication/literacy Key Skills across the curriculum in English and Welsh.

4. To organise meetings for pupils and parents who are being taught subjects bilingually and through the medium of Welsh.

5. To encourage pupils to develop their ability to speak Welsh in a range of contexts and situations beyond familiar contexts and the classroom.

6. To encourage all pupils to participate in extracurricular activities which offer the opportunity to make use of English and Welsh.

7. To encourage all staff to make use of incidental Welsh when talking to pupils and provide support for them to do so.

8. To use some written Welsh in all books other than English and MFL.

9. To use Welsh in assemblies. 
10. To promote the correct use of English and Welsh and establish a bilingual ethos in terms of the school's general environment through displays in classrooms and other areas of the school.

11. To work in collaboration with primary schools to ensure quality of provision in KS2.

12. To develop the skill of dual literacy therefore enhancing learners' confidence to translate.

13. To continue to make Welsh courses available for pupils to study at GCSE, AS and A levels.

14. To enable fast track pupils to sit GCSE Welsh as a second language examinations at the end of KS3 and extend their study of Welsh to Advanced Level in KS4.

15. To enable pupils who have attended Welsh Medium primary schools to sit first language Welsh GCSE in Year 11.

16. To enable pupils who have attended Welsh Medium primary schools to sit first language Welsh Advanced Level in Key Stage 5 if the course is viable.

17. To ensure that pupils, parents and all stakeholders are aware of advantages of bilingualism in terms of getting jobs in Wales, broadening horizons and promoting intellectual development.

18. To employ staff to further develop bilingual provision and monitoring within the school and its feeder primary schools.

NB Please also see the Curriculum Policy and the Literacy Policy.

\begin{tabular}{|l|l|}
\hline Compiled by: [Enw], Asst Headteacher & Revision Number: 4 \\
\hline $\begin{array}{l}\text { Approved by: SMT, Leadership Forum, Governing } \\
\text { Body }\end{array}$ & $\begin{array}{l}\text { Revision date: November 2015 } \\
\text { Person Responsible for Revision: [Enw] }\end{array}$ \\
\hline
\end{tabular}


Atodiad 11. Gwasgariad sgoriau cyfanswm yr holl gyfranogwyr
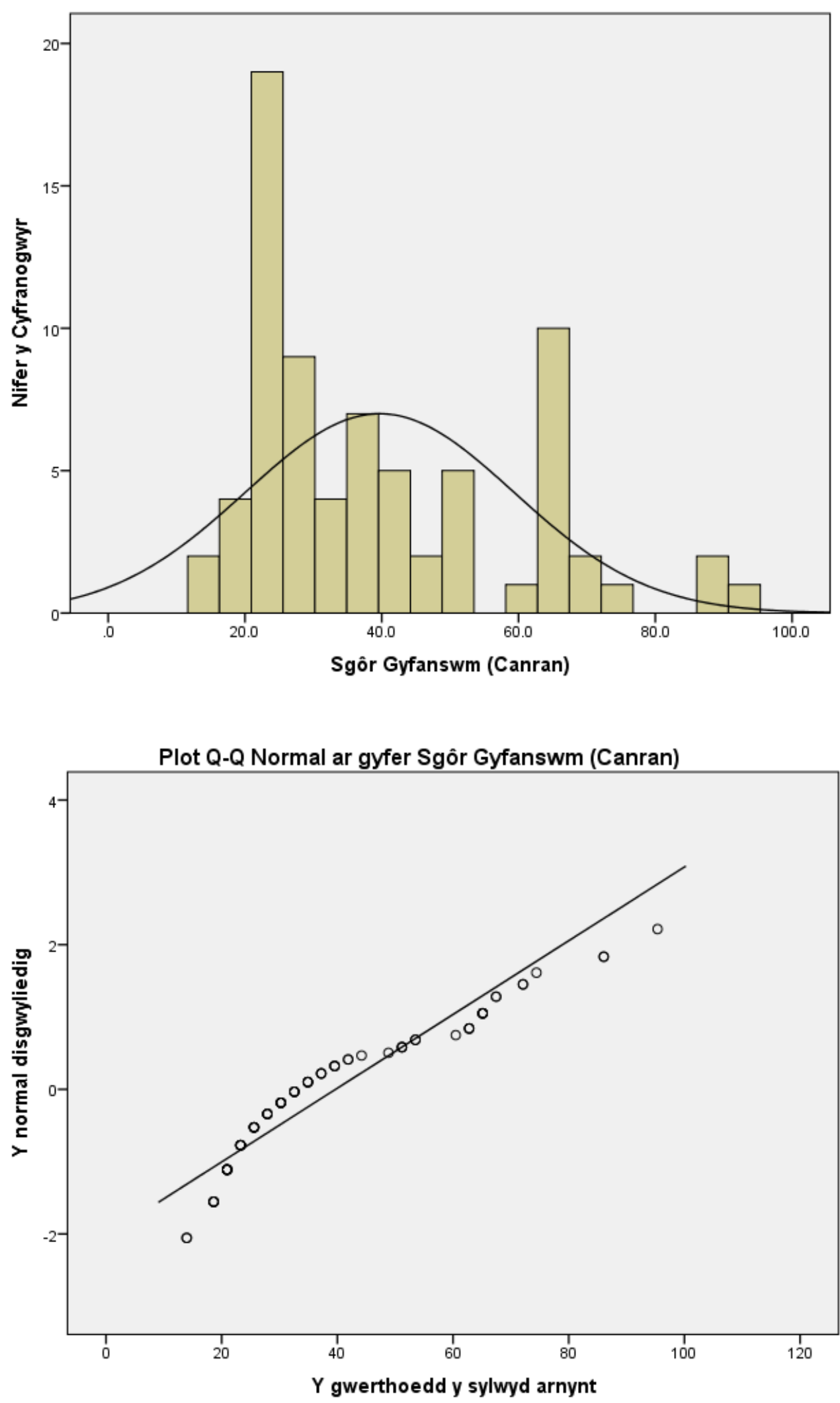
Atodiad 12. Gwasgariad sgoriau cyfanswm Dosbarth A: Set 1 Ysgol X
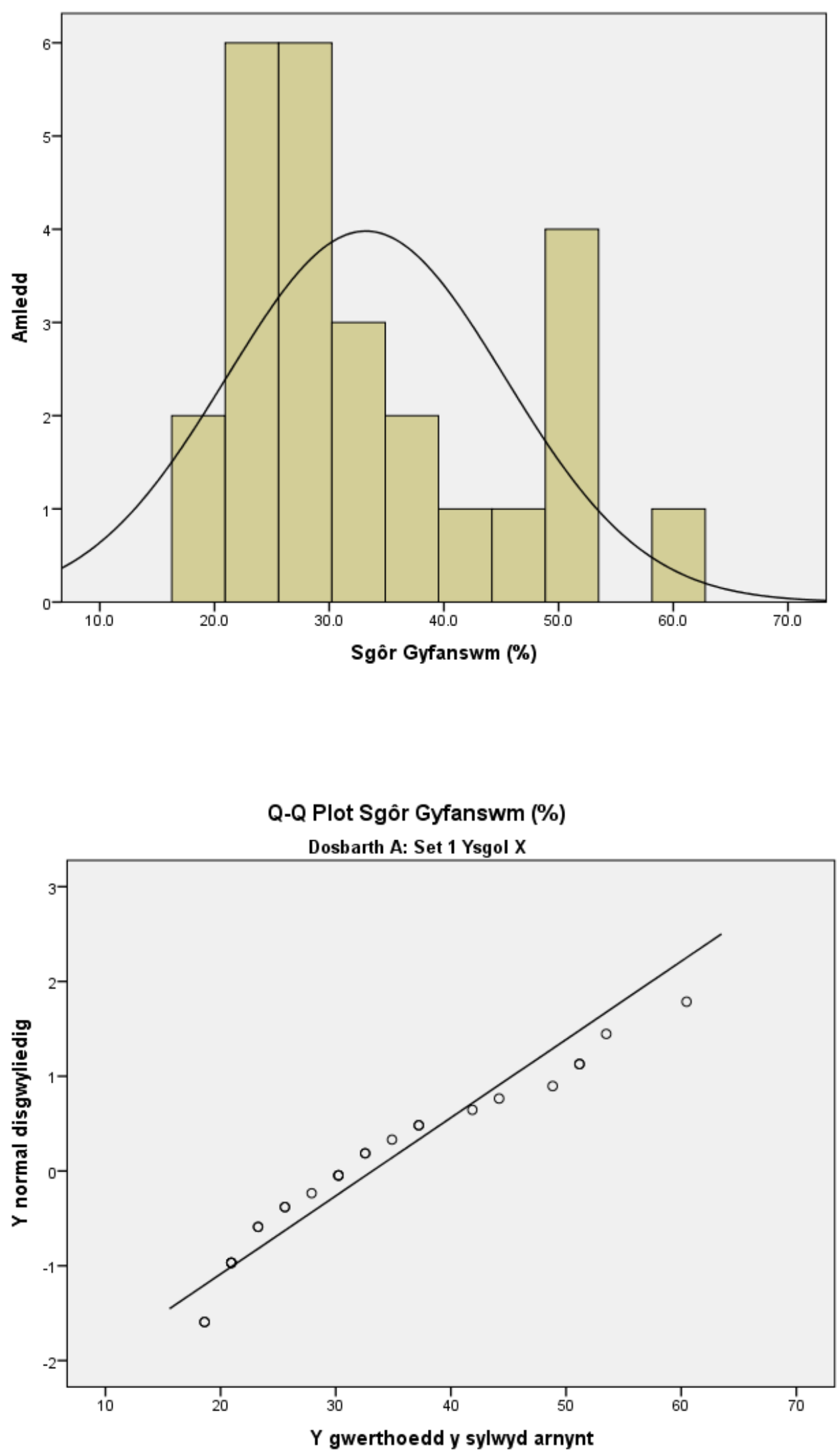
Atodiad 13. Gwasgariad sgoriau cyfanswm Dosbarth B: Cwrs Carlam Ysgol X

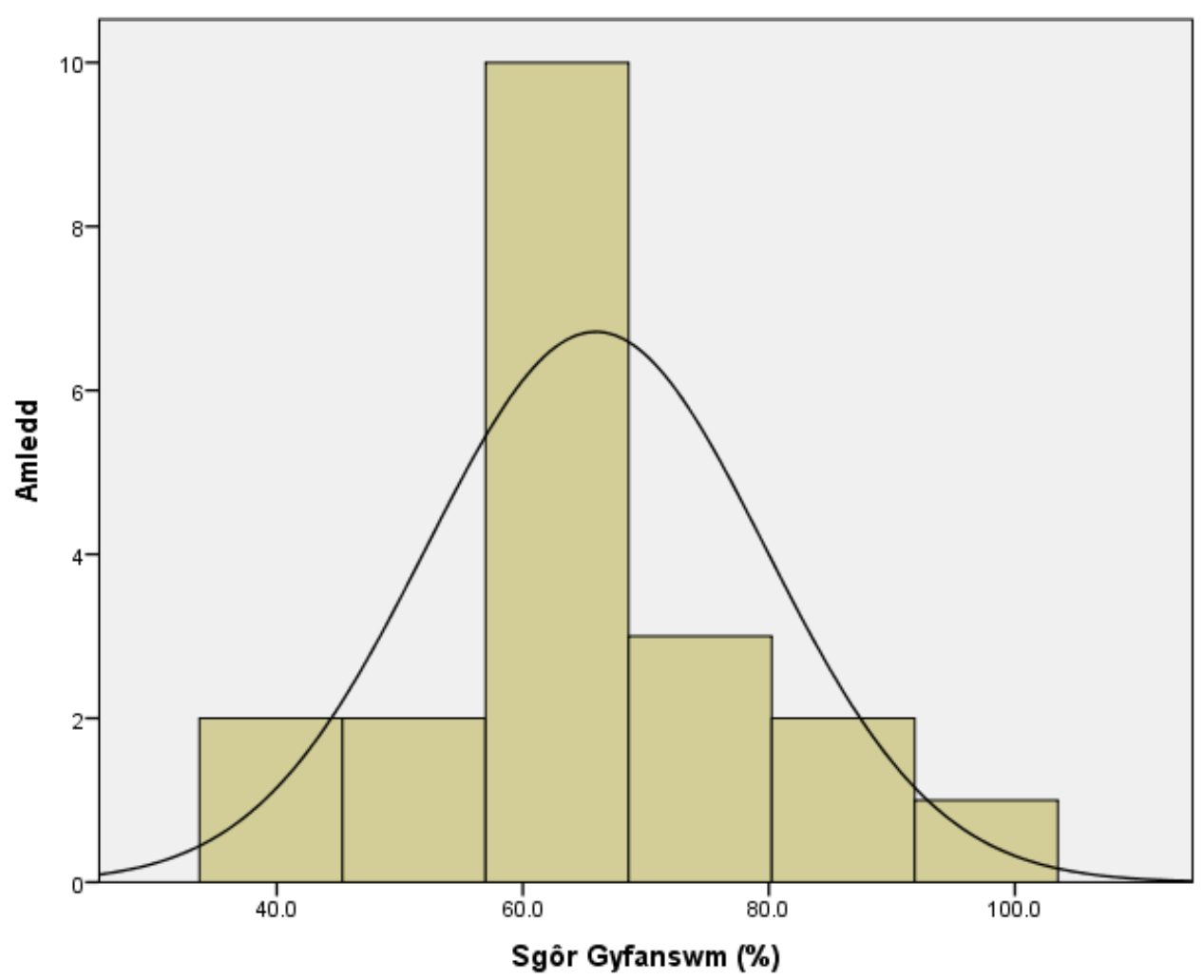

Q-Q Plot Sgôr Gyfanswm (\%)

Dosbarth B: Cwrs Carlam Ysgol X

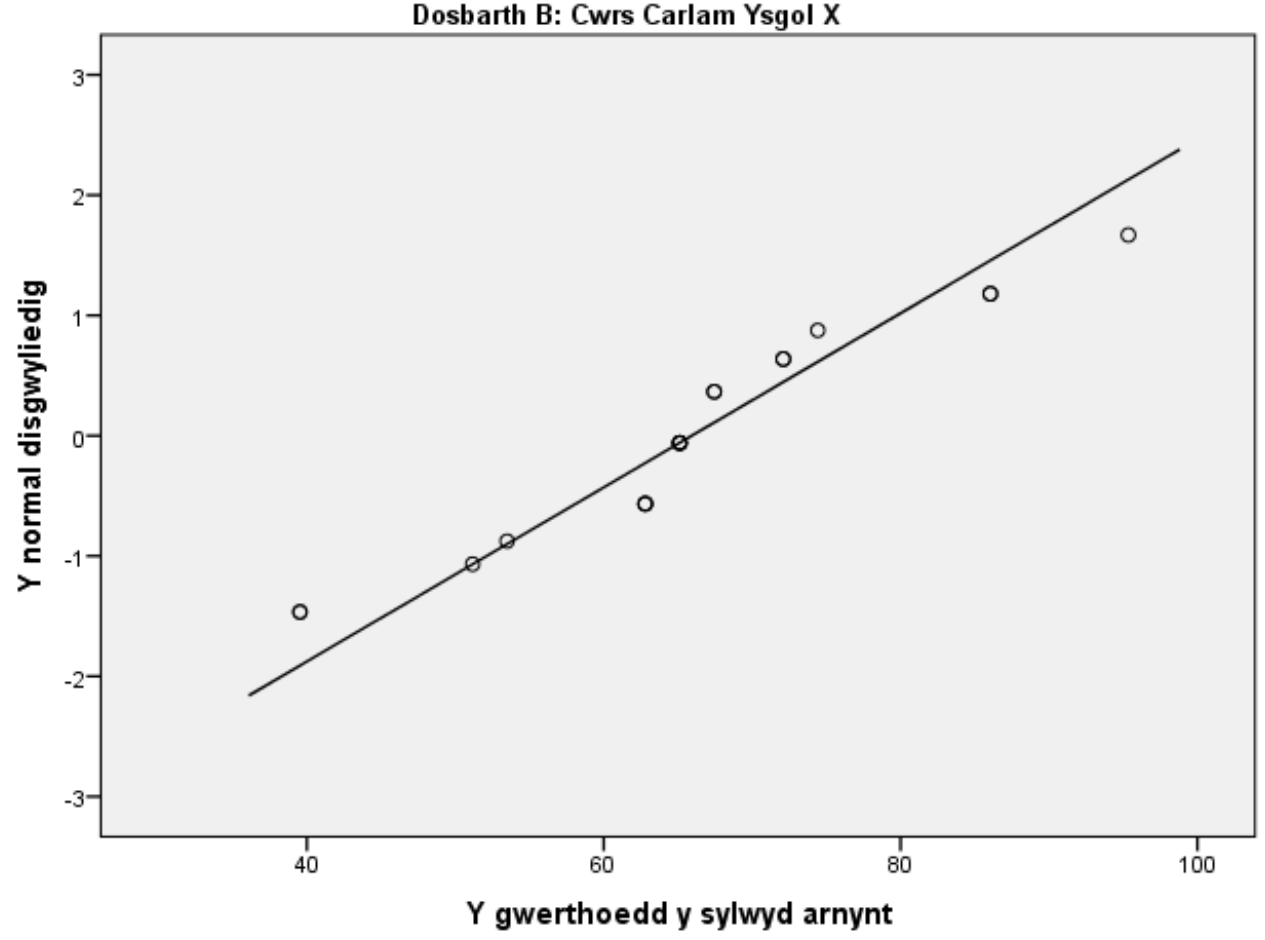


Atodiad 14. Gwasgariad sgoriau cyfanswm Dosbarth C: Set 1 Ysgol Y
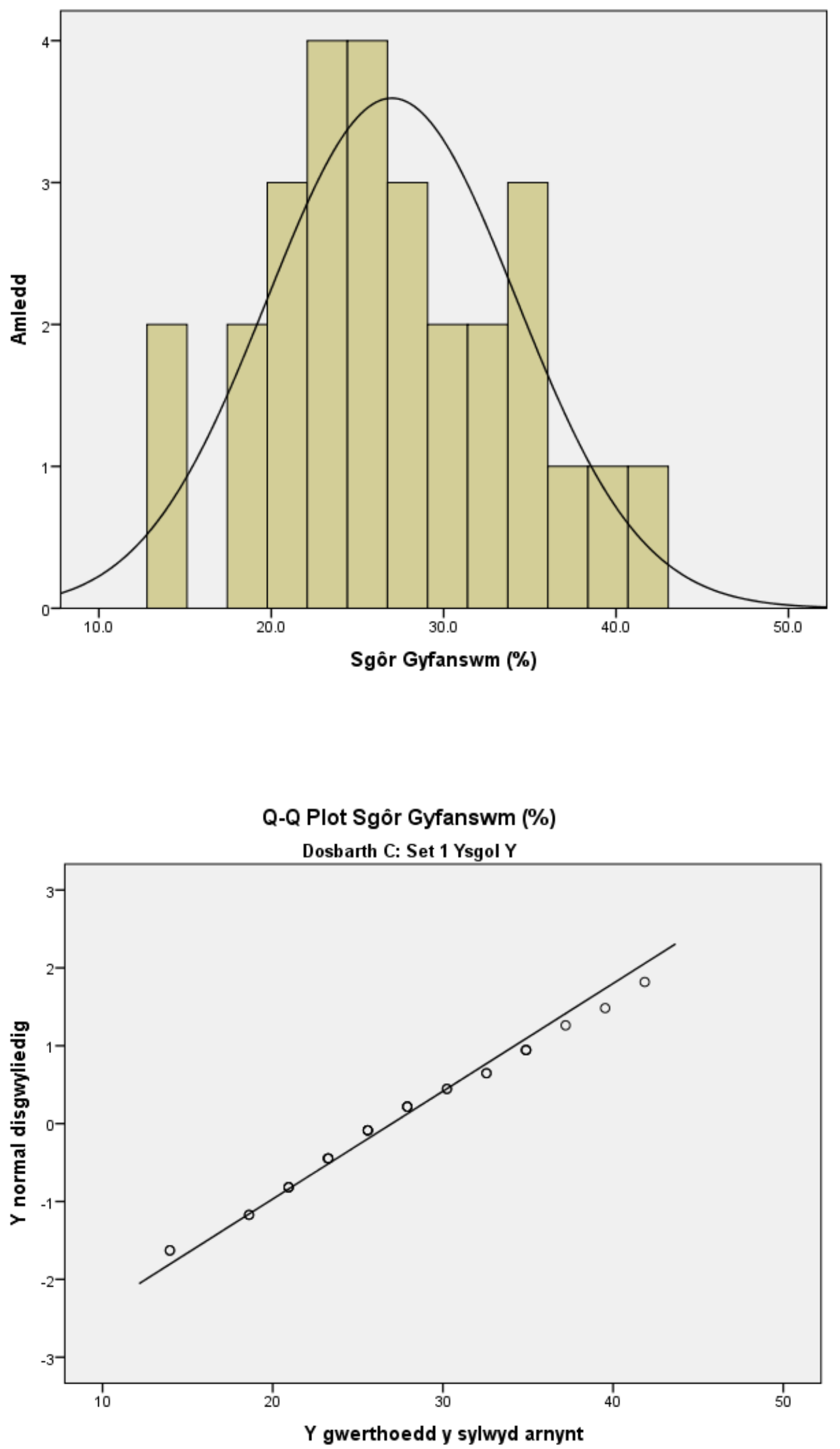
Atodiad 15. Eitemau'r prawf llenwi bylchau fesul anhawster.

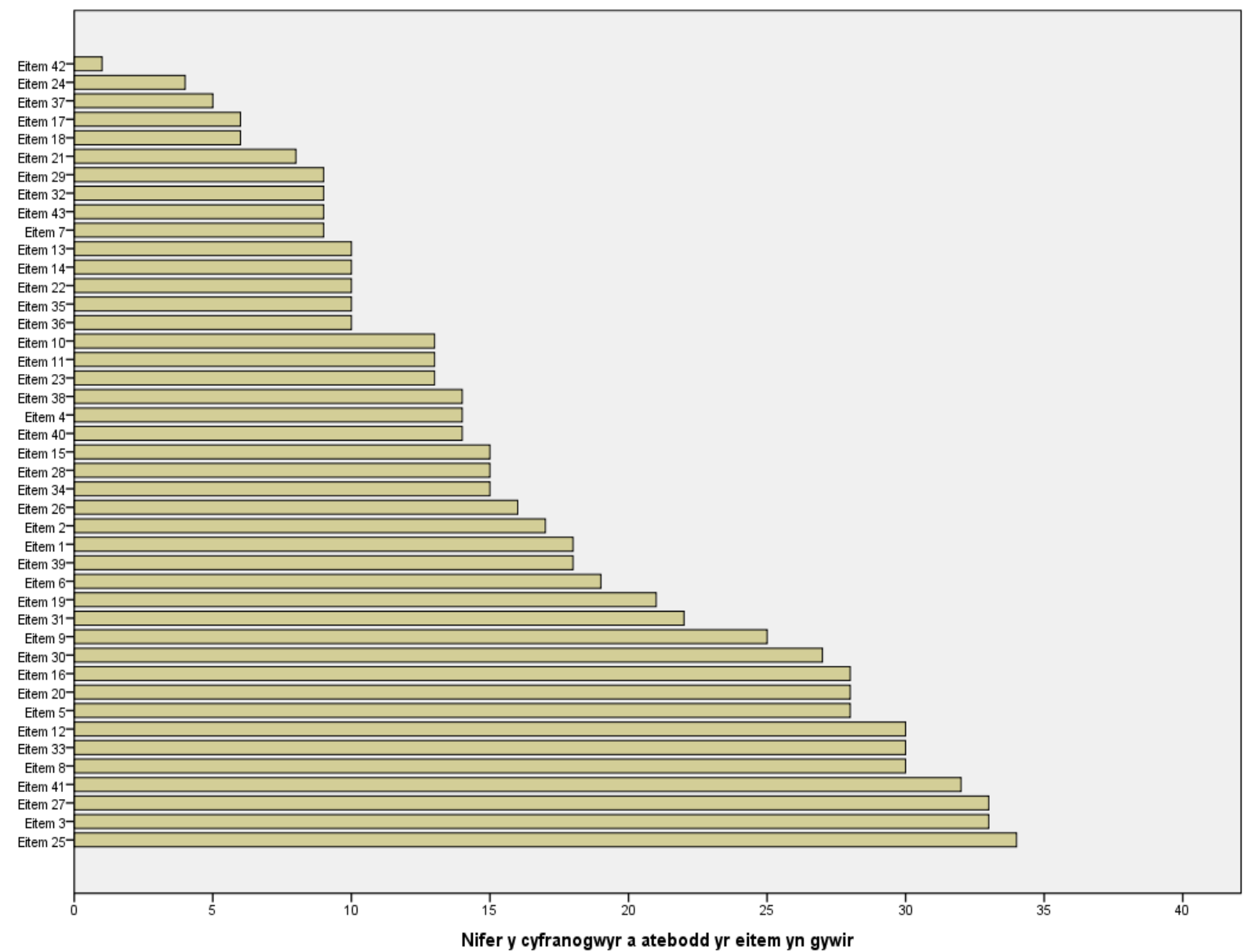


Atodiad 16. Plot gwasgariad ar gyfer sgoriau Rhan A a Rhan B

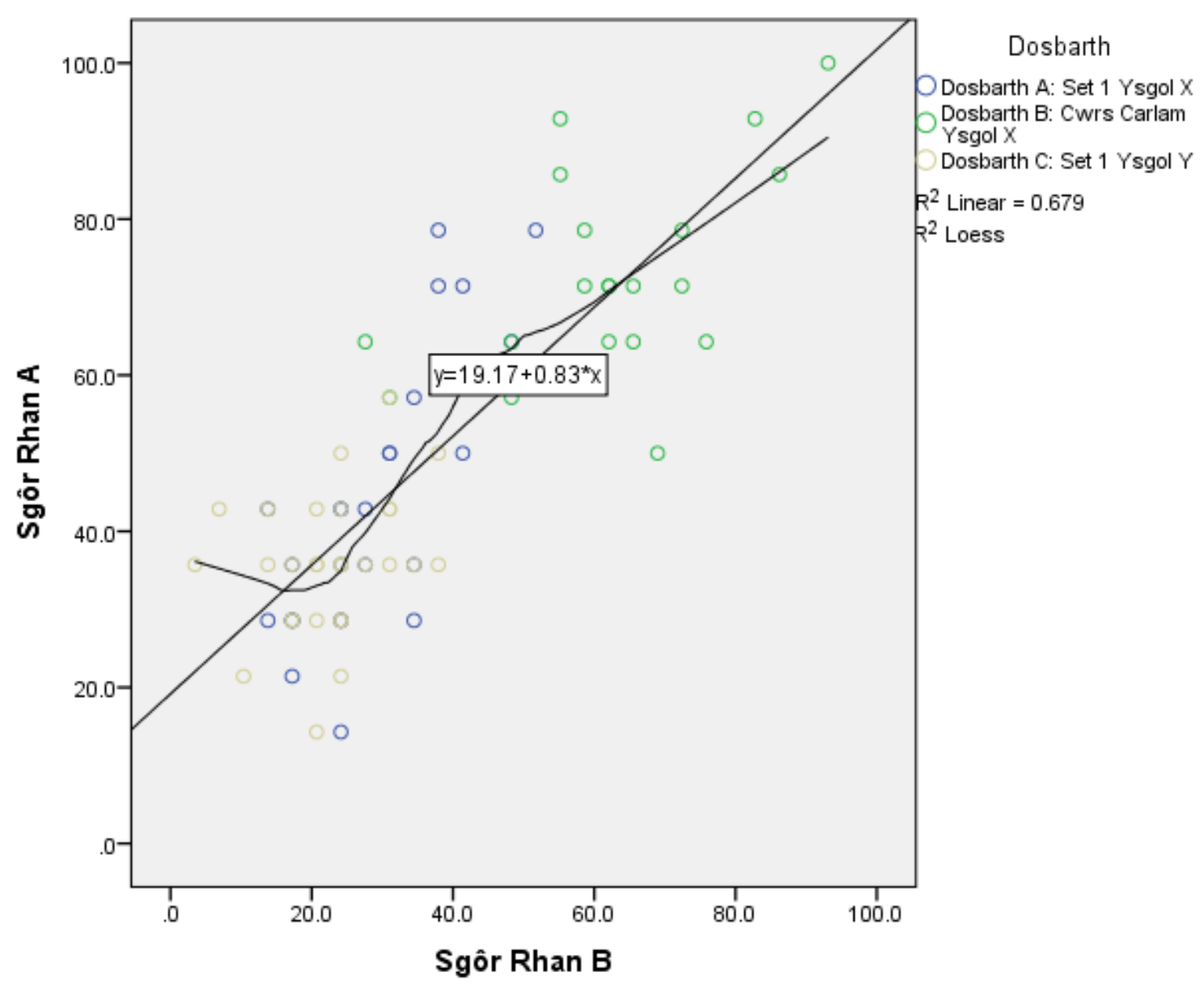


Atodiad 17. Plot gwasgariad ar gyfer Sgôr Rhan A a Sgôr Gyfanswm

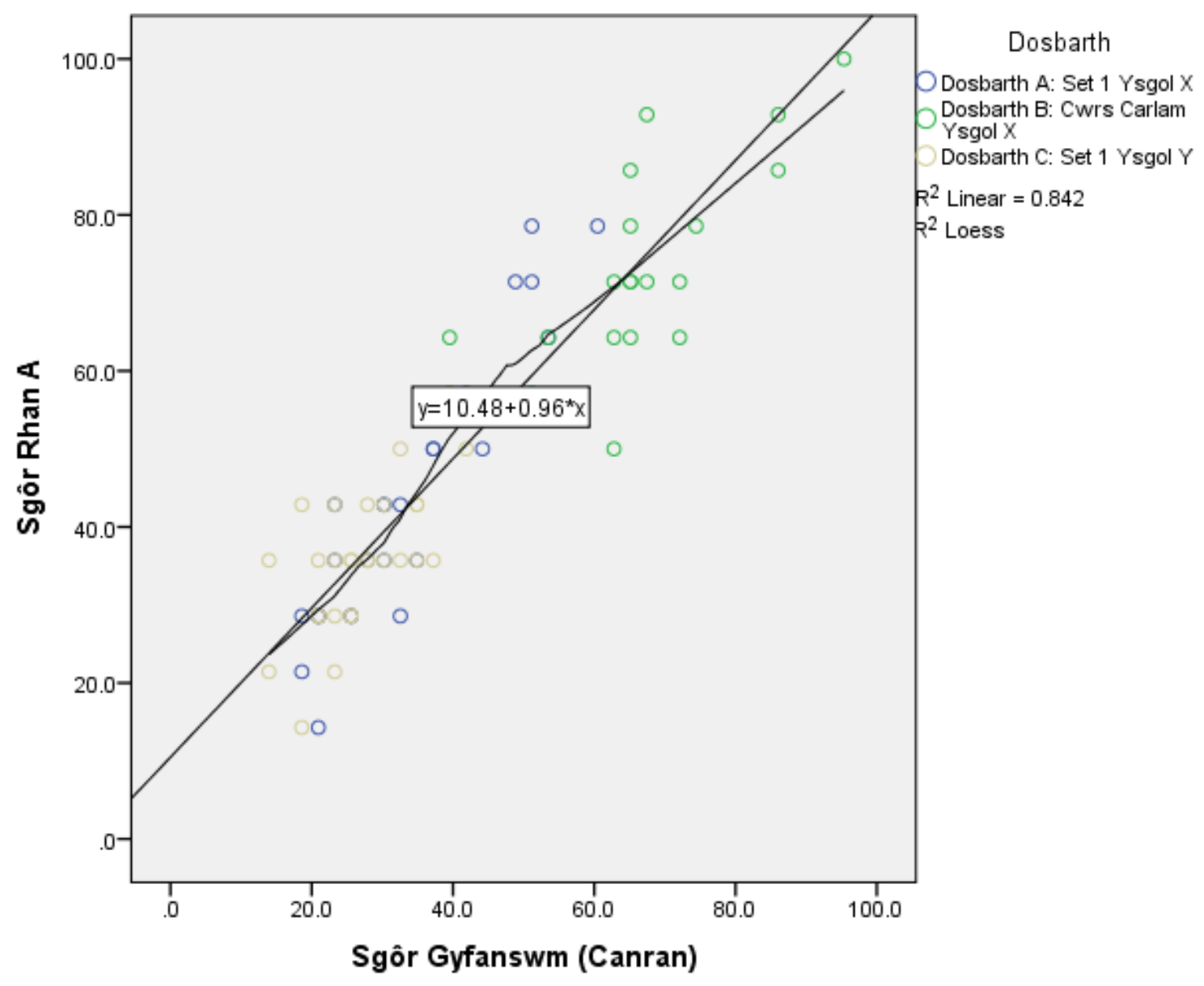


Atodiad 18. Plot gwasgariad ar gyfer Sgôr Rhan B a Sgôr Gyfanswm

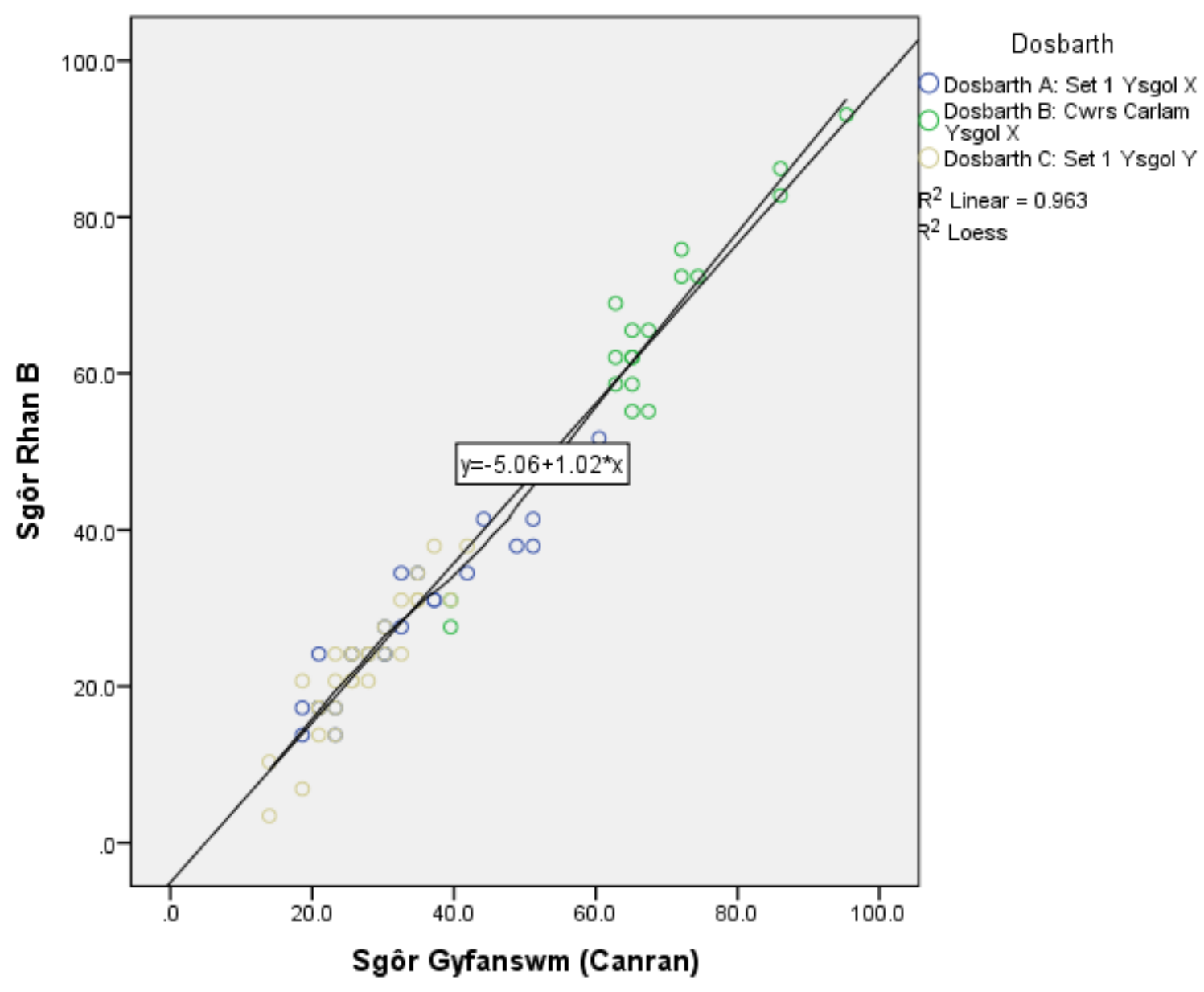


Atodiad 19. Cyfeirnodau didoli pob eitem ar y prawf llenwi bylchau.

\begin{tabular}{|c|c|c|c|c|c|c|c|}
\hline \multirow[b]{2}{*}{ Rhif yr eitem } & \multicolumn{3}{|c|}{ Cywirdeb (\%) } & \multirow[b]{2}{*}{$\begin{array}{l}\text { Rhif yr } \\
\text { eitem }\end{array}$} & \multicolumn{3}{|c|}{ Cywirdeb (\%) } \\
\hline & $\begin{array}{l}\mathrm{Y} \text { traean } \\
\text { uchaf }\end{array}$ & \begin{tabular}{|l}
$Y$ traean \\
isaf \\
\end{tabular} & Gwahaniaeth & & $\begin{array}{l}Y \text { traean } \\
\text { uchaf }\end{array}$ & $\begin{array}{l}\mathrm{Y} \text { traean } \\
\text { isaf }\end{array}$ & Gwahaniaeth \\
\hline 1 & 88 & 8 & 80 & 22 & 60 & 4 & 56 \\
\hline 2 & 76 & 12 & 64 & 25 & 92 & 72 & 20 \\
\hline 3 & 96 & 92 & 4 & 26 & 44 & 8 & 36 \\
\hline 4 & 60 & 28 & 32 & 27 & 96 & 76 & 20 \\
\hline 5 & 84 & 76 & 8 & 28 & 64 & 0 & 64 \\
\hline 6 & 68 & 16 & 52 & 29 & 44 & 0 & 44 \\
\hline 7 & 52 & 8 & 44 & 30 & 92 & 68 & 24 \\
\hline 8 & 92 & 72 & 20 & 31 & 76 & 24 & 52 \\
\hline 9 & 92 & 32 & 60 & 32 & 36 & 0 & 36 \\
\hline 10 & 56 & 0 & 56 & 33 & 96 & 48 & 48 \\
\hline 11 & 60 & 0 & 60 & 34 & 64 & 0 & 64 \\
\hline 12 & 96 & 64 & 32 & 35 & 56 & 0 & 56 \\
\hline 13 & 44 & 0 & 44 & 36 & 48 & 0 & 48 \\
\hline 14 & 44 & 12 & 32 & 37 & 20 & 0 & 20 \\
\hline 15 & 72 & 0 & 72 & 38 & 76 & 0 & 76 \\
\hline 16 & 76 & 52 & 24 & 39 & 92 & 12 & 80 \\
\hline 17 & 24 & 0 & 24 & 40 & 68 & 4 & 64 \\
\hline 18 & 32 & 4 & 28 & 41 & 96 & 60 & 36 \\
\hline 19 & 92 & 16 & 76 & 42 & 8 & 0 & 8 \\
\hline 20 & 92 & 36 & 56 & 43 & 32 & 8 & 24 \\
\hline 21 & 24 & 4 & 20 & & & & \\
\hline
\end{tabular}


Atodiad 20. Gwasgariad sgoriau darllen Saesneg Dosbarth A
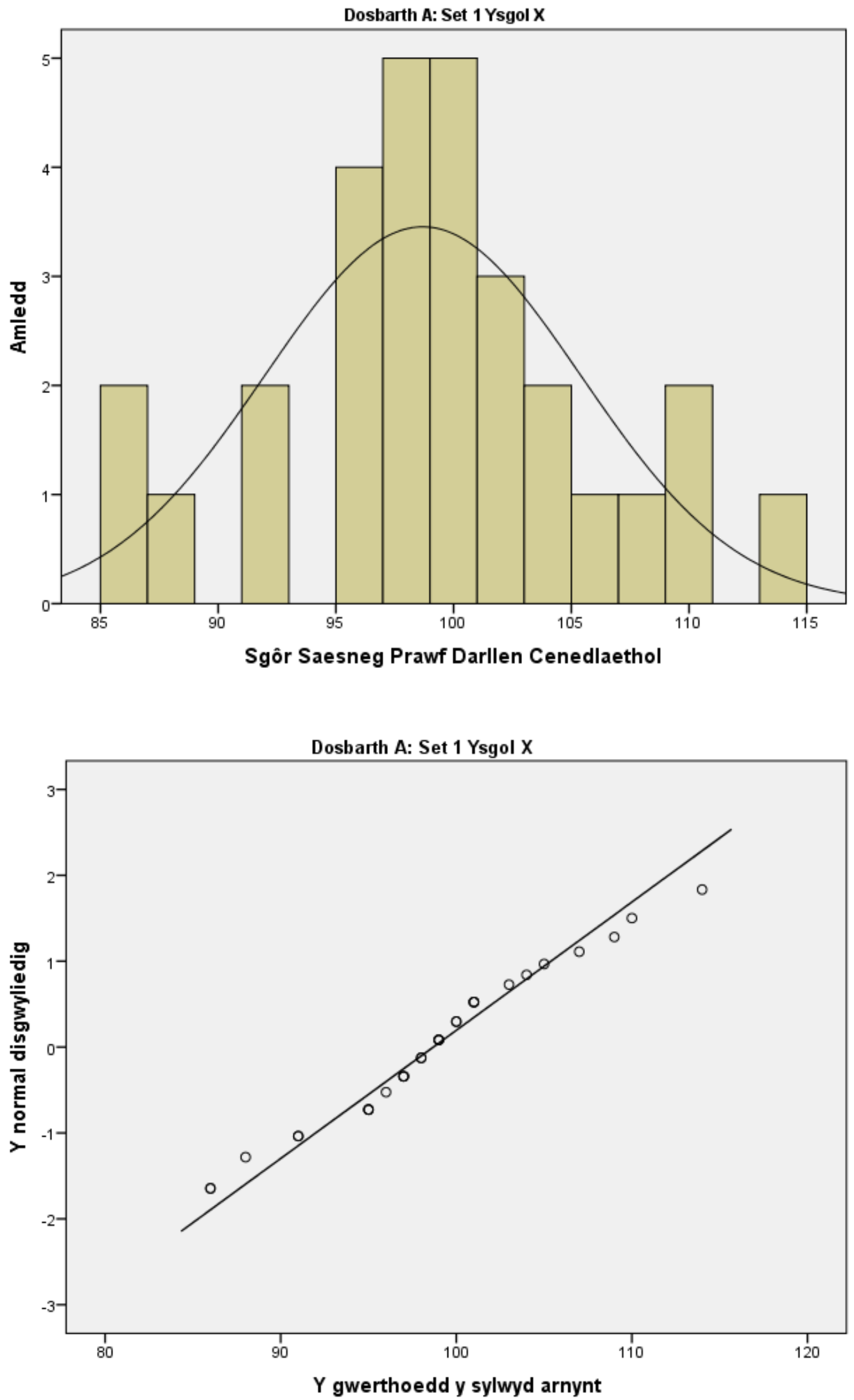
Atodiad 21. Gwasgariad sgoriau darllen Saesneg Dosbarth B.
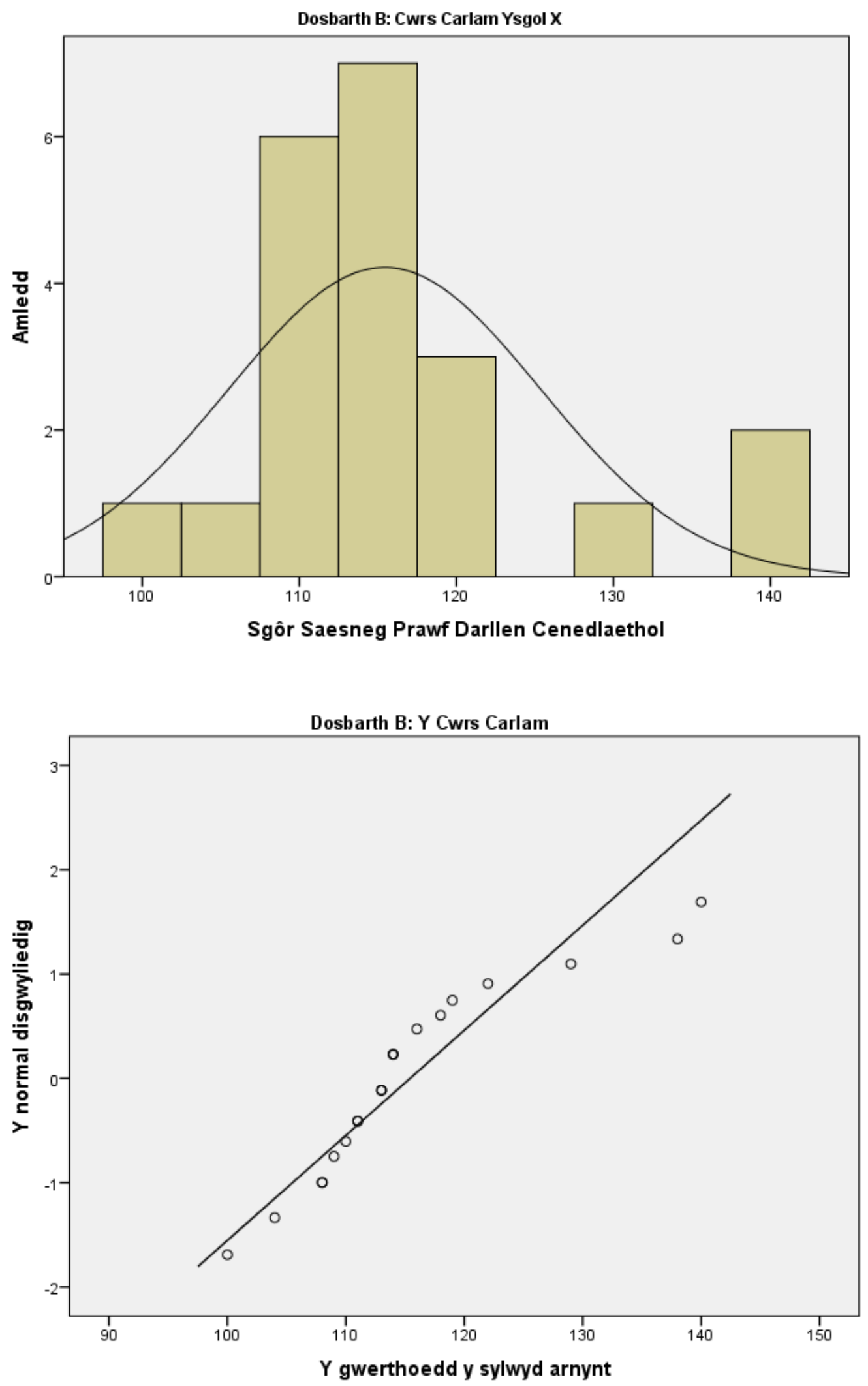
Atodiad 22. Gwasgariad sgoriau darllen Saesneg Dosbarth C.
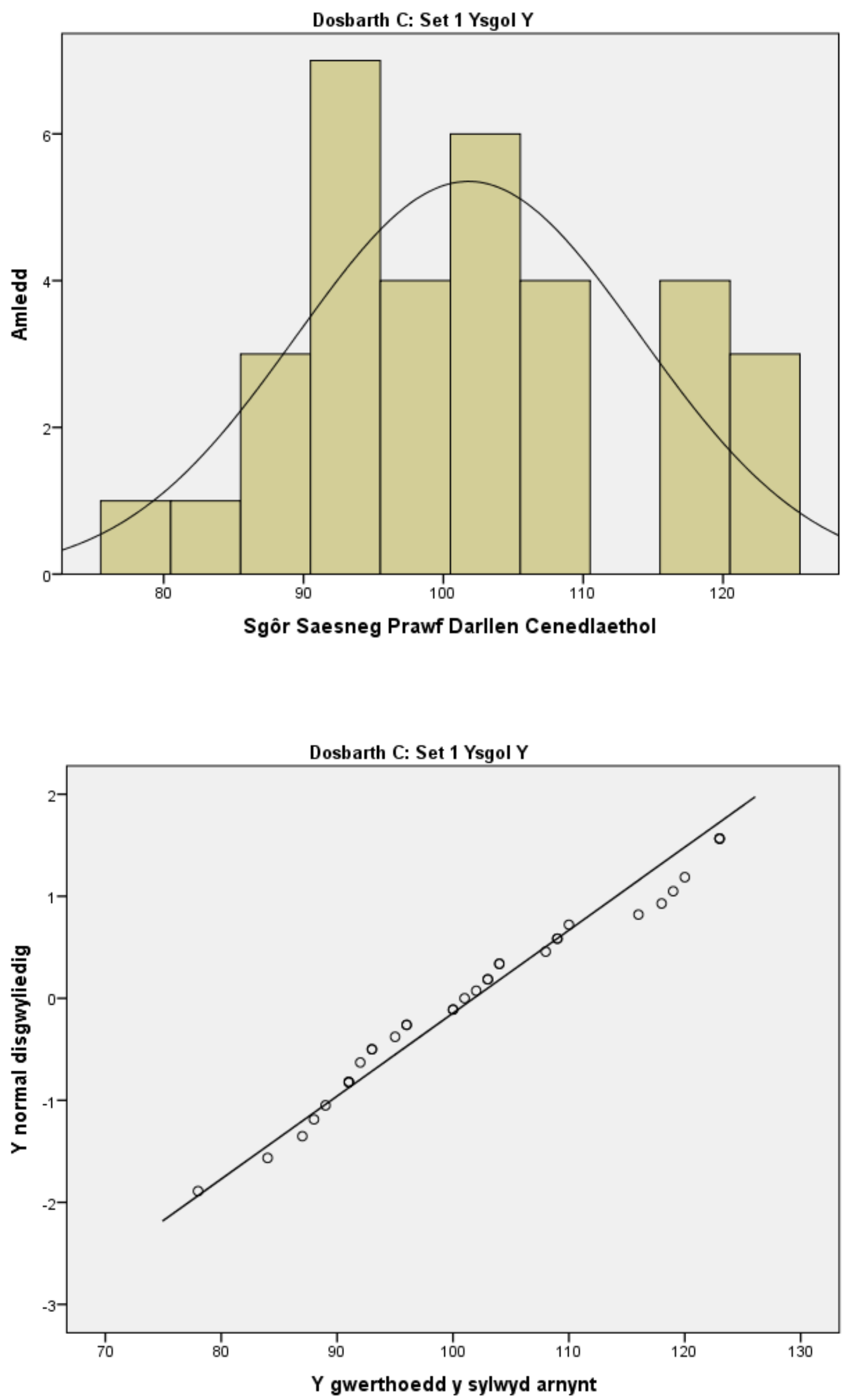


\section{Llyfryddiaeth}

Abu-Rabia, S., \& Sanitsky, E. (2010). Advantages of bilinguals over monolinguals in learning a third language. Bilingual Research Journal, 33(2), 173-199. doi: $10.1080 / 15235882.2010 .502797$

Ackroyd, S. \& Hughes, J.A. (1981). Data collection in context. Llundain: Longman.

Adelman, C., Kemmis, S. and Jenkins, D. (1980). Rethinking case study: Notes from the Second Cambridge Conference. Yn H.Simons (Gol.) Towards a Science of the Singular (tt. 45-61). Centre for Applied Research in Education, Prifysgol East Anglia.

Adran y Celfyddydau, Treftadaeth a'r Wyddeleg, Llywodraeth Iwerddon. (21 Rhagfyr, 2010). 20-year strategy for the Irish language: 2010 - 2030. Cyrchwyd 4 Tachwedd, 2014: http://www.ahg.gov.ie/en/20-YearStrategyfortheIrishLanguage20102030/Publications/20-Year\%20Strategy\%20-\%20English\%20version.pdf.

Agustín-Llach, M. P., \& Canga Alonso, A. (2016). Vocabulary growth in young CLIL and traditional EFL learners: Evidence from research and implications for education. International Journal of Applied Linguistics, 26(2), 211-227. doi: https://doi.org/10.1111/ijal.12090

Aierbe, P., Arregi, P., Etxeberria Balerdi, F., \& Etxeberria-Sagastume, F. (1989). UrretxuLegazpi-Zumarraga eskoletako euskararen egoera. Kilometroak, 85.

Aierbe, P., Etxezarreta, J., \& Satrustegi, L. M. (1974). Ikastoletako aurren jakite mailaren azterketa konparatiboa. Zeruko Argia, 603, 1.

Aitchison, J. W., \& Carter, H. (2000). Language, economy, and society: The changing fortunes of the Welsh language in the twentieth century (arg. diwygiedig). Caerdydd: Gwasg Prifysgol Cymru.

Aitken, K. G. (1977). Using Cloze procedure as an overall language proficiency test. TESOL Quarterly, 11(1), 59-67. Cyrchwyd 8 Rhagfyr, 2015: http://www.jstor.org/stable/3585592

Alderson, J. C. (1979). The Cloze procedure and proficiency in English as a foreign language. TESOL Quarterly, 13(2), 219-227. Cyrchwyd 19 Ionawr, 2016: http://www.jstor.org/stable/3586211 
Aldridge, A., \& Levine, K. (2001). Surveying the social world: Principles and practice in survey research. Buckingham: Gwasg Y Brifysgol Agored.

Alliance Française de Cebu. (d.d.). How long will it take me to speak French? Cyrchwyd Mai 18, 2015: http://www.alliancefr.ph/en/how-long-will-it-take-me-to-speak-french

Allder, M. (1993). The meaning of 'school ethos'. Westminster Studies in Education, 16(1), 59-69. doi: 10.1080/0140672930160109

Andersson, T., \& Boyer, M. (1978). Bilingual schooling in the United States (2 $2^{\text {ail }}$ arg.). Austin, Texas: National Educational Laboratory Publishers.

Andrews, L. (2012, 17 Gorffennaf). Datganiad ysgrifenedig - Adolygiad o Gymraeg ail iaith yng Nghyfnodau Allweddol 3 a 4. Cyrchwyd 9 Mawrth, 2015: http://gov.wales/?lang=cy

Apsel, C. (2012). Coping with CLIL: Dropouts from CLIL streams in Germany. International CLIL Research Journal, 1(4), 47-56. Cyrchwyd 7 Rhagfyr, 2015: http://www.icrj.eu/14/article5.html

Archibald, J., Roy, S., Harmel, S., Jesney, K., Dewey, E., Moisik, S., \& Lessard, P. (2006). A review of the literature on second language learning. Alberta Education. Cyrchwyd 8 Medi, 2016: https://files.eric.ed.gov/fulltext/ED498248.pdf

Artieda G., Roquet, H. \& Nicolás-Conesa, F. (2017). The impact of age and exposure on EFL achievement in two learning contexts: formal instruction and formal instruction + content and language integrated learning (CLIL), International Journal of Bilingual Education and Bilingualism. doi: 10.1080/13670050.2017.1373059

Arzamendi, J., \& Genesee, F. (1997). Reflections on immersion education in the Basque Country. Yn R. K. Johnson \& M. Swain (Gol.), Immersion education: International perspectives (tt. 151-166). Caergrawnt: Gwasg Prifysgol Caergrawnt.

Atkinson, D. (2011). Alternative approaches to second language acquisition. Llundain: Routledge.

Atkinson J. M. \& J. Heritage (Gol.), Structures of social action. Caergrawnt: Gwasg Prifysgol Caergrwant.

Bachman, L. F. (1982). The trait structure of cloze test scores. TESOL Quarterly, 16(1), 6170. http://www.jstor.org/stable/3586563 
Bachman, L. F. (1985). Performance on cloze tests with fixed-ratio and rational deletions. TESOL Quarterly, 19(3), 535-556.

Baetens-Beardsmore, H. (1986). Bilingualism: Basic principles. Clevedon: Multilingual Matters.

Baker, C. (1985). Aspects of bilingualism in Wales. Clevedon: Multilingual Matters.

Baker, C. (1992). Attitudes and language. Clevedon: Multilingual Matters

Baker, C. (1993). Bilingual education in Wales. Yn H. Baetens-Beardsmore (Gol.), European models of bilingual education (tt. 7-29). Clevedon: Multilingual Matters.

Baker, C. (1993). Foundations of bilingual education and bilingualism. Clevedon: Multilingual Matters.

Baker, C. (1996). Foundations of bilingual education and bilingualism (2ail arg.). Clevedon: Multilingual Matters.

Baker, C. (2001). Foundations of bilingual education and bilingualism (3 $\left.3^{\mathrm{ydd}} \mathrm{arg}.\right)$. Clevedon: Multilingual Matters.

Baker, C. (2004a). Bilingual education. Yn M. Byram (Gol.), Routledge encyclopedia of language teaching and learning (tt. 79-82). Llundain; Efrog Newydd: Routledge.

Baker, C. (2004b). Bilingualism. Yn M. Byram (Gol.), Routledge encyclopedia of language teaching and learning (tt. 82-84). Llundain; Efrog Newydd: Routledge.

Baker, C. (2010). The advantages of bilingualism in Welsh and English. Papur a gyflwynwyd i Gynulliad Cenedlaethol Cymru. Cyrchwyd 16 Mehefin, 2015: http://www.assembly.wales/cy/bus-home/bus-third-assembly/3-committees/3scrutiny/3-els/bus-committees-third-els-report/Pages/el_3_-10-r08.aspx.

Baker, C. (2011). Foundations of bilingual education and bilingualism (5 $5^{\mathrm{ed}}$ arg.). Clevedon: Multilingual Matters.

Baker, C., \& Jones, S. P. (Gol.). (1998). Encyclopedia of bilingualism and bilingual education. Clevedon: Multilingual Matters.

Basque Cultural Institute. (d.d.). Population in the Northern Basque Country. Cyrchwyd 16 Tachwedd, 2015: http://www.eke.eus/en 
Bassey, M. (1981). Pedagogic research: on the relative merits of search for generalisation and study of single events, Oxford Review of Education, 7 (1), 73-93.

Bassey, M. (1999). Case study research in educational settings. Doing qualitative research in educational settings. Buckingham: Gwasg Prifysgol Agored.

BBC Cymru Fyw. (2014, 6 Awst). £1.25 miliwn ar gyfer canolfannau Cymraeg. Cyrchwyd 17 Mawrth, 2015: http://www.bbc.co.uk/cymrufyw/28662081

BBC Cymru Fyw. (2014, 15 Mai). Angen newid addysg Gymraeg ail-iaith medd gweinidogion. Cyrchwyd 20 Awst, 2015: http://www.bbc.co.uk/cymrufyw/27426179

BBC News Wales. (2011). Welsh schools' GCSE performances revealed. Cyrchwyd 22 Chwefror, 2016: http://www.bbc.co.uk/news/uk-wales-13478504

Beard, A. C. (2016). Addysg Gymraeg ail iaith mewn Ysgolion cyfrwng-Saesneg: Astudiaeth $i$ archwilio i ba raddau y mae amodau dysgu'r rhaglen Gymraeg ail iaith yng Nghyfnodau Allweddol 2 a 3 yn gymwys $i$ gynhyrchu siaradwyr yr iaith (traethawd PhD). Caerfyrddin: Prifysgol Cymru Y Drindod Dewi Sant.

Beaufort Research. (2013). Ymchwilio i ddefnydd iaith siaradwyr Cymraeg yn eu bwydau bob dydd. Llywodraeth Cymru. Cyrchwyd 29 Mawrth, 2016: http://gov.wales/topics/welshlanguage/research/exploring-welsh-lang-in-dailylives/?lang=cy.

Bell, J. (1999). Doing your research project: A guide for first-time researchers in education and social science ( $3^{\mathrm{ydd}}$ arg.). Buckingham: Gwasg Prifysgol Agored.

Benjama, N., \& Palanukulwong, T. (2015). Rational cloze test: A valid measure of vocabulary? Veridian E-Journal: International humanities, social sciences and arts, 8(4), 113-126. Cyrchwyd 20 Ebrill, 2017: http://www.tci-thaijo.org/index.php/Veridian-E-Journal/article/view/40059

Ben-Zeev, S. (1977). The effect of bilingualism in children from Spanish-English low economic neighborhoods on cognitive development and cognitive strategy. Working Papers on Bilingualism, 14, 83-122.

Bialystok, E. (1987a). Influences of bilingualism on metalinguistic development. Second Language Research, 3(2), 154-166. Cyrchwyd 29 Gorffennaf, 2015: http://www.jstor.org/stable/43104362 
Bialystok, E. (1987b). Words as things: Development of word concept by bilingual children. Studies in Second Language Acquisition, 9(2), 133-140. doi: http://dx.doi.org/10.1017/S0272263100000437

Bialystok, E. (1993). Metalinguistic awareness: The development of children's representations of language. Yn C. Pratt \& A. Garton (Gol.), Systems of representation in children: Development and use, 211-33. Llundain: Wiley.

Bialystok, E. (1997). Effects of bilingualism and biliteracy on children's emerging concepts of print. Developmental Psychology, 33(3), 429-440. doi: http://dx.doi.org/10.1037/0012-1649.33.3.429

Bialystok, E. (1999). Cognitive complexity and attentional control in the bilingual mind. Child Development 70(5). 636-44. doi: https://doi.org/10.1111/1467-8624.00046

Bialystok, E. (2001a). Bilingualism in development: Language, literacy, and cognition. Cyrchwyd 13 Gorffennaf, 2015: http://site.ebrary.com/lib/swansea/reader.action?docID=5006349

Bialystok, E. (2001b). Metalinguistic aspects of bilingual processing. Annual Review of Applied Linguistics, 21, 169-181. doi: http://dx.doi.org/10.1017/S0267190501000101

Bialystok, E., \& Codd, J. (1997). Cardinal limits: Evidence from language awareness and bilingualism for developing concepts of number. Cognitive Development, 12(1), 85106. doi: http://dx.doi.org/10.1016/S0885-2014(97)90031-9

Bialystok, E., \& Craik, F., I. M. (2010). Cognitive and linguistic processing in the bilingual mind. Current Directions in Psychological Science, 19(1), 19-23. doi: $10.1177 / 0963721409358571$

Bialystok, E., Craik, F. I. M., \& Freedman, M. (2007). Bilingualism as a protection against the onset of symptoms of dementia. Neuropsychologia, 45(2), 459-464. Cyrchwyd 16 Gorffennaf, 2015: http://www.sciencedirect.com/science/article/pii/S0028393206004076

Bialystok, E., Craik, F. I. M., \& Luk, G. (2012). Bilingualism: Consequences for mind and brain. Trends in Cognitive Sciences, 16(4), 240-250. doi: http://dx.doi.org/10.1016/j.tics.2012.03.001 
Bialystok, E., \& Herman, J. (1999). Does bilingualism matter for early literacy?

Bilingualism: Language and Cognition, 2(1), 35-44. Cyrchwyd 22 Gorffennaf, 2015: http://journals.cambridge.org/abstract_S1366728999000139

Bialystok, E., Luk, G., Peets, K. F., \& Yang, S. (2010). Receptive vocabulary differences in monolingual and bilingual children. Bilingualism: Language and Cognition, 13(4), 525-531. doi: 10.1017/S1366728909990423

Bilki, U. (2011). The effectiveness of cloze tests in assessing the speaking/writing skills of university EFL learners. (traethawd MA). Prifysgol Bilkent, Ankara, Twrci. Cyrchwyd 26 Chwefror, 2016: http://library.bilkent.edu.tr/

Blackledge, A., \& Creese, A. (2010). Multilingualism. Llundain: Continnum International Publishing Group.

Bley-Vroman, R. (1983). The comparative fallacy in interlanguage studies: The case of systematicity. Language Learning, 33, 1-17. doi: https://doi.org/10.1111/j.14671770.1983.tb00983.x

Bloomfield, L. (1933). Language. Efrog Newydd: Holt.

Blom, E., \& Unsworth, S. (Gol.). (2010). Experimental methods in language acquisition research. Cyrchwyd 17 Medi, 2015: https://www.dawsonera.com

Board, K., \& Tinsley, T. (2015). Ieithoedd tramor modern mewn ysgolion uwchradd yng Nghymru: Canfyddiadau o arolwg tueddiadau ieithoedd 2014/15. Ymddiriedolaeth Addysg CfBT.

Bredenbroker, W. (2000). Förderung der fremdsprachlichen Kompetenz durch bilingualen Unterricht: Empirische Untersuchungen. Frankfurt am Main: Peter Lang.

Breidbach, S., \& Viebrock, B. (2012). CLIL in Germany: Results from recent research in a contested field of education. International CLIL Research Journal, 1(4), 5-16. Cyrchwyd 20 Tachwedd, 2015: http://www.icrj.eu/14/article1.html

Brenner, M., Brown, J. \& Canter, D. (1985). The research interview: Uses and approaches. Llundain: Academic Press, Inc.

Brinton, D., Snow, M. A., \& Wesche, M. (1989). Content-based second language instruction. Efrog Newydd: Newbury House. 
Brown, J. D. (1980). Relative merits of four methods for scoring cloze tests. The Modern Language Journal, 64(3), 311-317. Cyrchwyd 26 Ionawr, 2016: http://www.jstor.org/stable/324497

Brown, J. D. (2001). Surveys in language programs. Caergrawnt: Gwasg Prifysgol Caergrawnt.

Brown, J. D. (2002). Do cloze tests work? or, is it just an illusion? Second Language Studies, 21(1), 79-125. Cyrchwyd 25 Chwefror, 2016: http://www.hawaii.edu/sls/wp-content/uploads/2014/09/BrownCloze.pdf

Brown, J. D. (2003). Norm-referenced item analysis (item facilitation and item discrimination). Shiken: The JALT Testing and Evaluation SIG Newsletter, 7, 16-19. Cyrchwyd 5 Ionawr, 2018: http://www.jalt.org/test/PDF/Brown17.pdf

Brown, J. D. \& Rodgers, T. S. (2002). Doing second language research. Rhydychen: Gwasg Prifysgol Rhydychen.

Bruton, A. (2013). CLIL: Some of the reasons why ... and why not. System, 41, 587-597. doi: https://doi.org/10.1016/j.system.2013.07.001

Būdvytytě-Gudieně, A., \& Toleikieně, R. (2008). Content and language integrated learning: Features of educational methods. Social Sciences (1392-0758) 60, 90-97. Cyrchwyd 30 Hydref, 2015: http://etalpykla.lituanistikadb.lt/fedora/objects/LT-LDB0001:J.04 2008 1367165185610/datastreams/DS.002.1.01.ARTIC/content

Burgess, T. F. (2001). Guide to the design of questionnaires: A general introduction to the design of questionnaires for survery research. Cyrchwyd 8 Ionawr, 2016: http://iss.leeds.ac.uk/site/index.php

Burmeister, P., \& Daniel, A. (2002). How effective is late partial immersion? Some findings from a secondary school program in Germany. Yn P. Burmeister, T. Piske \& A. Rohde (Gol.), An integrated view of language development. Papers in honor of Henning Wode (tt. 499-515). Trier, yr Almaen: Wissenschaftlicher Verlag Trier. Cyrchwyd 18 Tachwedd, 2015: http://etalpykla.lituanistikadb.lt/fedora/objects/LTLDB-0001:J.04 2008 1367165185610/datastreams/DS.002.1.01.ARTIC/content

Burrowes, E., Bryer, N., Bryer, H., Campbell, C., \& Charles, E. (2011). Arolwg o'r gwasanaeth cymorth iaith Gymraeg i ysgolion. Old Bell 3 Cyf a Dateb ar ran 
Llywodraeth Cymru. Cyrchwyd 16 Mawrth, 2016: http://gov.wales/statistics-andresearch/review-welsh-language-support-services-schools/?lang=cy.

Cameron, L. (2001). Children learning a foreign language. Yn L. Cameron (Gol.), Teaching languages to young learners (tt. 1-20). Caergrawnt: Gwasg Prifysgol Caergrawnt.

Canadian Council on Learning. (2007). French-immersion education in Canada. Cyrchwyd 29 Hydref, 2014: http://www.ccl-cca.ca/pdfs/LessonsInLearning/May-17-07-Frenchimmersion.pdf.

Carleton Board of Education. (1996). Comparative outcomes and impacts of early middle and late entry French immersion options: Review of recent research and annotated bibliography. Cyrchwyd 8 Rhagfyr, 2015: http://eric.ed.gov/?id=ED402778

CBAC. (d.d.). Ystadegau canlyniadau. Cyrchwyd 1 Medi, 2015:

http://www.cbac.co.uk/students/results-and-research/resultsstatistics.html?language_id=2

CBAC. (Ionawr 2008). Canllawiau athrawon; Manylebau TAG newydd i'w dysgu o Fedi 2008: Cymraeg ail iaith. Cyrchwyd 10 Hydref, 2014: http://www.wjec.co.uk/uploads/publications/4724.pdf?language_id=1.

CBAC. (2017). Cymorth i Athrawon. Cyrchwyd 18 Mai, 2017: http://www.cbac.co.uk/teachers/teacher-support/?language_id=2

Cenoz, J. (1998). Multilingual education in the Basque Country. Yn J. Cenoz \& F. Genesee (Gol.), Beyond bilingualism: Multilingualism and multilingual education (tt. 175191). Clevedon: Multilingual Matters.

Cenoz, J. (2005). English in bilingual programs in the Basque Country. International Journal of the Sociology of Language, (2005)(171), 41-56. doi: 10.1515/ijsl.2005.2005.171.41

Cenoz, J. (2009). Identities and attitudes. Yn J. Cenoz (Gol.), Towards multilingual education: Basque educational research from an international perspective (tt. 170188). Clevedon: Multilingual Matters.

Cenoz, J. (2013). The influence of bilingualism on third language acquisition: Focus on multilingualism. Language teaching, 46(1), 71-86. doi: 10.1017/S0261444811000218 
Cenoz, J. (2015). Content-based instruction and content and language integrated learning: The same or different? Language, Culture and Curriculum, 28(1), 8-24. doi: http://dx.doi.org/10.1080/07908318.2014.1000922

Cenoz, J., Genesee, F., \& Gorter, D. (2014). Critical analysis of CLIL: Taking stock and looking forward. Applied Linguistics, 35(3), 243-262. doi: 10.1093/applin/amt011

Cenoz, J., \& Perales, J. (1997). Minority language learning in the administration: Data from the Basque Country. Journal of Multilingual and Multicultural Development, 18(4), 261-270. doi: http://dx.doi.org/10.1080/01434639708666319

Cenoz, J., \& Valencia, J. F. (1994). Additive trilingualism: Evidence from the Basque Country. Applied Psycholinguistics, 15(2), 195-207. doi: http://dx.doi.org/10.1017/S0142716400005324

Cherrington, R. (2004). Transfer. Yn M. Byram (Gol.), Routledge encyclopedia of language teaching and learning (tt. 633-635). Llundain; Efrog Newydd: Routledge.

Chertkow, H., Whitehead, V., Phillips, N., Wolfson, C., Atherton, J., \& Bergman, H. (2010). Multilingualism (but not always bilingualism) delays the onset of Alzheimer's disease: Evidence from a bilingual community. Alzheimer Dis. Assoc. Disord, 24(2), 118-125. doi: 10.1097/WAD.0b013e3181ca1221

Chomsky, N. (1965). Aspects of the Theory of Syntax. Caergrawnt: MA: MIT Press.

Chomsky, N. (1975). Reflections on language. Efrog Newydd: Pantheon Books.

Chomsky, N. (1993). Lectures on government and binding: The Pisa lectures. Cyrchwyd 25 Awst, 2015: http://site.ebrary.com/lib/swansea/reader.action?docID=10597900

Chomsky, N. (2007). Approaching UG from below. Yn U. Sauerland \& G. Hans-Martin (Gol.), Studies in generative grammar : Interfaces + recursion = language? : Chomsky's minimalism and the view from syntax-semantics (tt. 1-29). Berlin; Efrog Newydd: Mouton de Gruyter. Cyrchwyd 26 Awst, 2015: http://site.ebrary.com/lib/swansea/detail.action?docID=10256700

Christian, D., Spanos, G., Crandall, J., Simich-Dudgeon, C., \& Willets, K. (1990). Combining language and context for second-language students. Yn A. M. Padilla, H. H. Fairchild \& C. M. Valadez (Gol.), Bilingual Education: Issues and strategies (tt. 141-156) California: Sage Publications. 
Clare, L., Whitaker, C. J., Martyr, A., Martin-Forbes, P. A., Bastable, A. J. M., Pye, K. L., Quinn, C., Thomas, E. M., Gathercole, V. C. M. \& Hindle, J. V. (2016) Executive control in older Welsh monolinguals and bilinguals. Journal of Cognitive Psychology, 28(4), 412-426, doi: 10.1080/20445911.2016.1148041

Cohen, L., Manion, L., \& Morrison, K. (2003). Research Methods in Education (5 ${ }^{\mathrm{ed}}$ arg.). Llundain: Routledge.

Cohen, L., Manion, L., \& Morrison, K. (2011). Research methods in education ( $7^{\mathrm{fed}}$ arg.). Oxon: Routledge.

Colegau Cymru. (2010). Strategaeth genedlaethol ar ddwyieithrwydd mewn Addysg Bellach. Cyrchwyd 7 Ionawr, 2016: http://www.colegaucymru.ac.uk/cyhoeddiadau-24.aspx.

Comisiynydd y Gymraeg. (2014). Canllawiau dylunio dwyieithog. Cyrchwyd 6 Ionawr, 2016: http://www.comisiynyddygymraeg.cymru/hybu/SiteCollectionDocuments/CYG1754 \%20Canllaw\%20Dylunio\%20CYMRAEG.pdf.

Comisiynydd y Gymraeg. (d.d.). Cyfrifiad 2011: canlyniadau yn ôl Cymuned. Cyrchwyd 8 Mehefin, 2018:

http://www.comisiynyddygymraeg.cymru/Cymraeg/Polisi,\%20ymchwil\%20a\%20dat a/Data\%20Cyfrifiad/Pages/Cyfrifiad2011canlyniadauynolCymuned.aspx continwwm. (d.d.). Yn Geiriadur Termau'r Coleg Cymraeg Cenedlaethol. Cyrchwyd 21 Rhagfyr, 2017: http://www.colegcymraeg.ac.uk/cy/adnoddau/termau/\#continwwm continwwm. (d.d.). Yn Gweiadur. Cyrchwyd 21 Rhagfyr, 2017: https://www.gweiadur.com/cy/Pawb/continwwm

Cook, V. (2010). The relationship between first and second language acquisition revisited. Yn E. Macaro (Gol.), Continuum companion to second language acquisition. (tt. 137157). Cyrchwyd 5 Ionawr, 2018: http://www.ebrary.com/corp/index.jsp

Coyle, D. (2006). Content and Language Integrated Learning: Motivating learners and teachers. Scottish Languages Review, 13, 1-18. Cyrchwyd 2 Awst, 2018: https://www.scilt.org.uk/Library/ScottishLanguagesReview/ScottishLanguagesRevie wBackIssues/Issue1301052006/tabid/2839/Default.aspx

Coyle, D., Hood, P., \& Marsh, D. (2010). Content and Language Integrated Learning. Caergrawnt: Gwasg Prifysgol Caergrawnt. 
Craik, F. I. M., Bialystok, E., \& Freedman, M. (2010). Delaying the onset of Alzheimer disease: Bilingualism as a form of cognitive reserve. Neurology, 75(19), 1726-1729. Cyrchwyd 16 Gorffennaf, 2015: http://www.ncbi.nlm.nih.gov/pmc/articles/PMC3033609/pdf/8193.pdf

Crystal, D. (1997a). Yn D. Crystal (Gol.), The Cambridge encyclopedia of language (2 ${ }^{\text {ail }}$ arg.). Caergrawnt: Gwasg Prifysgol Caergrawnt.

Crystal, D. (1997b). Child language acquisition: Investigating childrens' language. Yn D. Crystal (Gol.), The Cambridge encyclopedia of language (2 $2^{\text {ail }}$ arg., tt. 230-237). Caergrawnt: Gwasg Prifysgol Caergrawnt.

Crystal, D. (2010a). Multilingualism. Yn D. Crystal (Gol.), The Cambridge encyclopedia of language (3 $3^{\mathrm{ydd}}$ arg., tt. 372-375). Caergrawnt: Gwasg Prifysgol Caergrawnt.

Crystal, D. (2010b). The Cambridge encyclopedia of language (3ydd arg.). Caergrawnt: Gwasg Prifysgol Caergrawnt.

Cummins, J. (1976). The influence of bilingualism on cognitive growth: A synthesis of research findings and explanatory hypothesis. Working Papers on Bilingualism 9, (tt. 1-43). Cafwtd 26 Ionawr, 2018: https://files.eric.ed.gov/fulltext/ED125311.pdf

Cummins, J. (1979a). Cognitive/academic language proficiency, linguistic interdependence, the optimum age question and some other matters. Working Papers on Bilingualism 19, (tt. 198-205). Cyrchwyd 27 Gorffennaf, 2015:

http://www.researchgate.net/publication/234573070_CognitiveAcademic_Language_ Proficiency_Linguistic_Interdependence_the_Optimum_Age_Question_and_Some_O ther_Matters._Working_Papers_on_Bilingualism_No._19

Cummins, J. (1979b). Linguistic interdependence and the educational development of bilingual children. Review of Educational Research, 49(2), 222-251. Cyrchwyd 1 Rhagfyr, 2015: www.jstor.org

Cummins, J. (1980). The construct of language proficiency in bilingual education. Yn J. A. Alatis (Gol.), Georgetown University Round Table on Language and Linguistics 1980. Washington DC: Gwasg Prifysgol Georgetown. Cyrchwyd 26 Hydref, 2015: https://repository.library.georgetown.edu

Cummins, J. (1991). Language development and academic Learning. Yn L. M. Malave \& G. Duquette (Gol.), Language culture and cognition. Clevedon: Multilingual Matters. 
Cymdeithas yr Iaith Gymraeg. (2015, 25 Chwefror). Donaldson: Angen gweithredu fel nad oes "Welsh Not" 21ain ganrif. Cyrchwyd 10 Mawrth, 2015:

http://cymdeithas.org/newyddion/donaldson-angen-gweithredu-fel-nad-oes-welshnot-21ain-ganrif

Cymdeithas yr Iaith Gymraeg. (2015, 8 Mai). Ymateb i adroddiad Donaldson. Cyrchwyd 1 Medi, 2015: http://cymdeithas.cymru/dogfen/ymateb-i-adroddiad-donaldson

Cymdeithas yr Iaith Gymraeg. (d.d.a). 6 pheth. Cyrchwyd 16 Ionawr, 2015: http://cymdeithas.org/6pheth

Cymdeithas yr Iaith Gymraeg. (d.d.b). Addysg. Cyrchwyd 31 Gorffennaf, 2015: http://cymdeithas.cymru/addysg

Cymwysterau Cymru. (d.d.). TGAU Cymraeg Ail Iaith: Crynodeb o'r penderfyniadau a dadansoddiad o'r ymatebion i'n hymgynghoriad ar ddiwygio TGAU Cymraeg Ail Iaith i’w haddysgu am y tro cyntaf ym mis Medi 2017. Cyrchwyd 31 Mai, 2018: http://qualificationswales.org/media/2024/tgau-cymraeg-ail-iaith.pdf

Cyngor Ewropeaidd. (2001). Common European framework of reference for languages: Learning, teaching, assessment. Caergrawnt: Gwasg Prifysgol Caergrawnt.

Cyngor Gwynedd. (2014). Cynllun strategol y Gymraeg mewn addysg 2014-17. Cyrchwyd 30 Mawrth, 2016: https://www.gwynedd.llyw.cymru/cy/Cyngor/Strategaethau-apholis\%C3\%AFau/Addysg/Polisi-Iaith.aspx.

Cynulliad Cenedlaethol Cymru. (2010, 30 Medi). Mae angen hyrwyddo dwyieithrwydd mewn ysgolion yn ôl adroddiad pwyllgor. Cyrchwyd 6 Mawrth 2015:

http://www.assembly.wales/cy/newhome/Pages/newsitem.aspx ?itemid=673

Cynulliad Cenedlaethol Cymru. (2015). Cofnod y trafodion 01/07/2015. Cyrchwyd 2 Medi, 2015: http://www.cynulliad.cymru/cy/bushome/Pages/Plenary.aspx?assembly=4\&category=Record\%20of\%20Proceedings.

Cynulliad Cenedlaethol Cymru. (2016, 21 Medi). Cyfnod y trafodion 21/09/2016. Cyrchwyd 20 Ionawr, 2017: http://www.cynulliad.cymru/cy/bushome/Pages/Plenary.aspx?assembly=5\&category=Record $\% 20$ of $\% 20$ Proceedings

Dąbrowska, E. (2015). What exactly is Universal Grammar, and has anyone seen it? Frontiers in Psychology, 6(852). doi:10.3389/fpsyg.2015.00852 
Dale, L., van der Es, W., \& Tanner, R. (2011). CLIL skils. European Platform Internationalising Education. Cyrchwyd 1 Awst, 2018: https://www.nuffic.nl/en/publications/find-a-publication/clil-skills.pdf

Dalton-Puffer, C. (2007). Discourse in content and language integrated learning (CLIL) classrooms. Amsterdam: John Benjamins.

Dalton-Puffer, C. (2008). Outcomes and processes in Content and Language Integrated Learning (CLIL): Current research from Europe. Yn W. Delanoy \& L. Volkmann (Gol.), Future perspectives for English language teaching (tt. 139-158). Heidelberg: Carl Winter.

Dalton-Puffer, C. (2011). Content-and-Language Integrated Learning: From practice to principle? Annual Review of Applied Linguistics, 31, 182-204. doi: $10.1017 / \mathrm{S} 0267190511000092$

Dalton-Puffer, C., Nikula, T., \& Smit, U. (2010). Language use and language learning in CLIL: Current findings and contentious issues. Yn C. Dalton-Puffer, T. Nikula \& U. Smit (Gol.), Language use and language learning in CLIL classrooms (tt. 279-291). Amsterdam: John Benjamins.

Darnell, D. K. (1968). The development of an English language proficiency test of foreign students, using a clozentropy procedure. Final report. U.S. Department of Health, Education and Welfare. Cyrchwyd 29 Chwefror, 2016: http://eric.ed.gov/?id=ED024039.

Davidson, J. (2001). Addysg drochi a dysgu iaith drwy ddulliau dwys: Adroddiad ar ymweliad â Newfounland a Labrador a New Brunswick. Caerdydd: Cynulliad Cenedlaethol Cymru.

Davidson, J. (2001). Y Gymraeg mewn addysg yng Nghymru. (ELL 19-01(p.2)). Caerdydd: Y Pwyllgor Addysg a Dysgu Gydol Oes.

Davies, C. (d.d.). [tystiolaeth ysgrifenedig a ddarparwyd i Gynulliad Cenedlaethol Cymru]. Cyrchwyd Mehefin 16, 2015: http://www.assembly.wales

Davies, S. (2013). Un Iaith i bawb: Adolygiad o Gymraeg ail iaith yng Nghyfnodau Allweddol 3 a 4. Cyrchwyd 10 Hydref, 2014: http://wales.gov.uk/docs/dcells/publications/130926-review-of-welsh-second-lancy.pdf. 
Day, E. M., \& Shapson, S. M. (1996). Studies in immersion education. Clevedon: Multilingual Matters.

Deuchar, M., P. Davies, J. Herring, M. Parafita Couto, and D. Carter (2014). Building bilingual corpora. Yn E. M. Thomas and I. Mennen (Gol.), Advances in the Study of Bilingualism (tt. 93-111). Bryste: Multilingual Matters

de Vaus, D.A. (1996). Surveys in social research. (4 ${ }^{\mathrm{ydd}}$ arg.). Llundain: UCL Press.

Diaz, R. M., \& Klingler, C. (1991). Towards an explanatory model of the interaction between bilingualism and cognitive development. Yn E. Bialystok (Gol.), Language processing in bilingual children (tt. 167-192). Caergrawnt: Gwasg Prifysgol Caergrawnt.

Donaldson, G. (2015). Dyfodol llwyddiannus: Adolygiad annibynnol o'r cwricwlwm a'r trefniadau asesu yng Nghymru. Cyrchwyd 16 Mawrth, 2015: http://gov.wales/docs/dcells/publications/150225-successful-futures-cy.pdf.

Donnelly, C. (2000). In pursuit of school ethos. British Journal of Educational Studies, 48(2), 134-154. Cyrchwyd 5 Ionawr, 2016: http://www.jstor.org/stable/1556001

Dörnyei, Z. (2003). Questionnaires in second language research: Construction, administration and processing. New Jersey: Lawrence Erlbaum Associates.

Douglas, M. O. (1994). Japanese cloze tests: Toward their construction. Japanese Language Education around the Globe, 4, 117-131. Cyrchwyd 9 Mawrth, 2016: http://dl.ndl.go.jp/info:ndljp/pid/8220976

Duñabeitia, J. A., Hernández, J. A., Antón, E., Macizo, P., Estévez, A., Fuentes, L. J., Carreiras, M. (2013). The inhibitory advantage in bilingual children revisited: myth or reality? Experimental Psychology, 61(3), 234-251. doi: 10.1027/1618-3169/a000243.

Dunmore, S. S. (2014). Bilingual life after school? Language use, ideologies and attitudes among Gaelic-medium educated adults, (traethawd PhD). Prifysgol Caeredin.

Durgunoğlu, A. Y., Nagy, W. E., \& Hancin-Bhatt, B. J. (1993). Cross-language transfer of phonological awareness. Journal of Educational Psychology, 85(3), 453-465. doi: http://dx.doi.org/10.1037/0022-0663.85.3.453

dwyieitheg (1964). Yn Geiriadur Prifysgol Cymru Ar Lein (arg. laf). Cyrchwyd Awst 21, 2015: http://geiriadur.ac.uk/gpc/gpc.html?dwyieitheg 
Dyfodol i'r Iaith. (2014). Datganiad polisi drafft Iaith fyw: iaith byw - bwrw mlaen: Ymateb Dyfodol i'r Iaith. Cafwyd, 9 Mawrth, 2015: http://www.dyfodol.net/wpcontent/uploads/2014/07/Ymateb-Dyfodol-i-Bwrw-Mlaen.docx

Dyfodol i'r Iaith. (2015). Ymateb Dyfodol i'r Iaith i adroddiad Donaldson. Cyrchwyd 18 Mai, 2015: http://www.dyfodol.net/2015/02/26/ymateb-dyfodol-ir-iaith-i-adroddiaddonaldson/

Dysgu Cymru. (2016, Ebrill 15). Hyfforddiant cyfrwng Cymraeg i ymarferwyr. Cyrchwyd Mawrth 16, 2017 o: http://learning.gov.wales/?lang=cy

Eaton, S. E. (2011). How long does it take to learn a second language?: Applying the “10,000-Hour Rule” as a model for fluency. Cafwyd, 8 Mai, 2015: http://files.eric.ed.gov/fulltext/ED516761.pdf

Elias, O. (2014, Rhagfyr). Dadansoddiad o elfennau ymddygiadol Bwrw 'Mlaen. Papur a gyflwynwyd yn Newid Ymddygiad a'r Gymraeg, Sefydliad Gwleidyddiaeth Rhyngwladol Prifysgol Aberystwyth. [Cyflwyniad PowerPoint]. Cafwyd, 15 Medi, 2015: http://www.iaith.cymru/cy/prosiectau/cynllunwyr-iaith-cymru/newidymddygiad-ar-gymraeg

Ellis, R. (2008). The study of second language acquisition (2 $2^{\text {ail }}$ arg.). Rhydychen: Gwasg Prifysgol Rhydychen.

Ellis, R. (2015). Understanding second language acquistion. Rhydychen: Gwasg Prifysgol Rhydychen.

Ericsson, K. A., Prietula, M. J., \& Cokely, E., T. (2007). The making of an expert. Harvard Business Review - Managing for the Long Term, Gorffennaf-Awst. Cyrchwyd 8 Ionawr, 2018: https://www.researchgate.net/publication/6196703_The_making_of_an_expert ERW. (2016). Llawlyfr Siarter Iaith Cymraeg Campus. Cyrchwyd 30 Awst, 2018: http://www.erw.cymru/ysgolion/dysgu-ac-addysgu/siarter-iaith/ Estyn. (2003). Arolwg o waith Athrawon Bro yng Nghymru. Caerdydd.

Estyn. (2004). Arolwg o'r Gymraeg fel ail iaith yng Nghyfnodau Allweddol 2 a 3 a'r Cyfnod Pontio. Cafwyd, 4 Mehefin, 2015: http://dera.ioe.ac.uk/5629/2/cy_WelshSecondLanguage.pdf. 
Estyn. (2006a). Adroddiad ar brosiectau peilot addysg drochi Cymraeg a dysgu iaith trwy ddulliau dwys mewn ysgolion. Cyrchwyd 29 Medi, 2015:

http://www.webarchive.org.uk/wayback/archive/20060501120000/http://www.wales. gov.uk/keypubassemedlearnskills/content/0706-paper1-annexd-e.pdf.

Estyn. (2006b). Adroddiad arolwg [Ysgol X]. Cyrchwyd 3 Mehefin, 2015: https://www.estyn.llyw.cymru

Estyn. (2007). Arfarniad o'r cwrs byr TGAU Cymraeg ail iaith. Cyrchwyd 15 Ionawr, 2015: https://www.estyn.llyw.cymru/thematic-reports/arfarniad-or-cwrs-byr-tgau-cymraegail-iaith-hydref-2007

Estyn. (2008). Adroddiad blynyddol Prif Arolygydd Ei Mawrhydi dros addysg a hyfforddiant yng Nghymru 2006-2007. Cyrchwyd 7 Mai, 2015:

http://www.estyn.gov.uk/download/publications/6279/the-annual-report-of-hermajestys-chief-inspector-of-education-and-training-in-wales-2006-2007/.

Estyn. (2009). Adroddiad blynyddol Prif Arolygydd Ei Mawrhydi dros addysg a hyfforddiant yng Nghymu 2007-2008. Cyrchwyd o:

http://www.estyn.gov.uk/download/publications/189871.5/annual-report-2007-08/.

Estyn. (2010). Adroddiad blynyddol Prif Arolygydd Ei Mawrhydi dros addysg a hyfforddiant yng Nghymru 2008-2009 - Cynnydd wrth wella ansawdd ym mhob un o'r sectorau rydyn ni'n eu harolygu. Cyrchwyd 7 Mai, 2015:

http://www.estyn.gov.uk/download/publications/187796.7/progress-in-improvingquality-in-each-of-the-sectors-we-inspect/.

Estyn. (2011). Adroddiad blynyddol Prif Arolygydd Ei Mawrhydi dros addysg a hyfforddiant yng Nghymu 2009-2010 - Adran 3: Cynydd wrth wella ansawdd ym mhob un o'r sectorau rydyn ni'n eu harolygu. Cyrchwyd 7 Mai, 2015:

http://www.estyn.gov.uk/download/publications/180616.8/2009-2010-annual-reportthe-progress-made-by-each-of-the-sectors/.

Estyn. (2012a). Adroddiad arolwg [Ysgol X]. Cyrchwyd 4 Mehefin, 2015: http://www.estyn.llyw.cymru

Estyn. (2012b). Adroddiad blynyddol Prif Arolygydd Ei Mawrhydi dros addysg a hyfforddiant yng Nghymu 2010-2011 - Adran 2: Adroddiad Sector. Cyrchwyd 30 
Ionawr, 2015: http://www.estyn.gov.uk/download/publication/233988.3/adroddiadsector/.

Estyn. (2012c). Balchder yn y Gymraeg yn sicrhau llwyddiant. Cyrchwyd 17 Mehefin, 2015: http://www.estyn.llyw.cymru

Estyn. (2012ch). Cymraeg ail iaith sy'n dod yn gyntaf. Cyrchwyd 17 Mehefin, 2015: http://www.estyn.llyw.cymru

Estyn. (2012d). Inspection report on [Ysgol X]. Cyrchwyd 4 Mehefin, 2015: http://www.estyn.llyw.cymru

Estyn. (2012dd). Yr iaith Gymraeg ar flaen y gad. Cyrchwyd 17 Mehefin, 2015: http://www.estyn.llyw.cymru

Estyn. (2013a). Adroddiad blynyddol Prif Arolygydd Ei Mawrhydi dros addysg a hyfforddiant yng Nghymru 2011-2012. Cyrchwyd 31 Ionawr, 2015: http://www.estyn.gov.uk/download/publications/268359/ar-2011-2012-compendium/.

Estyn. (2013b). Arweiniad atodol: Cymraeg. Cyrchwyd 2 Chwefror, 2016: http://www.estyn.llyw.cymru/.

Estyn. (2013c). Adroddiad arolwg [Ysgol Y]. Cyrchwyd o: http://www.estyn.llyw.cymru Estyn. (2014a). Adroddiad arolwg St John Baptist C.I.W. High School. Cafwyd, 15 Mawrth, 2015, o:http://www.estyn.llyw.cymru/darparwr/6744604.

Estyn. (2014b). Adroddiad blynyddol Prif Arolygydd Ei Mawrhydi dros addysg a hyfforddiant yng Nghymru 2012-2013. Cyrchwyd 11 Tachwedd, 2014: http://www.estyn.gov.uk/download/publications/300409.2/the-annual-report-of-hermajestys-chief-inspector-of-education-and-training-in-wales-2012-2013/.

Estyn. (2015). Adroddiad blynyddol Prif Arolygydd Ei Mawrhydi dros addysg a hyfforddiant yng Nghymru 2013-2014. Cyrchwyd 30 Ionawr, 2015: http://www.estyn.gov.uk/download/publications/345044.7/annual-report-2013-2014/.

Estyn. (2016a). Adroddiad arolwg [Ysgol X]. Cyrchwyd 23 Ionawr, 2017: https://www.estyn.llyw.cymru.

Estyn. (2016b). Adroddiad blynyddol Prif Arolygydd Ei Mawrhydi dros addysg a hyfforddiant yng Nghymru 2014-2015. Cyrchwyd 26 Ionawr, 2016: http://www.estyn.llyw.cymru/. 
Estyn. (2016c). Cyflymu dysgu disgyblion. Cyrchwyd 30 Ionawr, 2017:

https://www.estyn.llyw.cymru/arfer-orau/cyflymu-dysgu-disgyblion

Estyn. (2017). Adroddiad blynyddol Prif Arolygydd Ei Mawrhydi dros Addysg a Hyfforddiant yng Nghymru 2015-2016. Cyrchwyd 30 Ionawr, 2017: https://www.estyn.llyw.cymru.

Estyn. (2018). Adroddiad blynyddol Prif Arolygydd Ei Mawrhydi dros Addysg a Hyfforddiant yng Nghymru 2016-2017. Cyrchwyd 24 Mai, 2018: https://www.estyn.llyw.cymru.

Estyn. (d.d.). Y fframwaith arolygu cyffredin o Fedi 2010. Cyrchwyd 2 Chwefror, 2016: http://www.estyn.llyw.cymru/.

ethos. (1966). Yn Geiriadur Prifysgol Cymru Ar Lein (arg. laf). Cyrchwyd Ionawr 4, 2016: http://geiriadur.ac.uk/gpc/gpc.html?ethos

ethos. (d.d.). Yn Oxford Dictionaries Online. Cyrchwyd Ionawr 4, 2016:

http://www.oxforddictionaries.com/definition/english/ethos

Etxeberria-Sagastume, F. (2006). Attitudes towards language learning in different linguistic models of the Basque Autonomous Community. Yn O. García, T. Skutnabb-Kangas \& M. E. Torres-Guzman (Gol.), Imagining multilingual schools: Language in education and globalization (tt. 111-133). Clevedon: Multilingual Matters.

Eustat Municipal Inhabitant Statistics. (2014). Population of the Basque Country by province, according to sex and population density: 2014. Cyrchwyd 28 Hydref, 2015: http://en.eustat.eus/elementos/ele0011400/ti_population-of-the-basque-country-byprovince-according-to-sex-and-population-density/tb10011429_i.html\#axzz3pr1lqpBt

Everard, K. B. (1986). Developing management in schools. Rhydychen: Basil Blackwell.

Ferguson, C. (1959). Diglossia. Word, Rhif 15, 325-340.

Fishman, J. A. (1972). The sociology of language: An interdisciplinary social science approach to language in society. Rowley, MA: Newbury House.

Fishman, J. A. (1980). Bilingualism and biculturalism as individual and as societal phenomena. Journal of Multilingual and Multicultural Development, 1(1), 3-15. doi: $10.1080 / 01434632.1980 .9993995$

Fishman, J. A. (1991). Reversing language shift. Clevedon: Multilingual Matters. 
Fitzpatrick, T., \& Clenton, J. (2017). Making Sense of Learner Performance on Tests of Productive Vocabulary Knowledge. TESOL Quarterly, 51(4), 844-867. doi: 10.1002/tesq. 356

Fotos, S. (1991). The cloze test as an integrative measure of EFL proficiency: A substitute for essays on college entrance examinations? Language Learning, 41, 313-336. Cyrchwyd 26 Chwefror, 2016: https://www.deepdyve.com/

Freiberg, H. J. (1998). Measuring school climate: Let me count the ways. Educational Leadership, 56(1), 22-26. Cyrchwyd 5 Chwefror, 2016: http://search.proquest.com/

Freiberg, H. J. (1999). Three creative ways to measure school climate and next steps. Yn H. J. Freiberg (Gol.), School climate: Measuring, improving and sustaining healthy learning environments (tt. 214-224). Llundain: Falmer Press.

Frühe Mehrsprachigkeit an Kitas und Schulen. (2014). Bilinguale Grundschulen in Deutschland 2014. Cyrchwyd 26 Tachwedd, 2015: http://www.fmksonline.de/_wd_showdoc.php?pic=1118.

Gabiña, J. J., Gorostidi, R., Iruretagoiena, R., Olaziregi, I., \& Sierra, J. (1986). E.I.F.E. 1 Influence of factors on the learning of Basque. Cyrchwyd 16 Medi, 2015: http://www.hezkuntza.ejgv.euskadi.eus/r43573/en/contenidos/informacion/dia6/en_2027/adjuntos/publications_in_english/EIFE_ 1_en.pdf

García, O. (2009). Bilingual education in the 21st century: A global perspective. Malden, MA: Wiley-Blackwell.

García, O., \& Baker, C. (Gol.). (1995). Policy and practices in bilingual education. Clevedon: Multilingual Matters.

Gardner, N. (2002). Basque in education: In the Basque Autonomous Community. VitoriaGasteiz: Llywodraeth Gwlad y Basg. Cyrchwyd 27 Ebrill, 2015: http://www.euskadi.eus/r332732/es/contenidos/informacion/dih5/eu_2092/adjuntos/Basque\%20in\%20Education_ i.pdf.

Gardner, N. (2005). Basque: The Basque language in education in Spain. Mercator European Research Centre on Multilingualism and Language Learning. Cyrchwyd 31 Mawrth, 
2015: http://www.mercator-

research.eu/fileadmin/mercator/dossiers_pdf/basque_in_spain2nd.pdf.

Gardner, N. (2008). Profiad y Basg. Yn J. Osmond (Gol.), Creu Cymru ddwyieithog: Rôl y Gymraeg mewn addysg (tt. 38-50). Caerdydd: Y Sefydliad Materion Cymreig.

Gathercole, V. C. M. \& Hoff, E. (2007). Input and the acquisition of language: three questions, Yn E. Hoff \& M. Shatz (Gol.), The Handbook of Language Development (tt. 107-127). Efrog Newydd: Blackwell Publishers.

Gathercole, V. C. M. \& Thomas, E. M. (2005). Minority language survival: Input factors influencing the acquisition of Welsh, Yn J. Cohen, K. McAlister, K. Rolstad, and J. MacSwan (Gol.), ISB4: Proceedings of the 4th International Symposium on Bilingualism (tt. 852-874). Somerville: Cascadilla Press.

Gathercole, V. C. M. \& Thomas, E. M. (2009). Bilingual first language development: Dominant language takeover, threatened minority language take-up. Bilingualism: Language and Cognition, 12(2), 213-237. doi: 10.1017/S1366728909004015

Gathercole, V. C. Mueller, Thomas, E. M., Jones, L., Viñas Guasch, N., Young, N. \& Hughes, E. K. (2010). Cognitive effects of bilingualism: Digging deeper for the contributions of language dominance, linguistic knowledge, socioeconomic status, and cognitive abilities. Journal of Bilingual Education and Bilingualism, Special Issue 13 (5), 617-664.

Gathercole, V. C. Mueller, Thomas, E. M., Kennedy, I., Prys, C., Young, N., Viñas Guasch, N., Roberts, E. J., Hughes, E. K. a Jones, L. (2014). Does language dominance affect cognitive performance in Bilinguals? Lifespan evidence from preschoolers through older adults on Card Sorting, Simon, and Metalinguistic Tasks. Special issue of Frontiers in Psychology on the Development of Executive Function in Children. Frontiers in Psychology: Developmental Psychology 5, erthygl 11.

Gathercole, V. C. Mueller, Thomas, E. M., Viñas Guasch, N., Kennedy, I., Prys, C., Young, N. E., Roberts, E. J., Hughes, E. K. a Jones, L. (2016). Teasing apart factors influencing Executive Function performance in bilinguals and monolinguals at different ages. Linguistic Approaches to Bilingualism 6 (5), 605-647. 
Gellert, A. S., \& Elbro, C. (2013). Cloze tests may be quick, but are they dirty? Development and preliminary validation of a cloze test of reading comprehension. Journal of Psychoeducational Assessment, 31(1), 16-28. doi: 10.1177/0734282912451971

Genesee, F. (1983). Bilingual education of majority-language children: The Immersion experiments in review. Applied Psycholinguistics, 4(01), 1-46. doi: $10.1017 / \mathrm{S} 0142716400001739$

Genesee, F. (1984). Historical and theorectical foundations of immersion education. Yn California State Department of Education (Gol.), Studies on immersion education: A collection for United States educators (tt. 32-57). Sacremento: California State Department of Education. Cyrchwyd 5 Ionawr, 2018: http://eric.ed.gov/?id=ED239509.

Genesee, F., \& Gándara, P. (1999). Bilingual education programs: A cross-national perspective. Journal of Social Issues, 55(4), 665-685. doi: 10.1111/0022-4537.00141

Genesee, F., \& Hamayan, E. (2016). CLIL in context. Practical guidance for educators. Caergrawnt: Gwasg Prifysgol Caergrawnt.

Genesee, F., \& Lindholm-Leary, K. (2013). Two case studies of content-based language education. Journal of Immersion and Content-Based Language Education, 1(1), 3-33. doi: 10.1075/jicb.1.1.02gen

Genesee, F., Tucker, G. R., \& Lambert, W. E. (1975). Communication skills of bilingual children. Child Development, 46(4), 1010-1014. doi: 10.2307/1128415

Gierlinger, E. M., \& Wagner, T. A. (2016). The more the merrier - Revisiting CLIL-based vocabulary growth in secondary education. Latin American Journal of Content \& Language Integrated Learning, 9(1), 37-63. doi:10.5294/laclil.2016.9.1.3

Gillham, B. (2000). Developing a questionnaire. Llundain; Efrog Newydd: Continuum.

Glover, D., \& Coleman, M. (2005). School culture, climate and ethos: Interchangeable or distinctive concepts? Journal of In-service Education, 31(2), 251-272. doi: $10.1080 / 13674580500200278$

Goetz, J. P. \& LeCompte, M. D. (1984). Ethnography and qualitative design in educational research. Llundain: Academic Press, Inc. 
Gollan, T. H., \& Acenas, L.-a. R. (2004). What is a TOT? Cognate and translation effects on tip-of-the-tongue states in Spanish-English and Tagalog-English bilinguals. Journal Of Experimental Psychology: Learning, Memory And Cognition, 30(1), 246-269. doi: 10.1037/0278-7393.30.1.246

Gollan, T. H., Montoya, R. I., Fennema-Notestine, C., \& Morris, S. K. (2005). Bilingualism affects picture naming but not picture classification. Memory \& Cognition, 33(7), 1220-1234. doi: 10.3758/BF03193224

Gollan, T. H., Salmon, D. P., Montoya, R. I., \& Galasko, D. R. (2011). Degree of bilingualism predicts age of diagnosis of Alzheimer's disease in low-education but not in highly educated Hispanics. Neuropsychologia, 49(14), 3826-3830. doi: http://dx.doi.org/10.1016/j.neuropsychologia.2011.09.041

Golwg360. (2015, 23 Hydref). Leanne Wood yn herio record Llywodraeth Cymru. Cyrchwyd 26 Hydref, 2015: http://golwg360.cymru/newyddion/cymru/203676-leanne-wood-ynherio-record-llywodraeth-cymru

Grosjean, F. (1985). The bilingual as a competent but specific speaker-hearer. Journal of Multilingual and Multicultural Development 6(6). 467-477. doi: 10.1080/01434632.1985.9994221.

Grosjean, F. (2008). Studying bilinguals. Rhydychen: Gwasg Prifysgol Rhydychen. Cyrchwyd 5 Ionawr, 2018: http://www.ebrary.com/corp/index.jsp.

Grosjean, F. (2010). Bilingual: Life and reality. Massachusetts: Gwasg Prifysgol Harvard. Cyrchwyd 5 Ionawr, 2018: http://www.ebrary.com

Gruffudd, H., \& Morris, S. (2012). Canolfannau Cymraeg a rhwydweithiau cymdeithasol oedolion sy'n dysgu'r Gymraeg: Ymdrechion i wrthdroi shifft ieithyddol mewn cymunedau cymharol ddi-Gymraeg. Abertawe: Canolfan Cymraeg i Oedolion Deorllewin Cymru / Academi Hywel Teifi Prifysgol Abertawe.

Guilfoyle, B. (2008). [Ysgol X]: Y Gymraeg fel cyfrwng addysgu a dysgu o fewn y sector ail iaith. Yn J. Osmond (Gol.), Creu Cymru ddwyieithog: Rôl y Gymraeg mewn addysg (tt. 30-32). Caerdydd: Y Sefydliad Materion Cymreig.

Hadley, G. S., \& Naaykens, J. E. (1997). An investigation of the selective deletion cloze test as a valid measure of grammar-based proficiency in second language learning. Niigata University Research in Linguistics and Culture, 3, 111-118. Cyrchwyd 29 
Chwefror, 2016:

https://www.academia.edu/10555602/An_Investigation_of_the_Selective_Deletion_C loze_Test_as_a_Valid_Measure_of_GrammarBased_Proficiency_in_Second_Language_Learning

Hakuta, K. (1990). Bilingualism and bilingual education: A research perspective NCBE FOCUS. Occasional papers in bilingual education: Rhif 1, Gwanwyn. (cyhoeddiad cyfresol ar-lein). Cyrchwyd 23 Ebrill, 2015: http://www.ncela.us/files/rcd/BE017493/Hakuta.pdf.

Hamers, J. F., \& Blanc, M. H. A. (2000). Bilinguality and bilingualism. Cyrchwyd 16 Ebrill, 2015: http://www.ebrary.com/corp/index.jsp.

Hammerly, H. (1988). French immersion (does it work?) and the development of bilingual proficiency report. Canadian Modern Language Review, 45(3), 567-578.

Hammerly, H. (1991). Fluency and accuracy: Toward balance in language teaching and learning. Clevedon: Multilingual Matters.

Hanania, E., \& Shikhani, M. (1986). Interrelationships among three tests of language proficiency: Standardized ESL, cloze, and writing TESOL Quarterly, 20(1), 97-109. Cyrchwyd 25 Chwefror, 2016: http://www.jstor.org/stable/3586391

Harley, B., \& Swain, M. (1978). An analysis of the verb system used by young learners of French. Interlanguage Studies Bulletin, 3(1), 35-79. Cyrchwyd 5 Ionawr, 2018: http://www.jstor.org/stable/43135190

Harris, J. \& Ó Duibhir, P. (2011). Effective Language Teaching: A Synthesis of Research. Dulyn: National Council for Curriculum and Assessment.

Haugen, E. (1953). The Norwegian language in America. Philadelphia: Gwasg Prifysgol Philadelphia.

Haznedar, B., \& Gavruseva, E. (2013). Childhood second language acquisition. Yn J. Herschensohn \& M. Young-Scholten (Gol.), The Cambridge handbook of second language acquisition (tt. 338-352). Caergrawnt: Gwasg Prifysgol Caergrawnt.

Henley, A., \& Jones, R. E. (2003). Earnings and linguistic proficiency in a bilingual economy. Aberystwyth: Ysgol Reoli a Busnes, Prifysgol Aberystwyth. Cyrchwyd 2 Mehefin, 2015: http://repec.org/res2003/Henley.pdf 
Herschensohn, J. R., \& Young-Scholten, M. (Gol.). (2013). The Cambridge handbook of second language acquisition. Efrog Newydd: Gwasg Prifysgol Caergrawnt.

Hindle, J., Martin-Forbes, P., Bastable, A., Pye, K., Martyr, A., Whitaker, C., Craik, F. I. M., Bialystok, E., Thomas, E., Gathercole, V. C. M., \& Clare, L. (2015). Cognitive reserve in Parkinson's disease: The effects of Welsh-English bilingualism on executive function. Parkinson's Disease. Article ID 943572. doi: http://dx.doi.org/10.1155/2015/943572

Hinofotis, F. B. (1980). Cloze as an alternative method of ESL placement and proficiency testing. Yn J. W. J. Oller \& K. Perkins (Gol.), Research in language testing (tt. 121128). Rowley, MA: Newbury House.

Hitchcock, G. and Hughes, D. (1995). Research and the Teacher (2 $2^{\text {ail }}$ arg.). Llundain: Routledge.

Hodges, R. (2009). Welsh Language Use among Young People in the Rhymney Valley. Contemporary Wales, 22(1), 16-35.

Hoffmann, C. (1991). An introduction to bilingualism. Clevedon, Avon: Multilingual Matters.

Holmes, J. (1992). An introduction to Sociolinguistics. Llundain: Longman.

Hopkins, K. (2006). Achub ein hiaith. Caerdydd: Y Sefydliad Materion Cymreig.

Hopwood, M., Meek, E., Lloyd, L., \& Ebbsworth, M. (2018). Trosolwg o ddulliau asesu cymhwysedd iaith mewn detholiad o awdurdodaethau dwyieithog. Cymwysterau Cymru. Cyrchwyd 30 Awst, 2018:

https://www.qualificationswales.org/cymraeg/cyhoeddiadau/bilingual-languageproficiency-2018/

Hornberger, N. (Gol.). (2003). Continua of biliteracy: An ecological framework for educational policy, research and practice in multilingual settings. Clevedon: Multilingual Matters.

Howard, M. (2012). The advanced learner's sociolinguistic profile: On issues of individual differences, second language exposure conditions, and type of sociolinguistic variable. The Modern Language Journal, 96(1), 20-33. doi: 10.1111/j.15404781.2012.01293.x 
Ianco-Worrall, A. D. (1972). Bilingualism and cognitive development. Child Development, 43(4), 1390-1400. doi: 10.2307/1127524

Ibarrola, A. L. (2012). Faster and further morphosyntactic development of CLIL vs. EFL Basque-Spanish bilinguals learning English in high-school. International Journal of English Studies, 12(1), 79-96. doi: https://doi.org/10.6018/ijes.12.1.125621

Institut d'Estadística de Catalunya. (2013, 21 Tachwedd). Knowledge of Catalan: Catalonia. 2011. Cyrchwyd 27 Hydref, 2015: http://www.idescat.cat/economia/inec?tc=3\&id=da04\&lang=en

Institut d'Estadística de Catalunya. (2015). Population. 1900-2015: Provinces. Cyrchwyd 28 Hydref, 2015: http://www.idescat.cat/pub/?id=aec\&n=245\&lang=en

Instituto Nacional de Estadística. (2015). Population figures at 1 January 2015 - Migration statistics 2014. Cyrchwyd 14 Tachwedd, 2015: www.ine.es/en/prensa/prensa_en.htm.

Iorwerth, R. (2014, Tachwedd 5). Bwrw mlaen - I'r cyfeiriad cywir er budd y Gymraeg? [Gwe-log]. Cyrchwyd 16 Mawrth, 2015: https://pigion.wordpress.com/2014/11/05/bwrw-mlaen-ir-cyfeiriad-cywir-er-budd-ygymraeg/

Irvine, F. E., Roberts, G. W., Jones, P., Spencer, L. H., Baker, C., \& Williams, C. (2006). Communicative sensitivity in the bilingual healthcare setting: A qualitative study of language awareness. Journal of Advanced Nursing, 53(4), 422-434. doi: http://dx.doi.org/10.1111/j.1365-2648.2006.03733.x

Ivanova, I., \& Costa, A. (2008). Does bilingualism hamper lexical access in speech production? Acta Psychologica, 127(2), 277-288. doi: 10.1016/j.actpsy.2007.06.003

James, C. (1998). Errors in language learning and use: Exploring error analysis. Harlow, Lloegr: Pearson Education.

Jäppinen, A. K. (2005). Thinking and content learning of mathematics and science as cognitional development in content and language integrated learning (CLIL): Teaching through a foreign language in Finland. Language and Education, 19(2), 148-169. Cyrchwyd 7 Awst, 2018: https://www.unifg.it/sites/default/files/allegatiparagrafo/20-012014/jappinen_thinking_and_content_learning_in_mathematics_and_science.pdf 
Jefferson, G. (1984). Transcription Notation. Yn J.M. Atkinson \& J. Heritage (Gol.), Structures of social action (tt. ix-xvi). Efrog Newydd: Gwasg Prifysgol Caergrawnt.

Jiménez-Catalán, R. M., \& Ruiz de Zarobe, Y. (2009). The receptive vocabulary of EFL learners in two instructional contexts: CLIL versus non-CLIL instruction. Yn Y. Ruiz de Zarobe \& R. M. Jiménez Catalán (Gol.), Content and language integrated learning: Evidence from research in Europe (tt.81-92). Bryste: Multilingual Matters.

Johnson, R. K., \& Swain, M. (Gol.). (1997). Immersion education: International perspectives. Caergrawnt: Gwasg Prifysgol Caergrawnt.

Johnstone, R. (2002). Immersion in a second or additional language in school: A review of international research. Cyrchwyd 4 Tachwedd, 2015: http://www.scilt.org.uk/

Johnstone, R. (2004). Early language learning. Yn M. Byram (Gol.), Routledge encyclopedia of language teaching and learning (tt. 188-193). Llundain; Effrog Newydd: Routledge.

Johnstone, R. (2007). Characteristics of immersion programmes. Yn O. García \& C. Baker (Gol.), Bilingual education: An introductory reader (tt. 19-32). Clevedon: Multilingual Matters.

Jones, B. (2010). 'Amrywiaeth caleidosgopig': Addysg ddwyieithog yng Nghymru heddiw. Gwerddon, Rhifyn 5 (Ionawr 2010), 9-26. Cyrchwyd 16 Mehefin, 2015: http://www.gwerddon.org/cy/rhifynnau/rhifynnaugwerddon/teitl-3528-cy.aspx

Jones, C. (2015, Rhagfyr 4). [Llythyr at Colin Nosworthy]. Cymdeithas yr Iaith Gymraeg. Cyrchwyd 6 Ionawr, 2015: http://cymdeithas.cymru/sites/default/files/PW\%20Llythyr\%20Cymdeithas\%20yr\%20 Iaith\%20Rhagfyr\%202015.pdf

Jones, D. (1997). Cyflwyniad i ddwyieithrwydd. Aberystwyth: Canolfan Astudiaethau Addysg, Yr Hen Goleg.

Jones, D. R. (2013, 17 Ionawr). Cynllun strategol y Gymraeg mewn addysg. Eitem a gyflwynwyd yng nghyfarfod Pwyllgor Iaith y Cyngor: Caernarfon, Gwynedd. Cyrchwyd 30 Mawrth, 2016: https://www.gwynedd.llyw.cymru/cy/Cyngor/YCyngor.aspx.

Jones, D. V., Lewis, W. G., George, S., Thomas, M., \& Laugharne, J. (2009). Dysgu ail iaith yng nghyfnodau allweddol tri a phedwar: Addysgu'r Gymraeg mewn ysgolion 
cyfrwng Saesneg. Cylchgrawn Addysg Gymru, 14(2), 111-117. Cyrchwyd 18 Mehefin, 2015: http://ingentaconnect.com

Jones, G. W. (1993). Agweddau ar ddysgu iaith. Prifysgol Bangor: Canolfan Astudiaethau Iaith.

Jones, J. (1991). Addysg Uwchradd Ddwyieithog. Yn G. E. Jones (Gol.), Education, culture \& society: Some perspectives on the nineteenth and twentieth centuries; Essays presented to J. R. Webster. Caerdydd: Gwasg Prifysgol Cymru, cyhoeddwyd ar ran Cyfadran Addysg Prifysgol Cymru.

Jones, K., \& Bhatt, A. (2014). Cymraeg fel Iaith Ychwanegol: Ymchwil i lefel yr angen a' $r$ cymorth presennol a roddir $i$ ddisgyblion duon a lleiafrifoedd ethnig gydag anghenion cymorth yn y Gymraeg. Ymchwil Cymdeithasol Llywodraeth Cymru. Cyrchwyd 24 Chwefror, 2016: Retrieved from http://gov.wales/?lang=cy

Jones, M. (2001). Welsh: The Welsh language in the UK. Mercator-Education: European Network for Regional or Minority Languages and Education. Cyrchwyd 13 Mawrth, 2015: http://www.mercatorresearch.eu/fileadmin/mercator/dossiers_pdf/welsh_in_uk.pdf.

Jones, M. (2016). Briff ymchwil: Addysg cyfrwng Cymraeg a'r Gymraeg fel pwnc. Cynulliad Cenedlaethol Cymru. Cyrchwyd 31 Mawrth, 2017: http://www.assembly.wales/research\%20documents/rs16-048/16-048-welsh-web.pdf

Jones, M. L. N. (2013). Parent power. Yn H. S. Thomas \& C. H. Williams (Gol.), Parents, personalities and power: Welsh-medium schools in south-east Wales. Caerdydd: Gwasg y Brifysgol.

Jones, R. (2014). Newid ymddygiad a daearyddiaethau'r Gymraeg. Papur a gyflwynwyd yn Newid ymddygiad a'r Gymraeg, Prifysgol Aberystwyth. Cyrchwyd 5 Ionawr, 2018, o http://www.iaith.cymru/uploads/generaluploads/Trafodion_Newid_Ymddygiad_151214.pdf

Jones, R. M. (2011). A fydd y Cymry Cymraeg mewn pryd? Cyrchwyd 17 Hydref, 2014: http://www.rmjones-bobijones.net/llyfrau/afyddycymrycymraegmewnpryd.pdf

Jones, R. O. (1997). Hir oes i'r iaith: Agweddau ar hanes y Gymraeg a'r gymdeithas. Llandysul: Gwasg Gomer. 
Jones, S. M. (2010). Mesur y galw am addysg ôl-16 trwy gyfrwng y Gymraeg ymhlith disgyblion Blwyddyn 11 sydd yn astudio'r Gymraeg fel iaith gyntaf mewn tair ysgol uwchradd yng Ngheredigion. (Traethawd MA), Coleg Prifysgol y Drindod, Prifysgol Cymru Y Drindod Dewi Sant.

Jones, W. R. (1966). Bilingualism in Welsh education. Caerdydd: Gwasg Prifysgol Cymru. Jongsma, E. (1971). The cloze procedure: A survey of the research. Indiana University School of Education (Occasional Papers in Reading). Bloomington. Cyrchwyd 25 Chwefror, 2016: http://eric.ed.gov/?id=ED050893

Jonz, J. (1987). Textual cohesion and second language comprehension. Language Learning, 37(3), 409-438. Cyrchwyd 4 Mawrth, 2016: https://www.deepdyve.com

Jonz, J. (1990). Another turn in the conversation: What does cloze measure? TESOL Quarterly, 24(1), 61-83. Cyrchwyd 25 Chwefror, 2016: http://www.jstor.org/stable/3586852

Kavé, G., Eyal, N., Shorek, A., \& Cohen-Mansfield, J. (2008). Multilingualism and cognitive state in the oldest old. Psychology and Aging, 23(1), 70-78. doi: http://dx.doi.org/10.1037/0882-7974.23.1.70

Kharkhurin, A. V. (2007). The role of cross-linguistic and crosscultural experiences in bilinguals' divergent thinking. Yn I. Kecskes \& L. Albertazzi (Gol.), Cognitive aspects of bilingualism. Dordrecht, Yr Iseldiroedd: Springer.

Kharkhurin, A. V. (2011). The role of selective attention in bilingual creativity. Creativity Research Journal, 23(3), 329-254. doi: 10.1080/10400419.2011.595979

King, G. (2003). Modern Welsh: A comprehensive grammar (2 $2^{\text {ail }}$ arg.). Llundain: Routledge.

Koch, A. (2005). Bilingualer naturwissenschaftlicher anfangsunterricht: Entwicklung, erprobung, evaluation, (traethawd PhD). Prifysgol Kiel. Cyrchwyd 10 Gorffennaf, 2017: http://macau.unikiel.de/receive/dissertation_diss_00001531;jsessionid=5EBE62CAF103CF1D52EF0 BB83C1A56B8?lang=en

Korzilius, H. (2012). Quantitative Analysis in Case Study. Yn A. J. Mills, G. Durepos \& E. Wiebe (Gol.), Encyclopedia of Case Study Research (tt. 761-764). Thousand Oaks: Sage Publications. doi: http://dx.doi.org/10.4135/9781412957397 
Koster, J. (2013). Theories of language from a critical perspective. Yn J. Herschensohn \& M. Young-Scholten (Gol.), The Cambridge handbook to second language acquisition (tt. 9-25). Caergrawnt: Gwasg Prifysgol Caergrawnt.

Krashen, S. D. (1982). Principles and practice in second language acquisition. Cyrchwyd 13 Ebrill, 2015: http://www.sdkrashen.com/content/books/principles_and_practice.pdf

Kvale, S. (1996). Interviews: An introduction to qualitative research interviewing. Thousand Oaks: Sage Publications.

Laesch, K. B., \& Kleeck, A. v. (1987). The cloze test as an alternative measure of language proficiency of children considered for exit from bilingual education programs*. Language Learning, 37(2), 171-189. doi: 10.1111/j.1467-1770.1987.tb00564.x

Lambert, W. E. (1974). Culture and language as factors in learning and education. Yn Aboud, F.E. \& Meade, R.D (Gol.), Cultural Factors in Learning and Education, 5th Western Washington Symposium on Learning, Bellingham: Washington. Cyrchwyd 4 Mawrth, 2018: https://files.eric.ed.gov/fulltext/ED096820.pdf

Lambert, W. E., \& Tucker, G. R. (1972). Bilingual education of children: The St. Lambert experiment. Rowely, MA: Newbury House.

Larson-Hall, J. (2010). A guide to doing statistics in second language research using SPSS. Oxon: Routledge.

Lasagabaster, D. (2000). Three languages and three linguistic models in the Basque educational system. Yn J. Cenoz \& U. Jessner (Gol.), English in Europe: The acquisition of a third language (tt. 179-197). Clevedon: Multilingual Matters.

Lasagabaster, D. (2001). Bilingualism, immersion programmes and language learning in the Basque Country. Journal of Multilingual and Multicultural Development, 22(5), 401425.

Lasagabaster, D. (2007). Language use and language attitudes in the Basque Country. Yn D. Lasagabaster \& A. Huguet (Gol.), Multilingualism in European bilingual contexts: Language use and attitudes (tt. 65-89). Clevedon: Multilingual Matters. Cyrchwyd 5 Ionawr, 2018: http://site.ebrary.com/lib/swansea/Doc?id=10156063

Lasagabaster, D., \& Huguet, A. (2007). Multilingualism in European bilingual contexts Cyrchwyd 5 Ionawr, 2018: http://site.ebrary.com/lib/swansea/Doc?id=10156063 
Lasagabaster, D., \& Sierra, J. M. (2010). Immersion and CLIL in English: More differences than similarities. ELT Journal, 64(4), 367-375. doi: 10.1093/elt/ccp082

Lepage, J.-F., \& Corbeil, J.-P. (2013). The evolution of English-French bilingualism in Canada from 1961 to 2011. Minister of Industry, Statistics Canada. Cyrchwyd 29 Hydref, 2015: http://www.statcan.gc.ca/start-debut-eng.html.

Lewis, G. (2008). Hawl i'r Gymraeg. Talybont: Y Lolfa.

Lewis, H. (2014, 12 Mawrth). Datganiad ysgrifenedig - Adolygiad o'r Cwricwlwm a'r trefniadau asesu. Cyrchwyd 7 Mai, 2015: http://gov.wales/?lang=cy

Lewis, H. (2015, 15 Hydref). Datganiad ysgrifenedig - Cymraeg Ail Iaith yn ein cwricwlwm newydd. Llywodraeth Cymru. Cyrchwyd 21 Hydref, 2015: http://gov.wales/?lang=cy

Lewis, W. G. (2008). Addysg drochi yng Nghymru: Methodolegau a sialensau. Yn J. Osmond (Gol.), Creu Cymru ddwyieithog: Rôl y Gymraeg mewn addysg (tt. 8-18). Caerdydd: Y Sefydliad Materion Cymreig.

Lewis, W. G. (2010a). Raising standards in Welsh second language at ages 5-16: Project B. Tystiolaeth ysgrifenedig a ddarparwyd i Gynulliad Cenedlaethol Cymru. Cyrchwyd 17 Hydref, 2014: http://www.assembly.wales/NAfW\%20Documents/paper_3__dr_w_gwyn_lewis.pdf\%20-\%2002092010/paper_3_-_dr_w_gwyn_lewisEnglish.pdf\#search=W\%20Gwyn\%20Lewis.

Lewis, W. G. (2010b). Welsh second language in the National Curriculum: The case of teaching Welsh in English-medium schools. Cynulliad Cenedlaethol Cymru. Cyrchwyd 17 Hydref, 2014: http://www.assembly.wales/NAfW\%20Documents/paper_3__dr_w_gwyn_lewis.pdf\%20-\%2002092010/paper_3_-_dr_w_gwyn_lewisEnglish.pdf\#search=W\%20Gwyn\%20Lewis.

Lewis, W. G. (2011). Addysg Ddwyieithog yn yr unfed ganrif ar hugain: Adolygu'r cyddestun rhyngwladol. Gwerddon, Rhif 7, (tt. 66-88). Cyrchwyd 17 Hydref, 2014: http://www.gwerddon.org/en/media/main/gwerddon/rhifynnau/Gwerddon_07(terfynol).pdf

Lightbown, P. M., \& Spada, N. (2006). How languages are learned (3 $3^{\mathrm{ydd}}$ arg.). Rhydychen: Gwasg y Brifysgol. 
Lightbown, P. M., \& Spada, N. (2013). How languages are learned (4 ${ }^{\mathrm{ydd}}$ arg.). Rhydychen: Gwasg y Brifysgol.

Llywodraeth Cymru. (2011). Strategaeth ar gyfer y Gymraeg: Datganiad ar y camau nesaf. Cyrchwyd 6 Mawrth, 2015: http://gov.wales/?lang=cy

Llywodraeth Cymru. (2012a). Cynllun gweithredu Cymraeg ail iaith. Cyrchwyd 10 Hydref, 2014: http://wales.gov.uk/docs/dcells/publications/120711welsh2ndlangcy.pdf.

Llywodraeth Cymru. (2012b). Iaith fyw: Iaith byw: Strategaeth y Gymraeg 2012-2017. Cyrchwyd 12 Ionawr, 2015: http://wales.gov.uk/docs/dcells/publications/122902wls201217cy.pdf.

Llywodraeth Cymru. (2013a). Prydau ysgol am ddim. Cyrchwyd 14 Mai, 2017:

http://gov.wales/topics/educationandskills/schoolshome/foodanddrink/freeschoolmeal s/?skip=1\&lang=cy

Llywodraeth Cymru. (2013b). The cwricwlwm Cymreig, history and the story of Wales. Cyrchwyd 5 Mawrth, 2015:

http://learning.wales.gov.uk/docs/learningwales/news/130424-cwricwlwm-cymreigreport-en.pdf.

Llywodraeth Cymru. (2013, 5 Awst). Y Gynhadledd Fawr - Cyfle i ddweud eich dweud. Cyrchwyd Mai 21, 2015: http://gov.wales/?lang=cy

Llywodraeth Cymru. (2014). Iaith fyw: Iaith byw - bwrw mlaen. Cyrchwyd 9 Mawrth, 2015: http://gov.wales/docs/dcells/publications/140806-living-language-moving-forwardcy.pdf.

Llywodraeth Cymru. (2014, 4 Tachwedd). Prif Weinidog Cymru yn cyhoeddi £1m ychwanegol ar gyfer canolfannau i hybu'r defnydd o'r Gymraeg. Cyrchwyd 17 Mawrth, 2015: http://gov.wales/?lang=cy

Llywodraeth Cymru. (2015a). Arolwg i ganfod y ddarpariaeth ar gyfer y dysgwyr mwy galluog a thalentog drwy Gymru. Cyrchwyd 17 Gorffennaf, 2018: https://gov.wales/docs/dcells/publications/160106-mat-report-cy.pdf

Llywodraeth Cymru. (2015b). Canlyniadau'r cyfrifiad ysgolion, 2015. Datganiad Cyntaf, SDR 110/2015. Cyrchwyd 12 Awst, 2015: http://gov.wales/docs/statistics/2015/150723-school-census-results-2015-cy.pdf. 
Llywodraeth Cymru. (2016a). Fy ysgol leol. Cyrchwyd 23 Ionawr, 2017:

http://mylocalschool.wales.gov.uk/?lang=cy

Llywodraeth Cymru. (2016b). Gwerthusiad o'r Strategaeth Addysg cyfrwng Cymraeg.

Cyrchwyd 10 Ionawr, 2018: http://gov.wales/statistics-and-research/welsh-mediumeducation-strategy/?skip $=1 \&$ lang $=$ cy

Llywodraeth Cymru. (2016c). Strategaeth Addysg Cyfrwng Cymraeg: y camau nesaf. Cyrchwyd 10 Ionawr, 2018: http://gov.wales/docs/dcells/publications/160309-nextsteps-cy.pdf

Llywodraeth Cymru. (2017a). Canlyniadau'r cyfrifiad ysgolion, 2017. Datganiad Cyntaf, SFR 77/2017. Cyrchwyd 10 Ionawr, 2018:

http://gov.wales/docs/statistics/2017/170726-school-census-results-2017-cy.pdf

Llywodraeth Cymru. (2017b). Cymraeg 2050: Miliwn o siaradwyr. Cyrchwyd 10 Gorffennaf, 2017: http://gov.wales/topics/welshlanguage/welsh-language-strategy-andpolicies/cymraeg-2050-welsh-language-strategy/?skip=1\&lang=cy.

Llywodraeth Cymru. (2017c). Ymgynghoriad ar strategaeth ddrafft Llywodraeth Cymru: miliwn o siaradwyr Cymraeg erbyn 2050. Cyrchwyd 10 Ionawr, 2018: https://ymgyngoriadau.llyw.cymru/sites/default/files/consultation_doc_files/170110consultation-responses-cy.pdf

Llywodraeth Cymru. (2017ch). Y Gymraeg mewn addysg: Cynllun gweithredu 2017-2021. Cyrchwyd 20 Rhagfyr, 2017: http://gov.wales/docs/dcells/publications/171212-welshin-education-action-plan-2017-21-cy.pdf.

Llywodraeth Cymru. (2019a). Cwricwlwm Drafft i Gymru 2022: Canllaw i Gwricwlwm i Gymru 2022. Cyrchwyd 21 Mai, 2019: https://s3-eu-west-1.amazonaws.com/hwblive-storage/83/63/1e/da/ae534a18a9bec8c2a8a5be83/canllaw-i-gwricwlwm-i-gymru2022.pdf

Llywodraeth Cymru. (2019b). Cwricwlwm Drafft i Gymru 2022: Ieithoedd, Llythrennedd a Chyfathrebu. Cyrchwyd 21 Mai, 2019: https://s3-eu-west-1.amazonaws.com/hwblive-storage/df/28/8c/ab/27e745a2bba2108191795d16/canllawiau-statudol-drafftieithoedd-llythrennedd-a-chyfathrebu.pdf

Llywodraeth Cynulliad Cymru. (2002). Dyfodol dwyieithog: A bilingual future. Caerdydd: Llywodraeth Cynulliad Cymru. 
Llywodraeth Cynulliad Cymru. (2003). Iaith Pawb: Cynllun gweithredu cenedlaethol ar gyfer Cymru ddwyieithog. Cyrchwyd 9 Rhagfyr, 2014:

http://wales.gov.uk/depc/publications/welshlanguage/iaithpawb/iaithpawbw.pdf?lang $=\mathrm{cy}$.

Llywodraeth Cynulliad Cymru. (2007a). Cymru'n un: Rhaglen flaengar ar gyfer llywodraethu ar gyfer Cymru. Cyrchwyd 13 Ionawr, 2016:

https://www.hefcw.ac.uk/documents/publications/external_reports_and_studies/One_ Wales\%20Cy.pdf.

Llywodraeth Cynulliad Cymru. (2007b). Diffinio ysgolion yn ôl y ddarpariaeth cyfrwng Cymraeg. Cyrchwyd 25 Tachwedd, 2014:

http://wales.gov.uk/dcells/publications/publications/guidanceandinformation/pre2009/ defining-schools-welsh-medi1.pdf?lang=cy.

Llywodraeth Cynulliad Cymru. (2010a). Adroddiad blynyddol Iaith Pawb a'r Cynllun Iaith Gymraeg 2009-10. Cyrchwyd 5 Mawrth, 2015:

http://wales.gov.uk/docs/drah/report/20100713iaithpawbandwlsannualreport200910cym.pdf.

Llywodraeth Cynulliad Cymru. (2010b). Strategaeth Addysg Cyfrwng Cymraeg. Cyrchwyd 9 Ionawr, 2015:

http://wales.gov.uk/docs/dcells/publications/100420welshmediumstrategycy.pdf.

Llywodraeth Gwlad y Basg. (2013a). Fifth sociolinguistic survey. Vitoria-Gasteiz: Servicio Central de Publicaciones del Gobierno Vasco. Cyrchwyd 1 Ebrill, 2015:

http://www.euskara.euskadi.eus/contenidos/informacion/sociolinguistic_research2011 /en_2011/adjuntos/FithSociolingusticSurvey.pdf.

Llywodraeth Gwlad y Basg. (2013b). Talking pupils: The Arrue project 2011. VitoriaGasteiz: Servicio Central de Publicaciones del Gobierno Vasco. Cyrchwyd 6 Tachwedd, 2015: http://www.hezkuntza.ejgv.euskadi.eus/r43573/en/contenidos/informacion/dia6/en_2027/publicaciones_ingles.html.

Loewen, S., \& Reinders, H. (2011). Key concepts in second language acquisition. Efrog Newydd: Palgrave Macmillan.

Long, M. (2004). Acquisition and teaching. Yn M. Byram (Gol.), Routledge encyclopedia of language teaching and learning (tt. 4-5). Llundain; Efrog Newydd: Routledge. 
Lowie, W., \& Seton, B. (2013). Essential statistics for applied linguistics. Basingstoke: Palgrave Macmillan.

Lynch, J. (1987). Prejudice reduction and the schools. Llundain: Cassell Education.

Lyster, R. (2007). Learning and teaching languages through content: A counterbalanced approach. Cyrchwyd 5 Ionawr, 2018: http://site.ebrary.com/lib/swansea/docDetail.action?docID=10172327

Macaro, E. (2010). Second language acquisition: The landscape, the scholarship and the reader. Yn E. Macaro (Gol.), Continuum companion to second language acquisition (tt. 3-28). Cyrchwyd 5 Ionawr, 2018: http://www.ebrary.com/corp/index.jsp

Mackey, A., \& Gass, S. M. (2005). Second language research: Methodology and design. Mahwah; Llundain: Lawrence Erlbaum Associates.

MacNamara, J. (1969). How can one measure the extent of a person's bilingual proficiency? Yn L. G. Kelly (Gol.), Description and measurement of bilingualism (tt. 80-98). Toronto: Gwasg Prifysgol Toronto

Manterola, I., Almgren, M., \& Idiazabal, I. (2013). Basque L2 development in immersion school settings. International Journal of Bilingualism, 17(3), 275-391. doi: $10.1177 / 1367006912438996$

Marian, V., \& Shook, A. (2012). The cognitive benefits of being bilingual. Cyrchwyd 15 Gorffennaf, 2015: http://dana.org/Cerebrum/2012/The_Cognitive_Benefits_of_Being_Bilingual/

Marsh, C. (1982). The survey method: The contribution of surveys to sociological explanation. Llundain: George Allen \& Unwin.

Marsh, D. (Gol.). (2002). CLIL/EMILE the European dimension: Actions, trends and foresight potential. European Commission, DG EAC. Cyrchwyd 18 Mehefin, 2015: http://clilcd.ecml.at/LinkClick.aspx?fileticket=ekwp4udVLfQ\%3D\&tabid=947\&language=enGB

Martin-Jones, M. (2008). Rhagair. Yn J. Osmond (Gol.), Creu Cymru ddwyieithog: Rôl y Gymraeg mewn addysg (tt. 1-2). Caerdydd: Y Sefydliad Materion Cymreig. 
Massler, U., Stotz, D., \& Queisser, C. (2014). Assessment instruments for primary CLIL: The conceptualisation and evaluation of test tasks. The Language Learning Journal, 42, 137-150. doi: dx.doi.org/10.1080/09571736.2014.891371

May, T. (2011). Interviewing: Methods and process. Yn T. May (Gol.), Social research (4 ${ }^{\mathrm{ydd}}$ arg., tt. 131-160). Gwasg Y Brifysgol Agored. Cyrchwyd 22 Medi, 2016 o: https://www.dawsonera.com

McDonough. (2004). Psychology. Yn M. Byram (Gol.), Routledge encyclopedia of learning teaching and learning (tt. 491-498). Llundain; Efrog Newydd: Routledge.

McKamey, T. (2006). Getting closure on cloze: A validation study of the "rational deletion" method. Second Language Studies, 24(2), 114-164. Cyrchwyd 2 Mawrth, 2016: http://www.hawaii.edu/

McLeod, W. (2007). Foghlam tro mheadhan na Gàidhlig anns a' cho-theacsa eadarnàiseanta. Yn M. NicNeacail \& M. Macìomhair (Gol.), Foghlam tro mheadhan na Gàidhlig (tt. 16-38). Cyrchwyd 5 Ionawr, 2018: https://www.academia.edu/1587478/Foghlam_tro_mheadhan_na_G\%C3\%A0idhlig_a nns_a_cho-theacsa_eadar-n\%C3\%A0iseanta.

McLeod, W. (2014). Gaelic in contemporary Scotland: Contradictions, challenges and strategies. Europa Ethnica, 71(1/2), 3-12. Cyrchwyd 2 Medi, 2015: https://edinburgh.academia.edu/WMcLeod

McLuhan, M. \& Powers, B. R. (1989). The global village: Transformations in world life and media in the 21st century. Effrog Newydd; Rhydychen: Gwasg Prifysgol Rhydychen.

Mehisto, P. (2007). What a school needs to consider before launching a CLIL program: The Estonian experience. Yn D. Marsh \& D. Wolff (Gol.), Diverse contexts-converging goals: CLIL in Europe (tt. 61-77). Frankfurt-am-Main: Peter Lang.

Mehisto, P. (2013). Integrating CLIL with other mainstream discourses. Yn S. Breidbach \& B. Viebrock (Gol.), Content and Language Integrated Learning (CLIL) in Europe: Research perspectives on policy and practice (Cyfrol 14, tt. 25-50). Frankfurt am Main: Peter Lang.

Mehisto, P., Marsh, D., \& Frigols, M. J. (2008). Uncovering CLIL. Llundain: Macmillan Education. 
Merikivi, R., \& Pietilä, P. (2014). Vocabulary in CLIL and in mainstream education. Journal of Language Teaching \& Research, 5(3), 487-497. doi: 10.4304/j1tr.5.3.487-497

Merriam, S.B. (1988). Case Study Research in Education. San Francisco: Jossey Bass.

Milk, R. D. (1990). Integrating language and content: Implications for language distribution in bilingual classrooms. Yn R. Jacobson \& C. Faltis (Gol.), Language distribution issues in bilingual schooling. Clevedon: Multilingual Matters.

Milton, J. (2006a). Language Lite? Learning French Vocabulary in School. Journal of French Language Studies, 16, 187-205. doi: 10.1017/S0959269506002420

Milton, J. (2006b). X-Lex: The Swansea vocabulary levels test. Papur a gyflwynwyd yn The Proceedings of the 7th and 8th current trends in English language testing (CTELT) conferences: Volume 4., Dubai, Yr Emiraethau Arabaidd Unedig.

Morris, D. (2010). Young people and their use of the Welsh language. Yn D. Morris (Gol.), Welsh in the twenty-first century (tt. 80-98). Caerdydd: Gwasg Prifysgol Cymru.

Muñoz, C. (2002). Relevance \& potential of CLIL. Yn D. Marsh (Gol.), CLIL/EMILE the European dimension: Actions, trends and foresight potential: European Commission, DG EAC. Cafwyd18 Mehefin, 2015: http://clilcd.ecml.at/LinkClick.aspx ?fileticket=ekwp4udVLfQ\%3D\&tabid=947\&language =enGB

Muñoz, C. (2015). Time and Timing in CLIL: A Comparative Approach to Language Gains. Yn M. Juan-Garau \& J. Salazar-Noguera (Gol.), Content-based Learning in Multilingual Educational Environments, 87-105. Berlin: Springer.

Newcombe, L. P. (2007). Social context and fluency in L2 learners: The case of Wales. Cyrchwyd 17 Rhagfyr, 2015: http://site.ebrary.com/lib/swansea/home.action

Nisbet, J. \& Watt, J. (1984) Case study. Yn J. Bell, T. Bush, A. Fox, J. Goodey \& S. Goulding (Gol.), Conducting Small-scale Investigations in Educational Management (tt. 79-92). Llundain: Harper \& Row.

Ó Duibhir, P. (2009). The spoken Irish of sixth-class pupils in Irish immersion schools, (traethawd PhD). Coleg y Drindod.

Ó Duibhir, P. \& Cummins, J. (2012). Towards an integrated language curriculum in early childhood and primary education (3-12 years). National Council for Curriculum and 
Assessment. Cyrchwyd 12 Ionawr, 2018:

https://www.ncca.ie/media/2467/towards_an_integrated_language_curriculum_in_ear ly_childhood_and_primary_education.pdf

Ó Riagáin, P., Williams, G., \& Moreno, F. X. V. (2008). Young people and minority languages: Language use outside the classroom. Cyrchwyd 29 Mawrth, 2016: http://www.gaelscoileanna.ie/files/Young-People-and-Minority-Languages.pdf

OCR. (2016, 25 Medi). Withdrawal of NVQ Occupational Langauge qualifications. Cyrchwyd 25 Ionawr, 2017: http://www.ocr.org.uk/administration/support-andtools/siu/nvq-occupational-languages-230916/

Oestreicher, J. P. (1974). The early teaching of modern language, education and culture. Review of the Council for Cultural Cooperation of the Council of Europe, 24, tt. 9-16.

O'Grady, W., Dobrovolsky, M., \& Katamba, F. (1996). Contemporary linguistics: An introduction. Llundain: Longman.

O'Hanlon, F. (2012). Lost in transition? Celtic language revitalization in Scotland and Wales: The primary to secondary school stage, (traethawd PhD). Prifysgol Caeredin.

Oksaar, E. (1983). Multilingualism and multiculturalism from the linguist's point of view. In Husén \& Opper (Gol.), Multicultural and Multilingual Education in Immigrant Countries (Cyfrol 38, tt. 17-36). Rhydychen: Gwasg Pergamon.

Oldbell3 \& Dateb. (2008). Evaluation of the immersion and intensive language teaching pilot projects - Final evaluation report. Caerdydd: Ymchwil Gymdeithasol Llywodraeth Cymru. Cyrchwyd 30 Medi, 2015: http://gov.wales/statistics-and-research/evaluationimmersion-intensive-language-teaching-pilot-projects/?skip=1\&lang=cy.

Oller, J. W. J. (1972). Scoring methods and difficulty levels for Cloze tests of proficiency in English as a second language. The Modern Language Journal, 56(3), 151-158. Cyrchwyd 19 Ionawr, 2016: http://www.jstor.org/stable/324037

Oller, J. W. J., \& Conrad, C. A. (1971). The cloze technique and ESL proficiency. Language Learning, 21(2), 183-195. doi:10.1111/j.1467-1770.1971.tb00057.x

Osmond, J. (Gol.). (2008). Creu Cymru ddwyieithog: Rôl y Gymraeg mewn addysg. Caerdydd: Y Sefyliad Materion Cymreig 
Ouazizi, K. (2016). The effects of CLIL education on the subject matter (Mathematics) and the target language (English). LACLIL, 9(1), 110-137. doi: 10.5294/lacli1.2016.9.1.5

Owen, H. D. (2015). Yr iaith Gymraeg yn y gwasanaethau gofal iechyd a chymdeithasol, (traethawd PhD). Prifysgol Abertawe.

Owen, H. D., \& Morris, S. (2012). Effaith iaith ar adsefydlu corfforol: Astudiaeth o ddylanwad iaith ar effeithiolrwydd therapi mewn cymuned Gymraeg, Gwerddon, 10/11, Gorfennaf, 83-112. Cyrchwyd 4 Mawrth, 2018: http://www.gwerddon.cymru/en/media/main/gwerddon/rhifynnau/erthyglau/Rhifyn10 _e4.pdf

Owen, K. (2015, 10 Gorffennaf). Twf y Gymraeg yn ddibynnol ar dwf addysg Gymraeg., $Y$ Cymro, t. flaen.

Owens, M. (1992). The acquisition of Irish: A case study. Clevedon, Lloegr: Multilingual Matters.

Ozfidan, B. (2014). The Basque bilingual education system: A model for a Kurdish bilingual education system in Turkey. Journal of Language Teaching and Research, 5 (2), 382390. doi: 10.4304/jltr.5.2.382-390

Paran, A. (2013). Content and language integrated learning: Panacea or policy borrowing myth? Applied Linguistics Review, 4, 317-342. doi: 10.1515/applirev-2013-0014

Park, Chung-Yeol. (2011). Making Cloze Tests More Valid. Journal of the Korea AcademiaIndustrial cooperation Society, 12 (2), 640-645. doi: 10.5762/KAIS.2011.12.2.640.

Parliment UK. (d.d.). The Education Act of 1944. Living heritage: Going to school. Cyrchwyd 13 Mawrth, 2015: http://www.parliament.uk/about/livingheritage/transformingsociety/livinglearning/school/overview/educationact1944/

Parry, J. (2013, 19 Tachwedd). Addysg cyfrwng Cymraeg i bawb - Allwedd i dwf yr iaith. Cyrchwyd 15 Ionawr, 2015: http://cymdeithas.org/blog/addysg-cyfrwng-cymraeg-ibawb-allwedd-i-dwf-yr-iaith

Patton, M. Q. (1980). Qualitative evaluation methods. Califfornia: Sage Publications.

Pearson, B. Z., Fernandez, S. C., \& Oller, D. K. (1993). Lexical development in bilingual infants and toddlers: Comparison to monolingual norms. Language Learning, 43(1), 93-120. Cyrchwyd 30 Awst, 2018: https://scholarworks.umass.edu/adjunct_sw/7/ 
Pérez-Cañado, M. L. (2012). CLIL research in Europe: Past, present, and future.

International Journal of Bilingual Education and Bilingualism, 15(3), 315-341. doi: 10.1080/13670050.2011.630064

Pérez-Cañado, M. L. (2016). From the CLIL craze to the CLIL conundrum: Addressing the current CLIL controversy. Bellaterra Journal of Teaching \& Learning Language \& Literature, 9(1), 9-31. doi: http://dx.doi.org/10.5565/rev/jt13.667

Pérez-Cañado, M. L. (2018a). The effects of CLIL on L1 and content learning: Updated empirical evidence from monolingual contexts. Learning and Instruction. doi: 10.1016/j.learninstruc.2017.12.002.

Pérez-Cañado, M. L. (2018b). CLIL and educational level: A longitudinal study on the impact of CLIL on language outcomes. Porta Linguarum, 29, 51-70. Cyrchwyd 1 Awst, 2018:

http://www.ugr.es/ portalin/articulos/PL_numero29/3_MARIA\%20LUISA\%20PERE Z.pdf

Pérez-Cañado, M. L. \& Lancaster, N. K. (2017). The effects of CLIL on oral comprehension and production: A longitudinal case study. Language, Culture and Curriculum, 30(3), 300-316. doi: 10.1080/07908318.2017.1338717

Philpott, A. (2011). Blended learning in higher education. The Journal of The College of Foreign Languages 24, 39-54.

Pike, T. \& Young-Scholten, M. (Gol.). (2009). Input matters in SLA. Bryste: Multilingual Matters.

Pladevall-Ballester, E. \& Vallbona, A. (2016). CLIL in minimal input contexts: A longitudinal study of primary school learners' receptive skills. System, 58, 37-48. doi: http://dx.doi.org/10.1016/j.system.2016.02.009

Pritchard, J., \& Butt, G. (2009). Cwricwlwm cenedlaethol Cymru. Yn S. Capel, M. Leask \& T. Turner (Gol.), Learning to teach in the secondary school: A companion to school experience ( $5^{\mathrm{ed}}$ arg.). Llundain: Routledge.

Ransdell, S. E., \& Fischler, I. (1987). Memory in a monolingual mode: When are bilinguals at disadvantage? Journal of Memory and Language, 26(4), 392-405. Cyrchwyd 8 Medi, 2016: http://search.proquest.com/docview/1297346683?accountid=14680 
Reardon, S. F. (2011). The widening academic achievement gap between the rich and the poor: New evidence and possible explanations. Yn Duncan, G. J., \& Murnane, R. J. (Gol.), Whither opportunity? Rising inequality, schools, and children's life chances. Cyrchwyd 14 Mai, 2019: https://ebookcentral.proquest.com

Rhieni dros Addysg Gymraeg. (2015a). Adroddiad blynyddol 2013 - 2014. Cyrchwyd 20 Awst, 2015: http://www.rhag.net/dogfennau/Adroddiad\%20Blynyddol\%20201314.pdf.

Rhieni dros Addysg Gymraeg. (2015b). Angen gweledigaeth addysg Gymraeg: Ymateb $i$ adroddiad Donaldson. Cyrchwyd 18 Mai, 2015: http://www.rhag.net/stori.php?iaith=cym\&id=167

Rhys, M. (2013). Sgiliau iaith a gwybyddiaeth plant dwyieithog mewn cyd-destun iaith leiafrifol. (Traethawd PhD). Bangor: Prifysgol Bangor.

Rhys, M., \& Thomas, E. M. (2013). Bilingual Welsh-English children's acquisition of vocabulary and reading: Implications for bilingual education. International Journal of Bilingual Education and Bilingualism, 16(6), 633-656. doi: $10.1080 / 13670050.2012 .706248$

Ricciardelli, L. A. (1992). Creativity and bilingualism. Journal of Creative Behaviour, 26(4), 242-254.

Richards, J. C., \& Rodgers, T. S. (2001). Approaches and methods in language teaching (2 $2^{\text {ail }}$ arg.). Caergrawnt: Gwasg Prifysgol Caergrawnt.

Roberts, G. W. (2010, 25 Mawrth). Cyflwyniad i Bwyllgor Adrodd ar Ddwyieithrwydd LlCC. Tystiolaeth ysgrifenedig a ddarparwyd i Gynulliad Cenedlaethol Cymru. Cyrchwyd 15 Mehefin, 2015: http://www.cynulliad.cymru/NAfW\%20Documents/paper_1__gareth_wyn_roberts-e.pdf\%20-\%2002092010/paper_1_-_gareth_wyn_roberts-eCymraeg.pdf.

Roberts, G., \& Williams, C. (Gol.). (2003). Addysg Gymraeg, addysg Gymreig. Bangor: Ysgol Addysg, Prifysgol Cymru.

Romaine, S. (1995). Bilingualism (2 $2^{\text {ail }}$ arg.). Rhydychen: Blackwell Publishers.

Romaine, S. (2000). Language in society: An introduction to sociolinguistics. Cyrchwyd 8 Mehefin, 2015: http://www.ebrary.com 
Roy, S. (2008). French immersion studies: From Second-Language Acquisition (SLA) to social issues. The Alberta Journal of Educational Research, 54(4), 396-406. Cyrchwyd 29 Hydref, 2015: http://ajer.synergiesprairies.ca/index.php/ajer/index

Roy, S., \& Albert, G. (2011). Discourses on Bilingualism in Canadian French Immersion Programs. The Canadian Modern Language Review / La Revue canadienne des langues vivantes, 351-376. doi: 10.3138/cmlr.67.3.351

Rumlich, D. (2013). Students' general English proficiency prior to CLIL: Empirical evidence for substantial differences between prospective CLIL and non-CLIL students in Germany. Yn S. Breidbach \& B. Viebrock (Gol.), Content and Language Integrated Learning (CLIL) in Europe : Research perspectives on policy and practice (Cyfrol 14, tt. 181-201). Frankfurt am Main: Peter Lang.

Rumlich, D. (2014). Prospective CLIL and non-CLIL students' interest in English (classes): A quasi-experimental study on German sixth-graders. Yn R. Breeze, C. L. Saíz, C. M. Pasamar \& C. T. Sala (Gol.), Integration of theory and practice in CLIL (Cyfrol 28, Utrecht Studies in Language and Communication, tt. 75-96). Amsterdam: Rodopi.

Rutter, M., Maughan, B., Mortimore, P., Ouston, J., \& Smith, A. (1979). Fifteen thousand hours: Secondary schools and their effects on children. Caergrawnt, Mass.: Gwasg Prifysgol Harvard.

Sachs, J., Tung, P., \& Lam, R. Y. H. (1997). How to construct a Cloze test: Lessons from testing measurement theory models. Perspectives, 9, 145-160. Cyrchwyd 12 Medi, 2015:

http://www.ibrarian.net/navon/paper/How_to_construct_a_cloze_test_Lessons_from _testi.pdf?paperid=2160136

Safont, M. P. (2005). Third language learners: Pragmatic production and awareness.

Clevedon: Multilingual Matters. Cyrchwyd 16 Tachwedd, 2015: http://site.ebrary.com/lib/swansea/home.action?force=1

Sanz, C. (2000). Bilingual education enhances third language acquisition: Evidence from Catalonia. Applied Psycholinguistics, 21(1), 23-44. Cyrchwyd 24 Gorffennaf, 2015: http://journals.cambridge.org. 
Sasaki, M. (2000). Effects of cultural schemata on students' test-taking processes for cloze tests: A multiple data source approach. Language Testing, 17(1), 85-114. Cyrchwyd 8 Mawrth, 2016: http://www.proquest.com/

Saville-Troike, M. (2006). Introducing second language acquisition. Caergrawnt: Gwasg Prifysgol Caergrawnt. Cyrchwyd 5 Ionawr, 2018: http://www.dawsonera.com

Sayers, D., \& Selleck, C. (2015). Blindspots in policy and attrition in practice? Possible unintended consquences of Welsh language policy. Papur a gyflwynwyd yn the WISERD Annual Conference 2015, Caerdydd. Cyrchwyd 21 Medi, 2016: https://shu.academia.edu/DaveSayers/talks

Scott, D., \& Beadle, S. (2014). Improving the effectiveness of language learning: CLIL and computer assisted language learning. European Commission. Cyrchwyd 29 Hydref, 2015: http://ec.europa.eu/index_en.htm.

Selinker, L. (1972) 'Interlanguage', International Review of Applied Linguistics in Language Teaching, 10 (3), tt. 209-231. doi: https://doi.org/10.1515/iral.1972.10.1-4.209

Selleck, C. L. R. (2012). A comparative ethnographic study of students' experiences and perceptions of language ideologies in bilingual Welsh/English education: Inclusive policy and exclusionary practice, (traethawd $\mathrm{PhD}$ ). Prifysgol Caerdydd.

Selleck, C. L. R. (2013). Inclusive policy and exclusionary practice in secondary education in Wales. International Journal of Bilingual Education and Bilingualism, 16(1), 20-41. doi: 10.1080/13670050.2012.676622

Shanahan, T., Kamil, M. L., \& Tobin, A. W. (1982). Cloze as a measure of intersentential comprehension. Reading Research Quarterly, 17(2), 229-255. Cyrchwyd 26 Chwefror, 2016: http://www.jstor.org/stable/747485

Sierra, J. (1996). Los modelos de enseñanza bilingüe y el rendimiento escolar en educación primaria. Papur a gyflwynwyd yn The Third European Conference on Immersion Programs, Medi, 1996.

Sierra, J., \& Olaziregi, I. (1989). E.I.F.E. 2 Influence of factors on the learning of Basque. Cyrchwyd 16 Medi, 2015: http://www.hezkuntza.ejgv.euskadi.eus/r43573/en/contenidos/informacion/dia6/en_2027/adjuntos/publications_in_english/EIFE_ 2_en.pdf 
Sierra, J., \& Olaziregi, I. (1991). E.I.F.E 3 Influence of factors on the learning of Basque: Study of the models A, B and D in second year Basic General Education. Cyrchwyd 16 Medi, 2015: http://www.hezkuntza.ejgv.euskadi.eus/r43573/en/contenidos/informacion/dia6/en_2027/adjuntos/publications_in_english/EIFE_ 3_en.pdf

sign. (d.d.). Yn Oxford Dictionaries Online. Cyrchwyd 6 Chwefror, 2017: https://en.oxforddictionaries.com/definition/sign

Silverman, D. (200). Doing qualitative research: A practical handbook. Llundain: Sage Publications.

Simonton, D. K. (2008). Bilingualism and creativity. Yn J. Altarriba \& R. R. Heredia (Gol.), An Introduction to bilingualism: Principles and processes. Mahwah, NJ: Lawrence Erlbaum Associates.

Skutnabb-Kangas, T., \& McCarty, T. L. (2008). Key concepts in bilingual education: Ideological, historical, epistemological, and empirical foundations. Yn J. Cummins \& N. H. Hornberger (Gol.), Encyclopedia of language and education (2 $2^{\text {ail }}$ arg., Cyfrol 5: Bilingual education, tt. 3-17). Efrog Newydd: Springer.

Solvason, C. (2005). Investigating specialist school ethos ... or do you mean culture? Educational Studies, 31(1), 85-94. doi: 10.1080/0305569042000310985

Somers, T. (2017). Content and Language Integrated Learning and the inclusion of immigrant minority language students: A research review. International Review of Education, 63(4), 495-520. doi: https://doi.org/10.1007/s11159-017-9651-4

Somers, T. (2018a). CLIL and the quality of its criticism: Beyond a reply to Bruton (2015). Papur sydd wrthi'n cael ei baratoi.

Somers, T. (2018b). Content and Language Integrated Learning: A new direction for the present and future of Welsh language teaching? Papur a gyflwynwyd yn Y Gymraeg mewn Addysg cyfrwng Saesneg, Neuadd Gregynog.

Somers, T. \& Surmont, J. (2012). CLIL and immersion: how clear-cut are they? ELT Journal, 66(1), 113-116. doi:10.1093/elt/ccr079

Stake, R. E. (1995). The art of case study research. Llundain: Sage Publications. 
Stake, R. E. (2005). Qualitative case studies. Yn N. Denzin, \& Y. Lincoln (Gol.), The SAGE handbook of qualitative research, (3 ${ }^{\text {ydd }}$ arg.). Llundain: Sage Publications.

Statistics Canada. (2013, 17 Ionawr). Summary elmentary and secondary school indicators for Canada, the provinces and territories, 2006/2007 to 2010/2011, The Daily. Cyrchwyd 30 Hydref, 2015: http://www.statcan.gc.ca/start-debut-eng.html

Statistics Canada. (2015). Table 051-0001 - Estimates of population, by age group and sex for July 1, Canada, provinces and territories, annual (persons unless otherwise noted). [Set ddata]. Cyrchwyd 29 Hydref, 2015:

http://www5.statcan.gc.ca/cansim/a26?lang=eng\&retrLang=eng\&id=0510001\&\&patt ern $=\&$ stByVal=1\&p1=1\&p2=37\&tabMode=dataTable \&csid $=$

Stats Cymru. (2013). Siaradwyr Cymraeg yn ôl adrannau etholiadol, Cyfrifiad 2011. [Tabl ddata]. Llywodraeth Cymru. Cyrchwyd 23 Ionawr, 2017: https://statscymru.llyw.cymru/Catalogue/Welsh-Language/WelshSpeakers-by-Ediv2011Census

Stats Cymru. (2017a). Siarad Cymraeg gartref, asesiad rhieni, gan ddisgyblion o oed 5 neu drosodd mewn ysgolion cynradd, canol ac uwchradd yn ôl blwyddyn, sector a chategori. Llywodraeth Cymru. Cyrchwyd 29 Mai, 2018:

https://statscymru.cymru.gov.uk/Catalogue/Education-and-Skills/Schools-andTeachers/Schools-Census/Pupil-Level-Annual-School-Census/WelshLanguage/speakingwelshhomepupils5andover-by-year-sector-category

Stats Cymru. (2017b). Ysgolion yn ôl awdurdod lleol, rhanbarth a math o gyfrwng Cymraeg. SCHSO257. [Set ddata]. Cyrchwyd 21 Gorffennaf, 2017: https://statscymru.llyw.cymru/Catalogue/Education-and-Skills/Schools-andTeachers/Schools-Census/Pupil-Level-Annual-School-Census/WelshLanguage/Schools-by-LocalAuthorityRegion-WelshMediumType

Stats Cymru. (d.d.). Cyfnod allweddol 2. Cyrchwyd Mehefin 17, 2015: https://statscymru.cymru.gov.uk/Catalogue/Education-and-Skills/Schools-andTeachers/Examinations-and-Assessments/Key-Stage-2

Stenhouse, L. (1985), Case study methods. Yn T. Husen and T. N. Postlethwaite (Gol.), International Encyclopaedia of Education (tt. 640-6). Rhydychen: Pergamon. 
Stoller, F. (2008). Content-based instruction. Yn N. Van Deusen-Scholl \& N. H. Hornberger (Gol.), Encyclopedia of language and education. Vol. 4: Second and foreign language education (tt. 59-70). Efrog Newydd: Springer.

Stubbs, J. B., \& Tucker, G. R. (1974). The cloze test as a measure of English proficiency. The Modern Language Journal, 58(5/6), 239-241. Cyrchwyd 1 Mawrth, 2016: http://www.jstor.org/stable/325020

Stuijt, M., \& Sanchez, D. (2007). Basque: The Basque language in education in France. Leeuwarden: Mercator Education. Cyrchwyd 14 Tachwedd, 2015: http://www.mercatorresearch.eu/fileadmin/mercator/dossiers_pdf/basque_in_france2nd.pdf.

Surmont, J., Struys, E., van den Noort, M., van de Craen, Piet. (2016). The effects of CLIL on mathematical content learning: A longitudinal study. Studies in Second Language Learning and Teaching, 6(2), 319-337. doi: 10.14746/ssllt.2016.6.2.7

Sutton, C. (2011). Social surveys: Design to analysis. Yn T. May (Gol.), Social research (4ydd arg., tt. 93-130): Gwasg Y Brifysgol Agored. Cyrchwyd 5 Ionawr, 2018: https://www.dawsonera.com.

Swain, M. (1972). Bilingualism as a first language. (traethawd PhD heb ei gyhoeddi). Prifysgol California, Irvine.

Swain, M., \& Johnson, R. K. (1997). Immersion education: A category within bilingual education. Yn R. K. Johnson \& M. Swain (Gol.), Immersion education: International perspectives. Caergrawnt: Gwasg Prifysgol Caergrawnt.

Swain, M., \& Lapkin, S. (1982). Evaluating bilingual education: A Canadian case study. Clevedon: Multilingual Matters.

Swain, M., \& Lapkin, S. (2005). The evolving sociopolitical context of immersion education in Canada: Some implications for program development. International Journal of Applied Linguistics, 15(2), 169-186. doi: 10.1111/j.1473-4192.2005.00086.x

Sylven, L. K. (2010). Teaching in English or English teaching? On the effects of content and language integrated learning on Swedish learners' incidental vocabulary acquistion. Gothenburg Studies in English, 97, 1-294.

Takakuwa, M. (2005). Lessons from a Paradoxical Hypothesis: A Methodological Critique of the Threshold Hypothesis. ISB4: Proceedings of the 4th International Symposium on 
Bilingualism, gol. James Cohen, Kara T. McAlister, Kellie Rolstad \& Jeff MacSwan, 2222-2232. Somerville, MA: Cascadilla Press. Cyrchwyd 27 Ebrill, 2018: http://www.lingref.com/isb/4/173ISB4.PDF

Talfryn, I. (2001). Dulliau dysgu ail iaith: Eu hanes a'u datblygiad. Dinbych: Popeth Cymraeg.

Taylor, W. L. (1953). Cloze procedure: A new tool for measuring readability. Journalism Quarterly, 30, 414-433.

Thaler, R. H., \& Sunstein, C. R. (2008). Nudge: Improving decisions about health, wealth and happiness. New Haven; Llundain: Gwasg Prifysgol Yale.

Thomas, E. M., \& Mennen, I. (Gol.). (2014). Advances in the study of bilingualism. Bryste: Multilingual Matters.

Thomas, E. M. \& Webb-Davies, P. (2017). Agweddau ar Ddwyieithrwydd. Caerfyrddin: Y Coleg Cymraeg Cenedlaethol.

Thomas, E. M., Williams, N., Jones, L. 1. A., Davies, S., \& Binks, H. (2013). Acquiring complex structures under minority language conditions: bilingual acquisition of plural morphology in Welsh. Bilingualism: Language and Cognition 17 (3), 478-494. doi: $10.1017 / \mathrm{S} 1366728913000497$

Thompson, L. (2004). Mother tongue. Yn M. Byram (Gol.), Routledge encyclopedia of language teaching and learning (tt.418-420). Llundain; Effrog Newydd: Routledge.

Torrington, D., \& Weightman, J. (1989). The reality of school management. Rhydychen: Basil Blackwell.

Toukomaa, P. \& Skutnabb-Kangas, T. (1977). The intensive teaching of the mother tongue to migrant children at pre-school age (Adroddiad Ymchwil Rhif 26). Adran Sosioleg a Seicoleg Cymdeithasol, Prifysgol Tampere.

Tremblay, A. (2011). Proficiency assessment standards in second language acquisition research: "Clozing" the gap. Studies in Second Language Acquisition, 33, 339-372. doi: $10.1017 / \mathrm{S} 0272263111000015$

Tremblay, A., \& Garrison, M. D. (2010). Cloze tests: A tool for proficiency assessment in research on L2 French. Yn M. T. Prior, Y. Watanabe \& S.-K. Lee (Gol.), Selected 
proceedings of the 2008 Second Language Research Forum: Exploring SLA perspectives, positions, and practices (tt. 73-88). Somerville, MA: Cascadilla Proceedings Project. Cyrchwyd 8 Rhagfyr, 2015: http://www.lingref.com/.

Tucker, G. R., \& d'Anglejan, A. (1972). An approach to bilingual education: The St Lambert experiment. Yn M. Swain (Gol.), Bilingual schooling: Some experiences in Canada and the United States. Ontario: Ontario Institute for Studies in Education Symposium Series No. 1 (Mawrth, 1971).

Turner, D. W. (2010). Qualitative interview design: A practical guide for novice investigators. The Qualitative Report, 15(3), 754-760.

Ulusoy, M. (2008). Evaluating sixth graders' reading levels with different cloze test formats. Edgitim Arastirmalari-Eurasian Journal of Educational Research, 31, 115-133. Cyrchwyd 20 Ebrill, 2017: https://www.academia.edu/32072539/Evaluating_Sixth_Graders_Reading_Levels_wit h_Different_Cloze_Test_Formats

Uned Datblygu'r Gymraeg. (2010, 27 Mai). Welsh second language. Tystiolaeth ysgrifenedig a ddarparwyd i Gynulliad Cenedlaethol Cymru. Cyrchwyd 27 Ebrill, 2016: www.cynulliadcymru.org/paper_1_-_wldu.pdf.

Valdés, G. (2003). Expanding definitions of giftedness: The case of young interpreters from immigrant communities. Mahwah, N.J.: Lawrence Erlbaum Associates.

Van de Craen, P., Ceuleers, E., \& Mondt, K. (2007). Cognitive development and bilingualism in primary schools: Teaching maths in a CLIL environment. Yn D. Marsh \& D. Wolff (Gol.), Diverse contexts-converging goals: CLIL in Europe (tt. 185-200). Efrog Newydd: Peter Lang.

Van de Craen, P., Mondt, K., Allain, L., \& Ying, G. (2007). Why and how CLIL works. An outline for a CLIL theory. Vienna English Working Papers. CLIL Special Issue 2. 16(3), 70-78. Cyrchwyd 31 Awst, 2017: https://www.researchgate.net/publication/290828891_Why_and_how_CLIL_works_ An_outline_for_a_CLIL_theory

VanPatten, B., \& Williams, J. (2007). Theories in second language acquisition: An introduction. Mahwah, N.J.: Lawrence Erlbaum Associates. 
Walliman, N. (2006). Data collection methods. Yn N. Walliman (Gol.), Social research methods (tt. 83-100). Llundain: SAGE Publications Ltd. doi: http://dx.doi.org/10.4135/9781849209939

Walsh, J., O'Rourke, B., \& Rowland, H. (2015). Research report on new speakers of Irish. Oireachtas na Samhna, Dulyn: Foras na Gaeilge. Cyrchwyd 17 Rhagfyr, 2015: http://www.gaeilge.ie/?lang=en.

Warren, C. A. B. (2001). Qualitive interviewing. Yn J. F. Gubrium \& J. A. Holstein (Gol.), Handbook of interview research (tt. 83-102). Thousand Oaks, California: Sage Publications. doi: http://dx.doi.org/10.4135/9781412973588.n7

Weinreich, U. (1968). Languages in contact: Findings and problems. Yr Hag: Mouton Publishers.

Wells, F. G. H. (2000). Addysg Gymraeg: Achubydd yr iaith? Y Traethodydd, Cyf. 155, tt. 93-105. Cyrchwyd 8 Ionawr, 2018 o: https://journals.library.wales/browse

Welsh Government. (2016). Reading and numeracy tests in Wales - 2016: Information for parents and carers of children in Years 2 to 9. Cyrchwyd 5 Ebrill, 2017: http://learning.gov.wales/docs/learningwales/publications/140420-information-forparents-carers-2-9-en-v2.pdf.

Wesche, M. B. (1993). French immersion graduates at university and beyond: What difference has it made? In J. A. Alatis (Gol.), The Georgetown Roundtable on Languages and Linguistics 1992. Washington, DC: Gwasg Prifysgol Georgetown.

Wesche, M. B. (2002). Early French immersion: How has the original Canadian model stood the test of time? Papur a gyflwynwyd yn An Integrated View of Language Development. Papers in Honor of Henning Wode. Wissenschaftlicher Verlag Trier. Cyrchwyd 24 Awst, 2017: http://www.fmks-online.de/_wd_showdoc.php?pic=865

Whittaker, R., \& Llinares, A. (2011). Written discourse development in CLIL at secondary school. Language Teaching Research, 15(3), 343-362. doi: $10.1177 / 1362168811401154$

Williams, C. (1994). Arfarniad o ddulliau dysgu ac addysgu yng nghyd-destun addysg uwchradd ddwyieithog, (traethawd $\mathrm{PhD}$ heb ei gyhoeddi). Prifysgol Cymru, Bangor.

Williams, C. (1999). Cymraeg clir. Cyrchwyd 19 Ionawr, 2016: https://www.bangor.ac.uk/canolfanbedwyr/index.php.cy 
Williams, C. (2002). Ennill iaith: Astudiaeth o sefyllfa drochi yn 11-16 oed. Yn W. G. Lewis \& H. G. F. Roberts (Gol.), Trafodion Addysg. Bangor: Ysgol Addysg, Prifysgol Cymru Bangor. Cyrchwyd 1 Rhagfyr, 2014: http://www.bangor.ac.uk/addysg/publications/Ennill_Iaith.pdf

Williams, C. (2008). Trochi ieithyddol cyfnod canolig a hwyr: Modelau sefydledig a'r rhai sy’n ymddangos yng Nghymru. Yn J. Osmond (Gol.), Creu Cymru ddwyieithog: Rôl y Gymraeg mewn addysg (tt. 19-31). Caerdydd: Y Sefydliad Materion Cymreig.

Williams, C. (d.d.). Trothwy iaith bynciol: Cyflymu'r broses o'i gyrraedd. Cyrchwyd 23 Hydref, 2015: http://www.cbac.co.uk/?language_id=2

Williams, C. H. (2000). Language revitalization: Policy and planning in Wales. Caerdydd: Gwasg Prifysgol Caerdydd.

Williams, C. H. (2005). Iaith Pawb: The doctrine of plenary inclusion. Contemporary Wales, 17(1), tt. 1-27. Cyrchwyd 8 Medi, 2016: http://www.ingentaconnect.com

Williams, C. H. (2007). Deddfwriaeth Newydd a'r Gymraeg. Contemporary Wales, 19(1), 217-233. Cyrchwyd 8 Ionawr, 2018: http://www.ingentaconnect.com

Williams, C. H. (Awst 2013). Language strategies in comparative perspective. The Network to Promote Linguistic Diversity. Cyrchwyd 6 Mawrth, 2015: http://www.npld.eu/uploads/publications/139.pdf.

Williams, C. H. (2014). The lightening veil: Language revitalization in Wales. Review of Research in Education, 38(1), 242-272. doi: 10.3102/0091732X13512983

Williams, T. (2013). When teaching Welsh is a futile experiment. Cyrchwyd 15 Ionawr, 2015: http://www.clickonwales.org/2013/10/when-teaching-welsh-is-a-futile-experiment/

Wharton Dual Language Academy. (d.d.). Mission statement / Declaración de principios. Cyrchwyd 5 Mawrth, 2018: www.houstonisd.org/Page/159229

Wode, H. (1999). Language learning in European immersion classes. Yn J. Masih (Gol.), Learning through a foreign language: Models, methods and outcomes (tt. 16-29). Llundain: CILT.

Wolff, D. (2007). Germany. Yn A. Maljers, D. Marsh \& D. Wolff (Gol.), Windows on CLIL: Content and language integrated learning in the European spotlight. Graz, Awstria: 
European Centre for Modern Languages. Cyrchwyd 18 Tachwedd, 2015: http://archive.ecml.at/mtp2/CLILmatrix/EN/CLIL_windows_EN.htm

Wolff, D. (d.d.). Content and language integrated learning: An evaluation of the German approach. Cyrchwyd 16 Tachwedd, 2016:

http://share.dschola.it/dd4pinerolo/clil/Shared\%20Documents/Forms/AllItems.aspx?R ootFolder $=\% 2 \mathrm{fdd} 4$ pinerolo $\% 2 \mathrm{fclil} \% 2 \mathrm{fShared} \% 20$ Documents $\% 2 \mathrm{fTheory} \_s t r a t e g i e s \&$ View $=\{C C 2806 E 9-A 6 F B-4975-8834-B B 10 B D 9 A C 604\}$

Yamashita, J. (2003). Process of taking a gap-filling test: Comparison of skilled and less skilled EFL readers. Language Testing, 20(3), 267-293. doi:http://dx.doi.org/10.1191/02655322031t257oa

Yamashita, S. O. (1994). Is the reading comprehension performance of learners of Japanese as a second language the same as that of Japanese children? An analysis using a cloze test. Japanese Language Education around the Globe, 4, 133-146. Cyrchwyd 9 Mawrth, 2016, o : http://dl.ndl.go.jp/info:ndljp/pid/8220977

Yin, R. K. (1984). Case Study Research: Design and Methods. Beverly Hills: Sage Publications.

Yin, R. K. (2006). 'Case Study Methods', Yn Green, J., Camilli, G. \& Elmore, P. (Gol.), Handbook of Complementary Methods in Educational Research, Llundain: Lawrence Erlbaum Publishers.

Yow, W. Q., \& Markman, E. M. (2011). Young bilingual children's heightened sensitivity to referential cues. Journal of Cognition and Development, 12(1), 12-31. doi: $10.1080 / 15248372.2011 .539524$

Y Ganolfan Gynllunio Iaith. (2013). Iaith fyw: dweud eich dweud - Canlyniadau'r ymarferiadau ymgynghori. Cyrchwyd 21 Mai, 2015: http://gov.wales/docs/dcells/publications/131022-a-living-language-have-your-saycy.pdf.

Y Pwyllgor Diwylliant a'r Pwyllgor Addysg a Dysgu Gydol Oes. (2002). Ein Hiaith: Ei dyfodol - Adolygiad polisi o'r iaith Gymraeg. Cynulliad Cenedlaethol Cymru. Cyrchwyd 5 Mehefin, 2015: http://www.cynulliad.cymru/Laid\%20Documents/Culture\%20Committee\%20and\%20 Education\%20and\%20Lifelong\%20Learning\%20Committee\%20Policy\%20Review\% 
20of\%20the\%20Welsh\%20Language\%20-\%20'Our\%20Lang-01072002-24744/busGUIDE-N0000000000000000000000000001076-Cymraeg.pdf.

Y Pwyllgor Diwylliant, y Gymraeg a Chyfathrebu. (2017). Gwireddu'r uchelgais:

Ymchwiliad i strategaeth Gymraeg newydd Llywodraeth Cymru. Cynulliad

Cenedlaethol Cymru. Cyrchwyd 19 Mai, 2017: http://www.assembly.wales/cy/bushome/committees/Pages/Committee-Profile.aspx?cid=445.

Y Pwyllgor Menter a Dysgu. (2010). Dysgu ac addysgu Cymraeg fel ail iaith. Cynulliad Cenedlaethol Cymru. Cyrchwyd 10 Hydref, 2014:

http://www.cynulliadcymru.org/Laid\%20Documents/CR-LD8235\%20\%20Report\%20of\%20the\%20Enterprise\%20and\%20Learning\%20Committee\%20on $\% 20$ the $\% 20$ Teaching\%20and\%20Acquisition\%20of\%20Welsh\%20as\%20a\%20Seco n-30092010-197739/cr-ld8235-e-Cymraeg.pdf.

Ystadegau'r Boblogaeth a'r Cyfrifiad. (2012). Siaradwyr Cymraeg fesul awdurdod lleol a grwpiau oedran ehangach, Cyfrifiad 2001 a 2011. [Set ddata]. Llywodraeth Cymru. Cyrchwyd 16 Mehefin, 2016: https://statscymru.cymru.gov.uk/Catalogue/WelshLanguage/WelshSpeakers-by-LA-BroaderAge-2001And2011Census

Y Swyddfa Ystadegau Gwladol. (2012a). 2011 Census: Welsh language skills by age and sex, local authorities in Wales. [Set ddata]. Cyrchwyd 2 Mehefin, 2015: http://www.ons.gov.uk

Y Swyddfa Ystadegau Gwladol. (2012b). Cyfrifiad 2011: Proffil iaith Gymraeg, awdurdodau unedol yng Nghymru. [Set ddata]. Cyrchwyd 19 Chwefror, 2016: http://www.ons.gov.uk/

Y Swyddfa Ystadegau Gwladol. (2012c). Cyfrifiad 2011: Ystadegau allweddol ar gyfer Cymru, Mawrth 2011. Cyrchwyd 16 Ionawr, 2015: http://www.ons.gov.uk/ons/dcp171778_291480.pdf.

Y Swyddfa Ystadegau Gwladol. (2012ch). KS207WA Sgiliau iaith Gymraeg, 2011. [Set ddata]. Cyrchwyd 8 Ionawr, 2018: https://www.ons.gov.uk/

Zydatiß, W. (2012). Linguistic thresholds in the CLIL classroom? The Threshold Hypothesis revisited. International CLIL Research Journal, 1(4), 17-28. Cyrchwyd 1 Rhagfyr, 2015: www.icrj.eu/14/contents.html 